Universidade de São Paulo

Instituto de Física

\title{
Estudo da Influência de Modos Vibracionais \\ Localizados nas Propriedades de Transporte de Cargas em Sistemas de Escala Nanométrica
}

Pedro Brandimarte Mendonça

Orientador: Prof. Dr. Antônio José Roque da Silva (IF-USP/LNLS)

Coorientador: Prof. Dr. Alexandre Reily Rocha (IFT-UNESP)

Tese de doutorado apresentada ao Instituto

de Física como parte dos requisitos para

a obtenção do título de Doutor em Física

Banca Examinadora:

Prof. Dr. Antônio José Roque da Silva (IF-USP/LNLS)

Prof. Dr. Luis Gregório Dias da Silva (IF-USP)

Profa. Dra. Marilia Junqueira Caldas (IF-USP)

Prof. Dr. Rodrigo André Caetano (UFPR)

Prof. Dr. Roberto Hiroki Miwa (UFU) 
FICHA CATALOGRÁFICA

Preparada pelo Serviço de Biblioteca e Informação do Instituto de Física da Universidade de São Paulo

Mendonça, Pedro Brandimarte

Estudo da Influência de Modos Vibracionais Localizados nas Propriedades de Transporte de Cargas em Sistemas de Escala Nanométrica. São Paulo, 2014.

Tese (Doutorado) - Universidade de São Paulo. Instituto de Física. Departamento de Física dos Materiais e Mecânica.

Orientador: Prof. Dr. Antônio José Roque da Silva Coorientador: Prof. Dr. Alexandre Reily Rocha

Área de Concentração: Física

Unitermos: 1. Física da matéria condensada; 2. Fisica computacional; 3. Problemas de muitos corpos; 4. Interação elétron-fônon; 5. Nanotecnologia. 

"If you want to find the secrets of the universe, think in terms of energy, frequency and vibration"

Nikola Tesla 



\section{Agradecimentos}

Ao professor Alexandre Reily Rocha, que, com sua generosidade, paciência e dedicação, me incentivou ao longo de quatro anos a persistir na realização deste projeto. O desenvolvimento de um trabalho científico é, na maior parte do tempo, um caminho solitário e tortuoso. Nos momentos em que a pesquisa pareceu encontrar obstáculos intransponíveis, foram essenciais, para que eu avançasse nessa tese, suas palavras enconrajadoras e seu apoio, mesmo nos fins de semana, nos quais continuávamos em videoconferência discutindo problemas e resultados encontrados.

Ao grupo SAMPA e ao professor Antônio José Roque, pelo convívio e troca de experiências. Em especial aos colegas "da sala de baixo" Luana, Rodrigo e Alberto, pela amizade e por todo o auxílio e incentivo durante esse período.

Ao grupo TransSim, em especial ao meu mais recente colega e amigo, Cesar, pelo apoio e colaboração.

A minha companheira, Debora, por seu carinho e infinita paciência, e a meu pai. Por fim e acima de tudo, a minha mãe Vera, que sempre esteve ao meu lado e me apoiou em todos os meus projetos.

Também agradeço o CNPq pelo financiamento do projeto e o LCCA-USP, CENAPAD e Grid UNESP pelo suporte computacional. 


\section{Resumo}

Com o rápido avanço das técnicas experimentais observado nas últimas décadas, a fabricação de sistemas nanoestruturados se tornou uma realidade. Nessa escala de grandeza, as interações entre elétrons e vibrações nucleares têm um papel importante no transporte eletrônico, podendo causar a perda de coerência de fase dos elétrons, a abertura de novos canais de condução e a supressão de canais puramente elásticos. Neste trabalho, o problema do transporte eletrônico em escala nanométrica foi tratado considerando as interações elétron-fônon, o que resultou na implementação de ferramentas computacionais para simulação realística de materiais.

O transporte eletrônico foi abordado por meio do formalismo das Funções de Green Fora do Equilíbrio, onde as interações elétron-fônon foram tratadas por diferentes modelos. Para considerar o efeito dessas interações no transporte, é necessário, em princípio, incluir um termo de autoenergia de espalhamento na Hamiltoniana do sistema. Contudo, a forma exata dessa autoenergia é desconhecida e aproximações são necessárias.

O primeiro efeito da interação elétron-fônon estudado foi a perda de coerência de fase, o que foi abordado pelo modelo fenomenológico das sondas de Büttiker [1]. Foram realizadas duas implementações diferentes deste modelo, a primeira na forma usual, onde se considera uma aproximação elástica para o cálculo da corrente, e a segunda por meio de uma nova proposta sem a aproximação elástica. Entretanto, como a autoenergia de interação utilizada não contém informação a respeito da estrutura dos fônons, o modelo produz somente um alargamento do canal de condutância, simulando apenas o efeito de perda de coerência de fase dos elétrons devido à interação com fônons do material.

Para poder incluir as informações sobre a estrutura dos fônons, foi desenvolvido o programa PhOnonS ITeratIVE VIBRATIONS, para o cálculo das frequências e dos modos vibracionais de materiais e para calcular a matriz de acoplamento elétron-fônon, a partir de métodos de primeiros princípios. No cálculo da matriz de acoplamento elétron-fônon, além da implementação do código algumas intervenções foram realizadas no programa SIESTA [2,3] (uma implementação da Teoria do Funcional da Densidade).

Outra abordagem para a interação elétron-fônon consiste em expandir a autoenergia de inte- 
ração perturbativamente em diagramas de Feynman até a primeira ordem, o que é convencionalmente chamado de primeira aproximação de Born. Essa aproximação, assim como a sua versão autoconsistente, no qual uma classe mais ampla de diagramas é considerada, foram incorporadas ao programa SMEAGOL [4], um código de transporte eletrônico ab initio baseado na combinação DFT-NEGF e que utiliza como plataforma do cálculo da estrutura eletrônica o código SIESTA. Essas implementações, em conjunto com diversas mudanças realizadas no código SMEAGOL, deram origem ao programa Inelastic SMEAGOL para cálculos de transporte inelástico ab initio.

Nessa busca por uma descrição mais realista dos dispositivos eletrônicos, outro aspecto que deve ser considerado é o fato de que os dispositivos muitas vezes podem alcançar escalas de comprimento da ordem de $100 \mathrm{~nm}$ com um grande número de defeitos aleatoriamente distribuídos, o que pode levar a um novo regime fundamental de transporte, a saber, o de localização de Anderson [5]. Neste trabalho, foi desenvolvido o programa Inelastic DISORDER, que permite calcular, por primeiros princípios, as propriedades de transporte elástico e inelástico de sistemas com dezenas de milhares de átomos com um grande número de defeitos posicionados aleatoriamente. O método combina cálculos de estrutura eletrônica via DFT com o formalismo NEGF para o transporte, onde as interações elétron-fônon são incluídas por meio de teoria de perturbação com relação à matriz de acoplamento elétron-fônon (Lowest Order Expansion).

O método desenvolvido foi aplicado ao estudo de nanofitas de grafeno com impurezas hidroxílicas. Observou-se que, ao incluir a interação elétron-fônon, as propriedades de transporte sofrem mudanças significativas, indicando que estas interações podem influenciar nos efeitos de localização por desordem. 


\section{Abstract}

With the fast improvement of experimental techniques over the past decades, the synthesis of nanoscale systems has become a reality. At this length scales, the interaction between electrons and ionic vibrations plays an important role in electronic transport, and may cause the loss of the electron's phase coherence, the opening of new conductance channels and the suppression of purely elastic ones. In this work the electronic transport problem at nanoscale was addressed considering the electron-phonon interactions, resulting on the development of computational tools for realistic simulations of materials.

The electronic transport was approached with the Non-Equilibrium Green's Function formalism, where electron-phonon interactions were addressed by different models. To take into account the interaction's effects, one needs in principle to include a self-energy scattering term in the system Hamiltonian. Nevertheless, the exact form of this self-energy is unknown and approximations are required.

The first effect from electron-phonon interactions dealt was the loss of phase coherence, which was approached by the Büttiker's probes phenomenological model [1]. Two different implementations of this model were performed, the first in the standard form, where an elastic approximation is considered in order to compute the current, and the second by a new method without the elastic approximation. However, since the interaction self-energy used doesn't contains any information about the phonon's structure, this model only produces a broadening at the conducting channels, simulating just the effect of loss of phase coherence from the electrons due to their interactions with the phonons.

In order to be able to include information about the phonon's structure, the computational code PhOnonS ITeratIVE VIBRATIONS was developed, for calculating the frequencies and vibrational modes of the materials and to compute the electron-phonon coupling matrix, from first principles methods. In the calculation of the electron-phonon coupling matrix, besides the code implementation some changes were performed at the SIESTA program [2,3] (a Density Functional Theory implementation).

Another approach for the electron-phonon interactions consists of expanding the interaction 
self-energy perturbatively in Feynman diagrams until the first order, what is conventionally called the first Born approximation. This approximation, together with its self-consistent version, where a wider class of diagrams are regarded, have been incorporated into the SMEAGOL program [4], an ab initio electronic transport code based on the combination DFT-NEGF which uses the SIESTA code as a platform for electronic structure calculations. The implementations, together with many changes performed on SMEAGOL code, gave rise to the Inelastic SMEAGOL program for inelastic $a b$ initio transport calculations.

In this search for a more realistic description of electronic devices, another feature that should be taken into account is the fact that these devices most often can reach the $100 \mathrm{~nm}$ length scale with a large number of randomly distributed defects, which can lead to a fundamentally new transport regime, namely the Anderson localization regime [5]. In this work, the program Inelastic DISORDER was developed, which allows one to compute, by first principles, the elastic and inelastic transport properties from systems with tens of thousands of atoms with a large number of randomly positioned defects. The method combines electronic structure calculations via DFT with the NEGF formalism for transport, where the electron-phonon interactions are included with perturbation theory on the electron-phonon coupling matrix (Lowest Order Expansion).

The developed method was applied to the study of graphene nanoribbons with joint attachment of hydroxyl impurities. It was observed that, by including the electron-phonon interaction, the transport properties experience significant changes, indicating that these interactions can influence the effects of localization by disorder. 


\section{Índice}

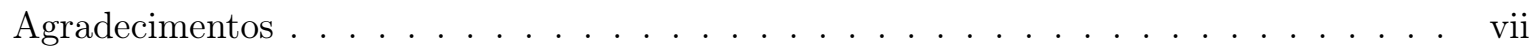

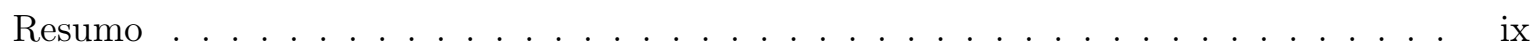

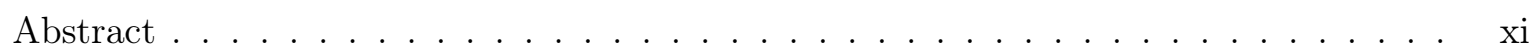

Índice . . . . . . . . . . . . . . . . . . . . . . . . . . . . . . . . . . . .

Lista de Figuras . . . . . . . . . . . . . . . . . . . . . . . . xxvii

Lista de Tabelas . . . . . . . . . . . . . . . . . . . . . . . xxix

\begin{tabular}{lll}
\hline & Introdução & 1
\end{tabular}

$1.1 \quad$ Objetivos e Organização da Tese $\ldots \ldots \ldots \ldots \ldots \ldots$. . . . . . . . . . . . 6

2 Formalismo das Funções de Green Fora do Equilíbrio 9

2.1 Segunda Quantização . . . . . . . . . . . . . . . . . . . . . . . . . . . . . 10

2.1 .1 Fônons $\ldots \ldots \ldots \ldots \ldots \ldots$

2.1 .2 Elétrons . . . . . . . . . . . . . . . . . . . . . . . . . . . . . . . . . 17

2.1 .3 Interação Elétron-Fônon $\ldots \ldots \ldots$. . . . . . . . . . . . . . 19

2.2 Funções de Green no Equilíbrio . . . . . . . . . . . . . . . . . . . . . . . . . . . . 21

2.3 Função Espectral e o Teorema de Flutuação-Dissipação . . . . . . . . . . . . . . . 26

2.4 Representação de Lehmann . . . . . . . . . . . . . . . . . . . . . . . . . 27

2.5 Valores Esperados de Observáveis Fora do Equilíbrio . . . . . . . . . . . . . . . . 29

2.6 Funções de Green Fora do Equilíbrio $\ldots \ldots \ldots$. . . . . . . . . . . . . . . . . . . . 35

2.7 Teorema de Wick e Diagramas de Feynman . . . . . . . . . . . . . . . . . 36

2.8 Continuação Analítica - Teorema de Langreth $\ldots \ldots \ldots$. . . . . . . . . . . . . . 38

2.9 Equação Keldysh $\ldots \ldots \ldots \ldots$. . . . . . . . . . . . . . . . . . . . 42

3 Estrutura Eletrônica e Cálculo Vibracional

3.1 Aproximação de Born-Oppenheimer $\ldots \ldots \ldots$. . . . . . . . . . . . 46

3.2 Estrutura Eletrônica $\ldots \ldots \ldots \ldots$

3.2 .1 Teoria do Funcional da Densidade . . . . . . . . . . . . . . . . . . . . . . 49 
$3.2 .2 \quad$ Equações de Kohn-Sham $\ldots \ldots \ldots \ldots$. . . . . . . . . . . . . . . . 51

$3.2 .3 \quad$ Funcionais de Troca e Correlação $\ldots \ldots \ldots$. . . . . . . . . . . . 53

$3.2 .4 \quad$ O Programa SIESTA $\ldots \ldots \ldots \ldots \ldots \ldots$

3.3 Análise Vibracional e Acoplamento Elétron-Fônon ～. . . . . . . . . . . . . . . . 58

$3.3 .1 \quad$ O Programa PhOnonS ITeratIVE VIBRATIONS $\ldots \ldots \ldots$. . . . . . . . 60

\begin{tabular}{|lll|}
\hline & Transporte Eletrônico em Escala Nanométrica & 63
\end{tabular}

4.1 Introdução $\ldots \ldots \ldots \ldots \ldots$. . . . . . . . . . . . . . . . . . . . 64

4.2 Modelo de Landauer . . . . . . . . . . . . . . . . . . . . . . . . . . . . . . . . . . 66

4.3 Hamiltoniana do Modelo . . . . . . . . . . . . . . . . . . . . . . . . . . . . . . . . . . 69

4.4 Expressão Geral para a Corrente . . . . . . . . . . . . . . . . . . . . . 70

4.5 Formalismo NEGF na Prática $\ldots \ldots \ldots \ldots \ldots \ldots \ldots$

$4.6 \quad$ O Programa SMEAGOL: NEGF + DFT . . . . . . . . . . . . . . . . . . 80

5 Perda de Coerência de Fase $\quad 85$

5.1 Formalismo de Landauer-Büttiker . . . . . . . . . . . . . . . . . . . . . . . . 86

5.2 Modelo Fenomenológico das Sondas de Büttiker . . . . . . . . . . . . . . . . . 87

5.3 Transporte Elástico Não-Coerente $\ldots \ldots \ldots$. . . . . . . . . . . . . . . . . . . . . . . . . 90

5.4 Transporte Inelástico Não-Coerente $\ldots \ldots \ldots$. . . . . . . . . . . . . . . . . . . . 90

5.5 Implementação $\ldots \ldots \ldots \ldots$. . . . . . . . . . . . . . . . . . . 92

5.5 .1 Sistema Modelo . . . . . . . . . . . . . . . . . . . . . . . . . . . 92

$5.5 .2 \quad$ Perfil do Potencial e Solução Autoconsistente . . . . . . . . . . . . . . . . 94

5.5 .3 Caso Particular . . . . . . . . . . . . . . . . . . . . . . . . . . . . . . . 94

5.6 Resultados . . . . . . . . . . . . . . . . . . . . . . . . . 96

$\begin{array}{lll}6 & \text { Aproximação de Born } & 105\end{array}$

6.1 Primeira Aproximação de Born - 1BA . . . . . . . . . . . . . . . 106

6.2 Aproximação de Born Autoconsistente - SCBA ． . . . . . . . . . . . . . . 110

6.3 Reformulação da Primeira Aproximação de Born . . . . . . . . . . . . . . . . . 110

6.4 Expressão para a Corrente e Conservação . . . . . . . . . . . . . . . . . . . . . . 114

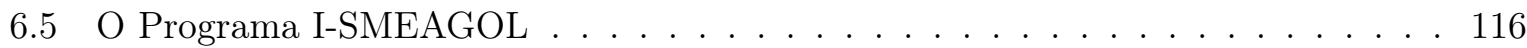

$6.5 .1 \quad$ Observações sobre Eficiência Computacional . . . . . . . . . . . . . . . . . 118

6.6 Resultados . . . . . . . . . . . . . . . . . . . . . . . . . . . . . . . . . . . 121

7 Transporte Inelástico em Sistemas Desordenados 129

7.1 Desordem e Localização . . . . . . . . . . . . . . . . . . . . . . . . . . . 130 
7.2 Transporte Elástico $\ldots \ldots \ldots$. . . . . . . . . . . . . . . . . . . . . . . . . 133

7.3 Cálculo Recursivo de Funções de Green . . . . . . . . . . . . . . . . . . . 136

7.4 Transporte Inelástico: Lowest Order Expansion - LOE . . . . . . . . . . . . . . . 141

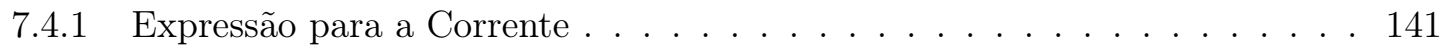

7.4 .2 Potência Dissipada . . . . . . . . . . . . . . . . . . . . . . . . . . . . . 144

7.4 .3 Ocupação dos Fônons $\ldots \ldots \ldots \ldots$. . . . . . . . . . . . 146

7.4 .4 Sistemas Desordenados . . . . . . . . . . . . . . . . . . . . . 147

$7.5 \quad$ O Programa I-DISORDER $\ldots \ldots \ldots \ldots \ldots \ldots \ldots$

7.5.1 Observações sobre Eficiência Computacional . . . . . . . . . . . . . . . . . 149

7.6 Resultados . . . . . . . . . . . . . . . . . . . . . . . . . . . . . . . . 152

8 Nanofitas de Grafeno com Impurezas Hidroxílicas $\quad 161$

8.1 Grafeno . . . . . . . . . . . . . . . . . . . . . . . . . 161

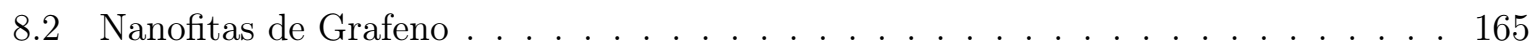

8.3 Cálculos de Estrutura Eletrônica ． . . . . . . . . . . . . . . . . . . . . . . 167

8.4 Propriedades de Transporte $\ldots \ldots \ldots \ldots$. . . . . . . . . . . . 170

8.5 Análise Vibracional . . . . . . . . . . . . . . . . . . . . . . . . . . . . . . . . . . . . . 173

8.6 Transporte Inelástico com LOE . . . . . . . . . . . . . . . . . . . . . . 174

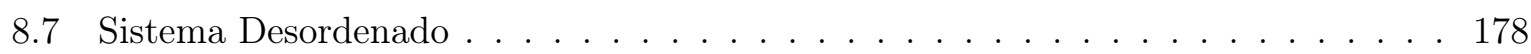

9 Conclusões Gerais 185

9.1 Perspectivas . . . . . . . . . . . . . . . . . . . . . . . . . . . . . . 186

\begin{tabular}{|l|l|}
\hline A Prova dos Teoremas de Hohenberg-Kohn & 189
\end{tabular}

B Cálculo de Integrais no Plano Complexo 193

B.1 Cálculo da Matriz Densidade Eletrônica . . . . . . . . . . . . . . . . . . . . . 195

B.2 Transformada de Hilbert . . . . . . . . . . . . . . . . . . . . . . . . . . . . . . . . 199

C Derivação da Expressão para a Corrente na LOE 203

\begin{tabular}{ll}
\hline D POSITIVE VIBRATIONS User's Guide & 217
\end{tabular}

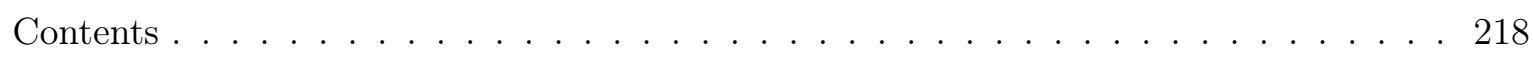

D.1 Introduction . . . . . . . . . . . . . . . . . . . . . . . 218

D.2 Compilation . . . . . . . . . . . . . . . . . . . . . . . . . . . . . . . . . 218

D.3 $\quad$ Execution Steps and Options … . . . . . . . . . . . . . . . . . . . . . . 219

D.3.1 Vibrational Modes and Frequencies . . . . . . . . . . . . . . . . . . . 219 
D.3.2 Electron-Phonon Coupling Matrices _ . . . . . . . . . . . . . . . . . . 220

D.4 Problem Handling . . . . . . . . . . . . . . . . . . . . . . . . . . . . . . . . . . 221

D.5 Acknowledgements . . . . . . . . . . . . . . . . . . . . . . 222

References. . . . . . . . . . . . . . . . . . . . . . . 222

E I-SMEAGOL User's Guide 223

Contents . . . . . . . . . . . . . . . . . . . . 225

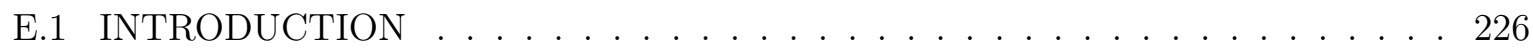

E.2 THE SYSTEM SET UP . . . . . . . . . . . . . . . . . . . . . . . . . . . . 226

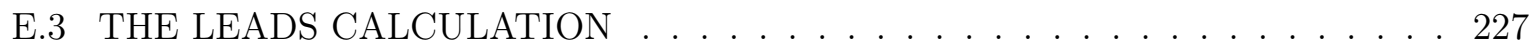

E.3.1 Input files . . . . . . . . . . . . . . . . . . . . . . . . . . . 228

E.3.2 Input flags . . . . . . . . . . . . . . . . . . . . . . . . . . . . 228

E.3.3 Output files . . . . . . . . . . . . . . . . . . . . . . . . . . . 228

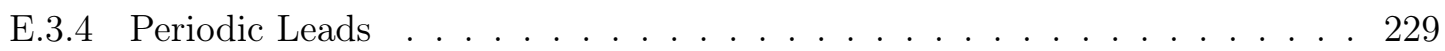

E.3.4.1 Input flags . . . . . . . . . . . . . . . . . . . . . . 229

E.3.4.2 Output files . . . . . . . . . . . . . . . . . . 230

E.3.4.3 Example . . . . . . . . . . . . . . . . . . 230

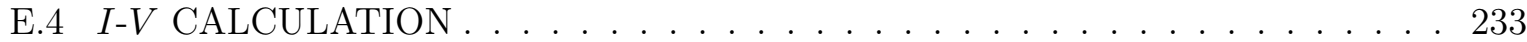

E.4.1 Input files . . . . . . . . . . . . . . . . . . . . . . . . 233

E.4.2 Input flags . . . . . . . . . . . . . . . . . . . . . . . . 234

E.4.2.1 Matching of the Hartree Potential . . . . . . . . . . . . . . . 238

E.4.2.2 Flags specific of $k$-point calculations . . . . . . . . . . . . 240

E.4.2.3 Flags specific of $\Gamma$ point calculations . . . . . . . . . . . . 241

E.4.2.4 Flags specific of calculations involving periodic leads . . . . . . . 241

E.4.2.5 Example for periodic lead calculation . . . . . . . . . . . . . 242

E.4.3 Output files . . . . . . . . . . . . . . . . . . . . . . . . . . 242

E.5 INELASTIC $I$ - $V$ CALCULATION $\ldots \ldots \ldots \ldots \ldots \ldots$

E.5.1 Input files . . . . . . . . . . . . . . . . . . . . . . . . . . 244

E.5.2 Input flags . . . . . . . . . . . . . . . . . . . . . . . . 245

E.5.3 Output files . . . . . . . . . . . . . . . . . . . . . . . . . 247

E.6 $\quad$ MOLECULAR DYNAMICS WITH TRANSPORT . . . . . . . . . . . . . . . . . 249

E.6.1 Input files . . . . . . . . . . . . . . . . . . . . . . . . . . . . . . 249

E.6.2 $\quad$ Input flags . . . . . . . . . . . . . . . . . . . . . . . . . . . . . . . . . . . . 249

E.6.3 Output files . . . . . . . . . . . . . . . . . . . . 250

E.7 HOW TO RUN SMEAGOL $\ldots \ldots \ldots \ldots \ldots \ldots$ 
E.8 $\quad$ PROBLEM HANDLING $\ldots \ldots \ldots \ldots$

E.9 ACKNOWLEDGEMENTS . . . . . . . . . . . . . . . . . . . . . . 252

References . . . . . . . . . . . . . . . . . . . . . . . . . 252

\begin{tabular}{lll}
\hline F I-DISORDER User's Guide & 255
\end{tabular}

Contents . . . . . . . . . . . . . . . . . . . . . 256

F.1 Introduction . . . . . . . . . . . . . . . . . . . . . . . . . . 256

F.2 Compilation . . . . . . . . . . . . . . . . . . . . . . 256

F.3 The System Set-Up . . . . . . . . . . . . . . . . . . . . . . . . . . 257

F.4 Input Files . . . . . . . . . . . . . . . . . . . . . . . . . . . . . 258

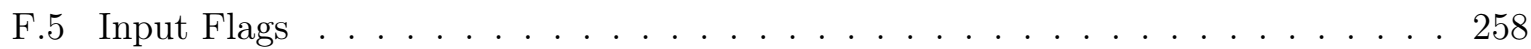

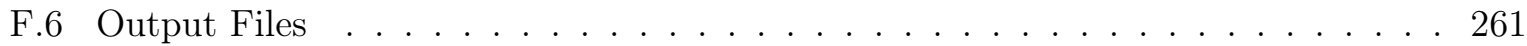

F.7 $\quad$ Running I-DISORDER $\ldots \ldots \ldots \ldots \ldots$

F.8 Problem Handling . . . . . . . . . . . . . . . . . . . . . . . . . . . . . . . . 263

F.9 Acknowledgements . . . . . . . . . . . . . . . . . . . . . . 263

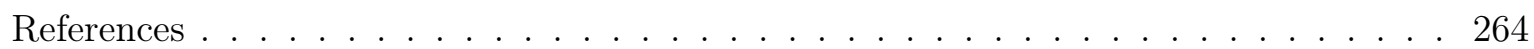

\begin{tabular}{ll}
\hline Referências Bibliográficas & 273
\end{tabular} 


\section{Lista de Figuras}

1.1 Condutância diferencial (acima à direita) e respectiva derivada (abaixo à direita) medidas de um contato $\mathrm{Pt} / \mathrm{H}_{2}$ (ilustração à esquerda). (Gráficos extraídos de $|6|$, com permissão de Macmillan Publishers Ltd: Nature 419, 906-909, 31 outubro 2002 (doi: 10.1038/nature01103), copyright (2002). $\ldots \ldots \ldots \ldots \ldots \ldots$

1.2 Valores de condutância em cadeias monoatômicas de ouro (abaixo à esquerda), medidas em função do distanciamento entre o substrato e a ponta do STM que foi afastada até a ruptura da cadeia (ilustração acima). Para cadeias com sete átomos, a condutância apresenta uma queda brusca em $\pm 15 \mathrm{mV}$ de $\sim 1 \%$ (à direita). (Gráficos extraídos de 77 com permissão de N. Agraït, C. Untiedt, G. Rubio-Bollinger e S. Vieira. Phys.

Rev. Lett. 88(21), 216803 (2002). Copyright (2002) by the American Physical Society. - http://dx.doi.org/10.1103/PhysRevLett.88.216803] . . . . . . . . . 3

1.3 Curvas do espectro $d^{2} I / d V^{2}$ obtidas com IETS para o $C_{2} H_{2}$ e o $C_{2} D_{2}$ adsorvidos a uma superfície de cobre (à esquerda). À direita, as imagens de espectroscopia espacial obtidas durante a varredura da ponta para gravar a topologia da superfície, onde o $\mathrm{C}_{2} \mathrm{H}_{2}$ está à esquerda e o $C_{2} D_{2}$, ̀̀ direita: Em (A), a imagem padrão de STM obtida no modo corrente

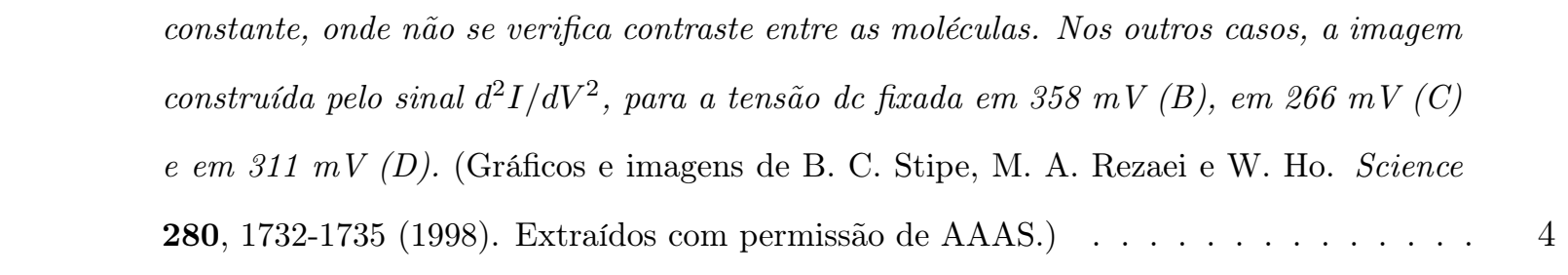

2.1 Cadeia harmônica unidimensional. . . . . . . . . . . . . . . . . . . . . . 11

2.2 Vértice básico da interação elétron-fônon: à esquerda, com a emissão de um fônon, e, $\grave{a}$ direita, com a absorção de um fônon. . . . . . . . . . . . . . . . . . 20

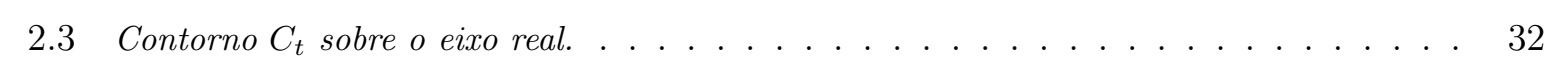

2.4 Contorno $\tilde{C}$ composto pelos contornos $C$ sobre o eixo real e $C_{\nu}$ perpendicular ao eixo real. 34

$\left.\begin{array}{|lll}\hline 2.5 & \text { Diagramas gerados no caso de Hamiltonianos de interação quárticos. (Adaptado de } & 8[.]\end{array}\right]$

2.6 Deformação do contorno C . . . . . . . . . . . . . . . . . . . . . . . . . . 39 
4.1 Desenho esquemático de um transistor, com um canal $\mathbf{C}$ de comprimento L conectado aos contatos fonte $(\mathbf{L})$ e dreno $(\mathbf{R})$ onde é aplicada uma diferença de potencial $V$ de forma a produzir uma corrente I. A quantidade de corrente que passa no dispositivo pode ser controlada por meio de uma tensão de porta $V_{G}$. . . . . . . . . . . . . . . . . . . . . 64

$4.2 \quad$ Esboço da curva $I \times V$ para três diferentes tensões de porta $V_{G} . \ldots \ldots \ldots$. . . . . . . 65

4.3 Esquema da situação fora do equilíbrio devido à tensão $V$ mantida por uma bateria. Abaixo observa-se as distribuições de Fermi-Dirac dos eletrodos e a densidade de estados do canal ao centro, ilustrando que são os estados com energias entre $\mu_{R}$ e $\mu_{L}$ os responsáveis pelo transporte. . . . . . . . . . . . . . . . . . . . . . . . . . . . . . . . 67

4.4 Esboço de um problema típico de transporte, com uma região central de espalhamento conectada a dois reservatórios de elétrons (eletrodos esquerdo e direito) em equilíbrio termodinâmico. . . . . . . . . . . . . . . . . . . .

4.5 Região de espalhamento com algumas células unitárias dos eletrodos, de forma que a queda na diferença de potencial ocorre somente nesta região. O efeito do acoplamento com os eletrodos é dado pelas autoenergias $\Sigma_{L / R} . \ldots \ldots \ldots \ldots \ldots$. . . . . . . . 77

4.6 Fluxograma do programa SMEAGOL, destacando a interface com o programa SIESTA. (Extraído de $[9]$, com permissão de A. R. Rocha.) .

5.1 Representação da interação elétron-fônon na região de espalhamento. a) Elétron se propaga coerentemente a partir do eletrodo esquerdo. b) Elétron muda para um nível molecular de mais baixa energia emitindo um fônon. c) Elétron se propaga coerentemente até o eletrodo direito. . . . . . . . . . . . . . . . . . . . . . . . . . . . . . . . . 88

$5.2 \quad$ Efeito obtido ao incluir uma sonda fictícia na região de espalhamento . . . . . . . . . . . 88

$5.3 \quad$ Ilustração das sondas fictícias acopladas à região de espalhamento. $\quad \ldots \ldots$. . . . . . . . 89

5.4 Cadeia atômica unidimensional. $\ldots \ldots \ldots \ldots \ldots \ldots$

5.5 Transmissão (esquerda) e densidade de estados (direita) para um sistema no equilíbrio. . 97

5.6 Corrente por tensão aplicada calculada por meio do formalismo NEGF (esquerda) e por expressões analíticas (direita). . . . . . . . . . . . . . . . . . . . . . . . . . . . . 97

5.7 Corrente por tensão aplicada (acima), condutância diferencial (meio), potenciais eletroquímicos dos eletrodos e da sonda e energia de sítio da molécula (abaixo), para um sistema com um sítio na região de espalhamento e onde foram utilizados os parâmetros $T=10 K, \beta=0,05 \mathrm{eV}, U_{0}=0,0$ (esquerda), $U_{0}=1,0$ (direita) e as energias e acoplamentos da Tabela 5.1] . . . . . . . . . . . . . . . . . . . . . . . 
5.8 Representação do sistema na situação em que $\varepsilon_{M}-\mu_{L}<0,05 e V=\gamma_{M L} / 2$, onde se \begin{tabular}{|c|}
\hline observa o acoplamento fraco entre os eletrodos e a região de espalhamento (largura estreita \\
\hline do nível na região de espalhamento) e que a diferença de potencial desloca a banda dos \\
\hline
\end{tabular}

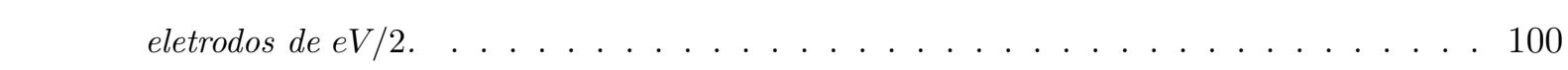

5.9 Corrente por tensão aplicada (acima), condutância diferencial (meio), potenciais ele-

\begin{tabular}{|c|}
\hline troquímicos dos eletrodos e da sonda e energia de sítio da molécula (abaixo), para um \\
\hline
\end{tabular}

sistema com um sítio na região de espalhamento e onde foram utilizados os parâmetros

$T=300 \mathrm{~K}, \beta=0,05 \mathrm{eV}, U_{0}=0,0$ (esquerda), $U_{0}=1,0$ (direita) e as energias e acopla-

mentos da Tabela 5.1 . . . . . . . . . . . . . . . . . . . . . . . . . . 101

5.10 Corrente por tensão aplicada (acima), condutância diferencial (meio) e potenciais ele-

\begin{tabular}{l}
\hline troquímicos dos eletrodos e sondas (abaixo), para um sistema com três sítios na região de \\
\begin{tabular}{|l}
\hline espalhamento e onde foram utilizados os parâmetros $T=10 K, U_{0}=1,0, \beta=0,05 e V$ e \\
\hline as energias e acoplamentos da Tabela 5 . 2 . . . . . . . . . . . . . . . . . . . . . . . . . . 102
\end{tabular}
\end{tabular}

5.11 Corrente por tensão aplicada (acima), condutância diferencial (meio), potenciais eletroquímicos dos eletrodos e da sonda e energia de sítio da molécula (abaixo), para um sistema com um sítio na região de espalhamento e onde foram utilizados os parâmetros

\begin{tabular}{l}
\hline$T=10 K, \beta=0,11 \mathrm{eV}, U_{0}=0,0$ (esquerda), $U_{0}=1,0$ (direita) e as energias e acopla- \\
\hline mentos da Tabela 5.1. . . . . . . . . . . . . . . . . . . . . . . . . . . 103
\end{tabular}

6.1 Expansão perturbativa da função de Green eletrônica em um sistema com interação elétron-

\begin{tabular}{|c|}
\hline fônon, onde as funções de Green são representadas por linha dupla para elétron intera- \\
\hline gente, linha simples para elétron livre e linha ondulada para fônon livre. . . . . . . . . . . . . . 106
\end{tabular}

6.2 Representação da Primeira Aproximação de Born com diagramas de Feynman, onde se identificam os diagramas tipo-Hartree à esquerda e tipo-Fock à direita. . . . . . . . . . . . 107

6.3 Representação da Aproximação de Born Autoconsistente com diagramas de Feynman. 110

6.4 Fluxograma do programa I-SMEAGOL. . . . . . . . . . . . . . . . . . . . . . . . . . . 117

6.5 Ilustração destacando os blocos da função de Green efetivamente utilizados nos cálculos com a aproximação de Born, onde $N L$ (NR) é a dimensão do eletrodo esquerdo (direito)

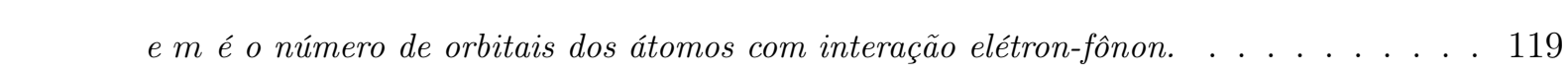

$6.6 \quad$ Paralelização do grid de energia em p processadores. . . . . . . . . . . . . . . . . . . . . 120

6.7 Representação da paralelização de uma matriz tridimensional entre quatro processadores $\left(P_{1}, P_{2}, P_{3}\right.$ e $\left.P_{4}\right)$, onde $N_{\epsilon}$ é o número de pontos de energia e $N_{\text {orb }}$ é o número de orbitais atômicos. À esquerda, a distribuição é realizada nos pontos de energia e, à direita, em termos dos orbitais atômicos. . . . . . . . . . . . . . . . . . . . . . . . . . . . . . . . 121 
6.8 Comparação da função espectral obtida a $k_{B} T=0,001 \mathrm{eV}$ e $V=0$ com a $1 B A$ (linha preta sólida), SCBA (linha azul) e sem interação elétron-fônon (linha hachurada). . . . . 122

6.9 Comparação da função espectral obtida a $k_{B} T=0,04 \mathrm{eV}$ com a 1BA (linha vermelha hachurada), SCBA (linha azul sólida) e sem interação elétron-fônon (linha preta pontilhada), para diversos valores de diferença de potencial aplicados. . . . . . . . . . . . . 123

6.10 Comparação das características $I$-V obtidas a $k_{B} T=0,04 \mathrm{eV}$ com as diferentes implementações para a interação elétron-fônon. . . . . . . . . . . . . . . . . . . . . . . . . 123

6.11 Comparação das características $I-V$ obtidas considerando-se eletrodos com largura de banda finita. . . . . . . . . . . . . . . . . . . . . . . . . . . . . . . . . . . . . . 124

6.12 Representação de um fio de ouro com o átomo central fracamente acoplado. . . . . . . . 125

6.13 Funções espectrais a $V=0$ de um fio de ouro com um sítio fracamente acoplado calculadas incluindo somente a autoenergia de Fock (esquerda) e incluindo ambas as autoenergias de Hartree e de Fock (direita), obtidas com a 1BA (linha vermelha), SCBA (linha azul tracejada) e sem interação elétron-fônon (linha preta tracejada). . . . . . . . . . . . . . 125

6.14 Curva I-V (acima) de um fio de ouro com um sítio fracamente acoplado, obtidas com a 1BA (linha vermelha), SCBA (linha azul) e sem interação elétron-fônon (linha preta).

Condutância diferencial (centro) e espectro $d^{2} I / d V^{2}$ (abaixo) obtidas com as correntes normalizadas pela corrente elástica e considerando somente os termos inelásticos na $1 B A$

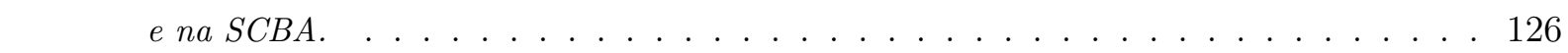

7.1 Representações da função de onda eletrônica espalhada por defeitos no material, onde $\grave{a}$ esquerda um estado estendido com livre caminho médio $\ell$ e à direita um estado localizado com comprimento de localização $\xi$. (Adaptado de $[10[$.$) . . . . . . . . . . . . . . . . . . . . . 131$

7.2 Região de espalhamento formada por uma nanofita de grafeno, onde se destacam segmentos pristinos $\left(H_{P}\right)$ e com defeito $\left(H_{D}^{1}\right.$ e $\left.H_{D}^{2}\right)$. Os eletrodos esquerdo e direito se acoplam ao primeiro e último segmentos, respectivamente. . . . . . . . . . . . . . . . . . . . . . 134

7.3 Primeira e segunda derivadas das funções $\mathcal{I}^{\text {Sym }}$ e $\mathcal{I}^{\text {Asym }}$ com relação à tensão $V$, para três temperaturas distintas, obtidas com o programa I-DISORDER (Seção 7.5 ). . . . . 144

7.4 Ilustração da matriz $M_{\lambda}^{(i)}$ destacando o bloco de elementos não-nulos correspondente ao subconjunto dos $m$ orbitais deslocados no cálculo de constantes de força. . . . . . . . . . 151

7.5 Representação dos dois tipos de defeitos permitidos pelo I-DISORDER para o cálculo de uma cadeia unidimensional. Acima, um defeito simples em um dos sítios da cadeia e, abaixo, o defeito simples com uma impureza "dangling bond". . . . . . . . . . . . . . . 152

7.6 Corrente total (esquerda) e condutância diferencial total (direita), em função da tensão e da energia, obtidas para uma cadeia contendo um único defeito simples. . . . . . . . . . 153 
7.7 Corrente por tensão aplicada (acima), condutância diferencial (meio) e derivada da condutância (abaixo), para a cadeia com somente um defeito simples. . . . . . . . . . . . . 154

7.8 Corrente e condutância diferencial, em função da tensão e da energia, obtidas para uma

cadeia contendo um único defeito com impureza "dangling bond". Acima: corrente to-

\begin{tabular}{|c|c|}
\hline tal (esquerda) e condutância diferencial total (direita). Abaixo: condutância diferencial \\
\hline
\end{tabular}

elástica (esquerda) e inelástica (direita). . . . . . . . . . . . . . . 155

7.9 Corrente por tensão aplicada (acima), condutância diferencial (meio) e derivada da condutância (abaixo), para a cadeia com somente um defeito com uma impureza "dangling

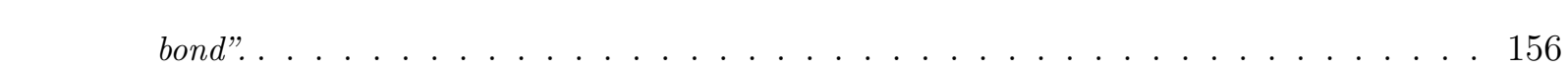

7.10 Valor médio da condutância diferencial versus o comprimento da cadeia, mantendo-se $10 \%$ dos sítios com o defeito simples. Acima, os valores obtidos para o transporte elástico e, abaixo, considerando a interação elétron-fônon. À direita, os gráficos são apresentados em escala logarítmica, para uma melhor visualização do ajuste exponencial. . . . . . . . 157

7.11 Valor médio da condutância diferencial versus o comprimento da cadeia, mantendo-se $10 \%$ dos sítios com a "dangling bond". Acima, os valores obtidos para o transporte elástico e, abaixo, considerando a interação elétron-fônon. À direita, os gráficos são apresentados em escala logarítmica, para uma melhor visualização do ajuste exponencial. . . . . . . . 158

7.12 Distribuição do logaritmo da condutância adimensional obtida com 1000 realizaçôes de uma cadeia com $L=300$ contendo $10 \%$ de defeitos simples. À esquerda, a distribuição

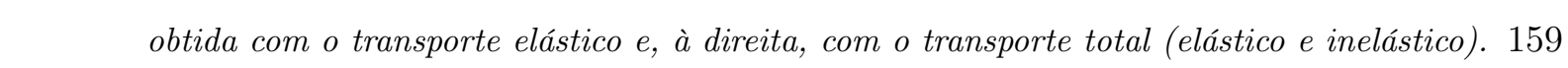

7.13 Distribuição do logaritmo da condutância adimensional obtida com 1000 realizações de uma cadeia com $L=300$ contendo $10 \%$ de defeitos com a "dangling bond". À esquerda, a distribuição obtida com o transporte elástico e, à direita, com o transporte total (elástico e inelástico). . . . . . . . . . . . . . . . . . . . . . . . 160

8.1 Orbitais de dois carbonos com hibridização $s p^{2}$. Os orbitais híbridos, no plano xy, formam ligaçôes $\sigma$ e os orbitais p, perpendiculares ao plano xy, formam ligações $\pi$. . . . . . . . 162

8.2 Representação da rede hexagonal do grafeno. À esquerda, observa-se a célula unitária da rede de Bravais, formada por dois átomos, com vetores base $\mathbf{a}_{1} e \mathbf{a}_{2}$. À direita, observa-se a rede recíproca, com a primeira zona de Brillouin ao centro e vetores base $\mathbf{b}_{1}$ e $\mathbf{b}_{2}$, onde se destacam dois pontos de alta simetria $K_{+} e K_{-}$. Nota-se também dois possiveis cortes do grafeno, um com terminação "armchair" e outro em "zigzag". . . . . . . . . . . . . 163

8.3 Bandas de valência e de condução do grafeno, destacando a relação de dispersãa linear próxima aos pontos $K_{+} e K_{-}$(Adaptado de 11$\left.].\right)$. . . . . . . . . . . . . . . . 164 
8.4 Ilustração das nanofitas "armchair" $N$-AGNR à esquerda e "zigzag" $N$-ZGNR à direita, onde são destacadas as respectivas células unitárias. . . . . . . . . . . . . . . . . . . 166

8.5 Estrutura de bandas obtidas com o programa SIESTA para uma nanofita 4-ZGNR sem polarização de spin (esquerda) e com polarização de spin (meio) e para uma nanofita 14 -AGNR (direita)

8.6 Representação dos quatro tipos de defeitos estudados, onde os grupos $\mathrm{H}$ e $\mathrm{OH}$ foram adsorvidos na borda da nanofita em (A) e (B), no meio da nanofita em $(C)$ e em ambos, borda e meio da nanofita, em (D). Os átomos considerados na análise vibracional de cada defeito estão destacados por uma linha pontilhada . . . . . . . . . . . . . . . . . 168

$8.7 \quad$ Densidade de estados obtida com o programa SIESTA para os defeitos $(A),(B),(C)$ e $(D)$ da Figura 8.6, onde a linha tracejada corresponde à densidade de estados na ausência

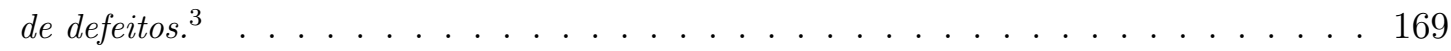

8.8 Estrutura de bandas (acima), densidade de estados (meio) e transmissão elástica a $V=0$ (abaixo) de uma nanofita 14 -AGNR. . . . . . . . . . . . . . . . . . . . 170

8.9 Transmissão elástica a $V=0$ obtida para os defeitos $(A),(B),(C)$ e $(D)$ da Figura 8 8.6, onde a linha tracejada corresponde à transmissão na ausência de defeitos. .

8.10 Transmissão elástica $a V=0$ (acima) e densidade de estados projetada nas ligações

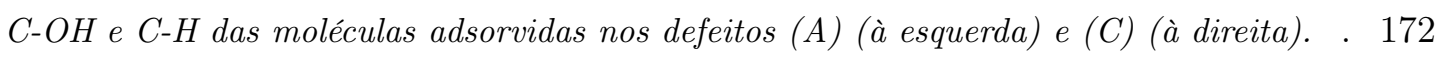

8.11 Frequências calculadas para os defeitos $(A),(B),(C)$ e $(D)$, onde foram considerados somente os átomos destacados na Figura $|8.6| \ldots \ldots \ldots$. . . . . . . . . . . 173

8.12 Valores de corrente obtidos para um único defeito (A) variando-se a tensão e a energia. Acima: contribuição elástica (à esquerda) e corrente total (à direita). Abaixo: componentes simétrica (à esquerda) e assimétrica (à direita) da corrente inelástica. . . . . . . . 174

8.13 Valores de condutância diferencial obtidos para um único defeito $(A)$ variando-se a tensão e a energia. Acima: contribuição elástica (à esquerda) e condutância total (à direita). Abaixo: componentes simétrica (à esquerda) e assimétrica (à direita) da contribuição inelástica.

8.14 Superfícies $d^{2} I / d V^{2}$ obtidas para um único defeito $(A)$ variando-se a tensão e a energia. Acima: contribuição elástica (à esquerda) e $d^{2} I / d V^{2}$ total (à direita). Abaixo: componentes simétrica (à esquerda) e assimétrica (à direita) da contribuição inelástica. . . . . . 176

8.15 Superfícies $d^{2} I / d V^{2}$ obtidas para um único defeito $(A)$ variando-se a tensão e a energia, para os modos vibracionais $\hbar \omega_{23}=0.0940 \mathrm{eV}$ (acima à esquerda), $\hbar \omega_{14}=0.1434 \mathrm{eV}$

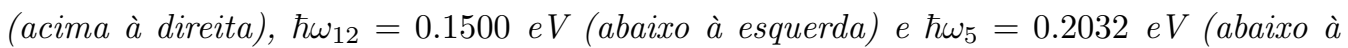
direita). . 
8.16 Exemplo de um sistema desordenado contendo dois segmentos com o defeito (A) e seis segmentos pristinos. . . . . . . . . . . . . . . . . . . . . . . . . . . . . . . . . . 178

8.17 Condutância diferencial elástica média computada a partir de 20 realizações de nanofitas com comprimentos $L_{2}=19,89 \mathrm{~nm}$ (acima à esquerda), $L_{5}=49,73 \mathrm{~nm}$ (acima à direita),

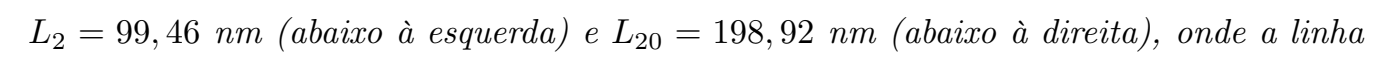
tracejada corresponde à condutância diferencial calculada com somente um defeito (A). . 179

8.18 Resultados obtidos a partir de 20 realizações de uma nanofita de comprimento $L_{2}=19,89$ nm com dois defeitos do tipo (A), onde foi considerado somente o modo vibracional M14 $\left(\hbar \omega_{14}=0.1434 \mathrm{eV}\right)$, ilustrado ao centro. Acima: valores médios de corrente (esquerda) e da superfície $d^{2} I / d V^{2}$ (direita), obtidos variando-se a tensão e a energia. Abaixo: condutância diferencial média elástica (esquerda) e total (direita). . . . . . . . . . . . . . 180

8.19 Resultados obtidos a partir de 20 realizações de uma nanofita de comprimento $L_{5}=49,73$

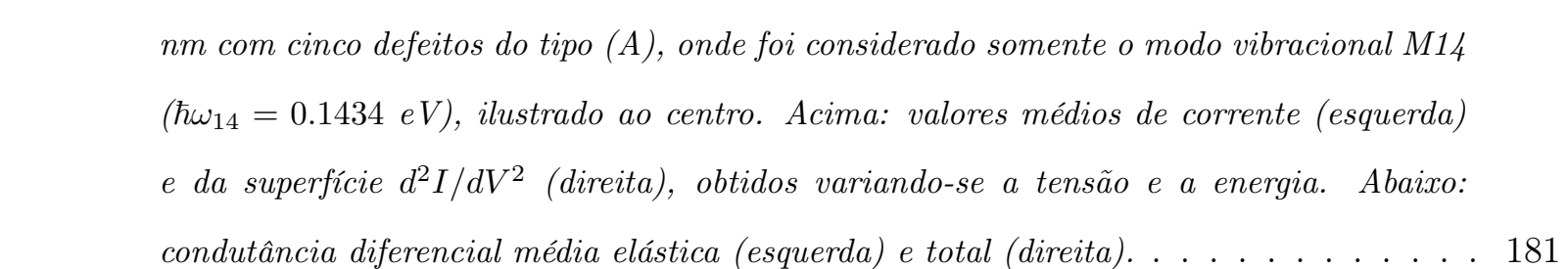

8.20 Resultados obtidos a partir de 20 realizações de uma nanofita de comprimento $L_{10}=99,46$

\begin{tabular}{|c|}
\hline$n m$ com dez defeitos do tipo (A), onde foi considerado somente o modo vibracional M14 \\
\hline$\left(\hbar \omega_{14}=0.1434 \mathrm{eV}\right)$, ilustrado ao centro. Acima: valores médios de corrente (esquerda) \\
\hline e da superficie $d^{2} I / d V^{2}$ (direita), obtidos variando-se a tensão e a energia. Abaixo: \\
\hline
\end{tabular}

8.21 Valores médios das componentes simétrica (esquerda) e assimétrica (direita) da superfície \begin{tabular}{|c|}
\hline$d^{2} I / d V^{2}$ obtidos a partir de 20 realizaçôes de nanofitas com comprimentos $L_{2}=19,89$ \\
\hline$n m$ (acima), $L_{5}=49,73 \mathrm{~nm}$ (meio) e $L_{10}=99,46 \mathrm{~nm}$ (abaixo), onde foi considerado \\
\hline
\end{tabular} somente o modo vibracional M14 $\left(\hbar \omega_{14}=0.1434 \mathrm{eV}\right)$. . . . . . . . . . . . . . . . . . . . 183

B.1 Contorno semicircular (anti-horário) na metade superior do plano complexo com semicírculo em torno de $x_{0}$. (Extraído de 12$]$, com permissão de Cambridge University Press.) 194

B.2 Esquema da integral de contorno para o cálculo de $\rho_{e q}$. . . . . . . . . . . . . . . . . . 196

B.3 Contorno próximo aos polos da função distribuição de Fermi-Dirac do eletrodo esquerdo. 197

C.1 Representação da função $f_{-}(\epsilon)-f_{+}(\epsilon)$ (em vermelho) e respectiva transformada de Hilbert $\mathcal{H}_{\epsilon^{\prime}}\left\{f_{-}\left(\epsilon^{\prime}\right)-f_{+}\left(\epsilon^{\prime}\right)\right\}(\epsilon)($ em azul). . . . . . . . . . . . . . . . . . . 211

C.2 Representação da transformada de Hilbert $\mathcal{H}_{\epsilon^{\prime}}\left\{f_{-}\left(\epsilon^{\prime}\right)-f_{+}\left(\epsilon^{\prime}\right)\right\}(\epsilon)$ e funções $f(\epsilon-e V)-$

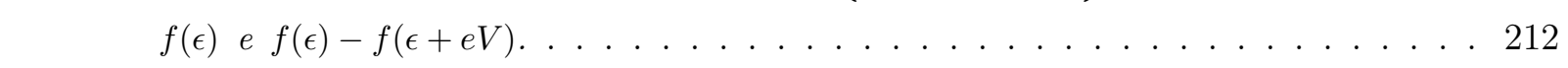


E.1 (a) Schematic two terminal device. Two leads are kept at the chemical potentials $\mu_{L}$ and $\mu_{R}$ and the transport is through the extended molecule $E M$. (b) The Hamiltonian is an infinite matrix comprising two block diagonal parts describing the leads and a part (finite) describing the extended molecule $H_{E M}$. (c) Typical potential profile. . . . . . . 227

E.2 Periodic structure of the leads. $H_{0}$ and $S_{0}$ are the Hamiltonian and overlap matrix describing the interaction within one unit cell and $H_{1}$ and $S_{1}$ are the coupling and overlap matrix describing the interaction between adjacent unit cells. The arrow shows the di-

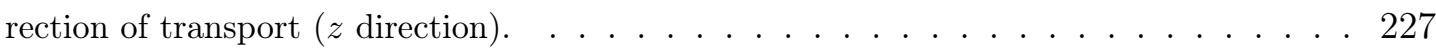

E.3 Example of solid lead supercell constituted by 27 gold atoms which are repeated periodically in all directions. The lead's unit cell containing 3 gold atoms is highlighted. .. . 231

E.4 a) Sketch of a typical system simulated using SMEAGOL: two semi-infinite electrodes connected to a central scattering region. b) The typical unit cell for a SMEAGOL calculation. The reader must note that we include at least one layer of the leads both to the left- and to the right-hand side of the scattering region. The order of the atoms in the input file is only important in these two layers. The first atoms of the list (see AtomicCoordinatesAndAtomicSpecies in the SIESTA user's guide) must be those contained in the left-hand side lead unit cell, and they should be input with the same order than in the bulk leads calculation. In an analogous way, the last atoms of the list must conform with the input file for the right-hand side lead. c) An example of a system setup for two semi-infinite zig-zag wires separated by a vacuum region. The numbers for each atom have been indicated as well as the two atoms on either side corresponding to the the unit cells for the left- and right-hand side lead. . . . . . . . . . . . . . . . 233

E.5 Sketches for the Green Function integration leading to the non-equilibrium charge density L $[1] 2]$. a) The non-equilibrium part must be performed along the real energy axis, but is bound by the left- and right-hand side Fermi distributions functions, $f_{L}$ and $f_{R}$. b) The equilibrium component is performed over a semicircular path in the complex energy \begin{tabular}{|c|}
\hline plane. Here we show the starting position EnergLowestBound, the energy mesh along the \\
\hline
\end{tabular} two segments of the curve and the poles of the Fermi distribution. . . . . . . . . . . . 234

E.6 Shift of the Hartree potential between two SIESTA calculations: bulk gold (the leads) \begin{tabular}{c} 
with two atoms in the unit cell (red line) and the parallel plate capacitor (scattering \\
\hline
\end{tabular}

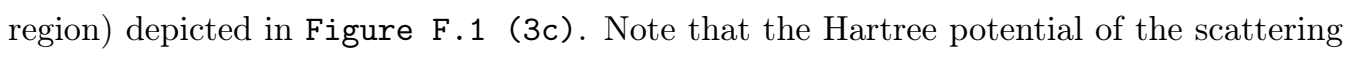
region has converged to the bulk value after the first atomic layer except for a constant. 239

E.7 Diagrammatic representation of the first Born approximation (left) and the self-consistent Born approximation (right). . . . . . . . . . . . . . . . . . . . . . 244 
F.1 Representation of a typical disordered system using a graphene nanoribbon as an example. One identifies a scattering region with three pristine segments $\left(H_{P}\right)$ and two with defects $\left(H_{D}^{1}\right.$ and $\left.H_{D}^{2}\right)$. The left and right leads, made by semi-infinite pristine nanoribbons, are coupled to the first and last segments, respectively. . . . . . . . . . . . . . . . . . . . . 257 


\section{Lista de Tabelas}

4.1 Tempos de execução em minutos de um mesmo sistema com as versões nova e antiga do SMEAGOL. . . . . . . . . . . . . . . . . . . . . . . . . . . . 81

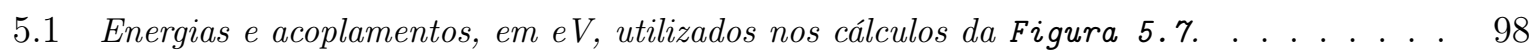

5.2 Energias e acoplamentos, em eV, utilizados nos cálculos da Figura 5.10 . . . . . . . . . 102

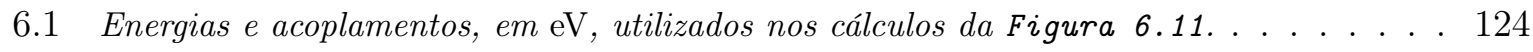

7.1 Energias e acoplamentos, em eV, utilizados nas simulações das cadeias unidimensionais na LOE, onde o indice 1 corresponde ao sistema pristino, 2, ao defeito simples e 3, ao defeito "dangling bond". . . . . . . . . . . . . . . . . . . . . . . . . . . . . . . 152 


\section{Capítulo 1}

\section{Introdução}

A compreensão de como ocorre o fluxo de elétrons passando por um material é essencial para se entender diversos processos na natureza. Comumente, aprende-se que a corrente que passa por um condutor é diretamente proporcional à diferença de potencial entre os dois pontos terminais e inversamente proporcional à sua resistência, o que é conhecido como lei de Ohm. De fato, esta lei é válida para uma grande quantidade de sistemas e pode ser utilizada para explicar uma vasta gama de fenômenos.

Contudo, com a enorme melhoria dos recursos tecnológicos e experimentais ocorrida nas últimas décadas, a fabricação e observação de sistemas em escala nanométrica se tornaram uma realidade e ficou claro que tais sistemas não podem ser modelados a partir de conceitos macroscópicos da matéria. Quando as dimensões de um condutor atingem, por exemplo, a ordem do livre caminho médio do material (i.e., a distância média que um elétron percorre antes de sofrer um espalhamento), novas propriedades de transporte emergem e a lei de Ohm claramente falha.

Do ponto de vista tecnológico, a busca por dispositivos cada vez menores, mais rápidos e mais eficientes é representada pela lei de Moore, que afirma que a densidade de transistores nos circuitos integrados dobra aproximadamente a cada dois anos [13]. Porém, essa miniaturização dos dispositivos está levando as tecnologias atuais, baseadas no silício, a alcançarem limites fundamentais a partir dos quais os efeitos quânticos, como tunelamento, se tornam predominantes. Além disso, acredita-se que as próprias técnicas atuais de fabricação em larga escala (litografia no caso de circuitos integrados) não serão capazes de atingir as resoluções que essas dimensões reduzidas demandam para o futuro próximo.

Neste contexto, existe uma necessidade urgente de se propor e desenhar novos sistemas em escala atômica e molecular capazes de incorporar efeitos quânticos e desempenhar tarefas semelhantes a dos seus equivalentes semicondutores. Entretanto, os desafios são grandes. Problemas de controle na fabricação e boa reprodutibilidade dos resultados em escala atômica e molecular 

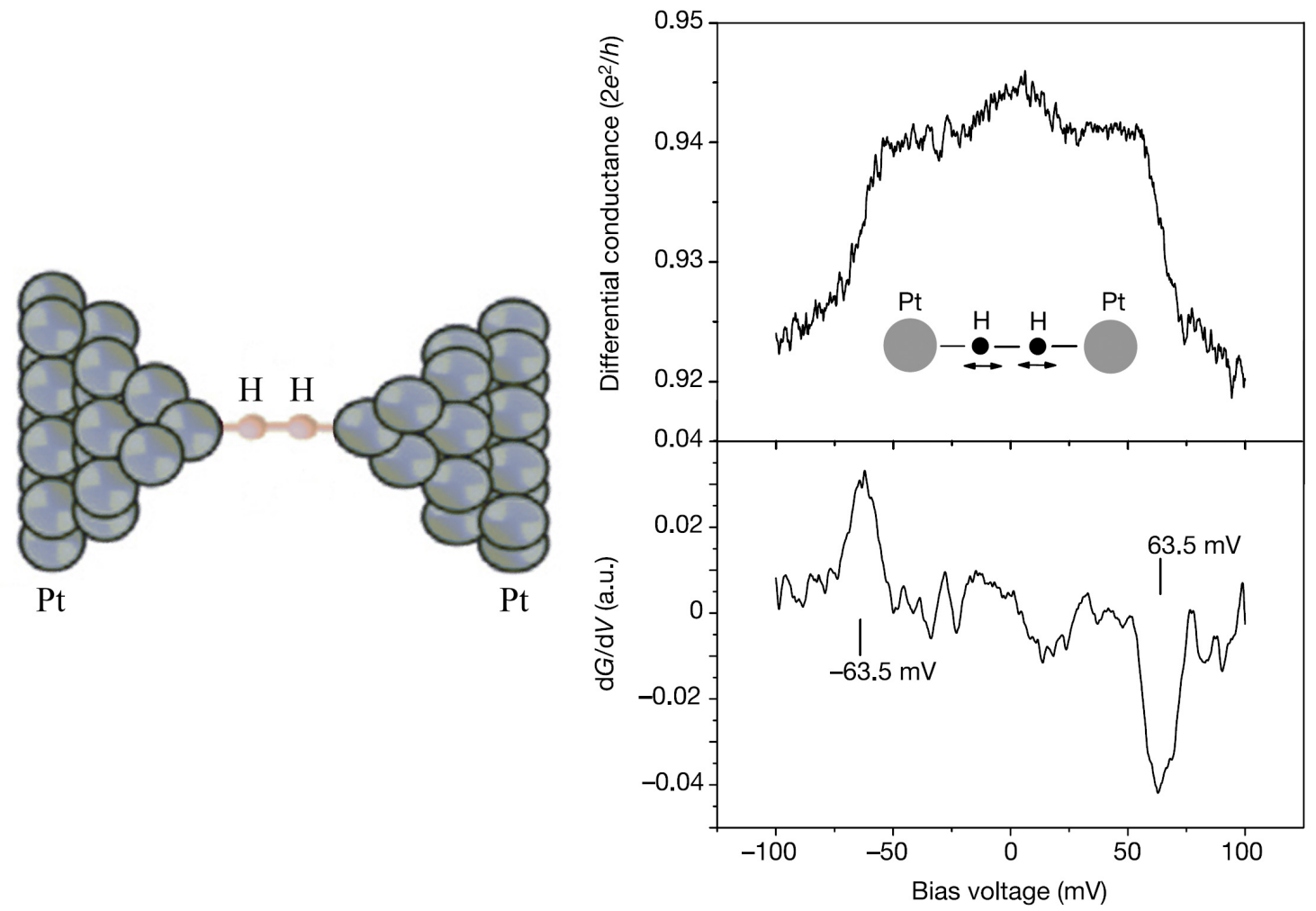

Figura 1.1: Condutância diferencial (acima à direita) e respectiva derivada (abaixo à direita) medidas de um contato $\mathrm{Pt} / \mathrm{H}_{2}$ (ilustração à esquerda). (Gráficos extraídos de [6], com permissão de Macmillan Publishers Ltd: Nature 419, 906-909, 31 outubro 2002 (doi: 10.1038/nature01103), copyright (2002).)

ainda terão que ser resolvidos.

Em particular, em eletrônica molecular propõe-se fabricar dispositivos utilizando uma única molécula entre eletrodos metálicos. Esta ideia foi introduzida por A. Aviran e M. Ratner, em 1974, que propuseram a utilização de uma molécula assimétrica como retificador de corrente, i.e., um diodo molecular [14]. Mas foi preciso mais vinte anos para essa ideia ser concretizada com M. Reed et al, que conseguiram medir o transporte de cargas de moléculas de benzeno-1,4ditiol entre eletrodos de ouro [15]. Um progresso substancial ocorreu na década passada [16 18$]$, mas ainda existem muitas dificuldades na reprodutibilidade dos resultados experimentais, devido principalmente à variedade de configurações em que as moléculas podem acoplar-se aos eletrodos.

Em dispositivos com escalas moleculares, os efeitos das vibrações nucleares podem ter um papel importante no transporte eletrônico. De fato, esses efeitos já vêm sendo investigados pela comunidade científica 19,20 e verificou-se que os fônons (modos vibracionais quantizados da matéria) são responsáveis por abrir novos canais de condutância, por onde os elétrons podem propagar-se, e por suprimir canais de transmissão puramente elásticos. Em experimento reportado em 2002, R. Smit et al mediram, por meio de espectroscopia de contato pontual, a con- 

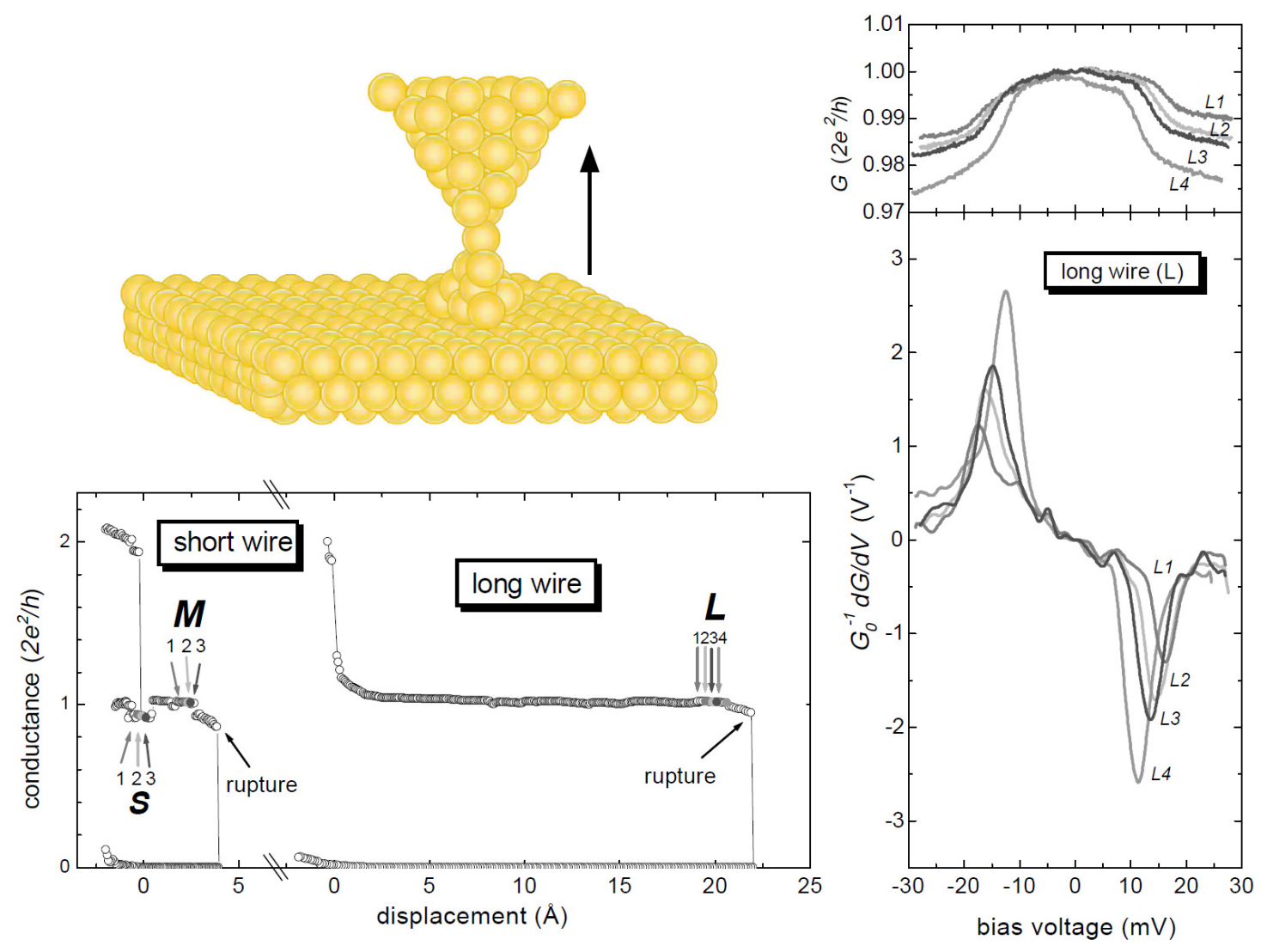

Figura 1.2: Valores de condutância em cadeias monoatômicas de ouro (abaixo à esquerda), medidas em função do distanciamento entre o substrato e a ponta do STM que foi afastada até a ruptura da cadeia (ilustração acima). Para cadeias com sete átomos, a condutância apresenta uma queda brusca em $\pm 15 m V$ de $\sim 1 \%$ (à direita). (Gráficos extraídos de 77 com permissão de N. Agraït, C. Untiedt, G. Rubio-Bollinger e S. Vieira. Phys. Rev. Lett. 88(21), 216803 (2002). Copyright (2002) by the American Physical Society. http://dx.doi.org/10.1103/PhysRevLett.88.216803)

dutância de uma única molécula de hidrogênio entre eletrodos de platina e obtiveram um valor muito próximo do quantum de condutância $G_{0}=2 e^{2} / h$, com $e$ a carga do elétron e $h$ a constante de Planck (Figura 1.1). Aumentando a diferença de potencial aplicada, observaram uma queda na condutância a tensões de $\pm 63,5 \mathrm{mV}$, o que foi posteriormente atribuído ao retroespalhamento dos elétrons devido à excitação do modo vibracional da molécula de hidrogênio.

A técnica utilizada neste experimento já havia sido aplicada no mesmo ano para a observação dos efeitos de modos vibracionais individuais nas propriedades de transporte em cadeias atômicas de ouro por N. Agraït et al [7]. Por meio de microscopia de tunelamento com varredura (STM) a baixas temperaturas, o transporte eletrônico foi medido em cadeias formadas por até sete átomos de ouro e observou-se o espalhamento inelástico dos elétrons, isto é, a queda brusca na condutância diferencial a tensões simétricas, devido à excitação de modos de vibração nos átomos da cadeia (Figura 1.2. 


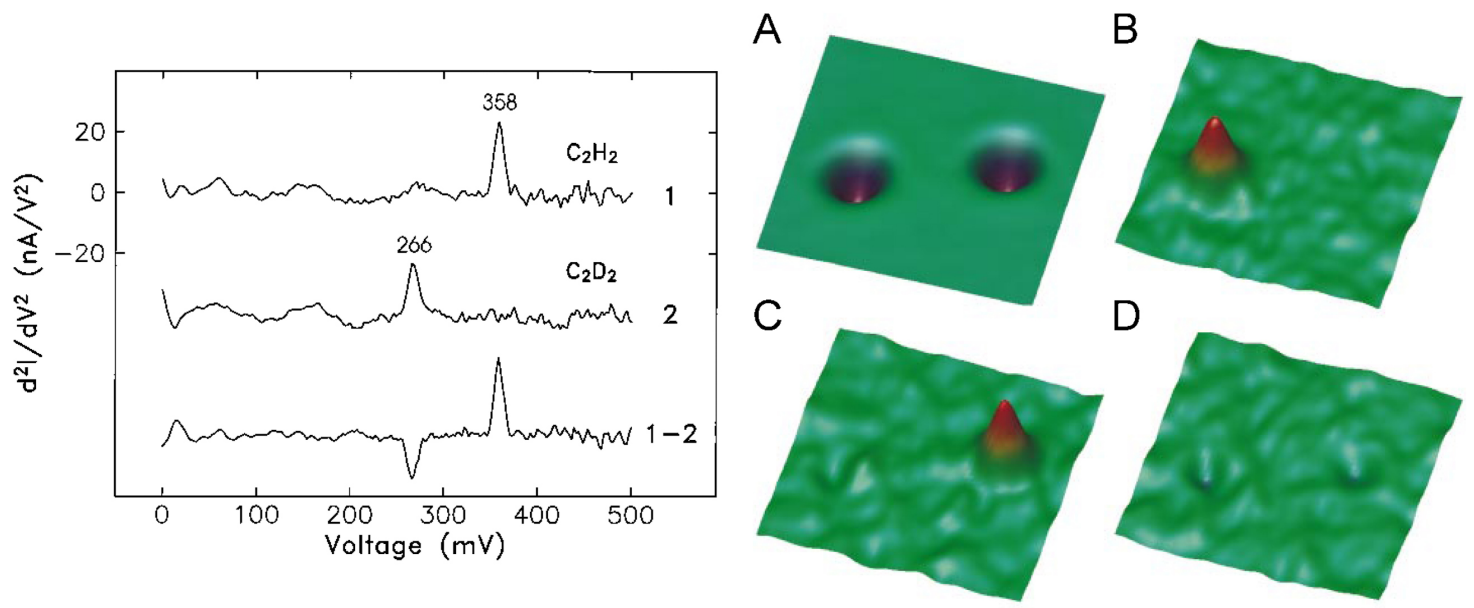

Figura 1.3: Curvas do espectro $d^{2} I / d V^{2}$ obtidas com IETS para o $C_{2} H_{2}$ e o $C_{2} D_{2}$ adsorvidos a uma

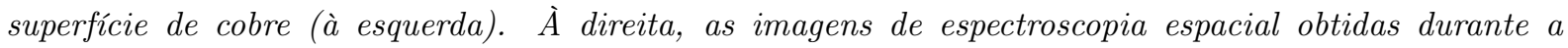
varredura da ponta para gravar a topologia da superfície, onde o $C_{2} H_{2}$ está à esquerda e o $C_{2} D_{2}$, à direita: Em (A), a imagem padrão de STM obtida no modo corrente constante, onde não se verifica contraste entre as moléculas. Nos outros casos, a imagem construída pelo sinal $d^{2} I / d V^{2}$, para a tensão dc fixada em $358 \mathrm{mV}(B)$, em $266 \mathrm{mV}(C)$ e em $311 \mathrm{mV}(\mathrm{D})$. (Gráficos e imagens de B. C. Stipe, M. A. Rezaei e W. Ho. Science 280, 1732-1735 (1998). Extraídos com permissão de AAAS.)

Os efeitos dos modos vibracionais no transporte eletrônico também vêm sendo estudados por meio da técnica de espectroscopia de tunelamento inelástico de elétrons (IETS). Nesse caso, o STM é utilizado para obter-se o espectro vibracional em conjunto com medidas de transporte. Em artigo publicado em 1998, B. Stipe, M. Rezaei e W. Ho utilizaram a IETS para investigar variações espaciais de processos de tunelamento inelástico por meio de moléculas adsorvidas em superfícies metálicas [21]. Nesse caso, foram investigados o acetileno $\left(\mathrm{C}_{2} \mathrm{H}_{2}\right)$ e o acetileno com deutério $\left(\mathrm{C}_{2} \mathrm{D}_{2}\right)$ sobre uma superfície de cobre. Verificou-se que a condutância aumenta quando os elétrons de tunelamento têm energia suficiente para excitar a vibração de estiramento $\mathrm{C}-\mathrm{H}$ (C-D) da molécula. De maneira análoga aos experimentos descritos nos parágrafos anteriores, este efeito aparece como um pico na curva $d^{2} I / d V^{2}$ quando a tensão corresponde ao quantum de vibração (Figura 1.3). Esses resultados sugerem que as interações entre elétrons e modos vibracionais podem ser utilizadas, simultaneamente com medidas de transporte, como uma ferramenta espectroscópica para se deduzir informações estruturais que são tipicamente inacessíveis por outras técnicas.

Além de sistemas formados por uma única molécula, outros candidatos em potencial para substituir as tecnologias baseados no silício são os materiais de baixa dimensionalidade baseados no grafeno [22]. Formado por uma única camada de átomos de carbono, o grafeno possui pro- 
priedades estruturais e eletrônicas únicas, sendo reportadas mobilidades de carga extremamente grandes como $10^{5} \mathrm{~cm}^{2} \mathrm{~V}^{-1} \mathrm{~s}^{-1}$ à temperatura ambiente [23]. Devido a suas propriedades peculiares, esse material tem sido objeto de intensa investigação científica desde sua descoberta em 2004 por K. Novoselov, A. Geim et al [24].

No entanto, um dos grandes problemas encontrados para sua utilização em dispositivos eletrônicos é o fato de que este material não possui um gap de energia entre as bandas de condução e de valência, o que é fundamental em dispositivos como o transistor, onde a corrente é ligada e desligada por meio de uma tensão de porta. Em face a esse problema, diversas propostas para se abrir o gap de energia do grafeno continuam surgindo, como, por exemplo, a utilização de nanofitas de grafeno [25, 26], camadas de grafeno sobrepostas [11,27] e a funcionalização química 28,29.

Contudo, a presença de defeitos é uma característica comum na fabricação desses materiais. Diferentes tipos de defeitos podem ser encontrados [30,31], tais como vacâncias na estrutura cristalina, presença de átomos substitucionais e bordas irregulares produzidas ao se cortar o grafeno. Em técnicas de fabricação por esfoliação química, tal como óxido-redução sequencial do grafite, os grafenos produzidos apresentam uma grande quantidade de grupos residuais contendo oxigênio adsorvidos na superfície [32,33]. Acredita-se que a adsorção de grupos hidroxílicos $(\mathrm{OH})$ na superfície de folhas de grafeno seja algo comum na maioria dos experimentos onde a presença de água é inevitável [34], sendo importante estudar seus efeitos nas propriedades do grafeno. A "desordem" gerada por defeitos pode levar a um novo regime fundamental de transporte, a saber, o de localização de Anderson [5, 10,35,36].

No caso de funcionalização química, além dos efeitos de desordem [37], modos vibracionais localizados nas moléculas adsorvidas à estrutura podem ter um papel importante nas propriedades do transporte eletrônico. De fato, alguns estudos têm sugerido que a combinação de sistemas desordenados com a interação elétron-fônon pode explicar questões em aberto, como o mecanismo de transporte nos polímeros condutores [38,39]. Nesses trabalhos, o efeito de perda de coerência de fase dos elétrons devido à interação com os fônons foi inserido por meio de um modelo fenomenológico simples e verificou-se que a inclusão deste efeito pode explicar a alta condutividade de polímeros fortemente desordenados, como polianilinas. Analogamente, ao mesmo tempo que moléculas adsorvidas na superfície do grafeno levam a um aumento do grau de desordem, o efeito de localização pode ser reduzido em razão da interação dos elétrons com os modos de vibração localizados nas moléculas adsorvidas. Em particular, acredita-se que esses modos localizados ocorram no caso de impurezas hidroxílicas, dado que o sinal do modo de estiramento O-H está sempre presente em medidas de espectroscopia de infravermelho [40,41]. 
Em paralelo a todo esse avanço experimental, um grande esforço tem sido dedicado ao desenvolvimento de teorias e métodos computacionais eficientes para se descrever corretamente o transporte eletrônico em escala nanométrica. Essas ferramentas são importantes pois, além de auxiliarem na interpretação dos resultados experimentais, podem prever e sugerir novos experimentos.

Modelar o transporte eletrônico nesta escala de grandeza envolve não apenas uma descrição microscópica da matéria por meio da mecânica quântica mas também conceitos de mecânica estatística fora do equilíbrio. O estado da arte em cálculos de transporte eletrônico em escala nanométrica consiste em combinar cálculos de estrutura eletrônica ab initio via Teoria do Funcional da Densidade [3, 42, com o formalismo das Funções de Green Fora do Equilíbrio [4, 8, 43].

Usualmente, esses cálculos são realizados desconsiderando-se as interações elétron-fônon. De fato, a inclusão dos efeitos das vibrações no transporte não é uma tarefa simples. Trabalhos vêm mostrando que as propriedades vibracionais em sistemas de escala nanométrica têm caráter eminentemente quântico, diferindo significativamente das predições semiclássicas, e que é necessário um estudo detalhado para se conseguir explicar a diversidade de fenômenos observados experimentalmente [44 51].

Nessa busca por simulações precisas e realísticas, outro desafio está relacionado ao tamanho dos sistemas. Dispositivos eletrônicos realísticos podem atingir escalas de comprimento de 100 nm com um grande número de defeitos distribuídos aleatoriamente [36]. Portanto, para realizar simulações quantitativamente significativas de dispositivos reais, o método utilizado deve ser capaz de considerar dezenas de milhares de átomos com os defeitos aleatoriamente posicionados, o que, em termos de cálculos ab initio, não é uma tarefa fácil.

\subsection{Objetivos e Organização da Tese}

De maneira geral, o objetivo desta tese consiste em estudar propriedades de transporte de cargas em sistemas de escala nanométrica considerando efeitos do espalhamento inelástico dos elétrons devido a sua interação com fônons do dispositivo. O trabalho desenvolvido pode ser visto como um projeto de implementação de diferentes ferramentas integradas para esse propósito. Distintas abordagens foram utilizadas resultando em diferentes implementações computacionais, permitindo a simulação do transporte inelástico por primeiros princípios, inclusive em sistemas desordenados.

Como o trabalho realizado envolve sobretudo muito desenvolvimento, em termos de implementação computacional, a compreensão da formulação teórica do problema é um passo fundamental. Por esse motivo, o Capítulo 2 foi dedicado à descrição do formalismo das Funções de 
Green Fora do Equilíbrio (NEGF), restringindo-se às partes relevantes ao tratamento do transporte com interações elétron-fônon. Este capítulo traz o arcabouço teórico principal da tese e base para os desenvolvimentos realizados.

Partindo da aproximação de Born-Oppenheimer, apresentaremos no Capítulo 3 a Teoria do Funcional da Densidade (DFT) e sua implementação no código SIESTA [2,3]. Uma boa compreensão deste código foi importante para o desenvolvimento deste trabalho, uma vez que ele foi utilizado como plataforma de cálculos de estrutura eletrônica de todos os programas implementados nesta tese. Nesse capítulo será descrito como o cálculo das frequências e dos modos vibracionais e do acoplamento elétron-fônon pode ser realizado a partir de métodos de primeiros princípios, o que culminou no programa PhOnonS ITeratIVE VIBRATIONS.

No Capítulo 4, o problema de transporte será abordado tanto do ponto de vista conceitual quanto do prático. Veremos como o formalismo NEGF pode ser aplicado ao problema de transporte eletrônico e discutiremos a implementação computacional do programa SMEAGOL [4], um código de transporte eletrônico ab initio baseado na combinação DFT-NEGF. Neste trabalho, diversas mudanças foram realizadas no SMEAGOL, sendo a principal delas a transição para uma versão atual do SIESTA.

O primeiro efeito da interação elétron-fônon estudado foi a perda de coerência de fase, o que foi abordado por meio do modelo fenomenológico das sondas de Büttiker [1]. Este modelo será apresentado no Capítulo [5, onde discutiremos a sua implementação computacional. Usualmente, o modelo de Büttiker é implementado considerando uma aproximação elástica no cálculo da corrente. Neste trabalho foi proposto um método para o cálculo da corrente sem essa aproximação. Veremos que o modelo produz somente um alargamento do canal de condutância, mas não inclui demais efeitos da interação elétron-fônon como os observados nos experimentos discutidos há pouco. Logo, o modelo de Büttiker é interessante quando se quer considerar somente o efeito de perda de coerência de fase.

Um modelo mais elaborado é apresentado no Capítulo 6, onde a autoenergia de interação é expandida perturbativamente em diagramas de Feynman até a primeira ordem, o que é conhecido como primeira aproximação de Born. Veremos como esta aproximação, assim como a sua versão autoconsistente, onde uma classe mais ampla de diagramas é considerada, foram incorporadas ao programa SMEAGOL, dando origem ao código Inelastic SMEAGOL para cálculos de transporte inelástico ab initio. Será apresentada, também, uma reformulação da expressão usualmente utilizada para o cálculo da autoenergia retardada de interação na primeira aproximação de Born, o que reduz drasticamente o custo computacional do cálculo.

No Capítulo 7, trataremos o problema do transporte inelástico em sistemas desordenados, 
onde serão discutidos os efeitos de localização por desordem. Será apresentado um método desenvolvido para o cálculo de transporte inelástico, por primeiros princípios, em dispositivos eletrônicos contendo um grande número de defeitos aleatoriamente distribuídos, o que foi chamado de Inelastic DISORDER. O código combina cálculos de estrutura eletrônica via DFT com o formalismo NEGF para o transporte, onde as funções de Green são obtidas por meio do cálculo recursivo de funções de Green [52]. A interação elétron-fônon é considerada perturbativamente com a Lowest Order Expansion 53 55].

A metodologia implementada no I-DISORDER foi aplicada ao estudo de nanofitas de grafeno com impurezas hidroxílicas e os resultados obtidos serão apresentados no Capítulo 8. Discutiremos de maneira sucinta as propriedades das nanofitas de grafeno, as quais motivaram a escolha do sistema a ser estudado. Analisaremos inicialmente os defeitos de maneira isolada, apresentando o cálculo das frequências vibracionais com o programa PhOnonS ITeratIVE VIBRATIONS e discutindo as propriedades de transporte elástico e inelástico. Veremos, então, os resultados para múltiplos defeitos considerando diferentes comprimentos para as nanofitas.

Por fim, no Capítulo 9 , serão expostas as conclusões gerais e serão listadas algumas perspectivas para futuros trabalhos. 


\section{Capítulo 2}

\section{Formalismo das Funções de Green Fora do Equilíbrio}

No curso de mecânica quântica básica [56], aprendemos que toda informação possível de se obter de um dado sistema (átomo, molécula ou sólido) está contida em sua função de onda $\Psi(\{\mathbf{r}\}, t)$, que, por sua vez, é solução da equação de Schrödinger:

$$
i \hbar \frac{\partial}{\partial t} \Psi=\mathscr{H} \Psi
$$

onde $\mathscr{H}$ é o operador Hamiltoniano e $\{\mathbf{r}\}=\left\{\mathbf{r}_{1}, \mathbf{r}_{2}, \ldots, \mathbf{r}_{n}\right\}$ é o conjunto de coordenadas das partículas que constituem o sistema. Portanto, na prática procura-se estabelecer o Hamiltoniano do sistema para, então, obter-se a função de onda a partir da resolução da equação de Schrödinger. Uma vez encontrada a função de onda do sistema, os observáveis físicos são dados pelos respectivos valores esperados de operadores hermitianos (i.e. $\langle\Psi|\hat{\mathbf{O}}| \Psi\rangle$ ).

Porém, a dificuldade para obter-se autofunções $\Psi(\{\mathbf{r}\}, t)$ do operador $\mathcal{H}$ cresce rapidamente com a quantidade de partículas constituintes. O átomo de hidrogênio, por exemplo, é um dos poucos sistemas realísticos que possuem soluções exatas na forma fechada e que podem ser obtidas analiticamente. A partir delas conseguimos, entre outras coisas, determinar todo o espectro de energia desse átomo.

Na maioria dos casos, porém, a Equação 2.1 é demasiadamente complexa e sua solução analítica é desconhecida. Com o aumento de partículas interagentes no sistema, procura-se utilizar métodos de aproximação, como o método variacional, teoria de perturbação, aproximação WKB etc. Todos se mostram muito precisos, e até exatos, em alguns casos (por exemplo, quando se compara com as soluções analíticas obtidas para o átomo de hidrogênio). No entanto, mesmo com esses métodos e com o auxílio da computação, o cálculo se torna inviável para sistemas mais 
complexos.

O formalismo das Funções de Green Fora do Equilíbrio (Non-Equilibrium Green's Function NEGF) serve justamente a esse propósito. Desenvolvido independentemente por L. V. Keldysh [57] e por L. P. Kadanoff e G. Baym [58], o formalismo NEGF permite, em princípio, resolver exatamente a equação de Schrödinger dependente do tempo para um sistema de muitos corpos interagentes. Na prática, é uma ferramenta poderosa para se calcular propriedades de sistemas de muitos corpos tanto em equilíbrio termodinâmico quanto fora do equilíbrio.

O objetivo deste capítulo é resumir alguns conceitos do formalismo NEGF relevantes à compreensão das metodologias utilizadas para o cálculo de transporte eletrônico em sistemas nanométricos (com e sem interação elétron-fônon), apresentadas nos capítulos seguintes. Iniciaremos listando alguns fatos básicos de segunda quantização, dando uma ênfase maior aos fônons, o que facilitará a compreensão do método implementado no programa PhOnonS ITeratIVE VIBRATIONS (Seção 3.3.1). Em seguida, descreveremos o formalismo NEGF, passando brevemente pelas funções de Green no equilíbrio para, então, chegar no formalismo de Keldysh. Todas as derivações a seguir são padrões, podendo ser encontradas nos livros texto da área [8,59 62], e, na parte fora do equilíbrio, em particular, seguiremos a notação do livro de Haug e Jauho [8]. Contudo, como esses resultados serão utilizados com frequência durante a tese, é útil tê-los aqui à disposição.

\subsection{Segunda Quantização}

O formalismo NEGF utiliza como base fundamental o formalismo de segunda quantização, no qual as partículas são representadas por operadores de campo $\psi$. Esses operadores, por sua vez, são dados por combinações lineares adequadas de operadores criação-aniquilação cujos coeficientes são funções de onda de partícula única. Por exemplo, na representação de posição, os operadores de campo:

$$
\psi^{\dagger}(\mathbf{r})=\sum_{k}\left\langle\phi_{k} \mid \mathbf{r}\right\rangle c_{k}^{\dagger} \quad \text { e } \psi(\mathbf{r})=\sum_{k}\left\langle\mathbf{r} \mid \phi_{k}\right\rangle c_{k}
$$

criam e destroem uma partícula na posição $\mathbf{r}$, respectivamente. Os operadores $c_{k}^{\dagger}$ e $c_{k}$ criam e destroem uma partícula no estado $k$, respectivamente, e as funções de onda $\phi_{k}$ formam um conjunto completo de autofunções de partícula única de número quântico $k$. A seguir, serão definidos os operadores criação-aniquilação para fônons (bósons) e para elétrons (férmions) e, em termos desses operadores, representaremos a interação elétron-fônon. 


\subsubsection{Fônons}

Os fônons surgem do movimento coletivo dos íons e podem ser entendidos como ondas que se propagam no material. Grosso modo, da mesma maneira que ondas de luz podem ser entendidas ora como oscilações de radiação quantizadas, ora como sendo constituídas de partículas (os fótons), a oscilação uniforme da rede numa certa frequência pode ser entendida como composta por um conjunto de (quasi) partículas, os fônons.

Para entender o tratamento quântico dos fônons, é instrutivo considerar inicialmente uma cadeia unidimensional com $N$ átomos unidos por molas de mesma constante elástica (Figura 2.1), para depois generalizar os resultados para três dimensões.

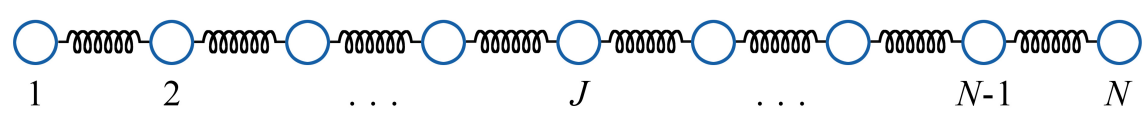

Figura 2.1: Cadeia harmônica unidimensional.

Seja $X_{J}$ o desvio do J-ésimo oscilador de sua posição de equilíbrio, $\omega$ a frequência de oscilação de cada mola e $M$ a massa de cada átomo. Considerando que os átomos realizam pequenos deslocamentos em torno de suas posições de equilíbrio, na aproximação harmônica ${ }^{1}$ o Hamiltoniano total desta cadeia será:

$$
H_{f}=\sum_{J} \frac{P_{J}^{2}}{2 M}+\frac{M \omega^{2}}{2} \sum_{J}\left(X_{J}-X_{J+1}\right)^{2},
$$

onde $J$ é a posição do J-ésimo íon. Em geral, em um problema de muitos corpos reais, fortemente acoplado, procura-se encontrar mudanças de coordenadas adequadas de forma a obter-se vários problemas de corpos fictícios (quasipartículas) fracamente acoplados [61]. No caso das vibrações da rede, uma mudança usual é a das coordenadas normais (transformada de Fourier dos operadores), assumindo-se condições periódicas de contorno:

$$
\begin{array}{rlrl}
X_{J} & =\frac{1}{\sqrt{N}} \sum_{q} e^{i q J a} X_{q} & \text { e } & X_{q}=\frac{1}{\sqrt{N}} \sum_{J} e^{-i q J a} X_{J}, \\
P_{J}=\frac{1}{\sqrt{N}} \sum_{q} e^{-i q J a} P_{q} & \text { e } & P_{q}=\frac{1}{\sqrt{N}} \sum_{J} e^{i q J a} P_{J},
\end{array}
$$

\footnotetext{
${ }^{1}$ A aproximação harmônica é obtida considerando-se somente os termos até a segunda ordem na expansão da superfície de energia potencial, o que é feito explicitamente mais à frente (Equação 2.28).
} 
onde $q$ é o vetor de onda e $a$ é a distância entre dois átomos na situação em que todas as molas se encontram em repouso, chamada de constante de rede. Para um número $N$ finito de átomos, o vetor de onda $q$ assume valores quantizados que, no caso das condições periódicas de contorno, são dados por:

$$
q=\frac{2 n \pi}{N a}, \quad n=0, \pm 1, \pm 2, \ldots, \pm \frac{N}{2} .
$$

A escolha das coordenadas normais mantém as relações de comutação tanto no espaço real quanto no espaço recíproco (espaço dos vetores de onda):

$$
\begin{aligned}
{\left[X_{J}, P_{I}\right] } & =i \hbar \delta_{J I} \\
{\left[X_{q}, P_{q^{\prime}}\right] } & =\frac{1}{N} \sum_{J, I} e^{-i q J a} e^{i q^{\prime} I a}\left[X_{J}, P_{I}\right]= \\
& =\frac{i \hbar}{N} \sum_{J} e^{i\left(q^{\prime}-q\right) J a}= \\
& =i \hbar \delta_{q q^{\prime}} .
\end{aligned}
$$

A partir dessas relações, obtém-se os seguintes resultados gerais:

$$
\begin{gathered}
\sum_{J} X_{J} X_{J+I}=\frac{1}{N} \sum_{q q^{\prime}} X_{q} X_{q^{\prime}} \sum_{J} e^{i\left(q+q^{\prime}\right) J a} e^{i q^{\prime} I a}=\sum_{q} X_{q} X_{-q} e^{i q I a} \\
\sum_{J} P_{J}^{2}=\sum_{q} P_{q} P_{-q} .
\end{gathered}
$$

Aplicando a mudança de coordenadas ao termo de energia potencial do Hamiltoniano obtémse:

$$
\begin{aligned}
\frac{M \omega^{2}}{2} \sum_{J}\left(X_{J}-X_{J+1}\right)^{2} & =\frac{M \omega^{2}}{2} \sum_{J}\left(X_{J}-X_{J+1}\right)\left(X_{J}-X_{J+1}\right)= \\
& =\frac{M \omega^{2}}{2} \sum_{J}\left(X_{J} X_{J}-X_{J} X_{J+1}-X_{J+1} X_{J}+X_{J+1} X_{J+1}\right)= \\
& =\frac{M \omega^{2}}{2}\left[\sum_{q} X_{q} X_{-q}-\sum_{q} X_{q} X_{-q} e^{i q a}-\sum_{q} X_{q} X_{-q} e^{-i q a}+\sum_{q} X_{q} X_{-q}\right]= \\
& =\frac{M}{2} \sum_{q} X_{q} X_{-q} \omega^{2}\left[2-e^{i q a}-e^{-i q a}\right]= \\
& =\frac{M}{2} \sum_{q} \omega_{q}^{2} X_{q} X_{-q},
\end{aligned}
$$


onde $\omega_{q}=\omega \sqrt{2-e^{i q a}-e^{-i q a}}=\omega \sqrt{2[1-\cos (q a)]}=2 \omega\left|\sin \frac{q a}{2}\right|$. Portanto, o Hamiltoniano no espaço recíproco se torna:

$$
H_{f}=\frac{1}{2 M} \sum_{q}\left[P_{q} P_{-q}+M^{2} \omega_{q}^{2} X_{q} X_{-q}\right]
$$

Pode-se definir operadores criação e destruição como:

$$
\begin{aligned}
b_{q}^{\dagger} & =\left(\frac{M \omega_{q}}{2 \hbar}\right)^{\frac{1}{2}}\left(X_{-q}-\frac{i P_{q}}{M \omega_{q}}\right), \\
b_{q} & =\left(\frac{M \omega_{q}}{2 \hbar}\right)^{\frac{1}{2}}\left(X_{q}+\frac{i P_{-q}}{M \omega}\right)
\end{aligned}
$$

que obedecem as seguintes relações de comutação:

$$
\begin{gathered}
{\left[b_{q}, b_{q^{\prime}}^{\dagger}\right]=-\frac{i}{2 \hbar}\left\{\left[X_{q}, P_{q^{\prime}}^{\dagger}\right]-\left[P_{-q^{\prime}}, X_{-q^{\prime}}^{\dagger}\right]\right\}=\delta_{q q^{\prime}},} \\
{\left[b_{q}, b_{q^{\prime}}\right]=\left[b_{q}^{\dagger}, b_{q^{\prime}}^{\dagger}\right]=0 .}
\end{gathered}
$$

Dessa forma, o Hamiltoniano pode ser reescrito em termos destes operadores como:

$$
H_{f}=\sum_{q} \hbar \omega_{q}\left[b_{q}^{\dagger} b_{q}+\frac{1}{2}\right]
$$

Nota-se que o Hamiltoniano tem a forma de um oscilador harmônico simples [56] para cada vetor de onda $q$. Logo, para um dado vetor de onda $q$, tem-se uma autoenergia correspondente:

$$
E_{q}=\hbar \omega_{q}\left(n_{q}+\frac{1}{2}\right)
$$

Seja $|0\rangle$ a autofunção de $H_{f}$ de menor autoenergia, então o estado do sistema num instante qualquer é dado por:

$$
\Psi=\left|n_{q_{1}}, \ldots, n_{q_{N}}\right\rangle=\prod_{q_{i}}\left|n_{q_{i}}\right\rangle=\prod_{q_{i}} \frac{\left(b_{q_{i}}^{\dagger}\right)^{n_{q_{i}}}}{\sqrt{n_{q_{i}} !}}|0\rangle
$$

onde $n_{q_{i}} \in \mathbb{N}$.

Podemos interpretar este resultado como um conjunto de $n_{q}$ fônons com energia $\hbar \omega_{q}$ cada, mais uma energia de estado fundamental $\hbar \omega_{q} / 2$ para cada número quântico $q$. Logo, a energia 
$\hbar \omega_{q}$ é a menor energia de excitação acima da energia de ponto zero $\hbar \omega_{q} / 2$ e por este motivo os fônons são chamados de excitações elementares.

Os operadores $b_{q}^{\dagger}$ e $b_{q}$ (Equações 2.13 e 2.14 podem ser interpretados como operadores de criação e de destruição de um fônon com vetor de onda $q$, respectivamente, e $\Psi=\left|n_{q_{1}}, \ldots, n_{q_{N}}\right\rangle$ descreve um sistema com $n_{q_{1}}$ fônons com vetor de onda $q_{1}, n_{q_{2}}$ fônons com vetor de onda $q_{2}$ etc.

A evolução temporal de $b$ e $b^{\dagger}$ na representação de Heisenberg é dada por:

$$
\begin{aligned}
i \hbar \frac{\partial b_{q}}{\partial t} & =\left[b_{q}, H_{f}\right]= \\
& =\hbar \omega_{q}\left(b_{q} b_{q}^{\dagger} b_{q}-b_{q}^{\dagger} b_{q} b_{q}\right)= \\
& =\hbar \omega_{q}\left[b_{q}, b_{q}^{\dagger}\right] b_{q}= \\
& =\hbar \omega_{q} b_{q},
\end{aligned}
$$

de onde se obtém:

$$
b_{q}(t)=e^{-i \omega_{q} t} b_{q}(0) \quad \Longrightarrow \quad b_{q}^{\dagger}(t)=e^{i \omega_{q} t} b_{q}^{\dagger}(0)
$$

Portanto, os operadores $b_{q}^{\dagger}$ criam uma distorção coletiva da rede com frequência $\omega_{q}$ e a resolução espacial desta distorção é dada no espaço recíproco e no espaço real respectivamente por:

$$
\begin{gathered}
X_{q}(t)=\left(\frac{\hbar}{2 M \omega_{q}}\right)^{\frac{1}{2}}\left(e^{-i \omega_{q} t} b_{q}+e^{i \omega_{q} t} b_{-q}^{\dagger}\right), \\
X_{J}(t)=\frac{1}{\sqrt{N}} \sum_{q}\left(\frac{\hbar}{2 M \omega_{q}}\right)^{\frac{1}{2}}\left(e^{-i \omega_{q} t} b_{q}+e^{i \omega_{q} t} b_{-q}^{\dagger}\right) e^{i q J a},
\end{gathered}
$$

o que fornece a amplitude quantizada de vibração da rede no íon $J$ no tempo $t$.

Devido à forma da relação de dispersão $\omega_{q}=2 \omega\left|\sin \frac{q a}{2}\right|$, estes fônons são chamados de acústicos, já que no limite $q \rightarrow 0$ tem-se $\omega_{q}=\omega q a=v_{S} q$, com $v_{S}$ a velocidade do som. Porém, se considerarmos dois sítios distintos por célula unitária, a simetria de translação dada pela constante de rede $a$ é quebrada e um novo ramo aparece na relação de dispersão (de maneira análoga ao tratamento dos elétrons numa rede de Bravais, onde surgem as bandas de Bloch). Como o novo ramo nunca se aproxima do zero de energia, para excitar estes fônons é necessário fornecer maiores energias através de, por exemplo, incidência de luz. Por essa razão, estes fônons são chamados de óticos.

A fim de generalizar os resultados anteriores para o caso de $p$ sítios por célula unitária, o número $N$ deve ser interpretado não mais como o número de átomos da rede, mas como o 
número de células unitárias. Nesse caso, teremos um ramo acústico e $(p-1)$ ramos óticos, e para identificá-los introduz-se um índice $\lambda$ no Hamiltoniano:

$$
H_{f}=\sum_{q, \lambda} \hbar \omega_{q, \lambda}\left[b_{q, \lambda}^{\dagger} b_{q, \lambda}+\frac{1}{2}\right] .
$$

A fim de generalizar o problema para três dimensões, procedemos de forma análoga ao caso unidimensional. Considera-se novamente que os íons realizam pequenos deslocamentos em torno de suas posições de equilíbrio, ou seja:

$$
\mathbf{R}_{J}=\mathbf{R}_{J}^{0}+\mathbf{Q}_{J}
$$

onde $\mathbf{R}_{J}^{0}$ é a posição de equilíbrio do $J$-ésimo íon e $\mathbf{Q}_{J}$ é o respectivo deslocamento. Assumindo que o potencial entre os íons seja do tipo campo de força central:

$$
V_{i o n}=\sum_{I<J} V\left(\mathbf{R}_{I}-\mathbf{R}_{J}\right)
$$

as posições de equilíbrio dos íons são determinadas pela condição de força nula:

$$
\mathbf{F}_{J}=\sum_{I} \nabla V\left(\mathbf{R}_{I}^{0}-\mathbf{R}_{J}^{0}\right)=0
$$

para cada íon $J$. A função potencial é obtida pela expansão em série de Taylor em torno das posições de equilíbrio:

$$
\begin{aligned}
V_{i o n}= & \sum_{I<J} V\left(\mathbf{R}_{I}^{0}-\mathbf{R}_{J}^{0}\right)+\sum_{I<J}\left(\mathbf{Q}_{I}-\mathbf{Q}_{J}\right) \nabla V\left(\mathbf{R}_{I}^{0}-\mathbf{R}_{J}^{0}\right)+ \\
& +\frac{1}{2} \sum_{I<J} \sum_{\mu, \nu}\left(\mathbf{Q}_{I}-\mathbf{Q}_{J}\right)_{\mu}\left(\mathbf{Q}_{I}-\mathbf{Q}_{J}\right)_{\nu} \frac{\partial^{2} V\left(\mathbf{R}_{I}^{0}-\mathbf{R}_{J}^{0}\right)}{\partial \mathbf{R}_{\mu} \partial \mathbf{R}_{\nu}}+O\left(\mathbf{Q}^{3}\right) .
\end{aligned}
$$

A matriz $\mathbf{C}(\mathbf{R})$, cujos elementos são dados por $C_{\mu \nu}(\mathbf{R})=\partial^{2} V\left(\mathbf{R}^{0}\right) / \partial \mathbf{R}_{\mu} \partial \mathbf{R}_{\nu}$, onde $\mathbf{R}^{0}=$ $\left\{\mathbf{R}_{I}^{0}-\mathbf{R}_{J}^{0}: I<J\right\}$, é conhecida como matriz de constantes de forças interatômicas e sua transformada de Fourier $\mathbf{C}(\mathbf{q})$ é a matriz dinâmica, com elementos $C_{\mu \nu}(\mathbf{q})=\sum_{\mathbf{R}^{0}} C_{\mu \nu}\left(\mathbf{R}^{0}\right) e^{-i \mathbf{q} \cdot \mathbf{R}^{0}}$. Nota-se que $\mathbf{C}(\mathbf{R})$ possui as seguintes propriedades:

$$
\mathbf{C}^{T}\left(\mathbf{R}^{0}\right)=\mathbf{C}\left(\mathbf{R}^{0}\right)
$$


pois $\partial^{2} V\left(\mathbf{R}^{0}\right) / \partial \mathbf{R}_{\nu} \partial \mathbf{R}_{\mu}=\partial^{2} V\left(\mathbf{R}^{0}\right) / \partial \mathbf{R}_{\mu} \partial \mathbf{R}_{\nu}$

$$
\sum_{\mathbf{R}^{0}} \mathbf{C}\left(\mathbf{R}^{0}\right)=0
$$

por conservação de momento, e

$$
\mathbf{C}\left(\mathbf{R}^{0}\right)=\mathbf{C}\left(-\mathbf{R}^{0}\right)
$$

devido à simetria de inversão da rede de Bravais monoatômica. A partir destas propriedades, pode-se obter uma expressão para a matriz dinâmica:

$$
\begin{aligned}
\mathbf{C}(\mathbf{q}) & =\sum_{\mathbf{R}^{0}} \mathbf{C}\left(\mathbf{R}^{0}\right) e^{-i \mathbf{q} \cdot \mathbf{R}^{0}}= \\
& =\frac{1}{2}\left[\sum_{\mathbf{R}^{0}} \mathbf{C}\left(\mathbf{R}^{0}\right) e^{-i \mathbf{q} \cdot \mathbf{R}^{0}}+\sum_{\mathbf{R}^{0}} \mathbf{C}\left(-\mathbf{R}^{0}\right) e^{i \mathbf{q} \cdot \mathbf{R}^{0}}\right]= \\
& =\frac{1}{2}\left[\sum_{\mathbf{R}^{0}} \mathbf{C}\left(\mathbf{R}^{0}\right) e^{-i \mathbf{q} \cdot \mathbf{R}^{0}}+\sum_{\mathbf{R}^{0}} \mathbf{C}\left(-\mathbf{R}^{0}\right) e^{i \mathbf{q} \cdot \mathbf{R}^{0}}\right]-\sum_{\mathbf{R}^{0}} \mathbf{C}\left(\mathbf{R}^{0}\right)= \\
& =\frac{1}{2} \sum_{\mathbf{R}^{0}} \mathbf{C}\left(\mathbf{R}^{0}\right)\left[e^{-i \mathbf{q} \cdot \mathbf{R}^{0}}+e^{i \mathbf{q} \cdot \mathbf{R}^{0}}-2\right]= \\
& =-\sum_{\mathbf{R}^{0}} \mathbf{C}\left(\mathbf{R}^{0}\right)\left[1-\cos \left(\mathbf{q} \cdot \mathbf{R}^{0}\right)\right]= \\
& =-2 \sum_{\mathbf{R}^{0}} \mathbf{C}\left(\mathbf{R}^{0}\right) \sin ^{2}\left(\frac{\mathbf{q} \cdot \mathbf{R}^{0}}{2}\right) .
\end{aligned}
$$

Dentro da aproximação harmônica (considerando somente os termos até a segunda ordem na expansão da superfície de energia potencial, Equação 2.28, , o deslocamento nuclear dependente do tempo pode ser escrito como:

$$
\mathbf{Q}_{J}(t)=\mathbf{R}_{J}(t)-\mathbf{R}_{J}^{0}(t) \equiv \xi e^{i\left(\mathbf{q} \cdot \mathbf{R}_{J}^{0}-\omega t\right)} .
$$

Inserindo na $2^{\underline{a}}$ lei de Newton:

$$
M \frac{\partial^{2} \mathbf{Q}_{J}(t)}{\partial t^{2}}=-\omega^{2} M \mathbf{Q}_{J}(t)=-\nabla V_{i o n}=-\sum_{\mathbf{R}_{J}^{0}} \mathbf{C}\left(\mathbf{R}_{I}^{0}-\mathbf{R}_{J}^{0}\right) \mathbf{Q}_{J}(t)
$$

e tomando a transformada de Fourier, podemos escrever este resultado em termos da matriz dinâmica $\mathbf{C}(\mathbf{q})$ :

$$
\omega^{2} M \xi=\mathbf{C}(\mathbf{q}) \xi
$$


Para cada valor de $\mathbf{q}$, pode-se obter um conjunto ortonormal de autovetores $\left\{\xi_{\mathbf{q}, 1}, \xi_{\mathbf{q}, 2}, \xi_{\mathbf{q}, 3}\right\}$, conhecidos como vetores de polarização, que especificam a direção vibracional do íon (para um fônon longitudinal $\xi_{\mathbf{q}, \lambda}$ é paralelo a $\mathbf{q}$, enquanto que para um fônon transversal $\xi_{\mathbf{q}, \lambda}$ é perpendicular a q).

Refazendo o mesmo procedimento de segunda quantização realizado para a cadeia unidimensional, obtemos:

$$
\begin{gathered}
\mathbf{Q}_{\mathbf{q}, \lambda}(t)=\left(\frac{\hbar}{2 M \omega_{\mathbf{q}, \lambda}}\right)^{\frac{1}{2}} \xi_{\mathbf{q}, \lambda}\left(e^{-i \omega_{\mathbf{q}, \lambda} t} b_{\mathbf{q}, \lambda}+e^{i \omega_{\mathbf{q}, \lambda} t} b_{-\mathbf{q}, \lambda}^{\dagger}\right) \\
\mathbf{Q}_{J, \lambda}(t)=\frac{1}{\sqrt{N}} \sum_{\mathbf{q}, \lambda}\left(\frac{\hbar}{2 M \omega_{\mathbf{q}, \lambda}}\right)^{\frac{1}{2}} \xi_{\mathbf{q}, \lambda}\left(e^{-i \omega_{\mathbf{q}, \lambda} t} b_{\mathbf{q}, \lambda}+e^{i \omega_{\mathbf{q}, \lambda} t} b_{-\mathbf{q}, \lambda}^{\dagger}\right) e^{i \mathbf{q} \cdot \mathbf{R}_{J}^{0}}
\end{gathered}
$$

Também de maneira análoga ao caso unidimensional, se tivermos $p$ íons por célula unitária, o valor $N$ passa a ser interpretado como o número de células unitárias e, nesse caso, o índice $\lambda$ percorre $3 p$ modos, sendo 3 acústicos e $3(p-1)$ óticos.

Por fim, desconsiderando-se o termo constante do potencial (Equação 2.28), o Hamiltoniano na aproximação harmônica pode ser escrito como:

$$
H_{f}=\frac{1}{2 M} \sum_{\mathbf{q}, \lambda}\left(\mathbf{P}_{\mathbf{q}, \lambda} \cdot \mathbf{P}_{-\mathbf{q}, \lambda}+M^{2} \omega_{\mathbf{q}, \lambda}^{2} \mathbf{Q}_{\mathbf{q}, \lambda} \cdot \mathbf{Q}_{-\mathbf{q}, \lambda}\right)=\sum_{\mathbf{q}, \lambda} \hbar \omega_{\mathbf{q}, \lambda}\left(b_{\mathbf{q}, \lambda}^{\dagger} b_{\mathbf{q}, \lambda}+\frac{1}{2}\right)
$$

onde $\mathbf{P}_{\mathbf{q}, \lambda}=M \dot{\mathbf{Q}}_{\mathbf{q}, \lambda}$ é o momento conjugado. Novamente, para cada vetor de onda $\mathbf{q}$ e modo $\lambda$, tem-se o Hamiltoniano de um oscilador harmônico.

\subsubsection{Elétrons}

Os elétrons são férmions, isto é, partículas que obedecem à estatística de Fermi-Dirac. Também se pode definir para estas partículas operadores de criação e aniquilação, mas estes devem obedecer ao princípio de exclusão de Pauli, ou seja, há que se escolher um conjunto ortonormal arbitrário de funções de partícula única em que cada estado $\mathbf{k}_{j}$ pode ser ocupado por 0 ou 1 partícula:

$$
\begin{aligned}
c_{\mathbf{k}_{j}}^{\dagger}\left|\ldots, 0_{\mathbf{k}_{j}}, \ldots\right\rangle & =\left|\ldots, 1_{\mathbf{k}_{j}}, \ldots\right\rangle, & & c_{\mathbf{k}_{j}}\left|\ldots, 0_{\mathbf{k}_{j}}, \ldots\right\rangle=0, \\
c_{\mathbf{k}_{j}}^{\dagger}\left|\ldots, 1_{\mathbf{k}_{j}}, \ldots\right\rangle & =0, & & c_{\mathbf{k}_{j}}\left|\ldots, 1_{\mathbf{k}_{j}}, \ldots\right\rangle=\left|\ldots, 0_{\mathbf{k}_{j}}, \ldots\right\rangle .
\end{aligned}
$$


A partir dessas relações verifica-se que $c_{\mathbf{k}_{j}}^{\dagger} c_{\mathbf{k}_{j}}$ atuando em um estado eletrônico qualquer do sistema $\Psi=\left|n_{\mathbf{k}_{1}}, \ldots, n_{\mathbf{k}_{j}}, \ldots\right\rangle$, age como um operador número do estado $\mathbf{k}_{j}$ :

$$
c_{\mathbf{k}_{j}}^{\dagger} c_{\mathbf{k}_{j}}\left|n_{\mathbf{k}_{1}}, \ldots, n_{\mathbf{k}_{j}}, \ldots\right\rangle=n_{\mathbf{k}_{j}}\left|n_{\mathbf{k}_{1}}, \ldots, n_{\mathbf{k}_{j}}, \ldots\right\rangle
$$

e, também, que:

$$
c_{\mathbf{k}_{j}} c_{\mathbf{k}_{j}}^{\dagger}\left|n_{\mathbf{k}_{1}}, \ldots, n_{\mathbf{k}_{j}}, \ldots\right\rangle=\left(1-n_{\mathbf{k}_{j}}\right)\left|n_{\mathbf{k}_{1}}, \ldots, n_{\mathbf{k}_{j}}, \ldots\right\rangle
$$

Com as Equações 2.40 2.41, verifica-se que os operadores criação e destruição de elétrons obedecem às seguintes relações de anticomutação:

$$
\begin{gathered}
\left\{c_{\mathbf{k}_{j}}, c_{\mathbf{k}_{i}}^{\dagger}\right\}=\delta_{\mathbf{k}_{j} \mathbf{k}_{i}}, \\
\left\{c_{\mathbf{k}_{j}}, c_{\mathbf{k}_{i}}\right\}=\left\{c_{\mathbf{k}_{j}}^{\dagger}, c_{\mathbf{k}_{i}}^{\dagger}\right\}=0 .
\end{gathered}
$$

Tipicamente, o Hamiltoniano para férmions tem a forma:

$$
H_{e}=\sum_{j}\left[\frac{\mathbf{p}_{j}^{2}}{2 m}+U\left(\mathbf{r}_{j}\right)+\frac{1}{2} \sum_{j \neq l} V\left(\mathbf{r}_{l}-\mathbf{r}_{j}\right)\right]
$$

onde os elétrons interagem com um potencial externo $U(\mathbf{r})$ e entre si por meio de interações elétron-elétron $\left.\right|^{2} V\left(\mathbf{r}_{l}-\mathbf{r}_{j}\right)$. Desconsiderando-se as interações elétron-elétron, as funções de partícula única definidas acima são autofunções do Hamiltoniano que, por sua vez, pode ser escrito como 3

$$
H_{e}=\sum_{\mathbf{k}} E_{\mathbf{k}} c_{\mathbf{k}}^{\dagger} c_{\mathbf{k}}
$$

A evolução temporal de $c$ e $c^{\dagger}$ na representação de Heisenberg é, então, dada por:

\footnotetext{
${ }^{2}$ Este termo aparece dividido por 2 , já que as somatórias sobre $l$ e $j$ contam cada par duas vezes.

${ }^{3}$ As interações elétron-elétron podem ser consideradas com uma aproximação de campo médio. Nesse caso, o Hamiltoniano tem a mesma forma, porém os autoestados serão estados de quasipartículas.
} 


$$
\begin{aligned}
i \hbar \partial_{t} c_{\mathbf{k}} & =\left[c_{\mathbf{k}}, H_{e}\right]= \\
& =\sum_{\mathbf{k}} E_{\mathbf{k}}\left[c_{\mathbf{k}}, c_{\mathbf{k}}^{\dagger} c_{\mathbf{k}}\right]= \\
& =\sum_{\mathbf{k}} E_{\mathbf{k}}\left(\left\{c_{\mathbf{k}}, c_{\mathbf{k}}^{\dagger}\right\} c_{\mathbf{k}}-c_{\mathbf{k}}^{\dagger}\left\{c_{\mathbf{k}}, c_{\mathbf{k}}\right\}\right)= \\
& =E_{\mathbf{k}} c_{\mathbf{k}},
\end{aligned}
$$

de onde se obtém:

$$
c_{\mathbf{k}}(t)=e^{-i \frac{E_{\mathbf{k}}}{\hbar} t} c_{\mathbf{k}}(0) \quad \Longrightarrow \quad c_{\mathbf{k}}^{\dagger}(t)=e^{i \frac{E_{\mathbf{k}}}{\hbar} t} c_{\mathbf{k}}^{\dagger}(0) .
$$

\subsubsection{Interação Elétron-Fônon}

Agora possuímos o arcabouço teórico para descrever cada tipo de partícula separadamente, onde, sem interagirem entre si, cada uma vive em seu espaço de Fock. Em um sistema de elétrons e fônons interagentes, o vetor de estado $|\Psi\rangle$ pode ser expandido em termos de uma base ortonormal de estados de multipartículas $|\mathcal{M}\rangle$ :

$$
|\Psi\rangle=\sum_{\mathcal{M}} \psi_{\mathcal{M}}|\mathcal{M}\rangle
$$

Um estado típico desta base pode parecer como algo do tipo:

$$
|\mathcal{M}\rangle=\left|1_{\mathbf{k}_{1}}, 0_{\mathbf{k}_{2}}, 1_{\mathbf{k}_{3}}, \ldots\right\rangle_{e}\left|0_{\mathbf{q}_{1}}, 8449_{\mathbf{q}_{2}}, 124_{\mathbf{q}_{3}}, \ldots\right\rangle_{f}
$$

A interação elétron-fônon acontece basicamente de duas formas, com a emissão ou com a absorção de fônons. Ou seja, o Hamiltoniano da interação $H_{e f}$ deve ser escrito como a soma das contribuições devido a estes dois processos:

$$
H_{e f}=\sum_{\mathbf{k}, \mathbf{q}}\left[H_{e f}^{\text {emissão }}(\mathbf{k}, \mathbf{q})+H_{e f}^{\text {absorção }}(\mathbf{k}, \mathbf{q})\right]
$$


Em termos dos operadores criação e destruição de fônons e de elétrons, os termos do Hamiltoniano serão dados por: 4

$$
\begin{gathered}
H_{e f}^{\text {emissão }}(\mathbf{k}, \mathbf{q})=M_{\mathbf{q}}^{\text {emissão }} b_{-\mathbf{q}}^{\dagger} c_{\mathbf{k}+\mathbf{q}}^{\dagger} c_{\mathbf{k}}, \\
H_{e f}^{\text {absorção }}(\mathbf{k}, \mathbf{q})=M_{\mathbf{q}}^{\text {absorção }} b_{-\mathbf{q}} c_{\mathbf{k}-\mathbf{q}}^{\dagger} c_{\mathbf{k}} .
\end{gathered}
$$

No processo de emissão (Figura 2.2 à esquerda), um elétron no estado $\mathbf{k}$ é aniquilado $\left(c_{\mathbf{k}}\right)$ e é criado novamente $\left(c_{\mathbf{k}+\mathbf{q}}^{\dagger}\right)$ no estado $\mathbf{k}+\mathbf{q}$ simultaneamente com a emissão de um fônon $\left(b_{-\mathbf{q}}^{\dagger}\right)$ com vetor de onda $-\mathbf{q}$, de forma a conservar energia-momento. No processo de absorção (Figura 2.2 à direita), um elétron no estado $\mathbf{k}$ é aniquilado $\left(c_{\mathbf{k}}\right)$ e é criado novamente $\left(c_{\mathbf{k}+\mathbf{q}}^{\dagger}\right)$ no estado $\mathbf{k}+\mathbf{q}$ simultaneamente com a absorção de um fônon $\left(b_{\mathbf{q}}\right)$ com vetor de onda $\mathbf{q}$, conservando energia-momento.
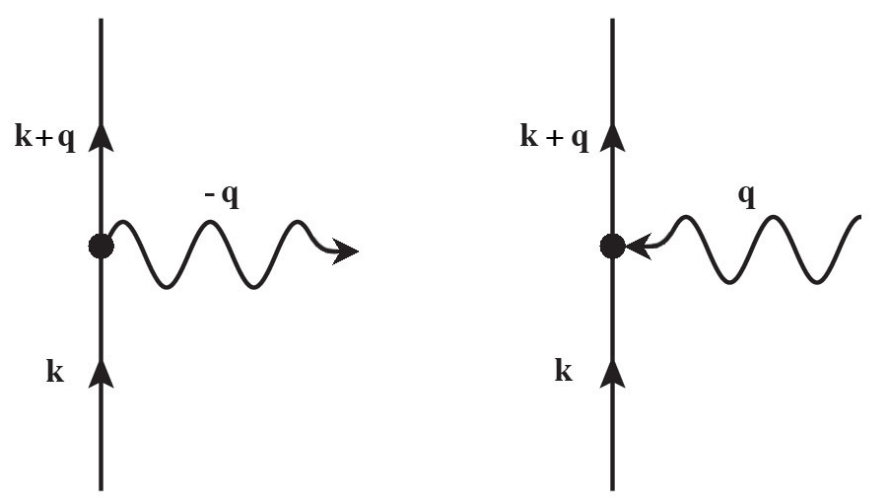

Figura 2.2: Vértice básico da interação elétron-fônon: à esquerda, com a emissão de um fônon, e, à direita, com a absorção de um fônon.

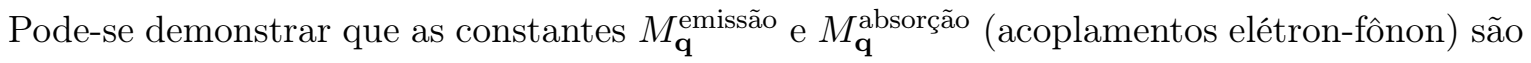
semelhantes e dadas por 60, 63]:

$$
M_{\mathbf{q}, \lambda}=K_{\mathbf{q}, \lambda}\left(\frac{\hbar}{2 M N \omega_{\mathbf{q}, \lambda}}\right)^{\frac{1}{2}}
$$

onde $K_{\mathbf{q}, \lambda}$ é uma constante que depende do vetor de polarização $\xi_{\mathbf{q}, \lambda}$ e do potencial de interação elétron-íon $V_{e i}(\mathbf{q})$.

\footnotetext{
${ }^{4}$ As somatórias nos modos normais de vibração $\lambda$ foram omitidas por enquanto para não tornar a notação demasiadamente carregada.
} 
Utilizando-se os resultados obtidos acima e nas Seções 2.1.1 e 2.1.2 anteriores, pode-se finalmente escrever o Hamiltoniano do sistema de elétrons e fônons acoplados:

$$
\begin{aligned}
\mathscr{H} & =H_{e}+H_{f}+H_{e f}, \\
H_{e} & =\sum_{\mathbf{k}} E_{\mathbf{k}} c_{\mathbf{k}}^{\dagger} c_{\mathbf{k}}, \\
H_{f} & =\sum_{\mathbf{q}, \lambda} \hbar \omega_{\mathbf{q}, \lambda}\left(b_{\mathbf{q}, \lambda}^{\dagger} b_{\mathbf{q}, \lambda}+\frac{1}{2}\right), \\
H_{e f} & =\sum_{\mathbf{k}} \sum_{\mathbf{q}, \lambda} M_{\mathbf{q}, \lambda} c_{\mathbf{k}+\mathbf{q}}^{\dagger} c_{\mathbf{k}}\left(b_{\mathbf{q}, \lambda}+b_{-\mathbf{q}, \lambda}^{\dagger}\right),
\end{aligned}
$$

onde foram desconsideradas as interações elétron-elétron ${ }^{5}\left(H_{e}\right.$ é dada pela soma de Hamiltonianos de partícula única) e fônon-fônon ( $H_{f}$ é dada pela soma de osciladores desacoplados). Entretanto, como discutido no início do capítulo, encontrar as autofunções desse Hamiltoniano é um problema extremamente difícil, cuja complexidade aumenta rapidamente com a quantidade de partículas que constituem o sistema. Uma alternativa é utilizar o formalismo das Funções de Green descrito a seguir.

\subsection{Funções de Green no Equilíbrio}

Antes de listar uma série de definições abstratas a respeito de funções de Green, vamos dar um passo para atrás e considerar o exemplo de partículas livres:

$$
i \hbar \frac{\partial}{\partial t}\left|\Psi_{0}(t)\right\rangle=H_{0}\left|\Psi_{0}(t)\right\rangle
$$

onde $H_{0}$ pode ser $H_{e}$ no caso de elétrons não-interagentes (Equação 2.55) ou $H_{f}$ no caso de fônons não-interagentes (Equação 2.56).

A igualdade acima pode ser escrita em termos de um operador diferencial linear $L=i \hbar \frac{\partial}{\partial t}-H_{0}$ como:

$$
L\left|\Psi_{0}(t)\right\rangle=f(t)
$$

onde $f(t)=0$. Logo, pela teoria geral de equações diferenciais, podemos associar à Equação 2.58 dois tipos de funções de Green, $G_{0}^{r}(t)$ e $G_{0}^{a}(t)$, tais que:

\footnotetext{
${ }^{5}$ As interações elétron-elétron podem ser consideradas com uma aproximação de campo médio e, neste caso, $c_{\mathbf{q}}^{\dagger}$ e $c_{\mathbf{q}}^{\dagger}$ são operadores criação e aniquilação de quasipartículas (ver Nota de Rodapé 3 .
} 


$$
L G_{0}^{r, a}(t)=\left(i \hbar \frac{\partial}{\partial t}-H_{0}\right) G_{0}^{r, a}(t)=\mathbb{1} \delta(t)
$$

com as seguintes condições de contorno:

$$
\begin{aligned}
& G_{0}^{r}(t)=0, \quad t<t_{0}=0, \\
& G_{0}^{a}(t)=0, \quad t>t_{0}=0 .
\end{aligned}
$$

Pode-se verificar por substituição direta que, com as condições de contorno acima, as soluções das equações de movimento (Equação 2.60) são dadas por:

$$
\begin{aligned}
& G_{0}^{r}(t)=\left\{\begin{array}{cl}
-\frac{i}{\hbar} e^{-i H_{0} t / \hbar}, & t>t_{0}=0, \\
0, & t<t_{0}=0,
\end{array}\right. \\
& G_{0}^{a}(t)=\left\{\begin{array}{cl}
0, & t>t_{0}=0, \\
\frac{i}{\hbar} e^{-i H_{0} t / \hbar}, & t<t_{0}=0 .
\end{array}\right.
\end{aligned}
$$

Nota-se que $G_{0}^{r}(t)$ é proporcional ao operador de evolução temporal $U\left(t, t_{0}=0\right)=e^{-i H_{0}\left(t-t_{0}\right) / \hbar}$ $=e^{-i H_{0} t / \hbar}$, para $t>t_{0}=0$. Logo, podemos escrever a evolução temporal do estado $\left|\Psi_{0}(t)\right\rangle$ em termos da $G_{0}^{r}(t)$ como:

$$
\left|\Psi_{0}(t)\right\rangle=i \hbar G_{0}^{r}\left(t, t_{0}\right)\left|\Psi_{0}\left(t_{0}\right)\right\rangle
$$

onde a função de Green $G_{0}^{r}\left(t, t_{0}\right)$ é identificada como um propagador do estado $\left|\Psi_{0}(t)\right\rangle$ de $t_{0}$ até $t$ e, por esse motivo, é chamada de função de Green retardada.

De modo análogo, para $t<t_{0}$, a função de Green $G_{0}^{a}(t)$ relaciona o estado $\left|\Psi_{0}\left(t_{0}\right)\right\rangle$ com seu estado passado $\left|\Psi_{0}(t)\right\rangle$ da seguinte maneira:

$$
\left|\Psi_{0}(t)\right\rangle=-i \hbar G_{0}^{a}\left(t, t_{0}\right)\left|\Psi_{0}\left(t_{0}\right)\right\rangle
$$

onde a função de Green $G_{0}^{a}\left(t, t_{0}\right)$ é identificada como um propagador do estado $\left|\Psi_{0}(t)\right\rangle$ de um tempo $t$ no passado até o presente $t_{0}$ e, por esse motivo, é chamada de função de Green avançada. 
No equilíbrio seria necessário, a princípio, somente uma função de Green, já que, como o Hamiltoniano é hermitiano, as funções de Green retardada e avançada são relacionadas por $\left[G_{0}^{r}\left(t, t_{0}\right)\right]^{\dagger}=G_{0}^{a}\left(t_{0}, t\right)$. Contudo, seguindo Langreth 64 , é conveniente trabalhar com o seguinte conjunto redundante de funções de Green de partícula única com diferentes ordenamentos temporais ${ }^{6}$

$$
\begin{aligned}
G^{<}\left(t, t^{\prime}\right) & \equiv \pm \frac{i}{\hbar}\left\langle\Psi_{\mathscr{H}}^{\dagger}\left(t^{\prime}\right) \Psi_{\mathscr{H}}(t)\right\rangle \\
G^{>}\left(t, t^{\prime}\right) & \equiv-\frac{i}{\hbar}\left\langle\Psi_{\mathscr{H}}(t) \Psi_{\mathscr{H}}^{\dagger}\left(t^{\prime}\right)\right\rangle \\
G^{r}\left(t, t^{\prime}\right) & \equiv-\frac{i}{\hbar} \theta\left(t-t^{\prime}\right)\left\langle\left[\Psi_{\mathscr{H}}(t), \Psi_{\mathscr{H}}^{\dagger}\left(t^{\prime}\right)\right]_{ \pm}\right\rangle=\theta\left(t-t^{\prime}\right)\left[G^{>}\left(t, t^{\prime}\right)-G^{<}\left(t, t^{\prime}\right)\right], \\
G^{a}\left(t, t^{\prime}\right) & \equiv \frac{i}{\hbar} \theta\left(t^{\prime}-t\right)\left\langle\left[\Psi_{\mathscr{H}}(t), \Psi_{\mathscr{H}}^{\dagger}\left(t^{\prime}\right)\right]_{ \pm}\right\rangle=-\theta\left(t^{\prime}-t\right)\left[G^{>}\left(t, t^{\prime}\right)-G^{<}\left(t, t^{\prime}\right)\right],
\end{aligned}
$$

onde $\Psi_{\mathscr{H}}(t)$ é o operador de campo na representação de Heisenberg e o sinal superior se aplica a férmions e o inferior a bósons ([, ]+ representa o anticomutador). As novas funções de $G^{<}\left(t, t^{\prime}\right)$ e $G^{>}\left(t, t^{\prime}\right)$ são chamadas de lesser e greater, respectivamente, e permitem manter o controle das partículas que entram e saem do sistema, também respectivamente. Estas funções estão diretamente relacionadas a observáveis e a propriedades cinéticas, como a densidade e o fluxo de partículas, o que ficará evidente no Teorema de Flutuação-Dissipação que será apresentado na Seção 2.3 .

Pelas equações acima, podemos explicitar a dependência linear entre as funções de Green da seguinte forma:

$$
G^{r}\left(t, t^{\prime}\right)-G^{a}\left(t, t^{\prime}\right)=G^{>}\left(t, t^{\prime}\right)-G^{<}\left(t, t^{\prime}\right)
$$

No caso dos elétrons não-interagentes, as funções de Green são dadas por:

$$
\begin{aligned}
G_{0}^{<}\left(\mathbf{k} t, \mathbf{k}^{\prime} t^{\prime}\right) & =\frac{i}{\hbar}\left\langle c_{\mathbf{k}^{\prime}}^{\dagger}\left(t^{\prime}\right) c_{\mathbf{k}}(t)\right\rangle=\frac{i}{\hbar} e^{-i \frac{E_{\mathbf{k}}}{\hbar}\left(t-t^{\prime}\right)}\left\langle n_{\mathbf{k}}\right\rangle \delta_{\mathbf{k k}^{\prime}}, \\
G_{0}^{>}\left(\mathbf{k} t, \mathbf{k}^{\prime} t^{\prime}\right) & =-\frac{i}{\hbar}\left\langle c_{\mathbf{k}}(t) c_{\mathbf{k}^{\prime}}^{\dagger}\left(t^{\prime}\right)\right\rangle=-\frac{i}{\hbar} e^{-i \frac{E_{\mathbf{k}}}{\hbar}\left(t-t^{\prime}\right)}\left(1-\left\langle n_{\mathbf{k}}\right\rangle\right) \delta_{\mathbf{k k}^{\prime}}, \\
G_{0}^{r}\left(\mathbf{k} t, \mathbf{k}^{\prime} t^{\prime}\right) & =-\frac{i}{\hbar} \theta\left(t-t^{\prime}\right)\left\langle\left\{c_{\mathbf{k}}(t), c_{\mathbf{k}^{\prime}}^{\dagger}\left(t^{\prime}\right)\right\}\right\rangle=-\frac{i}{\hbar} \theta\left(t-t^{\prime}\right) e^{-i \frac{E_{\mathbf{k}}}{\hbar}\left(t-t^{\prime}\right)} \delta_{\mathbf{k k}^{\prime}}, \\
G_{0}^{a}\left(\mathbf{k} t, \mathbf{k}^{\prime} t^{\prime}\right) & =\frac{i}{\hbar} \theta\left(t^{\prime}-t\right)\left\langle\left\{c_{\mathbf{k}}(t), c_{\mathbf{k}^{\prime}}^{\dagger}\left(t^{\prime}\right)\right\}\right\rangle=\frac{i}{\hbar} \theta\left(t^{\prime}-t\right) e^{-i \frac{E_{\mathbf{k}}}{\hbar}\left(t-t^{\prime}\right)} \delta_{\mathbf{k k}^{\prime}},
\end{aligned}
$$

onde $c_{\mathbf{k}}^{\dagger}$ e $c_{\mathbf{k}}$ são, respectivamente, os mesmos operadores criação e aniquilação do Hamiltoniano eletrônico e $\left\langle n_{\mathbf{k}}\right\rangle=\left\langle c_{\mathbf{k}}^{\dagger} c_{\mathbf{k}}\right\rangle$ é a densidade de partículas. Como no equilíbrio as funções de Green

\footnotetext{
${ }^{6}$ A origem destas quatro funções de Green ficará mais clara na Seção 2.8
} 
dependem somente da diferença de suas variáveis, muitas vezes é conveniente usar as transformadas de Fourier das funções:

$$
\begin{aligned}
G_{0}^{<}(\mathbf{k}, \epsilon) & =2 \pi i\left\langle n_{\mathbf{k}}\right\rangle \delta\left(\epsilon-E_{\mathbf{k}}\right), \\
G_{0}^{>}(\mathbf{k}, \epsilon) & =2 \pi i\left(\left\langle n_{\mathbf{k}}\right\rangle-1\right) \delta\left(\epsilon-E_{\mathbf{k}}\right) \\
G_{0}^{r}(\mathbf{k}, \epsilon) & =\frac{1}{\epsilon-E_{\mathbf{k}}+i \eta} \\
G_{0}^{a}(\mathbf{k}, \epsilon) & =\frac{1}{\epsilon-E_{\mathbf{k}}-i \eta}
\end{aligned}
$$

onde $\eta=0^{+}$é um infinitésimo positivo.

No equilíbrio termodinâmico, onde o sistema está em contato com um reservatório térmico à temperatura $T$ e um reservatório de partículas descrito por um potencial eletroquímico $\mu$, no ensemble grande canônico a matriz densidade é dada por:

$$
\rho=\frac{e^{-\beta H_{0}}}{\operatorname{Tr}\left[e^{-\beta H_{0}}\right]},
$$

onde $\beta=\left(k_{B} T\right)^{-1}$ e onde escolhemos medir a energia das partículas em relação a $\mu=0$, caso contrário teríamos que usar o operador número $N$ explicitamente. Neste caso, a densidade de elétrons será dada pela distribuição de Fermi-Dirac $f\left(E_{\mathbf{k}}, T\right)$ :

$$
\left\langle n_{\mathbf{k}}\right\rangle=\operatorname{Tr}\left[\rho n_{\mathbf{k}}\right]=\frac{\operatorname{Tr}\left[n_{\mathbf{k}} e^{-\beta H_{e}}\right]}{\operatorname{Tr}\left[e^{-\beta H_{e}}\right]}=\frac{\sum_{n_{\mathbf{k}}=0,1} n_{\mathbf{k}} e^{-\beta E_{\mathbf{k}} n_{\mathbf{k}}}}{\sum_{n_{\mathbf{k}}=0,1} e^{-\beta E_{\mathbf{k}} n_{\mathbf{k}}}}=\frac{1}{e^{\beta E_{\mathbf{k}}}+1}=f\left(E_{\mathbf{k}}, T\right) .
$$

Este resultado é válido no equilíbrio termodinâmico. Como veremos mais à frente, em um problema fora do equilíbrio, como o do transporte eletrônico em uma região de espalhamento acoplada a eletrodos submetidos a uma diferença de potencial $V$, o potencial eletroquímico da região de espalhamento não é bem definido e a ocupação eletrônica já não pode mais ser descrita em termos da distribuição de Fermi-Dirac.

A partir do Hamiltoniano descrevendo interações elétron-fônon (Equação 2.57), fica claro que os operadores de fônon relevantes não são os operadores individuais de criação e aniquilação, mas, sim, o operador $\left(b_{\mathbf{q}, \lambda}+b_{-\mathbf{q}, \lambda}^{\dagger}\right)$ que pode ser interpretado como a remoção de um momento q do sistema de fônons (ora pela aniquilação de um fônon de momento q ora pela criação de um fônon de momento $-\mathbf{q}$ ). Assim, as funções de Green dos fônons não-interagentes serão dadas 
por:7

$$
\begin{aligned}
D_{0}^{<}\left(\mathbf{q} t, \mathbf{q}^{\prime} t^{\prime}\right) & =-\frac{i}{\hbar}\left\langle\left(b_{\mathbf{q}^{\prime}}^{\dagger}+b_{\mathbf{q}^{\prime}}\right)\left(t^{\prime}\right)\left(b_{\mathbf{q}}^{\dagger}+b_{\mathbf{q}}\right)(t)\right\rangle= \\
& =-\frac{i}{\hbar}\left(e^{-i \omega_{\mathbf{q}}\left(t-t^{\prime}\right)}\left\langle n_{\mathbf{q}}\right\rangle+e^{i \omega_{\mathbf{q}}\left(t-t^{\prime}\right)}\left(\left\langle n_{\mathbf{q}}\right\rangle+1\right)\right) \delta_{\mathbf{q q}^{\prime}}, \\
D_{0}^{>}\left(\mathbf{q} t, \mathbf{q}^{\prime} t^{\prime}\right) & =-\frac{i}{\hbar}\left\langle\left(b_{\mathbf{q}}^{\dagger}+b_{\mathbf{q}}\right)(t)\left(b_{\mathbf{q}^{\prime}}^{\dagger}+b_{\mathbf{q}^{\prime}}\right)\left(t^{\prime}\right)\right\rangle= \\
& =-\frac{i}{\hbar}\left(e^{-i \omega_{\mathbf{q}}\left(t-t^{\prime}\right)}\left\langle n_{\mathbf{q}}\right\rangle+e^{i \omega_{\mathbf{q}}\left(t-t^{\prime}\right)}\left(\left\langle n_{\mathbf{q}}\right\rangle+1\right)\right) \delta_{\mathbf{q q}^{\prime}}, \\
D_{0}^{r}\left(\mathbf{q} t, \mathbf{q}^{\prime} t^{\prime}\right) & =-\frac{i}{\hbar} \theta\left(t-t^{\prime}\right)\left\langle\left[\left(b_{\mathbf{q}}^{\dagger}+b_{\mathbf{q}}\right)(t),\left(b_{\mathbf{q}^{\prime}}^{\dagger}+b_{\mathbf{q}^{\prime}}\right)\left(t^{\prime}\right)\right]\right\rangle= \\
& =-\frac{i}{\hbar} \theta\left(t-t^{\prime}\right)\left(-e^{i \omega_{\mathbf{q}}\left(t-t^{\prime}\right)}+e^{-i \omega_{\mathbf{q}}\left(t-t^{\prime}\right)}\right) \delta_{\mathbf{q q}^{\prime}}, \\
D_{0}^{a}\left(\mathbf{q} t, \mathbf{q}^{\prime} t^{\prime}\right) & =\frac{i}{\hbar} \theta\left(t^{\prime}-t\right)\left\langle\left[\left(b_{\mathbf{q}}^{\dagger}+b_{\mathbf{q}}\right)(t),\left(b_{\mathbf{q}^{\prime}}^{\dagger}+b_{\mathbf{q}^{\prime}}\right)\left(t^{\prime}\right)\right]\right\rangle= \\
& =\frac{i}{\hbar} \theta\left(t^{\prime}-t\right)\left(-e^{i \omega_{\mathbf{q}}\left(t-t^{\prime}\right)}+e^{-i \omega_{\mathbf{q}}\left(t-t^{\prime}\right)}\right) \delta_{\mathbf{q}_{\mathbf{q}^{\prime}}} .
\end{aligned}
$$

Aplicando a transformada de Fourier (temporal) nas funções, obtém-se:

$$
\begin{aligned}
& D_{0}^{<}(\mathbf{q}, \epsilon)=-2 \pi i\left(\left\langle n_{\mathbf{q}}\right\rangle \delta\left(\epsilon-\hbar \omega_{\mathbf{q}}\right)+\left(\left\langle n_{\mathbf{q}}\right\rangle+1\right) \delta\left(\epsilon+\hbar \omega_{\mathbf{q}}\right)\right) \\
& D_{0}^{>}(\mathbf{q}, \epsilon)=-2 \pi i\left(\left\langle n_{\mathbf{q}}\right\rangle \delta\left(\epsilon+\hbar \omega_{\mathbf{q}}\right)+\left(\left\langle n_{\mathbf{q}}\right\rangle+1\right) \delta\left(\epsilon-\hbar \omega_{\mathbf{q}}\right)\right) \\
& D_{0}^{r}(\mathbf{q}, \epsilon)=\frac{1}{\epsilon-\hbar \omega_{\mathbf{q}}+i \eta}-\frac{1}{\epsilon+\hbar \omega_{\mathbf{q}}+i \eta}, \\
& D_{0}^{a}(\mathbf{q}, \epsilon)=\frac{1}{\epsilon-\hbar \omega_{\mathbf{q}}-i \eta}-\frac{1}{\epsilon+\hbar \omega_{\mathbf{q}}-i \eta}
\end{aligned}
$$

Considerando-se que os fônons estão em equilíbrio termodinâmico, a densidade de partículas será dada pela distribuição de Bose-Einstein $n\left(\omega_{\mathbf{q}}, T\right)$ :

$$
\begin{aligned}
\left\langle n_{\mathbf{q}}\right\rangle=\operatorname{Tr}\left[\rho n_{\mathbf{q}}\right]=\frac{\operatorname{Tr}\left[n_{\mathbf{q}} e^{-\beta H_{f}}\right]}{\operatorname{Tr}\left[e^{-\beta H_{f}}\right]}=\frac{\sum_{n_{\mathbf{q}}=0}^{\infty} n_{\mathbf{q}} e^{-\beta \hbar \omega_{\mathbf{q}} n_{\mathbf{q}}}}{\sum_{n_{\mathbf{q}}=0}^{\infty} e^{-\beta \hbar \omega_{\mathbf{q}} n_{\mathbf{q}}}}= \\
=\frac{e^{-\beta \hbar \omega_{\mathbf{q}}} \sum_{n_{\mathbf{q}}=0}^{\infty} n_{\mathbf{q}} e^{-\beta \hbar \omega_{\mathbf{q}}\left(n_{\mathbf{q}}-1\right)}}{\sum_{n_{\mathbf{q}}=0}^{\infty} e^{-\beta \hbar \omega_{\mathbf{q}} n_{\mathbf{q}}}}=\frac{e^{-\beta \hbar \omega_{\mathbf{q}}} \frac{d}{d\left(e^{-\beta \hbar \omega_{\mathbf{q}}}\right)}\left[\sum_{n_{\mathbf{q}}=0}^{\infty} e^{-\beta \hbar \omega_{\mathbf{q}} n_{\mathbf{q}}}\right]}{\sum_{n_{\mathbf{q}}=0}^{\infty} e^{-\beta \hbar \omega_{\mathbf{q}} n_{\mathbf{q}}}}=
\end{aligned}
$$

\footnotetext{
${ }^{7}$ Foram omitidos os índices $\lambda$ sobre os modos normais para não sobrecarregar as equações.
} 


$$
\begin{aligned}
& =\frac{e^{-\beta \hbar \omega_{\mathbf{q}}} \frac{d}{d\left(e^{-\beta \hbar \omega_{\mathbf{q}}}\right)}\left[\frac{1}{1-e^{-\beta \hbar \omega_{\mathbf{q}}}}\right]}{\frac{1}{1-e^{-\beta \hbar \omega_{\mathbf{q}}}}}=\frac{e^{-\beta \hbar \omega_{\mathbf{q}}} \frac{1}{\left(1-e^{\left.-\beta \hbar \omega_{\mathbf{q}}\right)^{2}}\right.}}{\frac{1}{1-e^{-\beta \hbar \omega_{\mathbf{q}}}}}= \\
& =\frac{1}{e^{\beta \hbar \omega_{\mathbf{q}}}-1}=n\left(\omega_{\mathbf{q}}, T\right),
\end{aligned}
$$

onde as somatórias foram escritas como a soma dos termos de uma série geométrica convergente, o que é válido quando $e^{-\beta \hbar \omega_{\mathbf{q}}}<1$.

Na Seção 7.4.3, será apresentada uma maneira de se descrever a ocupação dos fônons na situação fora do equilíbrio, implementada no programa Inelastic DISORDER (Seção 7.5). No entanto, nos resultados que serão apresentados nesta tese, os fônons foram considerados no equilíbrio.

\subsection{Função Espectral e o Teorema de Flutuação-Dissipação}

Outra função muito útil em diversas aplicações e que será utilizada inúmeras vezes ao longo da tese é a função espectral $A$. Para férmions, vamos definir esta função como: 8

$$
A(\mathbf{k}, \epsilon)=i\left[G^{r}(\mathbf{k}, \epsilon)-G^{a}(\mathbf{k}, \epsilon)\right]=i\left[G^{>}(\mathbf{k}, \epsilon)-G^{<}(\mathbf{k}, \epsilon)\right]
$$

Esta função satisfaz a regra da soma, isto é:

$$
\frac{1}{2 \pi} \int_{-\infty}^{\infty} d \epsilon A(\mathbf{k}, \epsilon)=1
$$

o que pode ser mostrado a partir das definições das funções de Green. Para elétrons não interagentes, por exemplo, a integral é imediata, pois:

$$
\begin{aligned}
& A_{0}(\mathbf{k}, \epsilon)=i\left[G_{0}^{r}(\mathbf{k}, \epsilon)-G_{0}^{a}(\mathbf{k}, \epsilon)\right]=-2 \operatorname{Im}\left[G_{0}^{r}(\mathbf{k}, \epsilon)\right], \\
& A_{0}(\mathbf{k}, \epsilon)=i\left[G_{0}^{>}(\mathbf{k}, \epsilon)-G_{0}^{<}(\mathbf{k}, \epsilon)\right]=2 \pi \delta\left(\epsilon-E_{\mathbf{k}}\right) .
\end{aligned}
$$

Uma aplicação importante é no cálculo da densidade de estados no equilíbrio, que pode ser obtida integrando-se a função espectral sobre todos os possíveis vetores $\mathbf{k}$ :

\footnotetext{
${ }^{8}$ É comum também encontrar a definição da função espectral com o fator $(2 \pi)^{-1}$.
} 


$$
\rho(\epsilon)=\frac{1}{2 \pi} \int \frac{d \mathbf{k}}{(2 \pi)^{3}} A(\mathbf{k}, \epsilon)
$$

Nota-se também, pela Equação 2.93, que a função espectral é diretamente proporcional à parte imaginária da função de Green retardada, que determina domínio de tempo de decaimento, isto é, a dissipação da partícula. Esta observação justifica o nome dado a outro fato importante envolvendo a função espectral no equilíbrio, o Teorema de Flutuação-Dissipação que a relaciona com as funções de Green $G^{<}$e $G^{>}$(também chamadas de função de correlação, o que dá origem ao termo "flutuação" do teorema), da seguinte forma:

$$
\begin{aligned}
& G_{0}^{<}(\mathbf{k}, \epsilon)=i f\left(E_{\mathbf{k}}, T\right) A_{0}(\mathbf{k}, \epsilon), \\
& G_{0}^{>}(\mathbf{k}, \epsilon)=-i\left[1-f\left(E_{\mathbf{k}}, T\right)\right] A_{0}(\mathbf{k}, \epsilon),
\end{aligned}
$$

onde $f\left(E_{\mathbf{k}}, T\right)$ é a distribuição de Fermi-Dirac (Equação 2.81). Portanto, no equilíbrio a função de Green lesser é proporcional à dissipação $A_{0}$ com fator de proporcionalidade dado pela ocupação dos elétrons e pode ser interpretada como a densidade local de partículas. Da mesma maneira, a função de Green greater é proporcional à dissipação $A_{0}$ com fator de proporcionalidade dado pela ocupação de buracos e pode ser interpretada como a densidade local de buracos.

\subsection{Representação de Lehmann}

A representação de Lehmann é o nome dado quando usamos a base de autoestados $\{|n\rangle\}$ do Hamiltoniano total $\mathscr{H}$. Para escrever, por exemplo, a função de Green retardada de elétrons não interagentes nesta representação, partimos da Equação 2.74 e inserimos a igualdade $\sum_{n}|n\rangle\langle n|=1$ no anticomutador:

$$
\begin{aligned}
& G_{0}^{r}\left(\mathbf{k} t, \mathbf{k}^{\prime} t^{\prime}\right)=-\frac{i}{\hbar} \theta\left(t-t^{\prime}\right)\left\langle\left\{c_{\mathbf{k}}(t), c_{\mathbf{k}^{\prime}}^{\dagger}\left(t^{\prime}\right)\right\}\right\rangle= \\
&=-\frac{i}{\hbar} \frac{\theta\left(t-t^{\prime}\right)}{\operatorname{Tr}\left[e^{-\beta H_{0}}\right]} \sum_{n}\left\langle n\left|e^{-\beta \frac{H_{0}}{\hbar}}\left(c_{\mathbf{k}}(t) c_{\mathbf{k}}^{\dagger}\left(t^{\prime}\right)+c_{\mathbf{k}}^{\dagger}\left(t^{\prime}\right) c_{\mathbf{k}}(t)\right)\right| n\right\rangle= \\
&=-\frac{i}{\hbar} \frac{\theta\left(t-t^{\prime}\right)}{\operatorname{Tr}\left[e^{-\beta H_{0}}\right]} \sum_{n, n^{\prime}} e^{-\beta \frac{E_{n}}{\hbar}}\left[\left\langle n\left|c_{\mathbf{k}}\right| n^{\prime}\right\rangle\left\langle n^{\prime}\left|c_{\mathbf{k}}^{\dagger}\right| n\right\rangle e^{i \frac{E_{n}-E_{n^{\prime}}}{\hbar}\left(t-t^{\prime}\right)}+\right. \\
&\left.+\left\langle n\left|c_{\mathbf{k}}^{\dagger}\right| n^{\prime}\right\rangle\left\langle n^{\prime}\left|c_{\mathbf{k}}\right| n\right\rangle e^{-i \frac{E_{n}-E_{n^{\prime}}}{\hbar}\left(t-t^{\prime}\right)}\right] .
\end{aligned}
$$


Tomando a transformada de Fourier, podemos escrever a função de Green retardada com a representação de Lehmann no domínio de energia:

$$
\begin{aligned}
G_{0}^{r}(\mathbf{k}, \epsilon)=-\frac{i}{\hbar} \int_{0}^{\infty} d t \frac{e^{i(\epsilon+i \eta) t / \hbar}}{\operatorname{Tr}\left[e^{\left.-\beta H_{0}\right]}\right.} \sum_{n, n^{\prime}} e^{-\beta \frac{E_{n}}{\hbar}}\left[\left\langle n\left|c_{\mathbf{k}}\right| n^{\prime}\right\rangle\left\langle n^{\prime}\left|c_{\mathbf{k}}^{\dagger}\right| n\right\rangle e^{i \frac{E_{n}-E_{n^{\prime}}}{\hbar}\left(t-t^{\prime}\right)}+\right. \\
\quad+\left\langle n\left|c_{\mathbf{k}}^{\dagger}\right| n^{\prime}\right\rangle\left\langle n^{\prime}\left|c_{\mathbf{k}}\right| n\right\rangle e^{\left.-i \frac{E_{n}-E_{n^{\prime}}}{\hbar}\left(t-t^{\prime}\right)\right]=} \\
=\frac{1}{\operatorname{Tr}\left[e^{\left.-\beta H_{0}\right]}\right.} \sum_{n, n^{\prime}} e^{-\beta \frac{E_{n}}{\hbar}}\left[\frac{\left\langle n\left|c_{\mathbf{k}}\right| n^{\prime}\right\rangle\left\langle n^{\prime}\left|c_{\mathbf{k}}^{\dagger}\right| n\right\rangle}{\epsilon+E_{n}-E_{n^{\prime}}+i \eta}+\frac{\left\langle n\left|c_{\mathbf{k}}^{\dagger}\right| n^{\prime}\right\rangle\left\langle n^{\prime}\left|c_{\mathbf{k}}\right| n\right\rangle}{\epsilon-E_{n}+E_{n^{\prime}}+i \eta}\right]= \\
=\frac{1}{\operatorname{Tr}\left[e^{\left.-\beta H_{0}\right]}\right.} \sum_{n, n^{\prime}} \frac{\left\langle n\left|c_{\mathbf{k}}\right| n^{\prime}\right\rangle\left\langle n^{\prime}\left|c_{\mathbf{k}}^{\dagger}\right| n\right\rangle}{\epsilon+E_{n}-E_{n^{\prime}}+i \eta}\left(e^{-\beta \frac{E_{n}}{\hbar}}+e^{-\beta \frac{E_{n^{\prime}}}{\hbar}}\right) .
\end{aligned}
$$

Como vimos, a função espectral $A$ é essencialmente a parte imaginária da função de Green retardada (Equação 2.93). Portanto, deve haver uma maneira de relacionar a função espectral com o resultado que acabamos de obter para $G_{0}^{r}(\mathbf{k}, \epsilon)$. Tomemos, então, a parte imaginária da expressão acima:

$$
\begin{aligned}
A_{0}(\mathbf{k}, \epsilon) & =-2 \operatorname{Im}\left[G_{0}^{r}(\mathbf{k}, \epsilon)\right]= \\
& =\frac{2 \pi}{\operatorname{Tr}\left[e^{-\beta H_{0}}\right]} \sum_{n, n^{\prime}}\left\langle n\left|c_{\mathbf{k}}\right| n^{\prime}\right\rangle\left\langle n^{\prime}\left|c_{\mathbf{k}}^{\dagger}\right| n\right\rangle\left(e^{-\beta \frac{E_{n}}{\hbar}}+e^{-\beta \frac{E_{n^{\prime}}}{\hbar}}\right) \delta\left(\epsilon+E_{n}-E_{n^{\prime}}\right),
\end{aligned}
$$

onde a seguinte relação foi utilizada (ver Apêndice B):

$$
\frac{1}{\epsilon-i \eta}=\mathcal{P} \frac{1}{\epsilon}-i \pi \delta(\epsilon)
$$

Portanto, inserindo $\int d \epsilon^{\prime} \delta\left(\epsilon^{\prime}+E_{n}-E_{n^{\prime}}\right)=1$ na Equação 2.99 obtemos a seguinte relação:

$$
G_{0}^{r}(\mathbf{k}, \epsilon)=\int \frac{d \epsilon^{\prime}}{2 \pi} \frac{A_{0}(\mathbf{k}, \epsilon)}{\epsilon-\epsilon^{\prime}+i \eta}
$$

Esta relação é muito útil na análise de expressões envolvendo a função de Green eletrônica no equilíbrio. Em particular, ela viabilizou a reformulação da expressão usualmente utilizada para o cálculo da autoenergia de interação elétron-fônon na primeira aproximação de Born, resultado este que será discutido no Capítulo 6 . 


\subsection{Valores Esperados de Observáveis Fora do Equilíbrio}

Na Seção 2.2, obtivemos as funções de Green para um sistema em equilíbrio com um reservatório térmico à temperatura $T$ e um reservatório de partículas descrito por um potencial eletroquímico $\mu$. No entanto, existem inúmeros problemas nos quais o sistema estudado não se encontra no equilíbrio termodinâmico. Um exemplo típico é o caso do transporte eletrônico, no qual eletrodos descritos por diferentes potenciais eletroquímicos são acoplados a uma região de espalhamento, de forma que não há mais clareza sobre qual deva ser o potencial eletroquímico nessa região. Mesmo no caso de eletrodos descritos pelo mesmo potencial eletroquímico, se considerarmos, por exemplo, a interação entre elétrons e fônons, o problema eletrônico já não pode mais ser descrito pelas funções de Green $G_{0}$.

Nesta seção, veremos como abordar a situação fora do equilíbrio. Consideremos que o sistema estudado possa ser representado de forma geral pelo seguinte Hamiltoniano:

$$
\mathscr{H}(t)=H_{0}+H_{i}+H^{\prime}(t),
$$

onde a parte independente do tempo é composta por um termo não-interagente $H_{0}$ quadrático (bilinear, como é o caso das Hamiltonianas de elétrons e de fônons livres, Equações 2.55 e 2.56 , respectivamente) e por outro termo $H_{i}$ que descreve as diversas interações entre as partículas, e o termo $H^{\prime}(t)$ representa uma perturbação aplicada ao sistema a partir de um tempo $t_{0}$, ou seja:

$$
H^{\prime}(t)=0, \quad t<t_{0} .
$$

No caso do problema que iremos tratar nesta tese, essa perturbação tem origem ao conectarmos o sistema a contatos com diferentes potenciais eletroquímicos. Antes de a perturbação ser aplicada, considera-se que o sistema se encontra em equilíbrio termodinâmico com um reservatório térmico à temperatura $T$ e um reservatório de partículas descrito por um potencial eletroquímico $\mu$, ou seja, no ensemble grande canônico, o sistema é descrito pela seguinte matriz densidade:

$$
\rho(H)=\frac{e^{-\beta H}}{\operatorname{Tr}\left[e^{-\beta H}\right]},
$$

onde $H=H_{0}+H_{i}$ e onde escolhemos, novamente, medir a energia das partículas em relação a $\mu=0$ para não termos que explicitar o operador número $N$. 
Nosso objetivo, portanto, é calcular o valor esperado de observáveis $O$ para tempos $t>t_{0}$, o que, na representação de Heisenberg, é dado por 9

$$
\left\langle O_{\mathscr{H}}(t)\right\rangle=\operatorname{Tr}\left[\rho(H) O_{\mathscr{H}}(t)\right] .
$$

A dependência temporal de $O_{\mathscr{H}}$ costuma ser muito complicada e, por esse motivo, na descrição de problemas fora do equilíbrio é útil trabalhar com diferentes representações da mecânica quântica. Basicamente, busca-se eliminar as complicações trazidas pelos Hamiltonianos $H^{\prime}(t)$ e $H_{i}$.

O primeiro passo é remover a dependência explícita de $H^{\prime}(t)$, transformando-a na situação em que a evolução temporal dos operadores é governada somente por $H=H_{0}+H_{i}$. Escolhendo-se as representações de Schrödinger e de Heisenberg de forma a que elas coincidam no tempo $t_{0}$ (isto é, $\left|\Psi_{\mathscr{H}}\right\rangle=\left|\Psi_{S}\left(t_{0}\right)\right\rangle$ e $O_{\mathscr{H}}\left(t_{0}\right)=O_{S}$ ), a evolução temporal governada por $\mathscr{H}(t)$ é definida como:

$$
O_{\mathscr{H}}(t) \equiv u_{\mathscr{H}}^{\dagger}\left(t, t_{0}\right) O_{S} u_{\mathscr{H}}\left(t, t_{0}\right)
$$

onde o operador evolução temporal $u_{\mathscr{H}}\left(t, t_{0}\right)$ é dado por:

$$
u_{\mathscr{C}}\left(t, t_{0}\right)=\sum_{n=0}^{\infty} \frac{(-i)^{n}}{n ! \hbar^{n}} \int_{t_{0}}^{t} d t_{1} \cdots \int_{t_{0}}^{t} d t_{n} \mathrm{~T}\left\{\mathscr{H}\left(t_{1}\right) \cdots \mathscr{H}\left(t_{n}\right)\right\}=\mathrm{T}\left\{e^{-\frac{i}{\hbar} \int_{t_{0}}^{t} d t^{\prime} \mathscr{H}\left(t^{\prime}\right)}\right\}
$$

sendo $\mathrm{T}$ o operador de ordenamento temporal que organiza o produto de operadores de acordo com seus argumentos de tempo, colocando os mais recentes à esquerda:

$$
\mathrm{T}\left\{A_{1}\left(t_{1}\right) A_{2}\left(t_{2}\right) \cdots A_{n}\left(t_{n}\right)\right\}=( \pm 1)^{P} A_{i_{1}}\left(t_{i_{1}}\right) A_{i_{2}}\left(t_{i_{2}}\right) \cdots A_{i_{n}}\left(t_{i_{n}}\right), \quad t_{i_{1}}>t_{i_{2}}>\cdots>t_{i_{n}}
$$

com $P$ é o número de permutações realizadas entre os operadores na ordem original, onde o sinal "+" corresponde a bósons e o "-", a férmions.

Por outro lado, a evolução temporal governada por $H=H_{0}+H_{i}$ é definida como:

\footnotetext{
${ }^{9} \mathrm{~A}$ inclusão da matriz densidade do equilíbrio, independente no tempo, representa a situação em que os graus de liberdade termodinâmicos em $H$ não acompanham instantaneamente as variações de $H^{\prime}(t)$. Por outro lado, para que não ocorram transições entre estados do Hamiltoniano não-perturbado induzidas pelo meio em $t<t_{0}$, a perturbação $H^{\prime}(t)$ deve ser introduzida adiabaticamente (a inclusão direta de $H^{\prime}(t)$ na Equação 2.105 pode destruir a evolução unitária dos estados quânticos $60.65 \mid$ ).
} 


$$
O_{H}(t) \equiv u_{H}^{\dagger}\left(t, t_{0}\right) O_{S} u_{H}\left(t, t_{0}\right) \Longrightarrow O_{S} \equiv u_{H}\left(t, t_{0}\right) O_{H}(t) u_{H}^{\dagger}\left(t, t_{0}\right)
$$

onde o operador evolução temporal $u_{H}\left(t, t_{0}\right)$ é dado por:

$$
u_{H}\left(t, t_{0}\right)=e^{-i \frac{H}{\hbar}\left(t-t_{0}\right)} .
$$

Combinando as Equações 2.107 e 2.110, obtemos:

$$
O_{\mathscr{H}}(t)=u_{\mathscr{H}}^{\dagger}\left(t, t_{0}\right) u_{H}\left(t, t_{0}\right) O_{H}(t) u_{H}^{\dagger}\left(t, t_{0}\right) u_{\mathscr{H}}\left(t, t_{0}\right)=v_{H}^{\dagger}\left(t, t_{0}\right) O_{H}(t) v_{H}\left(t, t_{0}\right),
$$

onde $v_{H}\left(t, t_{0}\right)=u_{H}^{\dagger}\left(t, t_{0}\right) u_{\mathscr{H}}\left(t, t_{0}\right)$. Tomando a derivada de $v_{H}\left(t, t_{0}\right)$ com relação a $t$, tem-se:

$$
\begin{aligned}
i \hbar \frac{\partial}{\partial t} v_{H}\left(t, t_{0}\right) & =\left(i \hbar \frac{\partial}{\partial t} u_{H}^{\dagger}\left(t, t_{0}\right)\right) u_{\mathscr{H}}\left(t, t_{0}\right)+u_{H}^{\dagger}\left(t, t_{0}\right)\left(i \hbar \frac{\partial}{\partial t} u_{\mathscr{H}}\left(t, t_{0}\right)\right)= \\
& =H u_{H}^{\dagger}\left(t, t_{0}\right) u_{\mathscr{H}}\left(t, t_{0}\right)+u_{H}^{\dagger}\left(t, t_{0}\right) \mathscr{H}(t) u_{\mathscr{H}}\left(t, t_{0}\right)= \\
& =H v_{H}\left(t, t_{0}\right)+u_{H}^{\dagger}\left(t, t_{0}\right) \mathscr{H}(t) u_{H}\left(t, t_{0}\right) u_{H}^{\dagger}\left(t, t_{0}\right) u_{\mathscr{H}}\left(t, t_{0}\right)= \\
& =H v_{H}\left(t, t_{0}\right)+u_{H}^{\dagger}\left(t, t_{0}\right)\left(H+H^{\prime}(t)\right) u_{H}\left(t, t_{0}\right) v_{H}\left(t, t_{0}\right)= \\
& =u_{H}^{\dagger}\left(t, t_{0}\right) H^{\prime}(t) u_{H}\left(t, t_{0}\right) v_{H}\left(t, t_{0}\right)= \\
& =H_{H}^{\prime}(t) v_{H}\left(t, t_{0}\right)
\end{aligned}
$$

onde $H_{H}^{\prime}(t)=u_{H}^{\dagger}\left(t, t_{0}\right) H^{\prime}(t) u_{H}\left(t, t_{0}\right)$ é a representação de interação de $H^{\prime}(t)$. Integrando o resultado acima, obtém-se:

$$
v_{H}\left(t, t_{0}\right)-v_{H}\left(t_{0}, t_{0}\right)=-\frac{i}{\hbar} \int_{t_{0}}^{t} d t_{1} H_{H}^{\prime}(t) v_{H}\left(t_{1}, t_{0}\right) .
$$

Com a condição de contorno $v_{H}\left(t_{0}, t_{0}\right)=1$ e iterando, obtém-se o resultado:

$$
\begin{aligned}
v_{H}\left(t, t_{0}\right) & =\sum_{n=0}^{\infty} \frac{(-i)^{n}}{n ! \hbar^{n}} \int_{t_{0}}^{t} d t_{1} \int_{t_{0}}^{t_{1}} d t_{2} \cdots \int_{t_{0}}^{t_{n-1}} d t_{n}\left(H_{H}^{\prime}\left(t_{1}\right) H_{H}^{\prime}\left(t_{2}\right) \cdots H_{H}^{\prime}\left(t_{n}\right)\right)= \\
& =\sum_{n=0}^{\infty} \frac{(-i)^{n}}{n ! \hbar^{n}} \int_{t_{0}}^{t} d t_{1} \int_{t_{0}}^{t} d t_{2} \cdots \int_{t_{0}}^{t} d t_{n} \mathrm{~T}\left\{H_{H}^{\prime}\left(t_{1}\right) H_{H}^{\prime}\left(t_{2}\right) \cdots H_{H}^{\prime}\left(t_{n}\right)\right\}= \\
& =\mathrm{T}\left\{e^{-\frac{i}{\hbar} \int_{t_{0}}^{t} d t^{\prime} H_{H}^{\prime}\left(t^{\prime}\right)}\right\} .
\end{aligned}
$$


O ordenamento temporal na expressão acima pode ser escrito, de maneira equivalente, em termos do ordenamento nos ramos de um contorno sobre o eixo real (ou um infinitésimo distante) do tempo $t_{0}$ até $t$ e de volta a $t_{0}$ (Figura 2.3).

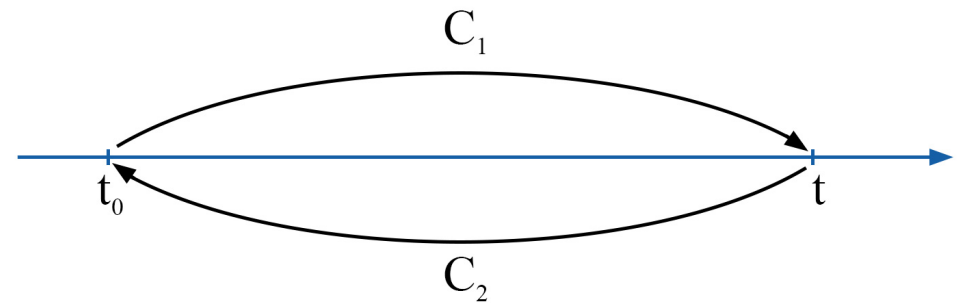

Figura 2.3: Contorno $C_{t}$ sobre o eixo real.

Logo, a Equação 2.112 toma a forma:

$$
\begin{aligned}
O_{\mathscr{H}}(t) & =v_{H}^{\dagger}\left(t, t_{0}\right) O_{H}(t) v_{H}\left(t, t_{0}\right)= \\
& =\mathrm{T}_{C_{2}}\left\{e^{-\frac{i}{\hbar} \int_{C_{2}} d \tau H_{H}^{\prime}(\tau)}\right\} O_{H}(t) \mathrm{T}_{C_{1}}\left\{e^{-\frac{i}{\hbar} \int_{C_{1}} d \tau H_{H}^{\prime}(\tau)}\right\}= \\
& =\mathrm{T}_{C_{t}}\left\{e^{-\frac{i}{\hbar} \int_{C_{t}} d \tau H_{H}^{\prime}(\tau)} O_{H}(t)\right\}
\end{aligned}
$$

onde $C_{t}=C_{1} \cup C_{2}$ e $\mathrm{T}_{C_{t}}$ é o operador de ordenamento de contorno que organiza o produto dos operadores de acordo com os argumentos de tempo no contorno $C_{t}$.

Conseguimos, portanto, remover a dependência explícita de $H^{\prime}(t)$, e, agora, a evolução temporal dos operadores é governada somente por $H=H_{0}+H_{i}$. Porém, ela ainda contém a complicação proveniente do termo $H_{i}$, que descreve as diversas interações possíveis entre as partículas.

O passo seguinte é, então, realizar uma nova transformação de modo que a evolução temporal dos operadores seja dada somente por $H_{0}$. O procedimento é análogo ao realizado para $H$, bastando substituir $H$ por $H_{0}$ nas Equações 2.110 2.112. Na diferenciação de $v_{H_{0}}\left(t, t_{0}\right)=$ $u_{H_{0}}^{\dagger}\left(t, t_{0}\right) u_{\mathscr{H}}\left(t, t_{0}\right)$, os termos com $H^{i}$ e $H(t)$ não se cancelam, restando:

$$
\begin{aligned}
i \hbar \frac{\partial}{\partial t} v_{H_{0}}\left(t, t_{0}\right) & =u_{H_{0}}^{\dagger}\left(t, t_{0}\right)\left(H^{i}+H^{\prime}(t)\right) u_{H_{0}}\left(t, t_{0}\right) v_{H_{0}}\left(t, t_{0}\right)= \\
& =\left(H_{H_{0}}^{i}(t)+H_{H_{0}}^{\prime}(t)\right) v_{H_{0}}\left(t, t_{0}\right) .
\end{aligned}
$$

Integrando a expressão acima e iterando, com a condição de contorno $v_{H_{0}}\left(t_{0}, t_{0}\right)=1$, obtémse: 


$$
v_{H_{0}}\left(t, t_{0}\right)=\mathrm{T}\left\{e^{-\frac{i}{\hbar} \int_{t_{0}}^{t} d t^{\prime} H_{H_{0}}^{i}\left(t^{\prime}\right)} e^{-\frac{i}{\hbar} \int_{t_{0}}^{t} d t^{\prime} H_{H_{0}}^{\prime}\left(t^{\prime}\right)}\right\}
$$

Por fim, em termos de $v_{H_{0}}\left(t, t_{0}\right)$, a evolução temporal de $\mathscr{H}(t)$ (Equação 2.107) passa a ser escrita como:

$$
O_{\mathscr{H}}(t)=\mathrm{T}_{C_{t}}\left\{e^{-\frac{i}{\hbar} \int_{C_{t}} d \tau H_{H_{0}}^{i}(\tau)} e^{-\frac{i}{\hbar} \int_{C_{t}} d \tau H_{H_{0}}^{\prime}(\tau)} O_{H_{0}}(t)\right\}=\mathrm{T}_{C_{t}}\left\{S_{C_{t}}^{i} S_{C_{t}}^{\prime} O_{H_{0}}(t)\right\}
$$

onde $S_{C_{t}}^{i}=e^{-\frac{i}{\hbar} \int_{C_{t}} d \tau H_{H_{0}}^{i}(\tau)}$ e $S_{C_{t}}^{\prime}=e^{-\frac{i}{\hbar} \int_{C_{t}} d \tau H_{H_{0}}^{\prime}(\tau)}$.

Para escrevermos o valor esperado de observáveis na representação de interação, resta ainda escrever a matriz densidade $\rho(H)$ em termos de $H_{0}$. Para tanto, é conveniente trabalhar com o seguinte operador:

$$
\nu\left(t, t_{0}\right) \equiv \mathrm{T}\left\{v_{H}\left(t, t_{0}\right) v_{H_{0}}^{\dagger}\left(t, t_{0}\right)\right\}
$$

Calculando sua derivada com relação a $t$, tem-se:

$$
\begin{aligned}
& i \hbar \frac{\partial}{\partial t} \nu\left(t, t_{0}\right)= \mathrm{T}\left\{H_{H}^{\prime}(t) v_{H}\left(t, t_{0}\right) v_{H_{0}}^{\dagger}\left(t, t_{0}\right)\right\}-\left(H_{H_{0}}^{i}(t)+H_{H_{0}}^{\prime}(t)\right) \mathrm{T}\left\{v_{H}\left(t, t_{0}\right) v_{H_{0}}^{\dagger}\left(t, t_{0}\right)\right\}= \\
&= \mathrm{T}\left\{u_{H}^{\dagger}\left(t, t_{0}\right) H^{\prime}(t) u_{H}\left(t, t_{0}\right) v_{H}\left(t, t_{0}\right) v_{H_{0}}^{\dagger}\left(t, t_{0}\right)\right\}-\left(H_{H_{0}}^{i}(t)+H_{H_{0}}^{\prime}(t)\right) \nu\left(t, t_{0}\right)= \\
&= \mathrm{T}\left\{u_{H}^{\dagger}\left(t, t_{0}\right) u_{H_{0}}\left(t, t_{0}\right) u_{H_{0}}^{\dagger}\left(t, t_{0}\right) H^{\prime}(t) u_{H_{0}}\left(t, t_{0}\right) u_{H_{0}}^{\dagger}\left(t, t_{0}\right) u_{H}\left(t, t_{0}\right)\right. \\
&\left.v_{H}\left(t, t_{0}\right) v_{H_{0}}^{\dagger}\left(t, t_{0}\right)\right\}-\left(H_{H_{0}}^{i}(t)+H_{H_{0}}^{\prime}(t)\right) \nu\left(t, t_{0}\right)= \\
&=\mathrm{T}\left\{u_{H}^{\dagger}\left(t, t_{0}\right) u_{H_{0}}\left(t, t_{0}\right) H_{H_{0}}^{\prime}(t) u_{H_{0}}^{\dagger}\left(t, t_{0}\right) u_{H}\left(t, t_{0}\right) v_{H}\left(t, t_{0}\right) v_{H_{0}}^{\dagger}\left(t, t_{0}\right)\right\}- \\
&-\left(H_{H_{0}}^{i}(t)+H_{H_{0}}^{\prime}(t)\right) \nu\left(t, t_{0}\right)= \\
&= H_{H_{0}}^{\prime}(t) \mathrm{T}\left\{v_{H}\left(t, t_{0}\right) v_{H_{0}}^{\dagger}\left(t, t_{0}\right)\right\}-\left(H_{H_{0}}^{i}(t)+H_{H_{0}}^{\prime}(t)\right) \nu\left(t, t_{0}\right)= \\
&=-H_{H_{0}}^{i}(t) \nu\left(t, t_{0}\right) .
\end{aligned}
$$

Integrando, com $\nu\left(t_{0}, t_{0}\right)=1$, e iterando, obtém-se:

$$
\nu\left(t, t_{0}\right)=\mathrm{T}\left\{e^{\frac{i}{\hbar} \int_{t_{0}}^{t} d t^{\prime} H_{H_{0}}^{i}\left(t^{\prime}\right)}\right\}
$$

Note que a partir de $v_{H}\left(t, t_{0}\right)=u_{H}^{\dagger}\left(t, t_{0}\right) u_{\mathscr{H}}\left(t, t_{0}\right)$, tem-se que $u_{H}\left(t, t_{0}\right)=u_{\mathscr{H}}\left(t, t_{0}\right) v_{H}^{\dagger}\left(t, t_{0}\right)$ e que, a partir de $v_{H_{0}}\left(t, t_{0}\right)=u_{H_{0}}^{\dagger}\left(t, t_{0}\right) u_{\mathscr{H}}\left(t, t_{0}\right)$, tem-se que $u_{H_{0}}\left(t, t_{0}\right)=u_{\mathscr{H}}\left(t, t_{0}\right) v_{H_{0}}^{\dagger}\left(t, t_{0}\right)$. Logo, podemos escrever $u_{H}\left(t, t_{0}\right)$ como: 


$$
u_{H}\left(t, t_{0}\right)=u_{H_{0}}\left(t, t_{0}\right) v_{H_{0}}\left(t, t_{0}\right) v_{H}^{\dagger}\left(t, t_{0}\right)=u_{H_{0}}\left(t, t_{0}\right) \nu\left(t, t_{0}\right) .
$$

Como $u_{H}\left(t, t_{0}\right)=e^{-i \frac{H}{\hbar}\left(t-t_{0}\right)}$ e $u_{H_{0}}\left(t, t_{0}\right)=e^{-i \frac{H_{0}}{\hbar}\left(t-t_{0}\right)}$, é conveniente tomar $t=t_{0}-i \hbar \beta$ a fim de recuperarmos a forma da matriz densidade [8] (Equação 2.105):

$$
e^{-\beta H}=e^{-\beta H_{0}} \nu\left(t_{0}-i \hbar \beta, t_{0}\right)
$$

O operador $\nu\left(t_{0}-i \hbar \beta, t_{0}\right)$ é definido em um contorno $C_{\nu}$ que vai de $t_{0}$ até $t=t_{0}-i \hbar \beta$ (Figura 2.4 .

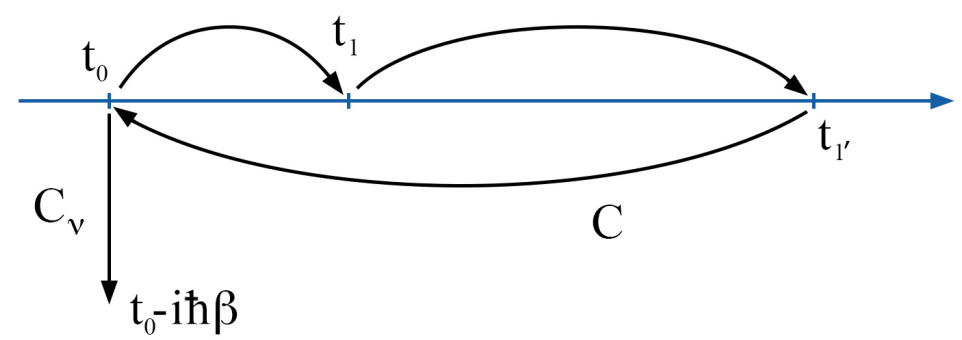

Figura 2.4: Contorno $\tilde{C}$ composto pelos contornos $C$ sobre o eixo real e $C_{\nu}$ perpendicular ao eixo real.

Como o contorno $C$, que vai de $t_{0}$ a $t_{1}$ e de volta a $t_{0}$, é fechado, segue que:

$$
\mathrm{T}_{C}\left\{e^{-\frac{i}{\hbar} \int_{C} d \tau H_{H_{0}}^{i}(\tau)} e^{-\frac{i}{\hbar} \int_{C} d \tau H_{H_{0}}^{\prime}(\tau)}\right\}=1
$$

Portanto, pode-se escrever o operador $\nu\left(t_{0}-i \hbar \beta, t_{0}\right)$ em termos de uma integral ao longo do contorno $\tilde{C}=C \cup C_{\nu}$ :

$$
\nu\left(t_{0}-i \hbar \beta, t_{0}\right)=\mathrm{T}_{\tilde{C}}\left\{e^{-\frac{i}{\hbar} \int_{C} d \tau H_{H_{0}}^{\prime}(\tau)} e^{-\frac{i}{\hbar} \int_{\tilde{C}} d \tau H_{H_{0}}^{i}(\tau)}\right\}=\mathrm{T}_{\tilde{C}}\left\{S_{C}^{\prime} S_{\tilde{C}}^{i}\right\}
$$

Com isso, podemos finalmente reescrever a matriz densidade $\rho(H)$ (Equação 2.105) em termos de $H_{0}$ e o valor esperado de observáveis físicos $O$ na representação de interação: 


$$
\begin{aligned}
\left\langle O_{\mathscr{H}}(t)\right\rangle & =\frac{\operatorname{Tr}\left[e^{-\beta H} O_{\mathscr{H}}(t)\right]}{\operatorname{Tr}\left[e^{-\beta H}\right]}=\frac{\operatorname{Tr}\left[e^{-\beta H_{0}} \nu\left(t_{0}-i \hbar \beta, t_{0}\right) O_{\mathscr{H}}(t)\right]}{\operatorname{Tr}\left[e^{-\beta H_{0}} \nu\left(t_{0}-i \hbar \beta, t_{0}\right)\right]}= \\
= & \frac{\left\langle\nu\left(t_{0}-i \hbar \beta, t_{0}\right) O_{\mathscr{H}}(t)\right\rangle_{0}}{\left\langle\nu\left(t_{0}-i \hbar \beta, t_{0}\right)\right\rangle_{0}}= \\
= & \frac{\left\langle\mathrm{T}_{\tilde{C}}\left\{S_{C}^{\prime} S_{\tilde{C}}^{i} O_{\mathscr{H}}(t)\right\}\right\rangle_{0}}{\left\langle\mathrm{~T}_{\tilde{C}}\left\{S_{C}^{\prime} S_{\tilde{C}}^{i}\right\}\right\rangle_{0}},
\end{aligned}
$$

onde \langle\rangle$_{0}$ indica que o valor esperado é calculado com respeito a $\rho\left(H_{0}\right)$.

Portanto, com as transformações realizadas conseguimos escrever toda a dependência temporal em termos do Hamiltoniano quadrático $H_{0}$. O fato de o valor esperado depender da matriz densidade $\rho\left(H_{0}\right)$ quadrática, e não mais de $\rho(H)$, nos permite descrever o problema fora do equilíbrio de forma diagramática, como veremos a seguir.

No problema do transporte de elétrons que trataremos nesta tese, vamos nos restringir à situação de estado estacionário, onde a perturbação (no caso uma bateria conectada aos eletrodos) é acionada adiabaticamente a um tempo suficientemente longo, isto é, no limite em que $t_{0} \rightarrow-\infty$. Neste caso, nota-se pela Equação 2.122 que a seguinte simplificação acontece $\nu\left(t_{0}-i \hbar \beta, t_{0}\right) \rightarrow 1$ e, portanto, os contornos $\tilde{C}$ e $C$ coincidem, de modo que somente o contorno $C$ precisa ser considerado.

\subsection{Funções de Green Fora do Equilíbrio}

Na Seção 2.2. definimos as funções de Green de partícula única com diferentes ordenamentos temporais. Fora do equilíbrio, porém, é conveniente trabalhar com a função de Green ordenada no contorno: 10

$$
G\left(1,1^{\prime}\right) \equiv-\frac{i}{\hbar}\left\langle\mathrm{T}_{C}\left\{\Psi_{\mathscr{H}}(1) \Psi_{\mathscr{H}}^{\dagger}\left(1^{\prime}\right)\right\}\right\rangle
$$

onde usamos a notação $(1) \equiv\left(\mathbf{r}_{1}, \sigma_{1}, t_{1}\right)$ e onde o contorno $C$ inicia em $t_{0}$, segue sobre o eixo real, passando uma única vez por $t_{1}$ e $t_{1^{\prime}}$ e, de volta, a $t_{0}$ (Figura 2.4.

Queremos reescrever a definição acima de modo que seja possível expandi-la perturbativamente. Para tanto, repetimos os passos da seção anterior para obter a função de Green fora do equilíbrio, no estado estacionário, na representação de interação:

\footnotetext{
${ }^{10}$ Nesta e nas próximas seções deste capítulo vamos trabalhar exclusivamente com férmions.
} 


$$
G\left(1,1^{\prime}\right)=-\frac{i}{\hbar} \frac{\left\langle\mathrm{T}_{C}\left\{S_{C}^{\prime} S_{C}^{i} \Psi_{H_{0}}(1) \Psi_{H_{0}}^{\dagger}\left(1^{\prime}\right)\right\}\right\rangle_{0}}{\left\langle\mathrm{~T}_{C}\left\{S_{C}^{\prime} S_{C}^{i}\right\}\right\rangle_{0}}
$$

\subsection{Teorema de Wick e Diagramas de Feynman}

Para explicitar a série perturbativa da função de Green fora do equilíbrio na representação de interação (Equação 2.129), note que o termo $\mathrm{T}_{C}\left\{S_{C}^{\prime} S_{C}^{i}\right\}$ possui a seguinte forma:

$$
\begin{aligned}
\mathrm{T}_{C}\left\{S_{C}\right\} & =\mathrm{T}_{C}\left\{e^{-\frac{i}{\hbar} \int_{C} d \tau \tilde{H}_{H_{0}}(\tau)}\right\}= \\
& =\sum_{n=0}^{\infty} \frac{(-i)^{n}}{n ! \hbar^{n}} \int_{C} d \tau_{1} \int_{C} d \tau_{2} \cdots \int_{C} d \tau_{n} \mathrm{~T}_{C}\left\{\tilde{H}_{H_{0}}\left(\tau_{1}\right) \tilde{H}_{H_{0}}\left(\tau_{2}\right) \cdots \tilde{H}_{H_{0}}\left(\tau_{n}\right)\right\},
\end{aligned}
$$

onde cada termo de $\tilde{H}_{H_{0}}(\tau)=H_{H_{0}}^{\prime}(\tau)+H_{H_{0}}^{i}(\tau)$ contém produtos de operadores de campo. Portanto, ao substituirmos $H_{H_{0}}^{\prime}(\tau)$ e $H_{H_{0}}^{i}(\tau)$ explicitamente na Equação 2.129 , teremos no integrando do numerador termos do seguinte tipo:

$$
\begin{aligned}
\left\langle\mathrm { T } _ { C } \left\{\Psi_{H_{0}}^{\dagger}\left(\tau_{1}\right) \Psi_{H_{0}}^{\dagger}\left(\tau_{2}\right) \Psi_{H_{0}}\left(\tau_{2}\right) \Psi_{H_{0}}\left(\tau_{1}\right) \cdots\right.\right. \\
\left.\left.\cdots \Psi_{H_{0}}^{\dagger}\left(\tau_{n-1}\right) \Psi_{H_{0}}^{\dagger}\left(\tau_{n}\right) \Psi_{H_{0}}\left(\tau_{n}\right) \Psi_{H_{0}}\left(\tau_{n-1}\right) \Psi_{H_{0}}(1) \Psi_{H_{0}}^{\dagger}\left(1^{\prime}\right)\right\}\right\rangle_{0} .
\end{aligned}
$$

Estes termos são calculados a partir do Teorema de Wick, que, em sua forma "fraca", afirma que o produto dos operadores de campo, nas condições da Equação 2.131 acima, pode ser escrito como a soma de todas as possíveis contrações de pares. Por exemplo, no caso de um Hamiltoniano de interação com quatro operadores de campo (como é o caso da interação coulombiana) obteríamos 6 termos:

$$
\begin{aligned}
\langle\mathrm{T} & \left.\left\{\Psi_{a}^{\dagger} \Psi_{b}^{\dagger} \Psi_{b} \Psi_{a} \Psi_{1} \Psi_{1^{\prime}}^{\dagger}\right\}\right\rangle_{0}= \\
= & \left\langle\mathrm{T}\left\{\Psi_{1} \Psi_{1^{\prime}}^{\dagger}\right\}\right\rangle_{0}\left(\left\langle\mathrm{~T}\left\{\Psi_{a} \Psi_{a}^{\dagger}\right\}\right\rangle_{0}\left\langle\mathrm{~T}\left\{\Psi_{b} \Psi_{b}^{\dagger}\right\}\right\rangle_{0}-\left\langle\mathrm{T}\left\{\Psi_{b} \Psi_{a}^{\dagger}\right\}\right\rangle_{0}\left\langle\mathrm{~T}\left\{\Psi_{a} \Psi_{b}^{\dagger}\right\}\right\rangle_{0}\right)+ \\
& +\left\langle\mathrm{T}\left\{\Psi_{1} \Psi_{a}^{\dagger}\right\}\right\rangle_{0}\left(\left\langle\mathrm{~T}\left\{\Psi_{b} \Psi_{1^{\prime}}^{\dagger}\right\}\right\rangle_{0}\left\langle\mathrm{~T}\left\{\Psi_{a} \Psi_{b}^{\dagger}\right\}\right\rangle_{0}-\left\langle\mathrm{T}\left\{\Psi_{b} \Psi_{b}^{\dagger}\right\}\right\rangle_{0}\left\langle\mathrm{~T}\left\{\Psi_{a} \Psi_{1^{\prime}}^{\dagger}\right\}\right\rangle_{0}\right)- \\
& -\left\langle\mathrm{T}\left\{\Psi_{1} \Psi_{b}^{\dagger}\right\}\right\rangle_{0}\left(\left\langle\mathrm{~T}\left\{\Psi_{b} \Psi_{a}^{\dagger}\right\}\right\rangle_{0}\left\langle\mathrm{~T}\left\{\Psi_{a} \Psi_{a}^{\dagger}\right\}\right\rangle_{0}-\left\langle\mathrm{T}\left\{\Psi_{a} \Psi_{1^{\prime}}^{\dagger}\right\}\right\rangle_{0}\left\langle\mathrm{~T}\left\{\Psi_{b} \Psi_{a}^{\dagger}\right\}\right\rangle_{0}\right)
\end{aligned}
$$


( a )

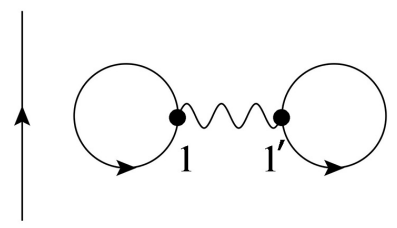

( c )

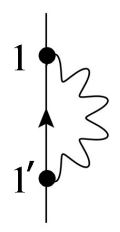

( e )

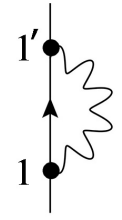

( b )

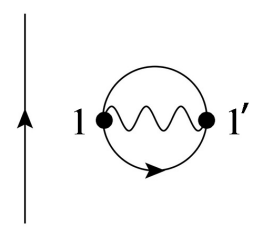

( d )

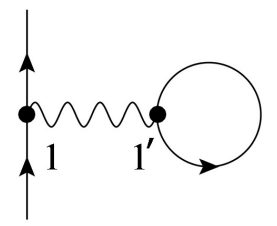

( f )

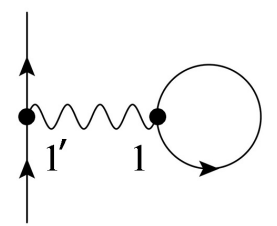

Figura 2.5: Diagramas gerados no caso de Hamiltonianos de interação quárticos. (Adaptado de |8.)

onde o sinal "-" aparece nos casos de um número ímpar de permutações dos operadores de campo fermiônicos. Cada um dos seis termos podem ser expressos em termos de Diagramas de Feynman como na Figura 2.5. A linha reta orientada representa a propagação de um férmion e a linha ondulada representa a interação (por exemplo, a emissão ou absorção de um bóson) que acontece nos instantes $t_{1}$ e $t_{1^{\prime}}$. Os dois primeiros diagramas (a) e (b) são desconexos e são cancelados pelo denominador da Equação 2.129. Os diagramas (c) e (e), assim como os diagramas (d) e (f), são topologicamente equivalentes, e apenas um de cada precisa ser considerado.

Por fim, considerando somente os diagramas conectados e topologicamente distintos, a função de Green fora do equilíbrio pode ser escrita como a seguinte expansão:

$$
\begin{aligned}
G\left(1,1^{\prime}\right)=-\frac{i}{\hbar} \sum_{n=0}^{\infty}\left(\frac{-i}{\hbar}\right)^{n} \int_{C} d \tau_{1} \int_{C} d \tau_{2} \cdots \int_{C} d \tau_{n} . \\
\cdot\left\langle\mathrm{T}_{C}\left\{\tilde{H}_{H_{0}}\left(\tau_{1}\right) \tilde{H}_{H_{0}}\left(\tau_{2}\right) \cdots \tilde{H}_{H_{0}}\left(\tau_{n}\right) \Psi_{H_{0}}(1) \Psi_{H_{0}}^{\dagger}\left(1^{\prime}\right)\right\}\right\rangle_{0} .
\end{aligned}
$$

A princípio, para obter a função de Green, seria preciso somar os infinitos termos da série perturbativa. Dessa forma, obteríamos o que é conhecido como autoenergia irredutível $\Sigma[G]$, um funcional da função de Green de partícula única contendo todas as interações de uma partícula com todas as demais e o espalhamento devido a um potencial externo. Porém, na prática, a autoenergia é determinada identificando-se os diagramas de Feynman mais relevantes para descrever um dado fenômeno 11 O procedimento de identificação dos diagramas é facilitado

\footnotetext{
${ }^{11}$ Esse procedimento parece razoável para descrever sistemas fracamente interagentes, onde se espera que a
} 
seguindo um conjunto de regras, conhecidas como regras de Feynman [59 62], que podem ser listadas como se segue:

(1) Interprete cada linha simples (dupla) de férmion como $i \hbar G_{0}(i \hbar G)$;

(2) Interprete cada linha simples (dupla) de fônon como $i \hbar D_{0}(i \hbar D)$;

(3) Atribua o fator $-i \hbar U$ para cada vértice (onde $U$ é um potencial externo) e iguale os índices;

(4) Tome o mesmo índice de spin ao longo de linhas de férmions conectadas;

(5) Multiplique por $(-1)^{P}$ onde $P$ é a quantidade de loops fermiônicos;

(6) Some sobre todas as variáveis internas;

(7) Variáveis de mesmo tempo devem ser interpretadas como $G\left(1,1^{+}\right)$, isto é, com o segundo argumento de tempo infinitesimalmente maior do que o primeiro.

\subsection{Continuação Analítica - Teorema de Langreth}

Apesar de a ordenação no contorno ser uma ferramenta útil para a formalização teórica, na prática os cálculos das integrais das funções de Green costumam ser efetuados no tempo real. O procedimento para passar de integrais no contorno para integrais no tempo real é conhecido como continuação analítica e, nesta seção, vamos utilizar a formulação de Langreth 64.

Tendo definido a autoenergia irredutível $\Sigma$ (supondo que ela exista) e assumindo que o termo fora do equilíbrio no Hamiltoniano possa ser representado por um potencial externo de partícula única $U$, a função de Green do estado estacionário ordenada no contorno $C$ (Equação 2.129) possui a seguinte equação de Dyson:

$$
G\left(1,1^{\prime}\right)=G_{0}\left(1,1^{\prime}\right)+\int_{C} d 2 G_{0}(1,2) U(2) G\left(2,1^{\prime}\right)+\int_{C} d 2 \int_{C} d 3 G_{0}(1,2) \Sigma(2,3) G\left(3,1^{\prime}\right)
$$

onde $\int_{C} d n=\sum_{\sigma_{n}} \int d \mathbf{r}_{n} \int_{C} d \tau_{n}$. Como as funções de Green possuem dois índices de tempo $t_{1}$ e $t_{1^{\prime}}$, em termos da posição destes instantes de tempo no contorno (ver Figura 2.3), existem quatro possibilidades distintas para escolha da função de Green:

série seja convergente. No entanto, mesmo nestes casos não se tem nenhuma garantia formal de que a convergência ocorra. 


$$
G\left(1,1^{\prime}\right)= \begin{cases}G_{c}\left(1,1^{\prime}\right), & t_{1}, t_{1^{\prime}} \in C_{1}, \\ G^{>}\left(1,1^{\prime}\right), & t_{1} \in C_{2}, t_{1^{\prime}} \in C_{1}, \\ G^{<}\left(1,1^{\prime}\right), & t_{1} \in C_{1}, t_{1^{\prime}} \in C_{2}, \\ G_{\tilde{c}}\left(1,1^{\prime}\right), & t_{1}, t_{1^{\prime}} \in C_{2} .\end{cases}
$$

As funções de Green $G^{>}\left(1,1^{\prime}\right)=-\frac{i}{\hbar}\left\langle\Psi_{\mathscr{H}}(1) \Psi_{\mathscr{H}}^{\dagger}\left(1^{\prime}\right)\right\rangle$ e $G^{<}\left(1,1^{\prime}\right) \equiv \frac{i}{\hbar}\left\langle\Psi_{\mathscr{H}}^{\dagger}\left(1^{\prime}\right) \Psi_{\mathscr{H}}(1)\right\rangle$ já foram apresentadas na Seção 2.2. A função de Green causal $G_{c}\left(1,1^{\prime}\right.$ ) (ou função de Green ordenada no tempo) e a função de Green antitemporalmente ordenada $G_{\tilde{c}}\left(1,1^{\prime}\right)$ são definidas como:

$$
\begin{aligned}
G_{c}\left(1,1^{\prime}\right) & \equiv-\frac{i}{\hbar}\left\langle\mathrm{T}\left\{\left[\Psi_{\mathscr{H}}(1), \Psi_{\mathscr{H}}^{\dagger}\left(1^{\prime}\right)\right]\right\}\right\rangle= \\
& =-\frac{i}{\hbar} \theta\left(t_{1}-t_{1^{\prime}}\right)\left\langle\Psi_{\mathscr{H}}(1) \Psi_{\mathscr{H}}^{\dagger}\left(1^{\prime}\right)\right\rangle+\frac{i}{\hbar} \theta\left(t_{1^{\prime}}-t_{1}\right)\left\langle\Psi_{\mathscr{H}}^{\dagger}\left(1^{\prime}\right) \Psi_{\mathscr{H}}(1)\right\rangle, \\
G_{\tilde{c}}\left(1,1^{\prime}\right) & \equiv-\frac{i}{\hbar}\left\langle\tilde{\mathrm{T}}\left\{\left[\Psi_{\mathscr{H}}(1), \Psi_{\mathscr{H}}^{\dagger}\left(1^{\prime}\right)\right]\right\}\right\rangle= \\
& =-\frac{i}{\hbar} \theta\left(t_{1^{\prime}}-t_{1}\right)\left\langle\Psi_{\mathscr{H}}(1) \Psi_{\mathscr{H}}^{\dagger}\left(1^{\prime}\right)\right\rangle+\frac{i}{\hbar} \theta\left(t_{1}-t_{1^{\prime}}\right)\left\langle\Psi_{\mathscr{H}}^{\dagger}\left(1^{\prime}\right) \Psi_{\mathscr{H}}(1)\right\rangle .
\end{aligned}
$$

Vamos analisar inicialmente o caso em que $t_{1}$ está na primeira metade do contorno e $t_{1^{\prime}}$ na segunda, isto é, $G\left(1,1^{\prime}\right)=G^{<}\left(1,1^{\prime}\right)$. Nota-se que, considerando a parte temporal, a equação de Dyson (Equação 2.133) contém produtos de dois ou mais termos do tipo:

$$
D^{<}\left(t_{1}, t_{1^{\prime}}\right)=\int_{C} d \tau A\left(t_{1}, \tau\right) B\left(\tau, t_{1^{\prime}}\right)
$$

O contorno $C$ pode ser deformado conforme indicado na Figura 2.6, de modo que $D^{<}\left(t_{1}, t_{1^{\prime}}\right)$ possa ser dividida em duas integrais:

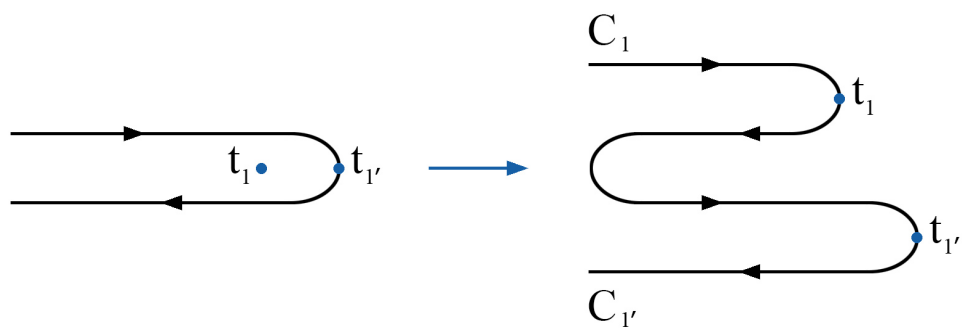

Figura 2.6: Deformação do contorno $C$. 


$$
\begin{aligned}
D^{<}\left(t_{1}, t_{1^{\prime}}\right) & =\int_{C_{1}} d \tau A\left(t_{1}, \tau\right) B\left(\tau, t_{1^{\prime}}\right)+\int_{C_{1^{\prime}}} d \tau A\left(t_{1}, \tau\right) B\left(\tau, t_{1^{\prime}}\right)= \\
& =\int_{C_{1}} d \tau A\left(t_{1}, \tau\right) B^{<}\left(\tau, t_{1^{\prime}}\right)+\int_{C_{1^{\prime}}} d \tau A^{<}\left(t_{1}, \tau\right) B\left(\tau, t_{1^{\prime}}\right),
\end{aligned}
$$

onde a primeira integral fica restrita ao contorno $C_{1}$ (e por esse motivo $B\left(\tau, t_{1^{\prime}}\right)=B^{<}\left(\tau, t_{1^{\prime}}\right)$ ) e a segunda fica restrita ao contorno $C_{1^{\prime}}$ (e por esse motivo $A\left(t_{1}, \tau\right)=A^{<}\left(t_{1}, \tau\right)$ ). O passo seguinte é dividir cada uma das integrais em dois ramos sobre o eixo real:

$$
\begin{aligned}
D^{<}\left(t_{1}, t_{1^{\prime}}\right)= & \int_{-\infty}^{t_{1}} d t A\left(t_{1}, t\right) B^{<}\left(t, t_{1^{\prime}}\right)+\int_{t_{1}}^{-\infty} d t A\left(t_{1}, t\right) B^{<}\left(t, t_{1^{\prime}}\right)+ \\
& +\int_{-\infty}^{t{ }_{1^{\prime}}} d t A^{<}\left(t_{1}, t\right) B\left(t, t_{1^{\prime}}\right)+\int_{t_{1^{\prime}}}^{-\infty} d t A^{<}\left(t_{1}, t\right) B\left(t, t_{1^{\prime}}\right)= \\
= & \int_{-\infty}^{t_{1}} d t A^{>}\left(t_{1}, t\right) B^{<}\left(t, t_{1^{\prime}}\right)-\int_{-\infty}^{t_{1}} d t A^{<}\left(t_{1}, t\right) B^{<}\left(t, t_{1^{\prime}}\right)+ \\
& +\int_{-\infty}^{t_{1^{\prime}}} d t A^{<}\left(t_{1}, t\right) B^{<}\left(t, t_{1^{\prime}}\right)-\int_{-\infty}^{t_{1^{\prime}}} d t A^{<}\left(t_{1}, t\right) B^{>}\left(t, t_{1^{\prime}}\right)= \\
= & \int_{-\infty}^{\infty} d t \theta\left(t_{1}-t\right)\left[A^{>}\left(t_{1}, t\right)-A^{<}\left(t_{1}, t\right)\right] B^{<}\left(t, t_{1^{\prime}}\right)- \\
& -\int_{-\infty}^{\infty} d t A^{<}\left(t_{1}, t\right) \theta\left(t_{1^{\prime}}-t\right)\left[B^{>}\left(t, t_{1^{\prime}}\right)-B^{<}\left(t, t_{1^{\prime}}\right)\right]= \\
= & \int_{-\infty}^{\infty} d t\left[A^{r}\left(t_{1}, t\right) B^{<}\left(t, t_{1^{\prime}}\right)+A^{<}\left(t_{1}, t\right) B^{a}\left(t, t_{1^{\prime}}\right)\right],
\end{aligned}
$$

onde usamos as definições das funções de Green retardada e avançada (Equações 2.69 e 2.70. respectivamente).

Analogamente, podemos refazer as passagens para o caso em que $t_{1}$ está na segunda metade do contorno e $t_{1^{\prime}}$ na primeira, isto é, $G\left(1,1^{\prime}\right)=G^{>}\left(1,1^{\prime}\right)$, obtendo:

$$
D^{>}\left(t_{1}, t_{1^{\prime}}\right)=\int_{-\infty}^{\infty} d t\left[A^{r}\left(t_{1}, t\right) B^{>}\left(t, t_{1^{\prime}}\right)+A^{>}\left(t_{1}, t\right) B^{a}\left(t, t_{1^{\prime}}\right)\right]
$$

Substituindo $D^{<}\left(t_{1}, t_{1^{\prime}}\right)$ e $D^{>}\left(t_{1}, t_{1^{\prime}}\right)$ na definição da função de Green retardada (Equação 2.69, obtém-se:

$$
\begin{aligned}
D^{r}\left(t_{1}, t_{1^{\prime}}\right)=\theta\left(t_{1}-t_{1^{\prime}}\right) \int_{-\infty}^{\infty} d t[ & A^{r}\left(t_{1}, t\right)\left(B^{>}\left(t, t_{1^{\prime}}\right)-B^{<}\left(t, t_{1^{\prime}}\right)\right)+ \\
& \left.+\left(A^{>}\left(t_{1}, t\right)-A^{<}\left(t_{1}, t\right)\right) B^{a}\left(t, t_{1^{\prime}}\right)\right]=
\end{aligned}
$$




$$
\begin{gathered}
=\theta\left(t_{1}-t_{1^{\prime}}\right) \int_{-\infty}^{\infty} d t\left[\theta\left(t_{1}-t\right)\left(A^{>}\left(t_{1}, t\right)-A^{<}\left(t_{1}, t\right)\right)\left(B^{>}\left(t, t_{1^{\prime}}\right)-B^{<}\left(t, t_{1^{\prime}}\right)\right)+\right. \\
\left.+\left(A^{>}\left(t_{1}, t\right)-A^{<}\left(t_{1}, t\right)\right) \theta\left(t_{1^{\prime}}-t\right)\left(B^{>}\left(t, t_{1^{\prime}}\right)-B^{<}\left(t, t_{1^{\prime}}\right)\right)\right]= \\
=\theta\left(t_{1}-t_{1^{\prime}}\right)\left[\int_{-\infty}^{t_{1}} d t\left(A^{>}\left(t_{1}, t\right)-A^{<}\left(t_{1}, t\right)\right)\left(B^{>}\left(t, t_{1^{\prime}}\right)-B^{<}\left(t, t_{1^{\prime}}\right)\right)+\right. \\
\left.\quad+\int_{-\infty}^{t_{1^{\prime}}} d t\left(A^{>}\left(t_{1}, t\right)-A^{<}\left(t_{1}, t\right)\right)\left(B^{>}\left(t, t_{1^{\prime}}\right)-B^{<}\left(t, t_{1^{\prime}}\right)\right)\right]= \\
=\int_{t_{1^{\prime}}}^{t_{1}} d t A^{r}\left(t_{1}, t\right) B^{r}\left(t, t_{1^{\prime}}\right) .
\end{gathered}
$$

Analogamente, substituindo $D^{<}\left(t_{1}, t_{1^{\prime}}\right)$ e $D^{>}\left(t_{1}, t_{1^{\prime}}\right)$ na definição da função de Green avançada (Equação 2.70, podemos refazer as passagens para obter:

$$
D^{a}\left(t_{1}, t_{1^{\prime}}\right)=\int_{t_{1^{\prime}}}^{t_{1}} d t A^{a}\left(t_{1}, t\right) B^{a}\left(t, t_{1^{\prime}}\right) .
$$

Os resultados obtidos podem ser generalizados para o produto de três elementos, como o terceiro termo da equação de Dyson (Equação 2.133), de onde se obtém:

$$
\begin{aligned}
D^{\lessgtr}\left(t_{1}, t_{1^{\prime}}\right)= & \int_{-\infty}^{\infty} d t \int_{-\infty}^{\infty} d t^{\prime}\left[A^{r}\left(t_{1}, t\right) B^{r}\left(t, t^{\prime}\right) C^{\lessgtr}\left(t^{\prime}, t_{1^{\prime}}\right)+\right. \\
& \left.+A^{r}\left(t_{1}, t\right) B^{\lessgtr}\left(t, t^{\prime}\right) C^{a}\left(t^{\prime}, t_{1^{\prime}}\right)+A^{\lessgtr}\left(t_{1}, t\right) B^{a}\left(t, t^{\prime}\right) C^{a}\left(t^{\prime}, t_{1^{\prime}}\right)\right], \\
D^{r(a)}\left(t_{1}, t_{1^{\prime}}\right)= & \int_{-\infty}^{\infty} d t \int_{-\infty}^{\infty} d t^{\prime}\left[A^{r(a)}\left(t_{1}, t\right) B^{r(a)}\left(t, t^{\prime}\right) C^{r(a)}\left(t^{\prime}, t_{1^{\prime}}\right)\right] .
\end{aligned}
$$

Frequentemente, na expansão diagramática da função de Green surgem termos paralelos $\left(D_{\Xi}\left(\tau, \tau^{\prime}\right)=A\left(\tau, \tau^{\prime}\right) B\left(\tau, \tau^{\prime}\right)\right)$ ou antiparalelos $\left(D \leftrightarrows\left(\tau, \tau^{\prime}\right)=A\left(\tau, \tau^{\prime}\right) B\left(\tau^{\prime}, \tau\right)\right)$ no tempo, como é o caso da autoenergia de interação elétron-fônon. O Teorema de Langreth também pode ser generalizado, utilizando-se a mesma análise feita até aqui, para obter-se:

$$
\begin{aligned}
& D_{\leftrightarrows}^{\lessgtr}\left(t, t^{\prime}\right)=A^{\lessgtr}\left(t, t^{\prime}\right) B^{\lessgtr}\left(t, t^{\prime}\right), \\
& D_{\leftrightarrows}^{\lessgtr}\left(t, t^{\prime}\right)=A^{\lessgtr}\left(t, t^{\prime}\right) B^{\lessgtr}\left(t^{\prime}, t\right), \\
& D_{\leftleftarrows}^{r}\left(t, t^{\prime}\right)=A^{<}\left(t, t^{\prime}\right) B^{r}\left(t, t^{\prime}\right)+A^{r}\left(t, t^{\prime}\right) B^{<}\left(t, t^{\prime}\right)+A^{r}\left(t, t^{\prime}\right) B^{r}\left(t, t^{\prime}\right), \\
& D_{\leftrightarrows}^{r}\left(t, t^{\prime}\right)=A^{<}\left(t, t^{\prime}\right) B^{a}\left(t^{\prime}, t\right)+A^{r}\left(t, t^{\prime}\right) B^{<}\left(t^{\prime}, t\right) .
\end{aligned}
$$




\subsection{Equação Keldysh}

Fecharemos este capítulo com a equação de Keldysh, que vem a ser uma equação de movimento que descreve a evolução da função de Green $G^{<}$na situação fora do equilíbrio. A derivação segue como uma aplicação imediata do Teorema de Langreth.

A equação de Dyson (Equação 2.133) pode ser escrita, ignorando-se potencial externo de partícula única $U$ (que pode ser absorvido na função de Green não-interagente $G_{0}$ ), como:

$$
G\left(1,1^{\prime}\right)=G_{0}\left(1,1^{\prime}\right)+\int_{C} d 2 \int_{C} d 3 G_{0}(1,2) \Sigma(2,3) G\left(3,1^{\prime}\right)
$$

Aplicando-se a regra de Langreth dada pela Equação 2.143, obtém-se:

$$
\begin{array}{rl}
G\left(1,1^{\prime}\right)^{<}=G_{0}^{<}\left(1,1^{\prime}\right)+\int_{t} & d 2 \int_{t} d 3\left[G_{0}^{r}(1,2) \Sigma^{r}(2,3) G^{<}\left(3,1^{\prime}\right)+\right. \\
& \left.+G_{0}^{r}(1,2) \Sigma^{<}(2,3) G^{a}\left(3,1^{\prime}\right)+G_{0}^{<}(1,2) \Sigma^{a}(2,3) G^{a}\left(3,1^{\prime}\right)\right],
\end{array}
$$

onde $\int_{t} d n=\sum_{\sigma_{n}} \int d \mathbf{r}_{n} \int_{t} d t_{n}$. Iterando indefinidamente chega-se na equação integral de Keldysh 8:

$$
\begin{aligned}
& G\left(1,1^{\prime}\right)^{<}= \int_{t} d 2 \int_{t} d 3 \int_{t} d 4 \int_{t} d 5\left[(\delta(1-2) \delta(2-3))+G^{r}(1,2) \Sigma^{r}(2,3)\right] G_{0}^{<}\left(3,1^{\prime}\right) \cdot \\
& \cdot\left[\left(\delta(4-5) \delta\left(5-1^{\prime}\right)\right)+\Sigma^{a}(4,5) G^{a}\left(5,1^{\prime}\right)\right]+ \\
&+\int_{t} d 2 \int_{t} d 3 G^{r}(1,2) \Sigma^{<}(2,3) G^{a}\left(3,1^{\prime}\right)
\end{aligned}
$$

onde $\delta(I V) \equiv \delta\left(\mathbf{r}_{i}-\mathbf{r}_{j}\right) \delta\left(t_{i}-t_{j}\right) \delta_{\sigma_{i} \sigma_{j}}$.

O primeiro termo da equação de Keldysh contém informações a respeito do estado inicial do sistema, antes da perturbação ser aplicada [66]. Como no problema de transporte que iremos tratar vamos considerar o estado estacionário, isto é, o limite em que $t_{0} \rightarrow-\infty$, este termo é desconsiderado e a equação de Keldysh se resume a:

$$
G\left(1,1^{\prime}\right)^{<}=\int_{t} d 2 \int_{t} d 3 G^{r}(1,2) \Sigma^{<}(2,3) G^{a}\left(3,1^{\prime}\right)
$$

Esta equação de movimento é o resultado central do formalismo NEGF, indicando que a evolução temporal da função de correlação eletrônica $G^{<}$é proporcional à autoenergia de espalhamento $\Sigma^{<}$que, por sua vez, está relacionada à taxa de incidência dos elétrons [67]. 
Conforme vimos na Seção 2.3 , no equilíbrio a função de correlação $G_{0}^{<}$é dada pela densidade de estados multiplicada pela distribuição estatística de ocupação desses estados e, portanto, corresponde a uma densidade de partículas. A mesma interpretação é válida para $G^{<}$fora do equilíbrio, embora, nesse caso, não haja uma relação clara como a Equação 2.96 . De fato, podese mostrar que a função de Green lesser é dada pela densidade de elétrons no limite $1^{\prime} \rightarrow 1$ [59]. Assim sendo, a equação de Keldysh pode ser compreendida, de certa maneira, como uma evolução temporal da ocupação eletrônica na situação fora do equilíbrio [8]. 


\section{Capítulo 3}

\section{Estrutura Eletrônica e Cálculo}

\section{Vibracional}

O formalismo da funções de Green fora do equilíbrio, descrito no capítulo anterior, independe da escolha do Hamiltoniano eletrônico. Contudo, para obter-se uma descrição quantitativa de sistemas realísticos, é necessário partir de uma descrição precisa da estrutura eletrônica do sistema de muitos corpos. Uma das ferramentas mais populares para este fim é a Teoria do Funcional da Densidade (DFT), que oferece uma descrição atomística ab initio da energia total do sistema, isto é, sem parâmetros de ajuste específicos do sistema estudado 68, 69.

A ideia fundamental da DFT é que toda informação contida na função de onda de $n$ corpos $\Psi(\{\mathbf{r}\})$, dependente de $3 n$ variáveis (mais spin), pode ser obtida a partir da densidade do estado fundamental $n_{0}(\mathbf{r})$, dependente de somente 3 variáveis. A existência e a unicidade da densidade do estado fundamental são garantidas por dois teoremas propostos e provados por P. Hohenberg e W. Kohn [68] em 1964.

Porém, antes de enunciar esses teoremas, iniciaremos este capítulo apresentando a aproximação adiabática de Born-Oppenheimer, que separa os graus de liberdade nucleares dos eletrônicos e que é utilizada extensivamente em cálculos de estrutura eletrônica.

Em seguida descreveremos a Teoria do Funcional da Densidade e o trabalho de W. Kohn e L. Sham [42], que forneceu um método para substituir o problema de muitos corpos interagentes em um problema equivalente de partículas independentes, onde todos os efeitos de muitos corpos são incluídos em um potencial de troca e correlação.

Descreveremos, do ponto de vista prático, o código SIESTA (Spanish Initiative for Electronic Simulations with Thousands of Atoms) [2,3], uma implementação da DFT que foi utilizada como plataforma de cálculo de estrutura eletrônica de todos os códigos que serão apresentados nesta tese. 
Por fim, apresentaremos o programa PhOnonS ITeratIVE VIBRATIONS, desenvolvido para o cálculo das frequências e dos modos vibracionais de materiais, assim como para calcular a matriz de acoplamento elétron-fônon, a partir de primeiros princípios.

\subsection{Aproximação de Born-Oppenheimer}

A análise de Born-Oppenheimer está diretamente relacionada à observação de que o espectro molecular é formado por termos de diferentes ordens de magnitude. Com base na grande diferença entre as massas eletrônica e nuclear, a energia cinética nuclear $T_{N}$ é usualmente pequena e pode ser tratada como uma perturbação do sistema. No trabalho de 1927, M. Born e J. Oppenheimer aplicaram sistematicamente a teoria de perturbação na equação de Schrödinger de elétrons e núcleos interagentes, onde utilizaram $(m / M)^{1 / 4}$ como o parâmetro de expansão [70], com $m$ a massa do elétron e $M$ a massa de um núcleo, ou a média das massas dos núcleos. Às diferentes ordens de expansão obtidas associam-se as diferentes partes do espectro e deduz-se que a maior contribuição provém do movimento dos elétrons em torno dos núcleos, seguida da vibração nuclear e, por fim, da rotação nuclear.

Nesta seção seguiremos a derivação padrão da aproximação adiabática de Born-Oppenheimer, que é mais sucinta e numa linguagem mais moderna, conforme descrita em [71.

A equação de Schrödinger independente do tempo para um sistema com $p$ elétrons e $l$ núcleos é dada por:

$$
\mathcal{H} \Psi_{n}(\mathbf{r}, \mathbf{R})=E_{n} \Psi_{n}(\mathbf{r}, \mathbf{R})
$$

onde $\mathbf{r}=\{\mathbf{r}\}=\left(\mathbf{r}_{1}, \mathbf{r}_{2}, \ldots, \mathbf{r}_{p}\right)$ e $\mathbf{R}=\{\mathbf{R}\}=\left(\mathbf{R}_{1}, \mathbf{R}_{2}, \ldots, \mathbf{R}_{l}\right)$ são os conjuntos de coordenadas dos elétrons e dos núcleos, respectivamente. O operador Hamiltoniano é dado pelas energias cinéticas dos núcleos $\left(T_{N}\right)$ e elétrons $\left(T_{e}\right)$, e pelas energias de interação coulombiana entre núcleos e elétrons $\left(V_{e N}\right)$, dos elétrons entre si $\left(V_{e}\right)$ e de cada núcleo com os demais núcleos $\left(V_{N}\right)$, ou seja:

$$
\mathcal{H}=\underbrace{\frac{-\hbar^{2}}{2 m} \sum_{i=1}^{p} \nabla_{i}^{2}}_{T_{e}} \underbrace{-\frac{1}{4 \pi \epsilon_{0}} \sum_{i=1}^{p} \sum_{I=1}^{l} \frac{Z_{I} e^{2}}{\left|\mathbf{r}_{i}-\mathbf{R}_{I}\right|}}_{V_{e N}} \underbrace{+\frac{1}{4 \pi \epsilon_{0}} \sum_{\substack{i, j=1 \\ j<i}}^{p} \frac{e^{2}}{\left|\mathbf{r}_{i}-\mathbf{r}_{j}\right|}}_{V_{e}} \underbrace{-\sum_{I=1}^{l} \frac{\hbar^{2}}{2 M_{I}} \nabla_{I}^{2}}_{T_{N}}+\underbrace{+\frac{1}{4 \pi \epsilon_{0}} \sum_{\substack{I, J=1 \\ J<I}}^{l} \frac{Z_{I} Z_{J} e^{2}}{\left|\mathbf{R}_{I}-\mathbf{R}_{J}\right|}}_{V_{N}} .
$$

Pode-se reescrever este Hamiltoniano representando a energia coulombiana total por $U(\mathbf{r}, \mathbf{R})$ e introduzindo a notação $T_{e}+U=H^{\prime}\left(\mathbf{r}, \frac{\partial}{\partial \mathbf{r}}, \mathbf{R}\right)$ : 


$$
\mathcal{H}=T_{e}+U+T_{N}=H^{\prime}+T_{N},
$$

onde $H^{\prime}$ não depende do movimento dos núcleos, isto é, podemos considerá-lo como o Hamiltoniano dos elétrons com os núcleos fixos. Desta forma, existe $\psi_{m}(\mathbf{r} ; \mathbf{R})$, que define uma base completa de autoestados eletrônicos para cada configuração dos núcleos $\mathbf{R}$ :

$$
H^{\prime} \psi_{m}(\mathbf{r} ; \mathbf{R})=\epsilon_{m}(\mathbf{R}) \psi_{m}(\mathbf{r} ; \mathbf{R})
$$

Portanto, para cada configuração nuclear $\mathbf{R}$, podemos expandir os autoestados do sistema total em termos da base $\left\{\psi_{m}(\mathbf{r} ; \mathbf{R})\right\}$ :

$$
\Psi_{n}(\mathbf{r}, \mathbf{R})=\sum_{m} \chi_{n m}(\mathbf{R}) \psi_{m}(\mathbf{r} ; \mathbf{R})
$$

onde os coeficientes $\chi_{n m}(\mathbf{R})$ especificam os estados $\Psi_{n}(\mathbf{r}, \mathbf{R})$ do sistema de elétrons e núcleos acoplados. Inserindo esta expansão na Equação 3.1 e projetando sobre um estado eletrônico $k$, obtém-se:

$$
\begin{gathered}
\left\langle\psi_{k}^{\dagger}(\mathbf{r} ; \mathbf{R})|\mathcal{H}| \sum_{m} \chi_{n m}(\mathbf{R}) \psi_{m}(\mathbf{r} ; \mathbf{R})\right\rangle_{\mathbf{r}}=\left\langle\psi_{k}^{\dagger}(\mathbf{r} ; \mathbf{R})\left|E_{n}\right| \sum_{m} \chi_{n m}(\mathbf{R}) \psi_{m}(\mathbf{r} ; \mathbf{R})\right\rangle_{\mathbf{r}} \\
\left\langle\psi_{k}^{\dagger}(\mathbf{r} ; \mathbf{R})\left|T_{N}\right| \sum_{m} \chi_{n m}(\mathbf{R}) \psi_{m}(\mathbf{r} ; \mathbf{R})\right\rangle_{\mathbf{r}}+\sum_{m} \chi_{n m}(\mathbf{R}) \epsilon_{m}(\mathbf{R}) \delta_{k m}=E_{n} \sum_{m} \chi_{n m}(\mathbf{R}) \delta_{k m} \\
\sum_{m}\left\langle\psi_{k}^{\dagger}(\mathbf{r} ; \mathbf{R})\left|T_{N}\right| \chi_{n m}(\mathbf{R}) \psi_{m}(\mathbf{r} ; \mathbf{R})\right\rangle_{\mathbf{r}}+\chi_{n k}(\mathbf{R}) \epsilon_{k}(\mathbf{R})=E_{n} \chi_{n k}(\mathbf{R}),
\end{gathered}
$$

no qual os autoestados eletrônicos são ortonormalizados segundo $\delta_{k m}=\left\langle\psi_{k}(\mathbf{r} ; \mathbf{R}) \mid \psi_{m}(\mathbf{r} ; \mathbf{R})\right\rangle_{\mathbf{r}}$, e onde a notação \langle\rangle$_{\mathbf{r}}$ representa a integração sobre todos os estados eletrônicos $\mathbf{r}$. O termo envolvendo a energia cinética dos núcleos fica: 


$$
\begin{aligned}
\sum_{m}\left\langle\psi_{k}^{\dagger}(\mathbf{r} ; \mathbf{R})\left|T_{N}\right| \chi_{n m}(\mathbf{R}) \psi_{m}(\mathbf{r} ; \mathbf{R})\right\rangle_{\mathbf{r}}= & \\
=- & -\sum_{I=1}^{l} \frac{\hbar^{2}}{2 M_{I}} \sum_{m}\left\langle\psi_{k}^{\dagger}(\mathbf{r} ; \mathbf{R})\left|\nabla_{I}^{2}\right| \chi_{n m}(\mathbf{R}) \psi_{m}(\mathbf{r} ; \mathbf{R})\right\rangle_{\mathbf{r}}= \\
= & -\sum_{I=1}^{l} \frac{\hbar^{2}}{2 M_{I}}\left[\sum_{m} \nabla_{I}^{2} \chi_{n m}(\mathbf{R}) \delta_{k m}+\sum_{m}\left\langle\psi_{k}^{\dagger}(\mathbf{r} ; \mathbf{R})\left|\nabla_{I}^{2}\right| \psi_{m}(\mathbf{r} ; \mathbf{R})\right\rangle_{\mathbf{r}} \chi_{n m}(\mathbf{R})+\right. \\
& \left.+2 \sum_{m}\left\langle\psi_{k}^{\dagger}(\mathbf{r} ; \mathbf{R})\left|\nabla_{I}\right| \psi_{m}(\mathbf{r} ; \mathbf{R})\right\rangle_{\mathbf{r}} \nabla_{I} \chi_{n m}(\mathbf{R})\right]= \\
= & T_{N} \chi_{n k}(\mathbf{R})-\sum_{m}\left[B_{k m}(\mathbf{R}) \chi_{n m}(\mathbf{R})+A_{k m}(\mathbf{R}) \chi_{n m}(\mathbf{R})\right]
\end{aligned}
$$

onde identificamos os elementos $C_{k m}(\mathbf{R})=A_{k m}(\mathbf{R})+B_{k m}(\mathbf{R})$ como:

$$
\begin{aligned}
& A_{k m}(\mathbf{R})=\sum_{I=1}^{l} \frac{\hbar^{2}}{M_{I}}\left\langle\psi_{k}^{\dagger}(\mathbf{r} ; \mathbf{R})\left|\nabla_{I}\right| \psi_{m}(\mathbf{r} ; \mathbf{R})\right\rangle_{\mathbf{r}} \nabla_{I} \\
& B_{k m}(\mathbf{R})=\sum_{I=1}^{l} \frac{\hbar^{2}}{2 M_{I}}\left\langle\psi_{k}^{\dagger}(\mathbf{r} ; \mathbf{R})\left|\nabla_{I}^{2}\right| \psi_{m}(\mathbf{r} ; \mathbf{R})\right\rangle_{\mathbf{r}}
\end{aligned}
$$

Substituindo os termos obtidos a partir de $T_{N}$ e reagrupando-os com os demais termos da Equação 3.6 , obtém-se:

$$
\left[T_{N}+\epsilon_{k}(\mathbf{R})-E_{n}\right] \chi_{n k}(\mathbf{R})=-\sum_{m} C_{k m}(\mathbf{R}) \chi_{n m}(\mathbf{R})
$$

A aproximação adiabática de Born-Oppenheimer consiste em considerar que os elementos fora da diagonal da matriz $C$ sejam nulos. Nesse caso, a Equação 3.10 se resume a um conjunto de equações desacopladas, de forma a que o movimento nuclear é governado somente por equações puramente nucleares:

$$
\left[T_{N}+C_{k k}(\mathbf{R})+\epsilon_{k}(\mathbf{R})-E_{n}\right] \chi_{n k}(\mathbf{R})=0 .
$$

Impor que $C_{k m}(\mathbf{R})=0$ para $k \neq m$ significa dizer que o elétron não faz transições entre estados, mas é o estado eletrônico que se deforma progressivamente com o deslocamento nuclear. Pode-se afirmar, então, que os elétrons seguem o movimento nuclear adiabaticamente. Dentro dessa aproximação, os estados do sistema como um todo são dados por:

$$
\Psi_{n}(\mathbf{r}, \mathbf{R})=\chi_{n k}(\mathbf{R}) \psi_{k}(\mathbf{r} ; \mathbf{R})
$$


Não é fácil estabelecer um limite de validade para a aproximação de Born-Oppenheimer. Uma possibilidade seria considerar a condição 72 :

$$
\left|\sum_{I=1}^{l} \frac{\hbar^{2}}{M_{I}}\left\langle\chi_{n k}(\mathbf{R})\left|\nabla_{I}\right| \chi_{n q}(\mathbf{R})\right\rangle\left\langle\psi_{k}(\mathbf{r} ; \mathbf{R})\left|\nabla_{I}\right| \psi_{q}(\mathbf{r} ; \mathbf{R})\right\rangle\right| \ll\left|\epsilon_{k}(\mathbf{R})-\epsilon_{q}(\mathbf{R})\right| .
$$

Nota-se que o primeiro termo é proporcional a $M_{I}^{-1}$, o que conta a favor da aproximação. O segundo termo é dado pela diferença entre duas superfícies de energia. Se a diferença for pequena, podemos concluir que a aproximação é inadequada. Contudo, a aproximação de BornOppenheimer é extensivamente utilizada para descrever sistemas metálicos, onde o gap de energia é nulo. Neste caso, porém, como as temperaturas típicas são baixas quando comparadas à temperatura eletrônica de Fermi, as excitações são confinadas a uma pequena região em torno da superfície de Fermi contendo poucos elétrons, de modo que as contribuições não-adiabáticas são desprezíveis no cálculo da maioria das propriedades [72].

Para ir além da aproximação adiabática de Born-Oppenheimer, costuma-se tratar a energia cinética dos núcleos $T_{N}$ perturbativamente de modo análogo ao que fizemos na Seção 2.1.1. A Equação 3.11 com $C_{k k}(\mathbf{R})=0$ é a base do método frozen phonons para determinar as frequências vibracionais dos núcleos. Voltaremos a esta discussão quando apresentarmos o método utilizado para o cálculo das energias dos fônons e do acoplamento elétron-fônon na Seção 3.3 .

\subsection{Estrutura Eletrônica}

\subsubsection{Teoria do Funcional da Densidade}

Com a aproximação de Born-Oppenheimer, conseguimos separar o problema eletrônico do nuclear. No entanto, mesmo com essa simplificação, a parte eletrônica por si só continua sendo um problema complicado de muitos corpos interagentes, já que, para um sistema com $p$ elétrons, o problema possui $3 p$ variáveis. Essa dificuldade pode ser remediada por meio da Teoria do Funcional da Densidade, cujos teoremas fundamentais enunciaremos nesta seção.

A primeira tentativa de formular um método baseado apenas na densidade, uma forma rudimentar da DFT, foi apresentada independentemente por L. H. Thomas e E. Fermi, em 1927. Três anos depois, P. Dirac adicionou ao funcional de Thomas-Fermi a aproximação local para a troca utilizada até os dias de hoje [71]. A aproximação de Thomas-Fermi-Dirac mostrou-se uma ferramenta proveitosa para uma descrição qualitativa de certas características, como a energia total dos átomos, mas pouco útil para a investigação da matéria de um modo geral. Não obstante, 
chamou a atenção de W. Kohn o fato de considerar os elétrons movendo-se num potencial externo e de propiciar uma relação entre este potencial e a distribuição da densidade de elétrons [69].

Apesar dos cálculos via modelo de Thomas-Fermi-Dirac resultarem numa aproximação grosseira da solução exata da equação de Schrödinger, a ideia de transformar um problema de $3 p$ variáveis ( $p$ elétrons) em outro com somente 3 era muito atrativa. Isso levou Kohn a questionar se era possível, em princípio, descrever de forma completa e exata a estrutura eletrônica em termos de $n(\mathbf{r})$, isto é, se a densidade contém todas as informações que se pode obter do estado fundamental de um sistema. Em 1964, P. Hohenberg e Kohn provaram que a resposta para esse questionamento era afirmativa [68] e formalizaram o que hoje é conhecido como Teoria do Funcional da Densidade (Density Functional Theory - DFT). Para tal, propuseram e provaram dois teoremas que enunciaremos a seguir (as demonstrações dos teoremas estão dispostas no Apêndice A).

Teorema I: A densidade $n_{0}(\mathbf{r})$ do estado fundamental de um sistema ligado de elétrons interagentes em um dado potencial externo $V_{\text {ext }}(\mathbf{r})$ determina unicamente este potencial.

Vale observar que o termo "unicamente" pode ser entendido como "a menos de uma constante aditiva". Além disso, no caso do estado fundamental ser degenerado, o Teorema I refere-se à densidade de qualquer um desses estados. Como consequência imediata do Teorema I, tem-se o seguinte corolário:

Corolário: Se o potencial externo $V_{\text {ext }}(\mathbf{r})$ é determinado, então também o serão o Hamiltoniano (a menos de um deslocamento rígido de energia) e, consequentemente, as funções de onda de cada elétron para todos os estados (fundamental e excitados). Logo, dada a densidade $n_{0}(\mathbf{r})$ do estado fundamental, todas as demais propriedades do sistema estarão completamente determinadas.

Já o segundo teorema discorre sobre a existência de um funcional da densidade $E[n]$ e sobre a unicidade da densidade do estado fundamental.

Teorema II: Existe um funcional universal para a energia $E[n]$ em termos da densidade $n(\mathbf{r})$ que é válido para qualquer potencial externo. Fixado um $V_{\text {ext }}(\mathbf{r})$, a energia do estado fundamental corresponde ao mínimo global deste funcional e a densidade $n(\mathbf{r})$ que o minimiza é exatamente a densidade do estado fundamental $n_{0}(\mathbf{r})$. 
O conceito de universalidade implica algo que é válido para qualquer sistema de elétrons

73. Por exemplo, os operadores energia cinética $T=-\frac{\hbar^{2}}{2 m} \sum_{i=1}^{p} \nabla_{i}^{2}$ e energia de interação elétron-elétron $U=\frac{1}{4 \pi \epsilon_{0}} \sum_{i=1}^{p} \sum_{j<i}^{p} \frac{e^{2}}{\mathbf{r}_{i}-\mathbf{r}_{j} \mid}$ são denominados universais, enquanto que o operador energia potencial $V=\sum_{i=1}^{p} V_{e x t}\left(\mathbf{r}_{i}\right)$ é dito não-universal, pois depende do tipo de sistema. Por conseguinte, é por meio da energia potencial que se distingue o problema de um átomo frente ao de uma molécula, um sólido etc.

Corolário: O funcional $E[n]$ sozinho é suficiente para determinar exatamente a energia e a densidade do estado fundamental.

Surgiu, porém, a dúvida de como obter-se esse funcional para se poder aplicar a DFT no cálculo de propriedades dos materiais. A resposta veio apenas um ano após a publicação da formulação de Hohenberg-Kohn com o método de Kohn-Sham [42], que popularizou o uso da DFT.

\subsubsection{Equações de Kohn-Sham}

Basicamente, a hipótese por trás do método de Kohn-Sham é a existência de um sistema de partículas não-interagentes cuja densidade é igual à densidade exata do estado fundamental do sistema original interagente. Em outras palavras, substitui-se o problema complicado de muitos corpos interagentes por um problema com equações de partículas independentes onde todos os termos complicados do problema de muitos corpos são incorporados em um funcional de troca e correlação.

Além disso, assume-se que esse sistema auxiliar de partículas não-interagentes possa ser definido por um Hamiltoniano formado pelo operador energia cinética e um potencial local efetivo atuando em um elétron de spin $\sigma$, ou seja:

$$
H_{\text {aux }}=-\frac{\hbar^{2}}{2 m} \sum_{i=1}^{p} \nabla_{i}^{2}+V_{e f}(\mathbf{r}, \sigma) .
$$

A densidade eletrônica do sistema auxiliar é dada por:

$$
n(\mathbf{r})=\sum_{\sigma} \sum_{i=1}^{p / 2}\left|\phi_{k}(\mathbf{r}, \sigma)\right|,
$$

onde o estado fundamental possui um elétron em cada um dos orbitais $\phi_{k}(\mathbf{r}, \sigma)$ com os menores autovalores de $H_{\text {aux }}$. 
Segundo os teoremas de Hohenberg-Kohn, energia total do estado fundamental é um funcional da densidade $n(\mathbf{r})$ dado pela seguinte expressão:

$$
E_{H K}[n(\mathbf{r})]=\langle T\rangle+\langle U\rangle+\int d \mathbf{r} V_{e x t}(\mathbf{r}) n(\mathbf{r})+E_{N N}(\mathbf{R}),
$$

onde $E_{N N}(\mathbf{R})$ é a energia de interação nuclear que, dentro da aproximação de Born-Oppenheimer, é apenas um número, e não um operador. Kohn e Sham reescreveram esta expressão da seguinte forma:

$$
\begin{aligned}
E_{K S}[n(\mathbf{r})] & =\int d \mathbf{r} V_{e x t}(\mathbf{r}) n(\mathbf{r})+\underbrace{\frac{1}{2} \iint d \mathbf{r} d \mathbf{r}^{\prime} \frac{n(\mathbf{r}) n\left(\mathbf{r}^{\prime}\right)}{\left|\mathbf{r}-\mathbf{r}^{\prime}\right|}}_{E_{\text {Hartree }}[n(\mathbf{r})]}+\mathbb{G}[n(\mathbf{r})]+E_{N N}(\mathbf{R})= \\
& =\int d \mathbf{r} V_{\text {ext }}(\mathbf{r}) n(\mathbf{r})+E_{\text {Hartree }}[n(\mathbf{r})]+T_{s}[n(\mathbf{r})]+E_{x c}[n(\mathbf{r})]+E_{N N}(\mathbf{R}),
\end{aligned}
$$

onde $V_{\text {ext }}(\mathbf{r})$ é o potencial estático devido aos núcleos e $\mathbb{G}[n(\mathbf{r})]=T_{s}[n(\mathbf{r})]+E_{x c}[n(\mathbf{r})]$ é um funcional universal da densidade, onde $T_{s}[n(\mathbf{r})]$ é a energia cinética de um sistema de elétrons não-interagentes com densidade $n(\mathbf{r})$ e $E_{x c}[n(\mathbf{r})]$ é a energia de troca e correlação de um sistema interagente com a mesma densidade $n(\mathbf{r})$.

Comparando o funcional de Kohn-Sham (Equação 3.17) com o de Hohenberg-Kohn (Equação 3.16), nota-se que a energia de troca e correlação pode ser escrita como:

$$
E_{x c}[n(\mathbf{r})]=\langle T\rangle-T_{s}[n(\mathbf{r})]+\langle U\rangle-E_{\text {Hartree }}[n(\mathbf{r})] .
$$

Portanto, a energia de troca e correlação de Kohn-Sham é um funcional da densidade que é dado pelas energias cinética e interna de interação do sistema de muitos corpos interagentes subtraídas da energia do sistema de partículas independentes, o que corresponde à energia cinética do sistema não interagente com interações elétron-elétron dadas pela energia de Hartree.

A minimização do funcional de Kohn-Sham (Equação 3.17) pode ser feita por meio do método dos multiplicadores de Lagrange utilizando as condições de ortonormalização dos orbitais $\phi_{k}(\mathbf{r}, \sigma)$, o que leva a equações tipo Schrödinger de partículas independentes:

$$
\left(H_{K S}-\varepsilon_{k}\right) \phi_{k}(\mathbf{r}, \sigma)=0
$$

onde $H_{K S}$ é o Hamiltoniano efetivo dado por: 


$$
H_{K S}(\mathbf{r}, \sigma)=-\frac{\hbar^{2}}{2 m} \sum_{i=1}^{p} \nabla_{i}^{2}+V_{K S}(\mathbf{r}, \sigma),
$$

onde $V_{K S}(\mathbf{r}, \sigma)$ é um potencial local efetivo atuando em um elétron de spin $\sigma$ e é dado por:

$$
\begin{aligned}
V_{K S}(\mathbf{r}, \sigma) & =V_{\text {ext }}(\mathbf{r})+\frac{\delta E_{\text {Hartree }}}{\delta n(\mathbf{r})}+\frac{\delta E_{x c}}{\delta n(\mathbf{r})}= \\
& =V_{\text {ext }}(\mathbf{r})+V_{\text {Hartree }}(\mathbf{r})+V_{x c}(\mathbf{r}, \sigma) .
\end{aligned}
$$

As Equações 3.19, 3.20 e 3.21 são conhecidas como equações de Kohn-Sham e, junto com a Equação 3.15, podem ser resolvidas de forma autoconsistente. A partir de um valor inicial para a densidade, calcula-se o potencial efetivo $V_{K S}(\mathbf{r}, \sigma)$, que, por sua vez, determina a Equação 3.19. Com as $p$ soluções $\phi_{k}(\mathbf{r}, \sigma)$, correspondentes aos $p$ menores autovalores, calcula-se a nova densidade (Equação 3.15). Uma vez atingida a autoconsistência, obtém-se a densidade e, consequentemente, a energia do estado fundamental do sistema interagente original com a precisão limitada somente pela aproximação no funcional de troca e correlação e pela base utilizada (na prática).

Segundo Kohn, esta teoria trouxe duas contribuições importantes para a ciência dos materiais. A primeira consiste na compreensão fundamental de sistemas quânticos de muitos corpos. Com o estabelecimento da equação de Schrödinger, os físicos se habituaram a pensar em termos de funções de onda de uma partícula. Entretanto, quanto mais complexo o sistema, mais difícil é manter esse ponto de vista. Nesse cenário, a DFT trouxe uma perspectiva complementar em termos de grandezas físicas no espaço real.

A outra contribuição se refere à aplicabilidade. Mesmo com o rápido avanço tecnológico que se observa atualmente, os métodos tradicionais possuem um limite no que se refere ao tamanho do sistema. Com a DFT, foi possível transpor esta barreira, tornando viável cálculos precisos de estrutura eletrônica para sistemas com $O\left(10^{2}\right)$ até $O\left(10^{3}\right)$ átomos 69].

$\mathrm{Na}$ prática, ao incorporar todos os termos complicados do problema de muitos corpos ao funcional de troca e correlação, o método de Kohn-Sham possibilitou que formulações aproximadas fossem realizadas. Na próxima seção, vamos discutir brevemente as aproximações mais conhecidas para este funcional.

\subsubsection{Funcionais de Troca e Correlação}

Se o funcional de troca e correlação fosse conhecido, o método de Kohn-Sham seria, em princípio, exato. Porém, a forma deste funcional é desconhecida e aproximações são necessárias. Com 
efeito, existe todo um campo de pesquisa dedicado a esse assunto e diversos funcionais já foram desenvolvidos 74. Dentre eles, o mais simples é a aproximação local para a densidade (Local Density Approximation - LDA), que consiste em considerar que a densidade de energia de troca e correlação é a mesma de um gás de elétrons homogêneo com esta densidade:

$$
E_{x c}^{L D A}[n(\mathbf{r})]=\int d \mathbf{r} n(\mathbf{r}) \epsilon_{x c}^{h o m}(n(\mathbf{r})),
$$

onde $\epsilon_{x c}^{\text {hom }}(n(\mathbf{r}))=\epsilon_{x}^{\text {hom }}(n(\mathbf{r}))+\epsilon_{c}^{\text {hom }}(n(\mathbf{r}))$ é a energia de troca e correlação associada a um gás de elétrons homogêneo de densidade $n(\mathbf{r})$. A energia de troca do gás homogêneo $\epsilon_{x}^{\text {hom }}(n(\mathbf{r}))$ possui uma forma analítica simples e a energia de correlação $\epsilon_{c}^{\text {hom }}(n(\mathbf{r}))$ foi calculada com grande precisão via métodos de Monte Carlo [71].

Por motivos óbvios, esta aproximação é boa para sistemas em que a densidade varia lentamente no espaço. Espera-se, portanto, que a LDA forneça resultados melhores para sólidos, porém esta aproximação mostra-se muito boa também para alguns átomos e moléculas [74]. Uma melhoria natural da LDA é considerar o gradiente da densidade e o valor da densidade em cada ponto, o que levou ao desenvolvimento das aproximações generalizadas com o gradiente (Generalized-Gradient Approximation - GGA):

$$
E_{x c}^{G G A}[n(\mathbf{r})]=\int d \mathbf{r} n(\mathbf{r}) \epsilon_{x c}\left(n, \nabla_{n}\right)
$$

Existem diferentes propostas para a energia de troca e correlação $\epsilon_{x c}\left(n, \nabla_{n}\right)$. Ao contrário da LDA, onde a forma deste funcional é única, as GGAs podem ser obtidas de diversas maneiras, ajustando-se a resultados experimentais ou por meio de limites conhecidos teoricamente. Neste trabalho, foi utilizada uma GGA baseada na parametrização não-empírica de PerdewBurke-Ernzerhof (PBE) [75]. De modo geral, as GGAs melhoram os resultados para sistemas moleculares, como distâncias e energias de ligação, quando comparados com os obtidos via LDA.

\subsubsection{O Programa SIESTA}

A implementação da DFT escolhida para ser utilizada neste trabalho foi a fornecida pelo código SIESTA [2:3] (Spanish Initiative for Electronic Simulations with Thousands of Atoms). Conforme ficará claro mais à frente, as metodologias utilizadas neste código, como a escolha da representação dos estados de Kohn-Sham, o tornam um candidato natural para ser utilizado como base de estrutura eletrônica no cálculo de transporte. Nesta seção, serão descritas resumidamente suas principais características. 
No código SIESTA, os elétrons de uma espécie atômica são separados em elétrons de caroço, mais ligados aos núcleos, e elétrons de valência. Dessa forma, o potencial sentido pelos elétrons de valência é dado pelo núcleo blindado pelos elétrons de caroço, o que é descrito por pseudopotenciais de norma conservada [76]. Isso tem como efeito a suavização das funções de onda quando próximas do caroço e, ao mesmo tempo, reduz o problema ao tratamento dos elétrons mais externos. Estes elétrons, por sua vez, são descritos por bases localizadas nos átomo@ ${ }^{1}$ e são estritamente confinados em uma região além da qual os orbitais são nulos. Dentro desta região de confinamento, para cada átomo $I$, localizado em $\mathbf{R}_{I}$, a base de orbitais atômicos é definida pela seguinte combinação de funções:

$$
\phi_{n l m}^{I}\left(\mathbf{r}_{I}\right)=\mathcal{R}_{n l}^{I}\left(r_{I}\right) Y_{l m}\left(\hat{\mathbf{r}}_{I}\right),
$$

onde $\mathcal{R}_{n l}^{I}\left(r_{I}\right)$ é a função radial do $n$-ésimo orbital e $Y_{l m}(\hat{\mathbf{r}})$ é o harmônico esférico real com momento angular $l$ e número quântico magnético $m$, e onde ambas as funções são convenientemente escritas em termos de $\mathbf{r}_{I}=\mathbf{r}-\mathbf{R}_{I}$.

Para obter-se uma base balanceada, onde o efeito do confinamento é semelhante para todos os orbitais, convém fixar um deslocamento de energia $\delta \epsilon$ (energy shift) ao invés de ajustar um raio de corte comum a todas as espécies atômicas e momentos angulares. Portanto, o método utilizado pelo programa considera que cada orbital terá um ganho particular em energia ao ser confinado (energy shift).

A quantidade mínima de orbitais a serem utilizados é dada pelo número de elétrons de valência, e, neste caso, a base é chamada "minimal". No entanto, para aumentar a liberdade variacional no processo de minimização, o programa permite que sejam incluídos orbitais vazios e que sejam utilizadas bases múltiplo- $\zeta$, onde todo orbital $\zeta$ possui o mesmo harmônico esférico que o orbital original, mas com diferentes dependências radiais.

Existem diversas vantagens em escolher um conjunto de bases localizadas, quando comparado, por exemplo, com uma base de ondas planas. Uma delas é o alcance finito de interação, o que faz com que as matrizes Hamiltoniana e de overlap se tornem relativamente esparsas, economizando memória e tempo computacional. Além disso, essas matrizes assumem uma forma "tight-binding" nesta representação, o que, como veremos no próximo capítulo, é ideal para a aplicação do problema de transporte com o formalismo NEGF. Com essa escolha de orbitais, os elementos da matriz de overlap e a maior parte dos elementos da matriz Hamiltoniana são dados por integrais

\footnotetext{
${ }^{1}$ Conforme veremos no próximo capítulo, o uso de bases localizadas torna o código conveniente para ser utilizado no tratamento do transporte onde o sistema é dividido em diferentes regiões (eletrodos e região de espalhamento).
} 
de dois centros. Por exemplo, a matriz de overlap é definida por:

$$
S_{i j}=\int d \mathbf{r} \phi_{i}^{I}\left(\mathbf{r}_{I}\right) \phi_{j}^{J}\left(\mathbf{r}_{J}\right)
$$

onde $i=(n, l, m)_{I}$ percorre todos os orbitais do $I$-ésimo átomo e onde, novamente, $\mathbf{r}_{I}=\mathbf{r}-\mathbf{R}_{I}$, com $\mathbf{R}_{I}$ a posição do $I$-ésimo átomo. Essas integrais são calculadas de maneira eficiente no espaço recíproco por meio de uma transformada de Fourier, onde a convolução se torna simplesmente um produto. Porém, alguns elementos da matriz Hamiltoniana têm que ser calculados no espaço real e a precisão destes cálculos é determinada pelo espaçamento da malha (grid) de energia utilizado.

Para obter a Hamiltoniana de Kohn-Sham, o programa calcula inicialmente o potencial efetivo. Isto é realizado partindo-se de uma densidade de carga inicial, usualmente dada pela densidade de carga dos átomos isolados que constituem o sistema. O potencial de troca e correlação é calculado usando a aproximação LDA ou a GGA, descritas na Seção 3.2.3. Já o potencial de Hartree é obtido resolvendo-se a equação de Poisson:

$$
\nabla^{2} V_{\text {Hartree }}(\mathbf{r})=-e^{2} n(\mathbf{r})
$$

Esta equação é computada no espaço recíproco, o que implica que o potencial seja periódico. O SIESTA utiliza condições periódicas de contorno correspondentes a uma supercélula com periodicidade nas três dimensões espaciais. A base da abordagem por supercélulas remete ao teorema de Bloch, onde, para um sistema submetido a um potencial periódico, afirma-se que a função de onda eletrônica pode ser escrita como o produto de uma onda plana $e^{i \mathbf{k} \cdot \mathbf{r}}$ por uma função $u_{\mathbf{k}}(\mathbf{r})$ com a periodicidade da rede:

$$
\psi_{j}(\mathbf{k}, \mathbf{r})=u_{j, \mathbf{k}}(\mathbf{r}) e^{i \mathbf{k} \cdot \mathbf{r}}
$$

onde $j$ é um índice de banda e $\mathbf{k}$ é o vetor da rede recíproca pertencente à primeira zona de Brillouin (BZ) correspondente à supercélula. Isto permite mapear o problema de autovalores de Kohn-Sham (Equação 3.19) no espaço recíproco, de modo que é possível obter, para cada ponto k, um conjunto discreto de autoestados do Hamiltoniano.

Os elementos da matriz Hamiltoniana de Kohn-Sham no espaço real são obtidos projetando-se a Equação 3.20 em cada orbital, o que resulta em integrais com a seguinte forma:

$$
H_{i j}=-\frac{\hbar^{2}}{2 m} \int d \mathbf{r} \phi_{i}^{I}\left(\mathbf{r}_{I}\right) \nabla^{2} \phi_{j}^{J}\left(\mathbf{r}_{J}\right)+\int d \mathbf{r} \phi_{i}^{I}\left(\mathbf{r}_{I}\right) V_{K S}(\mathbf{r}) \phi_{j}^{J}\left(\mathbf{r}_{J}\right) .
$$


Seguindo o teorema de Bloch, o Hamiltoniano para o sistema periódico é dado por:

$$
H_{i j}(\mathbf{k})=\sum_{l} H_{i j^{\prime}}^{l} e^{i \mathbf{k} \cdot \mathbf{r}_{l}}
$$

onde $\mathbf{r}_{l}$ é o vetor posição entre as células unitárias. Um procedimento análogo é realizado para a matriz de overlap.

Dessa forma, o problema se reduz às dimensões da célula unitária e a Hamiltoniana é diagonalizada, obtendo-se os coeficientes da expansão do estado de Bloch $c_{\mu j}(\mathbf{k})$ e, por conseguinte, os autoestados de Kohn-Sham:

$$
\psi_{j}(\mathbf{k}, \mathbf{r})=\sum_{\mu} c_{\mu j}(\mathbf{k}) \phi_{\mu}(\mathbf{r}) e^{i k R_{\mu}}
$$

onde a soma em $\mu$ se estende sobre todos os orbitais e $j$ é um índice de banda. Tendo os autoestados de Kohn-Sham, a nova densidade eletrônica é obtida com:

$$
n(\mathbf{r})=\sum_{\mu \nu} D_{\mu \nu} \phi_{\mu}^{*}(\mathbf{r}) \phi_{\nu}(\mathbf{r})
$$

onde, novamente, as somatórias se estendem sobre todos os orbitais e onde $D_{\mu \nu}$ são os elementos da matriz densidade dados por:

$$
D_{\mu \nu}=\sum_{j} \int_{B Z} d \mathbf{k} c_{\mu j}(\mathbf{k}) N_{j}(\mathbf{k}) c_{j \nu}(\mathbf{k}) e^{i k\left(R_{\nu}-R_{\mu}\right)},
$$

onde $N_{j}(\mathbf{k})$ é a ocupação do estado $j$.

A nova densidade eletrônica é, então, comparada com a densidade eletrônica anterior. Caso o critério de convergência não tenha sido atingido, isto é, se $\left|n^{i}-n^{i+1}\right|>\delta_{\text {conv }}$, os passos acima são repetidos a partir da nova densidade eletrônica, e assim sucessivamente até atingir a autoconsistência. Esse procedimento é válido para sistemas em equilíbrio, sendo necessário alterá-lo no caso de cálculos de transporte eletrônico, conforme veremos na Seção 4.5 .

As condições periódicas de contorno utilizadas pelo SIESTA são convenientes para cálculos de sistemas infinitos, como cristais. Para tratar sistemas finitos é necessário criar uma supercélula suficientemente grande para que não ocorra overlap entre orbitais de células unitárias vizinhas.

O processo de encontrar a geometria otimizada do sistema consiste em obter uma configuração em que a força entre os átomos é mínima. Ou seja, pequenos deslocamentos são aplicados aos núcleos de forma a encontrar o mínimo da superfície de energia de Born-Oppenheimer, onde a 
força em cada núcleo é nula. O código SIESTA possui alguns algoritmos para esta otimização de coordenadas, entre eles o Gradiente Conjugado. O cálculo das forças interatômicas é realizado com base no Teorema de Hellmann-Feynman que será discutido na próxima seção.

\subsection{Análise Vibracional e Acoplamento Elétron-Fônon}

Uma vez calculada a densidade do estado fundamental via DFT dentro da aproximação de BornOppenheimer, isto é, para uma configuração fixa dos núcleos, uma pergunta natural é como o movimento nuclear evolui no tempo. Para tanto, calcula-se a força conjugada à posição $\mathbf{R}_{I}$ do I-ésimo núcleo, o que pode ser escrita como:

$$
\mathbf{F}_{I}=-\frac{\partial E_{0}}{\partial \mathbf{R}_{I}}
$$

A partir da expressão geral para a energia total do estado fundamental (Equação 3.16), esta força é dada, segundo o Teorema de Hellmann-Feynman [71, por:

$$
\begin{aligned}
\mathbf{F}_{I} & =-\frac{\partial}{\partial \mathbf{R}_{I}}\left[\langle T\rangle+\langle U\rangle+\int d \mathbf{r} V_{e x t}(\mathbf{r}) n(\mathbf{r})+E_{N N}\right]= \\
& =-\int d \mathbf{r} \frac{\partial V_{e x t}(\mathbf{r})}{\partial \mathbf{R}_{I}} n(\mathbf{r})-\frac{\partial E_{N N}}{\partial \mathbf{R}_{I}} .
\end{aligned}
$$

Logo, a força agindo sobre o I-ésimo átomo depende somente da densidade de elétrons no estado fundamental $n(\mathbf{r})$ e dos demais núcleos (mantidos fixos).

De modo análogo ao que fizemos ao descrever a segunda quantização dos fônons em 3 dimensões (Seção 2.1.1 - Equação 2.27), a geometria de equilíbrio $\mathbf{R}^{0}$ é obtida pela condição $\mathbf{F}_{J}\left(\mathbf{R}^{0}\right)=0$.

Considerando pequenos deslocamentos em torno da configuração de equilíbrio $\mathbf{R}^{0}$, vimos que o cálculo dos modos e frequências vibracionais, dentro da aproximação harmônica, se dá a partir da matriz de constantes de força interatômica, que, por sua vez, é calculada com as segundas derivadas da superfície de energia de Born-Oppenheimer com relação às coordenadas nucleares:

$$
\left.C_{I \nu, J \mu} \equiv \frac{\partial^{2} E_{0}(\mathbf{R})}{\partial R_{I \nu} \partial R_{J \mu}}\right|_{\mathbf{R}=\mathbf{R}^{0}}
$$

onde os índices $I$ e $J$ pertencem ao conjunto de todos íons dinâmicos e $\nu$ e $\mu$ correspondem às coordenadas $x, y$ e $z$. 
Na Seção 2.1.1 consideramos que os núcleos possuíam a mesma massa. Para um sistema geral, porém, é conveniente escalar a Equação 2.34 pelas diferentes massas nucleares, da seguinte maneira:

$$
-\omega^{2} M_{I} Q_{I \nu}=-\sum_{J \mu} C_{I \nu, J \mu} Q_{J \mu} \quad \Longrightarrow \quad\left(\omega^{2} \mathbb{1}-W\right) \mathbf{v}=0
$$

onde $W_{I \nu, J \mu} \equiv C_{I \nu, J \mu} / \sqrt{M_{I} M_{J}}$ é matriz de constantes de força interatômica escalada por massas e $\mathbf{v}_{I}=\sqrt{M_{I}} \mathbf{Q}_{I}$. Portanto, a frequência vibracional $\omega_{\lambda}$ e o modo $\mathbf{v}^{\lambda}=\{\mathbf{v}\}_{\mathbf{I}}^{\lambda}$ pertencem ao conjunto das autossoluções $\left(\omega_{\lambda}^{2}, \mathbf{v}^{\lambda}\right)$.

Com a aproximação adiabática de Born-Oppenheimer, o Hamiltoniano eletrônico depende parametricamente das coordenadas nucleares $H_{e}=H_{e}(\mathbf{Q})$, onde $\mathbf{Q}$ é o vetor deslocamento dado por $\mathbf{R}-\mathbf{R}^{0}$, sendo $\mathbf{R}^{0}$ a configuração de equilíbrio. Para ir além desta aproximação e considerar o acoplamento elétron-fônon, devemos calcular a variação da Hamiltoniana eletrônica com o deslocamento nuclear. Considerando que o movimento nuclear esteja confinado em uma pequena região em torno $\mathbf{R}^{0}$, podemos tomar a expansão de $H_{e}$ até a mais baixa ordem em $\mathbf{Q}$ :

$$
H_{e} \approx H_{e}^{0}+\left.\sum_{I, \nu} \frac{\partial H_{e}}{\partial Q_{I \nu}}\right|_{Q=0} Q_{I \nu}
$$

onde o índice $I$ percorre todos os íons dinâmicos e $\nu$ corresponde às coordenadas $x, y$ e $z$. Impondo a transformação para as coordenadas dos modos normais e utilizando as quantizações canônicas usuais dos operadores posição e momento (Seção 2.1.1), obtém-se:

$$
H_{e} \approx H_{e}^{0}+\left.\sum_{I, \nu} \frac{\partial H_{e}}{\partial Q_{I \nu}}\right|_{Q=0} \sum_{\lambda} v_{I \nu}^{\lambda} \sqrt{\frac{\hbar}{2 M_{I} \omega_{\lambda}}}\left(b_{\lambda}^{\dagger}+b_{\lambda}\right)
$$

onde $\mathbf{v}^{\lambda}=\left\{v_{I \nu}^{\lambda}\right\}$ é o vetor deslocamento iônico do modo normal $\lambda$ de frequência $\omega_{\lambda}$ e $M_{I}$ é a massa do íon $I$.

Comparando esta equação com a Equação 2.57, infere-se que a matriz de acoplamento elétron-fônon é dada por:

$$
M_{i j}^{\lambda}=\sum_{I, \nu}\left\langle i\left|\frac{\partial H_{e}}{\partial Q_{I \nu}}\right| j\right\rangle_{Q=0} \mathbf{v}_{I \nu}^{\lambda} \sqrt{\frac{\hbar}{2 M_{I} \omega_{\lambda}}} .
$$

Portanto, temos uma maneira de realizar cálculos ab initio das frequências e do acoplamento elétron-fônon vistos na teoria do Capítulo 2 


\subsubsection{O Programa PhOnonS ITeratIVE VIBRATIONS}

Nesta tese foi desenvolvido o programa PhOnonS ITeratIVE VIBRATIONS, uma ferramenta para calcular modos e frequências vibracionais e a matriz de acoplamento elétron-fônon a partir de cálculos de primeiros princípios. Além de implementar um novo código, escrito com uma combinação de linguagem $C$ com scripts em Shell, algumas intervenções foram realizadas no SIESTA, cujos detalhes serão descritos a seguir.

O código SIESTA possui um loop externo em que a estrutura eletrônica é calculada para uma dada geometria (onde as forças atômicas $\mathbf{F}_{I}=\left\{F_{I \nu}\right\}$ são obtidas juntamente com o cálculo de energia total) e as posições atômicas são atualizadas. Na opção de execução FC (Force Constants), em cada ciclo do loop um íon dinâmico é movido em uma das direções $x, y$ ou $z$ por um pequeno deslocamento $Q_{J \mu}= \pm \Delta$ determinado pelo usuário e as componentes das forças exercidas sobre cada íon $\left(F_{I \nu} / Q_{J}\right)$ são computadas.

Dessa forma, a matriz de constantes de força interatômica pode ser aproximada por diferenças finitas, o que é conhecido como método frozen phonons:

$$
C_{I \nu, J \mu}=-\left.\frac{\partial F_{I \nu}(\mathbf{R})}{\partial R_{I \mu}}\right|_{\mathbf{R}=\mathbf{R}^{0}} \Longrightarrow C_{I \nu, J \mu} \approx-\frac{F_{I \nu}\left(Q_{J \mu}\right)-F_{I \nu}\left(-Q_{J \mu}\right)}{2 Q_{J \mu}}
$$

No entanto, um dos problemas conhecidos é que o código SIESTA pode errar a estimativa da variação da força no átomo que está sendo deslocado. Este problema está relacionado ao efeito conhecido como egg-box, que surge ao movimentar-se os orbitais base em relação ao grid de integração no espaço real [3]. Para calcular algumas integrais e para representar as densidades de carga e potenciais, o código SIESTA utiliza um grid finito em três dimensões, o que causa a quebra da simetria translacional. Esse fato é evidenciado ao mover-se um único átomo do sistema, onde se pode observar que a energia total e as forças oscilam de acordo com a periodicidade do grid (o que explica o nome dado ao efeito).

Para minimizar esse problema, uma solução seria aumentar a densidade do grid. Todavia, existe um limite operacional, já que o custo computacional cresce de forma aproximadamente linear com o aumento da densidade do grid. Outra forma de contornar o problema é impor a conservação do momento em cada direção $\nu$ (ver propriedade na Equação 2.30). Isso é feito via $\sum_{I} \Delta F_{I \nu}=0$, o que, então, determina os elementos da diagonal da matriz de constantes de força de acordo com: 


$$
\tilde{C}_{I \nu, J \mu}= \begin{cases}C_{I \nu, J \mu}, & I \neq J, \\ -\sum_{K \neq I} C_{I \nu, K \mu}, & I=J,\end{cases}
$$

onde $K$ varre todos os íons do sistema.

A matriz de constantes de força é simétrica (ver propriedade na Equação 2.30] e, portanto, possui autovalores reais. Pode ocorrer, no entanto, devido ao limite de precisão numérica, que alguns elementos sejam levemente diferentes de seus correspondentes simétricos. Portanto, antes de diagonalizar a matriz, aplica-se uma simetrização conforme $C_{i j}=\left(C_{i j}+C_{j i}\right) / 2$.

Outro problema, bastante conhecido na literatura, é que ao diagonalizar a matriz $W$ (Equação 3.36 pode-se encontrar alguns autovalores $\left\{\omega_{\lambda}^{2}\right\}$ negativos, levando a frequências imaginárias $\left\{\omega_{\lambda}\right\}$ e indicando que a configuração atômica $\mathbf{R}^{0}$ não está de fato descrevendo um verdadeiro mínimo de energia da superfície de Born-Oppenheimer. Para evitar que isso aconteça, é importante realizar a relaxação da estrutura de forma criteriosa, isto é, de forma a que as forças agindo sobre os átomos sejam inferiores a um certo valor limite (tipicamente o critério de força utilizado varia de $\max \left|F_{I \nu}\left(\mathbf{R}^{0}\right)\right|<0.01$ a $\left.\max \left|F_{I \nu}\left(\mathbf{R}^{0}\right)\right|<0.001 \mathrm{eV} \AA^{-1}\right)$.

Uma vez obtidas as frequências e os modos vibracionais, para calcular a matriz de acoplamento elétron-fônon resta, então, entender como obter-se os elementos de matriz dos gradientes do Hamiltoniano $\left\langle i\left|\partial H_{e} / \partial Q_{I \nu}\right| j\right\rangle$. Com o código SIESTA, temos acesso somente aos elementos de matriz $\left\langle i\left|H_{e}\right| j\right\rangle$ representados na base de orbitais atômicos. Contudo, podemos reescrever tais elementos da seguinte forma [77, 78]:

$$
\left\langle i\left|\frac{\partial H_{e}}{\partial Q_{I \nu}}\right| j\right\rangle=\frac{\partial\left\langle i\left|H_{e}\right| j\right\rangle}{\partial Q_{I \nu}}-\left\langle i^{\prime}\left|H_{e}\right| j\right\rangle-\left\langle i\left|H_{e}\right| j^{\prime}\right\rangle
$$

onde $\left|i^{\prime}\right\rangle \equiv \frac{\partial|i\rangle}{\partial Q_{I \nu}}$ representa a variação dos orbitais base com os deslocamentos.

A fim de calcular o primeiro termo da Equação 3.42 por diferenças finitas, o código SIESTA foi modificado para que, durante a execução FC (constantes de força), a Hamiltoniana de KohnSham seja armazenada a cada deslocamento.

Utilizando a identidade $\sum_{i, j}|i\rangle\left(\mathbf{S}^{-1}\right)_{i j}\langle j|=1$, onde $\mathbf{S}$ é a matriz de overlap, os demais termos podem ser reescritos como: 


$$
\begin{aligned}
& -\left\langle i^{\prime}\left|H_{e}\right| j\right\rangle=-\sum_{k, l}\left\langle i^{\prime} \mid k\right\rangle\left(\mathbf{S}^{-1}\right)_{k l}\left\langle l\left|H_{e}\right| j\right\rangle, \\
& -\left\langle i\left|H_{e}\right| j^{\prime}\right\rangle=-\sum_{k, l}\left\langle i\left|H_{e}\right| k\right\rangle\left(\mathbf{S}^{-1}\right)_{k l}\left\langle l \mid j^{\prime}\right\rangle .
\end{aligned}
$$

A Hamiltoniana de Kohn-Sham e a matriz de overlap são fornecidas diretamente pelo código SIESTA, restando somente calcular os termos $\left\langle i^{\prime} \mid k\right\rangle$ e $\left\langle l \mid j^{\prime}\right\rangle$. Estes elementos são obtidos por diferenças finitas a partir das matrizes de overlap resultantes de execuções do código SIESTA para o sistema duplicado, mas deslocando-se todos os átomos replicados de $\pm Q_{\nu}$ (totalizando seis execuções diferentes: $-x,+x,-y,+y,-z$ e $+z)$. Para automatizar esse processo, foi desenvolvido um script em linguagem Shell e o código SIESTA foi modificado de forma a inserir a opção de somente armazenar a matriz de overlap e encerrar a execução (isso torna muito rápida a obtenção destas matrizes, já que elas são definidas logo no início da execução).

Além de calcular as frequências e modos vibracionais, com as correções e cuidados descritos acima, e de calcular as matrizes de acoplamento elétron-fônon a partir do produto das intervenções no código SIESTA, o programa PhOnonS ITeratIVE VIBRATIONS também gera arquivos para a visualização de cada um dos modos vibracionais com o programa Jmol, um software livre em linguagem Java para visualização de estruturas químicas [79].

Portanto, com a implementação do código PhOnonS ITeratIVE VIBRATIONS, construímos uma ferramenta para a análise de vibrações e do acoplamento elétron-fônon a partir de métodos de primeiros princípios, o que é interessante não apenas para o transporte eletrônico mas também para o estudo de outras propriedades que envolvem fônons e elétrons. 


\section{Capítulo 4}

\section{Transporte Eletrônico em Escala Nanométrica}

A busca por dispositivos cada vez menores, mais rápidos e mais eficientes é representada pela lei de Moore, que afirma que a densidade de transistores nos circuitos integrados dobra aproximadamente a cada dois anos [13]. Atualmente, os microprocessadores dos computadores domésticos possuem cerca de 1 bilhão de transistores, sendo o mais comum o transistor de efeito de campo (FET - Field-Effect Transistor). Porém, essa miniaturização dos dispositivos está levando as tecnologias atuais, baseadas no silício, a alcançar limites fundamentais a partir dos quais os efeitos quânticos se tornam predominantes. Desta forma surgem diversos desafios, tanto do ponto de vista experimental como teórico.

A bem conhecida lei de Ohm, que apareceu em um tratado sobre eletricidade de Georg S. Ohm em 1827 [80], afirma que a corrente que passa por um condutor é diretamente proporcional à diferença de potencial entre os dois pontos terminais e inversamente proporcional a sua resistência. No entanto, apesar de esta lei simples ser válida em muitos dispositivos para uma vasta gama de valores de corrente e tensão, existem situações onde ela claramente falha. Um exemplo é quando as dimensões características do condutor se tornam suficientemente pequenas, de forma a que a natureza quântica dos portadores de carga já não possa mais ser negligenciada.

Neste capítulo, tomando um dispositivo com dois contatos (fonte e dreno) como objeto de estudo, serão apresentadas algumas das dificuldades encontradas ao se tentar descrever, a partir da lei de Ohm, a condutividade em dispositivos cujas dimensões são muito menores do que o próprio livre caminho médio. Será discutido, então, o modelo proposto por R. Landauer 81, onde a condutância é dada em termos da probabilidade de transmissão de elétrons espalhados por um potencial.

Em seguida, veremos como o formalismo NEGF, discutido no Capítulo 2, pode ser aplicado 
ao problema de transporte, onde será derivada uma expressão para a corrente similar ao modelo de Landauer. Por fim, discutiremos detalhes da implementação computacional do código SMEAGOL [4] (Spin and Molecular Electronics in Atomically Generated Orbital Landscapes), um código de transporte eletrônico ab initio baseado na combinação DFT-NEGF e que utiliza como plataforma do cálculo da estrutura eletrônica o código SIESTA (apresentado no Capítulo 3.

\subsection{Introdução}

Um transistor pode ser entendido, grosso modo, como um resistor (Canal) cuja resistência pode ser controlada (Figura 4.1). Para se medir a resistência, conecta-se o canal a dois contatos, fonte $(L)$ e dreno $(R)$, e aplica-se uma diferença de potencial $(V)$ para possibilitar um fluxo de elétrons $(I)$. Os contatos são constituídos por materiais de condutividade muito alta quando comparados ao canal, de modo que a resistência do dispositivo se dá basicamente pelo canal. Sob este coloca-se um material isolante por onde, idealmente, os elétrons não podem fluir, mas onde se pode aplicar uma tensão $\left(V_{G}\right)$ de forma a mudar a "condição" do canal. Variando-se a "condição" do canal, varia-se a quantidade de corrente que passa pelo dispositivo, ou seja, muda-se a resistência. Portanto, $V$ direciona a corrente e $V_{G}$ controla a resistência.

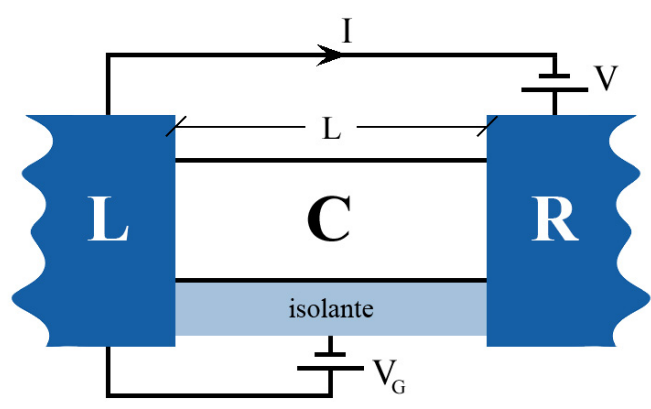

Figura 4.1: Desenho esquemático de um transistor, com um canal $\mathbf{C}$ de comprimento $L$ conectado aos contatos fonte $(\mathbf{L})$ e dreno $(\mathbf{R})$ onde é aplicada uma diferença de potencial $V$ de forma a produzir uma corrente I. A quantidade de corrente que passa no dispositivo pode ser controlada por meio de uma tensão de porta $V_{G}$.

A corrente $I$ no canal cresce linearmente com a tensão $V$ até atingir uma corrente de saturação. De acordo com a lei de Ohm, a inclinação da reta no regime linear corresponde ao inverso da resistência $\mathcal{R}$, isto é:

$$
\frac{d I}{d V}=\frac{1}{\mathcal{R}}
$$


A tensão de porta $\left(V_{G}\right)$ pode aumentar ou suprimir a corrente variando-se a resistência de tipicamente $10 k \Omega$ (canal aberto) a $100 k \Omega$ (canal fechado), conforme esboço na Figura 4.2 .

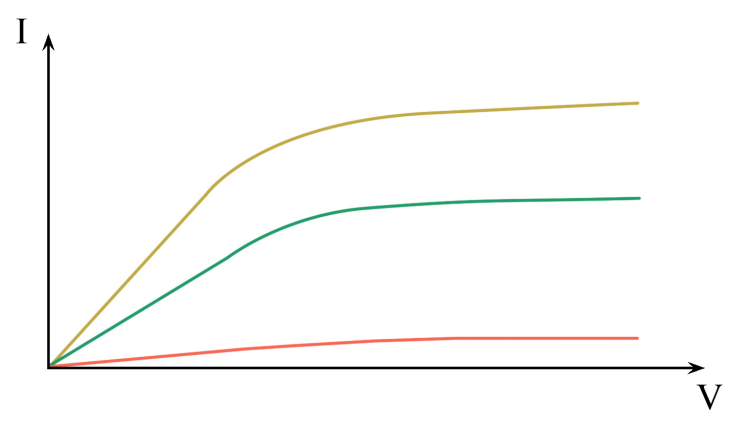

Figura 4.2: Esboço da curva $I \times V$ para três diferentes tensões de porta $V_{G}$.

O comprimento típico do canal em um transistor comercial é de $L>10 \mu m$. Já em laboratório chega-se em $L \sim 1 \mathrm{~nm}$ (por exemplo, coloca-se contatos em uma molécula de hidrogênio e medese sua resistência [6]). Porém, o que é resistência nesta ordem de grandeza? Para $L \sim 10 \mu m$ vale a lei de $\mathrm{Ohm}$, de onde se pode escrever a condutância como:

$$
\mathcal{G}=\sigma \frac{A}{L}
$$

onde $A$ é a seção transversal e $\sigma=1 / \mathfrak{R}$ é a condutividade (inverso da resistividade $\mathfrak{R}$ ), que, por sua vez, é normalmente encontrada na forma semiclássica como 82]:

$$
\sigma=e \rho \frac{e \tau}{m^{*}},
$$

onde $e$ é a carga do elétron, $\rho$ é a densidade eletrônica, $\tau$ é o tempo livre médio entre colisões e $m^{*}$ é a massa efetiva. No entanto, ao se tentar descrever, com estas equações, um dispositivo de dimensão nanométrica, vários problemas são encontrados. Quando se quer, por exemplo, calcular o fluxo de corrente através de uma molécula, passa a ser complicado definir a massa efetiva, já que não se tem um sólido periódico. O conceito de tempo livre médid $\rrbracket^{1}$ também se torna problemático, já que o comprimento do dispositivo pode ser menor do que o livre caminho médio $\ell$.

\footnotetext{
${ }^{1} \mathrm{O}$ tempo livre médio é o intervalo de tempo médio decorrido entre espalhamentos consecutivos sofridos pelo elétron, sem distinção entre espalhamentos elástico ou inelástico, e o livre caminho médio é a distância percorrida pelo elétron nesse intervalo.
} 
Além disso, a Equação 4.2 sugere que, quanto menor o comprimento $L$ do canal, maior é a condutância. No entanto, experimentalmente se verifica que medidas de condutância se aproximam de um valor fundamental $\mathcal{G}_{o}$ quando o comprimento do resistor é muito menor do que o livre caminho médio $\ell$ [6, 67]. Portanto, por menor que seja o dispositivo, existe um valor mínimo de resistência, que, como será discutido à frente, provém do contato entre o canal e os eletrodos.

\subsection{Modelo de Landauer}

Uma alternativa para se descrever o transporte eletrônico é partir de um modelo para a escala nanométrica e generalizá-lo para escalas maiores (bottom-up view 83]). Nesta seção será apresentada uma derivação heurística do Modelo de Landauer que descreve a corrente de elétrons não interagentes em termos de propriedades locais de uma região finita (canal) e das funções distribuições dos reservatórios conectados a esta região (eletrodos esquerdo e direito).

Assumindo que os contatos metálicos são relativamente grandes ( 5 a 10 vezes o comprimento do canal), podemos considerá-los como reservatórios de elétrons em equilíbrio termodinâmico e a fração de estados de energia ocupados será dada pela função distribuição de Fermi-Dirac:

$$
f(\epsilon)=\frac{1}{e^{\beta(\epsilon-\mu)}+1},
$$

onde $\mu$ é o potencial eletroquímico e $\beta=\left(k_{B} T\right)^{-1}$, com $k_{B}$, a constante de Boltzmann e $T$, a temperatura. Se os contatos estiverem acoplados ao canal e não existir uma diferença de potencial entre eles $(V=0)$, após algum tempo todo o sistema atingirá o equilíbrio com um mesmo potencial eletroquímico (neste caso, a energia de Fermi, $\mu=\varepsilon_{F}$ ). No entanto, se conectarmos aos contatos uma bateria tal que $V>0$, os níveis de energia do contato esquerdo (fonte) sobem e os do contato direito (dreno) descem, de forma que os potenciais eletroquímicos são mantidos separados de $\mu_{L}-\mu_{R}=e V$. Esta situação fora do equilíbrio está esquematizada na Figura 4.3. onde foram esboçadas as distribuições de Fermi-Dirac dos contatos e a densidade de estados do canal.

Nesta situação, ambos os contatos tentam fazer com que o canal fique em equilíbrio consigo, isto é, tenham o mesmo potencial eletroquímico. Porém, enquanto houver uma bateria que mantenha a diferença de potencial $V$, o equilíbrio nunca é estabelecido com nenhum dos dois. Se houver níveis de energia disponíveis no canal em torno de $\mu_{R}$ até $\mu_{L}$, o contato fonte $L$ permanecerá enviando elétrons ao canal e o dreno $R$ permanecerá removendo-os, levando o sistema para um estado de corrente estacionária. Portanto, para que ocorra um fluxo de corrente 

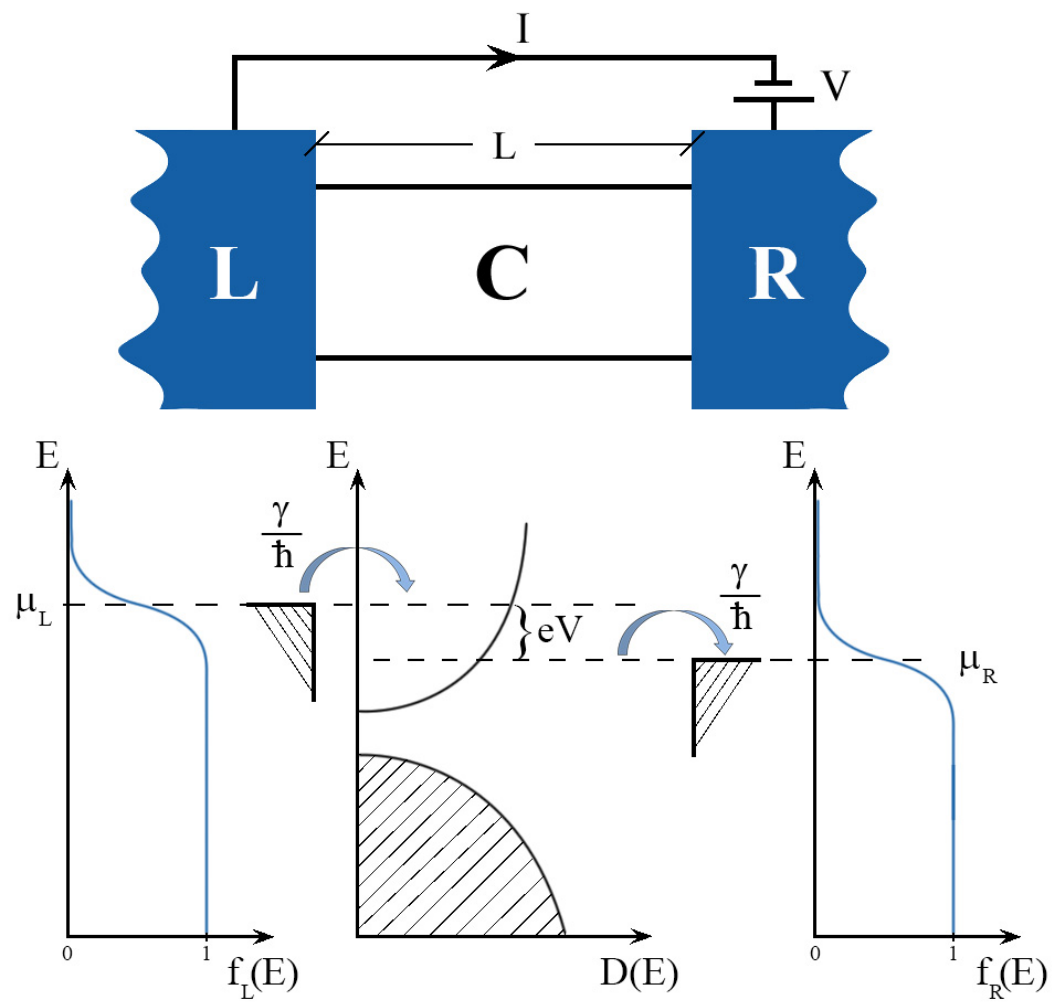

Figura 4.3: Esquema da situação fora do equilíbrio devido à tensão $V$ mantida por uma bateria. Abaixo observa-se as distribuições de Fermi-Dirac dos eletrodos e a densidade de estados do canal ao centro, ilustrando que são os estados com energias entre $\mu_{R}$ e $\mu_{L}$ os responsáveis pelo transporte.

é necessário, além da diferença de potencial, que haja estados disponíveis no intervalo de energia, ou seja:

$$
I \propto \int d \epsilon D(\epsilon)\left[f_{L}(\epsilon)-f_{R}(\epsilon)\right]
$$

onde $D(\epsilon)$ é a densidade de estados do canal.

Se tomarmos energias muito abaixo de $\mu_{R}$, então $f_{L}(\epsilon)=f_{R}(\epsilon)=1$ e, portanto, $f_{L}(\epsilon)-$ $f_{R}(\epsilon)=0$. Do mesmo modo, se tomarmos energias muito acima de $\mu_{L}$, então $f_{L}(\epsilon)=f_{R}(\epsilon)=0$ e, portanto, $f_{L}(\epsilon)-f_{R}(\epsilon)=0$. Logo, ao multiplicarmos a densidade de estados do canal $D(\epsilon)$ pela diferença das distribuições de Fermi-Dirac dos contatos, estamos dizendo que somente os níveis de energia em torno do intervalo $\left[\mu_{R}, \mu_{L}\right]$ participam da condução. Ao integrar nesta janela de energia, assume-se que todo elétron que entra no canal com uma dada energia $\epsilon$ atravessa-o e sai com esta mesma energia (como se cada intervalo de energia pudesse ser considerado como um canal independente). Isto é conhecido como transporte elástico, já que o elétron não perde energia ao atravessar o canal (mas pode sofrer espalhamentos elásticos, i.e., relaxação de momento). 
Espera-se também que a corrente seja proporcional à carga do elétron $e$ e à taxa com que os elétrons entram e saem do canal. Esta taxa pode ser representada pela razão $\frac{\gamma}{2 \hbar}$, onde $\gamma$ está relacionado a quão facilmente elétrons podem entrar e sair do canal e o fator 2 está relacionado ao fato de que o elétron leva um tempo $t$ para entrar no canal e o mesmo tempo $t$ para sair. 2 Portanto devemos ter:

$$
I=\frac{e}{h} \int d \epsilon(\pi D(\epsilon) \gamma(\epsilon))\left[f_{L}(\epsilon)-f_{R}(\epsilon)\right]
$$

Isto funciona bem para condutores pequenos, como alguns nanômetros, mas para condutores maiores $T(\epsilon)=\pi D(\epsilon) \gamma(\epsilon)$ tem que ser reavaliado. Esta quantidade é chamada de transmissão e pode ser entendida como a probabilidade de um elétron atravessar o canal. Se assumíssemos que $T(\epsilon)=\bar{T}$ é praticamente independente da energia, poderíamos escrever:

$$
I \approx \frac{e}{h} \bar{T} \int d \epsilon\left[f_{L}(\epsilon)-f_{R}(\epsilon)\right]=\frac{e}{h} \bar{T} e V
$$

o que nos leva à fórmula de Landauer:

$$
\mathcal{G}=\frac{I}{V}=\frac{2 e^{2}}{h} \bar{T}
$$

onde $\mathcal{G}_{o}=\frac{2 e^{2}}{h} \approx \frac{1}{13 k \Omega}$ é o quantum de condutância e o fator 2 se deve aos dois estados de spin degenerados. Portanto, segundo o modelo de Landauer, a condutância é igual ao quantum de condutância multiplicada pela probabilidade de transmissão.

Quando aumentamos as dimensões do dispositivo (alguns micros), se o condutor for balístico então $\gamma \sim \frac{1}{L}$ (i.e., quanto maior $L$ mais "difícil" será o elétron atravessar o canal). Como a densidade de estados é proporcional ao volume do sólidd $3(D \sim L A)$, teremos $\mathcal{G} \propto A$, onde $A$ é a área da seção reta do dispositivo. Ou seja, num condutor balístico, a condutância independe do comprimento $L$ do canal. Para um transporte difusivo $\gamma \sim \frac{1}{L^{2}}$ e, nesse caso, $\mathcal{G} \propto \frac{A}{L}$, o que está de acordo com a lei de Ohm [84].

No transistor, todo elétron perde em algum lugar uma quantidade de energia $\Delta \epsilon=\mu_{L}-\mu_{R}$. Em dispositivos grandes, a ideia que se tem é que essa dissipação ocorre em todo lugar continuamente. No entanto, em dispositivos pequenos, a ideia é que o elétron atravessa praticamente

\footnotetext{
${ }^{2}$ Estou considerando, por enquanto, que os contatos esquerdo e direito são idênticos para simplificar a derivação.

${ }^{3}$ Por exemplo, em um gás de elétrons livres a densidade de estados é dada por $D(\epsilon)=\frac{V}{2 \pi^{2}}\left(\frac{2 m}{\hbar^{2}}\right)^{3 / 2} \epsilon^{1 / 2}$.
} 
sem perder energia dentro do canal e todo aquecimento ocorre nos pontos terminais. De fato, existem evidências experimentais de que isso realmente ocorre. Mais precisamente, esta resistência está relacionada ao fato de haver aproximadamente quatro ordens de grandeza mais modos transversais nos contatos do que no canal [85].

O modelo de Landauer fornece uma abordagem para o transporte balístico de elétrons nãointeragentes. No entanto, muitas vezes pretende-se estudar as propriedades do transporte no regime não-linear e com interações. Uma alternativa é utilizar o formalismo das Funções de Green Fora do Equilíbrio (apresentado no Capítulo 2), que fornece uma estrutura conceitual e computacional poderosa para incluir espalhamento inelástico e efeitos de correlação no nível atomísticd ${ }^{4}$, que é o objetivo desta tese.

\subsection{Hamiltoniana do Modelo}

Nosso sistema de interesse (o transistor da seção anterior) pode ser representado pelo esboço na Figura 4.4, onde se observa uma região central de espalhamento conectada aos dois eletrodos (contatos).

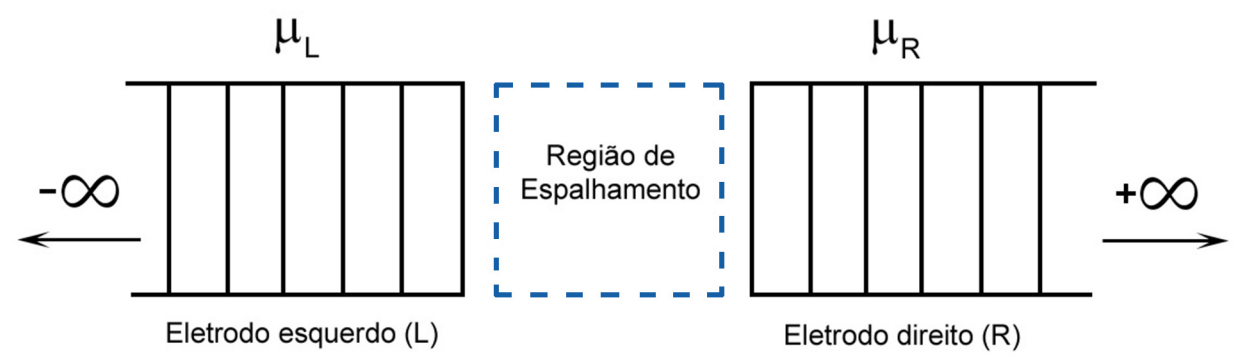

Figura 4.4: Esboço de um problema típico de transporte, com uma região central de espalhamento conectada a dois reservatórios de elétrons (eletrodos esquerdo e direito) em equilíbrio termodinâmico.

A região de espalhamento é arbitrária, podendo ser formada por um átomo, uma molécula ou mesmo um sólido. Já os eletrodos, considera-se que são formados por materiais cristalinos perfeitos e semi-infinitos, e são considerados em equilíbrio termodinâmico, sendo caracterizados por potenciais eletroquímicos $\mu_{L}$ e $\mu_{R}$. Supõe-se ainda que os eletrodos são excelentes metais. Dessa forma, podemos assumir que a queda na diferença de potencial criada pela bateria ocorre na região de espalhamento, já que, para metais, o comprimento de blindagem é pequeno 82 .

Seguindo Haug e Jauho [8], assume-se que a Hamiltoniana total descrevendo o sistema de elétrons interagentes acima possa ser dividida em três partes, $H_{C}$ descrevendo os contatos $L$ e

\footnotetext{
${ }^{4}$ Existem problemas em que mesmo no regime linear este formalismo se mostra vantajoso 64
} 
$R, H_{T}$ descrevendo o acoplamento de tunelamento entre a região central e os contatos, e $H_{M}$ descrevendo a região central onde ocorrem as interações:

$$
\begin{aligned}
\mathscr{H} & =\left(H_{C}^{L}+H_{C}^{R}\right)+\left(H_{T}^{L}+H_{T}^{R}\right)+H_{M}, \\
H_{C}^{\alpha} & =\sum_{n} E_{n \alpha} d_{n \alpha}^{\dagger} d_{n \alpha}, \\
H_{T}^{\alpha} & =\sum_{n, k}\left(V_{n \alpha, k} d_{n \alpha}^{\dagger} c_{k}+\text { h.c. }\right), \\
H_{M} & =H_{M}\left(\left\{c_{k}^{\dagger}\right\} ;\left\{c_{k}\right\}\right),
\end{aligned}
$$

onde $d_{n \alpha}^{\dagger}\left(d_{n \alpha}\right)$ cria (destrói) um elétron no eletrodo $\alpha$ no estado $n$ e $c_{k}^{\dagger}\left(c_{k}\right)$ cria (destrói) um elétron na região de interação no estado $k$. Como os eletrodos são metálicos e estão no equilíbrio termodinâmico, os elétrons no contato são considerados não-interagentes (exceto por um potencial global autoconsistente). O potencial de acoplamento entre os eletrodos e a região de espalhamento $V_{n \alpha, k}$ depende, em princípio, das densidades de cargas que devem ser determinadas por meio de cálculos autoconsistentes (por exemplo, via DFT com a metodologia descrita na Seção 3.2.4. A forma da Hamiltoniana da região de interação depende da geometria e do tipo de comportamento físico que será investigado. Em particular, no próximo capítulo iremos considerar o caso em que a região central está acoplada a um reservatório de fônons, o que leva à Hamiltoniana da Equação 2.57.

\subsection{Expressão Geral para a Corrente}

A corrente eletrônica que flui do contato $\alpha$ para a região central pode ser calculada a partir da evolução temporal do operador número $\mathcal{N}_{\alpha}=\sum_{n} d_{n \alpha}^{\dagger} d_{n \alpha}$ da seguinte forma:

$$
I_{\alpha}=-e\left\langle\frac{\partial \mathcal{N}_{\alpha}}{\partial t}\right\rangle=-i \frac{e}{\hbar}\left\langle\left[\mathscr{H}, \mathcal{N}_{\alpha}\right]\right\rangle=i \frac{e}{\hbar} \sum_{n, k}\left(V_{n \alpha, k}\left\langle d_{n \alpha}^{\dagger} c_{k}\right\rangle-V_{n \alpha, k}^{*}\left\langle c_{k}^{\dagger} d_{n \alpha}\right\rangle\right)
$$

onde a última igualdade segue do fato de que $H_{C}$ e $H_{M}$ comutam com $\mathcal{N}_{\alpha}$.

Definindo duas novas funções de Green de partícula única no tempo real:

$$
\begin{aligned}
G_{k, n \alpha}^{<}\left(t, t^{\prime}\right) & \equiv \frac{i}{\hbar}\left\langle d_{n \alpha}^{\dagger}\left(t^{\prime}\right) c_{k}(t)\right\rangle, \\
G_{n \alpha, k}^{<}\left(t, t^{\prime}\right) & \equiv \frac{i}{\hbar}\left\langle c_{k}^{\dagger}\left(t^{\prime}\right) d_{n \alpha}(t)\right\rangle,
\end{aligned}
$$


a corrente pode ser escrita como:

$$
\begin{aligned}
I_{\alpha} & =e \sum_{n, k}\left(V_{n \alpha, k} G_{k, n \alpha}^{<}(t, t)-V_{n \alpha, k}^{*} G_{n \alpha, k}^{<}(t, t)\right)= \\
& =\frac{e}{\hbar} \int_{-\infty}^{+\infty} \frac{d \epsilon}{2 \pi} \sum_{n, k}\left(V_{n \alpha, k} G_{k, n \alpha}^{<}(\epsilon)-V_{n \alpha, k}^{*} G_{n \alpha, k}^{<}(\epsilon)\right) .
\end{aligned}
$$

A fim de obter-se expressões para as funções de Green $G_{k, n \alpha}^{<}(\epsilon)$ e $G_{n \alpha, k}^{<}(\epsilon)$, podemos definir as seguintes funções de Green ordenadas no contorno:

$$
\begin{aligned}
G_{k, n \alpha}\left(\tau, \tau^{\prime}\right) & \equiv-\frac{i}{\hbar}\left\langle\mathrm{T}_{C}\left\{c_{k}(\tau) d_{n \alpha}^{\dagger}\left(\tau^{\prime}\right)\right\}\right\rangle, \\
G_{n \alpha, k}\left(\tau, \tau^{\prime}\right) & \equiv-\frac{i}{\hbar}\left\langle\mathrm{T}_{C}\left\{d_{n \alpha}(\tau) c_{k}^{\dagger}\left(\tau^{\prime}\right)\right\}\right\rangle .
\end{aligned}
$$

Como estamos considerando que os eletrodos são não-interagentes, estas funções de Green possuem um conjunto fechado de equações de movimento, o que permite escrevê-las como as seguintes equações de Dyson:

$$
\begin{aligned}
& G_{k, n \alpha}\left(\tau, \tau^{\prime}\right)=\sum_{m} \int_{C} d \tau_{1} G_{k, m}\left(\tau, \tau_{1}\right) V_{n \alpha, m}^{*} G_{n \alpha, n \alpha}\left(\tau_{1}, \tau^{\prime}\right), \\
& G_{n \alpha, k}\left(\tau, \tau^{\prime}\right)=\sum_{m} \int_{C} d \tau_{1} G_{n \alpha, n \alpha}\left(\tau, \tau_{1}\right) V_{n \alpha, m} G_{m, k}\left(\tau_{1}, \tau^{\prime}\right),
\end{aligned}
$$

onde definimos a função de Green da região central ordenada no contorno $G_{k, m}\left(\tau, \tau^{\prime}\right) \equiv$ $-\frac{i}{\hbar}\left\langle\mathrm{T}_{C}\left\{c_{k}(\tau) c_{m}^{\dagger}\left(\tau^{\prime}\right)\right\}\right\rangle$. Aplicando a continuação analítica (Equação 2.139, podemos reescrever as integrais em termos de tempos reais da seguinte forma:

$$
\begin{aligned}
& G_{k, n \alpha}^{<}\left(t, t^{\prime}\right)=\sum_{m} V_{n \alpha, m}^{*} \int_{-\infty}^{+\infty} d t_{1}\left[G_{k, m}^{r}\left(t, t_{1}\right) G_{n \alpha, n \alpha}^{<}\left(t_{1}, t^{\prime}\right)+G_{k, m}^{<}\left(t, t_{1}\right) G_{n \alpha, n \alpha}^{a}\left(t_{1}, t^{\prime}\right)\right], \\
& G_{n \alpha, k}^{<}\left(t, t^{\prime}\right)=\sum_{m} V_{n \alpha, m} \int_{-\infty}^{+\infty} d t_{1}\left[G_{n \alpha, n \alpha}^{r}\left(t, t_{1}\right) G_{m, k}^{<}\left(t_{1}, t^{\prime}\right)+G_{n \alpha, n \alpha}^{<}\left(t, t_{1}\right) G_{m, k}^{a}\left(t_{1}, t^{\prime}\right)\right] .
\end{aligned}
$$

No estado estacionário, as funções de Green dependem somente das diferenças dos argumentos de tempo. Logo, podemos tomar a transformada de Fourier, de modo que as convoluções se tornam simples produtos no espaço de energia: 


$$
\begin{aligned}
G_{k, n \alpha}^{<}(\epsilon) & =\sum_{m} V_{n \alpha, m}^{*}\left[G_{k, m}^{r}(\epsilon) G_{n \alpha, n \alpha}^{<}(\epsilon)+G_{k, m}^{<}(\epsilon) G_{n \alpha, n \alpha}^{a}(\epsilon)\right], \\
G_{n \alpha, k}^{<}(\epsilon) & =\sum_{m} V_{n \alpha, m}\left[G_{n \alpha, n \alpha}^{r}(\epsilon) G_{m, k}^{<}(\epsilon)+G_{n \alpha, n \alpha}^{<}(\epsilon) G_{m, k}^{a}(\epsilon)\right] .
\end{aligned}
$$

Substituindo estes resultados na expressão para a corrente (Equação 4.16), obtém-se:

$$
\begin{array}{r}
I_{\alpha}=\frac{e}{h} \int_{-\infty}^{+\infty} d \epsilon \sum_{n, k, m}\left\{V_{n \alpha, k} V_{n \alpha, m}^{*}\left[G_{k, m}^{r}(\epsilon) G_{n \alpha, n \alpha}^{<}(\epsilon)+G_{k, m}^{<}(\epsilon) G_{n \alpha, n \alpha}^{a}(\epsilon)\right]-\right. \\
\left.-V_{n \alpha, k}^{*} V_{n \alpha, m}\left[G_{n \alpha, n \alpha}^{r}(\epsilon) G_{m, k}^{<}(\epsilon)+G_{n \alpha, n \alpha}^{<}(\epsilon) G_{m, k}^{a}(\epsilon)\right]\right\}= \\
=\frac{e}{h} \int_{-\infty}^{+\infty} d \epsilon \sum_{n, k, m}\left\{V _ { n \alpha , k } V _ { n \alpha , m } ^ { * } \left[G_{n \alpha, n \alpha}^{<}(\epsilon)\left(G_{k, m}^{r}(\epsilon)-G_{k, m}^{a}(\epsilon)\right)-\right.\right. \\
\left.\left.\quad-\left(G_{n \alpha, n \alpha}^{r}(\epsilon)-G_{n \alpha, n \alpha}^{a}(\epsilon)\right) G_{k, m}^{<}(\epsilon)\right]\right\}= \\
=\frac{e}{h} \int_{-\infty}^{+\infty} d \epsilon \sum_{n, k, m}\left\{V _ { n \alpha , k } V _ { n \alpha , m } ^ { * } \left[G_{n \alpha, n \alpha}^{<}(\epsilon) G_{k, m}^{>}(\epsilon)-G_{n \alpha, n \alpha}^{<}(\epsilon) G_{k, m}^{<}(\epsilon)-\right.\right. \\
=\frac{e}{h} \int_{-\infty}^{+\infty} d \epsilon \sum_{n, k, m}^{>}\left\{V_{n \alpha, k} V_{n \alpha, m}^{*}\left[G_{n \alpha, n \alpha}^{<}(\epsilon) G_{k, m}^{<}(\epsilon)+G_{n \alpha, n \alpha}^{<}(\epsilon) G_{k, m}^{<}(\epsilon)\right]\right\}= \\
\left.\left.\quad G_{n \alpha, n \alpha}^{<}(\epsilon) G_{k, m}^{<}(\epsilon)\right]\right\},
\end{array}
$$

onde usamos a relação fundamental da Equação 2.71. Definindo, no contorno, a autoenergia de tunelamento do eletrodo $\alpha$ para a região de interação:

$$
\Sigma_{\alpha ; m, k}=\sum_{n} V_{n \alpha, m}^{*} G_{n \alpha, n \alpha}\left(\tau, \tau^{\prime}\right) V_{n \alpha, k}
$$

podemos escrever a expressão geral para a corrente que flui entre o eletrodo $\alpha$ e a região central da seguinte forma:

$$
\begin{aligned}
I_{\alpha} & =\frac{e}{h} \int_{-\infty}^{+\infty} d \epsilon \sum_{k, m}\left[\Sigma_{\alpha ; m, k}^{<}(\epsilon) G_{k, m}^{>}(\epsilon)-\Sigma_{\alpha ; m, k}^{>}(\epsilon) G_{k, m}^{<}(\epsilon)\right]= \\
& =\frac{e}{h} \int_{-\infty}^{+\infty} d \epsilon \operatorname{Tr}\left[\Sigma_{\alpha}^{<}(\epsilon) G^{>}(\epsilon)-\Sigma_{\alpha}^{>}(\epsilon) G^{<}(\epsilon)\right] .
\end{aligned}
$$


Portanto, a corrente que flui do eletrodo $\alpha$ para a região central (primeiro termo da expressão) é proporcional a $\Sigma_{\alpha}^{<}(\epsilon)$, que fornece a taxa saída por tunelamento dos estados ocupados do eletrodo $\alpha$, e a $G^{>}(\epsilon)$, que fornece o número de estados disponíveis na região central. Da mesma forma, o segundo termo corresponde à corrente que flui da região central para o eletrodo $\alpha$.

A fim de encontrar-se expressões para as autoenergias $\Sigma_{\alpha ; m, k}^{\lessgtr}(\epsilon)$, convém converter a somatória nos momentos em integrais em energia:

$$
\Sigma_{\alpha ; m, k}^{\lessgtr}(\epsilon)=\int_{-\infty}^{+\infty} d \varepsilon_{n} \rho_{\alpha}\left(\varepsilon_{n}\right) V_{\alpha, m}^{*}\left(\varepsilon_{n}\right) V_{\alpha, k}\left(\varepsilon_{n}\right) G_{n \alpha, n \alpha}^{\lessgtr}(\epsilon)=\int_{-\infty}^{+\infty} \frac{d \varepsilon_{n}}{2 \pi} \Gamma_{m, k}^{\alpha}\left(\varepsilon_{n}\right) G_{n \alpha, n \alpha}^{\lessgtr}(\epsilon),
$$

onde definimos a função largura de nível $\Gamma_{m, k}^{\alpha}\left(\varepsilon_{n}\right)=2 \pi \rho_{\alpha}\left(\varepsilon_{n}\right) V_{\alpha, m}^{*}\left(\varepsilon_{n}\right) V_{\alpha, k}\left(\varepsilon_{n}\right)$ (também chamada de matriz de acoplamento). Por fim, como estamos considerando os eletrodos no equilíbrio termodinâmico, podemos substituir as funções de Green $G_{n \alpha, n \alpha}^{\lessgtr}(\epsilon)$ pelas Equações $2.76 \mathrm{e} 2.77$

$$
\begin{aligned}
& \Sigma_{\alpha ; m, k}^{<}(\epsilon)=\int_{-\infty}^{+\infty} \frac{d \varepsilon_{n}}{2 \pi} \Gamma_{m, k}^{\alpha}\left(\varepsilon_{n}\right) 2 \pi i f\left(\varepsilon_{n}-\mu_{\alpha}\right) \delta\left(\epsilon-\varepsilon_{n}\right)=i f\left(\epsilon-\mu_{\alpha}\right) \Gamma_{m, k}^{\alpha}(\epsilon), \\
& \Sigma_{\alpha ; m, k}^{>}(\epsilon)=\int_{-\infty}^{+\infty} \frac{d \varepsilon_{n}}{2 \pi} \Gamma_{m, k}^{\alpha}\left(\varepsilon_{n}\right) 2 \pi i\left[f\left(\varepsilon_{n}-\mu_{\alpha}\right)-1\right] \delta\left(\epsilon-\varepsilon_{n}\right)=i\left[f\left(\epsilon-\mu_{\alpha}\right)-1\right] \Gamma_{m, k}^{\alpha}(\epsilon),
\end{aligned}
$$

onde $f\left(\epsilon-\mu_{\alpha}\right)$ é a distribuição de Fermi-Dirac do eletrodo $\alpha$ (Equação 4.4). Substituindo estes resultados na expressão geral para a corrente, obtém-se:

$$
\begin{aligned}
I_{\alpha} & =\frac{e}{h} \int_{-\infty}^{+\infty} d \epsilon \operatorname{Tr}\left[i f\left(\epsilon-\mu_{\alpha}\right) \Gamma_{\alpha}(\epsilon) G^{>}(\epsilon)-i\left[f\left(\epsilon-\mu_{\alpha}\right)-1\right] \Gamma_{\alpha}(\epsilon) G^{<}(\epsilon)\right]= \\
& =\frac{e}{h} \int_{-\infty}^{+\infty} d \epsilon \operatorname{Tr}\left[f\left(\epsilon-\mu_{\alpha}\right) \Gamma_{\alpha}(\epsilon) A(\epsilon)+i \Gamma_{\alpha}(\epsilon) G^{<}(\epsilon)\right]
\end{aligned}
$$

onde usamos a definição da função espectral $A(\epsilon)$ (Equação 2.91). Esta expressão geral para a corrente através de uma região com interação foi obtida pela primeira vez por Y. Meir e N. Wingreen 86$]$.

Nos cálculos usuais de transporte eletrônico, considera-se o problema de um sistema nãointeragent $5^{5}$ acoplado a contatos metálicos, isto é, $H_{M}=E_{k} c_{k}^{\dagger} c_{k}$ (onde a interação elétronelétron pode estar incluída por meio de uma aproximação de campo médio). Neste caso, a equação de Keldysh (Equação 2.152) possui somente as autoenergias devido aos acoplamentos com os eletrodos esquerdo e direito, ou seja:

\footnotetext{
${ }^{5}$ Vamos usar a notação adotada no Capítulo 2, onde o índice 0 indica a função de Green não-interagente.
} 


$$
\begin{aligned}
G_{0}^{<}(\epsilon) & =G_{0}^{r}(\epsilon)\left[\Sigma_{L}^{<}(\epsilon)+\Sigma_{R}^{<}(\epsilon)\right] G_{0}^{a}(\epsilon)= \\
& =i f\left(\epsilon-\mu_{L}\right) G_{0}^{r}(\epsilon) \Gamma_{L}(\epsilon) G_{0}^{a}(\epsilon)+i f\left(\epsilon-\mu_{R}\right) G_{0}^{r}(\epsilon) \Gamma_{R}(\epsilon) G_{0}^{a}(\epsilon), \\
G_{0}^{>}(\epsilon) & =G_{0}^{r}(\epsilon)\left[\Sigma_{L}^{>}(\epsilon)+\Sigma_{R}^{>}(\epsilon)\right] G_{0}^{a}(\epsilon)= \\
& =i\left[f\left(\epsilon-\mu_{L}\right)-1\right] G_{0}^{r}(\epsilon) \Gamma_{L}(\epsilon) G_{0}^{a}(\epsilon)+i\left[f\left(\epsilon-\mu_{R}\right)-1\right] G_{0}^{r}(\epsilon) \Gamma_{R}(\epsilon) G_{0}^{a}(\epsilon) .
\end{aligned}
$$

Neste caso, tomando, por exemplo, a corrente fluindo do eletrodo esquerdo para a região central, teremos:

$$
\begin{aligned}
& I_{L}= \frac{e}{h} \int_{-\infty}^{+\infty} d \epsilon \operatorname{Tr}\left[\Sigma_{L}^{<}(\epsilon) G_{0}^{>}(\epsilon)-\Sigma_{L}^{>}(\epsilon) G_{0}^{<}(\epsilon)\right]= \\
&=\frac{e}{h} \int_{-\infty}^{+\infty} d \epsilon \operatorname{Tr}\left[i f_{L} \Gamma_{L}\left\{i\left(f_{L}-1\right) G_{0}^{r} \Gamma_{L} G_{0}^{a}+i\left(f_{R}-1\right) G_{0}^{r} \Gamma_{R} G_{0}^{a}\right\}-\right. \\
&\left.\quad-i\left[f_{L}-1\right] \Gamma_{L}\left\{i f_{L} G_{0}^{r} \Gamma_{L} G_{0}^{a}+i f_{R} G_{0}^{r} \Gamma_{R} G_{0}^{a}\right\}\right]= \\
&=\frac{e}{h} \int_{-\infty}^{+\infty} d \epsilon \operatorname{Tr}\left[\left(f_{R} f_{L}-f_{R}-f_{L} f_{R}+f_{L}\right) \Gamma_{L} G_{0}^{r} \Gamma_{R} G_{0}^{a}\right]= \\
&=\frac{e}{h} \int_{-\infty}^{+\infty} d \epsilon \operatorname{Tr}\left[\Gamma_{L} G_{0}^{r} \Gamma_{R} G_{0}^{a}\right]\left(f_{L}-f_{R}\right),
\end{aligned}
$$

onde utilizamos a notação $f_{L / R}=f\left(\epsilon-\mu_{L / R}\right)$ e omitimos os argumentos das funções " $(\epsilon)$ " para facilitar a visualização da equaçã ${ }^{6}$. Comparando este resultado com a fórmula de Landauer na Seção 4.2, reconhecemos o termo $T_{L R}(\epsilon)=\operatorname{Tr}\left[\Gamma_{L} G_{0}^{r} \Gamma_{R} G_{0}^{a}\right]$ como o análogo para a probabilidade de transmissão no formalismo NEGF.

\subsection{Formalismo NEGF na Prática}

Até aqui vimos a descrição teórica do transporte eletrônico através de uma região de espalhamento por meio do formalismo das funções de Green fora do equilíbrio, que, por sua vez, é geral e independe da escolha do Hamiltoniano eletrônico. Contudo, em um problema como o de transporte, que envolve redistribuições de cargas conforme uma diferença de potencial é aplicada,

\footnotetext{
${ }^{6}$ Daqui para frente os argumentos das funções " $(\epsilon)$ " serão omitidos e adotaremos a notação $f_{L / R}=f\left(\epsilon-\mu_{L / R}\right)$ como padrão.
} 
é conveniente trabalhar com uma Hamiltoniana descrita em termos da densidade de carga $\mathcal{H}=$ $\mathcal{H}[n(\mathbf{r})]$, como é o caso da Hamiltoniana de Kohn-Sham (Seção 3.2.2.

Nesta seção, vamos entender como este formalismo NEGF é utilizado na prática, onde o problema de redistribuição de cargas é calculado de forma autoconsistente. Para isso, consideremos que o sistema da Figura 4.4 possa ser descrito por uma matriz Hamiltoniana e uma matriz de overlap, representadas numa base de orbitais atômicos como na Seção 3.2 .4 .

O problema é construído de forma a que cada célula unitária dos eletrodos metálicos interajam somente com seus primeiros vizinhos 17 Portanto, os Hamiltonianos correspondentes aos eletrodos têm a forma tridiagonal:

$$
\mathcal{H}_{L}=\left(\begin{array}{ccccc}
\ddots & \vdots & \vdots & \vdots & \vdots \\
\cdots & H_{L}^{0} & H_{L}^{1} & 0 & 0 \\
\cdots & H_{L}^{1^{\dagger}} & H_{L}^{0} & H_{L}^{1} & 0 \\
\cdots & 0 & H_{L}^{1^{\dagger}} & H_{L}^{0} & H_{L}^{1} \\
\cdots & 0 & 0 & H_{L}^{1 \dagger} & H_{L}^{0}
\end{array}\right), \quad \mathcal{H}_{R}=\left(\begin{array}{ccccc}
H_{R}^{0} & H_{R}^{1} & 0 & 0 & \cdots \\
H_{R}^{1 \dagger} & H_{R}^{0} & H_{R}^{1} & 0 & \cdots \\
0 & H_{R}^{1 \dagger} & H_{R}^{0} & H_{R}^{1} & \cdots \\
0 & 0 & H_{R}^{1^{\dagger}} & H_{R}^{0} & \cdots \\
\vdots & \vdots & \vdots & \vdots & \ddots
\end{array}\right),
$$

onde $H_{L}^{0}\left(H_{R}^{0}\right)$ é o Hamiltoniano correspondente à célula unitária do eletrodo esquerdo (direito) e $H_{L}^{1}\left(H_{R}^{1}\right)$ é acoplamento entre as células adjacentes.

Como a definição da região de espalhamento é arbitrária, podemos adicionar a esta região algumas células unitárias dos eletrodos (Figura 4.5). Assim, garante-se que a queda na diferença de potencial criada pela bateria ocorra somente nesta parte central e que a estrutura eletrônica dos eletrodos não seja alterada pelo acoplamento com esta região (exceto pelo deslocamento rígido dos níveis devido à diferença de potencial aplicada pela bateria).

Chamando de $H_{M}$ o Hamiltoniano correspondente à região de espalhamento e de $H_{L M}\left(H_{M R}\right)$ o acoplamento com o eletrodo esquerdo (direito), o Hamiltoniano do sistema como um todo é dado por:

\footnotetext{
${ }^{7}$ As células unitárias do eletrodo podem ser redefinidas de forma a garantir a interação somente entre primeiros vizinhos. Por exemplo, em uma cadeia monoatômica com interação até os segundos vizinhos, a célula unitária pode ser redefinida com 2 átomos.
} 


$$
\mathcal{H}=\left(\begin{array}{ccccccc}
\ddots & \vdots & \vdots & \vdots & \vdots & \vdots & \cdot \\
\cdots & H_{L}^{0} & H_{L}^{1} & 0 & 0 & 0 & \cdots \\
\cdots & H_{L}^{1^{\dagger}} & H_{L}^{0} & H_{L M} & 0 & 0 & \cdots \\
\cdots & 0 & H_{M L} & H_{M} & H_{M R} & 0 & \cdots \\
\cdots & 0 & 0 & H_{R M} & H_{R}^{0} & H_{R}^{1} & \cdots \\
\cdots & 0 & 0 & 0 & H_{R}^{1 \dagger} & H_{R}^{0} & \cdots \\
. & \vdots & \vdots & \vdots & \vdots & \vdots & \ddots
\end{array}\right) .
$$

Nota-se que a matriz $\mathcal{H}$ é infinita e não periódica. Para uma dada energia $\epsilon$, o problema pode ser reescrito em termos da função de Green retardada:

$$
[\varepsilon \mathcal{S}-\mathcal{H}] G^{r}(\epsilon)=\mathbb{1}
$$

onde $\varepsilon=\lim _{\eta \rightarrow 0^{+}} \epsilon+i \eta$ e $\mathcal{S}$ é a matriz de overlap, cujos elementos seguem a mesma convenção dos elementos de $\mathcal{H}$. Em termos das Hamiltonianas dos eletrodos (Equação 4.35) e de seus acoplamentos com a região de espalhamento $\left(\mathcal{H}_{L M} \text { e } \mathcal{H}_{M R}\right)^{8}$, temos:

$$
\left(\begin{array}{ccc}
\varepsilon \mathcal{S}_{L}-\mathcal{H}_{L} & \varepsilon \mathcal{S}_{L M}-\mathcal{H}_{L M} & 0 \\
\varepsilon \mathcal{S}_{M L}-\mathcal{H}_{M L} & \varepsilon S_{M}-H_{M} & \varepsilon \mathcal{S}_{M R}-\mathcal{H}_{M R} \\
0 & \varepsilon \mathcal{S}_{R M}-\mathcal{H}_{R M} & \varepsilon \mathcal{S}_{R}-\mathcal{H}_{R}
\end{array}\right)\left(\begin{array}{ccc}
G_{L}^{r} & G_{L M}^{r} & G_{L R}^{r} \\
G_{M L}^{r} & G_{M}^{r} & G_{M R}^{r} \\
G_{R L}^{r} & G_{R M}^{r} & G_{R}^{r}
\end{array}\right)=\mathbb{1}
$$

Conforme discutido acima, foram introduzidas algumas células unitárias dos eletrodos à região de espalhamento e, portanto, esta região sozinha "sentirá" a interação dos eletrodos da mesma forma como se estivesse considerando o sistema como um todo (Figura 4.5). Desta forma, basta tratar a região de espalhamento, cujas dimensões são finitas. A partir do sistema de equações acima (Equação 4.38), selecionemos as seguintes igualdades:

$$
\begin{gathered}
\left(\varepsilon \mathcal{S}_{L}-\mathcal{H}_{L}\right) G_{L M}^{r}+\left(\varepsilon \mathcal{S}_{L M}-\mathcal{H}_{L M}\right) G_{M}^{r}=\mathbb{O} \\
\left(\varepsilon \mathcal{S}_{M L}-\mathcal{H}_{M L}\right) G_{L M}^{r}+\left(\varepsilon S_{M}-H_{M}\right) G_{M}^{r}+\left(\varepsilon \mathcal{S}_{M R}-\mathcal{H}_{M R}\right) G_{R M}^{r}=\mathbb{1}, \\
\left(\varepsilon \mathcal{S}_{R M}-\mathcal{H}_{R M}\right) G_{M}^{r}+\left(\varepsilon \mathcal{S}_{R}-\mathcal{H}_{R}\right) G_{R M}^{r}=\mathbb{O} .
\end{gathered}
$$

\footnotetext{
${ }^{8}$ Note que estamos usando " $\mathcal{H}$ " e "S $\mathcal{S}$ " caligráficos para denotar as hamiltonianas e matrizes de overlap com uma ou duas dimensões infinitas e " $H$ " e " $S$ " para denotar matrizes finitas.
} 


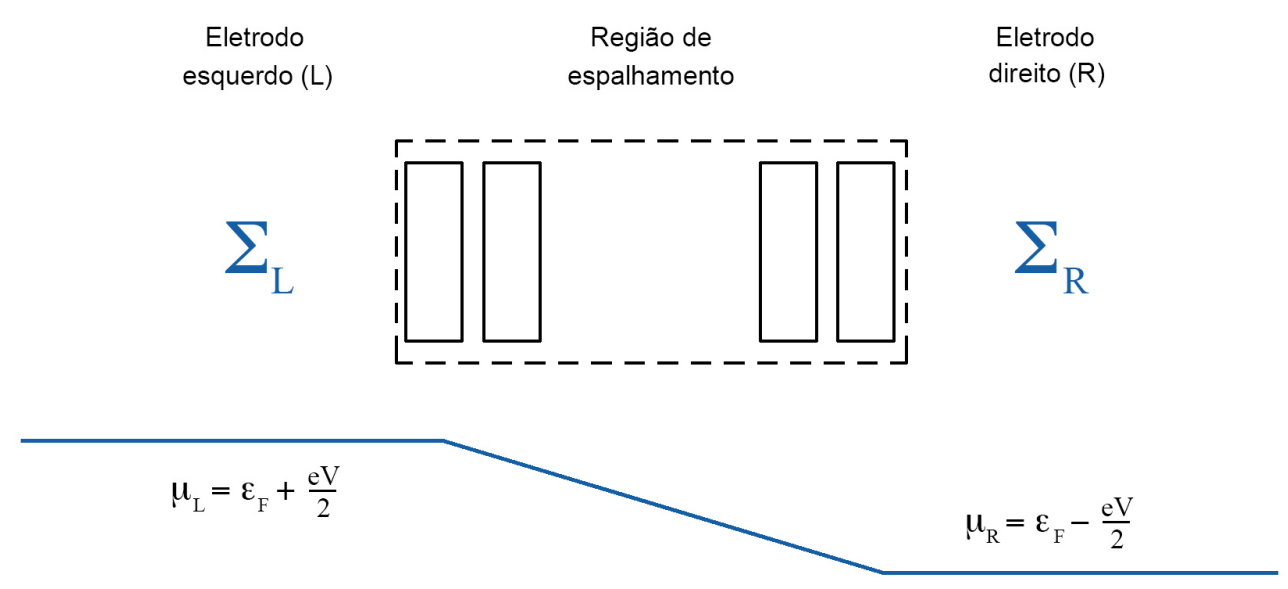

Figura 4.5: Região de espalhamento com algumas células unitárias dos eletrodos, de forma que a queda na diferença de potencial ocorre somente nesta região. O efeito do acoplamento com os eletrodos é dado pelas autoenergias $\Sigma_{L / R}$.

Substituindo as Equações 4.39 e 4.41 na Equação 4.40 , obtém-se a função de Green correspondente à região de espalhamento como sendo:

$$
G_{M}^{r}=\left[\varepsilon S_{M}-H_{M}-\Sigma_{L}^{r}-\Sigma_{R}^{r}\right]^{-1}
$$

onde $\Sigma_{L}^{r}$ e $\Sigma_{R}^{r}$ são, respectivamente, as autoenergias dos eletrodos esquerdo e direito dadas por:

$$
\begin{aligned}
& \Sigma_{L}^{r}=\left(\varepsilon \mathcal{S}_{M L}-\mathcal{H}_{M L}\right)\left(\varepsilon \mathcal{S}_{L}-\mathcal{H}_{L}\right)^{-1}\left(\varepsilon \mathcal{S}_{L M}-\mathcal{H}_{L M}\right), \\
& \Sigma_{R}^{r}=\left(\varepsilon \mathcal{S}_{M R}-\mathcal{H}_{M R}\right)\left(\varepsilon \mathcal{S}_{R}-\mathcal{H}_{R}\right)^{-1}\left(\varepsilon \mathcal{S}_{R M}-\mathcal{H}_{R M}\right) .
\end{aligned}
$$

Nota-se que a dificuldade passa a ser o cálculo das autoenergias dos eletrodos, pois estes são constituídos de multiplicações e inversões de matrizes de dimensões infinitas. Contudo, os acoplamentos dos eletrodos à região de espalhamento $\left(H_{L M}\right.$ e $\left.H_{M R}\right)$ são matrizes nulas exceto pelo bloco adjacente a esta região, que possui as dimensões da célula unitária do eletrodo. Logo, dos infinitos elementos de $\left(\varepsilon \mathcal{S}_{L}-\mathcal{H}_{L}\right)^{-1}$ e $\left(\varepsilon \mathcal{S}_{R}-\mathcal{H}_{R}\right)^{-1}$, estamos interessados somente naqueles pertencentes a um bloco com as dimensões da célula unitária do eletrodo. Esses elementos podem ser calculados analiticamente em alguns casos simples, mas em geral são obtidos ou por métodos semianalíticos ou por métodos recursivos $[9]$.

Uma vez calculadas as autoenergias dos eletrodos, obtém-se também as matrizes de acoplamento $\Gamma_{L / R}$, pois, subtraindo as Equações 4.29 e 4.30 , segue que: 


$$
\Gamma_{\alpha}=i\left[\Sigma_{\alpha}^{>}-\Sigma_{\alpha}^{<}\right]=i\left[\Sigma_{\alpha}^{r}-\Sigma_{\alpha}^{a}\right]
$$

onde na última igualdade usamos, novamente, a relação fundamental dada pela Equação 2.71 . Em posse das funções de Green retardada e avançada e das matrizes de acoplamento, podemos calcular a condutância no limite $V \rightarrow 0$ aplicando a relação de Fisher-Lee [67]:

$$
\mathcal{G}=\frac{e^{2}}{h} \operatorname{Tr}\left[\Gamma_{L} G^{r} \Gamma_{R} G^{a}\right]
$$

Conforme discutimos no início do capítulo, ao aplicarmos uma diferença de potencial por meio de uma bateria conectada aos eletrodos, os níveis de energia dos contatos sofrem um deslocamento rígido, de forma que os potenciais eletroquímicos são mantidos separados de $\left|\mu_{L}-\mu_{R}\right|=e V$. Ou seja, as Hamiltonianas correspondentes aos eletrodos não são modificadas pelo potencial externo, já que estes preservam a neutralidade de carga local.

Já na região central, a carga líquida e as polarizações elétricas serão afetadas pela diferença de potencial e, portanto, a distribuição de carga será diferente daquela no equilíbrio. A matriz densidade eletrônica $\rho$ fora do equilíbrio pode ser calculada a partir da função de correlação $G^{<}$ tomando $t=t^{\prime}$, 87 :

$$
\rho=-i G^{<}\left(t=t^{\prime}\right)=\frac{1}{2 \pi i} \int_{-\infty}^{+\infty} d \epsilon G_{M}^{<} .
$$

O cálculo da matriz densidade segue, portanto, da equação de Keldysh (Equação 2.152):

$$
\rho=\frac{1}{2 \pi i} \int_{-\infty}^{+\infty} d \epsilon i G_{M}^{r}\left[\Gamma_{L} f_{L}+\Gamma_{R} f_{R}\right] G_{M}^{a},
$$

onde $\mu_{L / R}=\varepsilon_{F} \pm \frac{e V}{2}, \Gamma_{L / R}=\Gamma_{L / R}\left(\epsilon \pm \frac{e V}{2}\right)$ e $f_{L / R}=f\left(\epsilon-\mu_{L / R}\right)$ é a função distribuição de Fermi-Dirac. Adicionando e subtraindo o termo $G_{M}^{r} \Gamma_{R} G_{M}^{a} f\left(\epsilon-\mu_{L}\right)$ à Equação 4.48 .

$$
\begin{aligned}
\rho & =\frac{1}{2 \pi} \int_{-\infty}^{+\infty} d \epsilon\left(G_{M}^{r} \Gamma_{L} G_{M}^{a} f_{L}+G_{M}^{r} \Gamma_{R} G_{M}^{a} f_{R}+G_{M}^{r} \Gamma_{R} G_{M}^{a} f_{L}-G_{M}^{r} \Gamma_{R} G_{M}^{a} f_{L}\right)= \\
& =\frac{1}{2 \pi} \int_{-\infty}^{+\infty} d \epsilon\left[G_{M}^{r}\left(\Gamma_{L}+\Gamma_{R}\right) G_{M}^{a} f_{L}+G_{M}^{r} \Gamma_{R} G_{M}^{a}\left(f_{R}-f_{L}\right)\right] .
\end{aligned}
$$


Mas $\Gamma_{L / R}=i\left[\Sigma_{L / R}^{r}-\Sigma_{L / R}^{a}\right]$, logo, podemos escrever:

$$
\begin{aligned}
\Gamma_{L}+\Gamma_{R} & =i\left(\Sigma_{L}^{r}-\Sigma_{L}^{a}+\Sigma_{R}^{r}-\Sigma_{R}^{a}\right)= \\
& =i\left(-\varepsilon S_{M}+H_{M}+\Sigma_{L}^{r}+\Sigma_{R}^{r}+\varepsilon S_{M}^{\dagger}-H_{M}^{\dagger}-\Sigma_{L}^{a}-\Sigma_{R}^{a}\right)+2 \eta S_{M}= \\
& =i\left[-G_{M}^{r}{ }^{-1}+\left(G_{M}^{a}\right)^{-1}\right]+2 \eta S_{M} .
\end{aligned}
$$

Inserindo este resultado na Equação 4.49, e desconsiderando o termo $2 \eta S_{M}$ que vai a zero em sistemas acoplados, obtém-se:

$$
\begin{aligned}
\rho & =\frac{1}{2 \pi} \int_{-\infty}^{+\infty} d \epsilon\left[i G_{M}^{r}\left(-G_{M}^{r}-1+\left(G_{M}^{a}\right)^{-1}\right) G_{M}^{a} f_{L}+G_{M}^{r} \Gamma_{R} G_{M}^{a}\left(f_{R}-f_{L}\right)\right]= \\
& =\frac{1}{2 \pi} \int_{-\infty}^{+\infty} d \epsilon\left[i\left(-G_{M}^{a}+G_{M}^{r}\right) f_{L}+G_{M}^{r} \Gamma_{R} G_{M}^{a}\left(f_{R}-f_{L}\right)\right] .
\end{aligned}
$$

Assumindo simetria de inversão temporal $G_{M}^{r}=\left(G_{M}^{r}\right)^{\top} \Rightarrow G_{M}^{a}=G_{M}^{r *}, \operatorname{logo} i\left(-G_{M}^{a}+G_{M}^{r}\right)$ $=i\left(-G_{M}^{r *}+G_{M}^{r}\right)=-2 \operatorname{Im}\left[G_{M}^{r}\right]$. Assim sendo:

$$
\begin{aligned}
\rho & =\frac{1}{2 \pi} \int_{-\infty}^{+\infty} d \epsilon\left\{-2 \operatorname{Im}\left[G_{M}^{r}\right] f_{L}+G_{M}^{r} \Gamma_{R} G_{M}^{a}\left[f_{L}-f_{R}\right]\right\}= \\
& =\underbrace{-\frac{1}{\pi} \int_{-\infty}^{+\infty} d \epsilon \operatorname{Im}\left[G_{M}^{r}\right] f_{L}}_{\rho_{e q}}+\underbrace{\frac{1}{2 \pi} \int_{-\infty}^{+\infty} d \epsilon G_{M}^{r} \Gamma_{R} G_{M}^{a}\left[f_{L}-f_{R}\right]}_{\rho_{V}} .
\end{aligned}
$$

Portanto, a matriz densidade de elétrons pode ser escrita como a soma $\rho=\rho_{e q}+\rho_{V}$, onde $\rho_{e q}$ pode ser interpretado como a densidade do sistema no equilíbrio (quando ambos os reservatórios têm o mesmo potencial eletroquímico $\mu_{L}$ ) e $\rho_{V}$, como a contribuição fora do equilíbrio, já que para $\mu_{L}=\mu_{R} \Rightarrow \rho_{V}=0$.

A integral na parte fora do equilíbrio da matriz densidade, $\rho_{V}$, é limitada pela subtração das funções distribuição de Fermi-Dirac dos eletrodos $\left(f_{L}-f_{R}\right)$. Já na parte no equilíbrio, $\rho_{e q}$, a integral é limitada somente superiormente pela função $f\left(\epsilon-\mu_{L}\right)$, sendo necessário integrar desde o nível ocupado de menor energia. Nesse caso, uma integração numérica padrão é inviável porque, como a função de Green $G_{M}^{r}$ possui polos próximos ao eixo real (Equação 4.42), seria necessário uma quantidade enorme de pontos. Uma alternativa é realizar a integração em um contorno no plano complexo, como detalhado no Apêndice B- Seção B.1. 
Com o traço da matriz densidade, obtém-se a nova densidade de carga. Compara-se, então, o valor obtido com a densidade eletrônica anterior e, caso o critério de convergência não tenha sido atingido $\left(\left|n^{i}-n^{i+1}\right|>\delta_{\text {conv }}\right)$, a Hamiltoniana é recalculada a partir da nova densidade eletrônica e os passos acima são repetidos. Após atingir a autoconsistência, calcula-se a corrente por meio da Equação 4.34 .

\subsection{O Programa SMEAGOL: NEGF + DFT}

O escopo deste trabalho nunca foi desenvolver um novo código de transporte eletrônico, mas, sim, realizar mudanças de forma a incluir os efeitos da interação elétron-fônon em um código já existente, a saber, o programa SMEAGOL [4] (Spin and Molecular Electronics in Atomically Generated Orbital Landscapes). Contudo, o SMEAGOL era baseado em uma versão ultrapassada, a 1.3, do código SIESTA (apresentado no Capítulo 3), utilizado como plataforma do cálculo da estrutura eletrônica $a b$ initio.

Desde a versão 1.3 até a versão 3.19 o SIESTA passou por uma série de melhorias e correções. As mudanças vão de melhorias e otimizações até a reformulação do código (com a modularização do programa principal e a reformulação de sub-rotinas fundamentais, como a de reutilização da matriz densidade, de diagonalização, do cálculo dos elementos das matrizes hamiltoniana e de overlap etc.). Entre as mudanças que surtiram grande efeito na eficiência do programa estão as melhorias e otimizações para convergência do ciclo autoconsistente, como novas opções para o algoritmo pulay mixing, generalização dos critérios de convergência, algoritmo fire e controle de passos. Também foram adicionados novos algoritmos para relaxação da geometria e para diagonalização, aperfeiçoando não apenas a eficiência mas, também, o uso de memória. Novos funcionais foram incluídos como RPBE, revPBE, PBEsol, Wu-Cohen, além dos funcionais BLYP, PBE, PW91 já existentes.

Portanto, decidiu-se que, antes de implementar o transporte inelástico, o primeiro passo deveria ser a transição para a versão mais atual do código SIESTA. Ao longo da transição, também foi realizada uma série de melhorias e otimizações nos algoritmos do SMEAGOL já existentes. Além disso, foram incluídas novas implementações, como dinâmica molecular com transporte, o cálculo eficiente das autoenergias para eletrodos periódicos, algoritmo multigrid para o cálculo da equação de Poisson com diferentes condições de contorno, além das implementações para o transporte com interação elétron-fônon que serão apresentadas no próximo capítulo.

Para exemplificar o ganho computacional obtido com o novo SMEAGOL, um mesmo cálculo

\footnotetext{
${ }^{9} \mathrm{Na}$ época em que este trabalho foi realizado, a versão mais recente do SIESTA era a 3.1, mas atualmente já se encontra na versão 3.2.
} 
foi realizado com a versão antiga, com a versão nova e com a versão nova com a opção de eletrodo periódico. O sistema simulado é constituído por uma molécula de benzeno-ditiol entre eletrodos de ouro, totalizando 102 átomos na região de espalhamento e a célula unitária de ambos os eletrodos é formada por 27 átomos de ouro. As duas versões do SMEAGOL utilizadas, antiga e nova, foram compiladas com as mesmas flags de otimização e todos os três cálculos foram executados na mesma máquina, com 32 processadores. Os resultados obtidos para o tempo de execução em minutos (walltime) estão dispostos na Tabela 4.1 .

\begin{tabular}{c|c|c}
\hline \hline SMEAGOL ANTIGO & SMEAGOL NOVO & $\begin{array}{c}\text { SMEAGOL NOVO } \\
\text { (eletrodo periódico) }\end{array}$ \\
\hline 1772.65 & 1333.11 & 845.27 \\
\hline \hline
\end{tabular}

Tabela 4.1: Tempos de execução em minutos de um mesmo sistema com as versões nova e antiga do SMEAGOL.

A integração entre a implementação do formalismo NEGF do SMEAGOL e o cálculo de estrutura eletrônica ocorre, sobretudo, por meio de três mudanças no código SIESTA. A primeira é a inclusão das autoenergias dos eletrodos na Hamiltoniana de Kohn-Sham. Para isso, um cálculo preliminar para cada eletrodo infinito tem que ser realizado (com o eletrodo repetido periodicamente na direção do transporte). Neste cálculo são armazenadas as matrizes Hamiltoniana $H_{L / R}^{0}$ e de overlap $S_{L / R}^{0}$ de cada célula unitária, assim como os respectivos acoplamentos $H_{L / R}^{1}$ e $S_{L / R}^{1}$. Ao executar o cálculo de transporte, essas matrizes são utilizadas para obter as funções de Green de superfície $\left(\left(\varepsilon \mathcal{S}_{L}-\mathcal{H}_{L}\right)^{-1}\right.$ e $\left.\left(\varepsilon \mathcal{S}_{R}-\mathcal{H}_{R}\right)^{-1}\right)$, que são computadas por meio de um algoritmo semianalítico exato. Como a estrutura eletrônica dos eletrodos não é afetada pelo acoplamento com a região de espalhamento, exceto pelo deslocamento rígido dos níveis devido à diferença de potencial aplicada pela bateria, o cálculo das autoenergias dos eletrodos tem que ser realizado somente uma vez.

A segunda alteração é introduzir no potencial de Hartree uma contribuição linear devido à diferença de potencial aplicada entre os eletrodos. O potencial de Hartree é obtido resolvendo-se a equação de Poisson (Equação 3.26) até o termo linear, que, por sua vez, é determinado por meio de condições periódicas de contorno nas extremidades da supercélula. Como o efeito do potencial externo é o deslocamento rígido da estrutura eletrônica dos eletrodos, basta adicionar um termo linear com a tensão $V$ da seguinte maneira: 


$$
V_{\text {Hartree }}(\mathbf{r})=\phi_{\text {Hartree }}(\mathbf{r})+\frac{V}{L}(z-a)+b,
$$

onde $z$ é a posição na direção de transporte, $L$ é o comprimento da célula nesta direção, $a$ é o centro da célula e $b$ é uma constante que determina o zero do potencial de Hartree.

Outro problema está relacionado ao algoritmo de transformada de Fourier utilizado para resolver a equação de Poisson no espaço recíproco. Este algoritmo descarta a componente $\mathbf{k}=0$ da transformada, que determina a carga total na célula, fazendo com que diferentes cálculos possam resultar em valores distintos para a constante $b$. No cálculo de transporte isto é um problema, pois é preciso que o potencial nas extremidades esquerda e direita da célula coincidam com o potencial dos eletrodos.

Para garantir esse casamento do potencial nas extremidades da célula, determina-se no cálculo preliminar dos eletrodos o valor do potencial em um ponto $z$ específico. Este valor é utilizado como parâmetro de entrada no cálculo de transporte. Durante o ciclo autoconsistente, comparase o valor do potencial de Hartree, no mesmo ponto $z$, com o calculado para o eletrodo. A diferença entre eles é considerada como sendo o valor da constante $b$.

Por fim, a última grande mudança consiste em trocar o algoritmo do cálculo da matriz densidade via DFT pelo procedimento apresentado na seção anterior via NEGF, onde a redistribuição de carga devido ao potencial externo é levada em consideração.

A interface entre o programa SMEAGOL e o programa SIESTA está esquematizada no fluxograma da Figura 4.6. Nota-se que o SIESTA fornece a Hamiltoniana de Kohn-Sham ao SMEAGOL, que, por sua vez, introduz as autoenergias dos eletrodos, de forma que o sistema passa de periódico a uma região central acoplada a eletrodos semi-infinitos, e calcula uma nova matriz densidade. As matrizes Hamiltoniana e densidade são trocadas entre o SIESTA e o SMEAGOL iterativamente até atingir a autoconsistência. Desta feita, o SMEAGOL calcula propriedades de transporte, como corrente e coeficientes de transmissão.

Vale mencionar que o SMEAGOL, assim como o SIESTA, são códigos paralelizados via Message Passing Interface (MPI) [87], o que permite maior capacidade computacional (já que os cálculos são distribuídos entre os processadores) e um melhor gerenciamento de memória (pois cada processador armazena somente os dados necessários para o seu cálculo).

O manual do programa, atualizado com as novas opções de execução, está disposto no Apêndice $\mathrm{E}$. onde estão detalhados os passos para se utilizar o programa, assim como as diferentes opções permitidas ao usuário. 


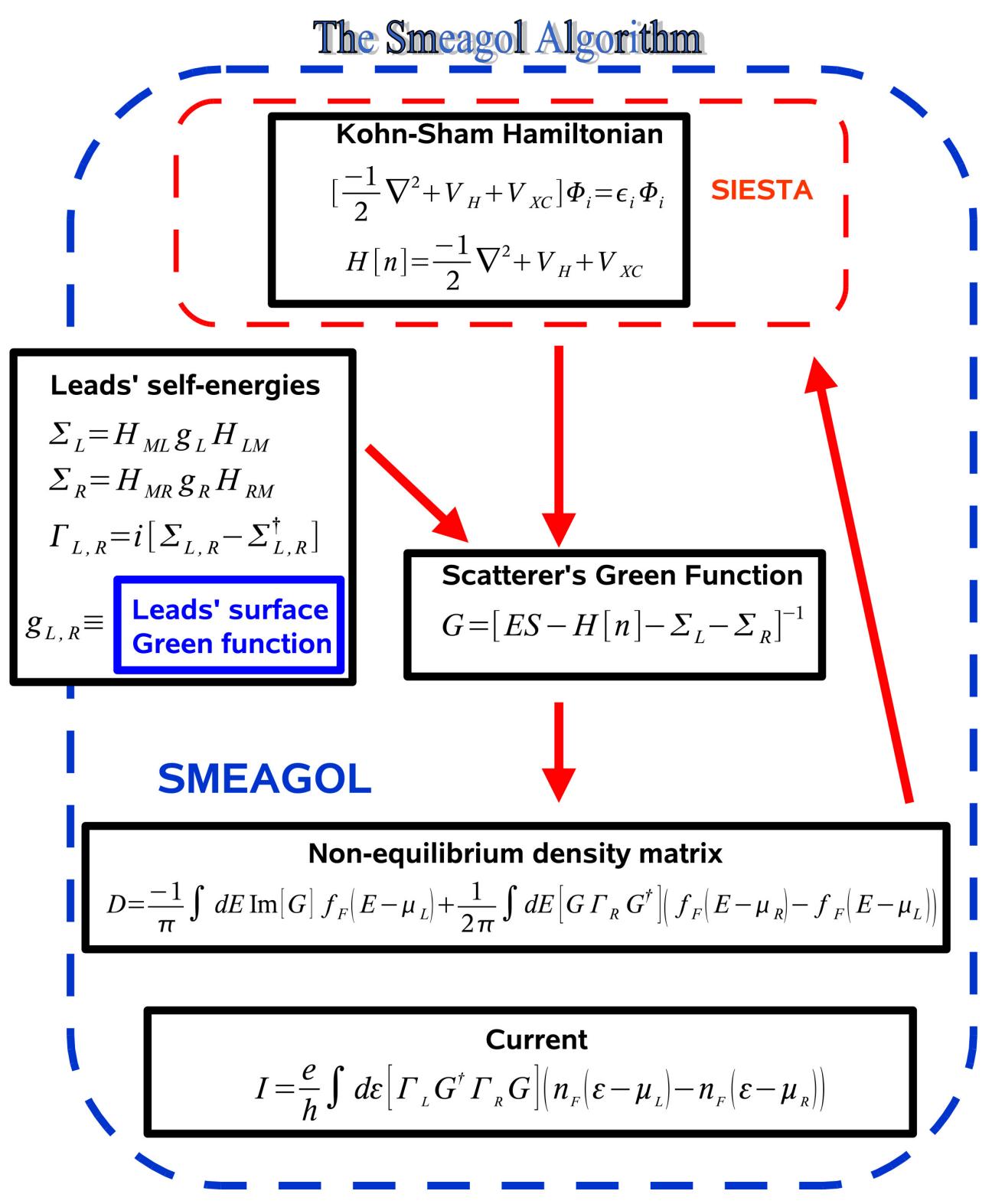

Figura 4.6: Fluxograma do programa SMEAGOL, destacando a interface com o programa SIESTA. (Extraído de [9], com permissão de A. R. Rocha.)

Apesar das intervenções discutidas acima, o código SMEAGOL foi implementado de maneira a preservar todas as capacidades do SIESTA, sendo possível realizar todos os tipos de cálculos oferecidos pelo SIESTA, sem a necessidade de uma nova compilação e sem perda de eficiência. Dessa forma, concilia-se em um único programa inúmeras ferramentas para o cálculo preciso tanto de estrutura eletrônica como de transporte. 


\section{Capítulo 5}

\section{Perda de Coerência de Fase}

Na abordagem apresentada no Capítulo 4, o transporte eletrônico ocorre sem que haja perda de energia no canal, ou seja, o elétron entra na região de espalhamento com uma dada energia $E$ e sai com esta mesma energia. No entanto, nos dispositivos reais, os elétrons interagem com as vibrações nucleares trocando energia, na maior parte dissipada na forma de calor. Observações experimentais indicam que essas interações podem ter um papel ainda mais relevante nas propriedades de transporte em escala atômica e molecular [19, 20], ocasionando a perda de coerência de fase dos elétrons, abertura de novos canais de condutância e supressão de canais puramente elásticos.

Para inserir a interação elétron-fônon no cálculo de transporte seria preciso, a princípio, resolver o problema eletrônico simultaneamente com o problema dos fônons na região de espalhamento. Ou seja, um cálculo rigoroso consideraria de forma autoconsistente o efeito causado pelos elétrons nos fônons e o efeito causado pelos fônons nos elétrons. Contudo, como primeira aproximação, pode-se considerar que a região de espalhamento está acoplada a um reservatório de fônons, onde estes se encontram em equilíbrio térmico.

A Hamiltoniana da região central $H_{M}$ tem a mesma forma daquela obtida no Capítulo 2 (Equações 2.54 2.57), onde foi considerada a aproximação harmônica para o movimento nuclear:

$$
\begin{aligned}
H_{M} & =H_{e}+H_{f}+H_{e f}, \\
H_{e} & =\sum_{\mathbf{k}, \sigma} E_{\mathbf{k}, \sigma} c_{\mathbf{k}, \sigma}^{\dagger} c_{\mathbf{k}, \sigma}, \\
H_{f} & =\sum_{\mathbf{q}, \lambda} \hbar \omega_{\mathbf{q}, \lambda}\left(b_{\mathbf{q}, \lambda}^{\dagger} b_{\mathbf{q}, \lambda}+\frac{1}{2}\right), \\
H_{e f} & =\sum_{\mathbf{k}, \mathbf{k}^{\prime}, \sigma} \sum_{\mathbf{q}, \lambda} M_{\mathbf{q}, \lambda} c_{\mathbf{k}, \sigma}^{\dagger} c_{\mathbf{k}^{\prime}, \sigma}\left(b_{\mathbf{q}, \lambda}+b_{-\mathbf{q}, \lambda}^{\dagger}\right),
\end{aligned}
$$


onde a forma utilizada para a Hamiltoniana de interação elétron-fônon não implica em conservação do momento cristalino, já que os sistemas que iremos considerar podem não ser invariantes por translação!

Porém, diferentemente do acoplamento da região central com os eletrodos, onde a autoenergia é conhecida e pode ser obtida de maneira exata, mesmo com a aproximação acima a forma da interação elétron-fônon é desconhecida, sendo inevitável a busca por modelos mais simples que incorporem os efeitos da interação ou por aproximações.

Neste capítulo, apresentaremos o modelo fenomenológico das sondas de Büttiker [1], onde o efeito da interação produz apenas um alargamento dos níveis de condutância, simulando o efeito de perda de coerência de fase dos elétrons devido à interação com fônons do material. Para tanto, iniciaremos apresentando a generalização do formalismo de Landauer para sistemas com multieletrodos devido a M. Büttiker et al [88, 89].

Serão apresentadas duas implementações diferentes para o modelo de Büttiker: na primeira, uma aproximação elástica é considerada no cálculo da corrente; a outra, sem esta aproximação.

Veremos, contudo, que neste modelo não são incluídos efeitos como abertura de novos canais de condutância e supressão de canais puramente elásticos, observados experimentalmente [90 91]. Isso nos levou à busca por outros modelos que serão apresentados nos capítulos seguintes.

\subsection{Formalismo de Landauer-Büttiker}

O formalismo de Landauer na forma com foi discutida na Seção 4.2 é restrito a dispositivos com dois terminais, os contatos fonte e dreno. No entanto, muitos experimentos são conduzidos com dispositivos com mais terminais, ora para criar uma simetria específica ora para eliminar graus de liberdade ou para realizar medidas diferentes simultaneamente. Este último caso foi o que levou M. Büttiker a considerar um dispositivo de quatro terminais quando investigava a ocorrência de magnetorresistências assimétricas [88]. Nesse dispositivo, dois terminais eram responsáveis pelo fluxo de corrente na região de espalhamento e os outros dois mediam a tensão.

Assim como os contatos, novos terminais também podem ser interpretados como reservatórios de elétrons em equilíbrio térmico e descritos por um potencial eletroquímico. Logo, Büttiker notou que todos os terminais poderiam ser tratados da mesma forma e simplesmente estendeu a fórmula de Landauer (Equação 4.8) somando sobre todos os terminais:

\footnotetext{
${ }^{1}$ A partir deste capítulo, diferentemente dos anteriores, vamos considerar explicitamente as componentes de spin nas expressões (antes elas estavam aglutinadas ao índice de estado $\mathbf{k}$ ).
} 


$$
I_{p}=\frac{2 e^{2}}{h} \sum_{q}\left[\bar{T}_{p q} V_{p}-\bar{T}_{q p} V_{q}\right]
$$

onde o fator 2 se refere às duas componentes de spin. Esta ideia é facilmente estendida para a fórmula da corrente obtida com o formalismo NEGF apresentada na Seção 4.4. somando-se também sobre todos os terminais:

$$
I_{p}=\frac{2 e}{h} \int_{-\infty}^{+\infty} d \epsilon \sum_{j=1}^{N} \operatorname{Tr}\left[\Gamma_{p} G_{M}^{r \dagger} \Gamma_{j} G_{M}^{r}\right]\left(f\left(\epsilon-\mu_{p}\right)-f\left(\epsilon-\mu_{j}\right)\right) .
$$

ou seja, a corrente que atravessa o terminal $p$ depende das contribuições dos demais terminais.

\subsection{Modelo Fenomenológico das Sondas de Büttiker}

Tipicamente, se considerarmos um dispositivo cuja dimensão $L$ seja suficientemente grande (muito maior do que o comprimento de relaxação de fase), um elétron se propagando no canal sofre transições devido a interações inelásticas. Isto é, o elétron se propaga coerentemente por um tempo até sofrer um espalhamento, trocando energia com um fônon do material, o que o transfere a outro nível de energia. Neste novo nível, o elétron se propaga coerentemente até ser espalhado novamente, e assim sucessivamente até alcançar o dreno (Figura 5.1).

Vimos que os eletrodos são reservatórios de elétrons em equilíbrio termodinâmico, de modo que ao alcançar um eletrodo o elétron é termalizado. Büttiker observou, então, que o efeito do espalhamento inelástico dos elétrons pode ser simulado introduzindo-se sondas fictícias (terminais) na região central (canal) do sistema coerente. Ou seja, no modelo de Büttiker, o efeito ocorre somente na região de espalhamento e os elétrons nos eletrodos são considerados não-interagentes. Ele, então, impôs que a corrente total em cada sonda fictícia tinha de ser nula, de forma que todo elétron que entra na sonda deve voltar ao dispositivo, porém sem uma relação de fase definida com os elétrons coerentes incidentes (Figura 5.2). Ou seja, introduzir sondas fictícias no sistema leva a uma perda da memória de fase no processo de espalhamento.

Da mesma maneira que o acoplamento dos eletrodos fonte e dreno com a região de espalhamento, o acoplamento das sondas fictícias é introduzido por meio de um termo de autoenergia de interação na função de Green $G_{M}^{r}$. Porém, como a forma dessa autoenergia é desconhecida, o modelo mais simples consiste em considerar que a autoenergia devido às sondas seja um número imaginário puro [92], de forma a produzir um efeito de alargamento dos canais de condutância simulando a quebra de coerência de fase: 
a)

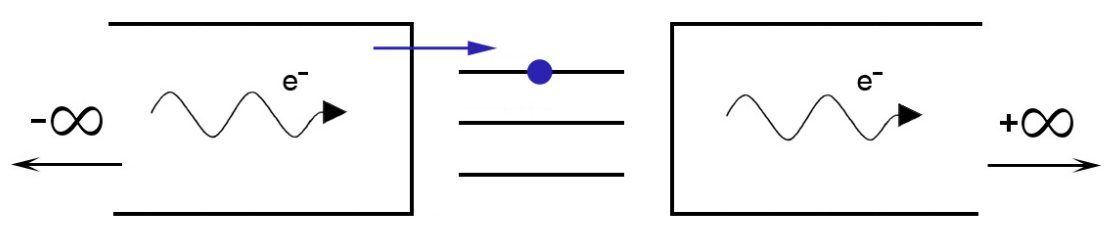

b)

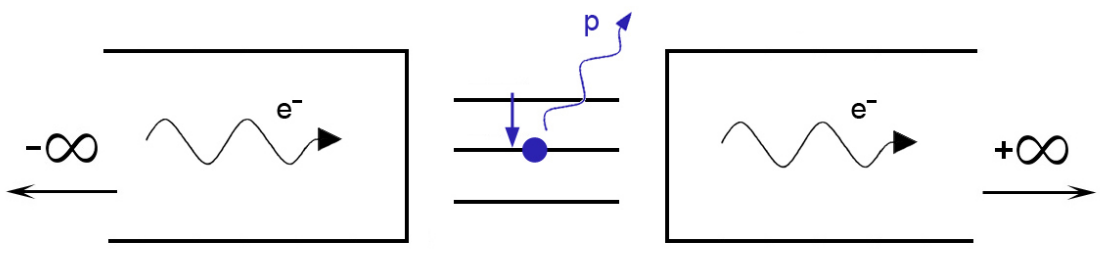

c)

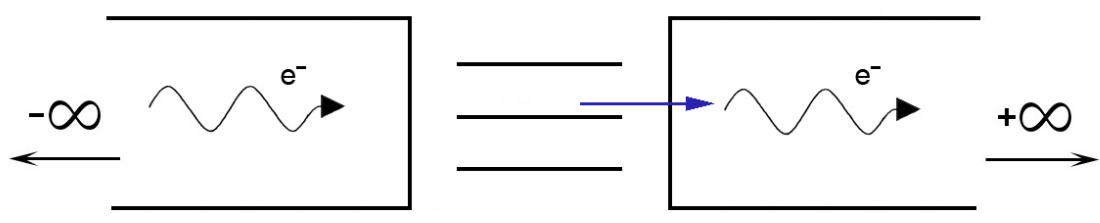

Figura 5.1: Representação da interação elétron-fônon na região de espalhamento. a) Elétron se propaga coerentemente a partir do eletrodo esquerdo. b) Elétron muda para um nível molecular de mais baixa energia emitindo um fônon. c) Elétron se propaga coerentemente até o eletrodo direito.

$$
\Sigma_{B}^{r}=-\frac{i}{2} \beta S_{M} \quad \Longrightarrow \quad G_{M}^{r}=\left[\varepsilon S_{M}-H_{M}-\Sigma_{L}^{r}-\Sigma_{R}^{r}-\Sigma_{B}^{r}\right]^{-1},
$$

onde $\beta$ é um parâmetro fenomenológico ajustável relacionado à intensidade da defasagem (acoplamento elétron-fônon) e $\Sigma_{B}^{r}$ é um operador imaginário puro, o que produz somente um alargamento dos níveis (ou seja, não é incluída a informação do espectro de energia dos fônons). A autoenergia de interação $\Sigma_{B}^{r}$ devido a todas as sondas é obtida somando-se o termo de autoenergia $\Sigma_{p}^{r}$ de

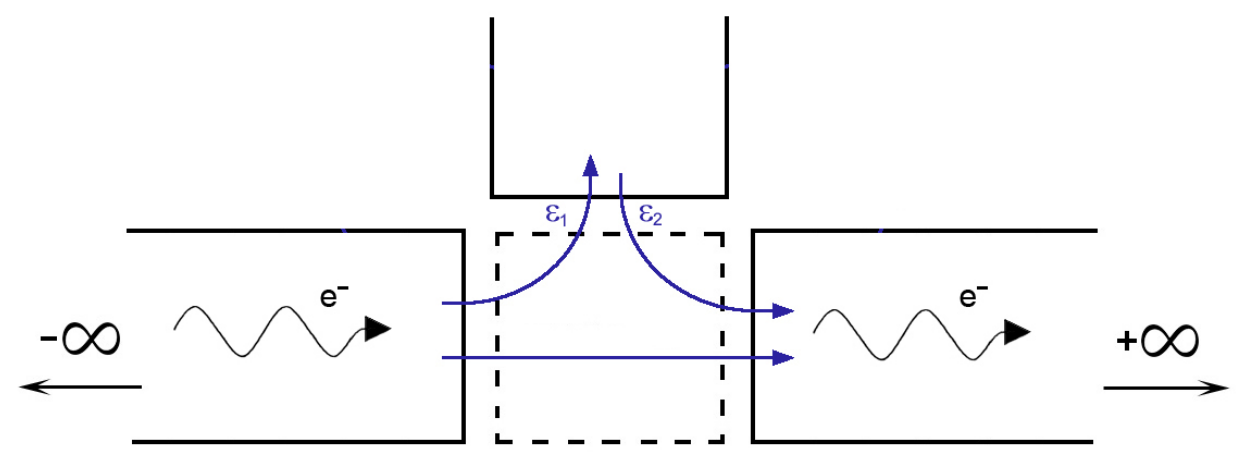

Figura 5.2: Efeito obtido ao incluir uma sonda fictícia na região de espalhamento 
cada sonda, sendo $p=1,2, \ldots, N$ e $N$ a quantidade de sondas.

Uma maneira pragmática de implementar este modelo é introduzir uma sonda em cada orbital atômico da região de espalhamento. Como as sondas são fictícias, elas podem trocar elétrons com a região de espalhamento, porém a corrente líquida em cada sonda deve ser nula. Portanto, os potenciais eletroquímicos das sondas $\mu_{p}$ devem ser ajustados de forma que a corrente total em cada uma seja nula. Uma ilustração do problema para o caso particular em que há somente um orbital por sítio pode ser observada na Figura 5.3

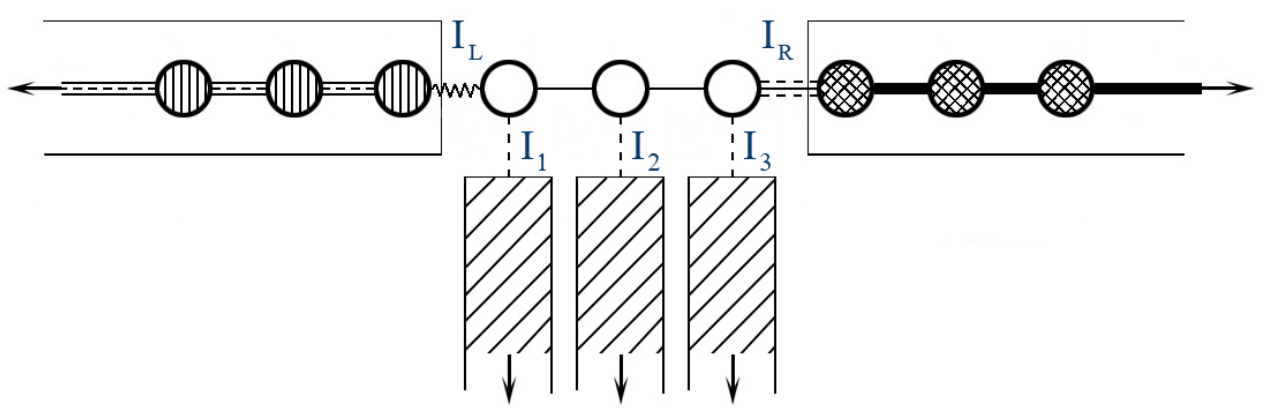

Figura 5.3: Ilustração das sondas fictícias acopladas à região de espalhamento.

Conforme discutido na seção anterior, a expressão para a corrente em cada sonda $p$ (incluindo os eletrodos esquerdo e direito) pode ser escrita como:

$$
I_{p}=\int_{-\infty}^{+\infty} d \epsilon \tilde{i}_{p}(\epsilon)
$$

onde $\tilde{i}_{p}$ é a corrente por unidade de energia, essencialmente equivalente a uma transmissão efetiva fora do equilíbrio:

$$
\tilde{i}_{p}(\epsilon)=\frac{2 e}{h} \sum_{j=1}^{N} \operatorname{Tr}\left[\Gamma_{p} G_{M}^{r} \Gamma_{j} G_{M}^{r}\right]\left(f\left(\epsilon-\mu_{p}\right)-f\left(\epsilon-\mu_{j}\right)\right) .
$$

Como a corrente em cada sonda depende do potencial eletroquímico de todas as outras sondas, é necessário encontrar o conjunto de potenciais eletroquímicos $\mu_{p}$ tais que a condição $I_{p}=0$ seja satisfeita para todas as sondas simultaneamente. Isso corresponde a resolver um sistema de $N$ equações não-lineares acopladas. Devido à integração na energia, isto não pode ser feito analiticamente. 


\subsection{Transporte Elástico Não-Coerente}

A alternativa mais simples para resolver este problema é impor que o integrando de cada uma das $N$ equações seja nulo [92, 93], isto é, $\tilde{i}_{p}(\epsilon)=0$. Isso corresponde a assumir conservação de energia no espalhamento, de forma que o elétron não sofre transições entre níveis de energia. Ou seja, na Figura 5.2, se o elétron entrar na sonda com energia $\varepsilon_{1}$, ele sairá com a mesma energia.

Outra forma de visualizar esta aproximação elástica é assumir que toda vez que um elétron sofrer um espalhamento indo de um nível de energia $\varepsilon_{1}$ para um nível $\varepsilon_{2}$, existirá outro elétron no nível $\varepsilon_{2}$ que sofrerá uma transição para o nível $\varepsilon_{1}[67$.

Apesar dessa aproximação elástica omitir efeitos de dissipação de energia devido à interação inelástica com os fônons do material, ela preserva o efeito da perda de fase, já que, ao entrar na sonda, o elétron termaliza dentro do eletrodo e o elétron que sai não terá a mesma relação de fase, por mais que a energia seja conservada.

Ao impor que $\tilde{i}_{p}(\epsilon)=0$ para toda sonda $p$ para cada energia $\epsilon$, o problema de encontrar o conjunto de potenciais eletroquímicos $\mu_{p}$ tais que a condição $I_{p}=0$ seja satisfeita para todas as sondas $p$ passa a ser linear e o sistema pode ser escrito como uma equação matricial linear com a forma:

$$
\mathbf{f}=A^{-1} \mathbf{b}
$$

onde:

$$
\begin{aligned}
A_{p p} & =T_{p L}+T_{p R}+\sum_{\substack{q=1 \\
q \neq p}}^{N} T_{p q}, \quad A_{p q}=-T_{p q} \\
\mathbf{f}_{p} & =f\left(\epsilon-\mu_{p}\right) \\
\mathbf{b}_{p} & =T_{p L} f\left(\epsilon-\mu_{L}\right)+T_{p R} f\left(\epsilon-\mu_{R}\right) .
\end{aligned}
$$

Dessa forma, calculando-se o sistema linear para cada energia, obtém-se um conjunto de potenciais eletroquímicos dependentes da energia (isto é, $\mathbf{f}=\mathbf{f}(\epsilon)$ ) e a corrente dada pela Equação 5.9 pode ser calculada.

\subsection{Transporte Inelástico Não-Coerente}

Neste trabalho, foi proposta uma nova maneira para encontrar o conjunto de potenciais eletroquímicos $\mu_{p}$ que satisfaça simultaneamente a condição $I_{p}=0$, que vem a ser resolver o sistema de $N$ 
equações não-lineares acopladas, sem a aproximação elástica. Para tanto, foi utilizado o método de Newton para sistemas não-lineares apresentado a seguir [94].

Pretende-se calcular o sistema $\vec{I}(\vec{\mu})=0$, onde $\vec{I}$ é um vetor de dimensão $N$ cujos elementos são dados por $I_{p}$ e $\vec{\mu}$ é o vetor cujos elementos $\mu_{p}$ se quer determinar. Define-se uma função $\vec{F}(\vec{\mu})$ como:

$$
\vec{F}(\vec{\mu})=\vec{\mu}-J(\vec{\mu})^{-1} \vec{I}(\vec{\mu}),
$$

onde $J(\vec{\mu})$ é a matriz jacobiana definida como:

$$
[J(\vec{\mu})]_{i j}=\frac{\partial I_{i}}{\partial \mu_{j}}(\vec{\mu})
$$

O procedimento de iteração funcional evolui a partir de um $\vec{\mu}^{(0)}$ selecionado ("chute" inicial), gerando para $k \geq 1$ :

$$
\vec{\mu}^{(k)}=\vec{F}\left(\vec{\mu}^{(k-1)}\right)=\vec{\mu}^{(k-1)}-J\left(\vec{\mu}^{(k-1)}\right)^{-1} \vec{I}\left(\vec{\mu}^{(k-1)}\right) .
$$

Se um "bom" valor inicial for conhecido e se a matriz jacobiana existir, o método de Newton para sistemas não-lineares possui convergência quadrática. Para o problema de se encontrar os potenciais eletroquímicos, garante-se um bom chute inicial calculando-se as propriedades de transporte a partir do equilíbrio $(V=0)$, sendo que a solução do problema é conhecida, e aumentando-se (ou diminuindo) gradativamente a tensão, onde a cada tensão o chute inicial é dado pelo conjunto de potenciais eletroquímicos calculados para a tensão anterior. Além disso, a matriz jacobiana existe e é dada por:

$$
[J(\vec{\mu})]_{i j}= \begin{cases}\frac{1}{2 k_{B} T} \int_{-\infty}^{+\infty} d \epsilon\left[\frac{T_{i L}+T_{i R}+\sum_{k=1}^{N} T_{i k}}{k \neq i}\right], \quad \text { para } i=j, \\ \frac{1+\cosh \left(\frac{\epsilon-\mu_{i}}{k_{B} T}\right)}{2 k_{B} T} \int_{-\infty}^{+\infty} d \epsilon\left[\frac{-T_{i j}}{1+\cosh \left(\frac{\epsilon-\mu_{j}}{k_{B} T}\right)}\right], & \text { para } i \neq j .\end{cases}
$$

De maneira geral, numericamente as integrais são somatórias nos quais para cada ponto calcula-se um peso correspondente 2 ou seja:

\footnotetext{
${ }^{2}$ A determinação dos pontos e dos pesos de cada ponto depende do método utilizado. Por exemplo, no caso da quadratura gaussiana dependerá do conjunto de polinômios ortogonais utilizado para o ajuste 95 .
} 


$$
\int_{x_{1}}^{x_{2}} d x f(x) \approx \sum_{j=0}^{N-1} w_{j} f\left(x_{j}\right) .
$$

Portanto, como o intervalo de integração dos elementos de $I_{p}$ e de $[J(\vec{\mu})]_{i j}$ são os mesmos, o custo computacional pode ser reduzido substancialmente computando-se previamente os coeficientes de transmissão $T_{i j}$ para cada ponto da integração.

Também é útil notar que $T_{i j}=T_{j i}$, o que é intuído fisicamente. Esta observação reduz pela metade os cálculos dos coeficientes de transmissão 3

Os dois métodos para o modelo fenomenológico de Büttiker com o formalismo NEGF foram implementados para um sistema modelo de uma cadeia linear, apresentado a seguir. Apesar do novo método proposto ser mais completo quando comparado ao da seção anterior (onde foi utilizada a aproximação elástica), como a autoenergia de interação não possui informação a respeito do espectro dos fônons, o único efeito obtido continua sendo o de um alargamento dos canais de condutância.

\subsection{Implementação}

Foi implementado um programa utilizando o formalismo NEGF e o modelo fenomenológico das sondas de Büttiker, com as duas abordagens descritas neste capítulo. Utilizou-se como modelo de teste o caso de uma cadeia atômica unidimensional, cuja vantagem é que a autoenergia do eletrodo possui uma expressão analítica. Em particular, no caso em que as energias de sítio dos átomos do sistema são iguais, os acoplamentos são os mesmos e a região de espalhamento contém somente um sítio, diversas propriedades podem ser obtidas analiticamente, o que torna este caso ideal para comparação com os resultados numéricos.

Nesta seção, serão discutidos os detalhes da implementação do sistema modelo, assim como a derivação das expressões analíticas de seu caso particular. Serão apresentados os resultados obtidos desde as primeiras implementações, onde foi abordado o caso no equilíbrio $(V=0)$, até o caso fora do equilíbrio e com os modelos para a perda de coerência de fase.

\subsubsection{Sistema Modelo}

O sistema modelo utilizado consiste em uma cadeia atômica unidimensional com um nível eletrônico por sítio, conforme pode ser observado na Figura 5.4 .

\footnotetext{
${ }^{3}$ Para provar que $T_{i j}=\operatorname{Tr}\left[\Gamma_{i} G_{M}^{r}{ }^{\dagger} \Gamma_{j} G_{M}^{r}\right]=\operatorname{Tr}\left[\Gamma_{j} G_{M}^{r}{ }^{\dagger} \Gamma_{i} G_{M}^{r}\right]=T_{j i}$, basta provar a igualdade $G_{M}^{r}{ }^{\dagger} \Gamma_{j} G_{M}^{r}=$
} 


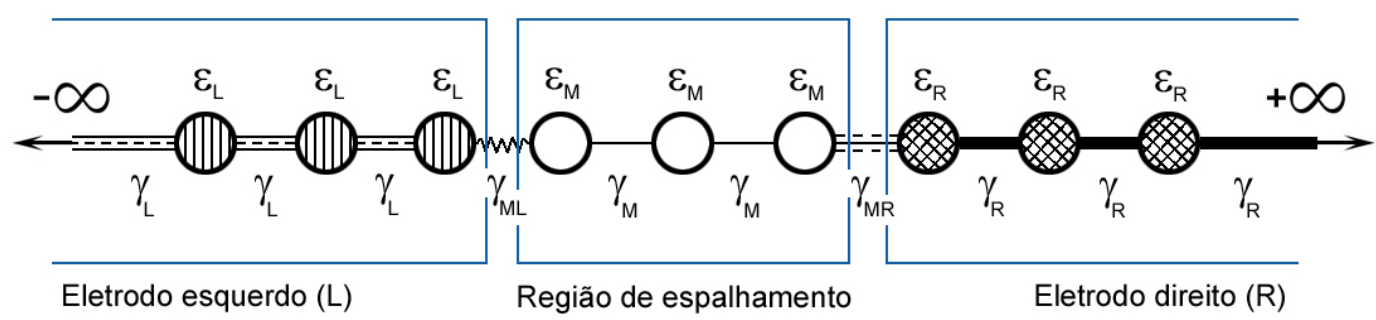

Figura 5.4: Cadeia atômica unidimensional.

Na Figura 5.4 $\varepsilon_{i}(i=L, M, R)$ representa a energia do sítio e $\gamma_{j}(j=L, M L, M, M R, R)$, o acoplamento entre dois sítios. Uma das vantagens de utilizar-se este modelo é que, para uma cadeia atômica unidimensional, a autoenergia do eletrodo tem uma expressão analítica dada por [67, 96]:

$$
\Sigma_{L}=\left(\gamma_{M L}\right)^{2} \frac{e^{i k_{L}}}{\gamma_{L}}
$$

onde o termo $e^{i k_{L}} / \gamma_{L}$ corresponde à função de Green de superfície do eletrodo esquerdo e $k_{L}$ é obtido da dispersão de uma cadeia unidimensional periódica $\epsilon=\mu_{L}+2 \gamma_{L} \cos \left(k_{L}\right)$, ou seja:

$$
k_{L}=\arccos \left(\frac{\epsilon-\mu_{L}}{2 \gamma_{L}}\right)
$$

onde $\mu_{L}=\varepsilon_{F}+\frac{e V}{2}$.

No entanto, um cuidado adicional deve ser tomado ao calcular $k_{L}$, pois $\left(\frac{\epsilon-\mu_{L}}{2 \gamma_{L}}\right) \in \mathbb{C}$ e, portanto, $k_{L} \in \mathbb{C}$. Além disso, a "função" arco-cosseno assume dois valores distintos para um mesmo ponto e o valor escolhido depende do ramo de corte no plano complexo adotado. Porém, uma forma de resolver esse problema, independentemente do método utilizado para o cálculo do arco-cosseno, é determinar o sinal de $k_{L}$ de forma a que a velocidade de grupo dos elétrons seja positiva, isto é:

$$
v=\frac{\partial \epsilon}{\partial k_{L}}=-2 \gamma_{L} \operatorname{sen}\left(k_{L}\right)>0 .
$$

Então, se $\operatorname{Re}\left[2 \gamma_{L} \operatorname{sen}\left(k_{L}\right)\right]>0$, toma-se $-k_{L}$. Os cálculos para o eletrodo direito são análogos, tomando-se o cuidado de definir o potencial eletroquímico como $\mu_{R}=\varepsilon_{F}-\frac{e V}{2}$.

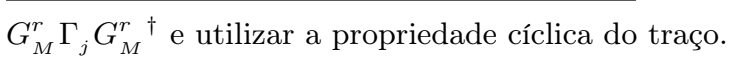




\subsubsection{Perfil do Potencial e Solução Autoconsistente}

Conforme vimos na Seção 4.5, a queda do potencial na região de espalhamento deve ser calculada de maneira autoconsistente levando em conta os rearranjos de carga, isto é, corrigindo os potenciais de cada sítio na região de espalhamento levando em consideração o potencial eletrostático e as interações elétron-elétron. Dentro do nosso modelo tomamos:

$$
H_{M}=H_{M}[\rho]=H_{0}+U_{S C F}
$$

onde $H_{0}$ é o Hamiltoniano não-perturbado e $U_{S C F}=U_{S C F}(\rho)$ é a contribuição do campo autoconsistente. A aproximação de mais baixa ordem para $U_{S C F}$ é dada pelo potencial de Hartree $V_{\text {Hartree }}$, que é obtido pela equação de Poisson:

$$
\nabla^{2} V_{\text {Hartree }}=-e^{2}\left(n-n_{0}\right)
$$

onde $n$ é a ocupação eletrônica sob uma diferença de potencial $V$ e $n_{0}$ é a ocupação no equilíbrio $(V=0)$. Então, o cálculo autoconsistente escolhido para $H$ tem a forma:

$$
H_{M}=H_{0}+U_{0}\left(\rho_{V \neq 0}-\rho_{V=0}\right),
$$

onde $U_{0}$ é uma constante relacionada à mudança na energia de Hartree devido à carga de um elétron extra. $4^{4}$ Dessa forma, constrói-se o seguinte ciclo autoconsistente:

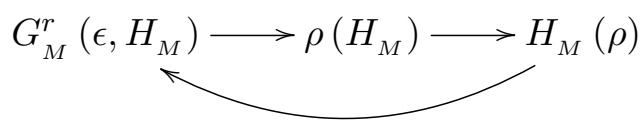

A convergência do ciclo ocorre quando $U_{S C F}^{\text {novo }}-U_{S C F}^{\text {antigo }}$ for menor do que uma fração de $k_{B} T$. Uma vez atingida a autoconsistência, pode-se calcular as propriedades de transporte.

\subsubsection{Caso Particular}

Quando a cadeia é constituída pelo mesmo tipo de átomo (i.e. $\gamma_{L}=\gamma_{R}=\gamma_{M L}=\gamma_{M R}=\gamma \quad$ e $\left.\varepsilon_{L}=\varepsilon_{R}=\varepsilon_{M}\right)$ e a região de espalhamento contém somente um sítio, diversas propriedades podem

\footnotetext{
${ }^{4}$ A Equação 5.24 corresponde ao Hamiltoniano de Hubbard na aproximação de campo médio sem polarização de spin.
} 
ser calculadas analiticamente. Portanto, este caso particular serve para verificar a consistência do programa.

Nota-se que, neste caso, a autoenergia do eletrodo simplifica-se a $\Sigma_{L}=\gamma e^{i k_{L}}$, logo:

$$
\Gamma_{L}=i \gamma\left(e^{i k_{L}}-e^{-i k_{L}}\right)=-2 \gamma \operatorname{sen}\left(k_{L}\right),
$$

e, analogamente,

$$
\Gamma_{R}=-2 \gamma \operatorname{sen}\left(k_{R}\right) .
$$

A transmissão é dada por $T_{L R}(\epsilon)=\operatorname{Tr}\left[\Gamma_{L} G_{M}^{r}{ }^{\dagger} \Gamma_{R} G_{M}^{r}\right]=\Gamma_{L} G_{M}^{r}{ }^{*} \Gamma_{R} G_{M}^{r}$, já que $\Gamma_{L / R}$ e $G_{M}^{r}$ têm dimensão 1. Considerando ainda que $\epsilon, k_{L}, k_{R} \in \mathbb{R}$, então:

$$
\begin{aligned}
\left(G_{M}^{r}\right)^{-1}\left(G_{M}^{r *}\right)^{-1} & =\left(\epsilon-\varepsilon_{M}-\gamma e^{i k_{L}}-\gamma e^{i k_{R}}\right)\left(\epsilon-\varepsilon_{M}-\gamma e^{-i k_{L}}-\gamma e^{-i k_{R}}\right)= \\
& =\left|\epsilon-\varepsilon_{M}-\gamma \cos \left(k_{L}\right)-i \gamma \operatorname{sen}\left(k_{L}\right)-\gamma \cos \left(k_{R}\right)-i \gamma \operatorname{sen}\left(k_{R}\right)\right|^{2}= \\
& =\left(\epsilon-\varepsilon_{M}-\gamma \cos \left(k_{L}\right)-\gamma \cos \left(k_{R}\right)\right)^{2}+\gamma^{2}\left(\operatorname{sen}\left(k_{L}\right)+\operatorname{sen}\left(k_{R}\right)\right)^{2}
\end{aligned}
$$

Assim, a transmissão se resume a:

$$
T_{L R}(\epsilon)=\frac{4 \gamma^{2} \operatorname{sen}\left(k_{L}\right) \operatorname{sen}\left(k_{R}\right)}{\left(\epsilon-\varepsilon_{M}-\gamma \cos \left(k_{L}\right)-\gamma \cos \left(k_{R}\right)\right)^{2}+\gamma^{2}\left(\operatorname{sen}\left(k_{L}\right)+\operatorname{sen}\left(k_{R}\right)\right)^{2}} .
$$

Mas usando o fato que $\cos \left(k_{L}\right)=\frac{\epsilon-\mu_{L}}{2 \gamma}=\frac{\epsilon-\varepsilon_{M}}{2 \gamma}-\frac{e V}{4 \gamma}$ e $\cos \left(k_{R}\right)=\frac{\epsilon-\mu_{R}}{2 \gamma}=\frac{\epsilon-\varepsilon_{M}}{2 \gamma}+\frac{e V}{4 \gamma}$, tem-se:

$$
\begin{aligned}
T_{L R}(\epsilon) & =\frac{4 \gamma^{2} \operatorname{sen}\left(k_{L}\right) \operatorname{sen}\left(k_{R}\right)}{\left(\epsilon-\varepsilon_{M}-\frac{\epsilon-\varepsilon_{M}}{2}-\frac{e V}{4}-\frac{\epsilon-\varepsilon_{M}}{2}+\frac{e V}{4}\right)^{2}+\gamma^{2}\left(\operatorname{sen}\left(k_{L}\right)+\operatorname{sen}\left(k_{R}\right)\right)^{2}}= \\
& =\frac{4 \gamma^{2} \operatorname{sen}\left(k_{L}\right) \operatorname{sen}\left(k_{R}\right)}{\left(\operatorname{sen}\left(k_{L}\right)+\operatorname{sen}\left(k_{R}\right)\right)^{2}} .
\end{aligned}
$$

No caso do modelo para a defasagem com aproximação elástica (Seção 5.3), a densidade de corrente da sonda será:

$$
0=\tilde{i}_{p}(\epsilon)=\frac{2 e}{h} \operatorname{Tr}\left[\Gamma_{p} G_{M}^{r \dagger} \Gamma_{L} G_{M}^{r}\right]\left(f_{p}-f_{L}\right)+\frac{2 e}{h} \operatorname{Tr}\left[\Gamma_{p} G_{M}^{r \dagger} \Gamma_{R} G_{M}^{r}\right]\left(f_{p}-f_{R}\right),
$$


onde $f_{x}=f_{x}\left(\epsilon-\mu_{x}\right)$ são as distribuições de Fermi-Dirac. Portanto, a ocupação da sonda pode ser escrita como:

$$
\begin{aligned}
f_{p} & =\frac{G_{M}^{r}{ }^{*} \Gamma_{L} G_{M}^{r} f_{L}+G_{M}^{r}{ }^{*} \Gamma_{R} G_{M}^{r} f_{R}}{G_{M}^{r}{ }^{*} \Gamma_{L} G_{M}^{r}+G_{M}^{r}{ }^{*} \Gamma_{R} G_{M}^{r}}=\frac{\Gamma_{L} f_{L}+\Gamma_{R} f_{R}}{\Gamma_{L}+\Gamma_{R}}= \\
& =\frac{-2 \gamma \operatorname{sen}\left(k_{L}\right) f_{L}-2 \gamma \operatorname{sen}\left(k_{R}\right) f_{R}}{-2 \gamma \operatorname{sen}\left(k_{L}\right)-2 \gamma \operatorname{sen}\left(k_{R}\right)}=\frac{\operatorname{sen}\left(k_{L}\right) f_{L}+\operatorname{sen}\left(k_{R}\right) f_{R}}{\operatorname{sen}\left(k_{L}\right)+\operatorname{sen}\left(k_{R}\right)} .
\end{aligned}
$$

\subsection{Resultados}

No programa implementado, supõe-se que as energias de sítio da região de espalhamento e eletrodos, a energia de Fermi e os acoplamentos são recebidos de um programa de estrutura eletrônica, como a DFT descrita no Capítulo 3 . Além das energias e acoplamentos, o programa recebe no arquivo de entrada os seguintes dados:

- NumberOfAtoms: número de átomos na região de espalhamento;

- Temperature: temperatura eletrônica do sistema em Kelvin. [valor padrão $300 \mathrm{~K}$ ]

- BiasFrom e BiasTo: valor inicial e final da diferença de potencial entre os eletrodos em eV. [valor padrão 0 e $5.0 \mathrm{eV}$, respectivamente]

- BiasPoints: quantidade de pontos em que o intervalo da diferença de potencial deve ser subdividido. [valor padrão 100]

- HartreeField: constante $U_{0}$ relacionada à mudança na energia de Hartree devido à carga de um elétron extra, Equação 5.24 . [valor padrão 1.0]

- Dephasing: parâmetro fenomenológico $\beta$ relacionado à intensidade da defasagem. [valor padrão 0.05]

- DensityRecovery: variável booleana (yes ou no) que pode ser utilizada no caso do programa por algum motivo parar de rodar antes do final. Se for escolhido yes, o programa recomeça de onde parou. Caso contrário (no), o programa começa do início. [valor padrão no]

A implementação ocorreu gradativamente. Inicialmente foi considerado o sistema no equilíbrio $(V=0)$, onde se calculou as autoenergias dos eletrodos e a função de Green, e, a partir delas, foram obtidas a transmissão e a densidade de estados (DOS) 5 . Veja Figura 5.5.

\footnotetext{
${ }^{5}$ A DOS para o caso no equilíbrio corresponde à função espectral e, portanto, é obtida tomando-se a parte imaginária da função de Green.
} 

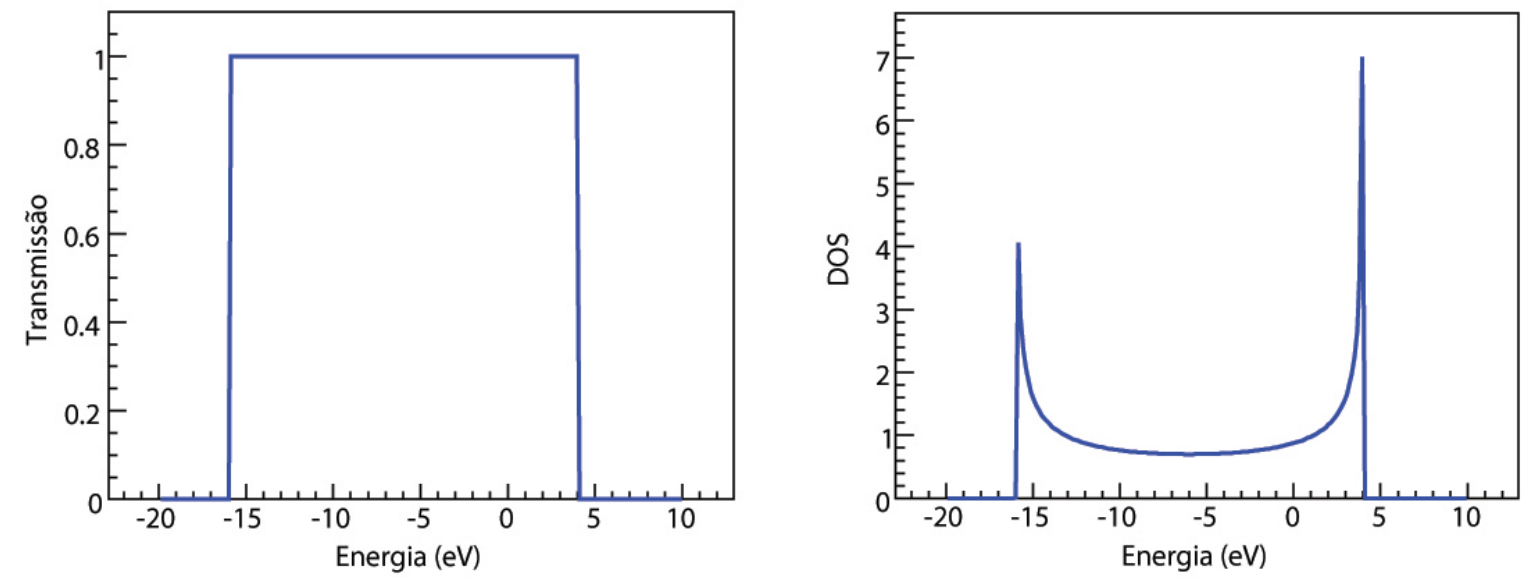

Figura 5.5: Transmissão (esquerda) e densidade de estados (direita) para um sistema no equilíbrio.

O passo seguinte foi implementar o formalismo NEGF. Conforme discutido acima (Seção 5.5.3), no caso particular de apenas um sítio na região de espalhamento, com acoplamentos e energias de sítio iguais, é possível calcular a corrente através de expressões analíticas a fim de comparar com os valores obtidos com o formalismo NEGF. Na Figura 5.6. podem ser observados os resultados obtidos de corrente por tensão aplicada, onde o gráfico à esquerda corresponde ao cálculo por meio do formalismo NEGF e o gráfico à direita, ao cálculo por meio das expressões analíticas.
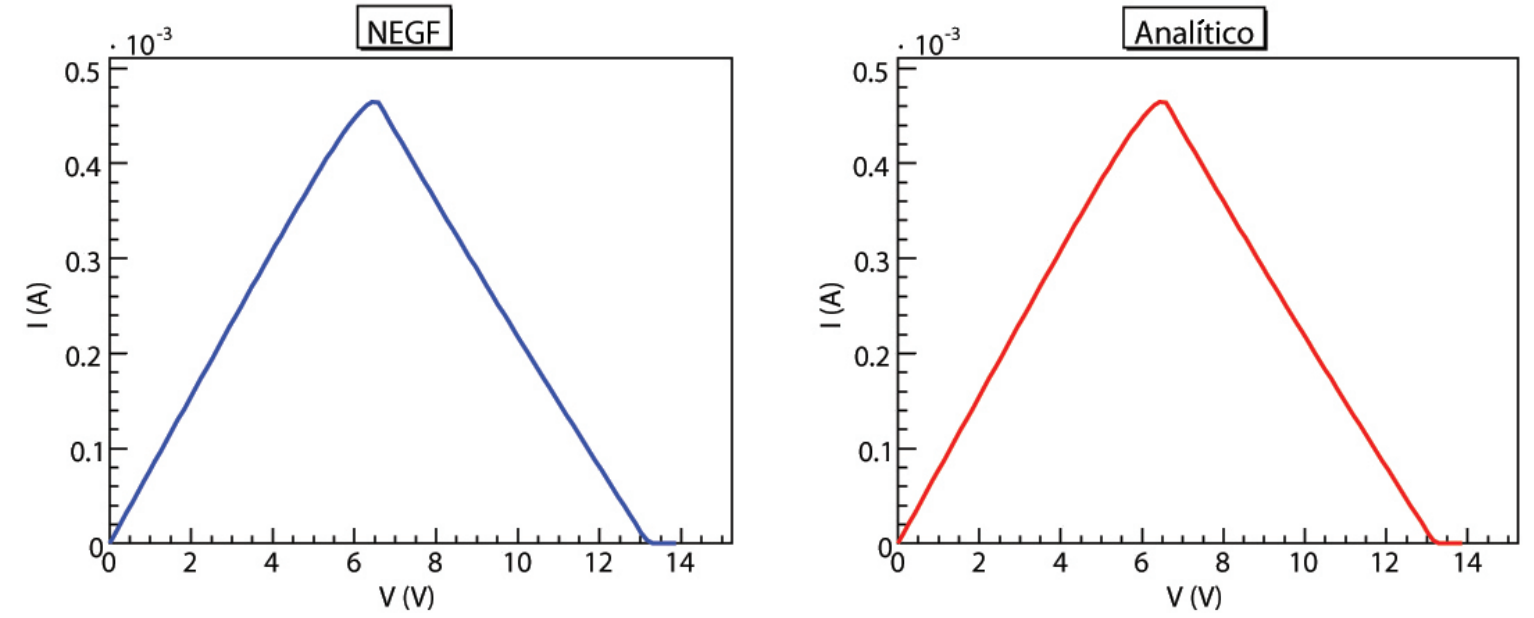

Figura 5.6: Corrente por tensão aplicada calculada por meio do formalismo NEGF (esquerda) e por expressões analíticas (direita). 
Nota-se que os resultados obtido pelos dois métodos são equivalentes, verificando-se a consistência da implementação do formalismo NEGF.

O modelo fenomenológico das sondas de Büttiker foi incorporado ao programa de duas formas, primeiro com a aproximação elástica (Seção 5.3) e segundo com a nova proposta apresentada na Seção 5.4 para o transporte inelástico não-coerente.

Na Figura 5.7 estão apresentados os resultados de corrente por tensão aplicada (acima) e a condutância diferencial (meio), para um sistema a $T=10 \mathrm{~K}$ com apenas um sítio na região de espalhamento e com intensidade de defasagem $\beta=0,05 \mathrm{eV}$. Os gráficos à esquerda foram calculados com $U_{0}=0,0$ (i.e. considera-se $H_{M}=H_{0}$ na Equação 5.22 e à direita com $U_{0}=1,0$. Também estão dispostos na parte inferior da Figura 5.7 a energia de sítio da região de espalhamento $\varepsilon_{M}$ e os potenciais eletroquímicos dos eletrodos e da sonda $\left(\mu_{L}, \mu_{R}\right.$ e $\mu_{p}$, respectivamente) obtidos por meio da proposta inelástica para a defasagem. As energias e acoplamentos de entrada utilizados estão dispostos na Tabela 5.1 .

\begin{tabular}{ccccccccc}
\hline$\varepsilon_{F}$ & $\varepsilon_{L}$ & $\varepsilon_{M}$ & $\varepsilon_{R}$ & $\gamma_{L}$ & $\gamma_{M L}$ & $\gamma_{M}$ & $\gamma_{M R}$ & $\gamma_{R}$ \\
\hline 0,5 & 0,0 & 1,0 & 0,0 & 5,0 & 0,1 & 0,5 & 0,1 & 5,0 \\
\hline
\end{tabular}

Tabela 5.1: Energias e acoplamentos, em eV, utilizados nos cálculos da Figura 5.7

Nota-se que, para este sistema, os efeitos de perda de coerência de fase pelos dois métodos são equivalentes, o que confirma que a aproximação elástica preserva a quebra de fase. No caso em que $U_{0}=0,0$ (gráficos à esquerda da Figura 5.7), nota-se que, ao incorporar a defasagem, ocorre um alargamento e uma redução à metade na intensidade da condutância diferencial no canal para uma tensão de $1 \mathrm{eV}$. Esse alargamento do canal, que pode ser observado tanto no gráfico de corrente como no da condutância, era esperado, pois o termo de autoenergia de espalhamento introduzido na função de Green devido à sonda é um número complexo (Equação 5.7). Ao mesmo tempo, por não haver uma parte real (ele é, por construção, um imaginário puro) a posição do pico permanece a mesma e corresponde aproximadamente à posição do nível do sítio central $\varepsilon_{M}$.

Observa-se também que, ao desprezar a contribuição do campo autoconsistente $\left(U_{0}=0,0\right)$, a corrente calculada sem defasagem cresce abruptamente quando a tensão aplicada atinge $1 \mathrm{~V}$. Ou seja, como o acoplamento entre o eletrodo esquerdo e a região de espalhamento é fraco $\left(\gamma_{M L}=0,1\right.$ eV), o sítio na região de espalhamento começa a ser ocupado pelos elétrons do eletrodo esquerdo somente quando $\varepsilon_{M}-\mu_{L}<0,05 \mathrm{eV}=\gamma_{M L} / 2$ (ver Figura 5.8). 

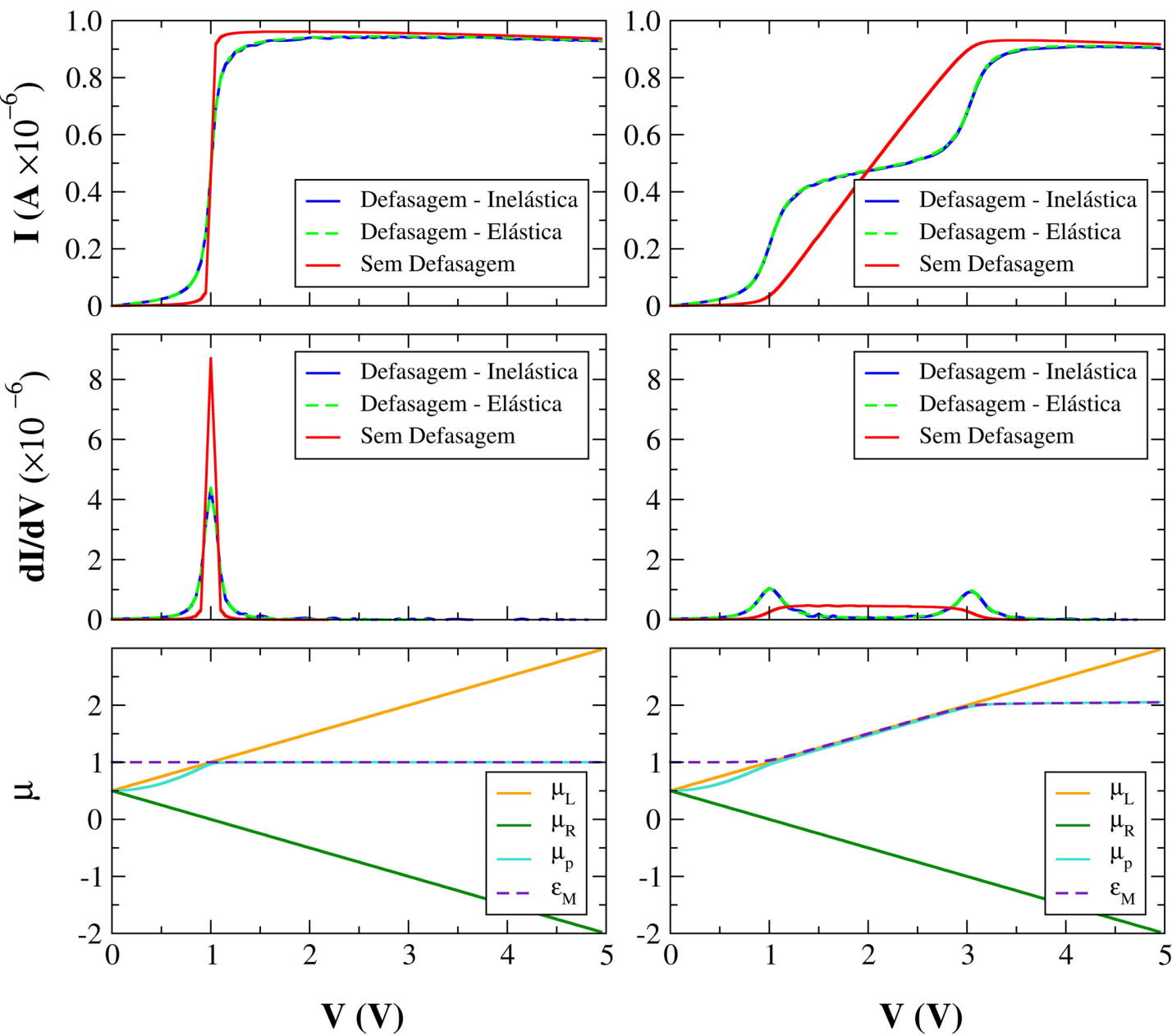

Figura 5.7: Corrente por tensão aplicada (acima), condutância diferencial (meio), potenciais eletroquímicos dos eletrodos e da sonda e energia de sítio da molécula (abaixo), para um sistema com um sítio na região de espalhamento e onde foram utilizados os parâmetros $T=10 \mathrm{~K}, \beta=0,05 \mathrm{eV}, U_{0}=0,0$ (esquerda), $U_{0}=1,0$ (direita) e as energias e acoplamentos da Tabela 5.1

Quando o potencial eletrostático e as interações elétron-elétron na região de espalhamento são considerados com $U_{0}=1,0$ (gráficos à direita da Figura 5.7), a corrente sem defasagem também surge somente quando a tensão se aproxima de $1 \mathrm{~V}$, porém cresce linearmente até a tensão alcançar $3 \mathrm{~V}$. A largura do intervalo corresponde ao dobro da constante $U_{0}$ utilizada, neste caso $2 \times U_{0}=2 \mathrm{eV}$. Devido à contribuição do campo autoconsistente ao Hamiltoniano não-perturbado, o nível de energia do sítio na região de espalhamento acompanha o potencial eletroquímico do eletrodo esquerdo (gráfico inferior à direita da Figura 5.7), o que faz com que este sítio possua uma ocupação fracionária ${ }^{6}$ que aumenta linearmente até atingir o valor 1.

\footnotetext{
${ }^{6}$ A ocupação no sítio central para o caso elástico neste modelo simples pode ser vista como uma média ponderada devido ao acoplamento em equilíbrio com o eletrodo esquerdo ou direito separadamente.
} 


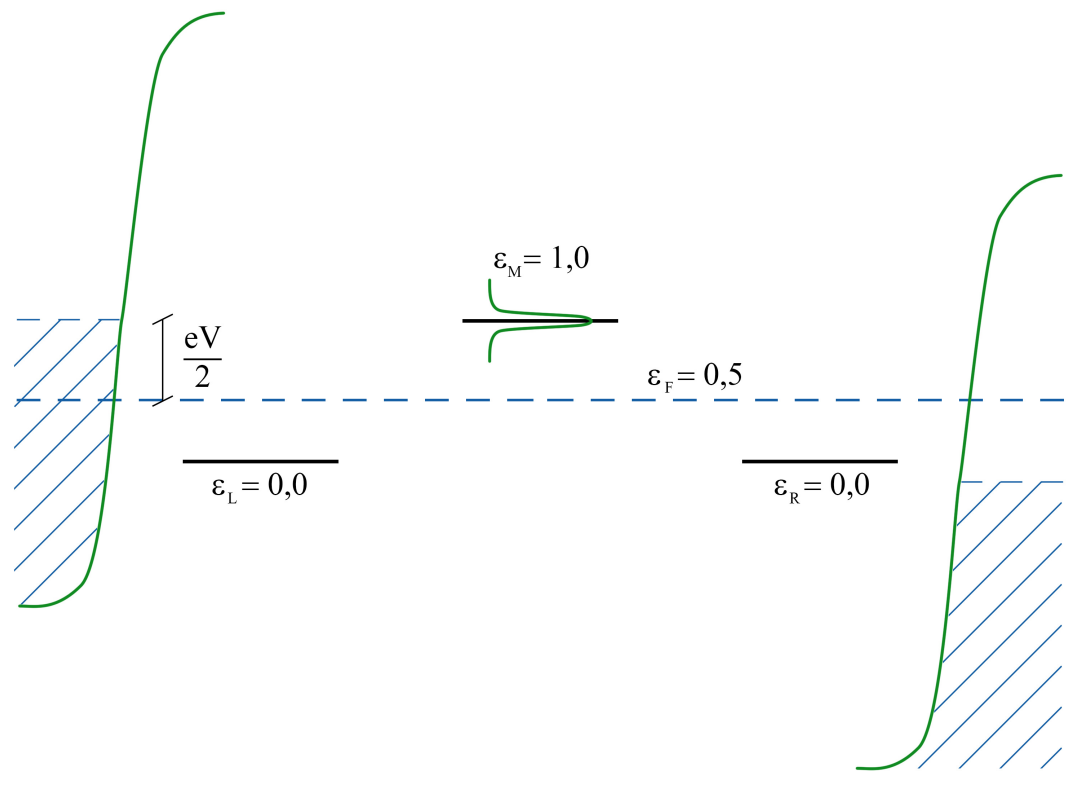

Figura 5.8: Representação do sistema na situação em que $\varepsilon_{M}-\mu_{L}<0,05 \mathrm{eV}=\gamma_{M L} / 2$, onde se observa o acoplamento fraco entre os eletrodos e a região de espalhamento (largura estreita do nivel na região de espalhamento) e que a diferença de potencial desloca a banda dos eletrodos de eV/2.

Um efeito interessante ocorre no cálculo da corrente com defasagem quando a contribuição do campo autoconsistente é considerada $\left(U_{0}=1,0\right)$. De maneira análoga ao que foi discutido nos parágrafos anteriores, há um onset de corrente quando a tensão se aproxima de 1 V. Porém, esta atinge um patamar que perdura até a tensão de $3 \mathrm{~V}$, onde a corrente praticamente dobra de valor.

Esse efeito está relacionado ao deslocamento do nível de energia devido à contribuição do campo autoconsistente. Tanto neste caso como no anterior, $U_{0}=0,0$, o potencial eletroquímico da sonda adquire o mesmo valor da energia do sítio $\varepsilon_{M}$ a partir de $V=1 \mathrm{~V}$. Porém, quando o potencial é considerado, a energia de sítio e o potencial eletroquímico da sonda se alinham com o potencial eletroquímico do eletrodo esquerdo.

Esta combinação de efeitos gera a estrutura observada na condutância diferencial. Contudo, o que se observa é, na verdade, uma propriedade do modelo das sondas de Büttiker e, não, um fenômeno físico. De fato, o aparecimento dessas estruturas indica que a sonda está influenciando no cálculo da recombinação de carga, ainda que a sonda não introduza ou retire elétrons do sistema.

O mesmo sistema da Figura 5.7 foi simulado à temperatura $T=300 K$. Os resultados desta simulação estão apresentados na Figura 5.9, onde novamente os gráficos à esquerda foram calculados com $U_{0}=0,0$ e à direita com $U_{0}=1,0$. 

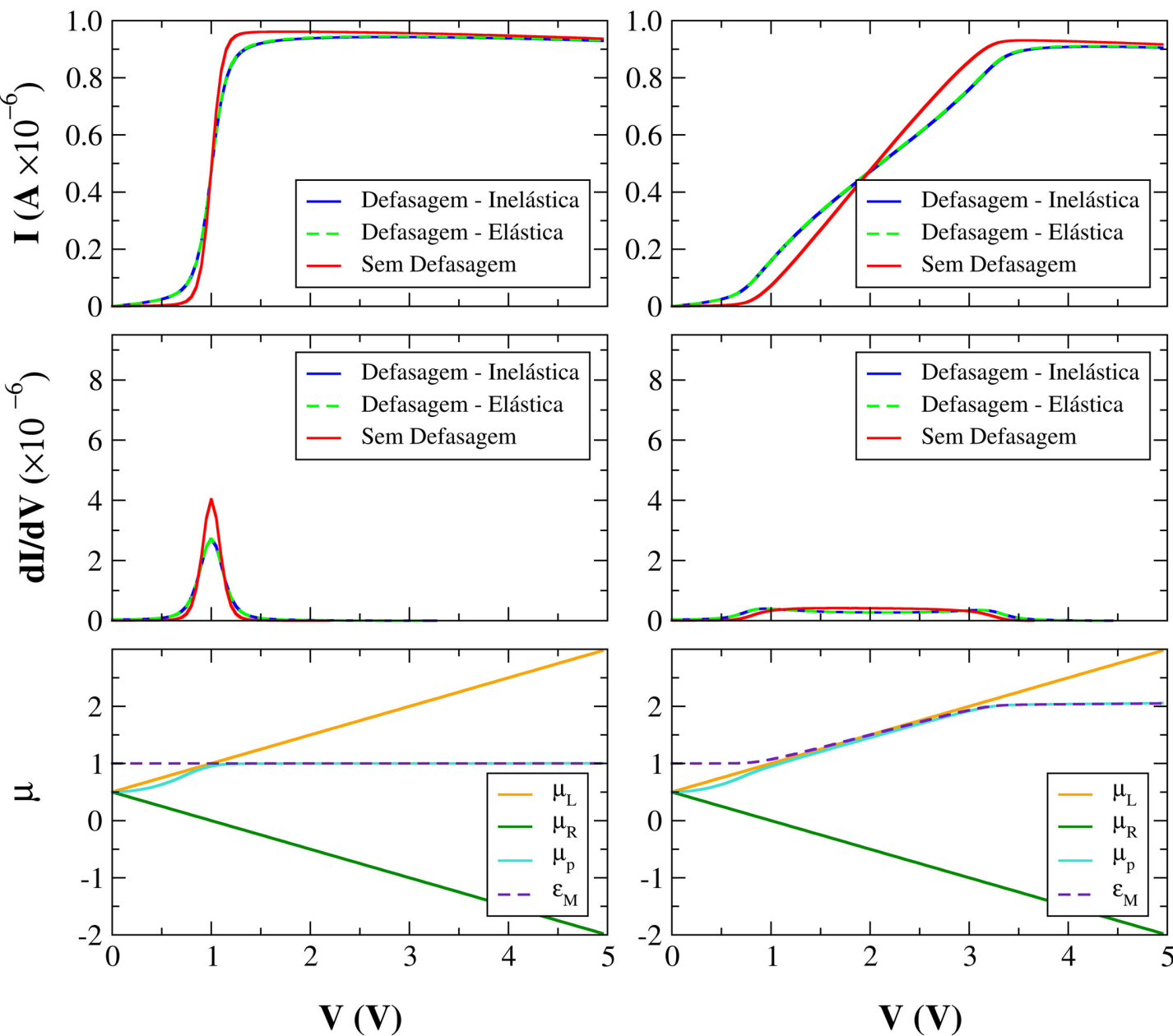

Figura 5.9: Corrente por tensão aplicada (acima), condutância diferencial (meio), potenciais eletroquímicos dos eletrodos e da sonda e energia de sítio da molécula (abaixo), para um sistema com um sítio na região de espalhamento e onde foram utilizados os parâmetros $T=300 \mathrm{~K}, \beta=0,05 \mathrm{eV}, U_{0}=0,0$ (esquerda), $U_{0}=1,0$ (direita) e as energias e acoplamentos da Tabela 5.1 .

Como o efeito da temperatura entra somente nas distribuições de Fermi-Dirac dos eletrodos utilizadas nos cálculos da densidade de cargas e da corrente, o aumento da temperatura produz um alargamento do canal. Grosso modo, o aumento da temperatura faz com que o trecho de ocupação fracionária do sítio na região de espalhamento seja maior.

O alargamento do canal também pode ser obtido aumentando-se o acoplamento entre os eletrodos e a região de espalhamento, $\gamma_{M L}$ e $\gamma_{M R}$. Na Figura 5.10 são apresentados os resultados obtidos para um sistema a $T=10 K$ com três sítios na região de espalhamento. A intensidade de defasagem $\beta=0,05 \mathrm{eV}$ e as energias de sítio utilizadas são as mesmas do exemplo anterior, mas os acoplamentos da molécula com os eletrodos são superiores, como pode ser verificado na Tabela 5.2, 


\begin{tabular}{ccccccccc}
\hline$\varepsilon_{F}$ & $\varepsilon_{L}$ & $\varepsilon_{M}$ & $\varepsilon_{R}$ & $\gamma_{L}$ & $\gamma_{M L}$ & $\gamma_{M}$ & $\gamma_{M R}$ & $\gamma_{R}$ \\
\hline 0,5 & 0,0 & 1,0 & 0,0 & 5,0 & 2,0 & 1,5 & 2,0 & 5,0 \\
\hline
\end{tabular}

Tabela 5.2: Energias e acoplamentos, em eV, utilizados nos cálculos da Figura 5.10.
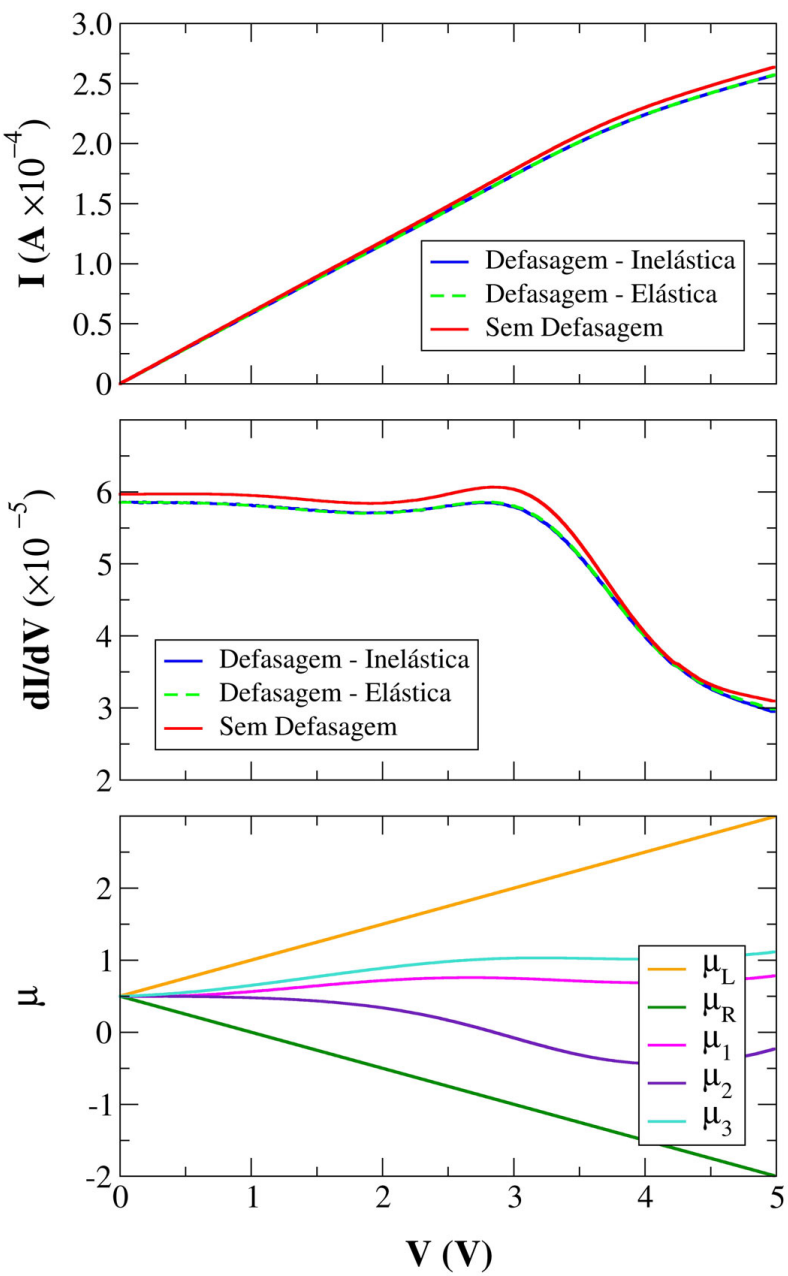

Figura 5.10: Corrente por tensão aplicada (acima), condutância diferencial (meio) e potenciais eletroquímicos dos eletrodos e sondas (abaixo), para um sistema com três sítios na região de espalhamento e onde foram utilizados os parâmetros $T=10 \mathrm{~K}, U_{0}=1,0, \beta=0,05 \mathrm{eV}$ e as energias e acoplamentos da Tabela 5.2

Nota-se que quando os acoplamentos são suficientemente fortes (maiores do que a diferença entre as energias de sítio da região de espalhamento $\varepsilon_{M}$ e os potenciais eletroquímicos dos eletrodos $\mu_{L}$ e $\mu_{R}$ ), devido ao efeito de alargamento dos níveis, a corrente passa a ser diferente de zero já nos valores iniciais de tensão. 
O parâmetro fenomenológico $\beta$ está relacionado à intensidade do acoplamento elétron-fônon, ou seja, aumentando-se seu valor aumenta-se o acoplamento das sondas à região de espalhamento. Na Figura 5.11 são apresentados os resultados da simulação de um sistema a $T=10 \mathrm{~K}$ com apenas um sítio na região de espalhamento e com intensidade de defasagem $\beta=0,11 \mathrm{eV}$, onde novamente os gráficos à esquerda foram calculados com $U_{0}=0,0$ e à direita com $U_{0}=1,0$. As energias e acoplamentos de entrada utilizados foram os mesmos da Tabela 5.1.
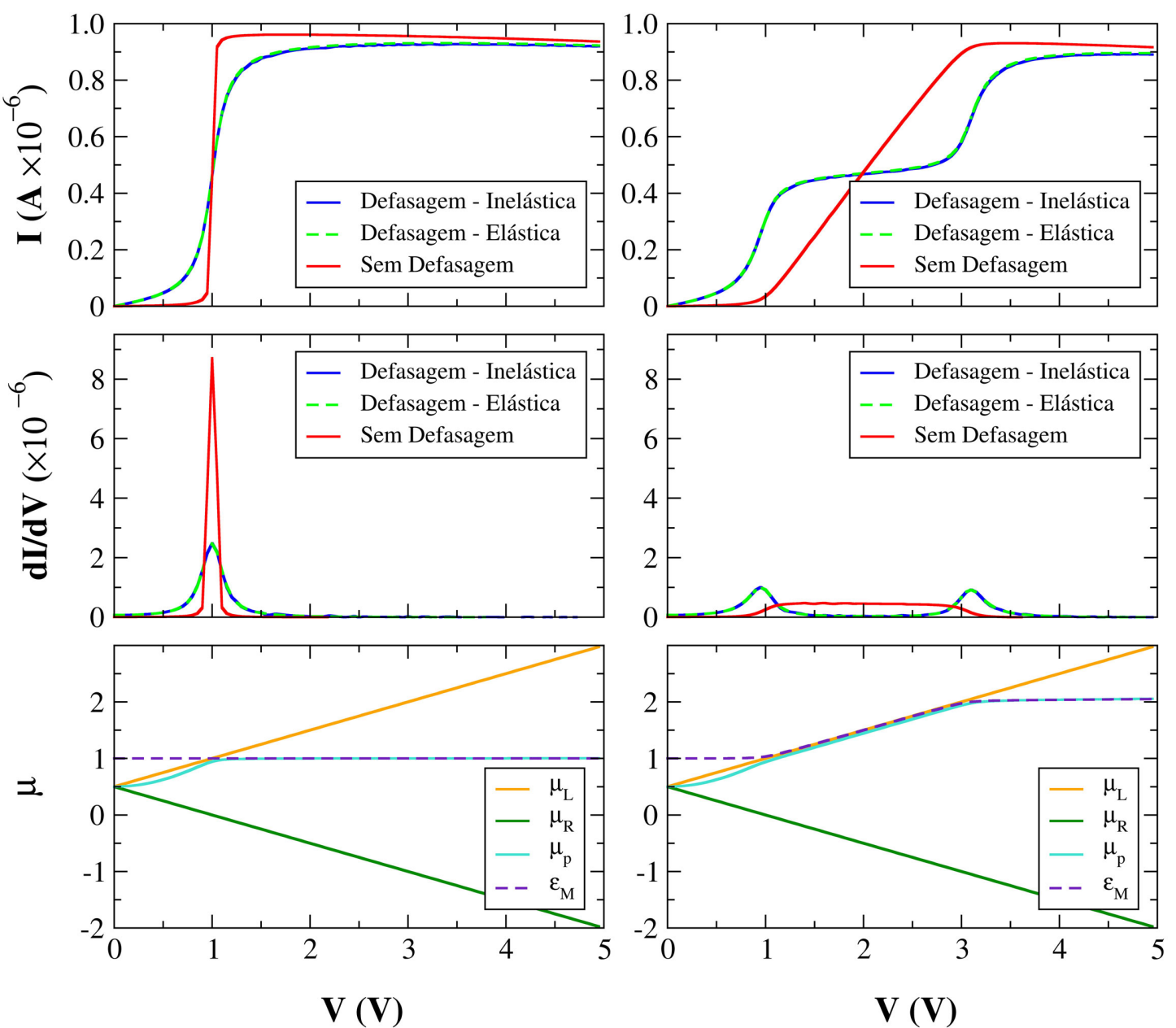

Figura 5.11: Corrente por tensão aplicada (acima), condutância diferencial (meio), potenciais eletroquímicos dos eletrodos e da sonda e energia de sítio da molécula (abaixo), para um sistema com um sítio na região de espalhamento e onde foram utilizados os parâmetros $T=10 \mathrm{~K}, \beta=0,11 \mathrm{eV}, U_{0}=0,0$ (esquerda), $U_{0}=1,0$ (direita) e as energias e acoplamentos da Tabela 5.1 .

Comparando-se os resultados obtidos com $\beta=0,11 \mathrm{eV}$ na Figura 5.11 com os resultados obtidos com $\beta=0,05 \mathrm{eV}$ na Figura 5.7, nota-se que o aumento do parâmetro $\beta$ produz somente um alargamento do canal. Isso era esperado, já que o termo de autoenergia de espalhamento introduzido na função de Green devido à sonda é diretamente proporcional ao parâmetro $\beta$ e é 
um número imaginário puro (Equação 5.7).

Como conclusão, podemos dizer que a metodologia de sondas de Büttiker permite introduzir efeitos de perda de coerência de fase na corrente por meio de um parâmetro fenomenológico $\beta$. O efeito deste parâmetro é introduzir um alargamento dos níveis moleculares ligados aos eletrodos.

Consequentemente, em geral se observa um onset da corrente anterior ao caso elástico e um valor terminal inferior. Ao mesmo tempo, efeitos espúrios não relacionados à estrutura da interação surgem na corrente.

Fica claro que, no modelo fenomenológico de Büttiker, é ignorado qualquer efeito que esteja relacionado à quantização dos fônons, sua estrutura e informações - além de um parâmetro fenomenológico de interação. Para tratar o problema com estas condições é necessário uma metodologia que inclua tais informações. 


\section{Capítulo 6}

\section{Aproximação de Born}

No modelo fenomenológico das sondas de Büttiker, apresentado no capítulo anterior, a autoenergia da interação utilizada produz um alargamento dos níveis de condutância, simulando o efeito de perda de coerência de fase dos elétrons devido à interação com fônons do material. Contudo, o modelo não inclui outros efeitos característicos desta interação, como abertura de novos canais de condutância e supressão de canais puramente elásticos, visto que a autoenergia utilizada não contém informações a respeito da estrutura dos fônons.

Outra abordagem, que veremos neste capítulo, consiste em considerar o acoplamento elétronfônon relativamente pequeno, de forma que a autoenergia possa ser aproximada por meio de métodos perturbativos. Dentro desta linha de raciocínio, uma proposta vem a ser expandir a autoenergia de interação em termos de diagramas de Feynman até a primeira ordem (ver Seção 2.7], o que é convencionalmente chamado de primeira aproximação de Born [8] (First Born Approximation - 1BA).

Neste trabalho foram implementadas a primeira aproximação de Born assim como a sua versão autoconsistente (Self-Consistent Born Approximation - SCBA), onde uma classe mais ampla de diagramas de Feynman é considerada. Essas implementações foram incorporadas ao programa SMEAGOL (Seção 4.6), o que deu origem ao código Inelastic SMEAGOL para cálculos de transporte inelástico $a b$ initio.

Um dos resultados importantes deste trabalho foi a reformulação da expressão usualmente utilizada para o cálculo da autoenergia retardada na 1BA [8,78, 96], o que diminui drasticamente o custo computacional do cálculo, viabilizando o uso desta aproximação na simulação de sistemas com uma quantidade relativamente grande de átomos. 


\subsection{Primeira Aproximação de Born - 1BA}

Para que mais efeitos característicos da interação elétron-fônon sejam observados nas simulações (tais como abertura, supressão e deslocamento de canais de condução), o modelo utilizado deve conter o espectro vibracional $\omega_{\lambda}$ e a matriz de acoplamento $M_{\mathbf{q}, \lambda}$ como ingredientes. Este é o caso da Primeira Aproximação de Born, apresentada a seguir.

Vamos continuar considerando que a região de espalhamento está acoplada a um reservatório de fônons em equilíbrio termodinâmico. Desta forma, os fônons podem ser descritos como partículas livres (Equação 5.3) e suas funções de Green são dadas pelas expressões analíticas nas Equações 2.86-2.89,

Conforme vimos no Capítulo 2, quando elétrons e fônons interagem segundo o Hamiltoniano da Equação 5.4 , podemos expandir diagramaticamente a função de Green de partícula única ordenada no contorno, na representação de interação, por meio do Teorema de Wick (Seção 2.7), onde somente os diagramas conexos precisam ser considerados (Figura 6.1).

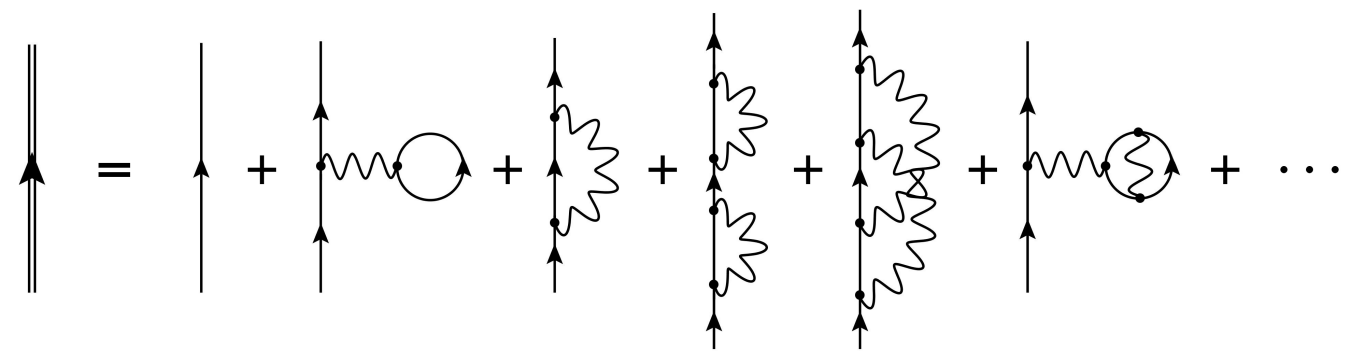

Figura 6.1: Expansão perturbativa da função de Green eletrônica em um sistema com interação elétronfônon, onde as funções de Green são representadas por linha dupla para elétron interagente, linha simples para elétron livre e linha ondulada para fônon livre.

No entanto, como existem infinitos diagramas deste tipo, é necessário truncar a expansão. A Primeira Aproximação de Born (1BA) consiste em considerar somente os termos de primeira ordem na expansão da função de Green eletrônica (dois primeiros diagramas da expansão na Figura 6.1 e desconsiderar interações elétron-elétron 8, 97. 1

Por analogia com o tratamento de interações elétron-elétron, os dois diagramas considerados na Figura 6.2 são chamados, da esquerda para direita, tipo-Hartree e tipo-Fock. Segundo P. Hyldgaard et al [97], a inclusão do diagrama de Hartree é essencial para obter-se uma descrição correta em sistemas sem simetria de invariância translacional. Aplicando as regras de Feynman nesse diagrama (Seção 2.7), nota-se que a energia e o momento ao longo da linha de interação

\footnotetext{
${ }^{1}$ Novamente, as interações elétron-elétron podem estar implícitas numa aproximação de campo médio.
} 


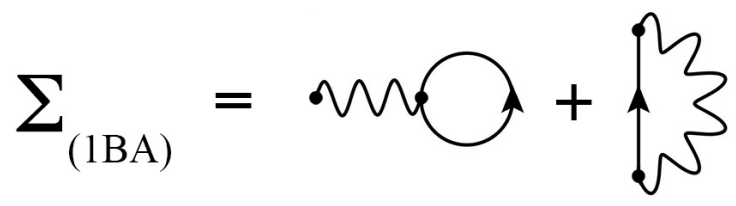

Figura 6.2: Representação da Primeira Aproximação de Born com diagramas de Feynman, onde se identificam os diagramas tipo-Hartree à esquerda e tipo-Fock à direita.

devem ser nulos por conservação, enquanto que no loop fermiônico a energia e o momento, assim como o spin, podem tomar qualquer valor. Considerando o fator $(-1)$ devido ao loop fermiônico e somando sobre todas as variáveis, obtém-se:

$$
\Sigma^{H}(\sigma)=-i \hbar \sum_{\lambda} \sum_{\sigma^{\prime}} M_{\lambda} D_{0}(\lambda, \epsilon=0) \operatorname{Tr}\left[G_{0}^{<}\left(\sigma^{\prime}, 0,0\right) M_{\lambda}\right]
$$

onde $M_{\lambda}=M_{\mathbf{q}, \lambda}$ é a matriz de acoplamento elétron-fônon do modo $\lambda, \sigma^{\prime}$ corresponde aos valores de spin do elétron ${ }^{2}, G_{0}$ e $D_{0}$ são as funções de Green para elétrons e para fônons, respectivamente, calculadas na ausência de interação $\left(M_{\lambda}=\mathbb{0}\right)$. Neste trabalho, os fônons foram considerados no equilíbrio termodinâmico, de modo que as funções de Green $D_{0}$ são dadas pelas expressões analíticas obtidas na Seção 2.2 .

Para o diagrama de Hartree, a continuação analítica (Seção 2.8) é imediata:

$$
\begin{aligned}
& \Sigma^{H, r}(\sigma)=-i \hbar \sum_{\lambda} \sum_{\sigma^{\prime}} M_{\lambda} D_{0}^{r}(\lambda, \epsilon=0) \operatorname{Tr}\left[G_{0}^{<}\left(\sigma^{\prime}, 0,0^{+}\right) M_{\lambda}\right], \\
& \Sigma^{H, \lessgtr}(\sigma)=-i \hbar \sum_{\lambda} \sum_{\sigma^{\prime}} M_{\lambda} D_{0}^{\lessgtr}(\lambda, \epsilon=0) \operatorname{Tr}\left[G_{0}^{<}\left(\sigma^{\prime}, 0,0^{+}\right) M_{\lambda}\right] .
\end{aligned}
$$

Substituindo as funções de Green dos fônons por suas expressões analíticas (Equações 2.862 .89 e tomando a transformada de Fourier, obtém-se:

\footnotetext{
${ }^{2} \mathrm{Na}$ descrição do formalismo NEGF (Capítulo 2), o índice correspondente ao spin foi omitido, porém sua inclusão nas equações é imediata. Como não estamos impondo conservação de momento, a função de Green dos fônons fica determinada somente pelo índice $\lambda$ sob os modos vibracionais, sendo omitidos os índices q. Também serão omitidos, desta parte em diante, os índices $M$ nas funções de Green, ficando subentendido que se tratam da região de espalhamento.
} 


$$
\begin{aligned}
& \Sigma^{H, r}(\sigma)=i \sum_{\lambda} \sum_{\sigma^{\prime}} \frac{2}{\hbar \omega_{\lambda}} \int_{-\infty}^{+\infty} \frac{d \epsilon^{\prime}}{2 \pi} M_{\lambda} \operatorname{Tr}\left[G_{0}^{<}\left(\sigma^{\prime}, \epsilon^{\prime}\right) M_{\lambda}\right], \\
& \Sigma^{H, \lessgtr}(\sigma)=0 .
\end{aligned}
$$

Nota-se que as expressões obtidas independem da energia, gerando somente uma contribuição constante para a autoenergia total, e é possível mostrar que esta contribuição vai a zero em sistemas com simetria translacional [98]. Verifica-se também a semelhança entre esta autoenergia e a matriz densidade (Equação 4.47), de modo que se pode utilizar a mesma técnica para calculála (ver Seção B.1).

Diferentemente do diagrama de Hartree, ao aplicar as regras de Feynman ao diagrama de Fock, verifica-se que o spin interno $\sigma^{\prime}$ é obrigado a ser igual ao spin externo $\sigma$ e que não há loop fermiônico, de forma que sua expressão fica sendo:

$$
\Sigma^{F}\left(\sigma, \tau, \tau^{\prime}\right)=i \hbar \sum_{\lambda} M_{\lambda} D_{0}\left(\lambda, \tau, \tau^{\prime}\right) G_{0}\left(\sigma, \tau, \tau^{\prime}\right) M_{\lambda}
$$

Utilizando as regras de Langreth dadas pelas Equações 2.147 e 2.145, a continuação analítica fica:

$$
\begin{aligned}
& \Sigma^{F, r}\left(\sigma, t, t^{\prime}\right)=i \hbar \sum_{\lambda} M_{\lambda}[ D_{0}^{r}\left(\lambda, t, t^{\prime}\right) G_{0}^{<}\left(\sigma, t, t^{\prime}\right)+ \\
&\left.+D_{0}^{r}\left(\lambda, t, t^{\prime}\right) G_{0}^{r}\left(\sigma, t, t^{\prime}\right)+D_{0}^{<}\left(\lambda, t, t^{\prime}\right) G_{0}^{r}\left(\sigma, t, t^{\prime}\right)\right] M_{\lambda}, \\
& \Sigma^{F, \lessgtr}\left(\sigma, t, t^{\prime}\right)=i \hbar \sum_{\lambda} M_{\lambda} D_{0}^{\lessgtr}\left(\lambda, t, t^{\prime}\right) G_{0}^{\lessgtr}\left(\sigma, t, t^{\prime}\right) M_{\lambda} .
\end{aligned}
$$

Tomando a transformada de Fourier (estado estacionário), obtém-se:

$$
\begin{aligned}
& \Sigma^{F, r}(\sigma, \epsilon)=i \sum_{\lambda} \int_{-\infty}^{+\infty} \frac{d \epsilon^{\prime}}{2 \pi} M_{\lambda}\left[D_{0}^{r}\left(\lambda, \epsilon-\epsilon^{\prime}\right) G_{0}^{<}\left(\sigma, \epsilon^{\prime}\right)+\right. \\
& \left.\quad+D_{0}^{r}\left(\lambda, \epsilon-\epsilon^{\prime}\right) G_{0}^{r}\left(\sigma, \epsilon^{\prime}\right)+D_{0}^{<}\left(\lambda, \epsilon-\epsilon^{\prime}\right) G_{0}^{r}\left(\sigma, \epsilon^{\prime}\right)\right] M_{\lambda}, \\
& \Sigma^{F, \lessgtr}(\sigma, \epsilon)=i \sum_{\lambda} \int_{-\infty}^{+\infty} \frac{d \epsilon^{\prime}}{2 \pi} M_{\lambda} D_{0}^{\lessgtr}\left(\lambda, \epsilon-\epsilon^{\prime}\right) G_{0}^{\lessgtr}\left(\sigma, \epsilon^{\prime}\right) M_{\lambda} .
\end{aligned}
$$

Substituindo as funções de Green dos fônons não-interagentes por suas expressões analíticas (Equações 2.86 2.89), as autoenergias lesser e greater do diagrama de Fock podem ser escritas 
em termos das funções de Green eletrônica retardada e avançada como:

$$
\begin{aligned}
& \Sigma^{F,<}(\sigma, \epsilon)=i \sum_{\lambda} M_{\lambda}\left\{n_{B_{\lambda}} G_{0_{-}}^{r}\left[\Gamma_{L_{-}} f_{L_{-}}+\Gamma_{R_{-}} f_{R_{-}}\right] G_{0_{-}}^{a}+\right. \\
&\left.+\left(n_{B_{\lambda}}+1\right) G_{0_{+}}^{r}\left[\Gamma_{L_{+}} f_{L_{+}}+\Gamma_{R_{+}} f_{R_{+}}\right] G_{0_{+}}^{a}\right\} M_{\lambda}, \\
& \Sigma^{F,>}(\sigma, \epsilon)=i \sum_{\lambda} M_{\lambda}\left\{n_{B_{\lambda}} G_{0_{+}}^{r}\left[\Gamma_{L_{+}}\left(f_{L_{+}}-1\right)+\Gamma_{R_{+}}\left(f_{R_{+}}-1\right)\right] G_{0_{+}}^{a}+\right. \\
&\left.+\left(n_{B_{\lambda}}+1\right) G_{0_{-}}^{r}\left[\Gamma_{L_{-}}\left(f_{L_{-}}-1\right)+\Gamma_{R_{-}}\left(f_{R_{-}}-1\right)\right] G_{0_{-}}^{a}\right\} M_{\lambda_{\lambda}},
\end{aligned}
$$

onde $n_{B_{\lambda}}$ é a função distribuição de Bose-Einstein (Equação 2.90 e o índice - (+) indica que a função é calculada em $\epsilon-\hbar \omega_{\lambda}\left(\epsilon+\hbar \omega_{\lambda}\right)$, sendo $\omega_{\lambda}$ a frequência do fônon de modo $\lambda$. Nota-se que as autoenergias $\Sigma^{F,<}$ e $\Sigma^{F,>}$ contêm contribuições de dois tipos de espalhamento. Na lesser (greater), elétrons com energia $\epsilon-\hbar \omega_{\lambda}$ podem absorver (emitir) um fônon com energia $\hbar \omega_{\lambda}$, e/ou elétrons com energia $\epsilon+\hbar \omega_{\lambda}$ podem emitir (absorver) um fônon com energia $\hbar \omega_{\lambda}$.

Nota-se, também, que no limite de baixas temperaturas $T \rightarrow 0$, a distribuição de BoseEinstein tende a 0 e, portanto, os elétrons só podem emitir fônons, o que é evidenciado nas expressões obtidas.

A função de Green retardada do diagrama de Fock, por sua vez, é usualmente escrita em termos das autoenergias lesser e greater da seguinte forma [78, 96:

$$
\Sigma^{F, r}(\sigma, \epsilon)=\frac{1}{2}\left[\Sigma^{F,>}(\epsilon)-\Sigma^{F,<}(\epsilon)\right]-\frac{i}{2} \mathcal{H}_{\epsilon^{\prime}}\left\{\Sigma^{F,>}\left(\epsilon^{\prime}\right)-\Sigma^{F,<}\left(\epsilon^{\prime}\right)\right\}(\epsilon),
$$

onde o funcional $\mathcal{H}_{\epsilon^{\prime}}\left\{\Sigma^{F,>}\left(\epsilon^{\prime}\right)-\Sigma^{F,<}\left(\epsilon^{\prime}\right)\right\}(\epsilon)$ é uma transformada de Hilbert definida pelo valor principal $\mathcal{P}$ da seguinte integral:

$$
\mathcal{H}_{\epsilon^{\prime}}\left\{\Sigma^{F,>}\left(\epsilon^{\prime}\right)-\Sigma^{F,<}\left(\epsilon^{\prime}\right)\right\}(\epsilon)=\frac{1}{\pi} \mathcal{P} \int_{-\infty}^{+\infty} d \epsilon^{\prime} \frac{\Sigma^{F,>}\left(\epsilon^{\prime}\right)-\Sigma^{F,<}\left(\epsilon^{\prime}\right)}{\epsilon^{\prime}-\epsilon}
$$

Esta transformada pode ser calculada numericamente em um grid equidistante de energia utilizando um algoritmo eficiente de transformada de Fourier discreta (Discret Fast Fourier Transform), tomando-se alguns cuidados, conforme descrito na Seção B.2. 


\subsection{Aproximação de Born Autoconsistente - SCBA}

Uma primeira extensão para a Primeira Aproximação de Born é considerar, nas expressões para as autoenergias (obtidas da seção anterior), as funções de Green eletrônicas completas:

$$
\begin{gathered}
G^{r}(\sigma, \epsilon)=\left[\varepsilon S-H(\sigma)-\Sigma_{L}^{r}(\sigma, \epsilon)-\Sigma_{R}^{r}(\sigma, \epsilon)-\Sigma^{H, r}(\sigma, \epsilon)-\Sigma^{F, r}(\sigma, \epsilon)\right]^{-1}, \\
G^{\lessgtr}(\sigma, \epsilon)=G^{r}(\sigma, \epsilon)\left[\Sigma_{L}^{\lessgtr}(\sigma, \epsilon)+\Sigma_{R}^{\lessgtr}(\sigma, \epsilon)+\Sigma^{F, \lessgtr}(\sigma, \epsilon)\right] G^{a}(\sigma, \epsilon) .
\end{gathered}
$$

Esse método é chamado de Aproximação de Born Autoconsistente (SCBA), pois, como $G^{r}$ depende das autoenergias de interação elétron-fônon $\left(\Sigma^{H, r}\right.$ e $\left.\Sigma^{F, r}\right)$ e estas, por sua vez, dependem da função de Green $\left(G^{r}\right)$, o cálculo tem que ser realizado de maneira autoconsistente.

Os diagramas tipo-Hartree e tipo-Fock nesta aproximação estão dispostos na Figura 6.3 . onde as linhas duplas representam as funções de Green eletrônicas interagentes, ampliando-se a classe de diagramas utilizados.

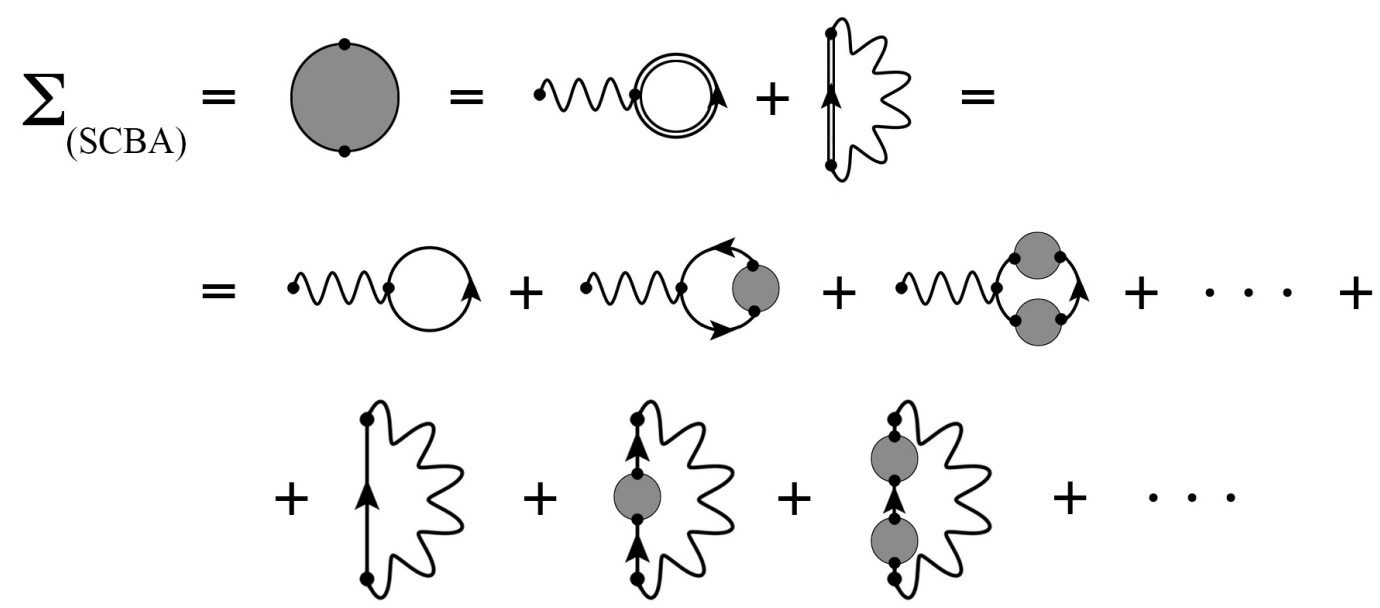

Figura 6.3: Representação da Aproximação de Born Autoconsistente com diagramas de Feynman.

\subsection{Reformulação da Primeira Aproximação de Born}

Do ponto de vista computacional, o cálculo da Equação 6.13 é extremamente custoso devido ao caráter não-local da transformada de Hilbert, isto é, o termo $\Sigma^{F,>}-\Sigma^{F,<}$ tem que ser calculado em todo o espectro de energia para realizar a integração. Isso inviabiliza a simulação de sistemas maiores do que algumas dezenas de átomos. Nesta seção, será apresentada uma nova abordagem que permite o cálculo da autoenergia de maneira mais eficiente e para uma quantidade bem maior de átomos. 
Voltando à expressão para a autoenergia retardada de Fock (Equação 6.9) e substituindo as funções de Green não interagentes dos fônons por suas expressões analíticas (Equações 2.86 e 2.88):

$$
\begin{aligned}
\Sigma^{F, r}(\sigma, \epsilon)= & -i \sum_{\lambda} M_{\lambda}\left\{\lim _{\eta \rightarrow 0^{+}} \int_{-\infty}^{+\infty} \frac{d \epsilon^{\prime}}{2 \pi}\left(G_{0}^{<}\left(\sigma, \epsilon^{\prime}\right)+G_{0}^{r}\left(\sigma, \epsilon^{\prime}\right)\right)\right. \\
& \left.\cdot\left[\frac{1}{\epsilon^{\prime}-\left(\epsilon-\hbar \omega_{\lambda}\right)-i \eta}-\frac{1}{\epsilon^{\prime}-\left(\epsilon+\hbar \omega_{\lambda}\right)-i \eta}\right]\right\} M_{\lambda}+ \\
& +\sum_{\lambda} M_{\lambda}\left\{\left(n_{B}\left(\hbar \omega_{\lambda}\right)+1\right) G_{0}^{r}\left(\sigma, \epsilon+\hbar \omega_{\lambda}\right)+n_{B}\left(\hbar \omega_{\lambda}\right) G_{0}^{r}\left(\sigma, \epsilon-\hbar \omega_{\lambda}\right)\right\} M_{\lambda} .
\end{aligned}
$$

A fim de evitar que as equações fiquem muito carregadas nos próximos passos da derivação, os argumentos das funções serão omitidos e adotaremos o índice - $(+)$ para indicar que a função é calculada em $\epsilon-\hbar \omega_{\lambda}\left(\epsilon+\hbar \omega_{\lambda}\right)$. Somando e subtraindo $i f_{L} G_{0}^{r} \Gamma_{R} G_{0}^{a}$ na expressão para função de Green lesser não-interagente (Equação 4.32), obtém-se:

$$
\begin{aligned}
G_{0}^{<} & =i f_{L} G_{0}^{r} \Gamma_{L} G_{0}^{a}+i f_{R} G_{0}^{r} \Gamma_{R} G_{0}^{a}+i f_{L} G_{0}^{r} \Gamma_{R} G_{0}^{a}-i f_{L} G_{0}^{r} \Gamma_{R} G_{0}^{a}= \\
& =i[G_{0}^{r} \underbrace{\left(\Gamma_{L}+\Gamma_{R}\right)}_{i\left(G_{0}^{a-1}-G_{0}^{r-1}\right)} G_{0}^{a} f_{L}+G_{0}^{r} \Gamma_{R} G_{0}^{a}\left(f_{R}-f_{L}\right)]= \\
& =-\left(G_{0}^{r}-G_{0}^{a}\right) f_{L}+i G_{0}^{r} \Gamma_{R} G_{0}^{a}\left(f_{R}-f_{L}\right) .
\end{aligned}
$$

Substituindo a expressão obtida para $G_{0}^{<}$na Equação 6.17 .

$$
\begin{aligned}
\Sigma^{F, r}=\frac{1}{2 \pi i} \sum_{\lambda} M_{\lambda} & \{\underbrace{\{\underbrace{}_{\eta \rightarrow 0^{+}}\left[\int_{-\infty}^{+\infty} d \epsilon^{\prime}\left(\frac{G_{0}^{a} f_{L}-G_{0}^{r} f_{L}+G_{0}^{r}}{\epsilon^{\prime}-\left(\epsilon-\hbar \omega_{\lambda}\right)-i \eta}-\frac{G_{0}^{a} f_{L}-G_{0}^{r} f_{L}+G_{0}^{r}}{\epsilon^{\prime}-\left(\epsilon+\hbar \omega_{\lambda}\right)-i \eta}\right)\right]}_{\mathcal{I}}+ \\
& \underbrace{+2 \pi i\left[\left(n_{B_{\lambda}}+1\right) G_{0_{+}}^{r}+n_{B_{\lambda}} G_{0_{-}}^{r}\right]}_{\mathcal{I} \mathcal{I}}+ \\
& \underbrace{\left.\lim _{L}\right)}_{\mathcal{I} \mathcal{I I}^{+\infty} \lim _{\eta \rightarrow 0^{+}} \int_{-\infty}^{+\infty} d \epsilon^{\prime}\left[\frac{G_{0}^{r} \Gamma_{R} G_{0}^{a}}{\epsilon^{\prime}-\left(\epsilon-\hbar \omega_{\lambda}\right)-i \eta}-\frac{G_{0}^{r} \Gamma_{R} G_{0}^{a}}{\epsilon^{\prime}-\left(\epsilon+\hbar \omega_{\lambda}\right)-i \eta}\right]\left(f_{R}-f_{L}\right)}\} M_{\lambda},
\end{aligned}
$$


onde podemos identificar as duas primeiras linhas $(\mathcal{I}$ e $\mathcal{I} \mathcal{I})$ como a parte correspondente ao equilíbrio (apenas conectado a um dos eletrodos, neste caso o esquerdo) e a terceira linha $(\mathcal{I} \mathcal{I} \mathcal{I}$ ) como a contribuição fora do equilíbrio, já que o termo $\left(f_{R}-f_{L}\right)$ é diferente de zero somente para $V \neq 0$. Consideremos inicialmente a primeira linha:

$$
\begin{aligned}
\mathcal{I}=\lim _{\eta \rightarrow 0^{+}} \int_{-\infty}^{+\infty} d \epsilon^{\prime} & {\left[\frac{G_{0}^{a} f_{L}}{\epsilon^{\prime}-\left(\epsilon-\hbar \omega_{\lambda}\right)-i \eta}-\frac{G_{0}^{r} f_{L}}{\epsilon^{\prime}-\left(\epsilon-\hbar \omega_{\lambda}\right)-i \eta}+\frac{G_{0}^{r}}{\epsilon^{\prime}-\left(\epsilon-\hbar \omega_{\lambda}\right)-i \eta}-\right.} \\
& \left.-\frac{G_{0}^{a} f_{L}}{\epsilon^{\prime}-\left(\epsilon+\hbar \omega_{\lambda}\right)-i \eta}+\frac{G_{0}^{r} f_{L}}{\epsilon^{\prime}-\left(\epsilon+\hbar \omega_{\lambda}\right)-i \eta}-\frac{G_{0}^{r}}{\epsilon^{\prime}-\left(\epsilon+\hbar \omega_{\lambda}\right)-i \eta}\right] .
\end{aligned}
$$

Essas integrais podem ser calculadas em um contorno semicircular na metade superior do plano complexo conforme a Figura B.1. Se tomarmos o limite para o raio do semicírculo que define o contorno $R \rightarrow \infty$, as integrais se resumem à soma dos resíduos interiores ao contorno:

$$
\begin{aligned}
\mathcal{I}= & -2 \pi i \sum_{j}\left(\operatorname{Res}\left(z_{j}\right)_{-}\right)^{\dagger}-2 \pi i \sum_{j} \operatorname{Res}\left(z_{j}\right)_{-}-2 \pi i G_{0_{-}}^{r} f_{L_{-}}+2 \pi i G_{0_{-}}^{r}+ \\
& +2 \pi i \sum_{j}\left(\operatorname{Res}\left(z_{j}\right)_{+}\right)^{\dagger}+2 \pi i \sum_{j} \operatorname{Res}\left(z_{j}\right)_{+}+2 \pi i G_{0_{+}}^{r} f_{L_{+}}-2 \pi i G_{0_{+}}^{r},
\end{aligned}
$$

onde foram utilizadas as igualdades obtidas no Apêndice B (segunda igualdade das Equações B.7 e B.8) e onde $\operatorname{Res}\left(z_{j}\right)$ é o resíduo calculado no $j$-ésimo polo da distribuição de Fermi-Dirac do eletrodo esquerdo $f_{L}$ (ver Equação B.14):

$$
\begin{aligned}
\sum_{j} \operatorname{Res}\left(z_{j}\right)_{ \pm} & =-k_{B} T \sum_{j} \frac{G_{0}^{r}\left(z_{j}\right)}{z_{j}-\left(\epsilon \pm \hbar \omega_{\lambda}\right)}= \\
& =-k_{B} T \sum_{j} \frac{G_{M}^{r}\left(\mu_{L}+i(2 j+1) \pi k_{B} T\right)}{\mu_{L}+i(2 j+1) \pi k_{B} T-\left(\epsilon \pm \hbar \omega_{\lambda}\right)} .
\end{aligned}
$$

Nota-se que foi considerada a soma sobre as frequências de Matsubara da distribuição de Fermi-Dirac, igualmente espaçadas ao longo do plano complexo (distando de $2 \pi i k_{B} T$ entre si). No entanto, essa série converge lentamente, sendo necessário considerar uma quantidade muito grande de polos na somatória [99], o que é inviável na prática. Uma alternativa é representar a distribuição de Fermi-Dirac na forma de fração continuada, derivada a partir de uma função hipergeométrica, o que garante uma convergência relativamente rápida [100]. Outra alternativa é utilizar a mesma técnica aplicada ao cálculo da matriz densidade (Seção B.1), onde a função integrada é calculada sobre o contorno no plano complexo e uma quantidade finita de polos da distribuição de Fermi Dirac é considerada. 
Para calcular a integral da contribuição fora do equilíbrio $(\mathcal{I} \mathcal{I} \mathcal{I})$, podemos utilizar outra relação derivada no Apêndice B (primeira igualdade das Equações B.7 e B.8):

$$
\begin{aligned}
& \mathcal{I I I}=i \lim _{\eta \rightarrow 0^{+}} \int_{-\infty}^{+\infty} d \epsilon^{\prime}\left[\frac{G_{0}^{r} \Gamma_{R} G_{0}^{a}}{\epsilon^{\prime}-\left(\epsilon-\hbar \omega_{\lambda}\right)-i \eta}-\frac{G_{0}^{r} \Gamma_{R} G_{0}^{a}}{\epsilon^{\prime}-\left(\epsilon+\hbar \omega_{\lambda}\right)-i \eta}\right]\left(f_{R}-f_{L}\right)=
\end{aligned}
$$

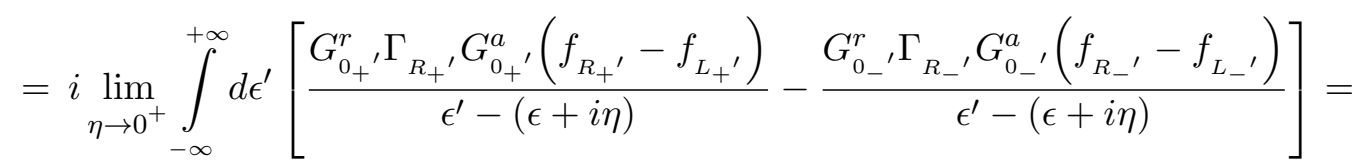

$$
\begin{aligned}
& =-\pi\left[G_{0_{+}}^{r} \Gamma_{R_{+}} G_{0_{+}}^{a}\left(f_{R_{+}}-f_{L_{+}}\right)-G_{0_{-}}^{r} \Gamma_{R_{-}} G_{0_{-}}^{a}\left(f_{R_{-}}-f_{L_{-}}\right)\right]+
\end{aligned}
$$

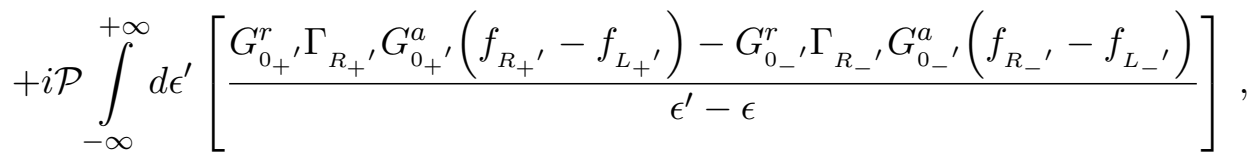

onde foram aplicadas as mudanças de variáveis $\epsilon^{\prime \prime}=\epsilon^{\prime}+\hbar \omega_{\lambda}$ no primeiro termo da integral e $\epsilon^{\prime \prime}=\epsilon^{\prime}-\hbar \omega_{\lambda}$ no segundo, e onde o índice $-^{\prime}\left(+^{\prime}\right)$ indica que a função é calculada em $\epsilon^{\prime}-\hbar \omega_{\lambda}$ $\left(\epsilon^{\prime}+\hbar \omega_{\lambda}\right)$. Multiplicando a expressão anterior pelo fator $1 / 2 \pi i$, obtém-se:

$$
\begin{aligned}
& \frac{1}{2 \pi i} \mathcal{I} \mathcal{I} \mathcal{I}=\frac{i}{2}\left[G_{0_{+}}^{r} \Gamma_{R_{+}} G_{0_{+}}^{a}\left(f_{R_{+}}-f_{L_{+}}\right)-G_{0_{-}}^{r} \Gamma_{R_{-}} G_{0_{-}}^{a}\left(f_{R_{-}}-f_{L_{-}}\right)\right]+
\end{aligned}
$$

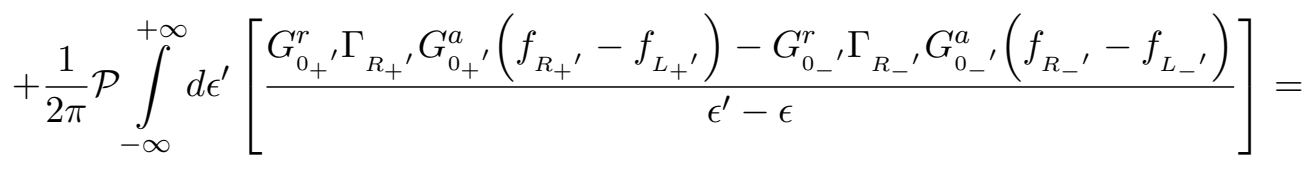

$$
\begin{aligned}
& =\frac{i}{2}\left[G_{0_{+}}^{r} \Gamma_{R_{+}} G_{0_{+}}^{a}\left(f_{R_{+}}-f_{L_{+}}\right)-G_{0_{-}}^{r} \Gamma_{R_{-}} G_{0_{-}}^{a}\left(f_{R_{-}}-f_{L_{-}}\right)\right]+
\end{aligned}
$$

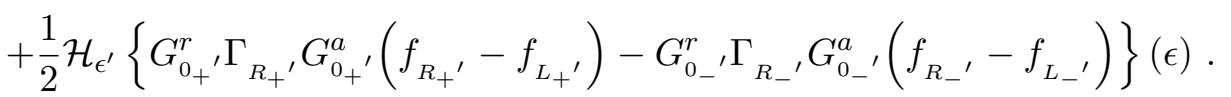

Novamente recai-se em uma transformada de Hilbert. Contudo, neste caso o intervalo de integração fica restrito a $\left[\mu_{R}-V / 2-\hbar \omega_{\lambda}, \mu_{L}+V / 2+\hbar \omega_{\lambda}\right]$, devido à subtração das distribuições de Fermi-Dirac dos eletrodos $\left(f_{R}-f_{L}\right)$ que se anula longe deste intervalo.

As expressões obtidas nesta seção também podem ser deduzidas partindo-se da expressão usual para a autoenergia retardada de Fock (Equação 6.13) e utilizando a relação entre a função espectral e a função de Green retardada derivada no Capítulo 2 (Equação 2.102). Porém, o caminho neste caso é mais longo e, por isso, será omitido. 
Portanto, com esta reformulação, o cálculo da autoenergia retardada de Fock que dependia de uma integral em todo o espectro de energia passa a ser simplesmente uma somatória e uma integral restrita a um pequeno intervalo de energia.

\subsection{Expressão para a Corrente e Conservação}

Ao substituir as funções de Green obtidas com a aproximação de Born (1BA ou SCBA) na expressão geral para a corrente (Equação 4.27) e escolhendo medir a corrente que flui entre o eletrodo esquerdo e a região de espalhamento, obtém-se:

$$
\begin{aligned}
I_{L}= & \frac{2 e}{h} \int_{-\infty}^{+\infty} d \epsilon \operatorname{Tr}\left[\Sigma_{L}^{<} G^{>}-\Sigma_{L}^{>} G^{<}\right]= \\
= & \frac{2 e}{h} \int_{-\infty}^{+\infty} d \epsilon \operatorname{Tr}\left[\Gamma_{L} G^{r} \Gamma_{R} G^{a}\right]\left(f_{L}-f_{R}\right)+ \\
& +i \frac{2 e}{h} \int_{-\infty}^{+\infty} d \epsilon \operatorname{Tr}\left[G^{a} \Gamma_{L} G^{r}\left(f_{L} \Sigma^{F,>}+\left(1-f_{L}\right) \Sigma^{F,<}\right)\right],
\end{aligned}
$$

onde se pode identificar o primeiro termo como o análogo ao formalismo de Landauer-Büttiker para o transporte elástico [4, 43, 89, 101], exceto que aqui as funções de Green são calculadas incluindo-se a autoenergia da interação elétron-fônon, e o segundo termo identifica-se como correspondendo ao transporte inelástico. Também podemos fazer uma analogia com o modelo das sondas de Büttiker descrito no Capítulo 5. Aqui a informação sobre cada sonda está contida nas autoenergias $\Sigma^{F,<}$ e $\Sigma^{F,>}$.

Uma pergunta natural é se, com as autoenergias de interação obtidas nas seções anteriores, a corrente se conserva, isto é, se $I_{L}+I_{R}=0$. Utilizando a expressão geral para a corrente (Equação 4.27, segue que:

$$
\begin{aligned}
I_{L}+I_{R} & =\frac{2 e}{h} \int_{-\infty}^{+\infty} d \epsilon \operatorname{Tr}\left[\left(\Sigma_{L}^{<}+\Sigma_{R}^{<}\right) G^{>}-\left(\Sigma_{L}^{>}+\Sigma_{R}^{>}\right) G^{<}\right]= \\
& =\frac{2 e}{h} \int_{-\infty}^{+\infty} d \epsilon \operatorname{Tr}\left[\left(\Sigma_{T o t}^{<}-\Sigma_{p h}^{<}\right) G^{>}-\left(\Sigma_{T o t}^{>}-\Sigma_{p h}^{>}\right) G^{<}\right]= \\
& =\frac{2 e}{h} \int_{-\infty}^{+\infty} d \epsilon \operatorname{Tr}\left[\left(\Sigma_{\text {Tot }}^{<} G^{>}-\Sigma_{T o t}^{>} G^{<}\right)-\left(\Sigma_{p h}^{<} G^{>}-\Sigma_{p h}^{>} G^{<}\right)\right],
\end{aligned}
$$


onde definimos a autoenergia de interação total $\Sigma_{\text {Tot }}^{\lessgtr}=\Sigma_{L}^{\lessgtr}+\Sigma_{R}^{\lessgtr}+\Sigma_{p h}^{\lessgtr}$. Vamos mostrar que o termo envolvendo explicitamente $\Sigma_{\text {Tot }}^{\lessgtr}$ na expressão anterior se anula. Para tanto, primeiramente vamos derivar uma relação envolvendo as equações de Keldysh para as funções de Green interagentes lesser e greater $G^{\lessgtr}=G^{r} \Sigma_{\text {Tot }}^{\lessgtr} G^{a}$. Multiplicando a relação fundamental da Equação 2.71 à esquerda por $\left(G^{r}\right)^{-1}$ e à direita por $\left(G^{a}\right)^{-1}$, tem-se:

$$
\begin{aligned}
\left(G^{r}\right)^{-1}\left[G^{r}-G^{a}\right]\left(G^{a}\right)^{-1} & =\left(G^{a}\right)^{-1}-\left(G^{r}\right)^{-1}=\left(G^{r}\right)^{-1}\left[G^{>}-G^{<}\right]\left(G^{a}\right)^{-1}= \\
& =\left(G^{r}\right)^{-1}\left[G^{r} \Sigma_{\text {Tot }}^{>} G^{a}-G^{r} \Sigma_{\text {Tot }}^{<} G^{a}\right]\left(G^{a}\right)^{-1}=\Sigma_{\text {Tot }}^{>}-\Sigma_{\text {Tot }}^{<},
\end{aligned}
$$

de onde se verifica:

$$
\Sigma_{\text {Tot }}^{>}=\left(G^{a}\right)^{-1}-\left(G^{r}\right)^{-1}+\Sigma_{T o t}^{<}
$$

Agora podemos utilizar este resultado para mostrar a condição de conservação de partículas:

$$
\begin{aligned}
\operatorname{Tr}\left[\Sigma_{\text {Tot }}^{<} G^{>}-\Sigma_{\text {Tot }}^{>} G^{<}\right]=\operatorname{Tr}\left[\Sigma_{\text {Tot }}^{<} G^{r} \Sigma_{\text {Tot }}^{>} G^{a}-\Sigma_{\text {Tot }}^{>} G^{r} \Sigma_{\text {Tot }}^{<} G^{a}\right]= \\
=\operatorname{Tr}\left[\Sigma_{\text {Tot }}^{<} G^{r}\left(\left(G^{a}\right)^{-1}-\left(G^{r}\right)^{-1}+\Sigma_{\text {Tot }}^{<}\right) G^{a}-\right. \\
\left.-\left(\left(G^{a}\right)^{-1}-\left(G^{r}\right)^{-1}+\Sigma_{T o t}^{<}\right) G^{r} \Sigma_{T o t}^{<} G^{a}\right]= \\
=\operatorname{Tr}\left[\Sigma_{\text {Tot }}^{<} G^{r}-\Sigma_{\text {Tot }}^{<} G^{a}+\Sigma_{\text {Tot }}^{<} G^{r} \Sigma_{\text {Tot }}^{<} G^{a}-\right. \\
\\
\left.\quad G^{r} \Sigma_{\text {Tot }}^{<}+G^{a} \Sigma_{\text {Tot }}^{<}+G^{a} \Sigma_{\text {Tot }}^{<} G^{r} \Sigma_{\text {Tot }}^{<}\right]=0,
\end{aligned}
$$

onde foi utilizada a propriedade cíclica do traço para obter-se as duas últimas igualdades. Desta forma, para que haja conservação de corrente, a autoenergia de interação utilizada no modelo deve satisfazer a condição:

$$
\mathcal{C}=\int_{-\infty}^{+\infty} d \epsilon \operatorname{Tr}\left[\Sigma_{p h}^{<} G^{>}-\Sigma_{p h}^{>} G^{<}\right]=0 .
$$

Substituindo na condição $\mathcal{C}$ as expressões para as autoenergias lesser e greater obtidas na aproximação de Born autoconsistente (SCBA), chega-se a: 


$$
\begin{aligned}
& \mathcal{C}=\int_{-\infty}^{+\infty} d \epsilon \operatorname{Tr}\left[\sum_{\lambda} M_{\lambda}\left\{n_{B_{\lambda}} G_{-}^{<}+\left(n_{B_{\lambda}}+1\right) G_{+}^{<}\right\} M_{\lambda} G^{>}-\right. \\
&\left.-\sum_{\lambda} M_{\lambda}\left\{n_{B_{\lambda}} G_{+}^{>}+\left(n_{B_{\lambda}}+1\right) G_{-}^{>}\right\} M_{\lambda} G^{<}\right]= \\
&=\sum_{\lambda} \int_{-\infty}^{+\infty} d \epsilon \operatorname{Tr}\left[n_{B_{\lambda}} M_{\lambda} G_{-}^{<} M_{\lambda} G^{>}+n_{B_{\lambda}} M_{\lambda} G_{+}^{<} M_{\lambda} G^{>}+M_{\lambda} G_{+}^{<} M_{\lambda} G^{>}-\right. \\
&\left.-n_{B_{\lambda}} M_{\lambda} G_{+}^{>} M_{\lambda} G^{<}-n_{B_{\lambda}} M_{\lambda} G_{-}^{>} M_{\lambda} G^{<}-M_{\lambda} G_{-}^{>} M_{\lambda} G^{<}\right] .
\end{aligned}
$$

Aplicando as mudanças de variáveis $\epsilon^{\prime}=\epsilon+\hbar \omega_{\lambda}$ ao primeiro termo da última linha e $\epsilon^{\prime}=$ $\epsilon-\hbar \omega_{\lambda}$ aos dois últimos termos e utilizando a propriedade cíclica do traço, obtém-se:

$$
\begin{aligned}
\mathcal{C}=\sum_{\lambda} \int_{-\infty}^{+\infty} d \epsilon \operatorname{Tr}[ & n_{B_{\lambda}} M_{\lambda} G_{-}^{<} M_{\lambda} G^{>}+n_{B_{\lambda}} M_{\lambda} G_{+}^{<} M_{\lambda} G^{>}+M_{\lambda} G_{+}^{<} M_{\lambda} G^{>}- \\
& \left.-n_{B_{\lambda}} M_{\lambda} G^{>} M_{\lambda} G_{-}^{<}-n_{B_{\lambda}} M_{\lambda} G^{>} M_{\lambda} G_{+}^{<}-M_{\lambda} G^{>} M_{\lambda} G_{+}^{<}\right]=0 .
\end{aligned}
$$

Portanto, demonstramos que a corrente é conservada na SCBA. Contudo, na primeira aproximação de Born (1BA) esse cancelamento não ocorre, pois as autoenergias são calculadas com as funções de Green não interagentes:

$$
\begin{aligned}
\mathcal{C}=\sum_{\lambda} \int_{-\infty}^{+\infty} d \epsilon \operatorname{Tr}[ & n_{B_{\lambda}} M_{\lambda} G_{0_{-}}^{<} M_{\lambda} G^{>}+n_{B_{\lambda}} M_{\lambda} G_{0_{+}}^{<} M_{\lambda} G^{>}+M_{\lambda} G_{0_{+}}^{<} M_{\lambda} G^{>}- \\
& \left.\quad n_{B_{\lambda}} M_{\lambda} G_{0}^{>} M_{\lambda} G_{-}^{<}-n_{B_{\lambda}} M_{\lambda} G_{0}^{>} M_{\lambda} G_{+}^{<}-M_{\lambda} G_{0}^{>} M_{\lambda} G_{+}^{<}\right] \neq 0 .
\end{aligned}
$$

Apesar disso, conforme veremos mais à frente, a 1BA apresenta bons resultados quando comparada à SCBA, além de ser extremamente menos custosa computacionalmente.

\subsection{O Programa I-SMEAGOL}

Nesta seção serão apresentados os detalhes da implementação da aproximação de Born (SCBA e 1BA) incorporada ao programa SMEAGOL (Seção 4.6), o que deu origem ao código Inelastic SMEAGOL para cálculos de transporte inelástico ab initio. 
Conforme explicado na Seção 4.6, a Hamiltoniana de Kohn-Sham calculada pelo SIESTA é fornecida ao SMEAGOL, que, por sua vez, introduz as autoenergias dos eletrodos e calcula uma nova matriz densidade. Esse processo ocorre iterativamente até atingir a autoconsistência, para cada voltagem $V$.

Uma vez convergida a densidade de carga, o programa está pronto para iniciar os cálculos das propriedades de transporte. Para tanto, o I-SMEAGOL precisa receber as autoenergias dos eletrodos $\left(\Sigma_{L}^{r}(\sigma, \epsilon)\right.$ e $\left.\Sigma_{R}^{r}(\sigma, \epsilon)\right)$ e as matrizes Hamiltoniana $(H(\sigma))$ e de overlap $(S)$, além das frequências dos fônons $\left(\omega_{\lambda}\right)$ e da matriz de acoplamento elétron-fônon $\left(M_{\lambda}\right)$ previamente calculadas com o programa PhOnonS ITeratIVE VIBRATIONS (Seção 3.3.1). Esta interface e os cálculos realizados pelo I-SMEAGOL estão representados no fluxograma da Figura 6.4.

\section{I-SMEAGOL}

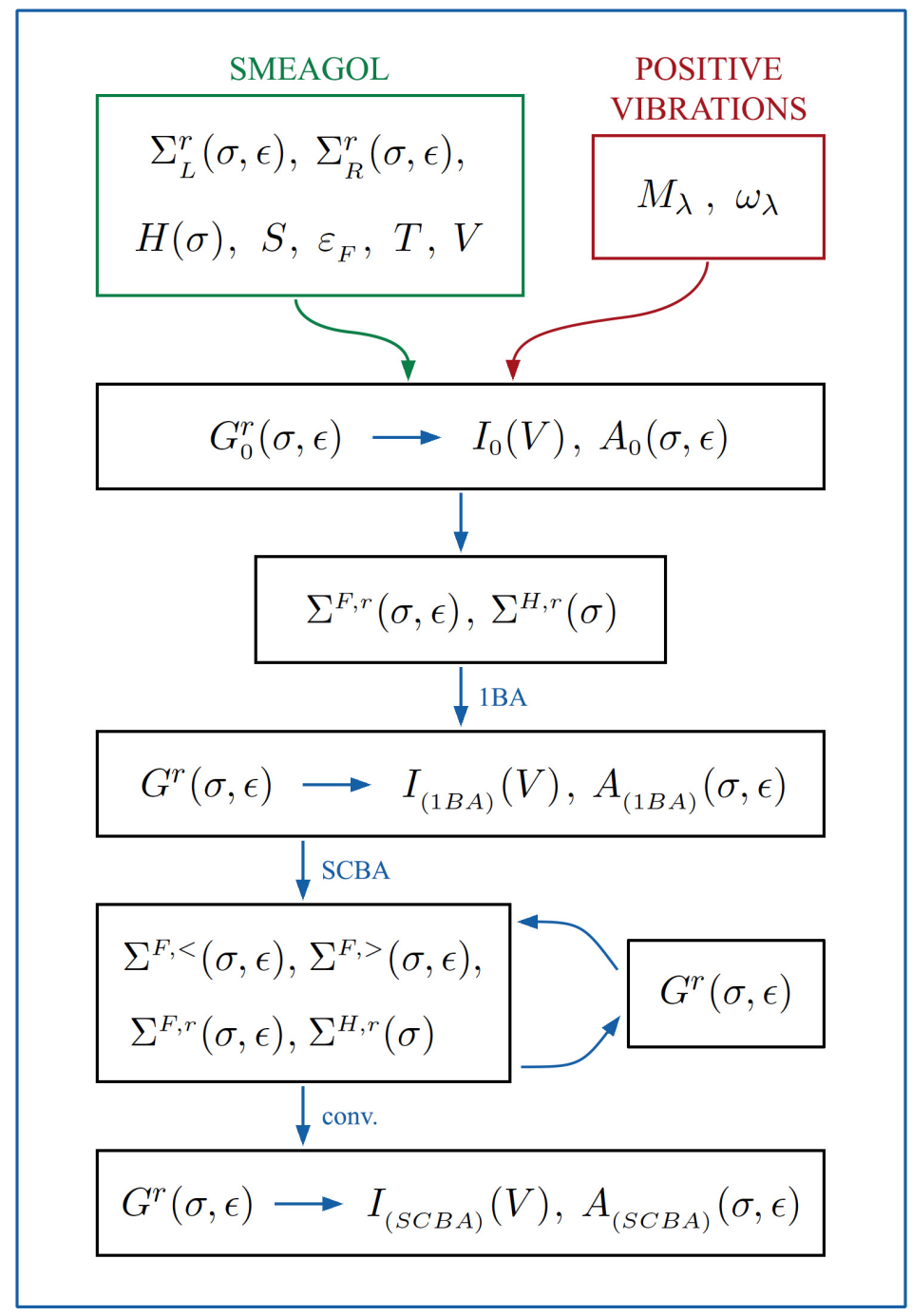

Figura 6.4: Fluxograma do programa I-SMEAGOL. 
Inicialmente, o código computa as propriedades de transporte sem interação elétron-fônon, calculando as funções de Green não-interagentes $\left(G_{0}^{r}(\sigma, \epsilon)\right)$ e, por conseguinte, a corrente elástica e a função espectral $\left(A_{0}(\sigma, \epsilon)\right)$.

O passo seguinte consiste em computar as autoenergias retardadas de interação elétron-fônon $\left(\Sigma^{F, r}(\sigma, \epsilon)\right.$ e $\left.\Sigma^{H, r}(\sigma)\right)$. O diagrama de Fock é calculado com o método descrito na Seção 6.3. Já o diagrama de Hartree é calculado com o mesmo método utilizado para a matriz densidade (Seção B.1), devido à semelhança desta autoenergia com a Equação 4.47 .

Em posse das autoenergias, calcula-se as funções de Green interagentes $\left(G^{r}(\sigma, \epsilon)\right)$ na primeira aproximação de Born e com elas computa-se a corrente com a Equação 6.25, assim como a função espectral do sistema interagente $(A(\sigma, \epsilon))$.

No cálculo da aproximação de Born autoconsistente, a autoenergia $\Sigma^{F, r}(\sigma, \epsilon)$ é calculada conforme a Equação 6.13. Portanto, neste caso é preciso calcular as autoenergias de interação lesser e greater explicitamente e em todo o espectro de energia (devido ao caráter não-local da transformada de Hilbert). Uma vez atingida a convergência, $\left|\left[\Sigma_{n}^{r}\right]_{i j}-\left[\Sigma_{n-1}^{r}\right]_{i j}\right|<\delta, \forall i, j$, calcula-se novamente a corrente e a função espectral do sistema interagente.

Pode-se observar, portanto, que a introdução do transporte inelástico gera alterações mínimas no programa original, o que implica em uma alta portabilidade do código, podendo ser facilmente incorporado a outros programas de transporte.

Na próxima seção, discutiremos alguns pontos mais técnicos envolvendo a implementação do código. Os detalhes de execução do programa I-SMEAGOL, assim como as diferentes opções permitidas ao usuário, podem ser encontrados no manual disposto no Apêndice E.

\subsubsection{Observações sobre Eficiência Computacional}

Ao incorporar os efeitos da interação elétron-fônon, uma demanda computacional significativa é introduzida no cálculo de transporte. Por este motivo, vários cuidados foram tomados durante a implementação para melhorar o desempenho do código. Nesta seção, alguns destes cuidados serão apontados de maneira sucinta.

Analisando as expressões para as autoenergias de interação, verifica-se que o maior custo computacional provem de produtos e inversões de matrizes, ambos com complexidade $O\left(n^{3}\right)$ (isto é, a quantidade de operações necessárias para realizar o produto ou a inversão é proporcional a $n^{3}$, onde $n$ é a dimensão principal da matriz).

Este custo pode ser reduzido utilizando algoritmos otimizados e levando em consideração as propriedades das matrizes envolvidas. Por exemplo, como os cálculos com interação elétron-fônon são realizados somente no ponto $\Gamma$ do espaço recíproco, as autoenergias dos eletrodos $\Sigma_{L / R}^{r}$ são 
simétricas e um algoritmo mais eficiente para a inversão pode ser utilizado no cálculo de $G_{0}^{r}$. Da mesma maneira, multiplicações envolvendo uma matriz simétrica (como é o caso de $M_{\lambda}$ e das autoenergias $\Sigma_{L / R}^{r}$ ) ou hermitiana (como é o caso de $H$ e $\Gamma_{L / R}$ ) podem ser realizadas por algoritmos que demandam menos operações quando comparados à multiplicação comum [95].

No I-SMEAGOL, todas essas operações são realizadas por meio de rotinas externas otimizadas para multiplicação (BLAS - Basic Linear Algebra Subprograms 102) e inversão (LAPACK Linear Algebra PACKage [103]) de matrizes.

Outro cuidado importante está relacionado às dimensões das matrizes envolvidas no problema. A princípio, a dimensão de todas elas é determinada pelo número de orbitais atômicos do sistema. No entanto, na maioria das vezes as interações elétron-fônon estão localizadas em um pequeno conjunto de átomos na região de espalhamento, o que define a dimensão da matriz $M_{\lambda}$ e, consequentemente, a dimensão das autoenergias de interação elétron-fônon (ou seja, estas matrizes possuem todos os elementos nulos, com exceção de um pequeno bloco referente aos orbitais dos átomos com interação elétron-fônon). Da mesma maneira, as autoenergias dos eletrodos (e, consequentemente, as matrizes $\Gamma_{L / R}$ ) também possuem todos os elementos nulos, com exceção de um bloco com as dimensões do eletrodo, correspondendo ao seu acoplamento com a região de espalhamento.

Assim, ao analisarmos os produtos de matrizes nas expressões implementadas (autoenergias de interação e corrente), verifica-se que somente alguns blocos da função de Green são realmente necessários. Esses blocos estão representados na Figura 6.5, onde $m$ é o número de orbitais dos átomos com interação elétron-fônon e $N L$ e $N R$ são as dimensões dos eletrodos esquerdo e direito, respectivamente.

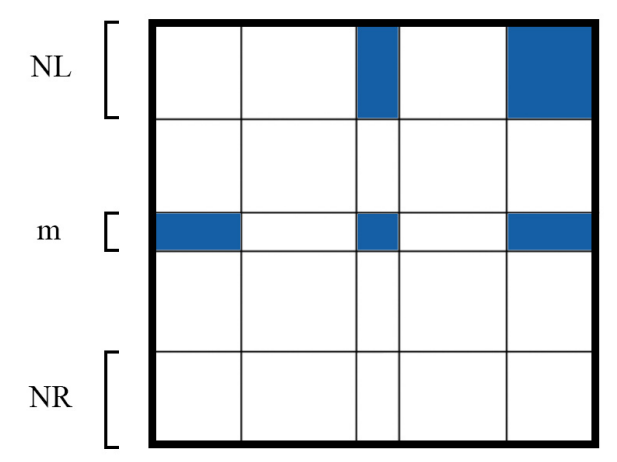

Figura 6.5: Ilustração destacando os blocos da função de Green efetivamente utilizados nos cálculos com a aproximação de Born, onde $N L$ (NR) é a dimensão do eletrodo esquerdo (direito) e $m$ é o número de orbitais dos átomos com interação elétron-fônon. 
Com esta observação, além de diminuir o custo computacional das multiplicações, reduz-se também a memória utilizada, visto que não é necessário armazenar toda a função de Green.

Por fim, vamos encerrar esta seção discutindo brevemente a respeito das dificuldades e soluções encontradas na paralelização do código. Conforme mencionado na Seção 4.6, o código SMEAGOL encontra-se paralelizado via Message Passing Interface (MPI) [87]. Como os cálculos de transporte eletrônico envolvem basicamente integrais em energia, essa paralelização consiste em dividir os pontos do grid de energia em conjuntos disjuntos, de forma a que cada processador fique responsável pelos cálculos envolvendo um desses conjuntos (Figura 6.6).

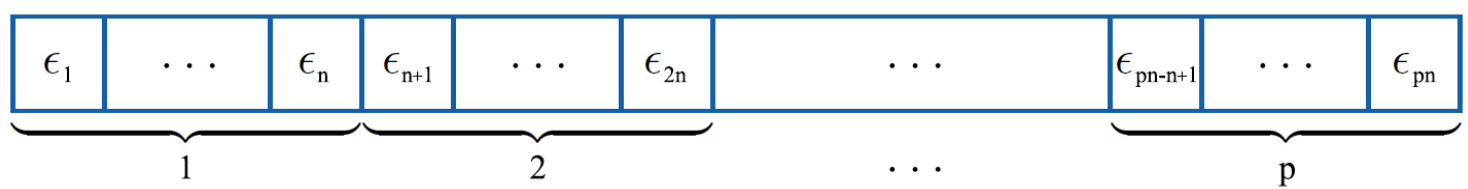

Figura 6.6: Paralelização do grid de energia em p processadores.

Porém, conforme veremos a seguir, essa paralelização se torna bem mais complexa no cálculo do transporte inelástico.

No I-SMEAGOL, as funções são calculadas em um grid equidistante de energia. Esta escolha se deve ao fato de que, neste caso, o núcleo da transformada de Hilbert pode ser simplificado e a transformada assume a forma de uma convolução discreta, podendo ser calculada de maneira eficiente por meio de algoritmos de transformada de Fourier discreta [95] (os detalhes do cálculo numérico da transformada de Hilbert podem ser encontrados no Apêndice B-Seção B.2.

Nos algoritmos disponíveis para transformada de Fourier discreta, é necessário fornecer a função a ser transformada calculada em todos os pontos de energia. No nosso caso, as funções correspondem a uma matriz para cada ponto de energia. Logo, primeiro é preciso coletar estas matrizes calculadas em todos os processadores. Após a coleta, a fim de não sobrecarregar um único processador e aumentar a eficiência do código, a solução encontrada foi redistribuir o cálculo das transformadas, paralelizando em termos dos orbitais atômicos (ver Figura 6.7).

Dessa forma, cada processador realiza transformadas de Hilbert unidimensionais em um conjunto de elementos da função. Uma vez que todos os processadores terminaram os respectivos cálculos, a função transformada é redistribuída novamente em termos dos pontos de energia.

Outra dificuldade relacionada à paralelização do código surge no cálculo das funções em $\epsilon-\hbar \omega_{\lambda}$ e $\epsilon+\hbar \omega_{\lambda}$, presentes nas expressões das autoenergias de interação elétron-fônon. Esses 

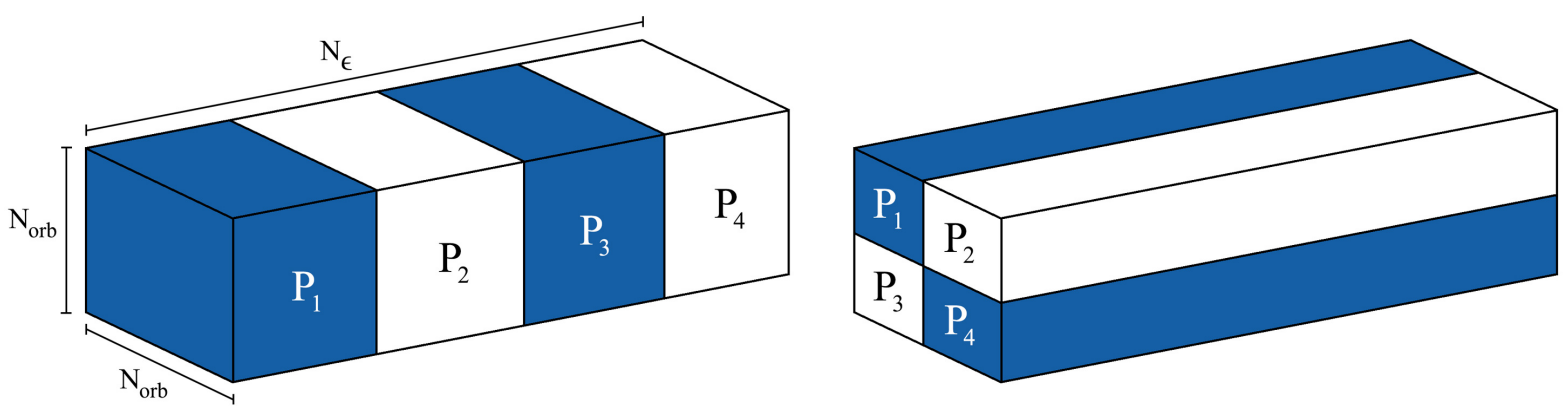

Figura 6.7: Representação da paralelização de uma matriz tridimensional entre quatro processadores $\left(P_{1}, P_{2}, P_{3}\right.$ e $\left.P_{4}\right)$, onde $N_{\epsilon}$ é o número de pontos de energia e $N_{o r b}$ é o número de orbitais atômicos. $\grave{A}$ esquerda, a distribuição é realizada nos pontos de energia e, à direita, em termos dos orbitais atômicos.

cálculos são realizados por meio de uma interpolação linear entre os valores da função calculada nos dois pontos de energia mais próximos.

Porém, pode ocorrer que esses pontos pertençam ao intervalo de energia referente a outro processador. Por exemplo, pode acontecer que, para calcular uma dada função no ponto $\epsilon+\hbar \omega_{\lambda}$, o processador $P_{1}$ precise interpolar a função calculada em dois pontos de energia pertencentes ao processador $P_{2}$. Nesse caso, $P_{1}$ deve saber a priori a quem "pedir" esses pontos, assim como $P_{2}$ deve saber a priori a quem "enviar" seus pontos.

Portanto, para solucionar esse problema, foi criada uma estrutura abstrata de dados comum a todos os processadores contendo, para cada modo vibracional $\lambda$, a quem pertence cada ponto de energia, a qual processador este ponto será enviado e por qual será recebido.

\subsection{Resultados}

Inicialmente, a aproximação de Born foi implementada para uma cadeia unidimensional com um único nível eletrônico por sítio, sistema modelo descrito na Seção 5.5.1. Esse primeiro passo é fundamental para compreender a natureza dos efeitos gerados pela aproximação nas propriedades do transporte, uma vez que nos cálculos de sistemas realísticos, com mais níveis atômicos e onde vários modos vibracionais estão envolvidos, a distinção desses efeitos pode tornar-se bastante complicada.

Vimos na Seção 5.5.1 que, para uma cadeia unidimensional, as autoenergias dos eletrodos possuem uma forma analítica simples (Equação 5.19). Contudo, em um primeiro momento, vamos considerá-las na aproximação Wide-Band Limit, que consiste em tomar somente a parte imaginária dessas autoenergias e considerar que os eletrodos possuem uma largura de banda 
infinita.

Vamos considerando o caso particular de um único nível eletrônico $\varepsilon_{0}$ na região de espalhamento e a interação com um fônon de energia $\hbar \omega=1,0 \mathrm{eV}$ e acoplamento $M_{\lambda}=0,32 \mathrm{eV}$. Nos cálculos descritos a seguir, os valores utilizados para energia de sítio dos eletrodos e do nível central, assim como os acoplamentos, foram escolhidos de forma a reproduzir o trabalho de $\mathrm{T}$. Frederiksen [104.

Considerando a uma temperatura baixa $\left(k_{B} T=0,001 \mathrm{eV}\right)$, calculou-se a função espectral a $V=0$ com a primeira aproximação de Born e com a aproximação de Born autoconsistente (Figura 6.8).

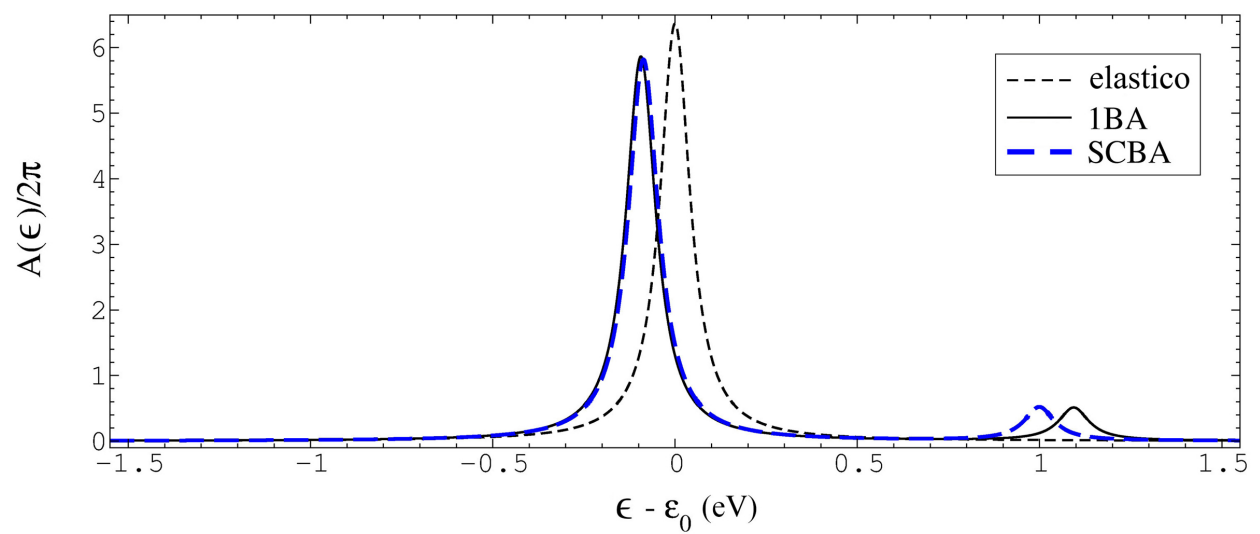

Figura 6.8: Comparação da função espectral obtida a $k_{B} T=0,001 \mathrm{eV}$ e $V=0$ com a 1BA (linha preta sólida), SCBA (linha azul) e sem interação elétron-fônon (linha hachurada).

Nota-se que, ao considerarmos o acoplamento elétron-fônon, o pico principal sofre um pequeno deslocamento para energias mais baixas. Esse efeito deve-se à contribuição da autoenergia de Hartree. Observa-se, também, que em energias aproximadamente iguais a $\epsilon-\varepsilon_{0}=\hbar \omega=1,0$ eV surge um pequeno pico, o que pode ser interpretado como um elétron incidente com energia $\varepsilon_{0}+m \hbar \omega$ emitindo $m$ fônons e se tornando ressonante com o nível localizado.

Como o sistema se encontra a baixa temperatura $\left(k_{B} T=0,001 \mathrm{eV}\right)$, um elétron incidente não pode absorver fônons (uma vez que a temperatura do banho de fônons é baixa) e, por isso, não se observa um pico a $-\hbar \omega$. Aumentando-se a temperatura para $k_{B} T=0,040 \mathrm{eV}$, este novo pico pode ser observado como apresentado na Figura 6.9.

Com a variação da diferença de potencial aplicada, observa-se na Figura 6.9 a existência de 


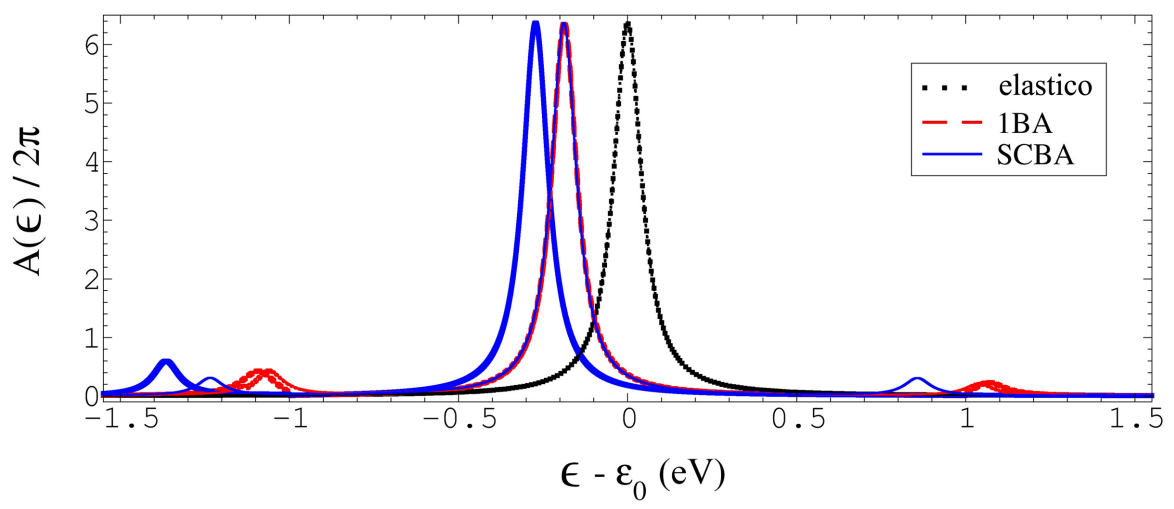

Figura 6.9: Comparação da função espectral obtida a $k_{B} T=0,04 \mathrm{eV}$ com a 1BA (linha vermelha hachurada), SCBA (linha azul sólida) e sem interação elétron-fônon (linha preta pontilhada), para diversos valores de diferença de potencial aplicados.

dois regimes diferentes na SCBA, o primeiro até $0,4 \mathrm{~V}$ com o pico principal a aproximadamente $-0,3 \mathrm{eV}$ e o segundo acima de $0,6 \mathrm{~V}$ com o pico principal em -0,2 eV. Entre estes dois regimes observa-se uma transição correspondente ao nível completamente preenchido para o nível preenchido exatamente pela metade.

As características $I-V$ foram calculadas com as diferentes implementações para a interação elétron-fônon (Figura 6.10).

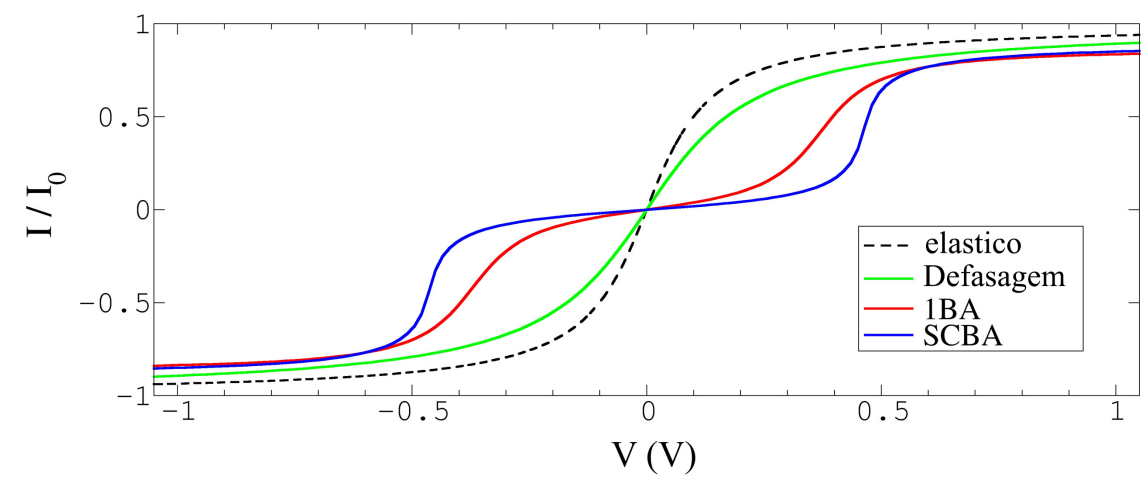

Figura 6.10: Comparação das características $I$-V obtidas a $k_{B} T=0,04 \mathrm{eV}$ com as diferentes implementações para a interação elétron-fônon.

Nota-se que o modelo fenomenológico de Büttiker produz um alargamento do nível, simulando o efeito de perda de coerência de fase dos elétrons. Já com a 1BA e a SCBA, observa-se que a forma da curva $I-V$ difere significativamente no intervalo de tensão $[-0,5 ; 0,5] \mathrm{V}$. Isso ocorre basicamente devido ao deslocamento do pico principal. 
Vamos considerar, agora, o caso em que os eletrodos possuem uma largura de banda finita, onde as autoenergias correspondem a Equação 5.19. As energias e acoplamentos de entrada utilizados estão dispostos na Tabela 6.1.

\begin{tabular}{cccccccc}
\hline$\varepsilon_{F}$ & $\varepsilon_{L}$ & $\varepsilon_{M}$ & $\varepsilon_{R}$ & $\gamma_{L}$ & $\gamma_{M L}$ & $\gamma_{M R}$ & $\gamma_{R}$ \\
\hline 0,0 & 0,0 & 0,51 & 0,0 & 0,70 & 0,05 & 0,05 & 0,70 \\
\hline
\end{tabular}

Tabela 6.1: Energias e acoplamentos, em eV, utilizados nos cálculos da Figura 6.11 .

Com esses dados, foi obtida a curva $I-V$ apresentada na Figura 6.11 para uma temperatura de $k_{B} T=0,01 \mathrm{eV}$. Ao considerar-se uma largura de banda finita dos eletrodos, nota-se o efeito de resistência diferencial negativa (diminuição da corrente com o aumento da tensão), característica fundamental em diodos de tunelamento ressonante. De fato, aqui o efeito surge porque, com a aplicação da diferença de potencial, as bandas dos dois eletrodos são deslocadas em energia em direções opostas. Para tensões $\sim 3 \mathrm{~V}$ já não há mais intersecção entre as bandas e o canal se fecha.

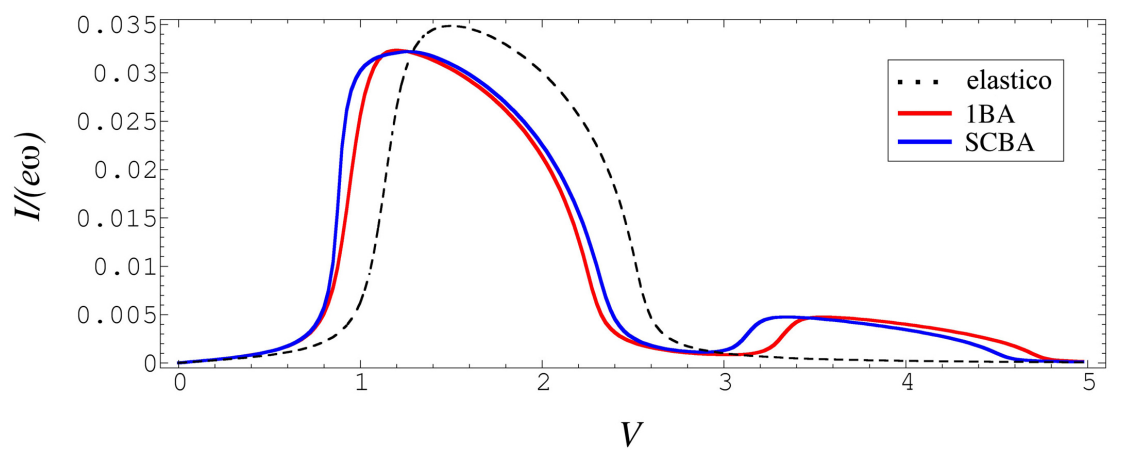

Figura 6.11: Comparação das características I-V obtidas considerando-se eletrodos com largura de banda finita.

A diferença mais saliente entre os cálculos com interação elétron-fônon (1BA e SCBA) e sem interação ocorre na região de queda da corrente, onde se observa o surgimento de um pico secundário devido à emissão de fônons. Nota-se também que, para este sistema simples, as aproximações 1BA e SCBA apresentam resultados qualitativamente semelhantes.

O sistema modelo pode ser reproduzido com DFT no programa I-SMEAGOL considerando um fio de ouro onde cada átomo é descrito somente com o orbital atômico $s$. Para gerar o 
acoplamento fraco do sítio central, aumentou-se a distância deste sítio com os átomos vizinhos de 2,7 para $3,8 \AA$, e manteve-se a estrutura fixa (Figura 6.12).

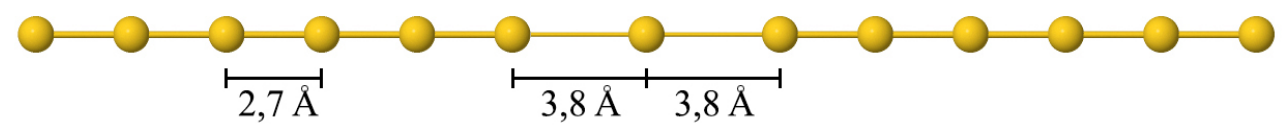

Figura 6.12: Representação de um fio de ouro com o átomo central fracamente acoplado.

Foi considerado um fônon de energia $\hbar \omega=1,0 \mathrm{eV}$ com acoplamento de $M_{\lambda}=0,32 \mathrm{eV}$ a uma temperatura de $T=116 \mathrm{~K}$. Na Figura 6.13 observa-se as funções espectrais obtidas em $V=0$, com e sem a contribuição da autoenergia de Hartree.

Fock

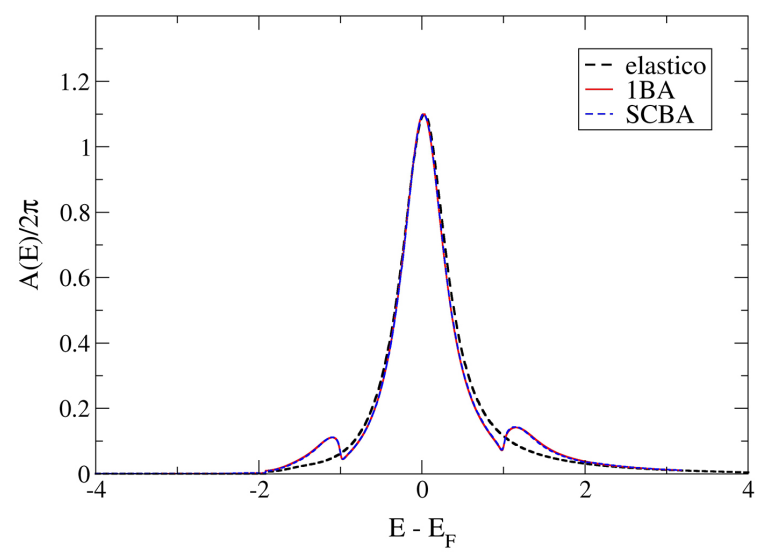

Fock + Hartree

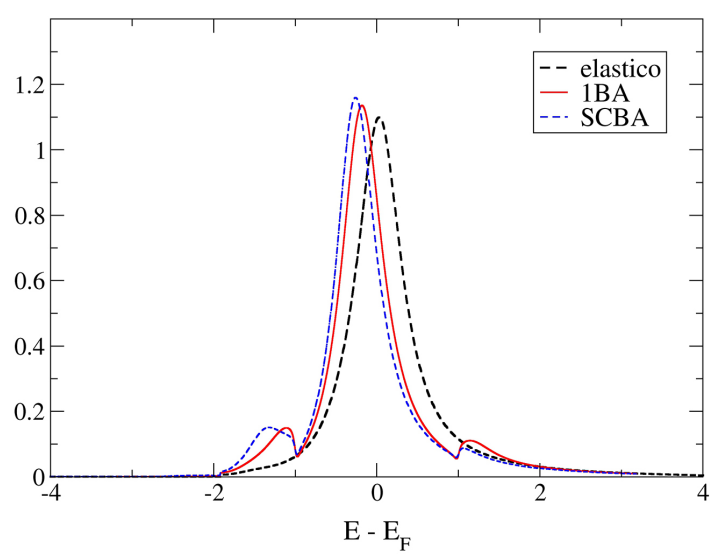

Figura 6.13: Funções espectrais a $V=0$ de um fio de ouro com um sítio fracamente acoplado calculadas incluindo somente a autoenergia de Fock (esquerda) e incluindo ambas as autoenergias de Hartree $e$ de Fock (direita), obtidas com a 1BA (linha vermelha), SCBA (linha azul tracejada) e sem interação elétron-fônon (linha preta tracejada).

Nota-se que a autoenergia de Fock é responsável pelas estruturas em $E-E_{F} \approx \pm 1,0 \mathrm{eV}$, correspondente a emissão e absorção de fônons. Ao incluir a contribuição do diagrama de Hartree, ocorre um deslocamento do pico principal para energias mais baixas e, consequentemente, uma renormalização das estruturas em $\pm 1,0 \mathrm{eV}$.

No gráfico superior da Figura 6.14 estão dispostas as características $I$ - $V$ obtidas. Nota-se que ocorre uma pequena redução no valor da corrente ao incluir a interação elétron-fônon, sendo mais significativa com a SCBA no intervalo $0 \leq V<1,0 \mathrm{~V}$, devido ao deslocamento maior do nível do átomo central (deslocamento do pico principal gerado pela contribuição de Hartree na Figura 6.13. 

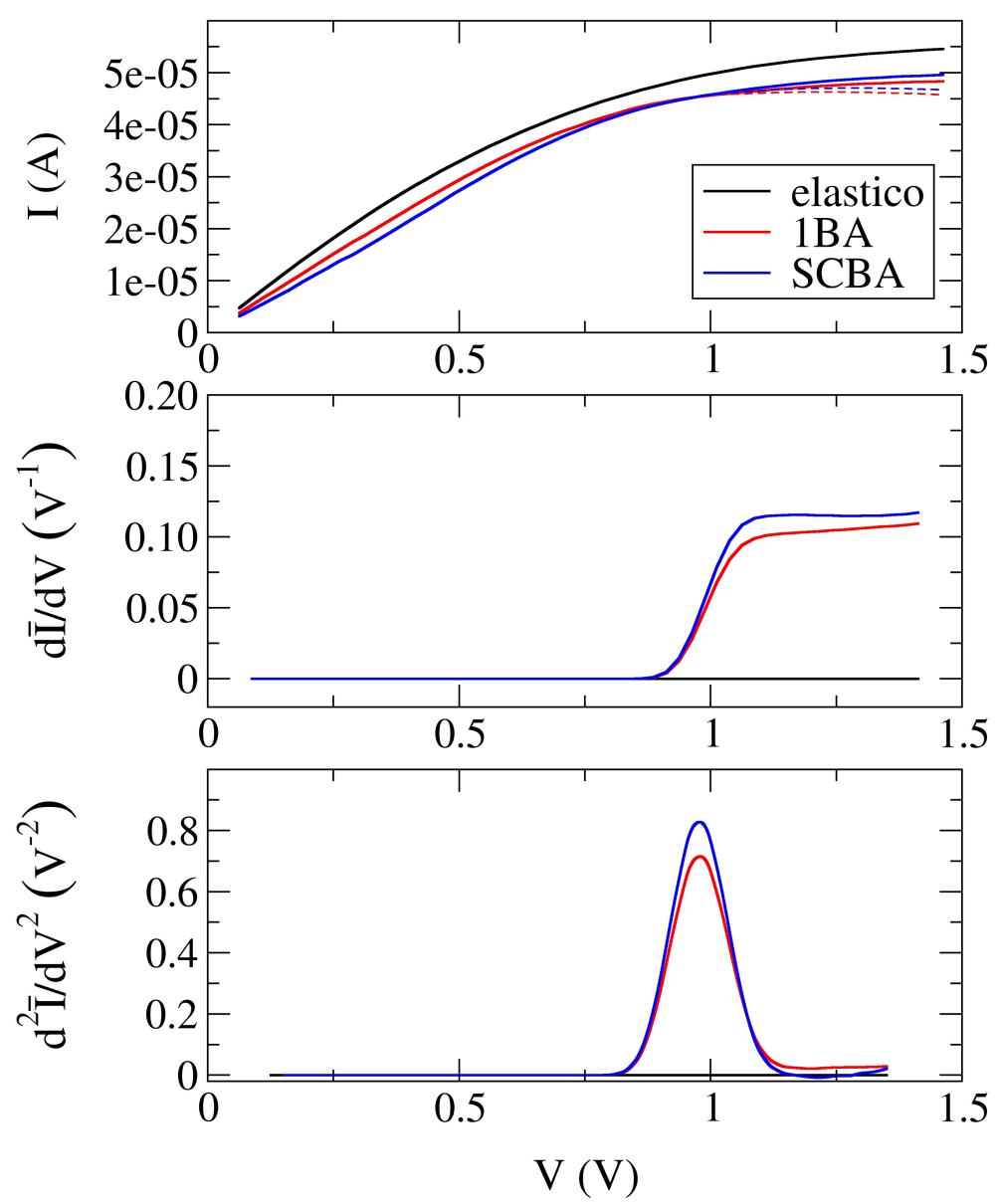

Figura 6.14: Curva I-V (acima) de um fio de ouro com um sitio fracamente acoplado, obtidas com a 1BA (linha vermelha), SCBA (linha azul) e sem interação elétron-fônon (linha preta). Condutância diferencial (centro) e espectro $d^{2} I / d V^{2}$ (abaixo) obtidas com as correntes normalizadas pela corrente elástica e considerando somente os termos inelásticos na $1 B A$ e na SCBA.

Os termos inelásticos da corrente começam a surgir quando a tensão atinge $1,0 \mathrm{~V}$, valor correspondente à energia do fônon. Para evidenciar esse efeito no cálculo da condutância diferencial e do espectro $d^{2} I / d V^{2}$ (gráficos ao centro e abaixo na Figura 6.14, respectivamente), as correntes foram normalizadas pela corrente elástica e considerou-se somente a componente inelástica da corrente na 1BA e na SCBA.

Verifica-se que ocorre a abertura de um novo canal de condutância devido à excitação do modo vibracional quando a tensão atinge o valor correspondente à energia do fônon e, consequentemente, surge um pico no espectro $d^{2} I / d V^{2}$ em $V \approx 1,0 \mathrm{~V}$.

Apesar de não conseguirmos reproduzir o desalinhamento dos níveis do sistema modelo (para isso poderíamos substituir o átomo central por outra espécie atômica), o sistema simulado consegue reproduzir qualitativamente algumas das características observadas no sistema modelo, como 
o deslocamento do pico principal para baixas energias na função espectral e o surgimento de novos estados em $\pm 1,0 \mathrm{eV}$. Além disso, com este sistema simplificado, novamente os resultados obtidos as aproximações 1BA e SCBA se mostraram qualitativamente semelhantes. 


\section{Capítulo 7}

\section{Transporte Inelástico em Sistemas Desordenados}

Nos capítulos anteriores, o problema de transporte foi descrito em termos de um sistema constituído por eletrodos metálicos perfeitos e semi-infinitos acoplados a uma região de espalhamento, quebrando a periodicidade do sistema. Essa região central foi tratada de maneira geral, podendo ser formada por um átomo, uma molécula ou por um defeito no material cristalino. Este último caso atrai um grande interesse, já que em dispositivos reais há uma grande probabilidade de haver defeitos. Em escala microscópica, como nos dispositivos eletrônicos atuais, a presença de defeitos pode ser tratada de forma média. No caso de dispositivos nanoscópicos isto deixa de ser verdade e distintos arranjos de defeitos podem levar a propriedades significativamente diferentes.

Os defeitos podem ser formados, por exemplo, por vacâncias na rede cristalina ou por impurezas adsorvidas no material. Dada a dificuldade em se determinar ou controlar a posição dos defeitos, experimentalmente estudam-se as propriedades estatísticas da condutância em uma amostra contendo várias realizações do sistema. Essa "desordem" pode levar a um novo regime fundamental de transporte, a saber, o regime de localização de Anderson [5].

A presença dos defeitos nos materiais também podem gerar modos vibracionais localizados, ocasionando o espalhamento inelástico dos elétrons. Recentemente, alguns estudos utilizando modelos têm sugerido que a combinação de sistemas desordenados com a interação elétronfônon pode ser a resposta para questões em aberto, como a natureza da condutividade em polímeros 38, 39].

Para prever e auxiliar o desenvolvimento de novos dispositivos seria interessante, portanto, ter uma ferramenta computacional capaz de simular dispositivos realísticos, considerando defeitos aleatoriamente posicionados e a interação elétron-fônon. No entanto, esses dispositivos podem atingir escalas de comprimento de $100 \mathrm{~nm}$ [36], o que corresponde a $O\left(10^{4}\right)$ átomos, de forma 
que simulações quantitativamente significativas a partir cálculos ab initio é algo extremamente custoso.

Neste trabalho, foi desenvolvido um método que permite calcular, por primeiros princípios, as propriedades de transporte de dispositivos eletrônicos com as duas características citadas acima: um grande número de defeitos aleatoriamente distribuídos em conjunto com a interação elétron-fônon. A implementação deste método deu origem ao programa Inelastic DISORDER.

Nosso método combina cálculos de estrutura eletrônica via DFT com o formalismo NEGF para o transporte, onde as funções de Green são obtidas recursivamente [52, 99]. Foi considerado o caso onde a interação elétron-fônon é fraca e localizada nos defeitos. Nestas circunstâncias, as expressões para a corrente e para a potência dissipada podem ser obtidas por meio de teoria de pertubação com relação à matriz de acoplamento elétron-fônon (Lowest Order Expansion) [53 55]. Essas expressões assumem uma forma simplificada quando as funções de Green e as funções largura de nível são consideradas como independentes da energia (Wide-Band Limit) e as integrais em energia são calculadas analiticamente.

Iniciaremos este capítulo discutindo os conceitos fundamentais envolvendo sistemas desordenados. Em seguida, descreveremos como o formalismo de transporte pode ser aplicado ao cálculo de sistemas com um grande número de defeitos. Mostraremos, então, como as interações elétron-fônon são incluídas neste cenário através da Lowest Order Expansion. Por fim, serão apresentados detalhes da implementação do programa I-DISORDER e exemplos de aplicação.

\subsection{Desordem e Localização}

A periodicidade em sistemas cristalinos nos permite descrever os elétrons por meio do teorema de Bloch, onde se afirma que, sob um potencial periódico, a função de onda eletrônica pode ser escrita como o produto de uma onda plana por uma função com a periodicidade da rede (Equação 3.27 .

Contudo, em sistemas reais esta periodicidade é geralmente quebrada pela presença de defeitos como, por exemplo, vacâncias na rede cristalina ou impurezas no material, gerando uma "desordem" no sistema cristalino. Essa desordem pode aparecer em diferentes graus, indo desde alguns poucos defeitos até o limite de sistemas fortemente desordenados, como ligas ou estruturas vítreas [10].

Com a introdução de um defeito no sistema periódico as funções de onda eletrônicas deixam de ser periódicas dentro da escala de comprimento do livre caminho médio $\ell$. Apesar disso, para um único defeito até o limite de baixa desordem, a função de onda mantém-se estendida ao longo do sistema (Figura 7.1 à esquerda), de forma que elétrons podem ser transmitidos por todo o 
sistema.
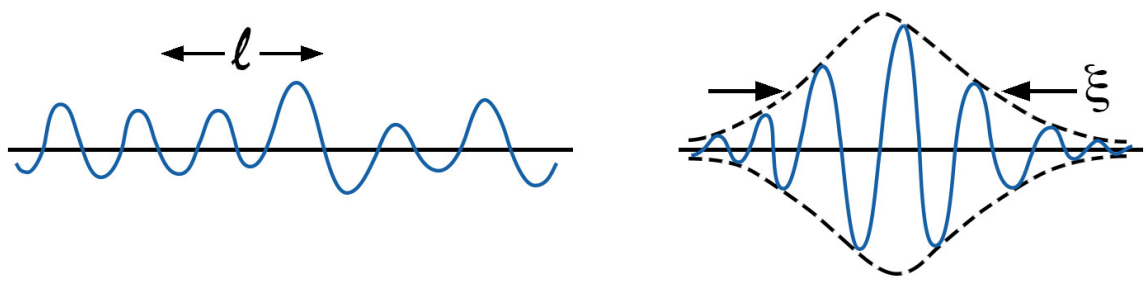

Figura 7.1: Representações da função de onda eletrônica espalhada por defeitos no material, onde à esquerda um estado estendido com livre caminho médio $\ell$ e à direita um estado localizado com comprimento de localização $\xi$. (Adaptado de [10].)

Porém, se o grau de desordem for muito forte, a função de onda eletrônica pode ser profundamente alterada (Figura 7.1 à direita), tornando-se localizada com uma função envelope que decai exponencialmente de acordo com:

$$
|\Psi(\mathbf{r})| \approx e^{-\left|\mathbf{r}-\mathbf{r}_{0}\right| / \xi}
$$

onde $\xi$ é o comprimento de localização. Nesse caso, o elétron fica confinado a uma pequena região e sua transmissão é completamente suprimida. Este conceito foi introduzido em 1958 por P. Anderson [5], sendo hoje conhecido como localização de Anderson.

Em uma situação intermediária, com um grau de desordem moderado, espera-se que os estados mais profundos da banda tendam a ser localizados e que os estados no centro da banda possuam maior chance de permanecerem estendidos. Logo, variando-se a energia dos autoestados, existe uma energia crítica, conhecida como borda de mobilidade, onde os estados passam de localizados a estendidos, o que define uma transição metal-isolante ${ }^{1}$

O estudo da transição metal-isolante induzida por desordem em sistemas com diferentes dimensões resultou em uma teoria de escala. A hipótese fundamental desta teoria é que, perto da transição entre estados localizados e estendidos, existe somente uma variável de escala, que, por sua vez, é suficiente para descrever o comportamento da condutividade e o comprimento de localização [35]. Essa variável de escala é dada pela condutância adimensional $g=\mathcal{G} / \mathcal{G}_{0}$.

Consideremos um sistema com dimensões $L^{\mathfrak{D}}$, onde $L$ é, por exemplo, o comprimento do canal na Figura 4.1 e $\mathfrak{D}$ é a dimensionalidade do sistema. Conforme foi discutido na Seção 4.2, no caso em que $L \gg \ell$ e o transporte é difusivo, ou seja, um sistema fracamente desordenado, a

\footnotetext{
${ }^{1}$ Isso é válido para sistemas $2 \mathrm{D}$ e $3 \mathrm{D}$. Em sistemas unidimensionais todos os estados são localizados, independentemente do grau de desordem [10].
} 
condutância obedece à lei de Ohm (Equação 4.2):

$$
\mathcal{G}=\sigma L^{\mathfrak{D}-2}
$$

onde $\sigma$ é a condutividade. Já no caso de sistemas fortemente desordenados, quando $L \gg \xi>\ell$, pode-se assumir que a condutância é proporcional a $|\Psi(L)|$ :

$$
\mathcal{G} \propto e^{-L / \xi}
$$

ou seja, a condutância tende a zero com o aumento de $L$ e o sistema se torna um isolante.

Para analisar as transições entre esses regimes de condutância, é conveniente trabalhar com uma função de escala definida como a seguinte derivada logarítmica:

$$
\beta(g)=\frac{d \ln (g)}{d \ln (L)}
$$

Seja $g_{c}$ a condutância adimensional crítica, a partir da qual podemos diferenciar os regimes de alta condutância $\left(g \gg g_{c}\right)$ e baixa condutância $\left(g \ll g_{c}\right)$. Nesses casos, identificam-se os seguintes limites assimptóticos para a função de escala:

$$
\beta(g) \propto \begin{cases}\mathfrak{D}-2, & g \gg g_{c}, \\ \ln (g), & g \ll g_{c} .\end{cases}
$$

Nota-se que no regime de alta condutância, onde vale a lei de Ohm (Equação 7.2), a função de escala tende a zero em duas dimensões. Isso reflete o fato da condutância ser proporcional à condutividade e independente das dimensões do sistema. Logo, em uma ou duas dimensões, para escalas de comprimento suficientemente grande não existem estados estendidos, independentemente do grau de desordem. Isso ocorre somente em três dimensões, onde a a função de escala pode assumir valores positivos. A mudança do sinal da função de escala de positivo para negativo em $\mathfrak{D}=3$ ocorre com o aumento do grau de desordem do sistema, de modo que para $g=g_{c}$ tem-se $\beta(g)=0$.

A teoria de escala apresentada sucintamente acima é aplicável para sistemas à temperatura $T=0$. A uma temperatura finita, porém, interações inelásticas introduzem flutuações randômicas na evolução temporal do estado eletrônico, o que limita a interferência quântica necessária para a localização [10]. Seja $\tau_{\text {in }}$ a meia-vida de um elétron em um dado estado de energia do 
potencial aleatório, e então, se esse tempo for muito maior do que o tempo de espalhamento elástico, $\tau_{i n} \gg \tau$, a distância percorrida por este elétron entre colisões inelásticas é dada por:

$$
\mathfrak{L}=\left(D \tau_{\text {in }}\right)^{1 / 2}
$$

onde $D=\left(v_{F}^{2} \tau / \mathfrak{D}\right)$ é a constante de difusão. Portanto, em escalas de comprimento além de $\mathfrak{L}$, os efeitos de localização pode ser suprimidos. Nota-se que $\mathfrak{L}$ depende da temperatura do sistema, uma vez que o tempo de espalhamento inelástico aumenta com a diminuição da temperatura.

Para determinar o comprimento de localização $\xi$ de um dado sistema, é necessário estudar a distribuição da condutância por meio de várias realizações do sistema, mantendo-se fixas a dimensão $L$ e a concentração de defeitos. Para uma estatística suficientemente grande, espera-se que $\langle\ln (g)\rangle=-L / \xi$.

No regime de localização, a condutância apresenta uma distribuição log-normal com média $\mu=\langle\ln (g)\rangle[35$ :

$$
\mathbb{P}\{g\} \propto \frac{1}{g} e^{-\frac{1}{2}\left(\frac{\ln (g)-\langle\ln (g)\rangle}{\Delta}\right)^{2}}
$$

Já no regime metálico, essa distribuição é aproximadamente:

$$
\mathbb{P}\{g\} \propto \frac{1}{g} e^{|\ln (g)-\langle\ln (g)\rangle|} .
$$

Portanto, ajustando-se uma gaussiana ao histograma de $\ln (g)$ gerado pelas diversas realizações do sistema, pode-se verificar se o sistema encontra-se no regime de localização e estimar a flutuação da condutância por meio da variância $\Delta^{2}$ do ajuste.

\subsection{Transporte Elástico}

Para tratar o problema de transporte em sistemas desordenados precisamos de um método que seja capaz de considerar sistemas com escalas de comprimento da ordem de $100 \mathrm{~nm}$ com dezenas de milhares de átomos. Além disso, o método deve ter um baixo custo computacional, visto que são necessárias varias realizações do cálculo para se obter a distribuição da condutância.

Podemos utilizar o formalismo NEGF para o transporte apresentado no Capítulo 4, já que a região de espalhamento é tratada de maneira geral e independe do tamanho do sistema. No entanto, a abordagem adotada pelo programa SMEAGOL (Seção 4.6) para combinar os cálculos de estrutura eletrônica via DFT com o formalismo NEGF é inviável em sistemas com 
essas dimensões. Portanto, é necessário utilizar uma nova metodologia para obter a função de Green.

Neste trabalho, nos restringiremos a sistemas unidimensionais (ou quasi-unidimensionais, como nanofitas de grafeno que serão nosso objeto de estudo no próximo capítulo) com defeitos distribuídos ao longo do sistema.

Para ilustrar o problema, vamos considerar o caso da nanofita de grafeno apresentada na Figura 7.2 .

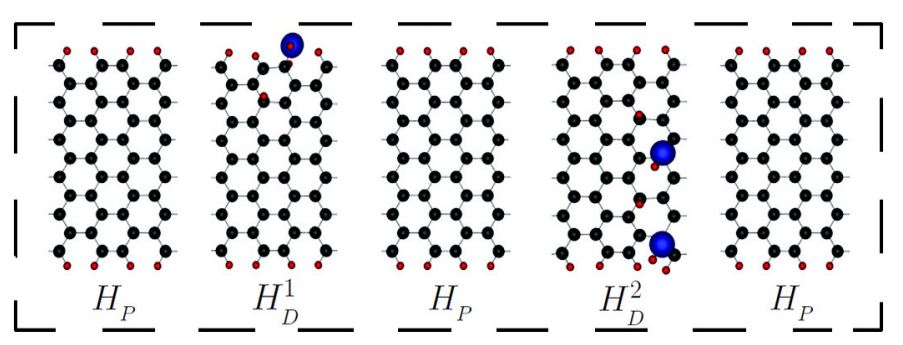

Figura 7.2: Região de espalhamento formada por uma nanofita de grafeno, onde se destacam segmentos pristinos $\left(H_{P}\right)$ e com defeito $\left(H_{D}^{1}\right.$ e $\left.H_{D}^{2}\right)$. Os eletrodos esquerdo e direito se acoplam ao primeiro e último segmentos, respectivamente.

Neste exemplo, a nanofita é composta por três blocos sem defeitos, chamados de pristinos, e outros dois segmentos com defeitos gerados pela adsorção de hidroxilas. Da mesma maneira que fizemos com os eletrodos ao descrever o problema de transporte (Seção 4.5), o problema deve ser construído de forma a que os blocos interajam somente com os primeiros vizinhos, o que nos leva a uma Hamiltoniana tridiagona $2^{2}$ para a região de espalhamento $3^{3}$

$$
H_{M}=\left(\begin{array}{ccccc}
H_{P} & V_{1,2} & 0 & 0 & 0 \\
V_{2,1} & H_{D}^{1} & V_{2,3} & 0 & 0 \\
0 & V_{3,2} & H_{P} & V_{3,4} & 0 \\
0 & 0 & V_{4,3} & H_{D}^{2} & V_{4,5} \\
0 & 0 & 0 & V_{5,4} & H_{P}
\end{array}\right),
$$

onde $V_{i, i-1}=V_{i-1, i}^{\dagger}$. Considerando-se que os eletrodos esquerdo e direito se acoplam somente com o primeiro e o último bloco, respectivamente, o problema que queremos resolver adquire a seguinte forma:

\footnotetext{
${ }^{2}$ Como ficará claro mais à frente, este é um ponto fundamental do método.

${ }^{3}$ No caso de uma base de orbitais localizados, é sempre possível construir um Hamiltoniano tridiagonal, bastando escolher o tamanho do bloco (fatia do sistema) de maneira apropriada.
} 


$$
\left(\begin{array}{ccccc}
\bar{H}_{P}-\Sigma_{L}^{r} & \bar{V}_{1,2} & 0 & 0 & 0 \\
\bar{V}_{2,1} & \bar{H}_{D}^{1} & \bar{V}_{2,3} & 0 & 0 \\
0 & \bar{V}_{3,2} & \bar{H}_{P} & \bar{V}_{3,4} & 0 \\
0 & 0 & \bar{V}_{4,3} & \bar{H}_{D}^{2} & \bar{V}_{4,5} \\
0 & 0 & 0 & \bar{V}_{5,4} & \bar{H}_{P}-\Sigma_{R}^{r}
\end{array}\right) G_{M}^{r}=\mathbb{1}
$$

onde foi adotada a notação $\bar{H}_{i}=\varepsilon S_{i}-H_{i}$ e $\bar{V}_{i, j}=\varepsilon S_{i, j}-H_{i, j}$, com $\varepsilon=\lim _{\eta \rightarrow 0^{+}} \epsilon+i \eta$, e $S_{M}$ é a matriz de overlap, cujos blocos seguem a mesma convenção dos blocos de $H_{M}$.

Até agora nada mudou comparado ao que fizemos na Seção 4.5, com exceção de termos detalhado a estrutura da região de espalhamento. A vantagem de dividir essa região em segmentos e escrever sua Hamiltoniana na forma tridiagonal fica evidente no cálculo da transmissão elástica $\operatorname{Tr}\left[\Gamma_{L} G_{M}^{r} \Gamma_{R} G_{M}^{a}\right]$. Analisando os produtos individualmente, tem-se que:

$$
\begin{aligned}
\Gamma_{L} G_{M}^{r}= & \left(\begin{array}{ccccc}
\Gamma_{L} & 0 & 0 & 0 & 0 \\
0 & 0 & 0 & 0 & 0 \\
0 & 0 & 0 & 0 & 0 \\
0 & 0 & 0 & 0 & 0 \\
0 & 0 & 0 & 0 & 0
\end{array}\right)\left(\begin{array}{ccccc}
G_{1,1}^{r} & G_{1,2}^{r} & G_{1,3}^{r} & G_{1,4}^{r} & G_{1,5}^{r} \\
G_{2,1}^{r} & G_{2,2}^{r} & G_{2,3}^{r} & G_{2,4}^{r} & G_{2,5}^{r} \\
G_{3,1}^{r} & G_{3,2}^{r} & G_{3,3}^{r} & G_{3,4}^{r} & G_{3,5}^{r} \\
G_{4,1}^{r} & G_{4,2}^{r} & G_{4,3}^{r} & G_{4,4}^{r} & G_{4,5}^{r} \\
G_{5,1}^{r} & G_{5,2}^{r} & G_{5,3}^{r} & G_{5,4}^{r} & G_{5,5}^{r}
\end{array}\right)= \\
= & \left(\begin{array}{ccccc}
\Gamma_{L} G_{1,1}^{r} & \Gamma_{L} G_{1,2}^{r} & \Gamma_{L} G_{1,3}^{r} & \Gamma_{L} G_{1,4}^{r} & \Gamma_{L} G_{1,5}^{r} \\
0 & 0 & 0 & 0 & 0 \\
0 & 0 & 0 & 0 & 0 \\
0 & 0 & 0 & 0 & 0 \\
0 & 0 & 0 & 0 & 0
\end{array}\right),
\end{aligned}
$$

onde $\Gamma_{L}=i\left(\Sigma_{L}^{r}-\Sigma_{L}^{a}\right)$. Analogamente, o produto $\Gamma_{R} G_{M}^{a}$ resulta em uma matriz com somente a última linha de blocos não-nulos, de modo que, ao calcular o produto das matrizes resultantes, verifica-se que a transmissão é dada somente por:

$$
\operatorname{Tr}\left[\Gamma_{L} G_{M}^{r} \Gamma_{R} G_{M}^{a}\right]=\operatorname{Tr}\left[\Gamma_{L} G_{1,5}^{r} \Gamma_{R} G_{1,5}^{a}\right]
$$

Portanto, para o cálculo da transmissão elástica, de todos os blocos da função de Green $G_{M}^{r}$ só nos interessa o bloco $G_{1,5}^{r}$, que efetivamente conecta o eletrodo esquerdo ao eletrodo direito. Este exemplo com cinco segmentos na região de espalhamento é facilmente generalizado para um sistema com $n$ segmentos, de onde se obtém: 


$$
\operatorname{Tr}\left[\Gamma_{L} G_{M}^{r} \Gamma_{R} G_{M}^{a}\right]=\operatorname{Tr}\left[\Gamma_{L} G_{1, n}^{r} \Gamma_{R} G_{1, n}^{a}\right]
$$

Assim sendo, ao invés de inverter toda a Hamiltoniana efetiva da região de espalhamento para obter-se a função de Green $G_{M}^{r}$ na Equação 7.10 (o que é impraticável em sistemas com as escalas de grandeza mencionadas acima), podemos usar o método recursivo apresentado a seguir com a finalidade de obter-se somente o termo $G_{1, n}^{r}$.

\subsection{Cálculo Recursivo de Funções de Green}

Neste trabalho, escolhemos utilizar o método proposto por C. Lewenkopf e E. Mucciolo para o cálculo recursivo de funções de Green [52]. Existem algoritmos mais eficientes para se obter o termo $G_{1, n}^{r}$ [36], porém, como ficará claro mais à frente, o método escolhido é ideal para o cálculo do transporte inelástico.

Grosso modo, a ideia do método consiste em obter relações de recorrência por meio da equação de Dyson (Equação 2.149) ao conectar-se iterativamente os blocos, partindo primeiro do eletrodo esquerdo até o fim da região de espalhamento e depois do eletrodo direito até o início dessa região. Esses dois procedimentos geram, respectivamente, os conjuntos de funções de Green $\left\{G^{L}\right\}$ e $\left\{G^{R}\right\}$, que são, então, utilizados para obter a função de Green total exata do sistema.

Para a derivação que se segue, é conveniente decompor a Hamiltoniana efetiva de um sistema com $n$ blocos em uma matriz $\mathbf{H}$ com as Hamiltonianas isoladas de cada segmento e outra $\mathbf{V}$ com os respectivos acoplamentos:

$$
\mathbf{H}+\mathbf{V}=\left(\begin{array}{ccccc}
\bar{H}_{1,1} & 0 & \cdots & 0 & 0 \\
0 & \bar{H}_{2,2} & \cdots & 0 & 0 \\
\vdots & \vdots & \ddots & \vdots & \vdots \\
0 & 0 & \cdots & \bar{H}_{n-1, n-1} & 0 \\
0 & 0 & \cdots & 0 & \bar{H}_{n, n}
\end{array}\right)+\left(\begin{array}{ccccc}
-\Sigma_{L}^{r} & \bar{V}_{1,2} & \cdots & 0 & 0 \\
\bar{V}_{2,1} & 0 & \cdots & 0 & 0 \\
\vdots & \vdots & \ddots & \vdots & \vdots \\
0 & 0 & \cdots & 0 & \bar{V}_{n-1, n} \\
0 & 0 & \cdots & \bar{V}_{n, n-1} & -\Sigma_{R}^{r}
\end{array}\right)
$$

de modo que o problema pode ser escrito da seguinte forma:

$$
(\mathbf{H}+\mathbf{V}) G_{M}^{r}=\mathbb{1}
$$


Partimos do sistema totalmente desacoplado, isto é, sem o acoplamento com os eletrodos e entre os segmentos $(\mathbf{V}=\mathbb{0})$. Nesse caso, o problema se resume a inverter uma matriz bloco diagonal, o que é equivalente a inverter cada um dos blocos. Assim, os únicos termos não-nulos da função de Green no caso desacoplado são os diagonais, para os quais adotaremos a seguinte notação:

$$
g_{i, i}^{r}=\left(\bar{H}_{i, i}\right)^{-1}=\left(\varepsilon S_{i, i}-H_{i, i}\right)^{-1} .
$$

A primeira etapa do algoritmo consiste em conectar os blocos partindo do eletrodo esquerdo até o último segmento da região de espalhamento. Vamos considerar, assim como fizemos nos outros capítulos, que as autoenergias dos eletrodos já foram previamente calculadas (por exemplo, pelo programa SMEAGOL). Dessa maneira, o primeiro passo, que corresponde a conectar o eletrodo esquerdo ao primeiro bloco, resulta na seguinte equação de Dyson para o primeiro elemento da função de Green:

$$
G_{1,1}^{L(1)}=g_{1,1}^{r}+g_{1,1}^{r} \Sigma_{L}^{r} G_{1,1}^{L(1)}=\left[\varepsilon S_{1,1}-H_{1,1}-\Sigma_{L}^{r}\right]^{-1},
$$

onde trocamos $G^{r} \rightarrow G^{L}$ para salientar que essa função de Green leva em consideração somente o acoplamento com o eletrodo esquerdo e o índice entre parênteses indica a primeira iteração.

O passo seguinte consiste em incluir o acoplamento com o segundo bloco, de forma que o problema passa a ser a inversão da seguinte matriz:

$$
\left(\begin{array}{ccccc}
\bar{H}_{1,1}-\Sigma_{L}^{r} & \bar{V}_{1,2} & \cdots & 0 & 0 \\
\bar{V}_{2,1} & \bar{H}_{2,2} & \cdots & 0 & 0 \\
\vdots & \vdots & \ddots & \vdots & \vdots \\
0 & 0 & \cdots & \bar{H}_{n-1, n-1} & 0 \\
0 & 0 & \cdots & 0 & \bar{H}_{n, n}
\end{array}\right)
$$

Novamente podemos aplicar a inversão por blocos, de onde se obtém as seguintes expressões para o primeiro bloco:

$$
\begin{gathered}
\left(\bar{H}_{1,1}-\Sigma_{L}^{r}\right) G_{1,2}^{L(2)}+V_{1,2} G_{2,2}^{L(2)}=\mathbb{0}, \\
V_{2,1} G_{1,2}^{L(2)}+\bar{H}_{2,2} G_{2,2}^{L(2)}=\mathbb{1} .
\end{gathered}
$$


Como $\left(\bar{H}_{1,1}-\Sigma_{L}^{r}\right)=\left[G_{1,1}^{L(1)}\right]^{-1}$, a primeira expressão pode ser reescrita como:

$$
G_{1,2}^{L(2)}=-G_{1,1}^{L(1)} V_{1,2} G_{2,2}^{L(2)}
$$

Substituindo este resultado e reorganizando os termos na Equação 7.20, obtém-se:

$$
G_{2,2}^{L(2)}=g_{2,2}^{r}-g_{2,2}^{r} V_{2,1} G_{1,2}^{L(2)}=g_{2,2}^{r}-g_{2,2}^{r} V_{2,1} G_{1,1}^{L(1)} V_{1,2} G_{2,2}^{L(2)}
$$

Multiplicando à direita por $\left[G_{2,2}^{L(2)}\right]^{-1}$ e rearranjando os termos:

$$
G_{2,2}^{L(2)}=\left[\left(g_{2,2}^{r}\right)^{-1}-V_{2,1} G_{1,1}^{L(1)} V_{1,2}\right]^{-1}=\left[\varepsilon S_{2,2}-H_{2,2}-V_{2,1} G_{1,1}^{L(1)} V_{1,2}\right]^{-1} .
$$

Ao procedimento de conectar sucessivamente os blocos partindo do eletrodo esquerdo até o último segmento dá-se o nome de varredura pela esquerda. Ao longo dessa varredura, uma família de funções de Green $\left\{G^{L}\right\}$ é obtida e verificam-se as seguintes relações de recorrência para os elementos da diagonal e para os elementos correspondentes à conexão com o eletrodo esquerdo:

$$
\begin{gathered}
G_{i, i}^{L(i)}=\left[\varepsilon S_{i, i}-H_{i, i}-V_{i, i-1} G_{i-1, i-1}^{L(i-1)} V_{i-1, i}\right]^{-1}, \forall i \in\{2,3, \ldots, n\}, \\
G_{1, i}^{L(i)}=-G_{1, i-1}^{L(i-1)} V_{i-1, i} G_{i, i}^{L(n)}, \quad \forall i \in\{2,3, \ldots, n\} .
\end{gathered}
$$

De maneira semelhante, os passos são refeitos partindo-se do eletrodo direito. Com o sistema totalmente desacoplado, conecta-se o eletrodo direito ao último segmento da região de espalhamento, resultando na seguinte equação de Dyson para o último elemento da função de Green:

$$
G_{n, n}^{R(n)}=g_{n, n}^{r}+g_{n, n}^{r} \Sigma_{R}^{r} G_{n, n}^{R(n)}=\left[\varepsilon S_{n, n}-H_{n, n}-\Sigma_{R}^{r}\right]^{-1},
$$

onde o índice $R$ na função de Green indica que o sistema está acoplado somente com o eletrodo direito e onde, nesse caso, a iteração é indexada em ordem decrescente.

De maneira análoga ao que fizemos para obter as Equações 7.21 e 7.23 , pode-se mostrar que, ao conectar o penúltimo bloco, obtém-se: 


$$
\begin{gathered}
G_{n, n-1}^{R(n-1)}=-G_{n, n}^{R(n)} V_{n, n-1} G_{n-1, n-1}^{R(n-1)}, \\
G_{n-1, n-1}^{R(n-1)}=\left[\varepsilon S_{n-1, n-1}-H_{n-1, n-1}-V_{n-1, n} G_{n, n}^{R(n)} V_{n, n-1}\right]^{-1} .
\end{gathered}
$$

Repetindo o algoritmo até o primeiro segmento da região de espalhamento (varredura pela direita), obtém-se uma família de funções de Green $\left\{G^{R}\right\}$ e verifica-se que valem as seguintes relações de recorrência para os elementos da diagonal e para os elementos referentes à conexão com o eletrodo direito:

$$
\begin{gathered}
G_{i, i}^{R(i)}=\left[\varepsilon S_{i, i}-H_{i, i}-V_{i, i+1} G_{i+1, i+1}^{R(i+1)} V_{i+1, i}\right]^{-1}, \forall i \in\{1,2, \ldots, n-1\}, \\
G_{n, i}^{R(i)}=-G_{i+1, i+1}^{R(i+1)} V_{i+1, i} G_{i, i}^{R(i)}, \quad \forall i \in\{1,2, \ldots, n-1\} .
\end{gathered}
$$

Resta agora entender como obter a função de Green total exata do sistema a partir de $\left\{G^{L}\right\}$ e $\left\{G^{R}\right\}$. Para tanto, suponha que chegamos ao $i$-ésimo bloco, com $i \in\{2, \ldots, n-1\}$, por ambas as varreduras descritas acima. Antes de conectarmos o bloco aos seus vizinhos, todos os elementos da função Green correspondentes são nulos ( $i$-ésimas linha e coluna), exceto o elemento diagonal $g_{i, i}^{r}$ :

$$
G_{M}^{r}=\left(\begin{array}{ccccccccc}
G_{1,1}^{L(1)} & G_{1,2}^{L(2)} & \cdots & G_{1, i-1}^{L(i-1)} & 0 & 0 & \cdots & 0 & 0 \\
G_{2,1}^{L(2)} & G_{2,2}^{L(2)} & \cdots & G_{2, i-1}^{L(i-1)} & 0 & 0 & \cdots & 0 & 0 \\
\vdots & \vdots & \ddots & \vdots & \vdots & \vdots & \ddots & \vdots & \vdots \\
G_{i-1,1}^{L(i-1)} & G_{i-1,2}^{L(i-1)} & \cdots & G_{i-1, i-1}^{L(i-1)} & 0 & 0 & \cdots & 0 & 0 \\
0 & 0 & \cdots & 0 & g_{i, i}^{r} & 0 & \cdots & 0 & 0 \\
0 & 0 & \cdots & 0 & 0 & G_{i+1, i+1}^{R(i+1)} & \cdots & G_{i+1, n-1}^{R(i+1)} & G_{i+1, n}^{R(i+1)} \\
\vdots & \vdots & \ddots & \vdots & \vdots & \vdots & \ddots & \vdots & \vdots \\
0 & 0 & \cdots & 0 & 0 & G_{n-1, i+1}^{R(i+1)} & \cdots & G_{n-1, n-1}^{R(n-1)} & G_{n-1, n}^{R(n-1)} \\
0 & 0 & \cdots & 0 & 0 & G_{n, i+1}^{R(i+1)} & \cdots & G_{n, n-1}^{R(n-1)} & G_{n, n}^{R(n)}
\end{array}\right) .
$$

Ao inserirmos os acoplamentos $V_{i-1, i}$ e $V_{i, i+1}$, todos os blocos se tornam conectados e do sistema de equações representado pela Equação 7.15 destaca-se a seguinte expressão envolvendo o elemento $G_{i, i}^{r}$ da função de Green:

$$
V_{i, i-1} G_{i-1, i}^{r}+\bar{H}_{i, i} G_{i, i}^{r}+V_{i, i+1} G_{i+1, i}^{r}=\mathbb{1}
$$


onde:

$$
\begin{gathered}
G_{i-1, i}^{r}=-G_{i-1, i-1}^{L(i-1)} V_{i-1, i} G_{i, i}^{r}, \\
G_{i+1, i}^{r}=-G_{i+1, i+1}^{R(i+1)} V_{i+1, i} G_{i, i}^{r} .
\end{gathered}
$$

Substituindo as duas últimas expressões na Equação 7.32 e rearranjando os termos, obtém-se finalmente a expressão exata para a função de Green total do $i$-ésimo bloco:

$$
G_{i, i}^{r}=\left[\varepsilon S_{i, i}-H_{i, i}-V_{i, i-1} G_{i-1, i-1}^{L(i-1)} V_{i-1, i}-V_{i, i+1} G_{i+1, i+1}^{R(i+1)} V_{i+1, i}\right]^{-1} .
$$

Expressões para a função de Green total análogas às Equações 7.25 e 7.30 , correspondendo ao segmento conectado aos eletrodos esquerdo e direito, respectivamente, também podem ser obtidas:

$$
\begin{aligned}
& G_{1, i}^{r}=-G_{1, i-1}^{L(i-1)} V_{i-1, i} G_{i, i}^{r}, \\
& G_{n, i}^{r}=-G_{n, i+1}^{R(i+1)} V_{i+1, i} G_{i, i}^{r},
\end{aligned}
$$

Nota-se, portanto, que, para calcular os elementos $G_{i, i}^{r}, G_{1, i}^{r}$ e $G_{n, i}^{r}$ da função de Green total, é preciso ter calculado previamente os conjuntos $\left\{G^{L}\right\}$ e $\left\{G^{R}\right\}$. Contudo, para o cálculo da transmissão no transporte elástico, onde o único elemento relevante da função de Green é o $G_{1, n}^{r}$, bastaria somente realizar o procedimento de conectar os blocos partindo do eletrodo esquerdo e, após o último passo, conectar prontamente com o eletrodo direito, ou seja:

$$
\begin{aligned}
G_{1, n}^{r} & =-G_{1, n-1}^{L(n-1)} V_{n-1, n} G_{n, n}^{r}= \\
& =-G_{1, n-1}^{L(n-1)} V_{n-1, n}\left[\varepsilon S_{n, n}-H_{n, n}-V_{n, n-1} G_{n-1, n-1}^{L(n-1)} V_{n-1, n}-\Sigma_{R}^{r}\right]^{-1} .
\end{aligned}
$$

No entanto, como veremos na próxima seção, para o cálculo da transmissão no transporte com interação elétron-fônon são necessários os elementos $G_{i, i}^{r}, G_{1, i}^{r}$ e $G_{n, i}^{r}$, para todo segmento $i$ com interação inelástica, o que torna esse método ideal para o problema que queremos tratar. 


\subsection{Transporte Inelástico: Lowest Order Expansion - LOE}

Até aqui já vimos algumas abordagens para se incluir a interação elétron-fônon no transporte. O modelo fenomenológico de Büttiker (apresentado no Capítulo 5) é relativamente simples e sua implementação computacional é sistemática e eficiente. Porém, esse modelo inclui somente o efeito de perda de coerência de fase dos elétrons devido à interação com os fônons do material, o que produz um alargamento dos canais de condutância.

Já na aproximação de Born (Capítulo 6), são incluídas as informações a respeito do espectro dos fônons e seus acoplamentos com os elétrons. Na sua versão autoconsistente, a corrente é conservada, porém é extremamente custosa do ponto de vista computacional, sendo inviável simular com precisão sistemas maiores do que algumas dezenas de átomos.

Mostramos, contudo, que é possível reformular a primeira aproximação de Born de forma a viabilizar o cálculo de sistemas relativamente grandes (Seção 6.3). No entanto, o ponto fraco dessa aproximação é que ela não conserva corrente, o que é fundamental para uma teoria de transporte.

Nesta seção vamos apresentar outra abordagem, a Lowest Order Expansion, que consiste em aplicar teoria de perturbação com relação à matriz de acoplamento elétron-fônon na expressão para a corrente com interações, utilizando as autoenergias de interação obtidas na 1BA [53 55]. Aproximando as funções de Green e as funções largura de nível como independentes da energia (Wide-Band Limit), isto é, $G_{0}(\epsilon) \approx G_{0}\left(\varepsilon_{F}\right)$ e $\Gamma_{L, R}(\epsilon) \approx \Gamma_{L, R}\left(\varepsilon_{F}\right)$, onde $\varepsilon_{F}$ é a energia de Fermi, chega-se a uma expressão relativamente simples para a corrente usando expressões analíticas para integração. Como veremos, este método contém as boas características das abordagens anteriores, pois é barato computacionalmente, os efeitos devido à estrutura dos fônons são incluídos e a corrente é conservada.

\subsubsection{Expressão para a Corrente}

Ao considerar a teoria de perturbação de mais baixa ordem com relação à matriz de acoplamento elétron-fônon $M_{\lambda}$, podemos expandir a função de Green eletrônica como:

$$
G^{r}=G_{0}^{r}+G_{0}^{r} \Sigma_{p h}^{r} G_{0}^{r}
$$

Substituindo esta expansão na equação exata para a corrente com interação elétron-fônon (Equação 6.25), obtém-se: 


$$
\begin{aligned}
I_{L}= & \frac{2 e}{h} \int_{-\infty}^{+\infty} d \epsilon \operatorname{Tr}\left[\Gamma_{L} G^{r} \Gamma_{R} G^{a}\right]\left(f_{L}-f_{R}\right)+ \\
& +i \frac{2 e}{h} \int_{-\infty}^{+\infty} d \epsilon \operatorname{Tr}\left[G^{a} \Gamma_{L} G^{r}\left(f_{L} \Sigma^{F,>}+\left(1-f_{L}\right) \Sigma^{F,<}\right)\right] \approx \\
\approx & \frac{2 e}{h} \int_{-\infty}^{+\infty} d \epsilon \operatorname{Tr}\left[\Gamma_{L}\left(G_{0}^{r}+G_{0}^{r} \Sigma_{p h}^{r} G_{0}^{r}\right) \Gamma_{R}\left(G_{0}^{r}+G_{0}^{r} \Sigma_{p h}^{r} G_{0}^{r}\right)^{\dagger}\right]\left(f_{L}-f_{R}\right)+ \\
& +i \frac{2 e}{h} \int_{-\infty}^{+\infty} d \epsilon \operatorname{Tr}\left[\left(G_{0}^{r}+G_{0}^{r} \Sigma_{p h}^{r} G_{0}^{r}\right)^{\dagger} \Gamma_{L}\left(G_{0}^{r}+G_{0}^{r} \Sigma_{p h}^{r} G_{0}^{r}\right)\left(f_{L} \Sigma^{F,>}+\left(1-f_{L}\right) \Sigma^{F,<}\right)\right] .
\end{aligned}
$$

Considerando as autoenergias de interação obtidas com a 1BA (Equações 6.11 a 6.13), nota-se que a mais baixa ordem é dada por termos $O\left(M_{\lambda}^{2}\right)$, já que estas autoenergias são todas proporcionais a $M_{\lambda}^{2}$. Ignorando os termos de ordem superior, a corrente simplifica-se a:

$$
\begin{aligned}
I_{L} \approx & \frac{2 e}{h} \int_{-\infty}^{+\infty} d \epsilon \operatorname{Tr}\left[\Gamma_{L} G_{0}^{r} \Gamma_{R} G_{0}^{a}\right]\left(f_{L}-f_{R}\right)+ \\
& +\frac{4 e}{h} \int_{-\infty}^{+\infty} d \epsilon \operatorname{Re}\left\{\operatorname{Tr}\left[\Gamma_{L} G_{0}^{r} \Sigma_{p h}^{r} G_{0}^{r} \Gamma_{R} G_{0}^{a}\right]\right\}\left(f_{L}-f_{R}\right)+ \\
& +i \frac{2 e}{h} \int_{-\infty}^{+\infty} d \epsilon \operatorname{Tr}\left[G_{0}^{a} \Gamma_{L} G_{0}^{r}\left(f_{L} \Sigma^{F,>}+\left(1-f_{L}\right) \Sigma^{F,<}\right)\right],
\end{aligned}
$$

de onde se nota que a componente elástica se divide em um termo puramente elástico (primeira linha) e uma correção devido à interação elétron-fônon (segunda linha).

Na aproximação Wide-Band Limit, onde as funções de Green e as funções largura de nível são consideradas como independentes da energia $\left(G_{0}(\epsilon) \approx G_{0}\left(\varepsilon_{F}\right)\right.$ e $\left.\Gamma_{L, R}(\epsilon) \approx \Gamma_{L, R}\left(\varepsilon_{F}\right)\right)$, a expressão para a corrente simplifica-se consideravelmente e os termos dependentes da energia, que envolvem subtrações e produtos de distribuições de Fermi-Dirac, podem ser calculados analiticamente, chegando-se ao seguinte resultado: 


$$
\begin{aligned}
I_{L} \approx & \frac{2 e^{2}}{h} V \operatorname{Tr}\left[\Gamma_{L} G_{0}^{r} \Gamma_{R} G_{0}^{a}\right]+ \\
& +\sum_{\lambda} \mathcal{I}^{S y m}\left(V, \hbar \omega_{\lambda}, T, n_{\lambda}\right) \operatorname{Tr}\left[G_{0}^{a} \Gamma_{L} G_{0}^{r}\left\{M_{\lambda} G_{0}^{r} \Gamma_{R} G_{0}^{a} M_{\lambda}+\frac{i}{2}\left(\Gamma_{R} G_{0}^{a} M_{\lambda} A_{0} M_{\lambda}-\text { h.c. }\right)\right\}\right]+ \\
& +\sum_{\lambda} \mathcal{I}^{\text {Asym }}\left(V, \hbar \omega_{\lambda}, T\right) \operatorname{Tr}\left[G_{0}^{a} \Gamma_{L} G_{0}^{r}\left\{\Gamma_{R} G_{0}^{a} M_{\lambda} G_{0}^{r}\left(\Gamma_{R}-\Gamma_{L}\right) G_{0}^{a} M_{\lambda}+\text { h.c. }\right\}\right]
\end{aligned}
$$

onde os coeficientes $\mathcal{I}^{\text {Sym }}$ e $\mathcal{I}^{\text {Asym }}$ são, respectivamente, simétricos e assimétricos sob reversão da diferença de potencial $V$ e dados por:

$$
\begin{gathered}
\mathcal{I}^{\text {Sym }}\left(V, \hbar \omega_{\lambda}, T, n_{\lambda}\right)=\frac{2 e}{h}\left(2 e V\left\langle n_{\lambda}\right\rangle+\frac{\hbar \omega_{\lambda}-e V}{e^{\beta\left(\hbar \omega_{\lambda}-e V\right)}-1}-\frac{\hbar \omega_{\lambda}+e V}{e^{\beta\left(\hbar \omega_{\lambda}+e V\right)}-1}\right), \\
\mathcal{I}^{A s y m}\left(V, \hbar \omega_{\lambda}, T\right)=\frac{e}{h} \int_{-\infty}^{+\infty} d \epsilon[f(\epsilon)-f(\epsilon-e V)] \mathcal{H}_{\epsilon^{\prime}}\left\{f\left(\epsilon^{\prime}+\hbar \omega_{\lambda}\right)-f\left(\epsilon^{\prime}-\hbar \omega_{\lambda}\right)\right\}(\epsilon),
\end{gathered}
$$

onde $\beta=\left(k_{B} T\right)^{-1}$ e $\left\langle n_{\lambda}\right\rangle$ é a ocupação dos fônons de modo $\lambda$ que será discutida mais à frente (Seção 7.4.3).

Nesta derivação, a contribuição do diagrama de Hartree foi omitida, pois ela aparece somente como correções no espalhamento elástico que não geram sinais adicionais na LOE nas energias dos fônons [55]. A demonstração da Equação 7.42 é bastante extensa. Porém, como não foi encontrada referência para se indicar, seus detalhes foram dispostos no Apêndice C.

É interessante notar que as funções $\mathcal{I}^{\text {Sym }}$ e $\mathcal{I}^{\text {Asym }}$, por si só, já levam a uma curva característica de espalhamento inelástico (Figura 7.3). O termo $\mathcal{I}^{\text {Sym }}$ gera degraus na condutância, simétricos com relação à reversão da tensão. O sinal desse degrau é determinado pelo sinal do traço do termo simétrico (segunda linha da Equação 7.42). Em geral, esse termo gera um aumento na condutância em sistemas com baixa condutividade, como moléculas, e um decréscimo em sistemas com alta condutividade, como fios atômicos.

Já o termo $\mathcal{I}^{\text {Asym }}$ gera picos e vales na condutância, assimétricos com relação à reversão da tensão. Em geral, esse termo produz uma contribuição pequena quando comparado ao termo simétrico e pode-se mostrar que em sistemas geometricamente simétricos esta contribuição é nula [54. Além disso, verifica-se na Figura 7.3 que ambas as funções simétrica e assimétrica também incorporam o efeito do alargamento do sinal vibracional devido ao aumento da temperatura.

A aproximação LOE é adequada a sistemas onde o acoplamento elétron-fônon é fraco e onde a densidade de estados varia lentamente com a energia. Isso é aplicável a uma grande quantidade 

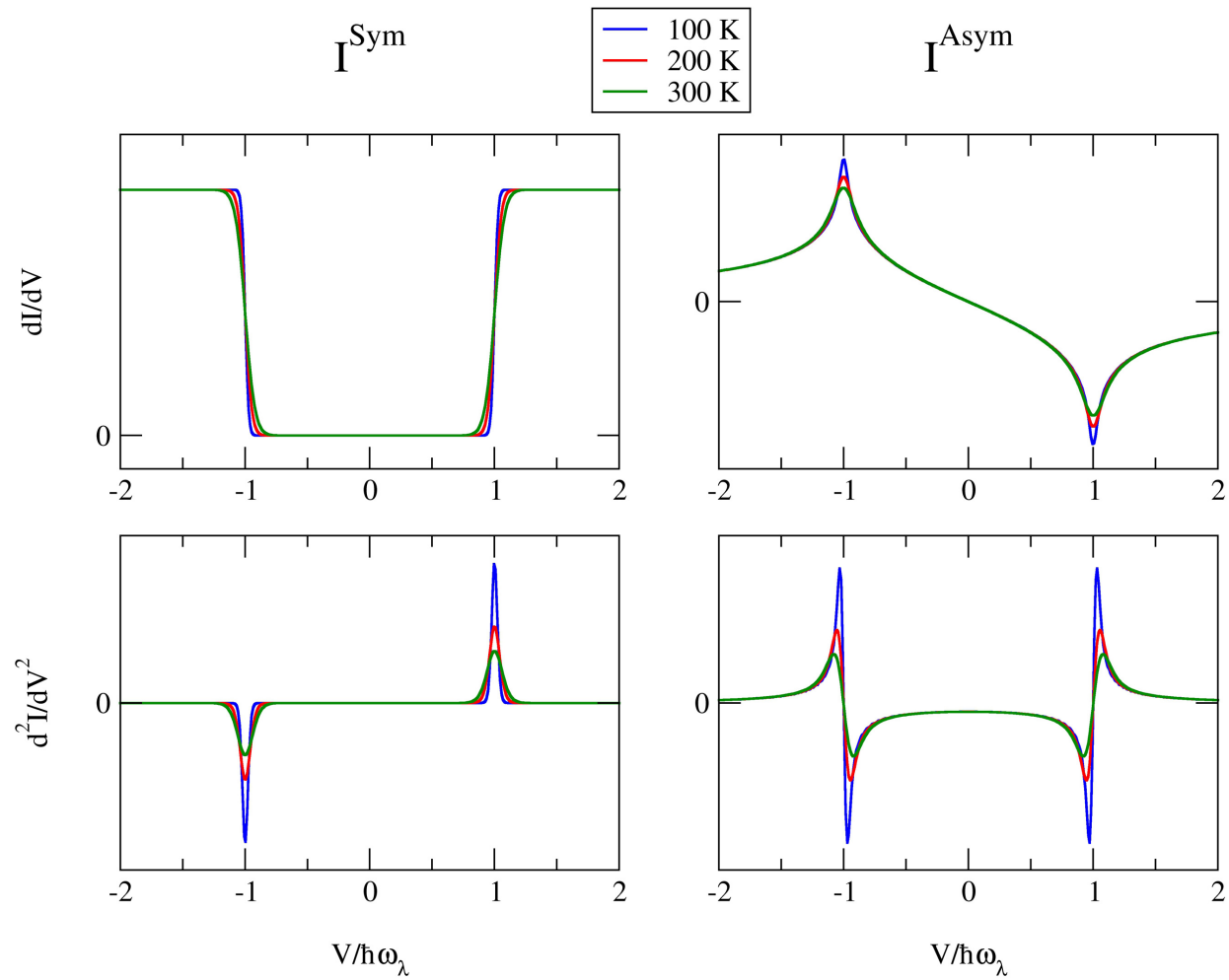

Figura 7.3: Primeira e segunda derivadas das funções $\mathcal{I}^{\text {Sym }}$ e $\mathcal{I}^{\text {Asym }}$ com relação à tensão $V$, para três temperaturas distintas, obtidas com o programa I-DISORDER (Seção 7.5).

de sistemas em escala nanométrica, já que em geral nesses sistemas o elétron permanece pouco tempo na região de espalhamento comparado ao tempo de espalhamento por fônons e os contatos são metálicos, com a densidade de estados variando lentamente com a energia. Contudo, mesmo em sistemas em que a variação da densidade de estados é da ordem das energias dos modos vibracionais, a aproximação LOE apresenta bons resultados quando comparada à SCBA 78.

\subsubsection{Potência Dissipada}

A derivação do fluxo de energia pode ser realizada de forma análoga à expressão geral para a corrente na Seção 4.4. A energia que flui do contato $\alpha$ para a região central é definida como:

$$
P_{\alpha} \equiv-\left\langle\frac{\partial H_{C}^{\alpha}}{\partial t}\right\rangle=-\frac{i}{\hbar}\left\langle\left[\mathscr{H}, H_{C}^{\alpha}\right]\right\rangle=\frac{i}{\hbar} \sum_{n, k}\left(E_{n \alpha}-\mu_{\alpha}\right)\left(V_{n \alpha, k}\left\langle d_{n \alpha}^{\dagger} c_{k}\right\rangle-V_{n \alpha, k}^{*}\left\langle c_{k}^{\dagger} d_{n \alpha}\right\rangle\right)
$$

onde, lembrando, $\mathscr{H}$ é a Hamiltoniana total do sistema dada pela Equação 4.9 e $\mu_{\alpha}$ é a referência pela qual as energias de partícula única são medidas no eletrodo $\alpha$. De modo análogo à derivação da corrente, as mesmas passagens podem ser realizadas de forma a obter-se: 


$$
\begin{aligned}
P_{\alpha} & =\frac{1}{h} \int_{-\infty}^{+\infty} d \epsilon\left(\epsilon-\mu_{\alpha}\right) \operatorname{Tr}\left[\Sigma_{\alpha}^{<}(\epsilon) G^{>}(\epsilon)-\Sigma_{\alpha}^{>}(\epsilon) G^{<}(\epsilon)\right]= \\
& =\frac{1}{h} \int_{-\infty}^{+\infty} d \epsilon \epsilon \operatorname{Tr}\left[\Sigma_{\alpha}^{<}(\epsilon) G^{>}(\epsilon)-\Sigma_{\alpha}^{>}(\epsilon) G^{<}(\epsilon)\right]-\mu_{\alpha} I_{\alpha}
\end{aligned}
$$

A potência total transferida à região de espalhamento é dada pela soma dos fluxos dos eletrodos:

$$
\begin{aligned}
P_{L}+P_{R}= & \frac{1}{h} \int_{-\infty}^{+\infty} d \epsilon \epsilon \operatorname{Tr}\left[\left(\Sigma_{L}^{<}(\epsilon)+\Sigma_{R}^{<}(\epsilon)\right) G^{>}(\epsilon)-\left(\Sigma_{L}^{>}(\epsilon)+\Sigma_{R}^{>}(\epsilon)\right) G^{<}(\epsilon)\right]- \\
& -\left(\mu_{L} I_{L}+\mu_{R} I_{R}\right) .
\end{aligned}
$$

Para o sistema como um todo, as energias de partícula única devem ser medidas em termos de um mesmo valor de referência, de forma que $\mu_{\alpha}=\mu_{r e f}$. Logo, o último termo da expressão desaparece por conservação de corrente. A partir da condição de conservação de partículas (Equação 6.29), a potência total pode ser escrita como:

$$
P_{L}+P_{R}=P_{p h}=-\frac{1}{h} \int_{-\infty}^{+\infty} d \epsilon \epsilon \operatorname{Tr}\left[\Sigma_{p h}^{<}(\epsilon) G^{>}(\epsilon)-\Sigma_{p h}^{>}(\epsilon) G^{<}(\epsilon)\right]
$$

Portanto, a potência transferida pelos elétrons nos eletrodos ao dispositivo é contrabalanceada pela potência transferida pelos elétrons do dispositivo aos fônons. Na ausência de interação elétron-fônon, não ocorre dissipação dentro da região de espalhamento e os elétrons dissipam sua energia somente nos reservatórios.

A mesma abordagem utilizada para a derivação da corrente na aproximação LOE pode ser aplicada para obter-se uma expressão da potência dissipada no sistema de fônons [54], de onde se obtém:

$$
P_{p h} \approx \sum_{\lambda} P_{\lambda}=\sum_{\lambda} \hbar \omega_{\lambda}\left\{\left[n_{B_{\lambda}}-\left\langle n_{\lambda}\right\rangle\right] \gamma_{e h}^{\lambda}+\gamma_{e m}^{\lambda}(V, T)\right\}
$$

onde os termos $\gamma_{e h}^{\lambda}$ e $\gamma_{e m}^{\lambda}(V, T)$ são:

$$
\gamma_{e h}^{\lambda}=\frac{\hbar \omega_{\lambda}}{\pi \hbar} \operatorname{Tr}\left[M_{\lambda} A_{0} M_{\lambda} A_{0}\right]
$$




$$
\gamma_{e m}^{\lambda}(V, T)=\frac{\hbar \omega_{\lambda}\left[\cosh \left(\frac{e V}{k_{B} T}\right)-1\right] \operatorname{coth}\left(\frac{\hbar \omega_{\lambda}}{2 k_{B} T}\right)-e V \sinh \left(\frac{e V}{k_{B} T}\right)}{\pi \hbar\left[\cosh \left(\frac{\hbar \omega_{\lambda}}{k_{B} T}\right)-\cosh \left(\frac{e V}{k_{B} T}\right)\right]} \operatorname{Tr}\left[M_{\lambda} G_{0}^{r} \Gamma_{L} G_{0}^{a} M_{\lambda} G_{0}^{r} \Gamma_{R} G_{0}^{a}\right] .
$$

O primeiro termo da expressão para a potência dissipada descreve a troca de energia no equilíbrio entre os graus de liberdade vibracional e eletrônico, com uma taxa de amortecimento de elétron-buraco $\gamma_{e h}^{\lambda}$ que tende a conduzir o sistema dos fônons à distribuição de Bose-Einstein $n_{B_{\lambda}}$. O termo fora do equilíbrio está relacionado a uma taxa de emissão efetiva $\gamma_{e m}^{\lambda}$ de fônons sob uma tensão finita. Esta taxa é desprezível a baixas tensões $\left(e V \ll \hbar \omega_{\lambda}\right)$, passa a ser considerável a partir da energia do fônon e torna-se linear a altas voltagens $\left(e V \gg \hbar \omega_{\lambda}\right)$ [55].

\subsubsection{Ocupação dos Fônons}

Para reproduzir curvas experimentais de condutância diferencial (como as curvas das Figuras 1.1 e 1.2, é preciso, em alguns casos, considerar o aquecimento do sistema de fônons 105. Uma primeira aproximação para este aquecimento fora do equilíbrio consiste em considerar a variação da ocupação dos fônons [54], incluindo uma taxa de amortecimento externo $\gamma_{d}^{\lambda}$ :

$$
\left\langle\frac{\partial n_{\lambda}}{\partial t}\right\rangle=\frac{P_{\lambda}}{\hbar \omega_{\lambda}}+\gamma_{d}^{\lambda}\left[n_{B_{\lambda}}-\left\langle n_{\lambda}\right\rangle\right]
$$

onde o amortecimento $\gamma_{d}^{\lambda}$ é um parâmetro fenomenológico relacionado à vida média do fônon. Na condição de estado estacionário ${ }^{4}$ tem-se:

$$
\frac{P_{\lambda}}{\hbar \omega_{\lambda}}=-\gamma_{d}^{\lambda}\left[n_{B_{\lambda}}-\left\langle n_{\lambda}\right\rangle\right]
$$

Comparando com expressão obtida para potência dissipada na LOE (Equação 7.49), podemos escrever:

$$
\left[n_{B_{\lambda}}-\left\langle n_{\lambda}\right\rangle\right] \gamma_{e h}^{\lambda}+\gamma_{e m}^{\lambda}=-\gamma_{d}^{\lambda}\left[n_{B_{\lambda}}-\left\langle n_{\lambda}\right\rangle\right]
$$

de onde, rearranjando os termos, obtém-se a ocupação do modo $\lambda$ :

\footnotetext{
${ }^{4}$ Como a potência dissipada inclui tanto o processo de emissão de fônons como o de absorção, a solução de estado estacionário sempre irá existir.
} 


$$
\left\langle n_{\lambda}\right\rangle=n_{B_{\lambda}}+\frac{\gamma_{e m}^{\lambda}}{\gamma_{d}^{\lambda}+\gamma_{e h}^{\lambda}}
$$

Analisando a expressão obtida, verifica-se a existência de dois limites distintos. Primeiro, temos o limite de amortecimento externo quando se considera $\gamma_{d}^{\lambda} \gg \gamma_{e h}^{\lambda}$. Neste caso, a energia transferida pelos elétrons aos fônons é instantaneamente absorvida por um reservatório térmico externo e a ocupação é fixada pela distribuição de Bose-Einstein, isto é, $\left\langle n_{\lambda}\right\rangle=n_{B_{\lambda}}$.

Já o limite sem amortecimento externo é obtido tomando-se $\gamma_{d}^{\lambda} \rightarrow 0$ :

$$
\left\langle n_{\lambda}\right\rangle=n_{B_{\lambda}}+\frac{\gamma_{e m}^{\lambda}}{\gamma_{e h}^{\lambda}}
$$

Nesse caso, a ocupação varia com a tensão de forma a que nenhuma potência seja dissipada no dispositivo $\left(P_{\lambda}=0\right)$.

\subsubsection{Sistemas Desordenados}

Para entender como a LOE pode ser aplicada a sistemas desordenados, vamos fazer uma análise semelhante à que fizemos para o transporte elástico, utilizando o exemplo da Figura 7.2 para ilustrar a metodologia. Para que o problema possa ser dividido em blocos, como na Equação 7.9 , é preciso considerar que as interações elétron-fônon sejam localizadas nos defeitos. Usualmente, a matriz de acoplamento elétron-fônon é calculada considerando uma pequena região em torno do defeito, possuindo, no máximo, as dimensões do segmento.

No entanto, espera-se que o grau de dificuldade neste caso seja maior, visto que, além do termo de transmissão elástica, a expressão para a corrente na LOE possui mais termos envolvendo a multiplicação de matrizes. De fato, analisando os produtos matriciais nas expressões de corrente e potência dissipada na LOE, verifica-se que, para cada segmento $i$ onde são consideradas as interações elétron-fônon, são necessários os elementos $G_{i, i}^{r}, G_{1, i}^{r}$ e $G_{n, i}^{r}$ da função de Green total, além do elemento $G_{1, n}^{r}$ usado no cálculo da transmissão elástica.

No exemplo da nanofita de grafeno representada pela Figura 7.2 , considerando que a interação elétron-fônon ocorra somente nos segmentos com defeito, isto é, no segundo e no quarto bloco, é preciso calcular os seguintes elementos da função de Green total destacados em vermelho: 


$$
\left(\begin{array}{ccccc}
\bar{H}_{P}-\Sigma_{L}^{r} & \bar{V}_{1,2} & 0 & 0 & 0 \\
\bar{V}_{2,1} & \bar{H}_{D}^{1} & \bar{V}_{2,3} & 0 & 0 \\
0 & \bar{V}_{3,2} & \bar{H}_{P} & \bar{V}_{3,4} & 0 \\
0 & 0 & \bar{V}_{4,3} & \bar{H}_{D}^{2} & \bar{V}_{4,5} \\
0 & 0 & 0 & \bar{V}_{5,4} & \bar{H}_{P}-\Sigma_{R}^{r}
\end{array}\right)\left(\begin{array}{ccccc}
G_{1,1}^{r} & \mathrm{G}_{1,2}^{\mathrm{r}} & G_{1,3}^{r} & \mathrm{G}_{1,4}^{\mathrm{r}} & \mathrm{G}_{1,5}^{\mathrm{r}} \\
G_{2,1}^{r} & \mathrm{G}_{2,2}^{\mathrm{r}} & G_{2,3}^{r} & G_{2,4}^{r} & G_{2,5}^{r} \\
G_{3,1}^{r} & G_{3,2}^{r} & G_{3,3}^{r} & G_{3,4}^{r} & G_{3,5}^{r} \\
G_{4,1}^{r} & G_{4,2}^{r} & G_{4,3}^{r} & \mathrm{G}_{4,4}^{\mathrm{r}} & G_{4,5}^{r} \\
G_{5,1}^{r} & \mathrm{G}_{5,2}^{\mathrm{r}} & G_{5,3}^{r} & \mathrm{G}_{5,4}^{\mathrm{r}} & G_{5,5}^{r}
\end{array}\right)=\mathbb{1} .
$$

Logo, o número de elementos da função de Green necessários para o cálculo do transporte inelástico cresce linearmente com a quantidade de segmentos com interação elétron-fônon. Porém, com o método recursivo apresentado na Seção 7.3 , uma vez realizadas as varreduras pela esquerda e pela direita, os elementos assinalados podem ser prontamente calculados por meio das Equações 7.357 .37 .

\subsection{O Programa I-DISORDER}

Nesta seção serão discutidos os detalhes e estratégias utilizadas na implementação do programa Inelastic DISORDER para cálculos ab initio de transporte elástico e inelástico com a aproximação LOE. Um pequeno manual, com os detalhes de execução e as opções permitidas ao usuário, está disposto no Apêndice F.

A princípio, a metodologia descrita neste capítulo independe da origem do Hamiltoniano eletrônico. Porém, conforme foi discutido nos capítulos anteriores, uma forma precisa de descrever a estrutura eletrônica dos dispositivos é por meio da Teoria do Funcional da Densidade. Dessa forma, no programa I-DISORDER escolheu-se utilizar como entrada as matrizes Hamiltoniana e de overlap geradas a partir do cálculo autoconsistente do código SIESTA (Seção 3.2.4).

Portanto, em uma etapa preliminar, para cada diferente espécie de segmento a ser utilizado, realiza-se um cálculo de estrutura eletrônica com o segmento repetido periodicamente na direção do transporte. Nesse cálculo são armazenadas as matrizes Hamiltoniana $H_{i}$ e de overlap $S_{i}$ de cada célula unitária assim como os respectivos acoplamentos $H_{i j}$ e $S_{i j}$. Além disso, todo bloco é construído de forma a que o acoplamento entre dois segmentos seja sempre igual ao de um sistema pristino $\bar{V}_{0}$, isto é:

$$
\bar{V}_{i, j}={ }_{N}^{M^{\prime}-N}\left(\begin{array}{cc}
N & M-N \\
0 & 0 \\
\bar{V}_{0} & 0
\end{array}\right),
$$


onde $N$ é a dimensão do sistema sem defeitos e $M$ e $M^{\prime}$ são as dimensões de dois blocos adjacentes. Assim, todos os blocos possuem ao menos um segmento do sistema pristino em suas extremidades e, portanto, as matrizes obtidas no cálculo de estrutura eletrônica podem ser identificadas diretamente como os blocos constituintes da Hamiltoniana total do sistema (como os da Equação 7.9).

Além das Hamiltonianas e acoplamentos, o programa também deve receber, para cada segmento com interação elétron-fônon, as frequências dos modos vibracionais $\omega_{\lambda}$ e as matrizes de acoplamento elétron-fônon $M_{\lambda}$ previamente calculadas com o programa PhOnonS ITeratIVE VIBRATIONS (Seção 3.3.1).

A quantidade de segmentos que constituirão a região de espalhamento e o número de blocos com defeito também devem ser passados ao programa. No caso de diferentes tipos de defeitos, o usuário fornece um percentual para cada tipo. Dessa forma, uma vez lidos os parâmetros de entrada, o programa gera aleatoriamente o sistema a ser calculado de acordo com as quantidades e as distribuições de probabilidades dos defeitos fornecidas.

O passo seguinte vem a ser o cálculo recursivo das funções de Green de acordo com o método descrito na Seção 7.3. Em posse dos elementos necessários da função de Green total do sistema, o programa calcula a corrente e a potência dissipada na aproximação Lowest Order Expansion (Seção 7.4).

Vimos que na LOE as funções de Green e as funções largura de nível são calculadas no nível de Fermi. Porém, o usuário pode fornecer um intervalo de energia em que estas funções são calculadas, simulando o efeito de deslocamento do nível de Fermi devido, por exemplo, a uma tensão de porta. Nesse caso, o grid de energia é paralelizado via Message Passing Interface (MPI) 87] e os cálculos para cada energia podem ser divididos pelos processadores.

\subsubsection{Observações sobre Eficiência Computacional}

Assim como nos demais programas implementados neste trabalho, diversos cuidados foram tomados no desenvolvimento do I-DISORDER a fim de obter um melhor desempenho. Porém, no caso de sistemas desordenados com transporte inelástico, uma atenção especial foi dedicada à utilização da memória. A maior demanda ocorre no cálculo dos elementos da função de Green total do sistema por meio das Equações 7.357 .37 , já que é necessário armazenar os conjuntos de funções de Green $\left\{G^{L}\right\}$ e $\left\{G^{R}\right\}$ obtidos nas varreduras pela esquerda e pela direita, respectivamente.

Para termos uma ideia quantitativa da utilização de memória, vamos supor um exemplo em que os blocos sejam descritos por matrizes com 2000 orbitais atômicos ( 170 átomos, como é o 
caso das nanofitas de grafeno simuladas neste trabalho, cujos resultados serão apresentados no próximo capítulo). Dessa forma, cada elemento da função de Green total é dado por uma matriz com 4 milhões de números complexos (16 B em precisão dupla), o que significa aproximadamente 60 MB de memória. Para um sistema constituído por 200 blocos, é preciso armazenar $2 \times 200$ elementos da função de Green em cada varredura (200 elementos diagonais e outros 200 elementos referentes à conexão com o eletrodo). Portanto, considerando somente a memória armazenada após as varreduras, para simular este sistema são necessários $\sim 47$ GB de memória. Se o cálculo for realizado com diferentes valores de nível de Fermi utilizando a paralelização do programa com 16 processadores, por exemplo, este saldo de memória sobe para 750 GB. Ou seja, o cálculo fica rapidamente impraticável para a maioria dos clusters atuais.

O primeiro passo para solucionar este problema foi armazenar somente as funções de Green $\left\{G^{L}\right\}$ e $\left\{G^{R}\right\}$ necessárias para o cálculo dos elementos da função de Green total utilizados na LOE. Dessa forma, ao invés de guardar quatro elementos de função de Green para cada um dos blocos, esses elementos são armazenados somente para os segmentos com interação elétron-fônon.

Com esta observação, a utilização de memória foi significativamente reduzida. No entanto, quando o sistema estudado contém uma grande concentração de blocos com interação elétronfônon, a demanda de memória continua a ser um problema. Logo, a solução encontrada foi o armazenamento das funções de Green em disco.

Como se sabe, o acesso a disco gera uma perda de performance quando comparado ao acesso a memória. Entretanto, dependendo da forma como os dados são armazenados e de como o programa acessa estes dados, sequencialmente ou de forma aleatória, o tempo gasto no acesso a disco pode ser de 6 a 100.000 vezes maior quando comparado à memória RAM [106]. No I-DISORDER, as funções de Green são armazenadas em arquivos não formatados (binários) com acesso direto, onde cada matriz armazenada representa um ponto de acesso indexado como um vetor. O acesso direto é importante para poder escrever na ordem inversa o arquivo contendo as funções de Green provenientes da varredura pela direita $\left\{G^{R}\right\}$. Dessa forma, todos os arquivos são lidos sequencialmente, minimizando os custos com o acesso a disco.

Além da questão da memória, outro fator importante no desempenho do código é o custo computacional. Neste caso, a maior demanda provém das multiplicações e inversões de matrizes realizadas no cálculo recursivo das funções de Green (Seção 7.3). Por exemplo, supondo um sistema com $n$ blocos de dimensão $p$, então cada uma das varreduras realizam $O\left(n p^{3}\right)$ operações.

Portanto, para uma boa performance do código, é fundamental utilizar algoritmos otimizados para inversão e multiplicação, sempre levando em consideração as propriedades das matrizes envolvidas (ver discussão na Seção 6.5.1). No I-DISORDER, todas essas operações são reali- 
zadas por meio de rotinas externas otimizadas para multiplicação (BLAS - Basic Linear Algebra Subprograms [102]) e inversão (LAPACK - Linear Algebra PACKage [103]) de matrizes. Inclusive a ordem das multiplicações de matrizes foi escolhida de forma a diminuir a quantidade de operações.

A dimensão das matrizes envolvidas também foi levada em consideração. Conforme discutido na Seção 7.4.4 para cada segmento $i$ com interação elétron-fônon, somente os elementos $G_{i, i}^{r}$, $G_{1, i}^{r}$ e $G_{n, i}^{r}$ da função de Green total são necessários para os cálculos na LOE (além do elemento $G_{1, n}^{r}$ da transmissão elástica). Se considerarmos, por exemplo, um sistema desordenado com defeitos de um único tipo, a matriz de acoplamento elétron-fônon total para um dado modo vibracional $\lambda$ possui a seguinte estrutura:

$$
\left(\begin{array}{cccccc}
0 & 0 & 0 & 0 & 0 & \cdots \\
0 & M_{\lambda}^{(2)} & 0 & 0 & 0 & \cdots \\
0 & 0 & 0 & 0 & 0 & \cdots \\
0 & 0 & 0 & 0 & 0 & \ldots \\
0 & 0 & 0 & 0 & M_{\lambda}^{(5)} & \ldots \\
\vdots & \vdots & \vdots & \vdots & \vdots & \ddots
\end{array}\right)
$$

Contudo, nos segmentos com o defeito somente alguns átomos são movidos durante o cálculo de constantes de força para a análise vibracional. Assim, a matriz $M_{\lambda}^{(i)}$ possui todos os elementos nulos, com exceção de um bloco diagonal correspondendo ao subconjunto dos $m$ orbitais que foram efetivamente deslocados, conforme ilustrado na Figura 7.4 .

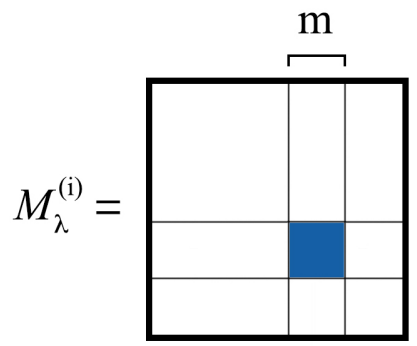

Figura 7.4: Ilustração da matriz $M_{\lambda}^{(i)}$ destacando o bloco de elementos não-nulos correspondente ao subconjunto dos $m$ orbitais deslocados no cálculo de constantes de força.

Portanto, as dimensões dos elementos $G_{i, i}^{r}, G_{1, i}^{r}$ e $G_{n, i}^{r}$ são restringidas pela dimensão $m$ da matriz de acoplamento elétron-fônon $M_{\lambda}^{(i)}$. Essa observação traz não só um ganho de performance mas também reduz a quantidade de memória utilizada. 


\subsection{Resultados}

Os objetivos desta seção são, primeiro, analisar os tipos de efeitos observados com a aproximação LOE no transporte eletrônico e, segundo, discutir o efeito de localização em sistemas desordenados. Para tanto, mostraremos os resultados obtidos para cadeias atômicas unidimensionais com um nível eletrônico por sítio contendo os defeitos ilustrados na Figura 7.5 .
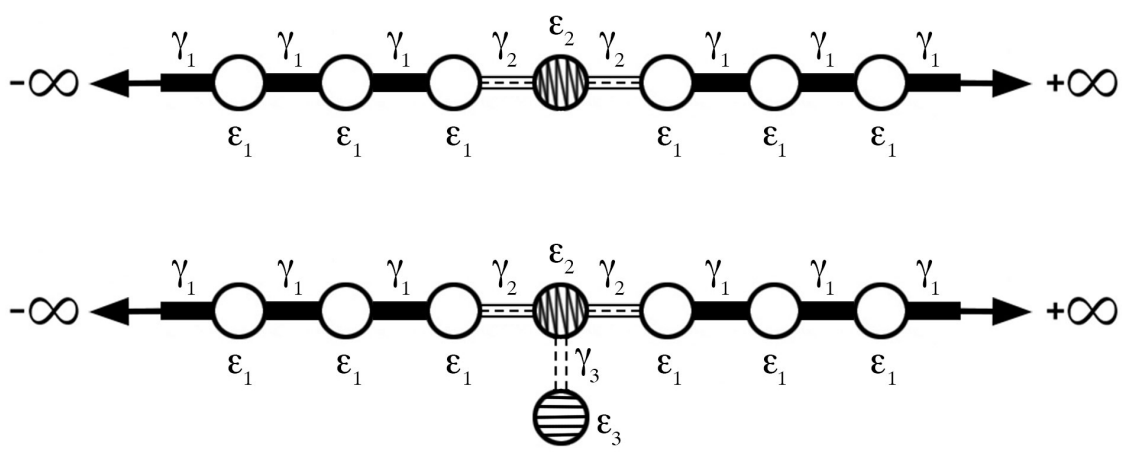

Figura 7.5: Representação dos dois tipos de defeitos permitidos pelo I-DISORDER para o cálculo de uma cadeia unidimensional. Acima, um defeito simples em um dos sítios da cadeia e, abaixo, o defeito simples com uma impureza "dangling bond".

Em vista disso, o I-DISORDER foi modificado de forma a oferecer a possibilidade de cálculos de cadeias unidimensionais com um nível eletrônico por sítio (ver Seção 5.5.1), onde podem ser utilizados os dois tipos de defeitos apresentados na Figura 7.5. Nesse caso, o usuário deve fornecer a energia de Fermi $\left(\varepsilon_{F}\right)$, as energias de sítio e acoplamentos do sistema pristino $\left(\varepsilon_{1} \mathrm{e}\right.$ $\left.\gamma_{1}\right)$ e dos defeitos $\left(\varepsilon_{2}\right.$ e $\gamma_{2}$ para o defeito simples e $\varepsilon_{3}$ e $\gamma_{3}$ para a dangling bond $)$ e o próprio programa constrói as Hamiltonianas e acoplamentos dos blocos.

Nas simulações cujos resultados serão apresentados a seguir foram utilizadas as energias e acoplamentos dispostos na Tabela 7.1

\begin{tabular}{ccccccc}
\hline$\varepsilon_{F}$ & $\varepsilon_{1}$ & $\gamma_{1}$ & $\varepsilon_{2}$ & $\gamma_{2}$ & $\varepsilon_{3}$ & $\gamma_{3}$ \\
\hline 0,51 & 0,01 & 5,00 & 0,51 & 0,50 & 0,61 & 0,20 \\
\hline
\end{tabular}

Tabela 7.1: Energias e acoplamentos, em $\mathrm{eV}$, utilizados nas simulações das cadeias unidimensionais na LOE, onde o indice 1 corresponde ao sistema pristino, 2, ao defeito simples e 3, ao defeito "dangling bond". 
Nota-se que os sítios do sistema puro $\varepsilon_{1}$ são fortemente acoplados $\left(\gamma_{1}=5,0 \mathrm{eV}\right)$, levando a uma largura de banda de $10 \mathrm{eV}$. Os defeitos, ao contrário, se acoplam fracamente a cadeia $\left(\gamma_{2}=0,5 \mathrm{eV}\right)$ e produzem uma quebra de simetria de translação do sistema.

Nos resultados apresentados a seguir, os sistemas foram simulados a uma temperatura de $77 \mathrm{~K}$ e foi considerado somente um fônon por defeito com energia $\hbar \omega_{\lambda}=1 \mathrm{eV}$ e acoplamento $M_{\lambda}=0,05 \mathrm{eV}$. No defeito simples, o fônon foi considerado no sítio $\varepsilon_{2}$, enquanto que, na dangling bond, o fônon foi considerado somente no sítio pendente $\varepsilon_{3}$.

Inicialmente foram estudados os efeitos da LOE nas características $I-V$, na condutância diferencial $d I / d V$ e na derivada da condutância $d^{2} I / d V^{2}$, considerando-se a cadeia com apenas um defeito. Na Figura 7.6 estão dispostos os resultados de corrente e condutância diferencial obtidos para o defeito simples variando-se a tensão e a energia.$^{5}$
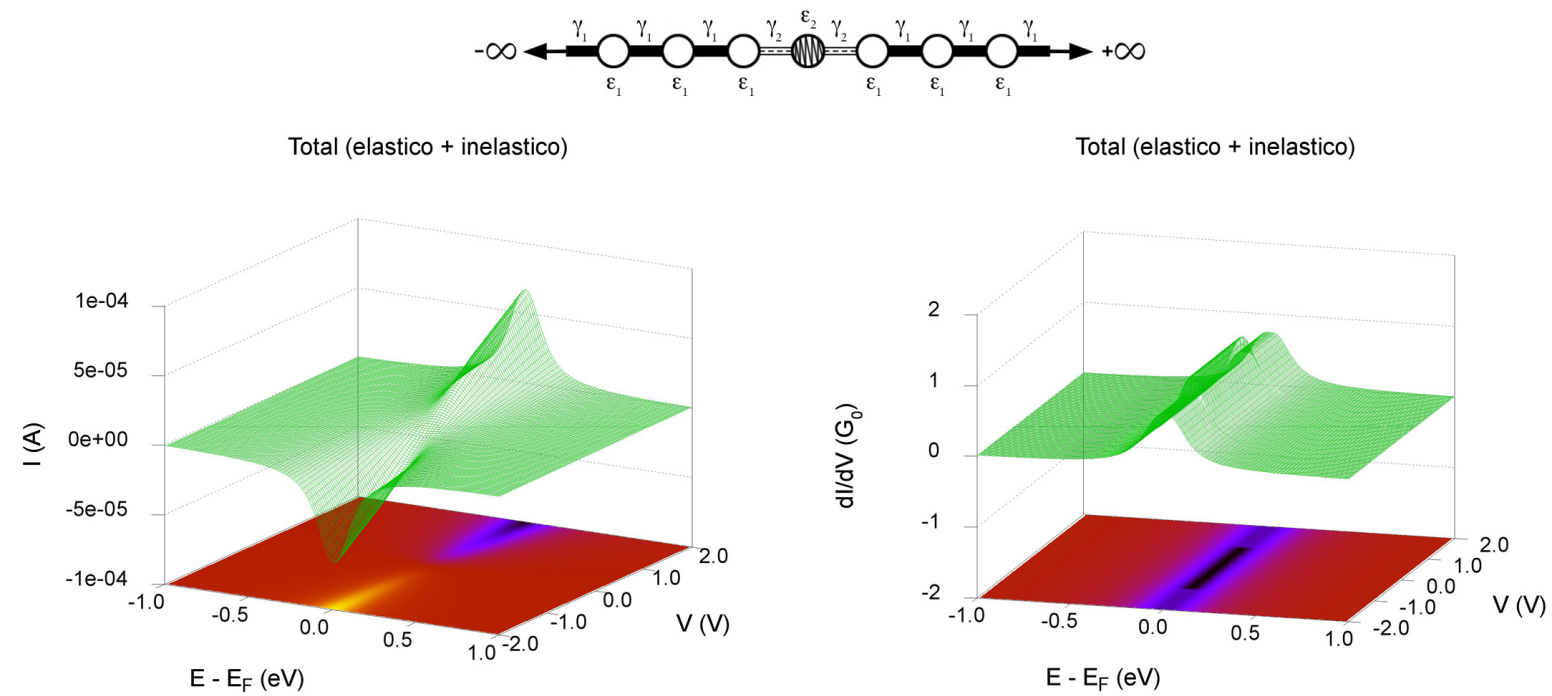

Figura 7.6: Corrente total (esquerda) e condutância diferencial total (direita), em função da tensão e da energia, obtidas para uma cadeia contendo um único defeito simples.

Nota-se que a corrente possui um valor máximo quando calculada na energia da ressonância $\left(E=\varepsilon_{2}\right)$ e apresenta um comportamento linear com a variação da tensão, sofrendo uma pequena mudança de inclinação em $V=1 \mathrm{~V}$. Essa mudança se torna evidente no gráfico da condutância diferencial, onde um degrau pode ser observado em $V= \pm 1 \mathrm{~V}$. O efeito observado tem origem na

\footnotetext{
${ }^{5}$ Conforme explicado na Seção 7.5 apesar de na LOE as funções de Green e as funções largura de nível serem calculadas no nível de Fermi, o usuário pode fornecer um intervalo de energia em que estas funções são calculadas, simulando o efeito do deslocamento do nível de Fermi devido, por exemplo, a uma tensão de porta.
} 


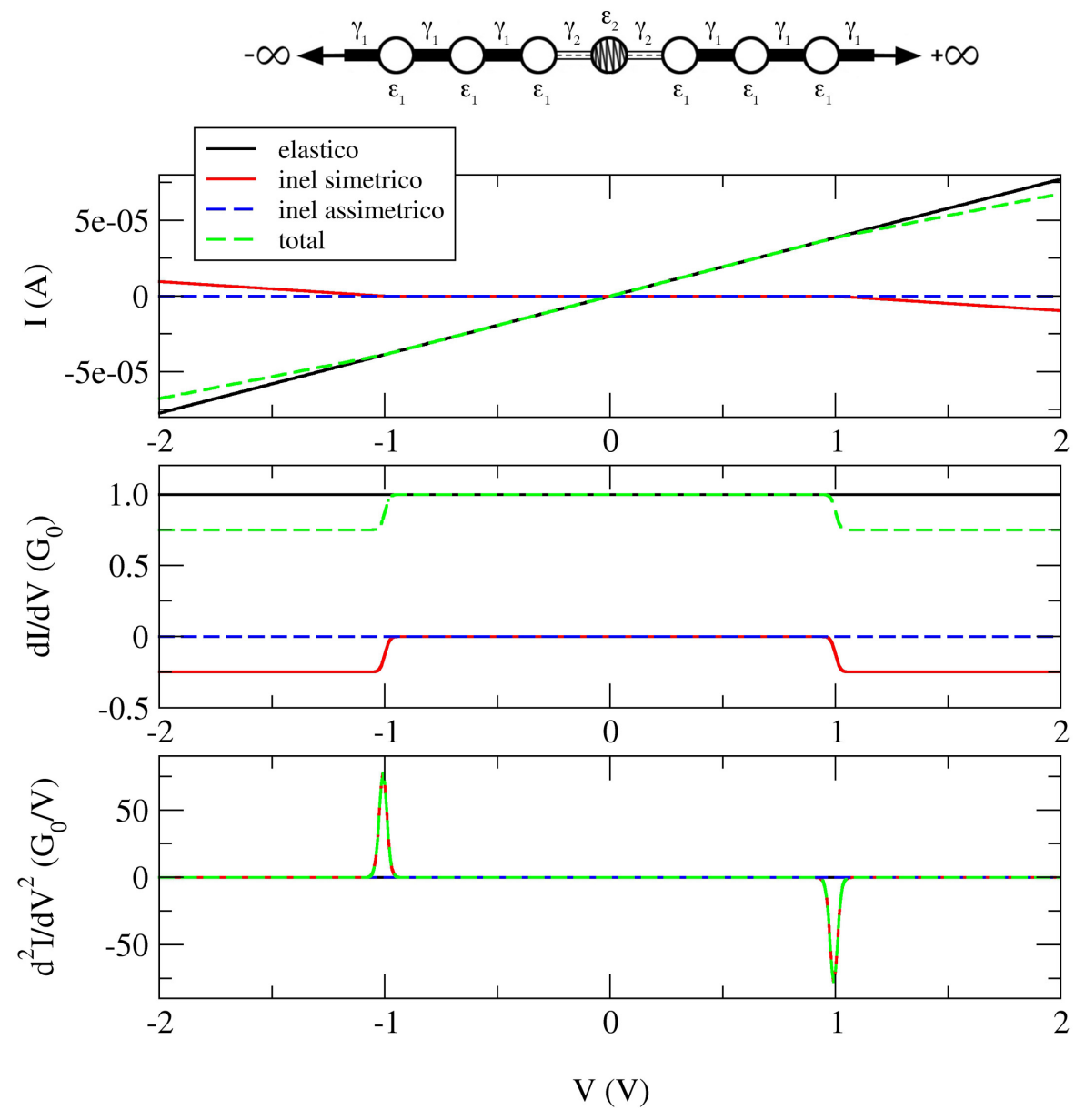

Figura 7.7: Corrente por tensão aplicada (acima), condutância diferencial (meio) e derivada da condutância (abaixo), para a cadeia com somente um defeito simples.

componente inelástica do transporte, conforme pode ser constatado na Figura 7.7, onde foram dispostos os resultados para a energia de Fermi $\left(E=\varepsilon_{F}=\varepsilon_{2}\right)$.

Se considerarmos somente o transporte elástico, o sistema apresenta um caráter metálico, com uma condutância de exatamente $1 \mathcal{G}_{0}$. Já no transporte inelástico, nota-se que o efeito da interação elétron-fônon se torna significativo quando o módulo da tensão aplicada atinge o valor da energia do fônon $(1 \mathrm{eV})$. Neste ponto ocorre uma diminuição da condutância para $0,75 \mathcal{G}_{0}$, simulando o efeito de retroespalhamento dos elétrons devido aos fônons. Em razão da geometria do sistema com simetria de espelho, a contribuição da componente assimétrica é nula (ver discussão na Seção 7.4.1), de forma que a única responsável pela queda na condutância é a componente simétrica.

Para a cadeia contendo um único defeito com a impureza dangling bond (Figura 7.8), observa-se a presença de dois canais de condutância correspondentes aos níveis $\varepsilon_{2}$ e $\varepsilon_{3}$. A ressonância em $E-\varepsilon_{F} \sim-0.1 \mathrm{eV}$ corresponde ao nível $\varepsilon_{2}$ e possui um alargamento maior comparado 


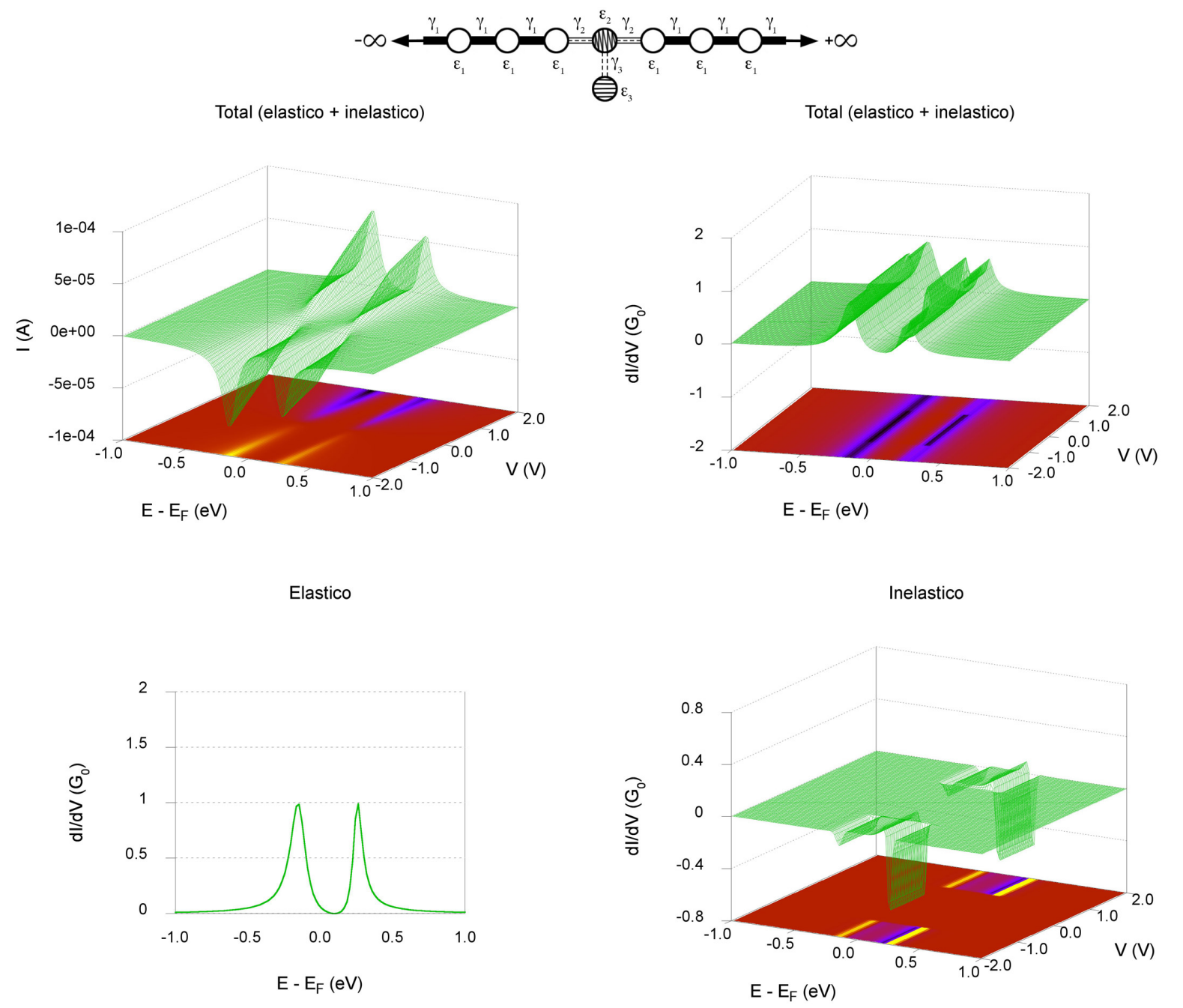

Figura 7.8: Corrente e condutância diferencial, em função da tensão e da energia, obtidas para uma cadeia contendo um único defeito com impureza "dangling bond". Acima: corrente total (esquerda) e condutância diferencial total (direita). Abaixo: condutância diferencial elástica (esquerda) e inelástica (direita).

à ressonância em $E-\varepsilon_{F} \sim 0.2 \mathrm{eV}$, correspondente ao nível $\varepsilon_{3}$, em razão da diferença entre os acoplamentos $\gamma_{2}=0.5 \mathrm{eV}>\gamma_{3}=0.2 \mathrm{eV}$.

Da mesma maneira que no caso anterior, a corrente apresenta um comportamento linear com a variação da tensão e uma mudança de inclinação é observada em $V=1$. No entanto, como o fônon foi considerado somente no sítio pendente $\varepsilon_{3}$, o efeito da interação elétron-fônon é mais pronunciado na ressonância em $E-\varepsilon_{F} \sim 0.2 \mathrm{eV}$, o que pode ser constatado pela condutância diferencial inelástica (gráfico inferior à direita na Figura 7.8).

Ao analisar os resultados para a energia de Fermi (Figura 7.9), observa-se um efeito reverso na simulação do sistema com a dangling bond quando comparado ao defeito simples. Novamente, 


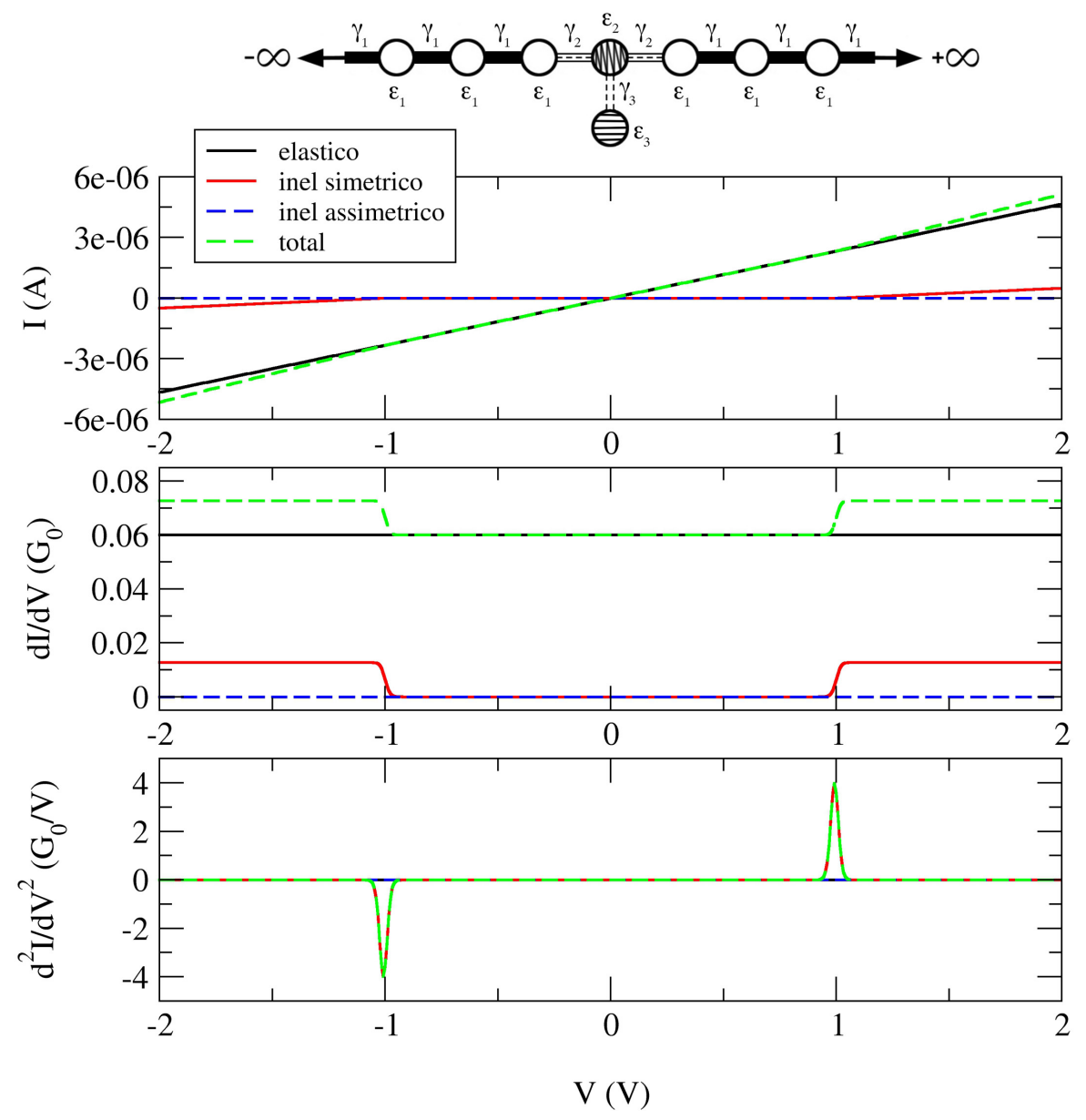

Figura 7.9: Corrente por tensão aplicada (acima), condutância diferencial (meio) e derivada da condutância (abaixo), para a cadeia com somente um defeito com uma impureza "dangling bond".

os fônons são excitados quando a tensão atinge $1 \mathrm{~V}$, mas, neste caso, ocorre um aumento da condutância de 0,06 para $\sim 0,07 \mathcal{G}_{0}$. Ou seja, enquanto que no caso do defeito simples os fônons atuam no sentido de suprimir a condutância em $\varepsilon_{F}$, quando consideramos os fônons no sítio pendente eles atuam no sentido de abrir o canal de condutância.

Para estudar os efeitos de localização decorrentes da desordem, o transporte eletrônico foi calculado em várias realizações das cadeias, mantendo-se a concentração de defeitos fixa em 10\% e variando-se o compriment ${ }^{6}$ da cadeia $L=\{20,40,60,80,100,200,300,400,500,600\}$. Foram consideradas 100 realizações para comprimentos até $L=100$ e para os demais comprimentos esta quantidade foi aumentada linearmente até 200 , com $L=600$.

Novamente, os dois tipos de defeitos foram considerados separadamente. Para a discussão que se segue, foram selecionados os resultados obtidos a uma tensão de $V=1,09 \mathrm{~V}$ (ou seja, acima

\footnotetext{
${ }^{6}$ Nesta seção, os comprimentos estão implicitamente descritos em termos do parâmetro de rede da cadeia (distância entre dois sítios vizinhos).
} 


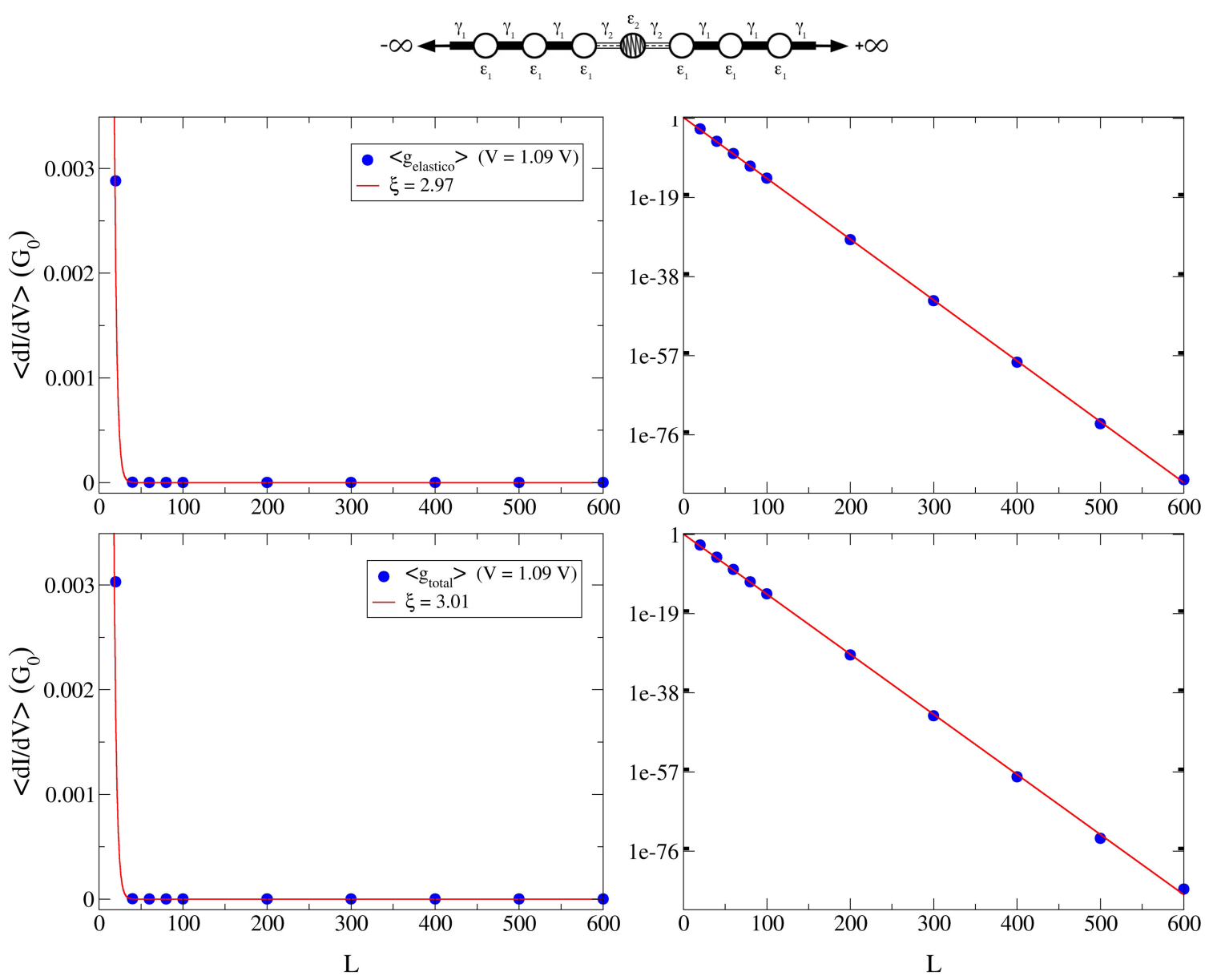

Figura 7.10: Valor médio da condutância diferencial versus o comprimento da cadeia, mantendo-se $10 \%$ dos sitios com o defeito simples. Acima, os valores obtidos para o transporte elástico e, abaixo, considerando a interação elétron-fônon. À direita, os gráficos são apresentados em escala logarítmica, para uma melhor visualização do ajuste exponencial.

da energia do fônon, de forma que o efeito da interação elétron-fônon já possa ser observado na condutância diferencial) e com a energia de Fermi em $\varepsilon_{F}=0,2 \mathrm{eV}$.

Na Figura 7.10 estão dispostos os valores médios da condutância diferencial obtidos para os diversos comprimentos do sistema, sendo que nos gráficos da parte superior foi considerada somente a contribuição elástica e nos da parte inferior a soma de todas as contribuições: elástica e inelástica. Nota-se que a condutância diferencial se aproxima de zero já para $L=40$, indicando que, com a concentração de defeitos em 10\% utilizada nos cálculos, os elétrons se tornam localizados rapidamente com o comprimento do sistema.

Conforme foi discutido na Seção 7.1, para uma amostragem suficientemente grande esperase que $\langle\ln (g)\rangle=-L / \xi$. Logo, para estimar o comprimento de localização $\xi$, ajustou-se uma função exponencial aos valores obtidos. O comportamento exponencial da condutância média fica evidente nos gráficos em escala logarítmica à direita da Figura 7.10 Para o transporte 

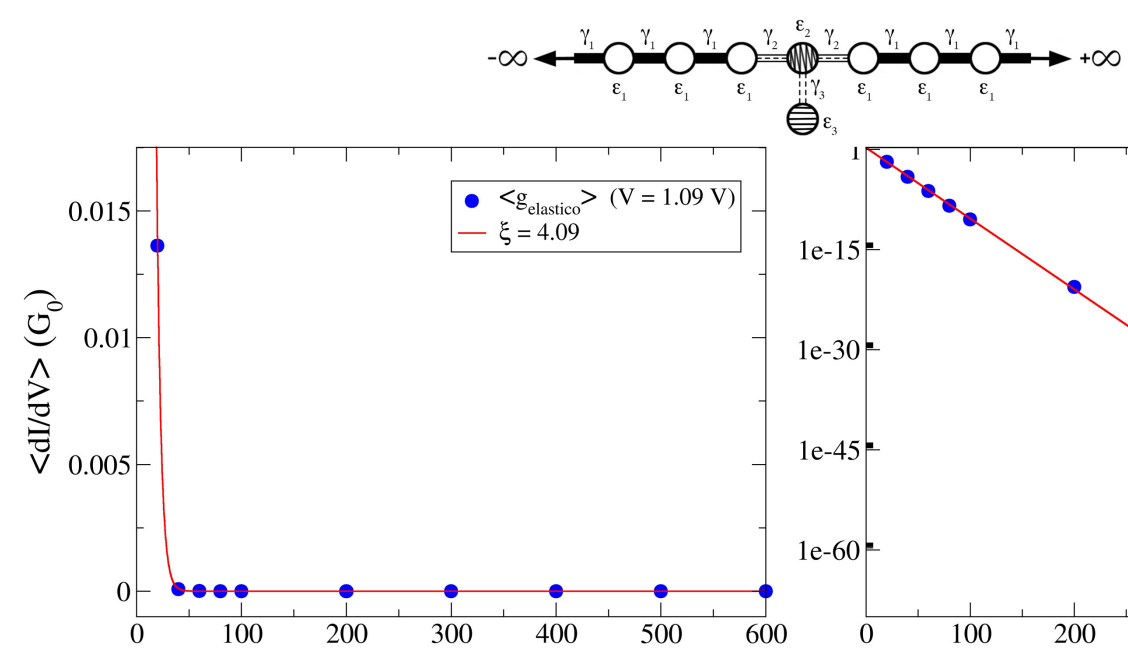

$\mathrm{\theta}_{3}$
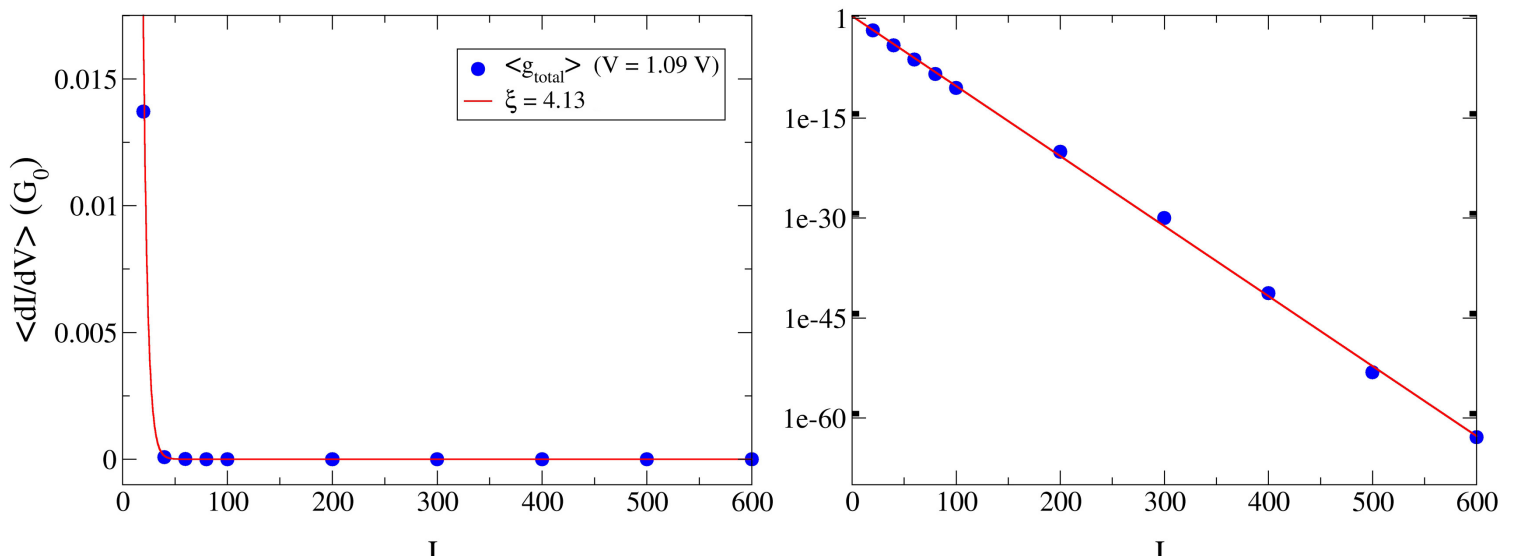

Figura 7.11: Valor médio da condutância diferencial versus o comprimento da cadeia, mantendo-se $10 \%$ dos sítios com a "dangling bond". Acima, os valores obtidos para o transporte elástico e, abaixo, considerando a interação elétron-fônon. À direita, os gráficos são apresentados em escala logarítmica, para uma melhor visualização do ajuste exponencial.

elástico, obteve-se um comprimento de localização de $\xi=2,97$. Quando se considera a interação elétron-fônon, este comprimento sofre um pequeno aumento para $\xi=3,01$.

A mesma análise foi realizada para cadeias contendo $10 \%$ dos sítios com a dangling bond. Os resultados obtidos estão dispostos na Figura 7.11. Novamente, quando se compara o ajuste para o transporte elástico com o do transporte total, observa-se um pequeno aumento no comprimento de localização de $\xi=4,09$ para $\xi=4,13$.

No entanto, tanto no caso do transporte elástico como no total, o comprimento de localização obtido é aproximadamente uma unidade maior quando comparado ao da cadeia com o defeito simples. Ou seja, a interferência entre a função de onda eletrônica transmitida e a que entra no sítio da dangling bond e retorna faz com que o comprimento de localização aumente.

Para verificar a consistência dos valores obtidos para o comprimento de localização, foram analisadas as distribuições do logaritmo da condutância adimensional nas cadeias com $L=300$. 
Para tanto, o transporte foi calculado em mais realizações, totalizando uma amostra de tamanho 1000 para cada um dos defeitos.

Na Figura 7.12 estão apresentadas as distribuições obtidas considerando somente o transporte elástico (esquerda) e o transporte total (direita), para a cadeia com defeitos simples.
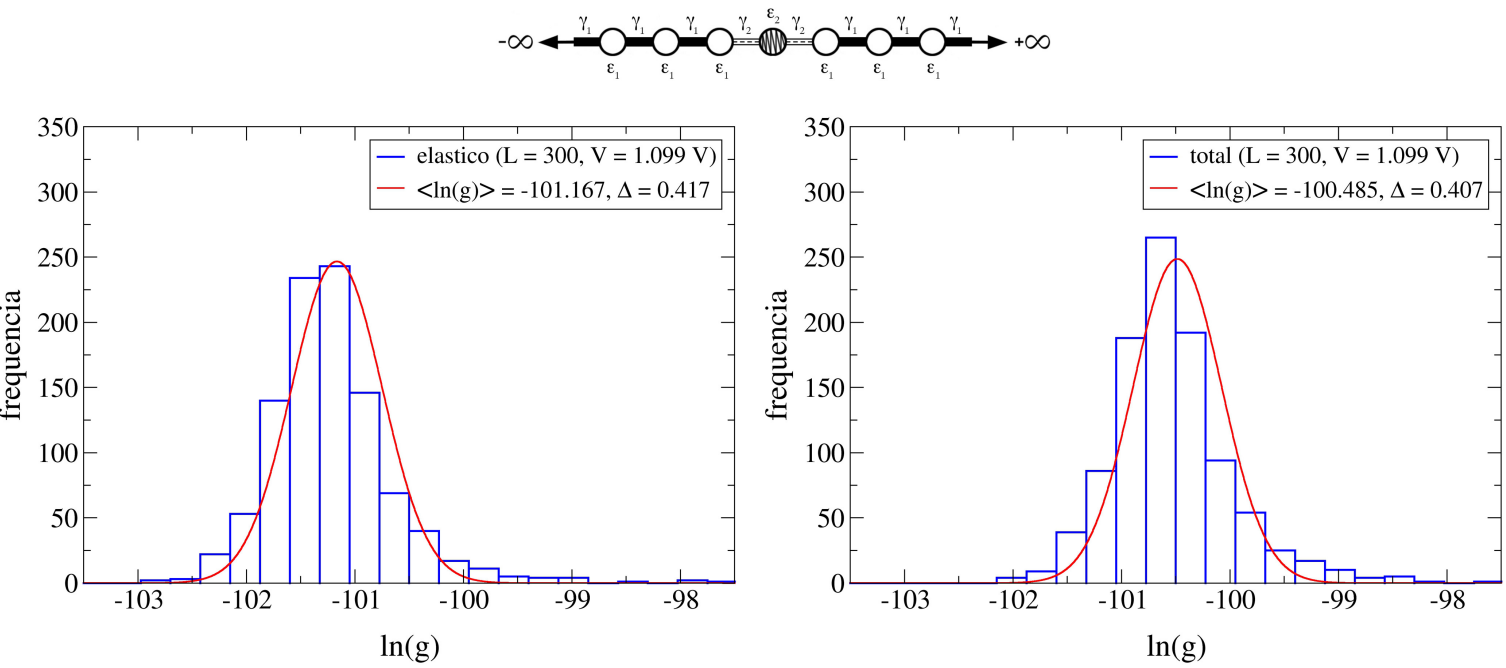

Figura 7.12: Distribuição do logaritmo da condutância adimensional obtida com 1000 realizações de uma cadeia com $L=300$ contendo $10 \%$ de defeitos simples. À esquerda, a distribuição obtida com o transporte elástico e, à direita, com o transporte total (elástico e inelástico).

Ajustando-se uma função gaussiana sobre as distribuições obteve-se para o transporte elástico $\langle\ln (g)\rangle=-101,167$ com desvio padrão $\Delta=0,417$. Se considerarmos o valor obtido para o comprimento de localização pelo ajuste exponencial, para uma cadeia com $L=300$ tem-se que $\langle\ln (g)\rangle=-100,989$, o que está a menos de $\Delta$ distante do obtido com a distribuição. Para o transporte inelástico, obteve-se $\langle\ln (g)\rangle=-100,485$ com desvio padrão $\Delta=0,407$, sendo aproximadamente $2 \Delta$ distante do valor estimado com o ajuste exponencial $\langle\ln (g)\rangle=-99,636$.

Os resultados obtidos para a cadeia contendo sítios com a dangling bond estão dispostos na Figura 7.13. Com o ajuste gaussiano obteve-se para o transporte elástico $\langle\ln (g)\rangle=-74,192$ com desvio padrão $\Delta=0,587$, sendo novamente compatível com o valor esperado de $\langle\ln (g)\rangle=$ $-73,308$. Para o transporte inelástico, obteve-se $\langle\ln (g)\rangle=-74,039$ com desvio padrão $\Delta=$ 0,597 distando menos de $3 \Delta$ do valor esperado pelo ajuste exponencial $\langle\ln (g)\rangle=-72,588$.

Portanto, aplicando a metodologia apresentada neste capítulo a um sistema simples de uma cadeia unidimensional com um nível eletrônico por sítio, foi possível estudar os efeitos característicos da interação elétron-fônon com a aproximação LOE, analisar o efeito de localização devido à desordem e estimar o comprimento de localização dos sistemas abordados. 


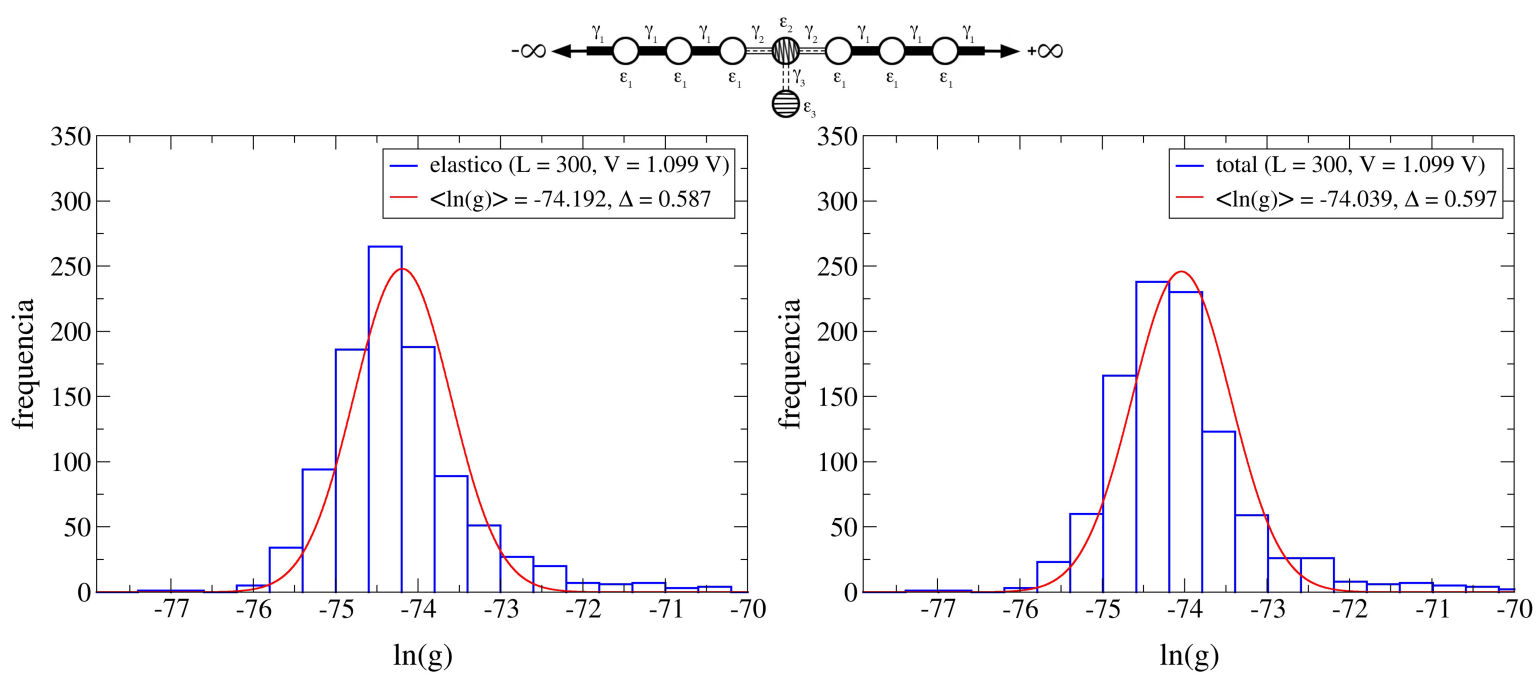

Figura 7.13: Distribuição do logaritmo da condutância adimensional obtida com 1000 realizações de uma cadeia com $L=300$ contendo $10 \%$ de defeitos com a "dangling bond". À esquerda, a distribuição obtida com o transporte elástico e, à direita, com o transporte total (elástico e inelástico).

É interessante notar que em ambos os casos ocorre um aumento no comprimento de localização. Este resultado pode estar associado à perda de coerência de fase dos elétrons devido à interação elétron-fônon, já que o fenômeno de localização de Anderson está ligado à fase da função de onda eletrônica se propagando no material. 


\section{Capítulo 8}

\section{Nanofitas de Grafeno com Impurezas Hidroxílicas}

No capítulo anterior, apresentamos o método implementado no programa I-DISORDER para cálculos ab initio das propriedades de transporte inelástico em sistemas desordenados. Mostramos como a interação elétron-fônon pode ser incluída com a Lowest Order Expansion e como o formalismo de transporte pode ser viabilizado para o cálculo de sistemas constituídos por uma grande quantidade de átomos com defeitos aleatoriamente posicionados. Também ilustramos as características do método aplicando-o em uma cadeia atômica unidimensional com um nível eletrônico por sítio.

Neste capítulo, vamos aplicar a metodologia no estudo de nanofitas de grafeno com impurezas hidroxílicas adsorvidas. Iniciaremos expondo de forma sucinta as propriedades das nanofitas de grafeno que tanto atraem a atenção da comunidade científica para, então, apresentar e discutir os resultados obtidos.

\subsection{Grafeno}

Por suas características e propriedades peculiares, o carbono é um foco permanente de interesse da comunidade científica em diversas áreas do conhecimento. Esse elemento químico, do grupo 14 da tabela periódica, é a base constituinte da química orgânica. Sua configuração eletrônica no estado fundamental é $1 s^{2} 2 s^{2} 2 p^{2}$, possuindo quatro elétrons na camada de valência. Dessa forma, seus orbitais podem hibridizar de maneiras diferentes, o que explica sua grande afinidade química. É possível encontrá-lo em diversas formas alotrópicas, sendo as mais conhecidas o diamante e o grafite.

Uma das formas alotrópicas mais interessantes é o grafeno, onde átomos de carbono formam 
uma rede hexagonal com um único átomo de espessura. Do ponto de vista conceitual, o grafeno pode ser considerado como o constituinte fundamental de diversos outros alótropos, como o fulereno $\mathrm{C}_{60}$, nanotubos de carbono e o grafite. Por esse motivo, seus estudos teóricos datam de mais de 60 anos atrás. Acreditava-se, porém, que qualquer cristal em duas dimensões seria termodinamicamente instável e, por isso, não poderia existir [23]. Não obstante, K. Novoselov, A. Geim e seu grupo de pesquisa relataram em 2004 a descoberta do grafeno, obtido de maneira relativamente simples, por meio da esfoliação mecânica de amostras de grafites pirolíticos altamente ordenados [24]. Um ano após a descoberta, eles obtiveram outros cristais bidimensionais [107 e observaram que esses cristais, além de serem contínuos, mostravam-se de alta qualidade. No caso do grafeno, os portadores de carga podem trafegar por milhares de distâncias interatômicas sem que ocorra espalhamento.

O grafeno é formado por carbonos com hibridização $s p^{2}$, onde três do quatro orbitais de valência se misturam (um orbital $2 s$ e dois orbitais $2 p$ ) e, devido ao princípio de exclusão de Pauli, se arranjam de forma a que os elétrons permaneçam o mais distante possível uns dos outros, gerando uma geometria planar trigonal com o ângulo de $120^{\circ}$ entre os orbitais. O orbital restante do tipo $p$ permanece perpendicular ao plano formado pelos orbitais híbridos.

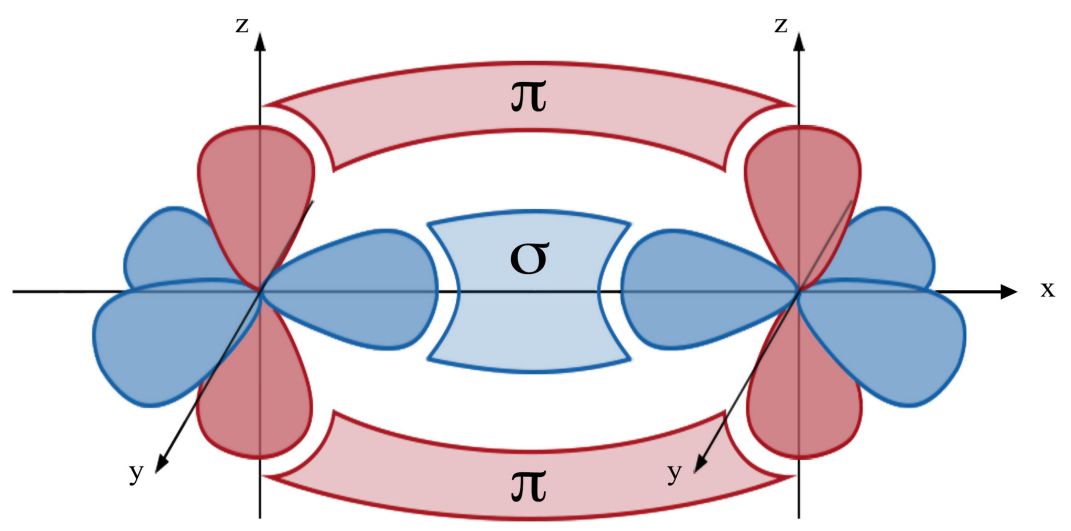

Figura 8.1: Orbitais de dois carbonos com hibridização $s p^{2}$. Os orbitais híbridos, no plano xy, formam ligaçôes $\sigma$ e os orbitais $p$, perpendiculares ao plano xy, formam ligações $\pi$.

Os três orbitais híbridos formam fortes ligações covalentes com os orbitais vizinhos no plano da rede (ligações do tipo $\sigma$ ) e são responsáveis pela estabilidade energética e pelas propriedades estruturais do grafeno, como a alta elasticidade. Já os orbitais do tipo $p$, perpendiculares ao plano, formam ligações do tipo $\pi$. Esses orbitais produzem as bandas de valência e de condução do grafeno, sendo responsáveis pela maioria de suas propriedades eletrônicas. 

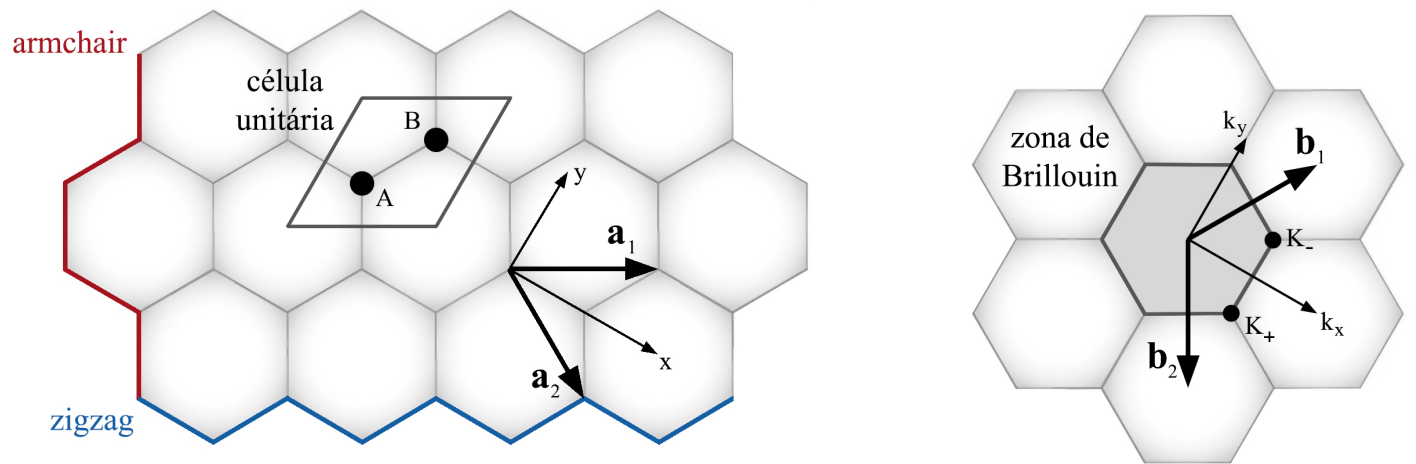

Figura 8.2: Representação da rede hexagonal do grafeno. À esquerda, observa-se a célula unitária da rede de Bravais, formada por dois átomos, com vetores base $\mathbf{a}_{1} e \mathbf{a}_{2}$. À direita, observa-se a rede recíproca, com a primeira zona de Brillouin ao centro e vetores base $\mathbf{b}_{1} e \mathbf{b}_{2}$, onde se destacam dois pontos de alta simetria $K_{+}$e $K_{-}$. Nota-se também dois possiveis cortes do grafeno, um com terminação "armchair" $e$ outro em "zigzag".

A rede hexagonal do grafeno, representada na Figura 8.2 à esquerda, pode ser descrita em termos de uma rede de Bravais triangular com dois átomos por célula unitária e vetores:

$$
\mathbf{a}_{1}=a\left(\frac{3}{2}, \frac{\sqrt{3}}{2}\right), \quad \mathbf{a}_{2}=a\left(\frac{3}{2},-\frac{\sqrt{3}}{2}\right),
$$

onde $a=1,42 \AA$ é o comprimento da ligação C-C no grafeno. Na Figura 8.2, também se pode observar a rede recíproca à direita, de onde se identificam nos cantos da zona de Brillouin os seguintes pontos de alta simetria:

$$
K_{+}=\frac{2 \pi}{3 a}\left(1,-\frac{\sqrt{3}}{3}\right), \quad K_{-}=\frac{2 \pi}{3 a}\left(1, \frac{\sqrt{3}}{3}\right) .
$$

Levando-se em consideração que as bandas dos elétrons envolvidos nas ligações $\sigma$ são fortemente separadas em energia, uma boa aproximação para descrever as bandas de condução e de valência consiste em tratar os elétrons dos orbitais $\pi$ de maneira independente (i.e., considerar o overlap nulo com as funções de onda nos orbitais híbridos). Assim, cada átomo fica definido por um único orbital $p$. Considerando-se interações somente entre primeiros vizinhos, as relações de dispersão para energia podem ser obtidas analiticamente dentro do formalismo tight-binding como: 


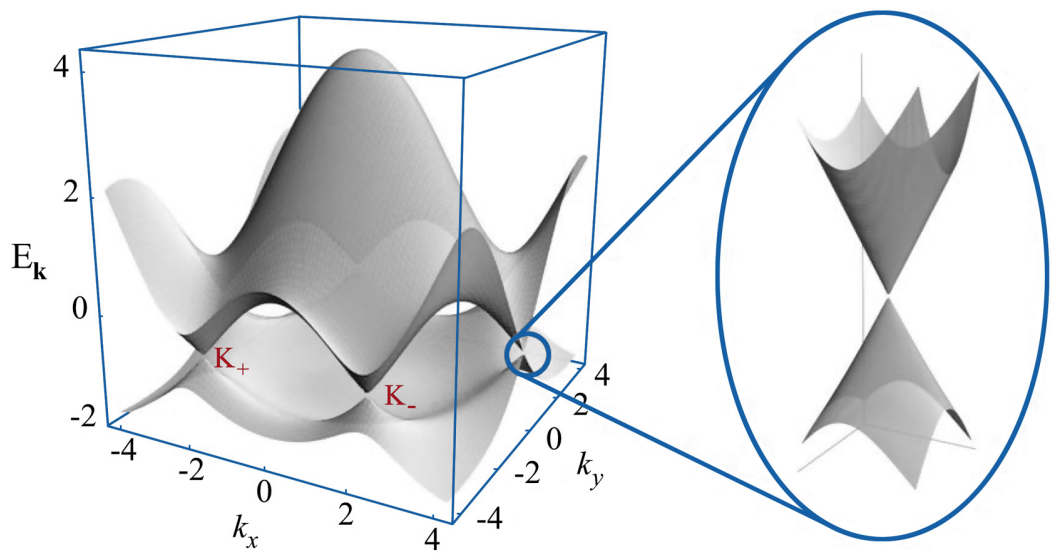

Figura 8.3: Bandas de valência e de condução do grafeno, destacando a relação de dispersão linear próxima aos pontos $K_{+}$e $K_{-}$. (Adaptado de [11.)

$$
E_{ \pm}(\mathbf{k})= \pm \gamma_{0} \sqrt{1+4 \cos \left(\frac{3 a k_{x}}{2}\right) \cos \left(\frac{\sqrt{3} a k_{y}}{2}\right)+4 \cos ^{2}\left(\frac{\sqrt{3} a k_{y}}{2}\right)}
$$

onde $\gamma_{0}$ é o acoplamento entre os primeiros vizinhos e os sinais $+\mathrm{e}-$ correspondem às bandas $\pi$ superior e inferior, respectivamente. Na Figura 8.3 , pode-se observar o gráfico da relação de dispersão característica obtida via tight-binding 1

Para o grafeno eletricamente neutro, a banda inferior (-) é totalmente preenchida, enquanto a banda superior $(+)$ é totalmente vazia. Dessa forma, a superfície de Fermi é constituída somente pelos pontos de alta simetria $K_{+}$e $K_{-}$, onde as bandas se tocam, e, por este motivo, o grafeno é considerado como um semicondutor de gap nulo. Expandindo a Equação 8.3 para $\mathbf{k}$ em torno de $K_{+}$ou $K_{-}$, obtém-se a seguinte relação linear (ver destaque na Figura 8.3):

$$
E_{ \pm}(\delta \mathbf{k}) \approx \pm \hbar v_{F}|\delta \mathbf{k}|
$$

onde $v_{F}=\frac{3 \gamma_{0} a}{2 \hbar}$ é a velocidade de grupo eletrônica. Devido a esta dispersão linear e à simetria elétron-buraco, os elétrons próximos aos pontos $K_{ \pm}$podem ser mapeados pela equação de Dirac relativística para férmions sem massa, onde $v_{F} \sim 10^{6} \mathrm{~m} \mathrm{~s}^{-1}$ representaria uma velocidade da luz efetiva, e, por esse motivo, os pontos onde as bandas se tocam são usualmente chamados de pontos de Dirac. Essas características levam a um novo grau de liberdade, o de pseudospin, cuja simetria

\footnotetext{
${ }^{1}$ No caso do espectro de energia da Figura 8.3 foram consideradas as interações entre segundos vizinhos. Contudo, as características fundamentais que queremos discutir aqui são as mesmas do espectro obtido considerando-se interações somente entre primeiros vizinhos.
} 
faz com que os estados eletrônicos sejam particularmente insensíveis a fontes de desordem elástica, como defeitos topológicos e eletrostáticos. De fato, já foram reportadas medidas de mobilidade de carga no grafeno extremamente elevadas como $10^{5} \mathrm{~cm}^{2} \mathrm{~V}^{-1} \mathrm{~s}^{-1}[24]$.

Em razão destas peculiaridades na estrutura eletrônica, diferentes propriedades fundamentais têm sido observadas no grafeno, como o efeito Hall quântico inteiro à temperatura ambiente e tunelamento de Klein [11]. Essas descobertas e as demais propriedades físicas do grafeno atraíram nos últimos anos um grande interesse tanto do ponto de vista de ciência básica como de aplicação tecnológica. Características como alta mobilidade e comprimentos de coerência longos fazem com que o grafeno seja considerado um forte candidato a ser o material fundamental no avanço da nanoeletrônica.

No entanto, um dos grandes empecilhos encontrados para o uso do grafeno em dispositivos eletrônicos é justamente a ausência do gap de energia, já que a condutância eletrônica não pode ser desligada aplicando-se, por exemplo, um potencial de porta (como nos transistores). Por esse motivo, um grande esforço na comunidade científica tem sido despendido no sentido de descobrir maneiras de se abrir o gap do grafeno. Uma das possíveis soluções encontradas consiste em utilizar tiras estreitas do grafeno, as chamadas nanofitas.

\subsection{Nanofitas de Grafeno}

Nanofitas de grafeno são estruturas estreitas que podem ser obtidas cortando-se uma folha de grafeno. Como consequência, as bordas produzidas no corte confinam as funções de onda eletrônica ao longo do comprimento da nanofita, formando um sistema quasi-unidimensional. Como efeito do confinamento, pode ocorrer a abertura do gap de energia, cujo tamanho depende fortemente da largura da nanofita $\left[\left.26\right|^{2}\right.$

Além da largura, outro fator que influencia nas propriedades eletrônicas das nanofitas é a geometria das bordas. Seguindo uma direção específica, dois tipos ideais de terminações podem ser obtidos, com borda armchair e em zigzag (Figura 8.2). Por convenção, as nanofitas armchair (AGNR) são classificadas pela quantidade de linhas de dímero (N-AGNR) e as zigzag (ZGNR), pelo número de linhas zigzag (N-ZGNR), conforme indicado na Figura 8.4

Estas terminações provocam diferentes condições de contorno e, consequentemente, as nanofitas apresentam diferentes propriedades eletrônicas [11]. Cálculos teóricos por primeiros prin-

\footnotetext{
${ }^{2}$ A princípio, dependendo da maneira como o corte do grafeno é realizado, seria possível obter nanofitas metálicas (como é o caso dos nanotubos de carbono). Porém, diferentemente dos nanotubos, isto levaria a estados confinados nas bordas e, nesse caso, haveria uma instabilidade de Stoner transformando essas nanofitas em antiferromagnéticas, abrindo o gap.
} 

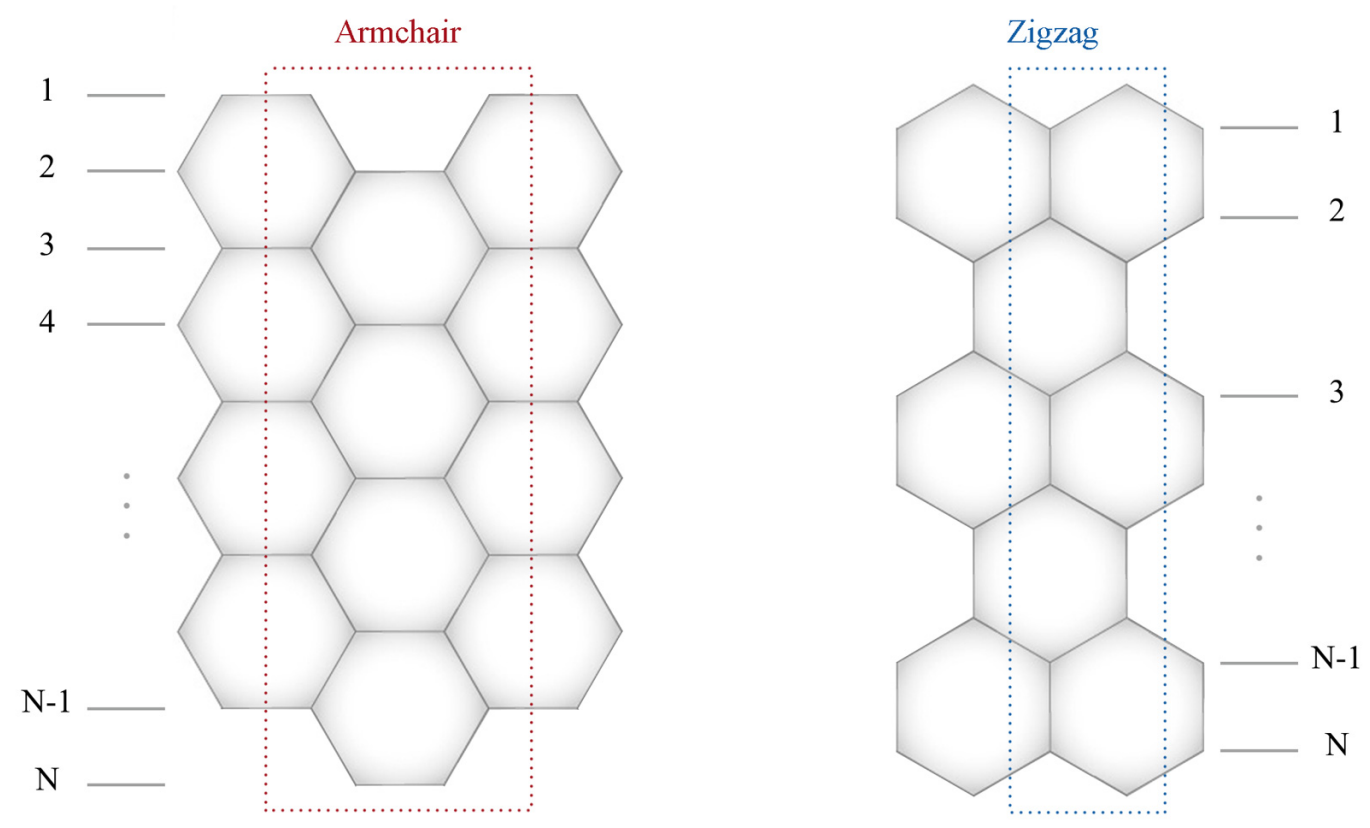

Figura 8.4: Ilustração das nanofitas "armchair" N-AGNR à esquerda e "zigzag" N-ZGNR à direita, onde são destacadas as respectivas células unitárias.

cípios indicam que o gap nas nanofitas armchair surge devido ao confinamento quântico e ao efeito crucial das bordas, enquanto nas nanofitas zigzag o gap é induzido pela interação de spin entre elétrons próximos da borda 25.

Na Figura 8.5, são apresentadas as estruturas de bandas de uma nanofita 4-ZGNR, com e sem polarização de spin, e uma 14-AGNR, todas obtidas via DFT pelo programa SIESTA. Notase que, sem considerar a polarização de spin nos cálculos, a nanofita zigzag apresenta um caráter metálico (Figura 8.5 à esquerda) e, quando a polarização é considerada, ocorre a abertura do gap (Figura 8.5 ao centro). Já na 14-AGNR, o gap surge no cálculo sem polarização de spin e não muda quando a polarização é considerada.

Atualmente existem diferentes técnicas experimentais para obter-se nanofitas de grafeno, podendo ser classificadas em duas categorias: as abordagens bottom-up e top-down. Os métodos top-down, tais como o corte do grafeno por litografia e o unzipping longitudinal de nanotubos de carbono, possuem uma resolução espacial limitada e as fitas obtidas apresentam diferentes padrões de bordas. Por outro lado, métodos bottom-up por síntese orgânica têm-se mostrado capazes de produzir nanofitas longas (da ordem de $200 \mathrm{~nm}$ ) e estruturalmente bem definidas [28, 29].

Outras técnicas experimentais se dedicam a obter um gap de energia a partir da funcionalização de materiais baseados no grafeno, sem precisar obter nanofitas com larguras drasticamente reduzidas. Em particular, foi reportada a fabricação de dispositivos de efeito de campo a partir 

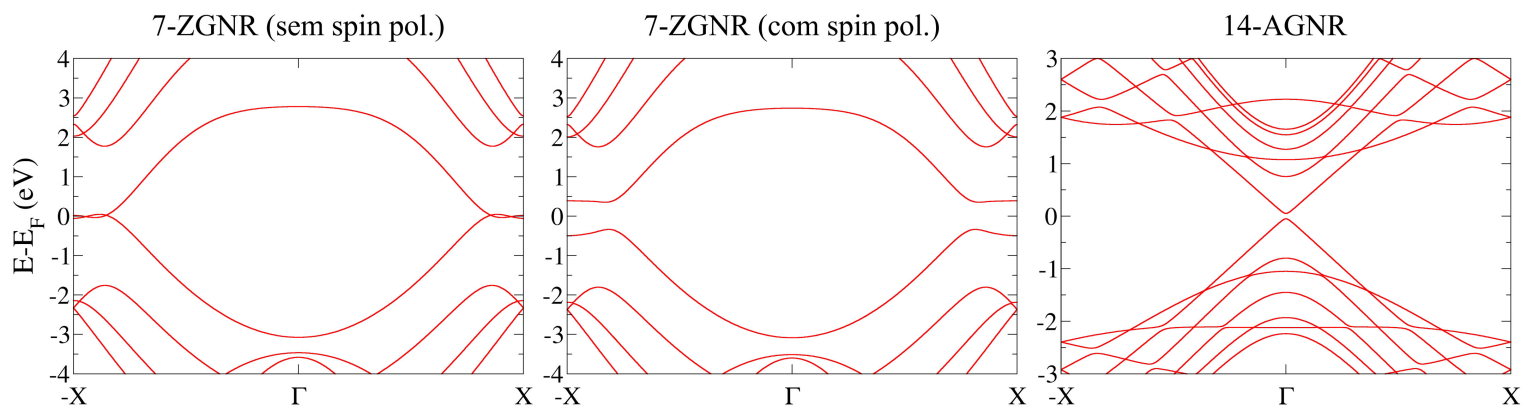

Figura 8.5: Estrutura de bandas obtidas com o programa SIESTA para uma nanofita 4-ZGNR sem polarização de spin (esquerda) e com polarização de spin (meio) e para uma nanofita 14-AGNR (direita).

da modificação química do grafeno [34]. Nesse experimento, moléculas de água presentes no ambiente ou na interface $\mathrm{SiO}_{2}$ /grafeno supostamente se dissociaram nos grupos $\mathrm{H}$ e OH, que, por sua vez, foram adsorvidos na superfície do grafeno, ocasionando a abertura de um largo gap na banda e explicando a modificação de seis ordens de magnitude na resistividade do canal medido.

Estudos indicam que a funcionalização química de materiais baseados no grafeno promovem a formação de ligações saturadas $s p^{3}$, induzindo uma dopagem significativa dos dispositivos e ocasionando fortes efeitos de retroespalhamento [37]. Dentro desse contexto, acredita-se que os modos vibracionais localizados nas moléculas adsorvidas à superfície do material também possam gerar um efeito significativo nas propriedades de transporte. A presença de água e, consequentemente, a oxidação pode ser inevitável nos experimentos e não há um controle sobre onde essas moléculas estarão. Em vista disso, neste trabalho foi estudado o efeito da adsorção de grupos $\mathrm{H}$ e $\mathrm{OH}$, com a introdução de modos localizados, no transporte de cargas de nanofitas armchair.

Foram estudados quatro tipos de defeitos em nanofitas 14-AGNR, representados na Figura 8.6. Em dois defeitos, (A) e (B), os grupos $\mathrm{H}$ e $\mathrm{OH}$ foram adsorvidos na borda da nanofita. No defeito (C), estes grupos foram adsorvidos no meio da nanofita, enquanto que, em (D), os grupos foram adsorvidos na borda e no meio.

\subsection{Cálculos de Estrutura Eletrônica}

Os cálculos de estrutura eletrônica foram realizados via DFT por meio do programa SIESTA (Seção 3.2.4). O funcional de troca e correlação escolhido foi uma GGA (Seção 3.2.3) baseada na parametrização não-empírica de Perdew-Burke-Ernzerhof (PBE) [75]. Foi utilizada uma base duplo $\zeta$ com orbitais polarizados (DZP) e um energy shift $\delta \epsilon=0.03 \mathrm{eV}$. Nesses cálculos, assim como nos de transporte que serão apresentados nas próximas seções, considerou-se uma 
(A)

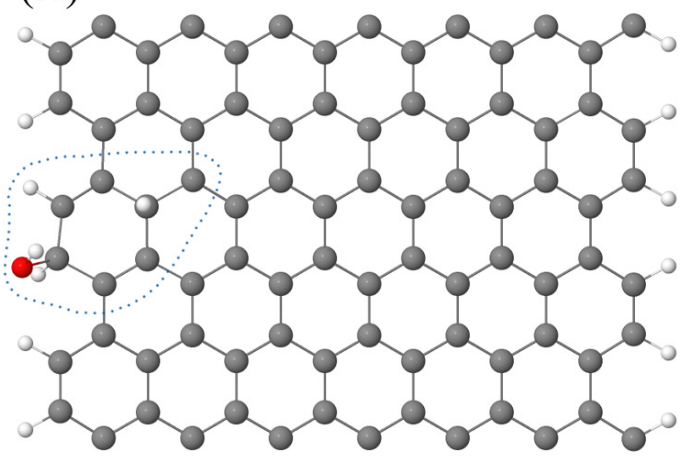

(C)

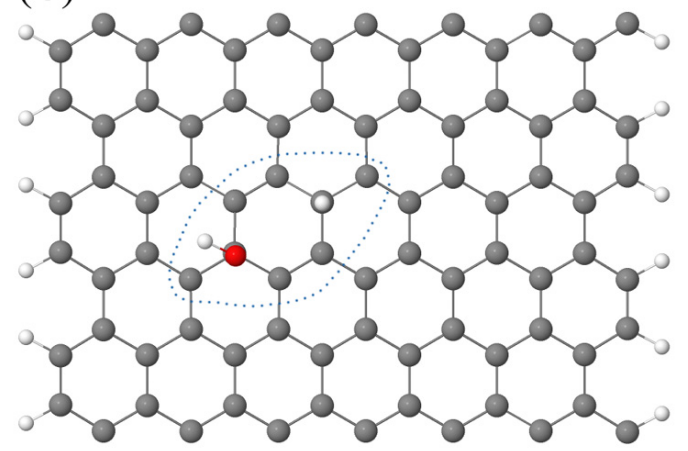

(B)

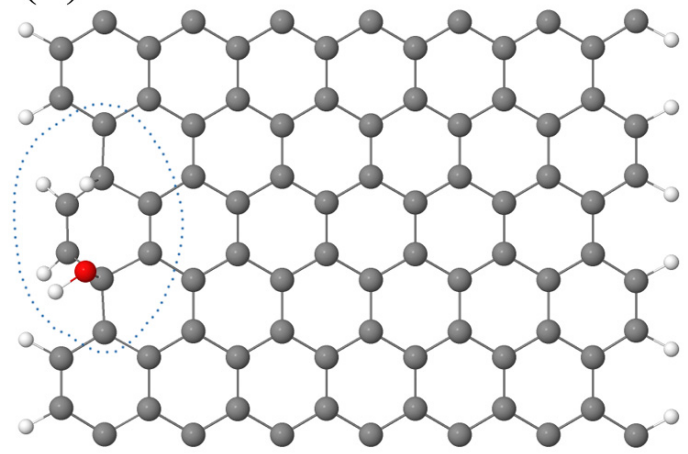

(D)

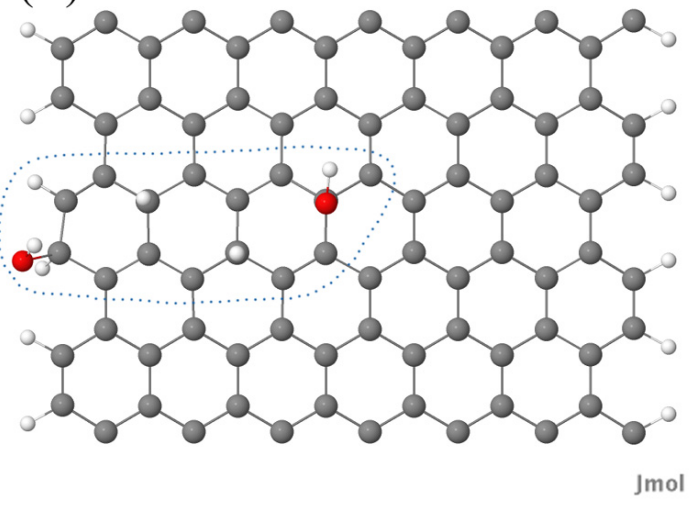

Figura 8.6: Representação dos quatro tipos de defeitos estudados, onde os grupos $\mathrm{H}$ e OH foram adsorvidos na borda da nanofita em (A) e (B), no meio da nanofita em (C) e em ambos, borda e meio da nanofita, em (D). Os átomos considerados na análise vibracional de cada defeito estão destacados por uma linha pontilhada

temperatura eletrônica de $300 \mathrm{~K}$.

Como o código SIESTA utiliza condições periódicas de contorno, é necessário criar uma supercélula suficientemente grande nas direções em que as estruturas possuem dimensões finitas, de forma que não ocorra overlap entre os orbitais de células unitárias vizinhas. As nanofitas 14AGNR estudadas possuem uma largura de $\sim 18.04 \AA$ e, portanto, nesta direção foi considerado 33.07 Å para a supercélula. Já na direção ortogonal ao plano das nanofitas, foi considerado 21.80 $\AA$.

No SIESTA, o espaçamento do grid de energia, utilizado nos cálculos de integrais e para a representação de densidades de cargas e potenciais, é determinado pelo parâmetro MeshCutoff, cujo valor padrão é 100 Ry. Porém, um valor baixo para esse parâmetro pode levar a um aumento do efeito de egg-box (ver discussão na Seção 3.3.1), o que pode comprometer o cálculo de vibrações. Em vista disso, nos cálculos o MeshCutoff foi fixado em 350 Ry.

O critério de convergência eletrônica considerado foi $10^{-6}$ (i.e. considera-se que a autoconsistência foi atingida quando as diferenças entre os elementos da nova matriz densidade com os 

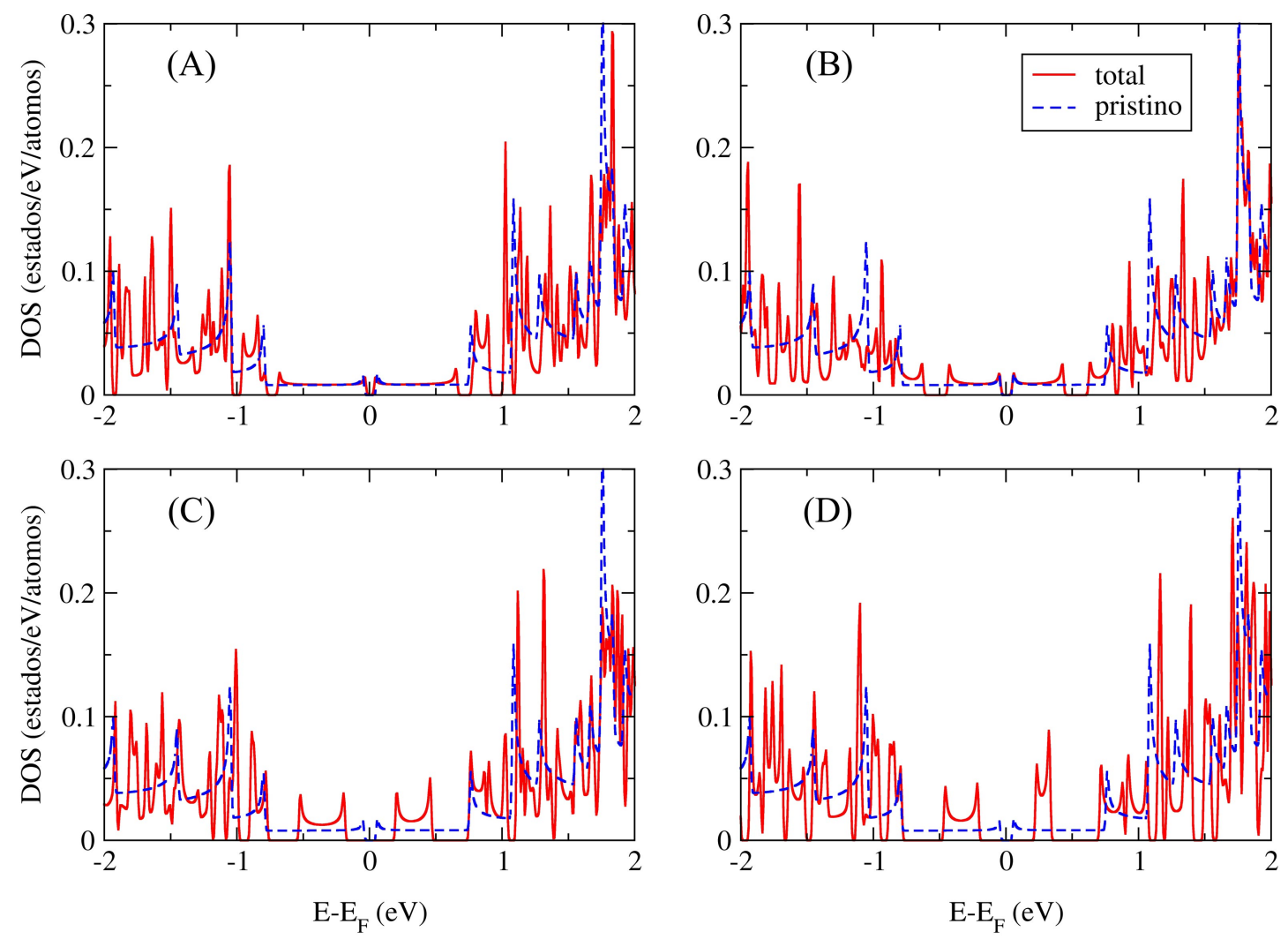

Figura 8.7: Densidade de estados obtida com o programa SIESTA para os defeitos $(A),(B),(C)$ e (D) da Figura 8.6 , onde a linha tracejada corresponde à densidade de estados na ausência de defeitos. ${ }^{3}$

da matriz do passo anterior forem todas inferiores a $10^{-6}$ ). Para a otimização das estruturas foi utilizado o método Gradiente Conjugado com uma tolerância máxima de força de $0.001 \mathrm{eV} \AA^{-1}$ (i.e., a estrutura é considerada relaxada quando as forças agindo sobre os átomos são inferiores a $0.001 \mathrm{eV} \AA^{-1}$ ). Esse rigor na relaxação das estruturas é importante para garantir que a configuração atômica obtida corresponda ao mínimo da superfície de energia de Born-Oppenheimer, o que é necessário para o cálculo de vibrações a partir da aproximação harmônica (Seção 3.3.).

Na Figura 8.7, estão dispostas as densidades de estados (DOS) de cada um dos defeitos obtidas com o programa SIESTA 3 Sobreposta a cada uma delas encontra-se a DOS da nanofita pristina (linha tracejada), onde se pode observar a presença de divergências características. Esses picos na DOS são conhecidos como singularidades de van Hove [82] e manifestam as propriedades de confinamento das nanofitas em 1-dimensão. Nota-se, também, a presença de um gap de aproximadamente $0.1 \mathrm{eV}$ ao redor do nível de Fermi, compatível com gap obtido no cálculo da estrutura de bandas (Figura 8.5 à direita).

\footnotetext{
${ }^{3}$ As densidades de estados foram divididas pelo número de átomos da célula unitária a fim de facilitar a comparação entre os gráficos.
} 
Nas densidades de estados calculadas para os defeitos observa-se a presença de mini-gaps. Este efeito surge devido ao potencial gerado pela adsorção das impurezas que, por sua vez, é repetido periodicamente pelo código SIESTA. No entanto, tal efeito não está presente nos cálculos de transporte, onde os defeitos são considerados somente na região de espalhamento e os eletrodos são pristinos, quebrando a periodicidade do sistema.

Ao comparar as densidades de estados obtidas para os diferentes defeitos, nota-se que conforme a ligação C-OH passa da borda para o centro da nanofita, maior é a mudança na DOS em energias próximas ao nível de Fermi em relação à estrutura pristina. Logo, espera-se que os defeitos na borda da nanofita provoquem um efeito menor nas propriedades de transporte, conforme veremos a seguir.

\subsection{Propriedades de Transporte}

As transmissões elásticas de cada um dos defeitos, bem como da nanofita pristina, foram calculadas com o programa SMEAGOL (Seção 4.6). Na Figura 8.8, pode-se comparar a transmissão elástica a $V=0$ (abaixo) obtida para a nanofita pristina com sua respectiva estrutura de bandas (acima) e densidade de estados (meio).

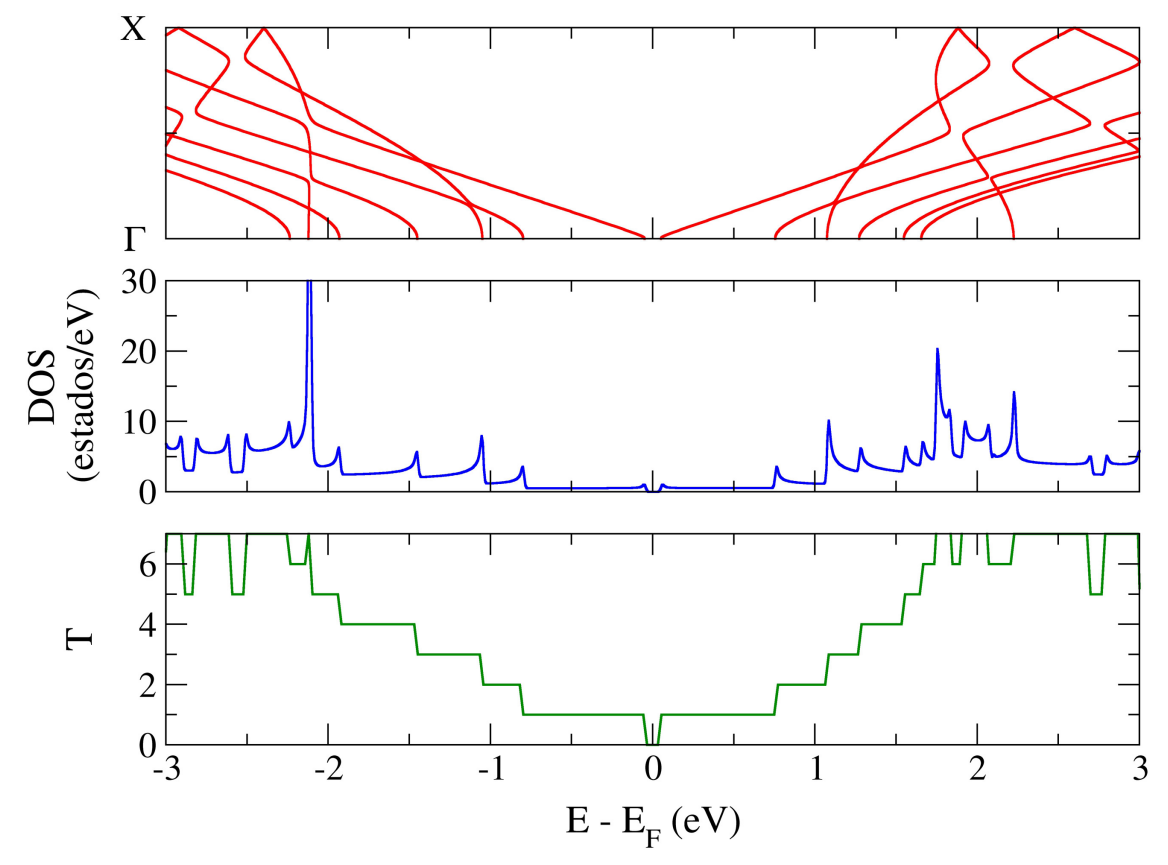

Figura 8.8: Estrutura de bandas (acima), densidade de estados (meio) e transmissão elástica a $V=0$ (abaixo) de uma nanofita 14-AGNR. 
Nota-se que, na ausência de defeitos, a nanofita apresenta uma condutância quantizada $\mathcal{G}=\mathcal{G}_{0} T(\epsilon)=\mathcal{G}_{0} N_{T}$, onde $N_{T}$ é o número de canais de condutância disponíveis a uma dada energia. Essa relação pode ser observada ao se comparar os gráficos da Figura 8.8, onde se verifica, por exemplo, que ao primeiro patamar de condutância $\left(N_{T}=1\right)$ corresponde uma única banda degenerada. Da mesma maneira, no intervalo de energia correspondente ao gap de aproximadamente $0.1 \mathrm{eV}$ ao redor do nível de Fermi, observa-se uma condutância nula $\left(N_{T}=0\right)$.

Na Figura 8.9, estão dispostas as transmissões elásticas em função da energia calculadas a $V=0$ para cada defeito. Para facilitar a visualização do efeito decorrente de cada defeito, a transmissão da nanofita pristina foi sobreposta aos gráficos (linha tracejada).
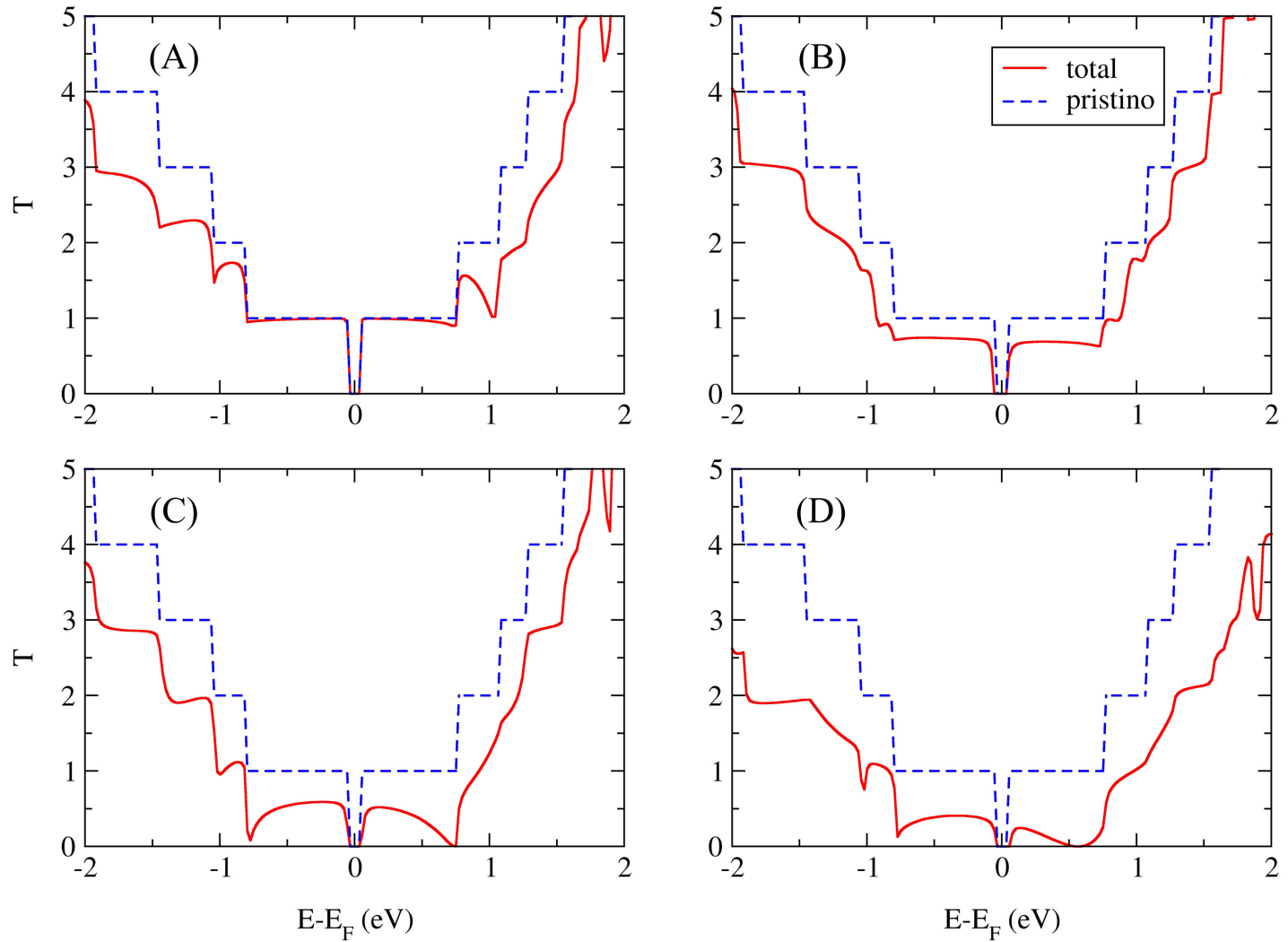

Figura 8.9: Transmissão elástica a $V=0$ obtida para os defeitos $(A),(B),(C)$ e (D) da Figura 8.6. onde a linha tracejada corresponde à transmissão na ausência de defeitos.

Quando a ligação C-OH está localizada na extremidade da nanofita, como é o caso do defeito (A), os efeitos de retroespalhamento começam a ser perceptíveis somente a partir do primeiro degrau de condutância, sendo mais pronunciados em energias maiores, devido ao aumento da probabilidade de retroespalhamento.

Conforme a ligação C-OH passa da borda para o centro, maior é o efeito de retroespalhamento observado próximo ao nível de Fermi. Isso pode ser verificado na Figura 8.10, onde são 

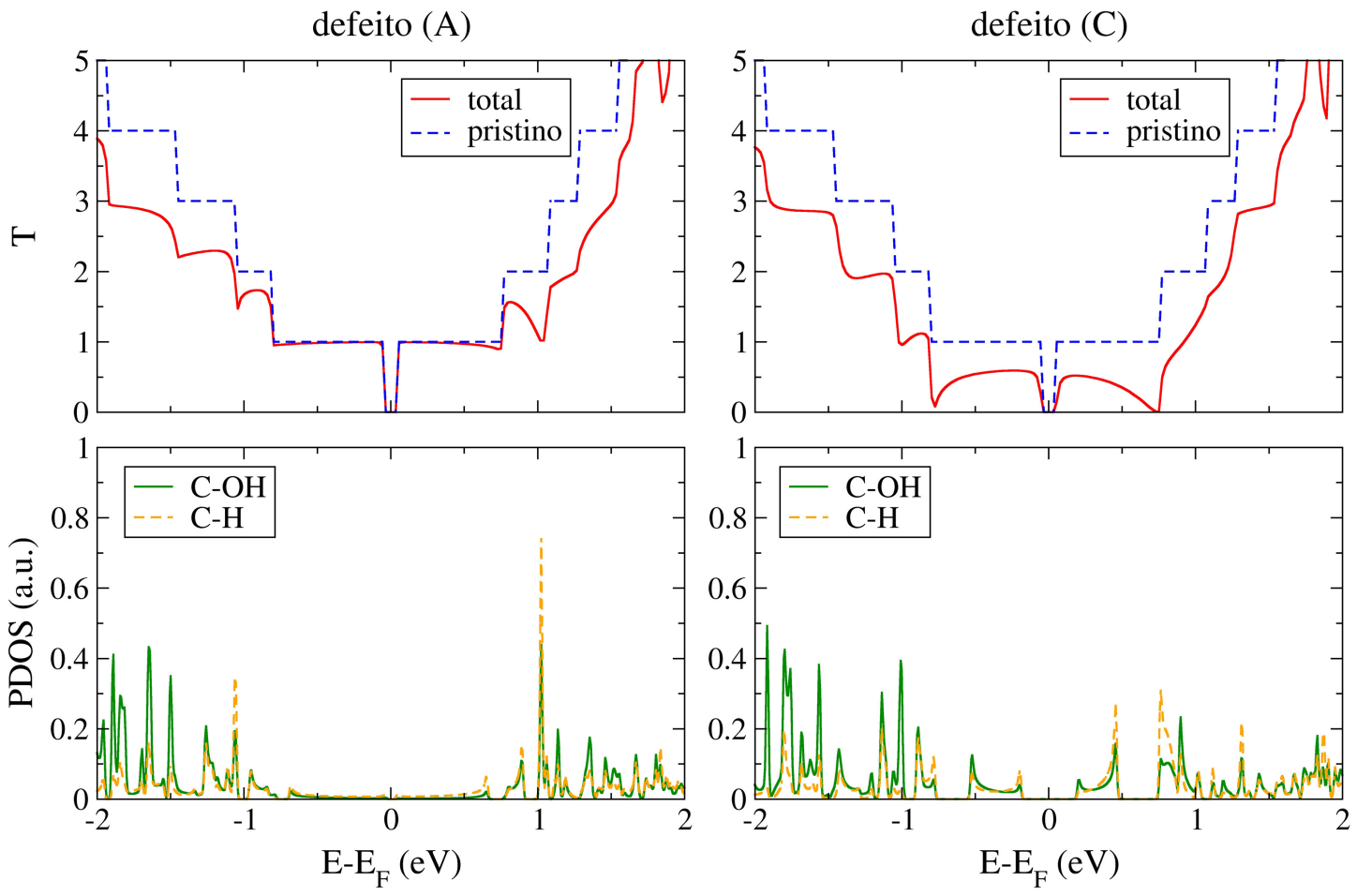

Figura 8.10: Transmissão elástica a $V=0$ (acima) e densidade de estados projetada nas ligações $\mathrm{C}$-OH e $C$-H das moléculas adsorvidas nos defeitos (A) (à esquerda) e (C) (à direita).

apresentadas as densidades de estados projetadas (PDOS) nas ligações C-OH e C-H das moléculas adsorvidas para o defeito (A) (onde os grupos $\mathrm{H}$ e $\mathrm{OH}$ foram adsorvidos na borda) e para o defeito $(\mathrm{C})$ (com os grupos $\mathrm{H}$ e $\mathrm{OH}$ adsorvidos no meio da nanofita).

Para o defeito (A), verifica-se que praticamente não há estados disponíveis ao redor do nível de Fermi. A densidade de estados começa a ficar significativa somente quando as energias se distanciam de aproximadamente $\pm 1 \mathrm{eV}$ do nível de Fermi, onde se observa um aumento pronunciado. Comparando os gráficos da PDOS com a transmissão elástica do defeito (A), nota-se que um aumento na densidade de estados das moléculas adsorvidas corresponde a um decréscimo na transmissão. O mesmo efeito também pode ser observado para o defeito $(\mathrm{C})$. Porém, nesse caso, a PDOS já apresenta picos pronunciados a valores mais próximos do nível de Fermi, o que explica o efeito de retroespalhamento observado já no primeiro degrau de condutância.

Portanto, os resultados obtidos indicam que a funcionalização nas extremidades da nanofita 14-AGNR tem uma baixa influência nas propriedades eletrônicas próximas ao nível de Fermi (o que já era esperado pela análise das densidades de estados na seção anterior). Além disso, a grande distorção na transmissão observada no defeito (D) sugere que, aumentando-se a quantidade de grupos $\mathrm{H}$ e $\mathrm{OH}$ adsorvidos, maior é a supressão da condutância. Esses resultados estão de acordo com os observados por A. López-Bezanilla et al [37]. 


\subsection{Análise Vibracional}

As frequências e os modo vibracionais, assim como a matriz de acoplamento elétron-fônon, foram obtidos para cada um dos defeitos com o programa PhOnonS ITeratIVE VIBRATIONS (Seção 3.3.1). Foram considerados para o cálculo vibracional os carbonos responsáveis pelas ligações com os grupos $\mathrm{H}$ e $\mathrm{OH}$ adsorvidos e os respectivos primeiros vizinhos, conforme destacado com a linha pontilhada na Figura 8.6 (no caso dos defeitos na borda da nanofita, também foram incluídos os hidrogênios da saturação). As frequências obtidas para cada um dos defeitos estão dispostas na Figura 8.11 .

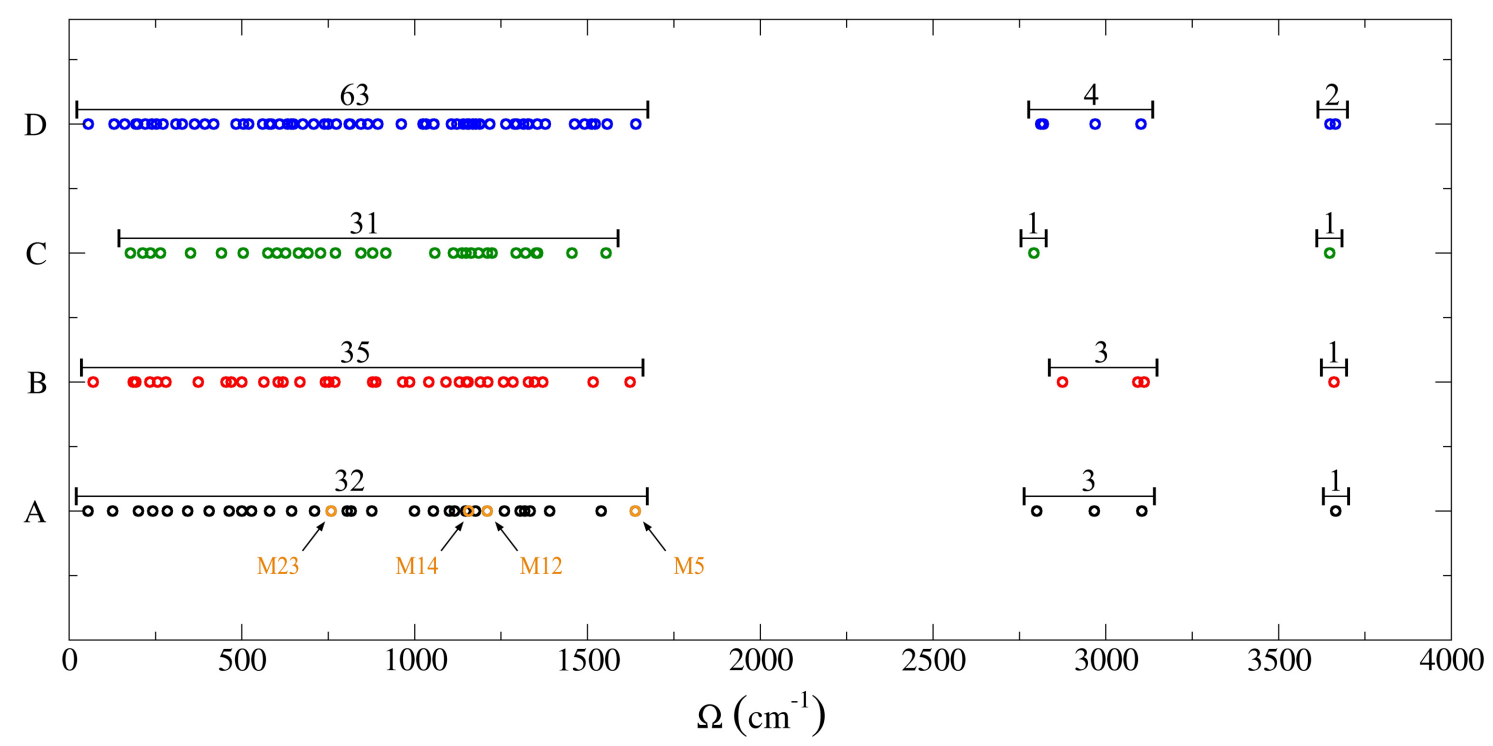

Figura 8.11: Frequências calculadas para os defeitos (A), (B), (C) e (D), onde foram considerados somente os átomos destacados na Figura 8.6 .

A quantidade de modos vibracionais computados depende do número de átomos dinâmicos considerados no cálculo vibracional (para cada átomo deslocado durante o cálculo de constante de forças são obtidos três modos vibracionais - ver Seção 3.3). Nos defeitos (A), (B) e (C), foram calculados 36, 39 e 33 modos vibracionais, respectivamente. Para a análise vibracional do defeito (D) foi preciso considerar uma quantidade superior de átomos (23), totalizando 69 modos vibracionais. Dada a grande diferença de energia entre as frequências e a existência de modos degenerados, essas quantidades foram assinaladas na Figura 8.11

Nota-se que a maioria das frequências encontra-se no intervalo $[0,1.700] \mathrm{cm}^{-1}$. As frequências em torno de $3.000 \mathrm{~cm}^{-1}$ correspondem a modos provenientes dos hidrogênios nas ligações $\mathrm{C}-\mathrm{H}$, enquanto as de maiores energias correspondem aos hidrogênios das ligações O-H. 


\subsection{Transporte Inelástico com LOE}

A interação elétron-fônon foi abordada com a aproximação Lowest Order Expansion discutida na Seção 7.4. Nesta seção, vamos nos restringir à análise do defeito (A), que, como vimos, demonstrou menor alteração na transmissão em energias próximas ao nível de Fermi. Na Figura 8.12, são apresentadas as características $I-V$ em função da energia para o transporte elástico, total (elástico e inelástico) e para as componentes simétrica e assimétrica da contribuição inelástica.
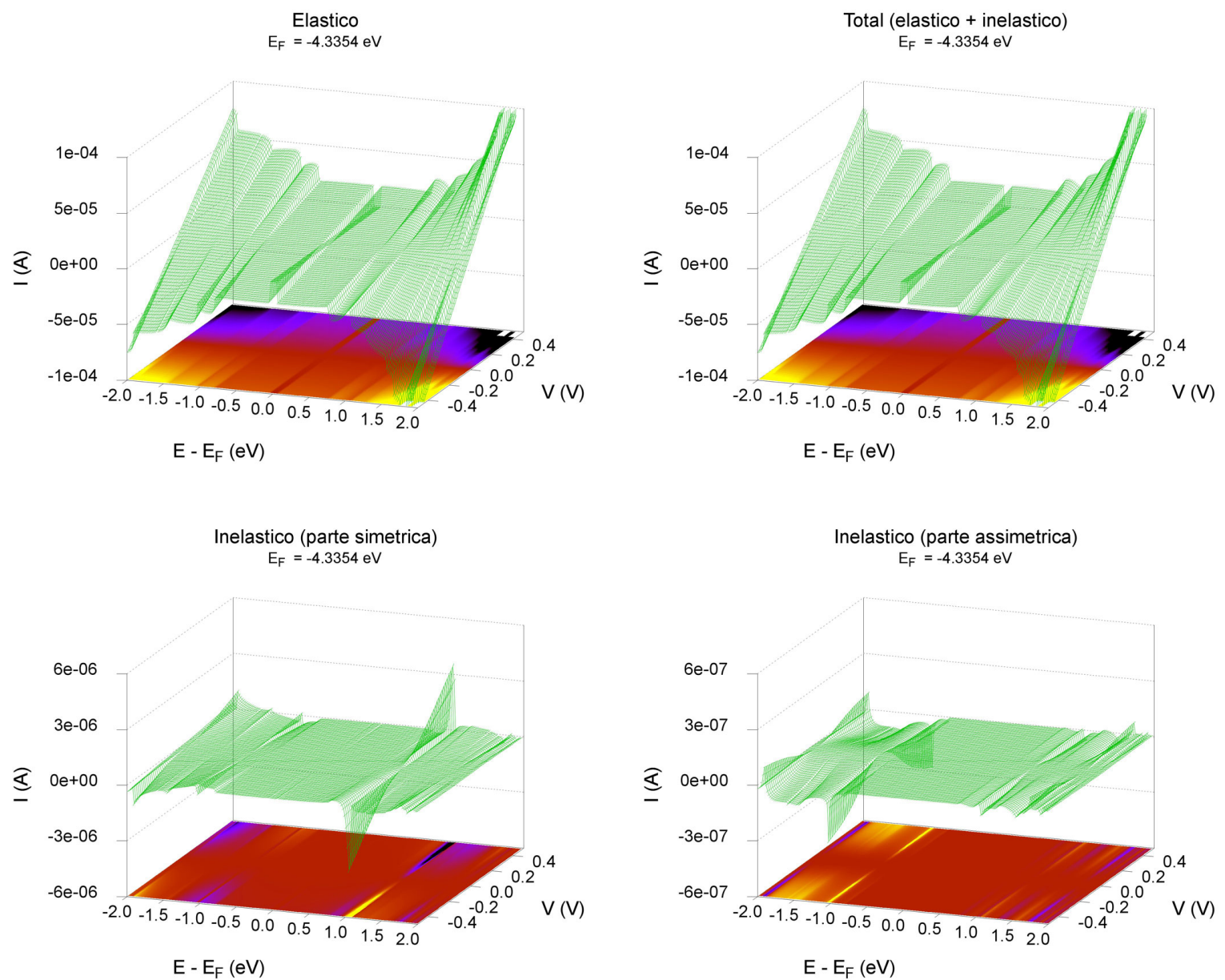

Figura 8.12: Valores de corrente obtidos para um único defeito (A) variando-se a tensão e a energia. Acima: contribuição elástica (à esquerda) e corrente total (à direita). Abaixo: componentes simétrica (à esquerda) e assimétrica (à direita) da corrente inelástica.

O efeito da interação elétron-fônon é praticamente imperceptível no gráfico da corrente total. De fato, verifica-se que as contribuições inelásticas são inferiores uma ordem de grandeza quando comparadas à contribuição elástica.

Ao analisar separadamente a corrente inelástica, verifica-se que a parcela mais expressiva provém da componente simétrica, sendo uma ordem de grandeza superior à componente assimétrica. 
Constata-se, também, que os fônons são excitados somente a partir de energias $\sim \pm 1 \mathrm{eV}$, onde um sinal acentuado é observado. Comparando esse resultado com os da Figura 8.10, nota-se que justamente nestas energias o efeito de retroespalhamento na transmissão é pronunciado e um aumento na PDOS do defeito é observado.

Na Figura 8.13, estão dispostas as condutâncias diferenciais obtidas para o defeito (A) variando-se a tensão e a energia.
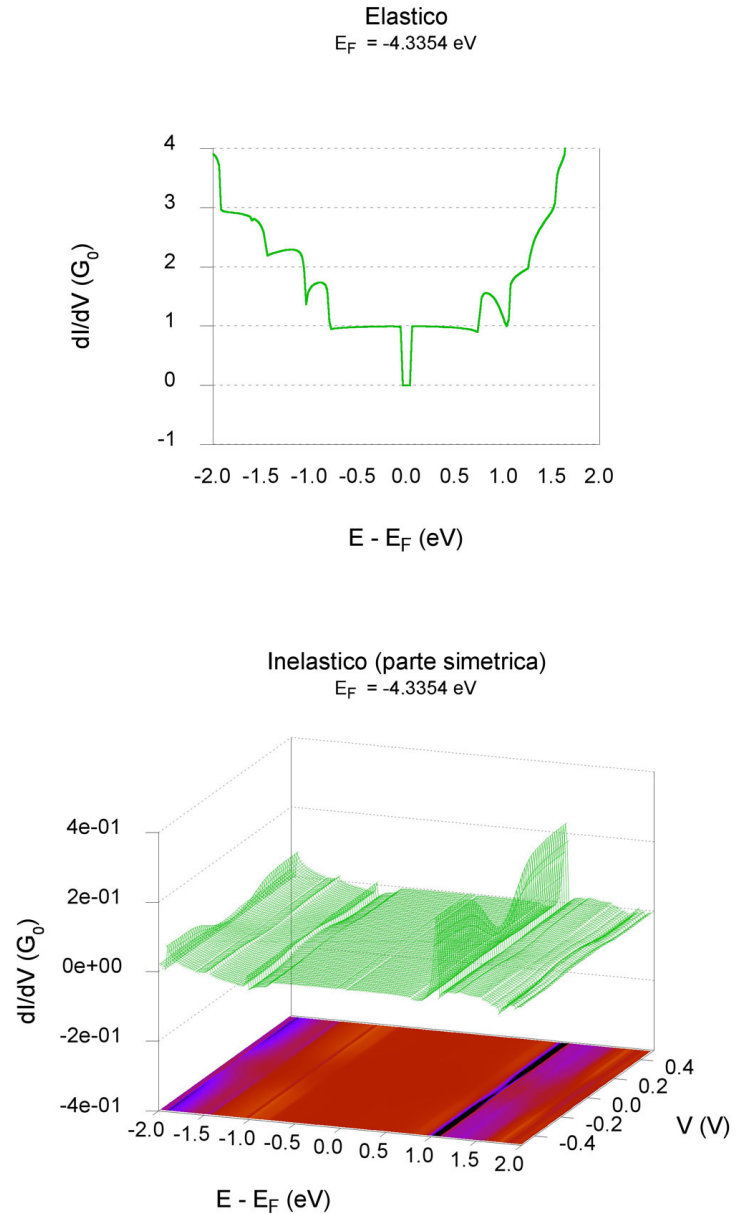
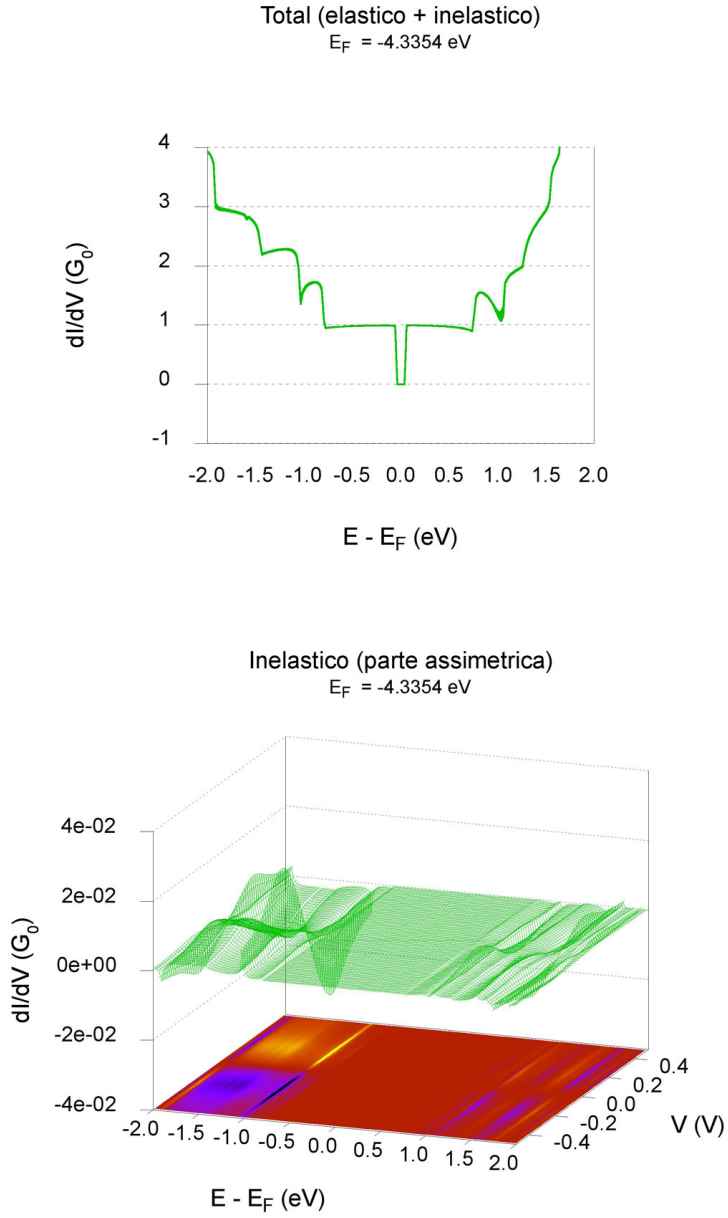

Figura 8.13: Valores de condutância diferencial obtidos para um único defeito (A) variando-se a tensão e a energia. Acima: contribuição elástica (à esquerda) e condutância total (à direita). Abaixo: componentes simétrica (à esquerda) e assimétrica (à direita) da contribuição inelástica.

Novamente, o efeito de espalhamento inelástico dos elétrons é praticamente imperceptível no gráfico da condutância diferencial total. Observa-se somente um pequeno aumento na condutância em $E-E_{F} \approx 1 \mathrm{eV}$. Pelos gráficos inferiores da Figura 8.13, verifica-se que esse efeito decorre da componente simétrica, que, por sua vez, atinge um patamar máximo a tensões de $\sim \pm 0.2 \mathrm{~V}$. 
Conforme discutido no Capítulo 1, uma das técnicas experimentais que vem sendo empregada no estudo do efeito dos modos vibracionais no transporte eletrônico é a espectroscopia de tunelamento inelástico de elétrons (IETS) [21]. No IETS, esse efeito aparece como picos na curva $d^{2} I / d V^{2}$ que surgem quando a tensão aplicada corresponde à energia de um modo vibracional do sistema estudado. Estas observações podem ser reproduzidas com a LOE, conforme pode ser verificado na Figura 8.14 .
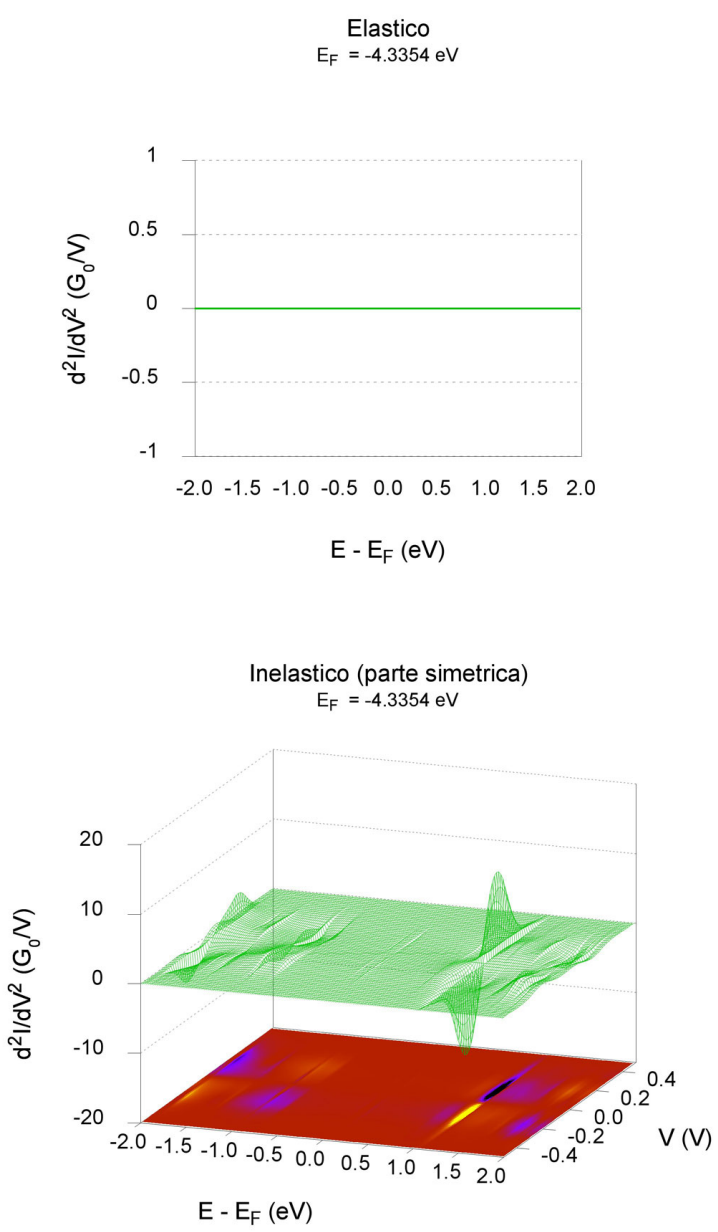

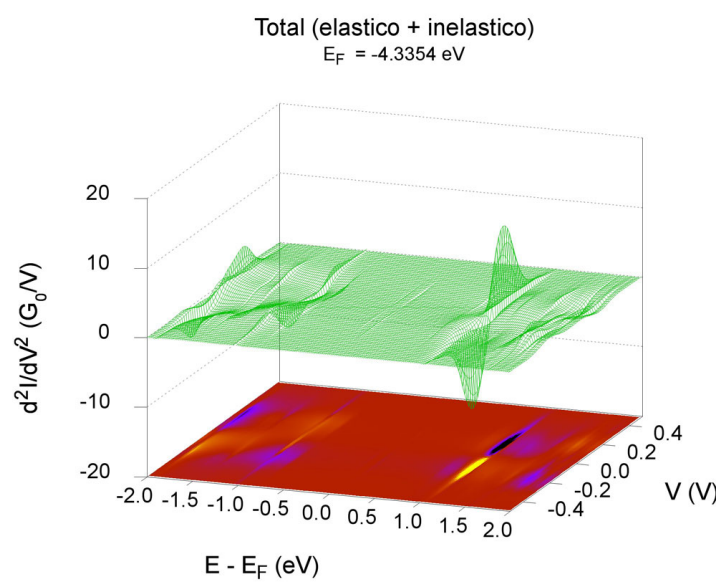

Inelastico (parte assimetrica) $E_{F}=-4.3354 \mathrm{eV}$

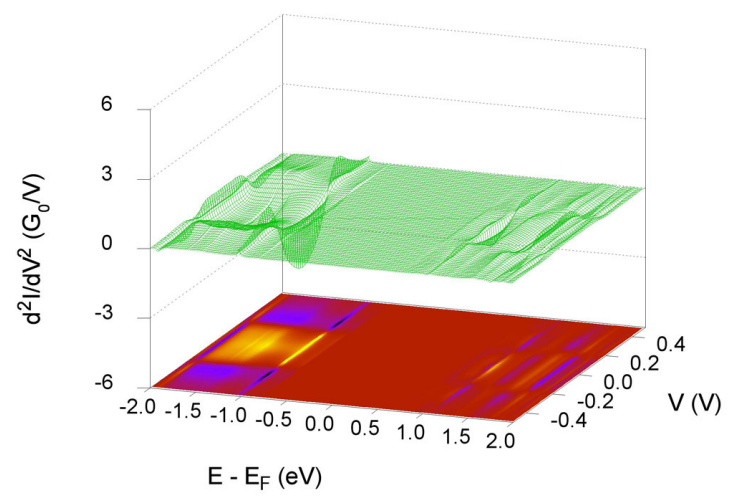

Figura 8.14: Superficies $d^{2} I / d V^{2}$ obtidas para um único defeito (A) variando-se a tensão e a energia. Acima: contribuição elástica (à esquerda) e $d^{2} I / d V^{2}$ total (à direita). Abaixo: componentes simétrica (à esquerda) e assimétrica (à direita) da contribuição inelástica.

Como a condutância diferencial elástica é constante com a variação da tensão, a componente elástica da LOE não fornece nenhuma contribuição para a superfície $d^{2} I / d V^{2}$ total. Novamente, o sinal observado em $E-E_{F} \approx 1 \mathrm{eV}$ provém da parte simétrica da componente inelástica e possui valores mínimo e máximo em $\sim-0.1 \mathrm{~V}$ e $\sim+0.1 \mathrm{~V}$, respectivamente.

Diferentemente da aproximação de Born discutida no Capítulo 6, a LOE permite analisar 
os efeitos da interação elétron-fônon no transporte considerando cada modo individualmente (ver Equação 7.42). Na Figura 8.15, estão dispostos os valores de $d^{2} I / d V^{2}$ obtidos considerando separadamente os modos vibracionais destacados na Figura 8.11. M23 $\left(\hbar \omega_{23}=0.0940 \mathrm{eV}\right)$, M14 $\left(\hbar \omega_{14}=0.1434 \mathrm{eV}\right)$, M12 $\left(\hbar \omega_{12}=0.1500 \mathrm{eV}\right)$ e M5 $\left(\hbar \omega_{5}=0.2032 \mathrm{eV}\right)$.
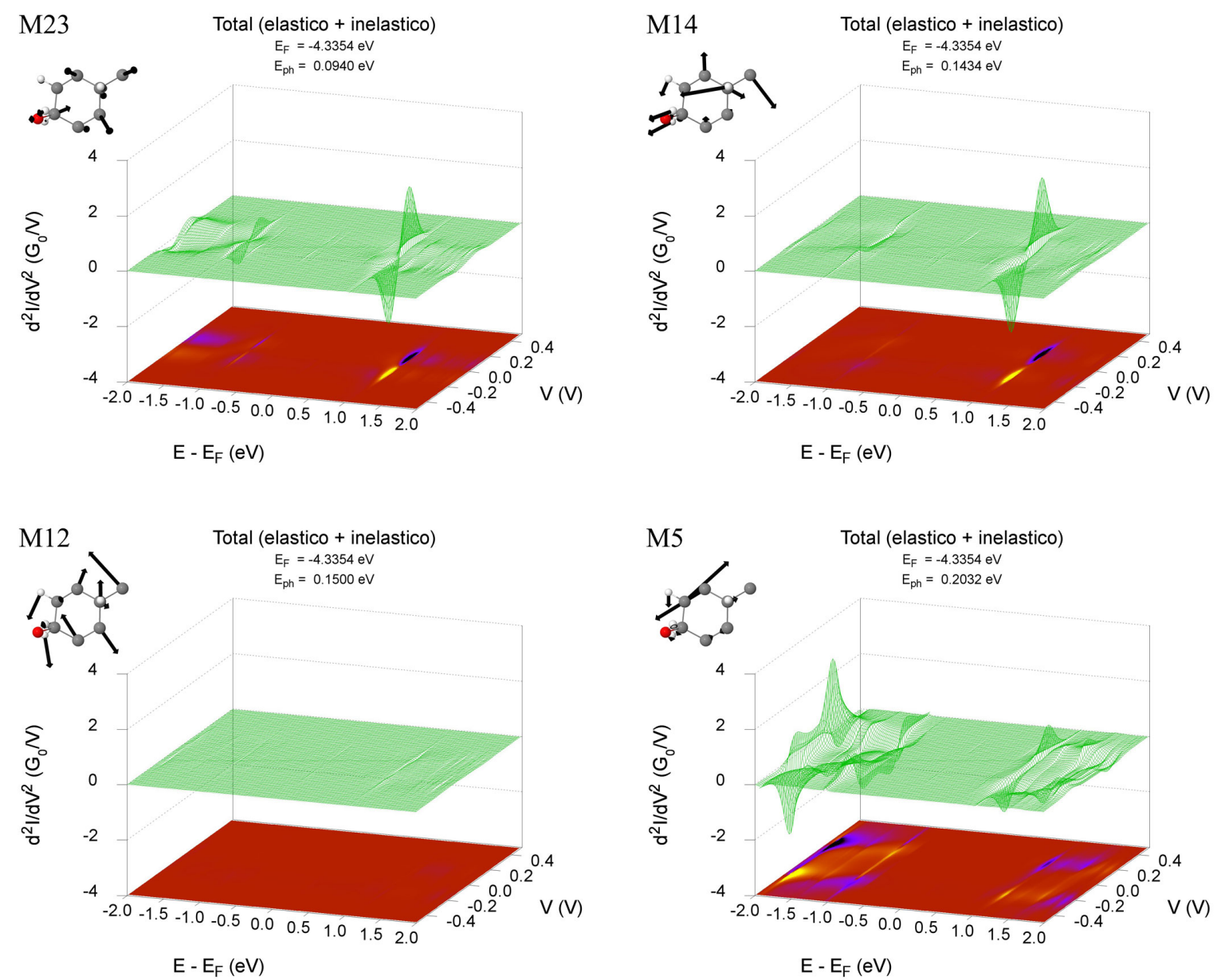

Figura 8.15: Superfícies $d^{2} I / d V^{2}$ obtidas para um único defeito (A) variando-se a tensão e a energia, para os modos vibracionais $\hbar \omega_{23}=0.0940 \mathrm{eV}$ (acima à esquerda), $\hbar \omega_{14}=0.1434 \mathrm{eV}$ (acima à direita), $\hbar \omega_{12}=0.1500 \mathrm{eV}$ (abaixo à esquerda) e $\hbar \omega_{5}=0.2032 \mathrm{eV}$ (abaixo à direita).

Os modos M23 e M14, na parte superior da Figura 8.15, apresentaram os sinais mais pronunciados na superfície $d^{2} I / d V^{2}$. Comparando-se esses gráficos com o obtido considerando-se todos os modos (Figura 8.14), verifica-se que os modos M23 e M14 são os maiores responsáveis pelo sinal observado em $E-E_{F} \approx 1 \mathrm{eV}$. A maioria dos 36 modos vibracionais calculados para o defeito (A) apresentaram sinais pequenos quando comparados ao M23 e M14, como é o caso do M12 $\left(\hbar \omega_{12}=0.1500 \mathrm{eV}\right)$ na parte inferior da Figura 8.15 à esquerda, onde o sinal é praticamente imperceptível quando mantemos os gráficos sob a mesma escala. 
Essa análise individual de cada modo nos permite verificar que a excitação do modo vibracional ocorre quando a tensão atinge a energia dos fônons, da mesma maneira como havíamos observado nos modelos simples apresentados na Seção 7.6. A seguir, discutiremos os resultados obtidos para sistemas desordenados, onde, para facilitar a discussão dos resultados, iremos considerar somente o modo M14 do defeito (A).

\subsection{Sistema Desordenado}

Uma vez analisado o efeito dos defeitos de maneira isolada, o próximo passo foi simular nanofitas com os defeitos distribuídos aleatoriamente com o programa I-DISORDER. Conforme foi discutido na Seção 7.5 , em uma etapa preliminar um cálculo de estrutura eletrônica para cada defeito e para a nanofita pristina é realizado com o segmento repetido periodicamente na direção do transporte. Nesses cálculos, as matrizes Hamiltoniana e de overlap de cada célula unitária e respectivos acoplamentos são armazenadas para, então, serem fornecidos como entrada ao programa I-DISORDER.

Nesta seção, assim como na anterior, vamos nos limitar à discussão dos resultados obtidos a partir da simulação de sistemas desordenados contendo somente o defeito (A). Um exemplo de sistema contendo oito blocos, sendo seis pristinos e dois com o defeito, pode ser observado na Figura 8.16 .

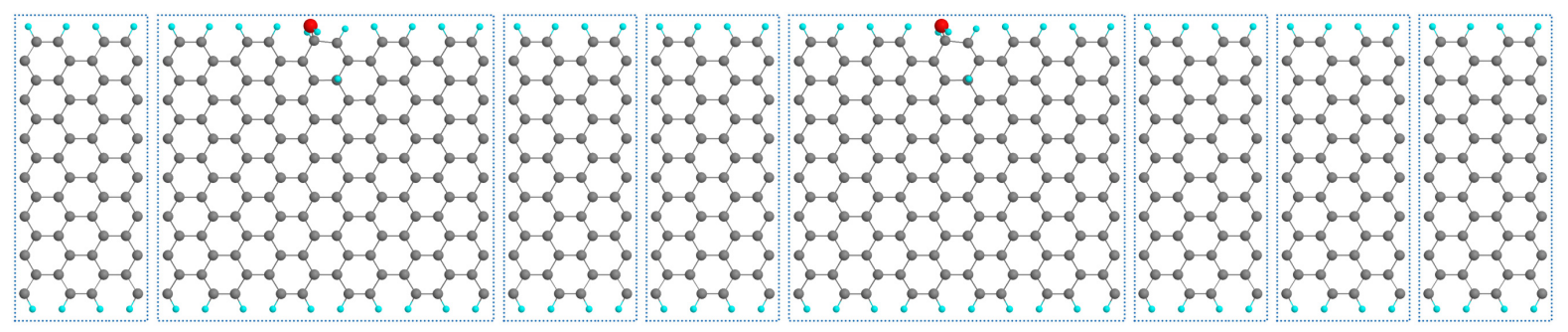

Figura 8.16: Exemplo de um sistema desordenado contendo dois segmentos com o defeito (A) e seis segmentos pristinos.

Nota-se que o bloco contendo o defeito (A) possui um comprimento maior quando comparado ao segmento apresentado na Figura 8.6. Isso é necessário no programa I-DISORDER para garantir que o acoplamento entre dois segmentos seja sempre igual ao de um sistema pristino.

Em posse das matrizes Hamiltoniana e de overlap dos segmentos, bem como da matriz de acoplamento elétron-fônon calculada para o defeito (A), a cada realização o programa gera aleatoriamente o sistema a ser simulado de acordo com a quantidade total de blocos e o número de 
defeitos especificados pelo usuário.

Nos resultados apresentados a seguir, a concentração de defeitos foi mantida fixa e variou-se o comprimento das nanofitas. Foram consideradas nanofitas com os comprimentos $L_{2}=19,89$ $\mathrm{nm}$ ( 2 blocos com defeito e 18 blocos pristinos), $L_{5}=49,73 \mathrm{~nm}$ ( 5 blocos com defeito e 45 blocos pristinos), $L_{10}=99,46 \mathrm{~nm}$ (10 blocos com defeito e 90 blocos pristinos) e $L_{20}=198,92 \mathrm{~nm}(20$ blocos com defeito e 180 blocos pristinos).

Na Figura 8.17 pode-se observar a condutância diferencial elástica média proveniente de 20 realizações das nanofitas $L_{2}, L_{5}, L_{10}$ e $L_{20}$.
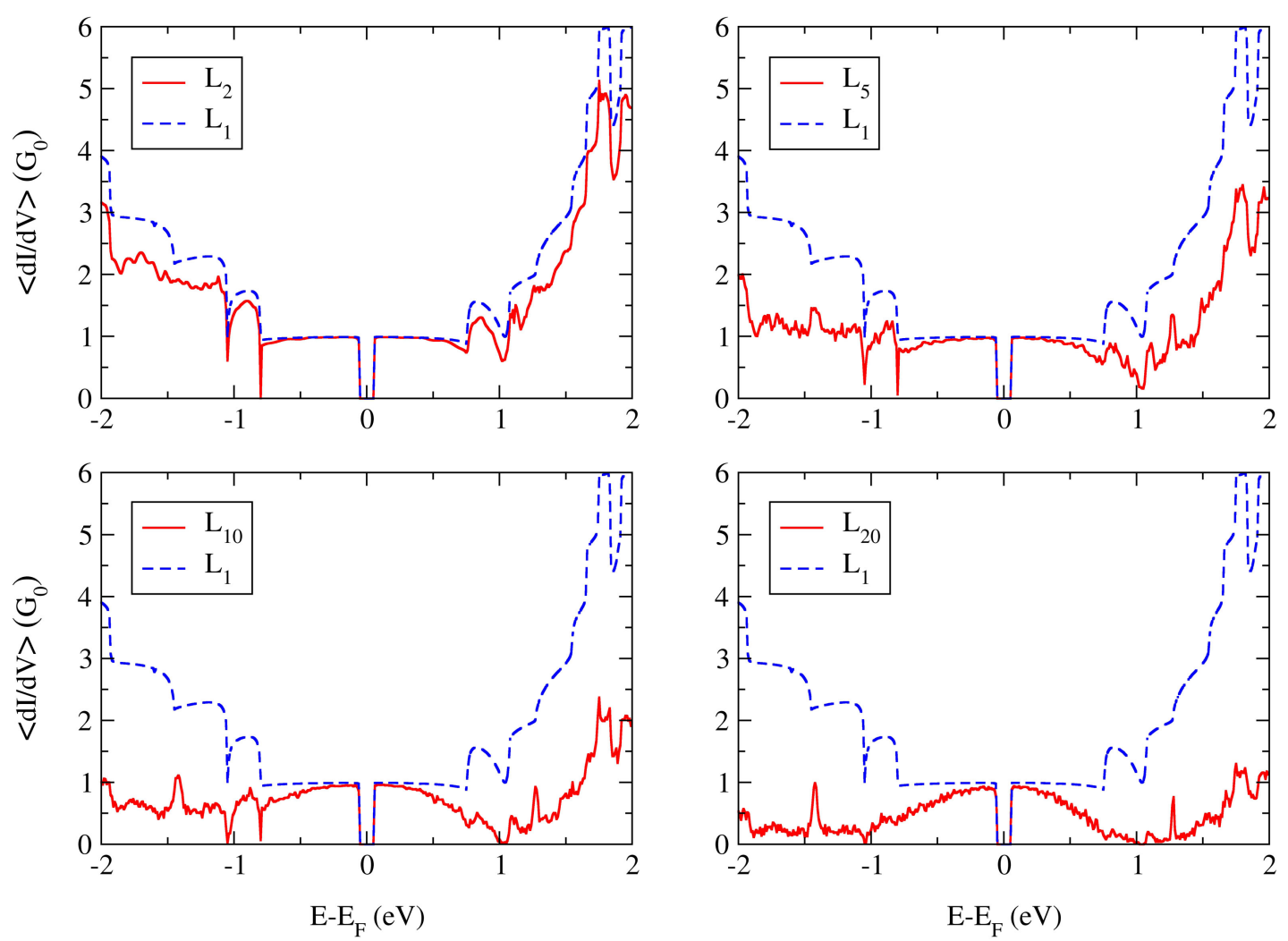

Figura 8.17: Condutância diferencial elástica média computada a partir de 20 realizações de nanofitas com comprimentos $L_{2}=19,89 \mathrm{~nm}$ (acima à esquerda), $L_{5}=49,73 \mathrm{~nm}$ (acima à direita), $L_{2}=99,46$ $n m$ (abaixo à esquerda) e $L_{20}=198,92 \mathrm{~nm}$ (abaixo à direita), onde a linha tracejada corresponde à condutância diferencial calculada com somente um defeito $(A)$.

Comparando-se esses resultados com a condutância diferencial calculada com um único defeito (A) (linha tracejada nos gráficos da Figura 8.17), verifica-se que, com o aumento do comprimento do sistema, ocorre a diminuição da condutância diferencial média. Nota-se, também, que o padrão de degraus na condutância fica cada vez mais distorcido, o que se deve à flutuação 
gerada pelas diferentes realizações.

Para facilitar a discussão dos resultados obtidos para o transporte inelástico de sistemas desordenados, vamos considerar somente o modo vibracional M14, que, como vimos, apresentou um efeito mais pronunciado nas propriedades de transporte. Na Figura 8.18, estão dispostos os resultados provenientes de 20 realizações das nanofitas $L_{2}$.
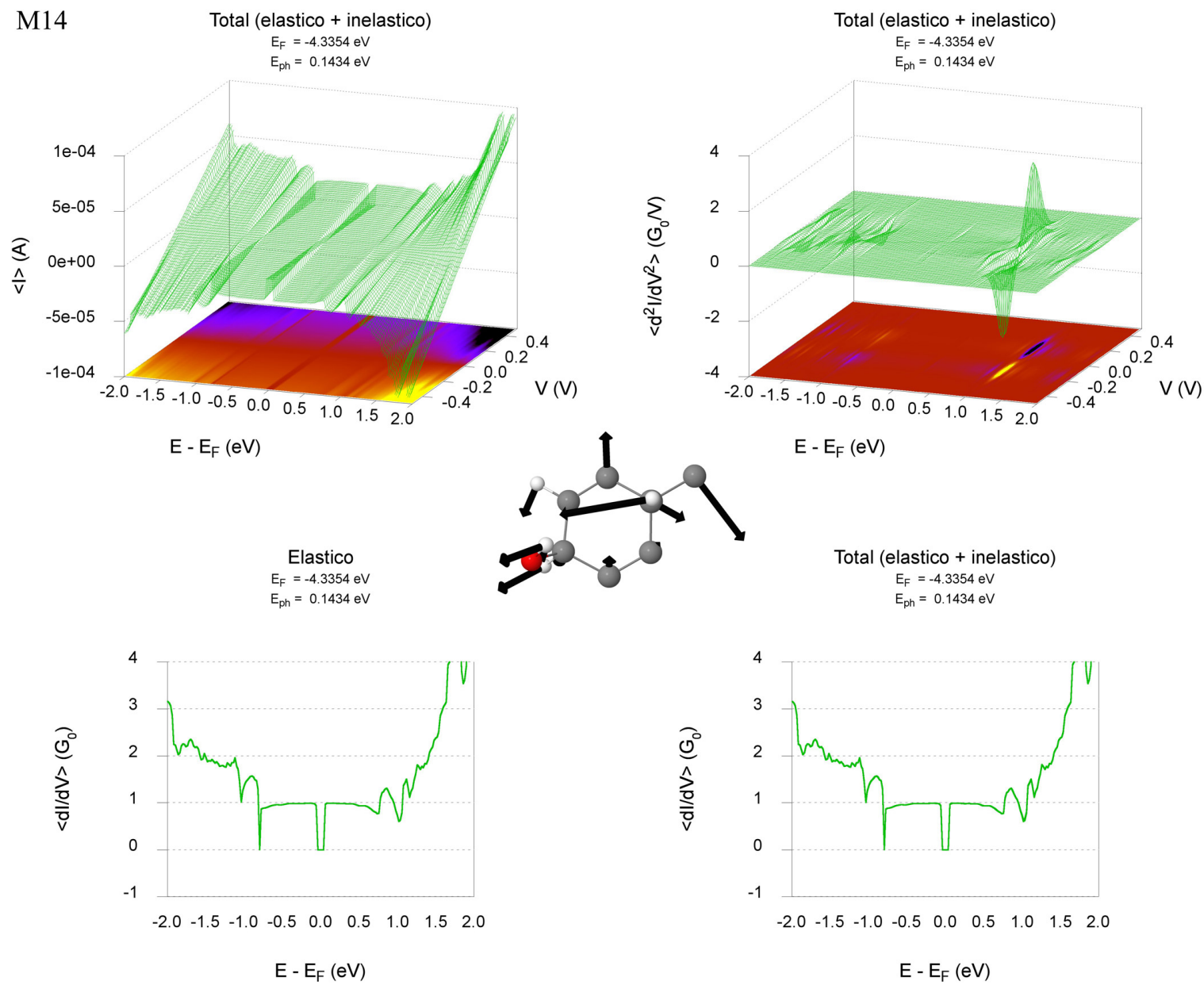

Figura 8.18: Resultados obtidos a partir de 20 realizaçôes de uma nanofita de comprimento $L_{2}=19,89$ $n m$ com dois defeitos do tipo (A), onde foi considerado somente o modo vibracional M14 ( $\hbar \omega_{14}=0.1434$ $e V$ ), ilustrado ao centro. Acima: valores médios de corrente (esquerda) e da superfície $d^{2} I / d V^{2}$ (direita), obtidos variando-se a tensão e a energia. Abaixo: condutância diferencial média elástica (esquerda) e total (direita).

Para as nanofitas com comprimento $L_{2}=19,89 \mathrm{~nm}$, com dois defeitos do tipo (A), os resultados apresentam pequenas diferenças quando comparados aos apresentados nas Figuras 8.12 8.14. Nota-se uma pequena redução nos valores de corrente e na condutância diferencial. Por outro lado, um pequeno aumento do sinal no espectro $d^{2} I / d V^{2}$ pode ser observado quando 
comparado ao resultado obtido para o modo M14 com um único defeito (Figura 8.15).

Com o aumento do comprimento do sistema para $L_{5}=49,73 \mathrm{~nm}$ (Figura 8.19), com cinco defeitos do tipo (A), uma mudança significativa ocorre na superfície $d^{2} I / d V^{2}$.
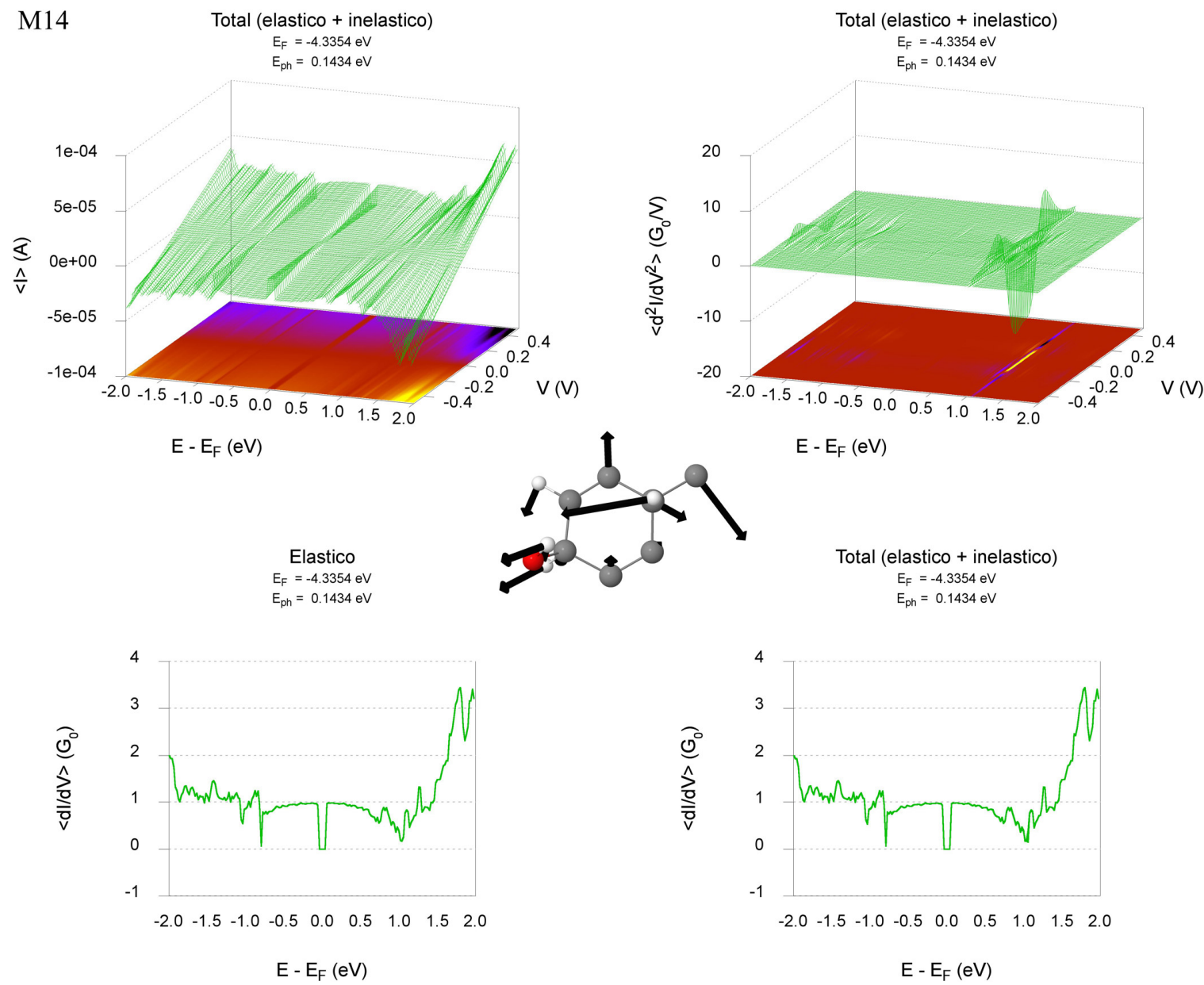

Figura 8.19: Resultados obtidos a partir de 20 realizações de uma nanofita de comprimento $L_{5}=49,73$ $n m$ com cinco defeitos do tipo (A), onde foi considerado somente o modo vibracional M14 ( $\hbar \omega_{14}=0.1434$ eV), ilustrado ao centro. Acima: valores médios de corrente (esquerda) e da superfície $d^{2} I / d V^{2}$ (direita), obtidos variando-se a tensão e a energia. Abaixo: condutância diferencial média elástica (esquerda) e total (direita).

Novamente, observa-se uma redução na corrente e na condutância diferencial média. Porém, um aumento significativo ocorre no sinal do espectro $d^{2} I / d V^{2}$. Além disso, nota-se que a contribuição assimétrica passa a dominar sobre a simétrica na componente inelástica do transporte. Essa tendência persiste quando passamos para as nanofitas de comprimento $L_{10}=99,46 \mathrm{~nm}$, conforme pode ser observado na Figura 8.20

No caso das nanofitas $L_{10}$, nota-se que, ao considerar a interação elétron-fônon, a condutância 

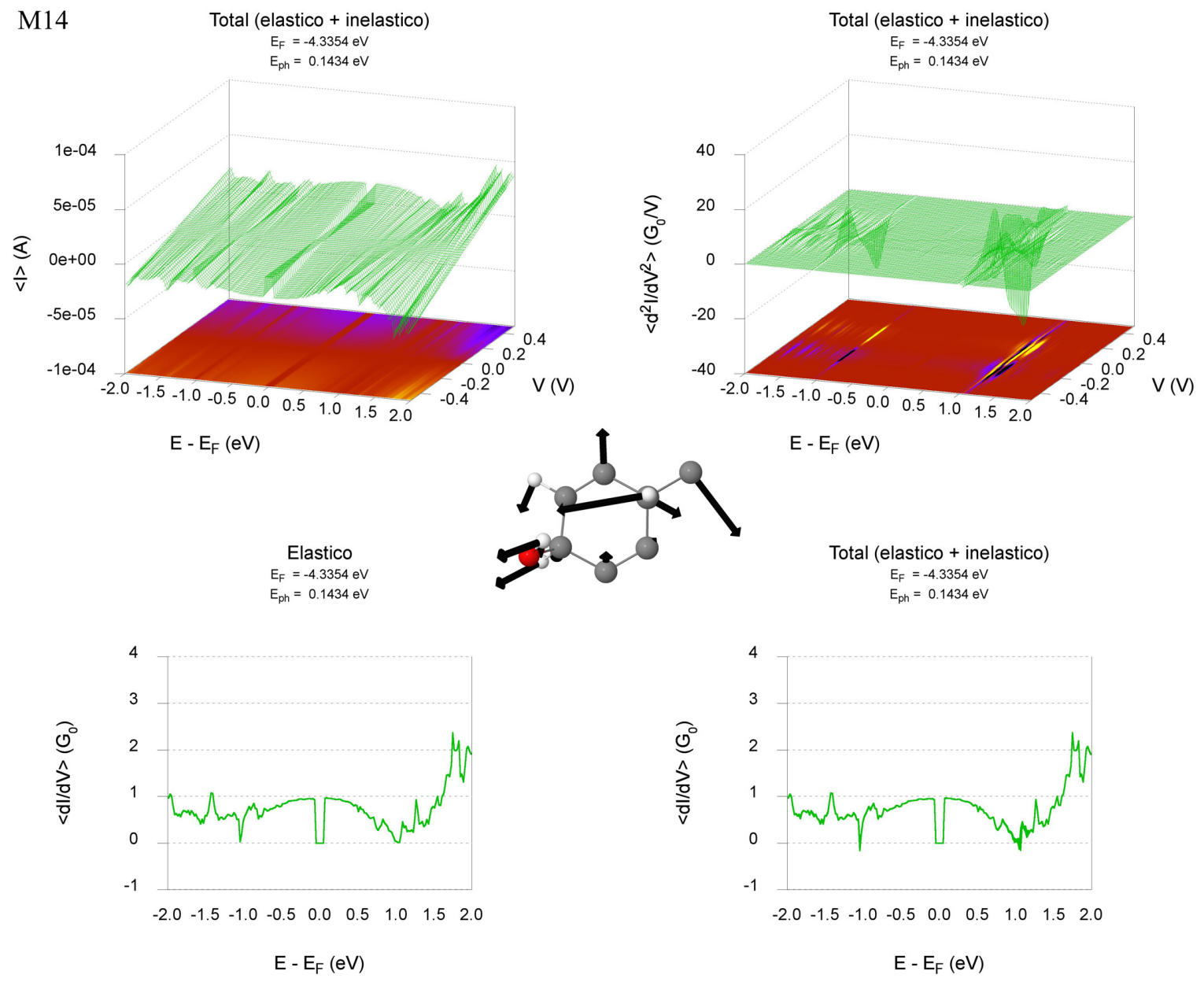

Figura 8.20: Resultados obtidos a partir de 20 realizações de uma nanofita de comprimento $L_{10}=99,46$ $n m$ com dez defeitos do tipo (A), onde foi considerado somente o modo vibracional M14 ( $\hbar \omega_{14}=0.1434$ eV), ilustrado ao centro. Acima: valores médios de corrente (esquerda) e da superfície $d^{2} I / d V^{2}$ (direita), obtidos variando-se a tensão e a energia. Abaixo: condutância diferencial média elástica (esquerda) e total (direita).

diferencial total média chega a atingir valores negativos. De fato, para energias $E-E_{F} \sim \pm 1 \mathrm{eV}$ a corrente inelástica passa a ser maior em valores absolutos do que a corrente elástica, levando a correntes negativas sob tensões positivas.

O comportamento das contribuições simétrica e assimétrica da superfície $d^{2} I / d V^{2}$ com o aumento do comprimento do sistema pode ser observado na Figura 8.21. Para a componente simétrica nota-se um aumento do ruído no sinal $d^{2} I / d V^{2}$ a partir de $L_{5}$ e um aumento significativo de magnitude quando passamos de $L_{5}$ para $L_{10}$. Já para a componente assimétrica observa-se um aumento substancial do sinal em $E-E_{F} \sim 1 \mathrm{eV}$ com o acréscimo do número de defeitos.

Esses resultados parecem indicar que o aumento da quantidade de defeitos gera um efeito aditivo na matriz de acoplamento elétron-fônon total, de forma que esse acoplamento já não pode 

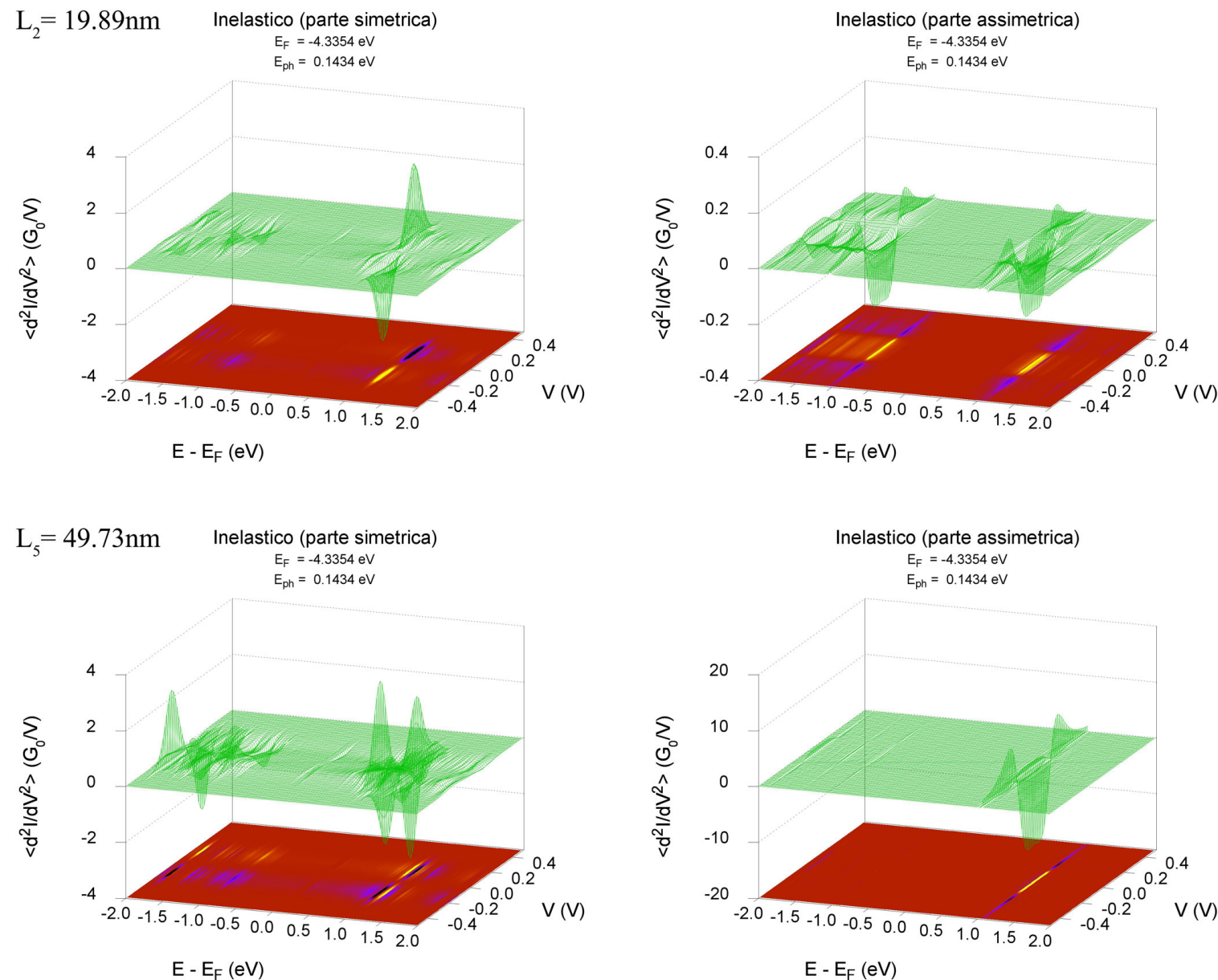

$\mathrm{L}_{10}=99.46 \mathrm{~nm} \quad$ Inelastico (parte simetrica)
$\mathrm{E}_{\mathrm{F}}=-4.3354 \mathrm{eV}$
$\mathrm{E}_{\mathrm{ph}}=0.1434 \mathrm{eV}$

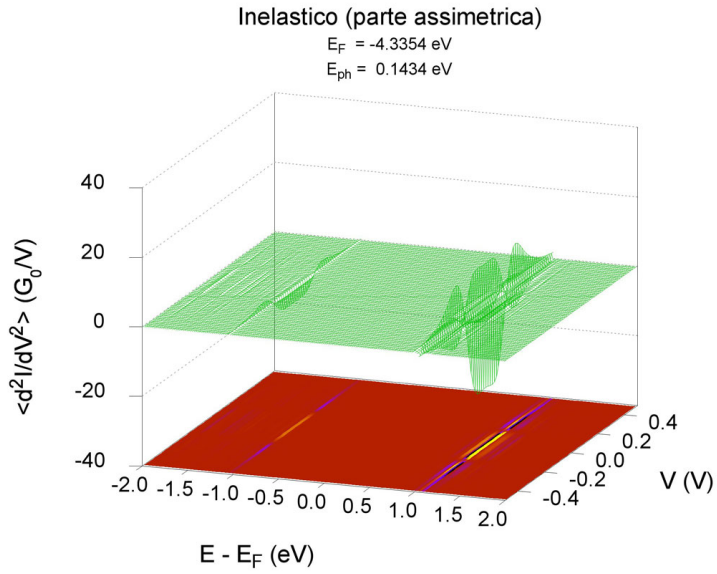

Figura 8.21: Valores médios das componentes simétrica (esquerda) e assimétrica (direita) da superfície $d^{2} I / d V^{2}$ obtidos a partir de 20 realizações de nanofitas com comprimentos $L_{2}=19,89 \mathrm{~nm}$ (acima), $L_{5}=49,73 \mathrm{~nm}$ (meio) e $L_{10}=99,46 \mathrm{~nm}$ (abaixo), onde foi considerado somente o modo vibracional M14 $\left(\hbar \omega_{14}=0.1434 \mathrm{eV}\right)$. 
mais ser tratado perturbativamente e a aproximação LOE parece falhar.

Por outro lado, para uma pequena quantidade de defeitos, observa-se que, enquanto a corrente elástica tende a diminuir com o acréscimo da desordem no sistema, os efeitos de interação elétronfônon tendem a aumentar. Esses resultados preliminares sugerem que considerar os efeitos de interação elétron-fônon pode influenciar nos efeitos de localização por desordem. 


\section{Capítulo 9}

\section{Conclusões Gerais}

Com o rápido avanço das técnicas experimentais observado nas últimas décadas, a fabricação e a observação de sistemas nanoestruturados se tornaram uma realidade. Nessa escala de grandeza, efeitos quânticos como coerência de fase e discretização da carga não podem ser negligenciados e novas propriedades de transporte emergem. Em particular, diversos experimentos evidenciam que as vibrações nucleares podem desempenhar um papel importante no transporte eletrônico. Essas observações motivaram a escolha do tema desta tese.

Para o cálculo das frequências e dos modos vibracionais de materiais e para calcular a matriz de acoplamento elétron-fônon, a partir de métodos de primeiros princípios, foi desenvolvido o programa PhOnonS ITeratIVE VIBRATIONS. No cálculo da matriz de acoplamento elétronfônon, além da implementação do código, algumas intervenções foram realizadas no programa SIESTA [2,3], uma implementação da DFT utilizada como plataforma de cálculos de estrutura eletrônica.

Diferentes abordagens para a interação elétron-fônon foram estudadas. O efeito de perda de coerência de fase devido à interação elétron-fônon foi considerado por meio do modelo fenomenológico das sondas de Büttiker [1]. Foram realizadas duas implementações diferentes desse modelo, a primeira na forma usual, onde se considera uma aproximação elástica para o cálculo da corrente, e a segunda por meio de uma nova proposta sem a aproximação elástica. Como a autoenergia de interação utilizada não contém informação a respeito da estrutura dos fônons, o modelo produz somente um alargamento do canal de condutância. Portanto, o modelo de Büttiker é interessante quando se quer considerar apenas o efeito de perda de coerência de fase dos elétrons devido à interação com fônons do material.

Uma abordagem mais elaborada consiste em expandir a autoenergia de interação perturbativamente em diagramas de Feynman até a primeira ordem, o que é convencionalmente chamado de primeira aproximação de Born. Essa aproximação assim como a sua versão autoconsis- 
tente, onde uma classe mais ampla de diagramas é considerada, foram incorporadas ao programa SMEAGOL [4], um código de transporte eletrônico ab initio baseado na combinação DFT-NEGF que utiliza como plataforma do cálculo da estrutura eletrônica o código SIESTA.

Porém, antes de incluir o transporte inelástico no SMEAGOL, que era baseado em uma versão obsoleta do SIESTA, foi realizada a transição do código para a versão 3.1 do programa SIESTA. Ao longo dessa transição, uma série de melhorias e novas implementações foram realizadas. Essas mudanças e as implementações da aproximação de Born deram origem ao programa Inelastic SMEAGOL para cálculos de transporte inelástico ab initio.

Um resultado importante do ponto de vista computacional foi a reformulação da expressão usualmente utilizada para o cálculo da autoenergia retardada de interação na primeira aproximação de Born, o que reduziu drasticamente o custo computacional do cálculo e viabilizou o uso desta aproximação na simulação de sistemas com uma quantidade relativamente grande de átomos.

Contudo, o resultado mais importante deste trabalho foi a implementação do programa Inelastic DISORDER. Este código permite calcular, por primeiros princípios, as propriedades de transporte elástico e inelástico de sistemas com dezenas de milhares de átomos com um grande número de defeitos aleatoriamente distribuídos. O método combina cálculos de estrutura eletrônica via DFT com o formalismo NEGF para o transporte e as interações elétron-fônon são incluídas por meio de teoria de perturbação com relação à matriz de acoplamento elétron-fônon.

Aplicando-se o método desenvolvido em nanofitas de grafeno com impurezas hidroxílicas, observou-se que, ao incluir a interação elétron-fônon, as propriedades de transporte sofrem mudanças significativas, indicando que essas interações podem influenciar nos efeitos de localização por desordem. Ao mesmo tempo, observamos que, enquanto a condutividade elástica diminui com o aumento da desordem (como é de se esperar), há um aumento relativo da condutância diferencial próxima à ressonância do fônon. Isso indica que a presença de diversas impurezas pode levar a uma melhora da relação sinal/ruído na IETS (espectroscopia de tunelamento inelástico de elétrons).

\subsection{Perspectivas}

O trabalho realizado forneceu ferramentas para o estudo sistemático do transporte de carga em sistemas com escala nanométrica, considerando efeitos da interação elétron-fônon e da desordem gerada por defeitos. Esses efeitos podem ser estudados em função de variáveis como temperatura e ocupação dos fônons.

Nos resultados apresentados nesta tese, os fônons foram considerados no limite de amorteci- 
mento externo, onde a energia transferida pelos elétrons aos fônons é instantaneamente absorvida por um reservatório térmico externo (de forma que a ocupação dos fônons é fixada pela distribuição de Bose-Einstein). No entanto, estudos podem ser realizados considerando o aquecimento do sistema de fônons, onde a ocupação depende da diferença de potencial aplicada. Outra possibilidade interessante é estudar a potência transferida pelos elétrons ao sistema de fônons. O cálculo da ocupação dos fônons fora do equilíbrio, assim como o cálculo da potência dissipada, já estão disponíveis no programa I-DISORDER e podem ser implementadas no código I-SMEAGOL.

Outra implementação efetuada no código I-SMEAGOL é a possibilidade de realizar dinâmica molecular e otimização de estrutura, conforme as opções permitidas no SIESTA, na situação fora do equilíbrio junto com cálculos de transporte. Em particular, o cálculo das forças fora do equilíbrio foi implementado segundo a metodologia descrita em 108. Portanto, um estudo interessante consiste em analisar as frequências vibracionais e o acoplamento elétron-fônon a partir de cálculos de constante de forças variando-se a diferença de potencial aplicada.

Do ponto de vista de novos desenvolvimentos nos códigos, existem diversas melhorias e novas implementações que podem ser realizadas. Por exemplo, vimos que a aproximação de Born autoconsistente (SCBA), além de ser custosa computacionalmente, demanda uma quantidade significativa de memória. Dessa forma, as ideias utilizadas no I-DISORDER para o armazenamento em disco podem ser aplicadas ao I-SMEAGOL, o que possibilitaria a simulação de sistemas maiores com a SCBA. Para diminuir o custo computacional, um estudo semelhante ao realizado na reformulação da primeira aproximação de Born (1BA) poderia ser feito.

Outras duas mudanças no I-SMEAGOL possibilitariam um impacto significativo na performance da SCBA. A primeira seria considerar um grid de energia assimétrico, sendo mais denso somente ao redor dos potenciais eletroquímicos dos eletrodos e rarefeito para energias mais baixas. Neste caso, a transformada de Hilbert pode ser realizada utilizando previamente uma interpolação na parte mais rarefeita do grid de energia. A segunda mudança seria implementar algoritmos para o controle e a aceleração da convergência do ciclo autoconsistente da SCBA.

Finalmente, uma mudança que pode trazer resultados interessantes seria considerar a aproximação de Born dentro do ciclo autoconsistente do I-SMEAGOL no cálculo da matriz densidade eletrônica. Como vimos, a autoenergia de Hartree promove um deslocamento dos níveis de energia, o que certamente influenciaria no cálculo da matriz densidade eletrônica. 


\section{Apêndice A}

\section{Prova dos Teoremas de}

\section{Hohenberg-Kohn}

Teorema I: A densidade $n_{0}(\mathbf{r})$ do estado fundamental de um sistema ligado de elétrons interagentes em um dado potencial externo $V_{\text {ext }}(\mathbf{r})$ determina unicamente este potencial.

Prova Do Teorema I: Seja $n(\mathbf{r})$ a densidade não-degenerada do estado fundamental de $p$ elétrons num potencial externo $V_{e x t}^{(1)}(\mathbf{r})$ com as correspondentes função de onda $\Psi^{(1)}$ e energia $E^{(1)}$. Logo:

$$
\begin{aligned}
E^{(1)} & =\left\langle\Psi^{(1)}\left|T+U+V^{(1)}\right| \Psi^{(1)}\right\rangle= \\
& =\int \Psi^{*(1)}(T+U) \Psi^{(1)} d \mathbf{r}+\sum_{i=1}^{p}\left\langle\Psi^{(1)}\left|V_{\text {ext }}^{(1)}(\mathbf{r}) \delta\left(\mathbf{r}-\mathbf{r}_{i}\right)\right| \Psi^{(1)}\right\rangle= \\
& =\int \Psi^{*(1)}(T+U) \Psi^{(1)} d \mathbf{r}+\int V_{\text {ext }}^{(1)}(\mathbf{r}) n(\mathbf{r}) d \mathbf{r} .
\end{aligned}
$$

Porém, como $\Psi^{(1)}$ é a função de onda do estado fundamental não-degenerado, então $E^{(1)}$ é a energia do estado fundamental, de modo que para qualquer $\Psi^{\prime} \neq e^{i \theta} \Psi^{(1)}$ resulta em $E^{\prime} \geqslant E^{(1)}$. Isso pode ser verificado a partir da equação de Schrödinger, Equação 3.1 . Seja $\left\{\left|\phi_{k}\right\rangle\right\}$ uma base ortonormal de autoestados do Hamiltoniano $\hat{H}^{(1)}=T+U+V^{(1)}$, então qualquer estado deste sistema pode ser escrito como uma combinação linear da forma $\left|\Psi^{\prime}\right\rangle=\sum_{k} c_{k}\left|\phi_{k}\right\rangle$. Considerando que a $\Psi^{\prime}$ é normalizada, tem-se:

$$
\left\langle\Psi^{\prime} \mid \Psi^{\prime}\right\rangle=1=\sum_{k, m} c_{k}^{*} c_{m} \underbrace{\left\langle\phi_{k} \mid \phi_{m}\right\rangle}_{\delta_{k m}}=\sum_{k}\left|c_{k}\right|^{2},
$$

de onde se conclui que $0 \leqslant\left|c_{k}\right| \leqslant 1, \forall n$. Portanto, a energia do estado $\left|\Psi^{\prime}\right\rangle$ será: 


$$
E^{\prime}=\left\langle\Psi^{\prime}|H| \Psi^{\prime}\right\rangle=\sum_{k, m} c_{k}^{*} c_{m}\left\langle\phi_{k}|H| \phi_{m}\right\rangle=\sum_{k, m} c_{k}^{*} c_{m} E_{m} \underbrace{\left\langle\phi_{k} \mid \phi_{m}\right\rangle}_{\delta_{k m}}=\sum_{k} E_{k}\left|c_{k}\right|^{2}
$$

Para qualquer autoestado $\left|\phi_{k}\right\rangle$ a respectiva autoenergia é tal que $E_{k} \geqslant E^{(1)}$, onde $E^{(1)}$ é a energia do estado fundamental, logo:

$$
E^{\prime}=\sum_{k} E_{k}\left|c_{k}\right|^{2} \geqslant E^{(1)} \sum_{\not k}\left|e_{k}\right|^{2}=E^{(1)} .
$$

Considere agora um potencial externo $V_{e x t}^{(2)}(\mathbf{r})$ distinto de $V_{e x t}^{(1)}(\mathbf{r})$ não somente por uma constante aditiva, cujo estado fundamental é $\Psi^{(2)} \neq e^{i \theta} \Psi^{(1)}$ e $E^{(2)}$ é a energia correspondente. Suponha por absurdo que tal potencial também leve à densidade $n(\mathbf{r})$, ou seja:

$$
E^{(2)}=\left\langle\Psi^{(2)}\left|T+U+V^{(2)}\right| \Psi^{(2)}\right\rangle=\left\langle\Psi^{(2)}|T+U| \Psi^{(2)}\right\rangle+\int V_{\text {ext }}^{(2)}(\mathbf{r}) n(\mathbf{r}) d \mathbf{r} .
$$

Conforme foi mostrado acima (Equação A.4), se $\Psi^{(1)}$ é a função de onda do estado fundamental não-degenerado de $H^{(1)}$, com energia $E^{(1)}$, então se pode afirmar que:

$$
\begin{aligned}
E^{(1)} & <\left\langle\Psi^{(2)}\left|T+U+V^{(1)}\right| \Psi^{(2)}\right\rangle= \\
& =\left\langle\Psi^{(2)}|T+U| \Psi^{(2)}\right\rangle+\int V_{\text {ext }}^{(1)}(\mathbf{r}) n(\mathbf{r}) d \mathbf{r}=\stackrel{\text { A.5 }}{\longrightarrow}\left\langle\Psi^{(2)}|T+U| \Psi^{(2)}\right\rangle=E^{(2)}-\int V_{\text {ext }}^{(2)}(\mathbf{r}) n(\mathbf{r}) d \mathbf{r} \\
& =E^{(2)}+\int\left[V_{\text {ext }}^{(1)}(\mathbf{r})-V_{\text {ext }}^{(2)}(\mathbf{r})\right] n(\mathbf{r}) d \mathbf{r} .
\end{aligned}
$$

Da mesma maneira, para o potencial externo $V_{e x t}^{(2)}(\mathbf{r})$, tem-se uma inequação para a energia $E^{(2)}$, porém aqui vale a igualdade, já que não foi assumido que $\Psi^{(2)}$ não é degenerado:

$$
E^{(2)} \leqslant\left\langle\Psi^{(1)}\left|T+U+V^{(2)}\right| \Psi^{(1)}\right\rangle=E^{(1)}+\int\left[V_{\text {ext }}^{(2)}(\mathbf{r})-V_{\text {ext }}^{(1)}(\mathbf{r})\right] n(\mathbf{r}) d \mathbf{r} .
$$

Somando a Equação A.6 com a Equação A.7 encontra-se a seguinte contradição:

$$
E^{(1)}+E^{(2)} \stackrel{!}{<} E^{(1)}+E^{(2)}
$$

Portanto, não podem haver dois potenciais externos $V_{\text {ext }}^{(1)}(\mathbf{r}) \neq V_{\text {ext }}^{(2)}(\mathbf{r})$, distintos não somente por uma constante aditiva, que levam a uma mesma densidade $n(\mathbf{r})$. 
Teorema II: Existe um funcional universal para a energia $E[n]$ em termos da densidade $n(\mathbf{r})$ que é válido para qualquer potencial externo. Fixado um $V_{\text {ext }}(\mathbf{r})$, a energia do estado fundamental corresponde ao mínimo global deste funcional, e a densidade $n(\mathbf{r})$ que o minimiza é exatamente a densidade do estado fundamental $n_{0}(\mathbf{r})$.

Prova Do Teorema II: A demonstração do teorema a seguir é conhecida como método de busca forçada (do inglês constrained search method) e é devida a M. Levy e E. Lieb.1

Considere uma função de prova normalizada $\tilde{\Psi}(\mathbf{r})$ de um sistema com $p$ elétrons. Em princípio, a energia do estado fundamental poderia ser obtida minimizando a energia com relação a todas as $3 p$ variáveis de $\tilde{\Psi}(\mathbf{r})$ :

$$
E_{0}=\min _{\tilde{\Psi}}\langle\tilde{\Psi}|T+U+V| \tilde{\Psi}\rangle
$$

Todavia, como para cada função de prova $\tilde{\Psi}(\mathbf{r})$ existe uma densidade correspondente, o problema pode ser dividido em duas etapas:

$1^{\text {a }}$. Fixa-se uma densidade de prova $\tilde{n}(\mathbf{r})$ e minimiza-se a energia somente com relação à classe de funções $\tilde{\Psi}_{\tilde{n}}(\mathbf{r})$ com mesma densidade $\tilde{n}(\mathbf{r})$ :

$$
E[\tilde{n}(\mathbf{r})]=\min _{\tilde{\Psi}_{\tilde{n}}}\left\langle\tilde{\Psi}_{\tilde{n}}|T+U+V| \tilde{\Psi}_{\tilde{n}}\right\rangle=F[\tilde{n}(\mathbf{r})]+\int V_{\text {ext }}(\mathbf{r}) \tilde{n}(\mathbf{r}) d \mathbf{r},
$$

onde $F[\tilde{n}(\mathbf{r})] \equiv \min _{\tilde{\Psi}_{\tilde{n}}}\left\langle\tilde{\Psi}_{\tilde{n}}|T+U| \tilde{\Psi}_{\tilde{n}}\right\rangle$ é um funcional universal da densidade $\tilde{n}(\mathbf{r})$, já que não possui dependência explícita com $V_{\text {ext }}(\mathbf{r})$.

$2^{\text {a }}$. Minimiza-se a energia com relação a todas as densidades $\tilde{n}(\mathbf{r})$ :

$$
E=\min _{\tilde{n}} E[\tilde{n}(\mathbf{r})]=\min _{\tilde{n}}\left\{F[\tilde{n}(\mathbf{r})]+\int V_{\text {ext }}(\mathbf{r}) \tilde{n}(\mathbf{r}) d \mathbf{r}\right\}
$$

Se o estado fundamental for não-degenerado, a minimização ocorre para $\tilde{n}(\mathbf{r})=n_{0}(\mathbf{r})$, a densidade do estado fundamental. No caso degenerado, a minimização ocorrerá com qualquer uma das densidades desses estados.

\footnotetext{
${ }^{1}$ M. Levy. Phys. Rev. A 26, 1200 (1982). E. Lieb. Physics as Natural Philosophy: Essays in Honor of Laszlo Tisza on His 75th Birthday. MIT Press, Cambridge, (1982). - citados em 69, 71
} 


\section{Apêndice B}

\section{Cálculo de Integrais no Plano}

\section{Complexo}

Os resultados a seguir serão utilizados na derivação de uma nova expressão para o cálculo da autoenergia retardada. Seja $f$ uma função analítica na metade superior do plano complexo, exceto por um número finito de pontos onde a função possui polos, e suponha que $f(z) \rightarrow 0$ para $z \rightarrow \infty$. Queremos calcular a integral:

$$
\int_{-\infty}^{+\infty} d x \frac{f(x)}{x-z_{0}}
$$

onde $z_{0}=x_{0}-i \eta$, com $x_{0}, \eta \in \mathbb{R}$ e $\eta \rightarrow 0^{+}$(isto é, $z_{0} \rightarrow x_{0}$ por baixo do eixo real). Portanto, o integrando possui uma singularidade em $z_{0}$. Mas podemos considerar a integral sobre um contorno semicircular anti-horário na metade superior do plano complexo (Figura B.1) e usar o teorema de Cauchy dos resíduos:

$$
\oint_{C} d z \frac{f(z)}{z-z_{0}}=2 \pi i \sum_{j} \operatorname{Res}\left(z_{j}\right)
$$

onde a somatória varre um número finito de polos $z_{j}$ no interior do contorno $C$ e $\operatorname{Res}\left(z_{j}\right)$ denota o resíduo correspondente ao polo em $z=z_{j}$.

Logo, podemos reescrever o problema da seguinte forma: 


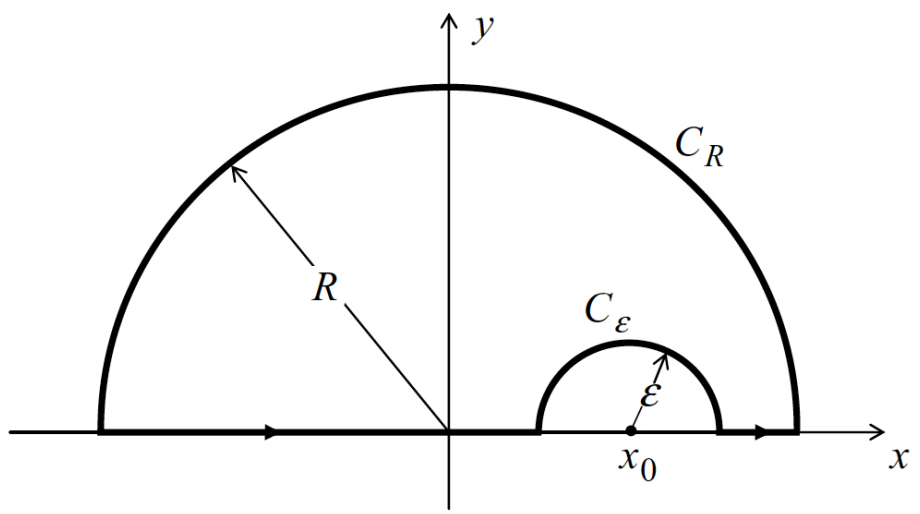

Figura B.1: Contorno semicircular (anti-horário) na metade superior do plano complexo com semicírculo em torno de $x_{0}$. (Extraído de [12], com permissão de Cambridge University Press.)

$$
\begin{aligned}
\oint_{C} d z \frac{f(z)}{z-z_{0}} & =2 \pi i \sum_{j} \operatorname{Res}\left(z_{j}\right)= \\
& =\int_{-R}^{x_{0}-\varepsilon} d x \frac{f(x)}{x-z_{0}}+\int_{C_{\varepsilon}} d z \frac{f(z)}{z-z_{0}}+\int_{x_{0}+\varepsilon}^{R} d x \frac{f(x)}{x-z_{0}}+\int_{C_{R}} d z \frac{f(z)}{z-z_{0}} .
\end{aligned}
$$

Estamos interessados em calcular as integrais do lado direito da equação anterior no caso em que $R \rightarrow \infty$ e $\varepsilon \rightarrow 0$. Para o primeiro e terceiro termos tem-se:

$$
\lim _{R \rightarrow \infty} \lim _{\varepsilon \rightarrow 0}\left\{\int_{-R}^{x_{0}-\varepsilon} d x \frac{f(x)}{x-z_{0}}+\int_{x_{0}+\varepsilon}^{R} d x \frac{f(x)}{x-z_{0}}\right\}=\mathcal{P} \int_{-\infty}^{+\infty} d x \frac{f(x)}{x-x_{0}}
$$

onde $\mathcal{P}$ é o valor principal de Cauchy. O último termo, envolvendo a integração sobre o contorno $C_{R}$, é simplificado se considerarmos $z=R e^{i \theta}$ :

$$
\lim _{R \rightarrow \infty} \int_{C_{R}} d z \frac{f(z)}{z-z_{0}}=\lim _{R \rightarrow \infty} \int_{0}^{\pi} i d \theta \frac{f\left(R e^{i \theta}\right) R e^{i \theta}}{R e^{i \theta}-z_{0}}=0,
$$

já que $f(z) \rightarrow 0$ para $z \rightarrow \infty$. Para o segundo termo, envolvendo a integração sobre o contorno $C_{\varepsilon}$, considere que $z-z_{0}=\varepsilon e^{i \theta}$ : 


$$
\begin{aligned}
\lim _{\varepsilon \rightarrow 0} \int_{C_{\varepsilon}} d z \frac{f(z)}{z-z_{0}} & =\lim _{\varepsilon \rightarrow 0} \int_{\pi}^{0} i d \theta \frac{f\left(z_{0}+\varepsilon e^{i \theta}\right) \varepsilon e^{i \theta}}{\varepsilon e^{i \theta}}= \\
& =-i \lim _{\varepsilon \rightarrow 0} \int_{0}^{\pi} d \theta f\left(z_{0}+\varepsilon e^{i \theta}\right)= \\
& =-i \pi f\left(z_{0}\right) .
\end{aligned}
$$

Portanto, para $z_{0}=x_{0}-i \eta, \operatorname{com} x_{0}, \eta \in \mathbb{R}$ e $\eta \rightarrow 0^{+}$, tem-se:

$$
\int_{-\infty}^{+\infty} d z \frac{f(z)}{z-z_{0}}=-i \pi f\left(z_{0}\right)+\mathcal{P} \int_{-\infty}^{+\infty} d x \frac{f(x)}{x-x_{0}}=2 \pi i \sum_{j} \operatorname{Res}\left(z_{j}\right)
$$

Agora, se considerarmos que $z_{0}=x_{0}+i \eta$, com $x_{0}, \eta \in \mathbb{R}$ e $\eta \rightarrow 0^{+}$(isto é, $z_{0} \rightarrow x_{0}$ por cima do eixo real), a contribuição do polo simples em $z_{0}$ tem que ser considerada no contorno. Isto é equivalente a tomar o contorno $C_{\varepsilon}$ abaixo da singularidade $z_{0}$ :

$$
\lim _{\varepsilon \rightarrow 0} \int_{C_{\varepsilon}} d z \frac{f(z)}{z-z_{0}}=\lim _{\varepsilon \rightarrow 0} \int_{\pi}^{2 \pi} i d \theta \frac{f\left(z_{0}+\varepsilon e^{i \theta}\right) \varepsilon e^{i \theta}}{\varepsilon e^{i \theta}}=i \pi f\left(z_{0}\right) .
$$

Portanto, para $z_{0}=x_{0}+i \eta$, com $x_{0}, \eta \in \mathbb{R}$ e $\eta \rightarrow 0^{+}$, tem-se:

$$
\int_{-\infty}^{+\infty} d z \frac{f(z)}{z-z_{0}}=i \pi f\left(z_{0}\right)+\mathcal{P} \int_{-\infty}^{+\infty} d x \frac{f(x)}{x-x_{0}}=2 \pi i \sum_{j} \operatorname{Res}\left(z_{j}\right)+2 \pi i f\left(z_{0}\right)
$$

\section{B.1 Cálculo da Matriz Densidade Eletrônica}

Na Seção 4.5, vimos que ao aplicar uma diferença de potencial entre os eletrodos $L$ e $R$, a matriz densidade eletrônica na região central é dada por:

$$
\rho=\frac{1}{2 \pi i} \int_{-\infty}^{+\infty} d E G_{M}^{<}=\frac{1}{2 \pi} \int_{-\infty}^{+\infty} d E\left\{-2 \operatorname{Im}\left[G_{M}^{r}\right] f_{L}+G_{M}^{r} \Gamma_{R} G_{M}^{a}\left(f_{L}-f_{R}\right)\right\} .
$$

A integral na parte fora do equilíbrio da matriz densidade, $\rho_{V}$, é limitada pela subtração das funções distribuição de Fermi-Dirac dos eletrodos $\left(f_{L}-f_{R}\right)$ e pode ser calculada diretamente no eixo real. Já na parte no equilíbrio, $\rho_{e q}$, a integral possui somente um limite superior dado 
pela função $f_{L}=f\left(E-\mu_{L}\right)$, sendo necessário integrar desde o nível ocupado de menor energia. Neste caso, uma integração numérica padrão é inviável porque, como a função de Green $G_{M}^{r}$ (Equação 4.42 possui polos próximos ao eixo real, seria necessário uma quantidade enorme de pontos. Uma alternativa é realizar a integração em um contorno no plano complexo, como o representado pela Figura B.2.

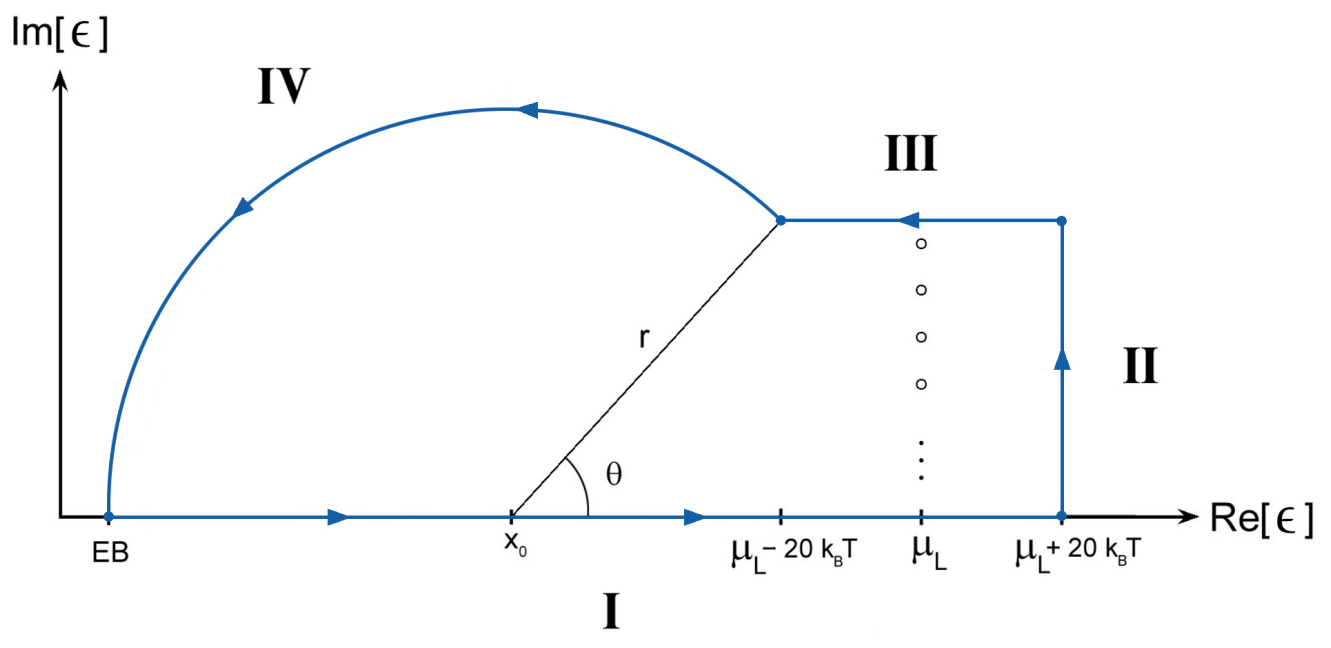

Figura B.2: Esquema da integral de contorno para o cálculo de $\rho_{\text {eq }}$.

Para tanto, deve-se considerar os resíduos da função distribuição de Fermi-Dirac, já que esta possui polos $z_{j}$ no plano complexo (polos de Matsubara):

$$
e^{\frac{z_{j}-\mu_{L}}{k_{B} T}}=-1 \quad \Longrightarrow \quad(2 j+1) \pi i=\frac{z_{j}-\mu_{L}}{k_{B} T} \quad \Longrightarrow \quad z_{j}=\mu_{L}+i(2 j+1) \pi k_{B} T
$$

Como não se conhece o comportamento ao se integrar próximo aos polos, a integração deve ser realizada entre dois polos (Figura B.3), onde para $\epsilon=\mu_{L}+i 2 n \pi k_{B} T$ tem-se que $f_{L} \in \mathbb{R}$.

No esquema da integral de contorno apresentado na Figura B.2, o valor $E B$ é a menor energia de estado ocupado (abaixo dela não há mais estados e, portanto, a contribuição para a integral é nula), $x_{0}$ é o centro da circunferência contendo os pontos $(E B ; 0)$ e $\left(\mu_{L}-20 k_{B} T ; 2 n \pi k_{B} T\right)$ e $r$, o respectivo raio. Pela equação da circunferência $\left(x-x_{0}\right)^{2}+y^{2}=r^{2}$ e utilizando os pontos conhecidos, $(E B ; 0)$ e $\left(\mu_{L}-20 k_{B} T ; 2 n \pi k_{B} T\right)$, verifica-se que o centro é dado por: 1

\footnotetext{
${ }^{1} \mathrm{O}$ valor $20 k_{B} T$ apresentado nas figuras e no cálculo do centro da circunferência é arbitrário, sendo utilizado aqui para ilustrar o problema. Este valor deve ser suficientemente grande para que $f\left(\epsilon-\mu_{L}-20 k_{B} T\right)=1$ e $f\left(\epsilon-\mu_{L}+20 k_{B} T\right)=0$.
} 


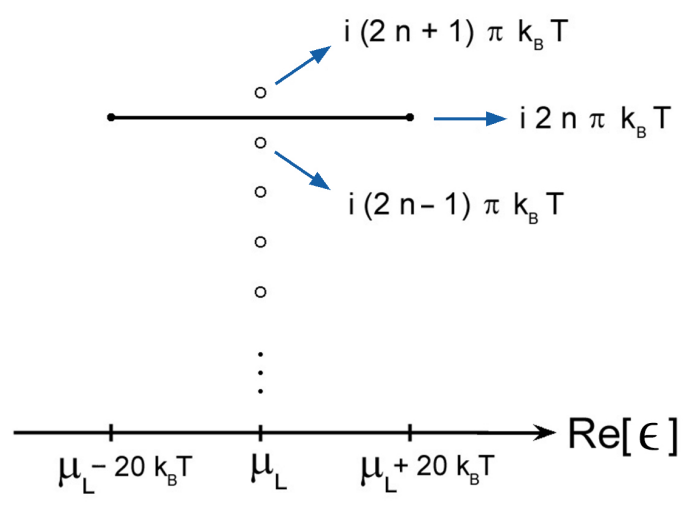

Figura B.3: Contorno próximo aos polos da função distribuição de Fermi-Dirac do eletrodo esquerdo.

$$
x_{0}=\frac{\left(\mu_{L}-20 k_{B} T\right)^{2}+\left(2 n \pi k_{B} T\right)^{2}-E B^{2}}{2\left(\mu_{L}-20 k_{B} T-E B\right)^{2}} .
$$

Com isso, na parte da integral de contorno da circunferência (IV), utiliza-se a parametrização $\epsilon=x_{0}+z=x_{0}+r e^{i \theta} \Longrightarrow d \epsilon=i r e^{i \theta} d \theta=i\left(\epsilon-x_{0}\right) d \theta$, com os limites de integração indo de $\theta_{0}=\arccos \left(\frac{\mu_{L}-20 k_{B} T-x_{0}}{\left|x_{0}-E B\right|}\right)$ a $\pi$.

Portanto, a integral de contorno no plano complexo para o cálculo de $\rho_{e q}$ representada na Figura B.2 será:

$$
\begin{aligned}
& \oint d \epsilon G_{M}^{r} f\left(\epsilon-\mu_{L}\right)=2 \pi i \sum_{j} \operatorname{Res}\left(z_{j}\right)=
\end{aligned}
$$

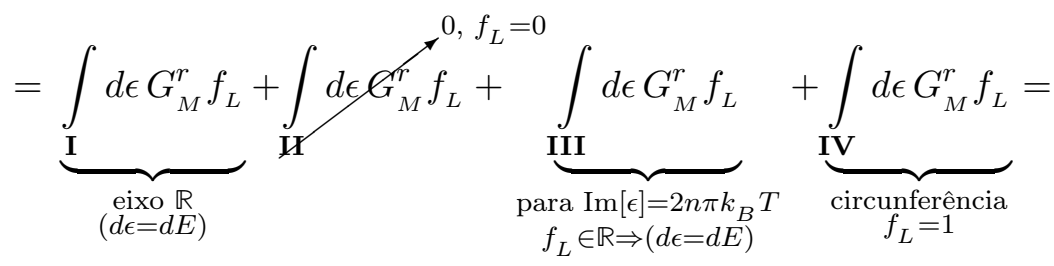

$$
\begin{aligned}
& =\int_{E B}^{\mu_{L}+20 k_{B}{ }^{T}} d E G_{M}^{r} f_{L}+\int_{\mu_{L}-20 k_{B} T}^{\mu_{L}+20 k_{B}{ }^{T}} d E G_{M}^{r} f_{L}+\int_{\theta_{0}}^{\pi} d \theta i\left(\epsilon-x_{0}\right) G_{M}^{r}(\epsilon) .
\end{aligned}
$$

O cálculo dos resíduos devido aos polos da função distribuição de Fermi-Dirac do eletrodo esquerdo pode ser obtido expandindo $e^{\left(\epsilon-\mu_{L}\right) /\left(k_{B} T\right)}$ até a primeira ordem em série de Taylor em torno de cada polo $z_{j}=\mu_{L}+i(2 j+1) \pi k_{B} T$, ou seja: 


$$
\begin{aligned}
e^{\frac{\epsilon-\mu_{L}}{k_{B} T}}+1 & \approx e^{i(2 j+1) \pi}+\frac{e^{i(2 j+1) \pi}}{k_{B} T}\left(\epsilon-\mu_{L}-i(2 j+1) \pi k_{B} T\right)+1= \\
& =-1-\frac{1}{k_{B} T}\left(\epsilon-\mu_{L}-i(2 j+1) \pi k_{B} T\right)+1= \\
& =-\frac{1}{k_{B} T}\left(\epsilon-\mu_{L}-i(2 j+1) \pi k_{B} T\right) .
\end{aligned}
$$

Assim, a somatória dos resíduos resulta em:

$$
\begin{aligned}
\sum_{j=0}^{n-1} \operatorname{Res}\left(z_{j}\right) & =\sum_{j=0}^{n-1}\left\{\lim _{\epsilon \rightarrow \mu_{L}+i(2 j+1) \pi k_{B} T}\left(\epsilon-\mu_{L}-i(2 j+1) \pi k_{B} T\right) G_{M}^{r}(\epsilon) f_{L}(\epsilon)\right\} \approx \\
& \approx \sum_{j=0}^{n-1}\left\{\lim _{\epsilon \rightarrow \mu_{L}+i(2 j+1) \pi k_{B} T} \frac{\left(\epsilon-\mu_{L}-i(2 j+1) \pi k_{B} T\right) G_{M}^{r}(\epsilon)}{\frac{-1}{k_{B} T}\left(\epsilon-\mu_{L}-i(2 j+1) \pi k_{B} T\right)}\right\}= \\
& =-k_{B} T \sum_{j=0}^{n-1} G_{M}^{r}\left(\mu_{L}+i(2 j+1) \pi k_{B} T\right),
\end{aligned}
$$

onde $n$ é o número de polos da função distribuição de Fermi-Dirac dentro da região de integração. Finalmente, a contribuição do sistema no equilíbrio para a matriz densidade pode ser obtida por:

$$
\begin{aligned}
\rho_{e q}=\operatorname{Im} & {\left[-\frac{1}{\pi} \int_{-\infty}^{+\infty} d E G_{M}^{r} f\left(E-\mu_{L}\right)\right]=} \\
= & \operatorname{Im}\left[-\frac{1}{\pi} 2 \pi i \sum_{j} \operatorname{Res}\left(z_{j}\right)+\frac{1}{\pi} \int_{\mu_{L}-20 k_{B} T}^{\mu_{L}+20 k_{B} T} d E G_{M}^{r} f_{L}+\frac{1}{\pi} \int_{\theta_{0}}^{\pi} d \theta i\left(\epsilon-x_{0}\right) G_{M}^{r}(\epsilon)\right]= \\
= & 2 k_{B} T \sum_{j=0}^{n-1} \operatorname{Re}\left[G_{M}^{r}\left(\mu_{L}+i(2 j+1) \pi k_{B} T\right)\right]+ \\
& +\frac{1}{\pi} \int_{\mu_{L}-20 k_{B} T}^{\mu_{L}+20 k_{B} T} d E \operatorname{Im}\left[G_{M}^{r}\right] f_{L}+\frac{1}{\pi} \int_{\theta_{0}}^{\pi} \underbrace{d \theta \operatorname{Im}\left[i\left(\epsilon-x_{0}\right) G_{M}^{r}(\epsilon)\right]}_{\epsilon=x_{0}+r e^{i \theta}=x_{0}+r(\cos \theta+i \operatorname{sen} \theta)}= \\
= & 2 k_{B} T \sum_{j=0}^{n-1} \operatorname{Re}\left[G_{M}^{r}\left(\mu_{L}+i(2 j+1) \pi k_{B} T\right)\right]+ \\
& +\frac{1}{\pi} \int_{\mu_{L}-20 k_{B} T}^{\mu_{L}+20 k_{B} T} d E \operatorname{Im}\left[G_{M}^{r}\right] f_{L}+\frac{1}{\pi} \int_{\theta_{0}}^{\pi} d \theta r\left(\operatorname{Re}\left[G_{M}^{r}\right] \cos \theta-\operatorname{Im}\left[G_{M}^{r}\right] \operatorname{sen} \theta\right) .
\end{aligned}
$$




\section{B.2 Transformada de Hilbert}

A transformada de Hilbert no eixo $\mathbb{R}$ de uma função $f(x)$ é definida como o valor principal $\mathcal{P}$ da seguinte integral: $:^{2}$

$$
\mathcal{H}_{x}\{f(x)\}(y)=\frac{1}{\pi} \mathcal{P} \int_{-\infty}^{+\infty} d x \frac{f(x)}{x-y}
$$

onde identifica-se a função núcleo:

$$
k(x, y)=\frac{1}{\pi(x-y)}
$$

A forma desta integral é semelhante à da Equação B.1 e, portanto, poderíamos cogitar uma integral sobre um contorno no plano complexo para computá-la. No entanto, queremos considerar o caso mais geral onde os polos da função $f(x)$ são desconhecidos, impossibilitando o cálculo dos resíduos.

Em integrais como a da Equação B.16, onde se distingue uma função núcleo $k(x, y)$, o primeiro impulso consiste em considerar a convolução da função $f(x) \operatorname{com} k(x, y)$, o que poderia ser calculado de maneira eficiente por meio da transformada de Fourier $\mathcal{F}$ com o teorema da convolução:

$$
\int_{-\infty}^{+\infty} d x k(x, y) f(x) \equiv f * k=\mathcal{F}^{-1}\{\mathcal{F}\{f * k\}\}=\mathcal{F}^{-1}\{\mathcal{F}\{f\} \mathcal{F}\{k\}\}
$$

Numericamente a transformada de Fourier é realizada em um grid discreto de pontos por meio do algoritmo DFFT [95] (Discrete Fast Fourier Transform) e um teorema de convolução análogo pode ser utilizado. Contudo, um cuidado especial deve ser tomado ao aproximar integrais de Fourier envolvendo funções contínuas singulares por transformadas de Fourier discretas, como é o caso da função núcleo $k(x, y)$ singular em $x=y$.

Seguindo T. Frederiksen et al [78], a convolução discreta pode ser aplicada a partir de um método um pouco mais sofisticado baseado na interpolação linear da função $f(x)$. Considerando um grid com $N$ pontos igualmente espaçados por $\Delta$ (i.e. $x_{i}=x_{0}+i \Delta$, para $i \in\{1,2, \ldots, N\}$ ), a função $f(x)$ pode ser aproximada por uma interpolação linear da seguinte maneira:

$$
f(x) \approx f_{I}(x) \equiv \sum_{i=1}^{N} \psi\left(x_{i}, x\right) f\left(x_{i}\right)
$$

\footnotetext{
${ }^{2}$ Também é comum encontrar a definição da transformada de Hilbert com o sinal oposto.
} 
onde a função núcleo associada a interpolação linear $\psi\left(x_{i}, x\right)$ é dada pela função triangular:

$$
\psi\left(x_{i}, x\right) \equiv \max \left\{1-\left|\frac{x-x_{i}}{\Delta}\right|\right\}=\left\{\begin{array}{cc}
1+\frac{x-x_{i}}{\Delta}, & -\Delta \leqslant x-x_{i} \leqslant 0 \\
1-\frac{x-x_{i}}{\Delta}, & 0 \geqslant x-x_{i} \geqslant \Delta \\
0, & \left|x-x_{i}\right|>\Delta .
\end{array}\right.
$$

Desta forma, a transformada de Hilbert na Equação B.16 pode ser aproximada pela transformada de Hilbert de $f_{I}(x)$ calculada no mesmo conjunto discreto de pontos $x_{j}=x_{0}+j \Delta$ :

$$
\begin{aligned}
\mathcal{H}_{x}\{f(x)\}\left(x_{j}\right) & \approx \mathcal{H}_{x}\left\{f_{I}(x)\right\}\left(x_{j}\right)= \\
& =\frac{1}{\pi} \mathcal{P} \int_{-\infty}^{+\infty} d x \frac{f_{I}(x)}{x-x_{j}}= \\
& =\frac{1}{\pi} \mathcal{P} \int_{-\infty}^{+\infty} d x \sum_{i=1}^{N} \frac{\psi\left(x_{i}, x\right)}{x-x_{j}} f\left(x_{i}\right)= \\
& =\sum_{i=1}^{N} K_{j i} f\left(x_{i}\right),
\end{aligned}
$$

onde $K_{j i}$ é o núcleo da transformação dado por:

$$
\begin{aligned}
K_{j i} & =\frac{1}{\pi} \mathcal{P} \int_{-\infty}^{+\infty} d x \frac{\psi\left(x_{i}, x\right)}{x-x_{j}}= \\
& =\frac{1}{\pi} \mathcal{P} \int_{x_{i}-\Delta}^{x_{i}} d x \frac{1+\frac{x-x_{i}}{\Delta}}{x-x_{j}}+\frac{1}{\pi} \mathcal{P} \int_{x_{i}}^{x_{i}+\Delta} d x \frac{1-\frac{x-x_{i}}{\Delta}}{x-x_{j}}=\frac{u=\frac{x-x_{i}}{\Delta}}{x=\Delta u+x_{i}} \\
& =\frac{1}{\pi} \mathcal{P} \int_{-1}^{0} \Delta d u \frac{1+u}{\Delta u+x_{i}-x_{j}}+\frac{1}{\pi} \mathcal{P} \int_{0}^{1} \Delta d u \frac{1-u}{\Delta u+x_{i}-x_{j}}=\frac{x_{i}=x_{0}+i \Delta}{x_{i}-x_{j}=-(j-i) \Delta} \\
& =\frac{1}{\pi} \mathcal{P} \int_{-1}^{0} d u \frac{1+u}{u-(j-i)}+\frac{1}{\pi} \mathcal{P} \int_{0}^{1} d u \frac{1-u}{u-(j-i)}= \\
& =\frac{1}{\pi} \mathcal{P} \int_{-1}^{0} d u \frac{1+u}{u-m}+\frac{1}{\pi} \mathcal{P} \int_{0}^{1} d u \frac{1-u}{u-m}= \\
& =\frac{1}{\pi}[-(m+1) \ln (-(m+1))+2 m \ln (-m)-(m-1) \ln (-(m-1))]= \\
& =\frac{1}{\pi}[-(m+1) \ln (m+1)+2 m \ln (-m)-(m-1) \ln (m-1)] .
\end{aligned}
$$


Portanto, a partir da interpolação linear, fica claro a maneira que a transformada de Hilbert pode ser calculada como uma convolução discreta por meio do algoritmo DFFT. Primeiro definese um conjunto de pontos equidistantes $\left\{x_{i}\right\}$ tal que a função $f_{I}(x)$ possa ser totalmente representada e possua suporte finito (i.e., a função deve ser evanescente nas extremidades do grid). Para todo ponto $x_{i}$, calcula-se o núcleo da transformada $K_{j i}$ e a função $f\left(x_{i}\right)$ e suas respectivas transformadas discretas de Fourier. As transformadas são, então, multiplicadas elemento a elemento e, por fim, computa-se a transformada inversa. 


\section{Apêndice C}

\section{Derivação da Expressão para a}

\section{Corrente na LOE}

Ao considerar a teoria de perturbação de mais baixa ordem com relação à matriz de acoplamento elétron-fônon $M_{\lambda}$, podemos expandir a função de Green eletrônica como $G^{r}=G_{0}^{r}+G_{0}^{r} \Sigma_{p h}^{r} G_{0}^{r}$. Substituindo esta expansão na equação para a corrente com interação elétron-fônon (Equação 6.25, obtém-se:

$$
\begin{aligned}
I_{L}= & \frac{2 e}{h} \int_{-\infty}^{+\infty} d \epsilon \operatorname{Tr}\left[\Gamma_{L} G^{r} \Gamma_{R} G^{a}\right]\left(f_{L}-f_{R}\right)+ \\
& +i \frac{2 e}{h} \int_{-\infty}^{+\infty} d \epsilon \operatorname{Tr}\left[G^{a} \Gamma_{L} G^{r}\left(f_{L} \Sigma^{F,>}+\left(1-f_{L}\right) \Sigma^{F,<}\right)\right] \approx \\
\approx & \frac{2 e}{h} \int_{-\infty}^{+\infty} d \epsilon \operatorname{Tr}\left[\Gamma_{L}\left(G_{0}^{r}+G_{0}^{r} \Sigma_{p h}^{r} G_{0}^{r}\right) \Gamma_{R}\left(G_{0}^{r}+G_{0}^{r} \Sigma_{p h}^{r} G_{0}^{r}\right)^{\dagger}\right]\left(f_{L}-f_{R}\right)+ \\
& +i \frac{2 e}{h} \int_{-\infty}^{+\infty} d \epsilon \operatorname{Tr}\left[\left(G_{0}^{r}+G_{0}^{r} \Sigma_{p h}^{r} G_{0}^{r}\right)^{\dagger} \Gamma_{L}\left(G_{0}^{r}+G_{0}^{r} \Sigma_{p h}^{r} G_{0}^{r}\right)\left(f_{L} \Sigma^{F,>}+\left(1-f_{L}\right) \Sigma^{F,<}\right)\right] .
\end{aligned}
$$

Considerando as autoenergias de interação obtidas com a 1BA (Equações 6.11 a 6.13), nota-se que a mais baixa ordem é dada por termos $O\left(M_{\lambda}^{2}\right)$, já que essas autoenergias são todas proporcionais a $M_{\lambda}^{2}$. Ignorando os termos de ordem superior, a corrente simplifica-se a: 


$$
\begin{aligned}
I_{L} \approx & \underbrace{\frac{2 e}{h} \int_{-\infty}^{+\infty} d \epsilon \operatorname{Tr}\left[\Gamma_{L} G_{0}^{r} \Gamma_{R} G_{0}^{a}\right]\left(f_{L}-f_{R}\right)}_{I_{e l}}+ \\
& +\underbrace{\frac{4 e}{h} \int_{-\infty}^{+\infty} d \epsilon \operatorname{Re}\left\{\operatorname{Tr}\left[\Gamma_{L} G_{0}^{r} \Sigma_{p h}^{r} G_{0}^{r} \Gamma_{R} G_{0}^{a}\right]\right\}\left(f_{L}-f_{R}\right)}_{\delta I_{e l}}+ \\
& +\underbrace{i \frac{2 e}{h} \int_{-\infty}^{+\infty} d \epsilon \operatorname{Tr}\left[G_{0}^{a} \Gamma_{L} G_{0}^{r}\left(f_{L} \Sigma^{F,>}+\left(1-f_{L}\right) \Sigma^{F,<}\right)\right]}_{I_{\text {inel }}}
\end{aligned}
$$

de onde se nota que a componente elástica se divide em um termo puramente elástico $I_{e l}$ e uma correção devido à interação elétron-fônon $\delta I_{e l}$.

Na aproximação Wide-Band Limit, onde as funções de Green e as funções largura de nível são consideradas como independentes da energia $\left(G_{0}(\epsilon) \approx G_{0}\left(\varepsilon_{F}\right)\right.$ e $\left.\Gamma_{L, R}(\epsilon) \approx \Gamma_{L, R}\left(\varepsilon_{F}\right)\right)$, a expressão para a corrente simplifica-se consideravelmente e os termos dependentes da energia, que envolvem subtrações e produtos de distribuições de Fermi-Dirac, podem ser calculados analiticamente. Para mostrar como isso ocorre, vamos analisar os termos separadamente, iniciando pelo termo elástico:

$$
I_{e l} \approx \frac{2 e}{h} \operatorname{Tr}\left[\Gamma_{L}\left(\varepsilon_{F}\right) G_{0}^{r}\left(\varepsilon_{F}\right) \Gamma_{R}\left(\varepsilon_{F}\right) G_{0}^{a}\left(\varepsilon_{F}\right)\right] \int_{-\infty}^{+\infty} d \epsilon\left(f_{L}-f_{R}\right)
$$

onde a integral pode ser calculada da seguinte forma:

$$
\begin{aligned}
\int_{-\infty}^{+\infty} d \epsilon\left(f_{L}-f_{R}\right) & =\int_{-\infty}^{+\infty} d \epsilon\left(\frac{1}{e^{\beta\left(\epsilon-\mu_{L}\right)}+1}-\frac{1}{e^{\beta\left(\epsilon-\mu_{R}\right)}+1}\right)=\frac{u=e^{\beta\left(\epsilon-\mu_{L}\right)}}{u^{\prime}=e^{\beta\left(\epsilon-\mu_{R}\right)}} \\
& =\frac{1}{\beta} \int_{0}^{+\infty} d u\left(\frac{1}{u}-\frac{1}{u+1}\right)-\frac{1}{\beta} \int_{0}^{+\infty} d u^{\prime}\left(\frac{1}{u^{\prime}}-\frac{1}{u^{\prime}+1}\right)= \\
& =\left.\frac{1}{\beta}\left[\ln (u)-\ln (u+1)-\ln \left(u^{\prime}\right)+\ln \left(u^{\prime}+1\right)\right]\right|_{0} ^{+\infty}= \\
& =\left.\left\{\epsilon-\mu_{L}-\epsilon+\mu_{R}+\frac{1}{\beta}\left[\ln \left(e^{\beta\left(\epsilon-\mu_{R}\right)}+1\right)-\ln \left(e^{\beta\left(\epsilon-\mu_{L}\right)}+1\right)\right]\right\}\right|_{-\infty} ^{+\infty}=
\end{aligned}
$$




$$
\begin{aligned}
& =\left(\mu_{R}-\mu_{L}\right)+\left.\frac{1}{\beta}\left[\frac{\ln \left(e^{\beta\left(\epsilon-\mu_{R}\right)}+1\right)}{\ln \left(e^{\beta\left(\epsilon-\mu_{L}\right)}+1\right)}\right]\right|_{-\infty} ^{+\infty}= \\
& =\left(\mu_{R}-\mu_{L}\right)-\left(\mu_{R}-\mu_{L}\right)+\frac{1}{\beta} \beta\left(\mu_{L}-\mu_{R}\right)= \\
& =\mu_{L}-\mu_{R}= \\
& =e V
\end{aligned}
$$

onde $\beta=\left(k_{B} T\right)^{-1}$ e $\mu_{L / R}=\varepsilon_{F} \pm \frac{e V}{2}$. De maneira geral, para a distribuição de Fermi-Dirac vale a seguinte igualdade:

$$
\int_{-\infty}^{+\infty} d \epsilon(f(\epsilon-a)-f(\epsilon-b))=a-b .
$$

Portanto, o termo elástico se torna:

$$
I_{e l} \approx \frac{2 e^{2} V}{h} \operatorname{Tr}\left[\Gamma_{L}\left(\varepsilon_{F}\right) G_{0}^{r}\left(\varepsilon_{F}\right) \Gamma_{R}\left(\varepsilon_{F}\right) G_{0}^{a}\left(\varepsilon_{F}\right)\right] .
$$

Para analisar a correção no termo elástico $\delta I_{e l}$, o primeiro passo é substituir $\Sigma_{p h}^{r}$ pela autoenergia retardada de Fock obtida pela primeira aproximação de Born (Equação 6.13):

$$
\begin{aligned}
& \delta I_{e l}= \frac{2 e}{h} \int_{-\infty}^{+\infty} d \epsilon \operatorname{Re}\left\{\operatorname{Tr}\left[\Gamma_{L} G_{0}^{r}\left(\Sigma^{F,>}-\Sigma^{F,<}-i \mathcal{H}_{\epsilon^{\prime}}\left\{\Sigma^{F,>}\left(\epsilon^{\prime}\right)-\Sigma^{F,<}\left(\epsilon^{\prime}\right)\right\}(\epsilon)\right) G_{0}^{r} \Gamma_{R} G_{0}^{a}\right]\right\} \\
&=\cdot\left(f_{L}-f_{R}\right)= \\
&=\underbrace{\frac{2 e}{h} \int_{-\infty}^{+\infty} d \epsilon \operatorname{Re}\left\{\operatorname{Tr}\left[\left(\Sigma^{F,>}-\Sigma^{F,<}\right) G_{0}^{r} \Gamma_{R} G_{0}^{a} \Gamma_{L} G_{0}^{r}\right]\right\}\left(f_{L}-f_{R}\right)}_{\delta I_{\text {el }}^{\text {sym }}}- \\
& \underbrace{-\frac{2 e}{h} \int_{-\infty}^{+\infty} d \epsilon \operatorname{Re}\left\{\operatorname{Tr}\left[\left(i \mathcal{H}_{\epsilon^{\prime}}\left\{\Sigma^{F,>}\left(\epsilon^{\prime}\right)-\Sigma^{F,<}\left(\epsilon^{\prime}\right)\right\}(\epsilon)\right) G_{0}^{r} \Gamma_{R} G_{0}^{a} \Gamma_{L} G_{0}^{r}\right]\right\}\left(f_{L}-f_{R}\right)}_{\delta I_{\text {el }}^{\text {asym }}}, \quad(C .7
\end{aligned}
$$

onde foi utilizada a propriedade cíclica do traço e onde identificamos os termos $\delta I_{e l}^{s y m}$ e $\delta I_{e l}^{a s y m}$, respectivamente simétrico e assimétrico sob reversão da diferença de potencial $V$.

Substituindo $\Sigma^{F,<}$ e $\Sigma^{F,>}$ por suas expressões dadas pelas Equações 6.11 e 6.12 , respectivamente, e considerando a aproximação Wide-Band Limit, obtém-se para a parte simétrica: 


$$
\begin{aligned}
& \delta I_{e l}^{\text {sym }}= i \frac{e}{h} \sum_{\lambda} \operatorname{Tr}\left[M_{\lambda} G_{0}^{r}\left(\varepsilon_{F}\right) \Gamma_{L}\left(\varepsilon_{F}\right) G_{0}^{a}\left(\varepsilon_{F}\right) M_{\lambda} G_{0}^{r}\left(\varepsilon_{F}\right) \Gamma_{R}\left(\varepsilon_{F}\right) G_{0}^{a}\left(\varepsilon_{F}\right) \Gamma_{L}\left(\varepsilon_{F}\right) G_{0}^{r}\left(\varepsilon_{F}\right)-\text { h.c. }\right] . \\
& \cdot \int_{-\infty}^{+\infty} d \epsilon\left(f_{L}-f_{R}\right)\left(f_{L_{-}}-f_{L_{+}}\right)+ \\
&+ i \frac{e}{h} \sum_{\lambda} \operatorname{Tr}\left[M_{\lambda} G_{0}^{r}\left(\varepsilon_{F}\right) \Gamma_{R}\left(\varepsilon_{F}\right) G_{0}^{a}\left(\varepsilon_{F}\right) M_{\lambda} G_{0}^{r}\left(\varepsilon_{F}\right) \Gamma_{R}\left(\varepsilon_{F}\right) G_{0}^{a}\left(\varepsilon_{F}\right) \Gamma_{L}\left(\varepsilon_{F}\right) G_{0}^{r}\left(\varepsilon_{F}\right)-\text { h.c. }\right] . \\
& \cdot \int_{-\infty}^{+\infty} d \epsilon\left(f_{L}-f_{R}\right)\left(f_{R_{-}}-f_{R_{+}}\right)- \\
&-i \frac{e}{h} \sum_{\lambda}\left(2\left\langle n_{\lambda}\right\rangle+1\right) \operatorname{Tr}\left[M_{\lambda} A_{0}\left(\varepsilon_{F}\right) M_{\lambda} G_{0}^{r}\left(\varepsilon_{F}\right) \Gamma_{R}\left(\varepsilon_{F}\right) G_{0}^{a}\left(\varepsilon_{F}\right) \Gamma_{L}\left(\varepsilon_{F}\right) G_{0}^{r}\left(\varepsilon_{F}\right)-\text { h.c. }\right] . \\
& \cdot \int_{-\infty}^{+\infty} d \epsilon\left(f_{L}-f_{R}\right),
\end{aligned}
$$

onde $A_{0}=G_{0}^{r}\left(\Gamma_{L}+\Gamma_{R}\right) G_{0}^{a}$ é essencialmente a função espectral não-interagente, $\left\langle n_{\lambda}\right\rangle$ é a ocupação dos fônons de modo $\lambda$ e o índice - (+) indica que a função distribuição é calculada em $\epsilon-\hbar \omega_{\lambda}$ $\left(\epsilon+\hbar \omega_{\lambda}\right)$, sendo $\omega_{\lambda}$ a frequência do fônon de modo $\lambda$. Já vimos como calcular a integral do último termo, restando somente calcular as integrais dos dois primeiros termos. Vamos analisar a segunda integral, que, por sua vez, pode ser dividida em quatro partes:

$$
\int_{-\infty}^{+\infty} d \epsilon\left(f_{L}-f_{R}\right)\left(f_{R_{-}}-f_{R_{+}}\right)=\underbrace{\int_{-\infty}^{+\infty} d \epsilon f_{L} f_{R_{-}}}_{\mathcal{I}} \underbrace{-\int_{-\infty}^{+\infty} d \epsilon f_{L} f_{R_{+}}}_{\mathcal{I I}} \underbrace{-\int_{-\infty}^{+\infty} d \epsilon f_{R} f_{R_{-}}}_{\mathcal{I} \mathcal{I} \mathcal{I}}+\underbrace{+\int_{-\infty}^{+\infty} d \epsilon f_{R} f_{R_{+}}}_{\mathcal{I} \mathcal{V}}
$$

O primeiro termo pode ser calculado da seguinte forma:

$$
\begin{aligned}
\mathcal{I} & =\int_{-\infty}^{+\infty} d \epsilon\left(\frac{1}{e^{\beta\left(\epsilon-\mu_{L}\right)}+1} \cdot \frac{1}{e^{\beta\left(\epsilon-\mu_{R}-\hbar \omega_{\lambda}\right)}+1}\right)= \\
& =\int_{-\infty}^{+\infty} d \epsilon\left(\frac{1}{e^{\beta\left(\epsilon-\varepsilon_{F}-e V / 2\right)}+1} \cdot \frac{1}{e^{\beta\left(\epsilon-\varepsilon_{F}+e V / 2-\hbar \omega_{\lambda}\right)}+1}\right)=\quad \stackrel{u=e^{\beta\left(\epsilon-\varepsilon_{F}+e V / 2-\hbar \omega_{\lambda}\right)}}{\longrightarrow}
\end{aligned}
$$




$$
\begin{aligned}
&= \frac{1}{\beta} \int_{0}^{+\infty} d u\left(\frac{1}{u} \cdot \frac{1}{u e^{-\beta\left(e V-\hbar \omega_{\lambda}\right)}+1} \cdot \frac{1}{u+1}\right)= \\
&=\left.\frac{1}{\beta}\left[\ln (u)-\frac{1}{1-e^{\beta\left(e V-\hbar \omega_{\lambda}\right)}} \ln \left(u e^{-\beta\left(e V-\hbar \omega_{\lambda}\right)}+1\right)-\frac{1}{1-e^{-\beta\left(e V-\hbar \omega_{\lambda}\right)}} \ln (u+1)\right]\right|_{0} ^{+\infty}= \\
&=\frac{1}{\beta}\left[\beta\left(\epsilon-\varepsilon_{F}+\frac{e V}{2}-\hbar \omega_{\lambda}\right)-\frac{1}{1-e^{\beta\left(e V-\hbar \omega_{\lambda}\right)}} \ln \left(e^{\beta\left(\epsilon-\varepsilon_{F}-e V / 2\right)}+1\right)-\right. \\
&\left.\quad-\frac{1}{1-e^{-\beta\left(e V-\hbar \omega_{\lambda}\right)}} \ln \left(e^{\beta\left(\epsilon-\varepsilon_{F}+e V / 2-\hbar \omega_{\lambda}\right)}+1\right)\right]\left.\right|_{-\infty} ^{+\infty} .
\end{aligned}
$$

Com substituições de variáveis adequadas $\left(u=e^{\beta\left(\epsilon-\varepsilon_{F}+e V / 2+\hbar \omega_{\lambda}\right)}\right.$ para a $\mathcal{I} \mathcal{I}, u=e^{\beta \hbar \omega_{\lambda}}$ para a $\mathcal{I} \mathcal{I} \mathcal{I}$ e $u=e^{-\beta \hbar \omega_{\lambda}}$ para a $\left.\mathcal{I} \mathcal{V}\right)$, os demais termos podem ser calculados de maneira semelhante e obtém-se:

$$
\begin{aligned}
& \mathcal{I I}=-\frac{1}{\beta}\left[\beta\left(\epsilon-\varepsilon_{F}+\frac{e V}{2}+\hbar \omega_{\lambda}\right)-\frac{1}{1-e^{\beta\left(e V+\hbar \omega_{\lambda}\right)}} \ln \left(e^{\beta\left(\epsilon-\varepsilon_{F}-e V / 2\right)}+1\right)-\right. \\
& \left.-\frac{1}{1-e^{-\beta\left(e V+\hbar \omega_{\lambda}\right)}} \ln \left(e^{\beta\left(\epsilon-\varepsilon_{F}+e V / 2+\hbar \omega_{\lambda}\right)}+1\right)\right]\left.\right|_{-\infty} ^{+\infty} \\
& \mathcal{I I I}=-\frac{1}{\beta}\left[\beta\left(\epsilon-\varepsilon_{F}+\frac{e V}{2}-\hbar \omega_{\lambda}\right)-\frac{1}{1-e^{-\beta \hbar \omega_{\lambda}}} \ln \left(e^{\beta\left(\epsilon-\varepsilon_{F}+e V / 2\right)}+1\right)-\right. \\
& \left.-\frac{1}{1-e^{\beta \hbar \omega_{\lambda}}} \ln \left(e^{\beta\left(\epsilon-\varepsilon_{F}+e V / 2-\hbar \omega_{\lambda}\right)}+1\right)\right]\left.\right|_{-\infty} ^{+\infty} \\
& \mathcal{I V}=\frac{1}{\beta}\left[\beta\left(\epsilon-\varepsilon_{F}+\frac{e V}{2}+\hbar \omega_{\lambda}\right)-\frac{1}{1-e^{\beta \hbar \omega_{\lambda}}} \ln \left(e^{\beta\left(\epsilon+\varepsilon_{F}+e V / 2\right)}+1\right)-\right. \\
& \left.-\frac{1}{1-e^{-\beta \hbar \omega_{\lambda}}} \ln \left(e^{\beta\left(\epsilon-\varepsilon_{F}+e V / 2+\hbar \omega_{\lambda}\right)}+1\right)\right]\left.\right|_{-\infty} ^{+\infty} .
\end{aligned}
$$

Inserindo os resultados obtidos, Equações C.10 C.13, na Equações C.9 


$$
\begin{aligned}
\int_{-\infty}^{+\infty} d \epsilon\left(f_{L}-f_{R}\right)\left(f_{R_{-}}-f_{R_{+}}\right)=\mathcal{I}+\mathcal{I} \mathcal{I}+\mathcal{I} \mathcal{I}+\mathcal{I} \mathcal{V}= \\
=\frac{1}{\beta}\left[-\frac{1}{1-e^{\beta\left(e V-\hbar \omega_{\lambda}\right)}} \ln \left(e^{\beta\left(\epsilon-\varepsilon_{F}-e V / 2\right)}+1\right)-\frac{1}{1-e^{-\beta\left(e V-\hbar \omega_{\lambda}\right)}} \ln \left(e^{\beta\left(\epsilon-\varepsilon_{F}+e V / 2-\hbar \omega_{\lambda}\right)}+1\right)+\right. \\
\quad+\frac{1}{1-e^{\beta\left(e V+\hbar \omega_{\lambda}\right)}} \ln \left(e^{\beta\left(\epsilon-\varepsilon_{F}-e V / 2\right)}+1\right)+\frac{1}{1-e^{-\beta\left(e V+\hbar \omega_{\lambda}\right)}} \ln \left(e^{\beta\left(\epsilon-\varepsilon_{F}+e V / 2+\hbar \omega_{\lambda}\right)}+1\right)+ \\
\quad+\frac{1}{1-e^{-\beta \hbar \omega_{\lambda}}} \ln \left(e^{\beta\left(\epsilon-\varepsilon_{F}+e V / 2\right)}+1\right)+\frac{1}{1-e^{\beta \hbar \omega_{\lambda}}} \ln \left(e^{\beta\left(\epsilon-\varepsilon_{F}+e V / 2-\hbar \omega_{\lambda}\right)}+1\right)- \\
\left.\quad-\frac{1}{1-e^{\beta \hbar \omega_{\lambda}}} \ln \left(e^{\beta\left(\epsilon-\varepsilon_{F}+e V / 2\right)}+1\right)-\frac{1}{1-e^{-\beta \hbar \omega_{\lambda}}} \ln \left(e^{\beta\left(\epsilon-\varepsilon_{F}+e V / 2+\hbar \omega_{\lambda}\right)}+1\right)\right]\left.\right|_{-\infty} ^{+\infty}
\end{aligned}
$$

Para poder reagrupar os termos de maneira a tornar conveniente a aplicação dos limites, podemos utilizar a seguinte igualdade:

$$
-\frac{1}{1-e^{-\beta\left(e V-\hbar \omega_{\lambda}\right)}}=\frac{1}{1-e^{\beta\left(e V-\hbar \omega_{\lambda}\right)}}-1 .
$$

Usando a igualdade acima e reagrupando os termos:

$$
\begin{aligned}
& \int_{-\infty}^{+\infty} d \epsilon\left(f_{L}-f_{R}\right)\left(f_{R_{-}}-f_{R_{+}}\right)=\mathcal{I}+\mathcal{I} \mathcal{I}+\mathcal{I} \mathcal{I}+\mathcal{I} \mathcal{V}= \\
&=-\frac{1}{\beta}\left[\frac{1}{1-e^{\beta\left(e V+\hbar \omega_{\lambda}\right)}} \ln \left(\frac{e^{\beta\left(\epsilon-\varepsilon_{F}+e V / 2+\hbar \omega_{\lambda}\right)}+1}{e^{\beta\left(\epsilon-\varepsilon_{F}-e V / 2\right)}+1}\right)+\frac{1}{1-e^{\beta \hbar \omega_{\lambda}}} \ln \left(\frac{e^{\beta\left(\epsilon-\varepsilon_{F}+e V / 2\right)}+1}{e^{\beta\left(\epsilon-\varepsilon_{F}+e V / 2-\hbar \omega_{\lambda}\right)}+1}\right)+\right. \\
& \quad+\frac{1}{1-e^{-\beta\left(e V-\hbar \omega_{\lambda}\right)}} \ln \left(\frac{e^{\beta\left(\epsilon-\varepsilon_{F}+e V / 2-\hbar \omega_{\lambda}\right)}+1}{e^{\beta\left(\epsilon-\varepsilon_{F}-e V / 2\right)}+1}\right)+\frac{1}{1-e^{-\beta \hbar \omega_{\lambda}}} \ln \left(\frac{e^{\beta\left(\epsilon-\varepsilon_{F}+e V / 2+\hbar \omega_{\lambda}\right)}+1}{e^{\beta\left(\epsilon-\varepsilon_{F}+e V / 2\right)}+1}\right)+ \\
&\left.\quad+\ln \left(\frac{\left.e^{\beta\left(\epsilon-\varepsilon_{F}\right.}-e V / 2\right)}{e^{\beta\left(\epsilon-\varepsilon_{F}+e V / 2+\hbar \omega_{\lambda}\right)}+1}+1\right)\right]\left.\right|_{-\infty} ^{+\infty}= \\
&=- \frac{1}{\beta}\left[\frac{\beta\left(e V+\hbar \omega_{\lambda}\right)}{1-e^{\beta\left(e V+\hbar \omega_{\lambda}\right)}}+\frac{\beta\left(e V-\hbar \omega_{\lambda}\right)}{1-e^{-\beta\left(e V-\hbar \omega_{\lambda}\right)}}+\frac{\beta \hbar \omega_{\lambda}}{1-e^{\beta \hbar \omega_{\lambda}}}+\frac{\beta \hbar \omega_{\lambda}}{1-e^{-\beta \hbar \omega_{\lambda}}}-\beta\left(e V+\hbar \omega_{\lambda}\right)\right]=
\end{aligned}
$$




$$
\begin{aligned}
& =-\frac{e V+\hbar \omega_{\lambda}}{1-e^{\beta\left(e V+\hbar \omega_{\lambda}\right)}}+\frac{-e V+\hbar \omega_{\lambda}}{1-e^{-\beta\left(e V-\hbar \omega_{\lambda}\right)}}-\hbar \omega_{\lambda}+e V+\hbar \omega_{\lambda}= \\
& =\frac{\hbar \omega_{\lambda}+e V}{e^{\beta\left(\hbar \omega_{\lambda}+e V\right)}-1}-\frac{\hbar \omega_{\lambda}-e V}{e^{\beta\left(\hbar \omega_{\lambda}-e V\right)}-1}+e V .
\end{aligned}
$$

Pode-se mostrar que a primeira integral da Equação C.8 é idêntica a integral que acabamos de calcular. Isso pode ser feito repetindo os mesmos passos ou analisando a simetria das funções distribuições de Fermi-Dirac. Uma vez verificada a identidade, a parte simétrica da correção no termo elástico pode ser escrita como:

$$
\begin{array}{r}
\delta I_{e l}^{\text {sym }}=i \frac{e}{h} \sum_{\lambda} \operatorname{Tr}\left[M_{\lambda} A_{0}\left(\varepsilon_{F}\right) M_{\lambda} G_{0}^{r}\left(\varepsilon_{F}\right) \Gamma_{R}\left(\varepsilon_{F}\right) G_{0}^{a}\left(\varepsilon_{F}\right) \Gamma_{L}\left(\varepsilon_{F}\right) G_{0}^{r}\left(\varepsilon_{F}\right)-\text { h.c. }\right] . \\
\cdot\left(\frac{\hbar \omega_{\lambda}+e V}{e^{\beta\left(\hbar \omega_{\lambda}+e V\right)}-1}-\frac{\hbar \omega_{\lambda}-e V}{e^{\beta\left(\hbar \omega_{\lambda}-e V\right)}-1}+e V-2\left\langle n_{\lambda}\right\rangle e V-e V\right)= \\
=i \frac{e}{h} \sum_{\lambda} \operatorname{Tr}\left[G_{0}^{a}\left(\varepsilon_{F}\right) \Gamma_{L}\left(\varepsilon_{F}\right) G_{0}^{r}\left(\varepsilon_{F}\right) \Gamma_{R}\left(\varepsilon_{F}\right) G_{0}^{a}\left(\varepsilon_{F}\right) M_{\lambda} A_{0}\left(\varepsilon_{F}\right) M_{\lambda}-\text { h.c. }\right] . \\
\cdot\left(2\left\langle n_{\lambda}\right\rangle e V+\frac{\hbar \omega_{\lambda}-e V}{e^{\beta\left(\hbar \omega_{\lambda}-e V\right)}-1}-\frac{\hbar \omega_{\lambda}+e V}{e^{\beta\left(\hbar \omega_{\lambda}+e V\right)}-1}\right) .
\end{array}
$$

Substituindo $\Sigma^{F,<}$ e $\Sigma^{F,>}$ por suas expressões (Equações 6.11 e 6.12 respectivamente) na parte assimétrica da correção no termo elástico $\delta I_{e l}^{a s y m}$ e considerando a aproximação Wide-Band Limit, obtém-se:

$$
\begin{aligned}
\delta I_{e l}^{a s y m}= & \frac{e}{h} \sum_{\lambda} \operatorname{Tr}\left[M_{\lambda} G_{0}^{r}\left(\varepsilon_{F}\right) \Gamma_{L}\left(\varepsilon_{F}\right) G_{0}^{a}\left(\varepsilon_{F}\right) M_{\lambda} G_{0}^{r}\left(\varepsilon_{F}\right) \Gamma_{R}\left(\varepsilon_{F}\right) G_{0}^{a}\left(\varepsilon_{F}\right) \Gamma_{L}\left(\varepsilon_{F}\right) G_{0}^{r}\left(\varepsilon_{F}\right)+\text { h.c. }\right] . \\
& \cdot \int_{-\infty}^{+\infty} d \epsilon\left(f_{L}-f_{R}\right) \mathcal{H}_{\epsilon^{\prime}}\left\{f_{L_{-}}\left(\epsilon^{\prime}\right)-f_{L_{+}}\left(\epsilon^{\prime}\right)\right\}(\epsilon)+ \\
& +\frac{e}{h} \sum_{\lambda} \operatorname{Tr}\left[M_{\lambda} G_{0}^{r}\left(\varepsilon_{F}\right) \Gamma_{R}\left(\varepsilon_{F}\right) G_{0}^{a}\left(\varepsilon_{F}\right) M_{\lambda} G_{0}^{r}\left(\varepsilon_{F}\right) \Gamma_{R}\left(\varepsilon_{F}\right) G_{0}^{a}\left(\varepsilon_{F}\right) \Gamma_{L}\left(\varepsilon_{F}\right) G_{0}^{r}\left(\varepsilon_{F}\right)+\text { h.c. }\right] . \\
& \cdot \int_{-\infty}^{+\infty} d \epsilon\left(f_{L}-f_{R}\right) \mathcal{H}_{\epsilon^{\prime}}\left\{f_{R_{-}}\left(\epsilon^{\prime}\right)-f_{R_{+}}\left(\epsilon^{\prime}\right)\right\}(\epsilon)- \\
& -\frac{e}{h} \sum_{\lambda}\left(2\left\langle n_{\lambda}\right\rangle+1\right) \operatorname{Tr}\left[M_{\lambda} A_{0}\left(\varepsilon_{F}\right) M_{\lambda} G_{0}^{r}\left(\varepsilon_{F}\right) \Gamma_{R}\left(\varepsilon_{F}\right) G_{0}^{a}\left(\varepsilon_{F}\right) \Gamma_{L}\left(\varepsilon_{F}\right) G_{0}^{r}\left(\varepsilon_{F}\right)+\text { h.c. }\right] . \\
& \cdot \int_{-\infty}^{+\infty} d \epsilon\left(f_{L}-f_{R}\right) \mathcal{H}_{\epsilon^{\prime}}\{1\}(\epsilon)
\end{aligned}
$$


Na primeira integral podemos aplicar a mudança de variável $\epsilon=\epsilon^{\prime \prime}+\mu_{L}$, de modo que:

$$
\begin{aligned}
\int_{-\infty}^{+\infty} & d \epsilon\left(f_{L}-f_{R}\right) \mathcal{H}_{\epsilon^{\prime}}\left\{f_{L_{-}}\left(\epsilon^{\prime}\right)-f_{L_{+}}\left(\epsilon^{\prime}\right)\right\}(\epsilon)= \\
= & \int_{-\infty}^{+\infty} d \epsilon^{\prime \prime}\left(\frac{1}{e^{\beta\left(\epsilon^{\prime \prime}\right)}+1}-\frac{1}{e^{\beta\left(\epsilon^{\prime \prime}+\mu_{L}-\mu_{R}\right)}+1}\right) \mathcal{H}_{\epsilon^{\prime}}\left\{f_{L_{-}}\left(\epsilon^{\prime}\right)-f_{L_{+}}\left(\epsilon^{\prime}\right)\right\}\left(\epsilon^{\prime \prime}+\mu_{L}\right)= \\
= & \int_{-\infty}^{+\infty} d \epsilon^{\prime \prime}\left(f\left(\epsilon^{\prime \prime}\right)-f\left(\epsilon^{\prime \prime}+e V\right)\right) \mathcal{H}_{\epsilon^{\prime}}\left\{f_{L_{-}}\left(\epsilon^{\prime}\right)-f_{L_{+}}\left(\epsilon^{\prime}\right)\right\}\left(\epsilon^{\prime \prime}+\mu_{L}\right)
\end{aligned}
$$

Com a mudança de variável, a transformada de Hilbert (Equação 6.14) pode ser escrita como:

$$
\begin{aligned}
\mathcal{H}_{\epsilon^{\prime}}\left\{f_{L_{-}}\left(\epsilon^{\prime}\right)-f_{L_{+}}\left(\epsilon^{\prime}\right)\right\}\left(\epsilon^{\prime \prime}+\mu_{L}\right) & =\frac{1}{\pi} \mathcal{P} \int_{-\infty}^{+\infty} d \epsilon^{\prime} \frac{f\left(\epsilon^{\prime}-\mu_{L}-\hbar \omega_{\lambda}\right)-f\left(\epsilon^{\prime}-\mu_{L}+\hbar \omega_{\lambda}\right)}{\epsilon^{\prime}-\left(\epsilon^{\prime \prime}+\mu_{L}\right)}= \\
& =\frac{1}{\pi} \mathcal{P} \int_{-\infty}^{+\infty} d \epsilon^{\prime \prime \prime} \frac{f\left(\epsilon^{\prime \prime \prime}-\hbar \omega_{\lambda}\right)-f\left(\epsilon^{\prime \prime \prime}+\hbar \omega_{\lambda}\right)}{\epsilon^{\prime \prime \prime}-\epsilon^{\prime \prime}}= \\
& =\mathcal{H}_{\epsilon^{\prime}}\left\{f_{-}\left(\epsilon^{\prime}\right)-f_{+}\left(\epsilon^{\prime}\right)\right\}\left(\epsilon^{\prime \prime}\right) .
\end{aligned}
$$

Dessa forma, a integral do primeiro termo da Equação C.18 pode ser escrita como:

$$
\int_{-\infty}^{+\infty} d \epsilon\left(f_{L}-f_{R}\right) \mathcal{H}_{\epsilon^{\prime}}\left\{f_{L_{-}}\left(\epsilon^{\prime}\right)-f_{L_{+}}\left(\epsilon^{\prime}\right)\right\}(\epsilon)=\int_{-\infty}^{+\infty} d \epsilon(f(\epsilon)-f(\epsilon+e V)) \mathcal{H}_{\epsilon^{\prime}}\left\{f_{-}\left(\epsilon^{\prime}\right)-f_{+}\left(\epsilon^{\prime}\right)\right\}(\epsilon) .
$$

O mesmo desenvolvimento pode ser repetido para o segundo termo da Equação C.18, aplicandose a mudança de variável $\epsilon=\epsilon^{\prime \prime}+\mu_{R}$ e obtendo-se:

$$
\int_{-\infty}^{+\infty} d \epsilon\left(f_{L}-f_{R}\right) \mathcal{H}_{\epsilon^{\prime}}\left\{f_{R_{-}}\left(\epsilon^{\prime}\right)-f_{R_{+}}\left(\epsilon^{\prime}\right)\right\}(\epsilon)=\int_{-\infty}^{+\infty} d \epsilon(f(\epsilon-e V)-f(\epsilon)) \mathcal{H}_{\epsilon^{\prime}}\left\{f_{-}\left(\epsilon^{\prime}\right)-f_{+}\left(\epsilon^{\prime}\right)\right\}(\epsilon) .
$$


A subtração das funções distribuições $f_{-}(\epsilon)-f_{+}(\epsilon)$ é uma função par com relação ao ponto $\epsilon$, isto é, $f_{-}(\epsilon-x)-f_{+}(\epsilon-x)=f_{-}(\epsilon+x)-f_{+}(\epsilon+x), \forall x$. Além disso, ela é estritamente positiva e vai a zero para $x<\epsilon-\hbar \omega_{\lambda}$ e $x>\epsilon+\hbar \omega_{\lambda}$. Nesse caso, a transformada de Hilbert $\mathcal{H}_{\epsilon^{\prime}}\left\{f_{-}\left(\epsilon^{\prime}\right)-f_{+}\left(\epsilon^{\prime}\right)\right\}(\epsilon)$ é uma função ímpar com relação ao ponto $\epsilon$, 12 , sendo estritamente negativa para $x<\epsilon$ e estritamente positiva para $x>\epsilon$ conforme representado na Figura C.1.

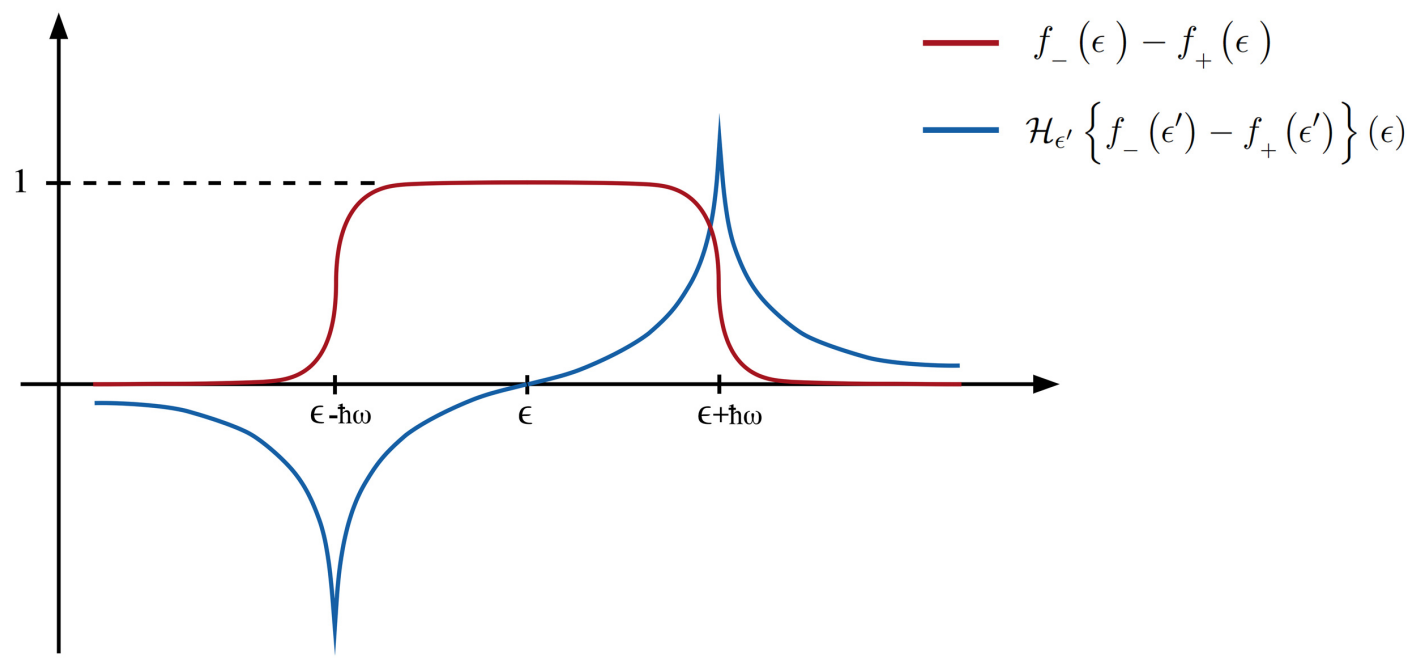

Figura C.1: Representação da função $f_{-}(\epsilon)-f_{+}(\epsilon)$ (em vermelho) e respectiva transformada de Hilbert $\mathcal{H}_{\epsilon^{\prime}}\left\{f_{-}\left(\epsilon^{\prime}\right)-f_{+}\left(\epsilon^{\prime}\right)\right\}(\epsilon)$ (em azul).

Da mesma maneira, as funções $f(\epsilon)-f(\epsilon+e V)$ e $f(\epsilon-e V)-f(\epsilon)$, nos integrandos das Equações C.21 e C.22, respectivamente, são estritamente positivas e simétricas entre si com relação ao ponto $\epsilon$, conforme pode ser observado na Figura C.2.

Portanto, com essa análise dos integrandos nas Equações C.21 e C.22 pode-se concluir que:

$$
\begin{aligned}
& \int_{-\infty}^{+\infty} d \epsilon(f(\epsilon)-f(\epsilon+e V)) \mathcal{H}_{\epsilon^{\prime}}\left\{f_{-}\left(\epsilon^{\prime}\right)-f_{+}\left(\epsilon^{\prime}\right)\right\}(\epsilon)= \\
& =-\int_{-\infty}^{+\infty} d \epsilon(f(\epsilon-e V)-f(\epsilon)) \mathcal{H}_{\epsilon^{\prime}}\left\{f_{-}\left(\epsilon^{\prime}\right)-f_{+}\left(\epsilon^{\prime}\right)\right\}(\epsilon) .
\end{aligned}
$$

Logo, a parte assimétrica da correção no termo elástico fica resumida a: 


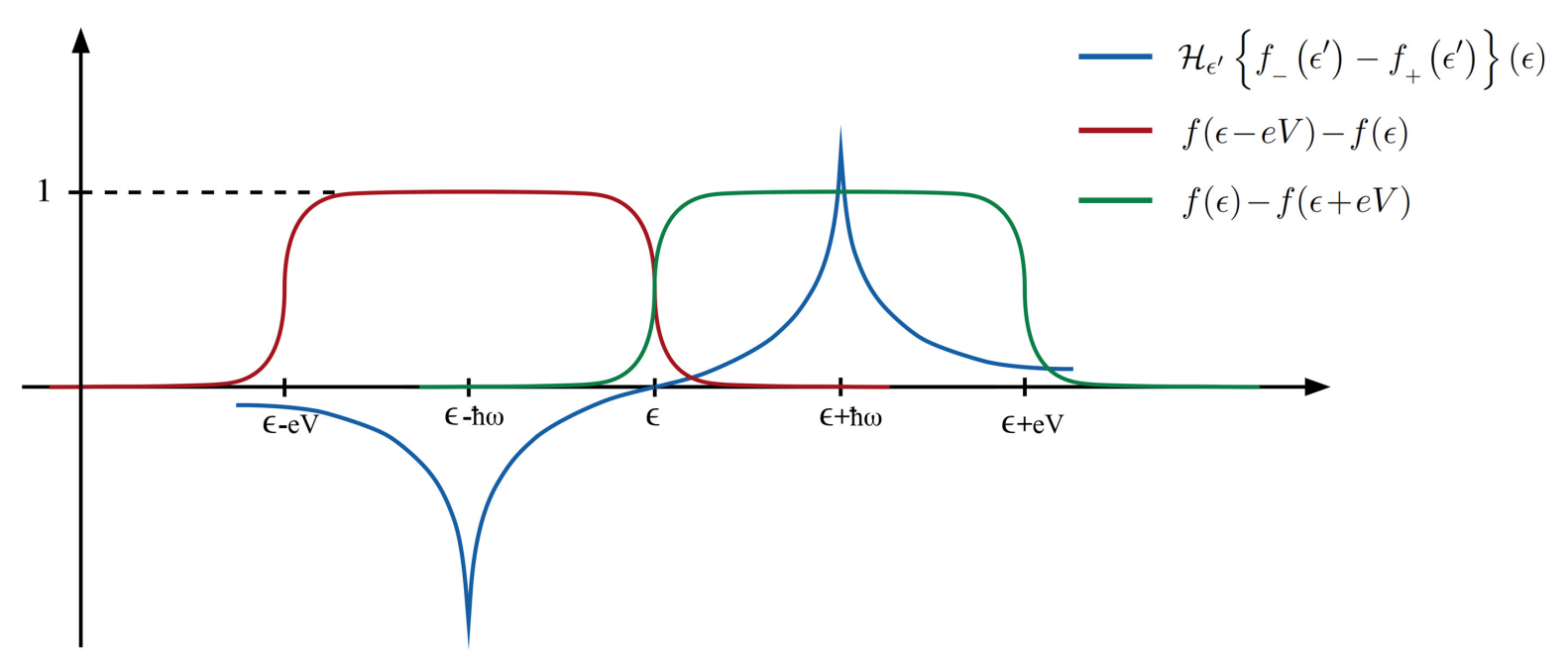

Figura C.2: Representação da transformada de Hilbert $\mathcal{H}_{\epsilon^{\prime}}\left\{f_{-}\left(\epsilon^{\prime}\right)-f_{+}\left(\epsilon^{\prime}\right)\right\}(\epsilon)$ e funções $f(\epsilon-e V)-$ $f(\epsilon)$ e $f(\epsilon)-f(\epsilon+e V)$.

$$
\begin{aligned}
\delta I_{e l}^{\text {asym }}=\frac{e}{h} \sum_{\lambda} \operatorname{Tr}[ & M_{\lambda} G_{0}^{r}\left(\varepsilon_{F}\right)\left(\Gamma_{R}\left(\varepsilon_{F}\right)-\Gamma_{L}\left(\varepsilon_{F}\right)\right) G_{0}^{a}\left(\varepsilon_{F}\right) M_{\lambda} . \\
& \left.\cdot G_{0}^{r}\left(\varepsilon_{F}\right) \Gamma_{R}\left(\varepsilon_{F}\right) G_{0}^{a}\left(\varepsilon_{F}\right) \Gamma_{L}\left(\varepsilon_{F}\right) G_{0}^{r}\left(\varepsilon_{F}\right)+\text { h.c. }\right] . \\
& \cdot \int_{-\infty}^{+\infty} d \epsilon(f(\epsilon)-f(\epsilon-e V)) \mathcal{H}_{\epsilon^{\prime}}\left\{f_{+}\left(\epsilon^{\prime}\right)-f_{-}\left(\epsilon^{\prime}\right)\right\}(\epsilon) .
\end{aligned}
$$

Para terminar a derivação resta somente tratar o termo inelástico $I_{\text {inel }}$ da Equação C.2. Substituindo $\Sigma^{F,<}$ e $\Sigma^{F,>}$ por suas expressões (Equações 6.11 e 6.12, respectivamente) e considerando a aproximação Wide-Band Limit, o termo inelástico se torna:

$$
\begin{aligned}
I_{\text {inel }}= & i \frac{2 e}{h} \int_{-\infty}^{+\infty} d \epsilon \operatorname{Tr}\left[G_{0}^{a} \Gamma_{L} G_{0}^{r}\left(f_{L} \Sigma^{F,>}+\left(1-f_{L}\right) \Sigma^{F,<}\right)\right]= \\
= & -\frac{2 e}{h} \sum_{\lambda} \operatorname{Tr}\left[G_{0}^{a}\left(\varepsilon_{F}\right) \Gamma_{L}\left(\varepsilon_{F}\right) G_{0}^{r}\left(\varepsilon_{F}\right) M_{\lambda} G_{0}^{r}\left(\varepsilon_{F}\right) \Gamma_{L}\left(\varepsilon_{F}\right) G_{0}^{a}\left(\varepsilon_{F}\right) M_{\lambda}\right] . \\
& \cdot \int_{-\infty}^{+\infty} d \epsilon\left(-2\left\langle n_{\lambda}\right\rangle f_{L}+f_{L}\left(f_{L_{-}}-f_{L_{+}}\right)+\left\langle n_{\lambda}\right\rangle f_{L_{-}}+\left\langle n_{\lambda}\right\rangle f_{L_{+}}+f_{L_{+}}-f_{L_{-}}\right)- \\
& -\frac{2 e}{h} \sum_{\lambda} \operatorname{Tr}\left[G_{0}^{a}\left(\varepsilon_{F}\right) \Gamma_{L}\left(\varepsilon_{F}\right) G_{0}^{r}\left(\varepsilon_{F}\right) M_{\lambda} G_{0}^{r}\left(\varepsilon_{F}\right) \Gamma_{R}\left(\varepsilon_{F}\right) G_{0}^{a}\left(\varepsilon_{F}\right) M_{\lambda}\right] . \\
& \cdot \int_{-\infty}^{+\infty} d \epsilon\left(\left\langle n_{\lambda}\right\rangle\left(f_{R_{-}}+f_{R_{+}}-2 f_{L}\right)-\left(f_{L}-f_{R_{+}}\right)+f_{L}\left(f_{R_{-}}-f_{R_{+}}\right)\right) .
\end{aligned}
$$


A primeira integral pode ser dividia em quatro integrais da seguinte forma:

$$
\begin{aligned}
\int_{-\infty}^{+\infty} d \epsilon & \left(-2\left\langle n_{\lambda}\right\rangle f_{L}+f_{L}\left(f_{L_{-}}-f_{L_{+}}\right)+\left\langle n_{\lambda}\right\rangle f_{L_{-}}+\left\langle n_{\lambda}\right\rangle f_{L_{+}}+f_{L_{+}}-f_{L_{-}}\right)= \\
= & \underbrace{-\left\langle n_{\lambda}\right\rangle \int_{-\infty}^{+\infty} d \epsilon\left(f_{L}-f_{L_{-}}\right)}_{\mathcal{A}} \underbrace{-\left\langle n_{\lambda}\right\rangle \int_{-\infty}^{+\infty} d \epsilon\left(f_{L}-f_{L_{+}}\right)}_{\mathcal{C}}- \\
& \underbrace{-\int_{-\infty}^{+\infty} d \epsilon f_{L}\left(1-f_{L_{-}}\right)}_{\mathcal{- \infty}}+\underbrace{\int_{-\infty}^{+\infty} d \epsilon f_{L_{+}}\left(1-f_{L}\right)}_{\mathcal{D}} .
\end{aligned}
$$

Utilizando a igualdade da Equação C.5 nota-se que as integrais $\mathcal{A}$ e $\mathcal{B}$ se cancelam, pois:

$$
\mathcal{A}+\mathcal{B}=-\left\langle n_{\lambda}\right\rangle\left[\int_{-\infty}^{+\infty} d \epsilon\left(f_{L}-f_{L_{-}}\right)+\int_{-\infty}^{+\infty} d \epsilon\left(f_{L}-f_{L_{+}}\right)\right]=-\left\langle n_{\lambda}\right\rangle\left[\hbar \omega_{\lambda}-\hbar \omega_{\lambda}\right]=0
$$

Para mostrar que as integrais $\mathcal{C}$ e $\mathcal{D}$ também se cancelam, basta aplicar a mudança de variável $\epsilon=\epsilon^{\prime}+\hbar \omega_{\lambda}$ em $\mathcal{C}:$

$$
\begin{aligned}
\mathcal{C} & =-\int_{-\infty}^{+\infty} d \epsilon f\left(\epsilon-\mu_{L}\right)\left[1-f\left(\epsilon-\mu_{L}-\hbar \omega_{\lambda}\right)\right]=\quad \stackrel{\epsilon=\epsilon^{\prime}+\hbar \omega_{\lambda}}{\longrightarrow} \\
& =-\int_{-\infty}^{+\infty} d \epsilon^{\prime} f\left(\epsilon^{\prime}-\mu_{L}+\hbar \omega_{\lambda}\right)\left[1-f\left(\epsilon^{\prime}-\mu_{L}\right)\right]= \\
& =-\mathcal{D} .
\end{aligned}
$$

Portanto, a integral no primeiro termo da Equação C.25 é nula, restando somente o último termo. Para analisar a integral do termo restante, é conveniente separá-la em quatro integrais da seguinte forma: 


$$
\begin{aligned}
\int_{-\infty}^{+\infty} d \epsilon & \left(\left\langle n_{\lambda}\right\rangle\left(f_{R_{-}}+f_{R_{+}}-2 f_{L}\right)-\left(f_{L}-f_{R_{+}}\right)+f_{L}\left(f_{R_{-}}-f_{R_{+}}\right)\right)= \\
= & -\left\langle n_{\lambda}\right\rangle\left[\int_{-\infty}^{+\infty} d \epsilon\left(f_{L}-f_{R_{-}}\right)+\int_{-\infty}^{+\infty} d \epsilon\left(f_{L}-f_{R_{+}}\right)\right]- \\
& -\int_{-\infty}^{+\infty} d \epsilon\left(f_{L}-f_{R_{+}}\right)+\int_{-\infty}^{+\infty} d \epsilon f_{L}\left(f_{R_{-}}-f_{R_{+}}\right)= \\
= & -\left\langle n_{\lambda}\right\rangle\left[e V-\hbar \omega_{\lambda}+e V+\hbar \omega_{\lambda}\right]-\left(e V+\hbar \omega_{\lambda}\right)+\int_{-\infty}^{+\infty} d \epsilon f_{L}\left(f_{R_{-}}-f_{R_{+}}\right)= \\
= & -2\left\langle n_{\lambda}\right\rangle e V-\left(e V+\hbar \omega_{\lambda}\right)+\int_{-\infty}^{+\infty} d \epsilon f_{L}\left(f_{R_{-}}-f_{R_{+}}\right)
\end{aligned}
$$

onde foi utilizada a igualdade da Equação C.5. A integral restante já foi calculada antes, correspondendo aos termos $\mathcal{I}$ e $\mathcal{I} \mathcal{I}$ da Equação C.9

$$
\begin{aligned}
& \int_{-\infty}^{+\infty} d \epsilon f_{L}\left(f_{R_{-}}-f_{R_{+}}\right)= \mathcal{I}+\mathcal{I} \mathcal{I}= \\
&=-\frac{1}{\beta}\left[\frac{1}{1-e^{\beta\left(e V+\hbar \omega_{\lambda}\right)}} \ln \left(\frac{e^{\beta\left(\epsilon-\varepsilon_{F}+e V / 2+\hbar \omega_{\lambda}\right)}+1}{e^{\beta\left(\epsilon-\varepsilon_{F}-e V / 2\right)}+1}\right)+\right. \\
&+\frac{1}{1-e^{-\beta\left(e V-\hbar \omega_{\lambda}\right)}} \ln \left(\frac{e^{\beta\left(\epsilon-\varepsilon_{F}+e V / 2-\hbar \omega_{\lambda}\right)}+1}{e^{\beta\left(\epsilon-\varepsilon_{F}-e V / 2\right)}+1}\right)+ \\
&\left.\quad+\ln \left(\frac{e^{\beta\left(\epsilon-\varepsilon_{F}-e V / 2\right)}}{e^{\beta\left(\epsilon-\varepsilon_{F}+e V / 2+\hbar \omega_{\lambda}\right)}+1}+1\right)\right]\left.\right|_{-\infty} ^{+\infty}= \\
&=-\frac{1}{\beta}\left[\frac{\beta\left(e V+\hbar \omega_{\lambda}\right)}{1-e^{\beta\left(e V+\hbar \omega_{\lambda}\right)}}+\frac{\beta\left(e V-\hbar \omega_{\lambda}\right)}{1-e^{-\beta\left(e V-\hbar \omega_{\lambda}\right)}-\beta\left(e V+\hbar \omega_{\lambda}\right)}\right]= \\
&=\frac{\hbar \omega_{\lambda}+e V}{e^{\beta\left(\hbar \omega_{\lambda}+e V\right)}-1}-\frac{\hbar \omega_{\lambda}-e V}{e^{\beta\left(\hbar \omega_{\lambda}-e V\right)}-1}+\left(e V+\hbar \omega_{\lambda}\right) .
\end{aligned}
$$

Portanto, a integral do termo inelástico é dada por: 


$$
\begin{aligned}
& \int_{-\infty}^{+\infty} d \epsilon\left(\left\langle n_{\lambda}\right\rangle\left(f_{R_{-}}+f_{R_{+}}-2 f_{L}\right)-\left(f_{L}-f_{R_{+}}\right)+f_{L}\left(f_{R_{-}}-f_{R_{+}}\right)\right)= \\
& \quad=-2\left\langle n_{\lambda}\right\rangle e V-\left(e V+\hbar \omega_{\lambda}\right)+\frac{\hbar \omega_{\lambda}+e V}{e^{\beta\left(\hbar \omega_{\lambda}+e V\right)}-1}-\frac{\hbar \omega_{\lambda}-e V}{e^{\beta\left(\hbar \omega_{\lambda}-e V\right)}-1}+\left(e V+\hbar \omega_{\lambda}\right)= \\
& \quad=-\left(2\left\langle n_{\lambda}\right\rangle e V+\frac{\hbar \omega_{\lambda}-e V}{e^{\beta\left(\hbar \omega_{\lambda}-e V\right)}-1}-\frac{\hbar \omega_{\lambda}+e V}{e^{\beta\left(\hbar \omega_{\lambda}+e V\right)}-1}\right) .
\end{aligned}
$$

Finalmente, a componente inelástica da corrente é dada por:

$$
\begin{gathered}
I_{\text {inel }}=\frac{2 e}{h} \sum_{\lambda} \operatorname{Tr}\left[G_{0}^{a}\left(\varepsilon_{F}\right) \Gamma_{L}\left(\varepsilon_{F}\right) G_{0}^{r}\left(\varepsilon_{F}\right) M_{\lambda} G_{0}^{r}\left(\varepsilon_{F}\right) \Gamma_{R}\left(\varepsilon_{F}\right) G_{0}^{a}\left(\varepsilon_{F}\right) M_{\lambda}\right] . \\
\cdot\left(2\left\langle n_{\lambda}\right\rangle e V+\frac{\hbar \omega_{\lambda}-e V}{e^{\beta\left(\hbar \omega_{\lambda}-e V\right)}-1}-\frac{\hbar \omega_{\lambda}+e V}{e^{\beta\left(\hbar \omega_{\lambda}+e V\right)}-1}\right) .
\end{gathered}
$$

Reagrupando as componentes $I_{e l}$ (Equação C.6), $\delta I_{e l}^{\text {sym }}$ (Equação C.17), $\delta I_{e l}^{a s y m}$ (Equação C.24) e $I_{\text {inel }}$ (Equação C.32), obtém-se a expressão para corrente na Lowest Order Expansion:

$$
\begin{aligned}
I_{L} \approx & I_{e l}+\delta I_{e l}^{s y m}+\delta I_{e l}^{a s y m}+I_{\text {inel }}= \\
= & \frac{2 e^{2}}{h} V \operatorname{Tr}\left[\Gamma_{L} G_{0}^{r} \Gamma_{R} G_{0}^{a}\right]+ \\
& +\sum_{\lambda} \mathcal{I}^{S y m}\left(V, \hbar \omega_{\lambda}, T, n_{\lambda}\right) \operatorname{Tr}\left[G_{0}^{a} \Gamma_{L} G_{0}^{r}\left\{M_{\lambda} G_{0}^{r} \Gamma_{R} G_{0}^{a} M_{\lambda}+\frac{i}{2}\left(\Gamma_{R} G_{0}^{a} M_{\lambda} A_{0} M_{\lambda}-\text { h.c. }\right)\right\}\right]+ \\
& +\sum_{\lambda} \mathcal{I}^{A s y m}\left(V, \hbar \omega_{\lambda}, T\right) \operatorname{Tr}\left[G_{0}^{a} \Gamma_{L} G_{0}^{r}\left\{\Gamma_{R} G_{0}^{a} M_{\lambda} G_{0}^{r}\left(\Gamma_{R}-\Gamma_{L}\right) G_{0}^{a} M_{\lambda}+\text { h.c. }\right\}\right]
\end{aligned}
$$

onde os coeficientes $\mathcal{I}^{\text {Sym }}$ e $\mathcal{I}^{\text {Asym }}$ são, respectivamente, simétricos e assimétricos sob reversão da diferença de potencial $V$ e dados por:

$$
\begin{gathered}
\mathcal{I}^{S y m}\left(V, \hbar \omega_{\lambda}, T, n_{\lambda}\right)=\frac{2 e}{h}\left(2 e V\left\langle n_{\lambda}\right\rangle+\frac{\hbar \omega_{\lambda}-e V}{e^{\beta\left(\hbar \omega_{\lambda}-e V\right)}-1}-\frac{\hbar \omega_{\lambda}+e V}{e^{\beta\left(\hbar \omega_{\lambda}+e V\right)}-1}\right), \\
\mathcal{I}^{A s y m}\left(V, \hbar \omega_{\lambda}, T\right)=\frac{e}{h} \int_{-\infty}^{+\infty} d \epsilon[f(\epsilon)-f(\epsilon-e V)] \mathcal{H}_{\epsilon^{\prime}}\left\{f\left(\epsilon^{\prime}+\hbar \omega_{\lambda}\right)-f\left(\epsilon^{\prime}-\hbar \omega_{\lambda}\right)\right\}(\epsilon) .
\end{gathered}
$$




\section{Apêndice D}

\section{POSITIVE VIBRATIONS User's Guide}

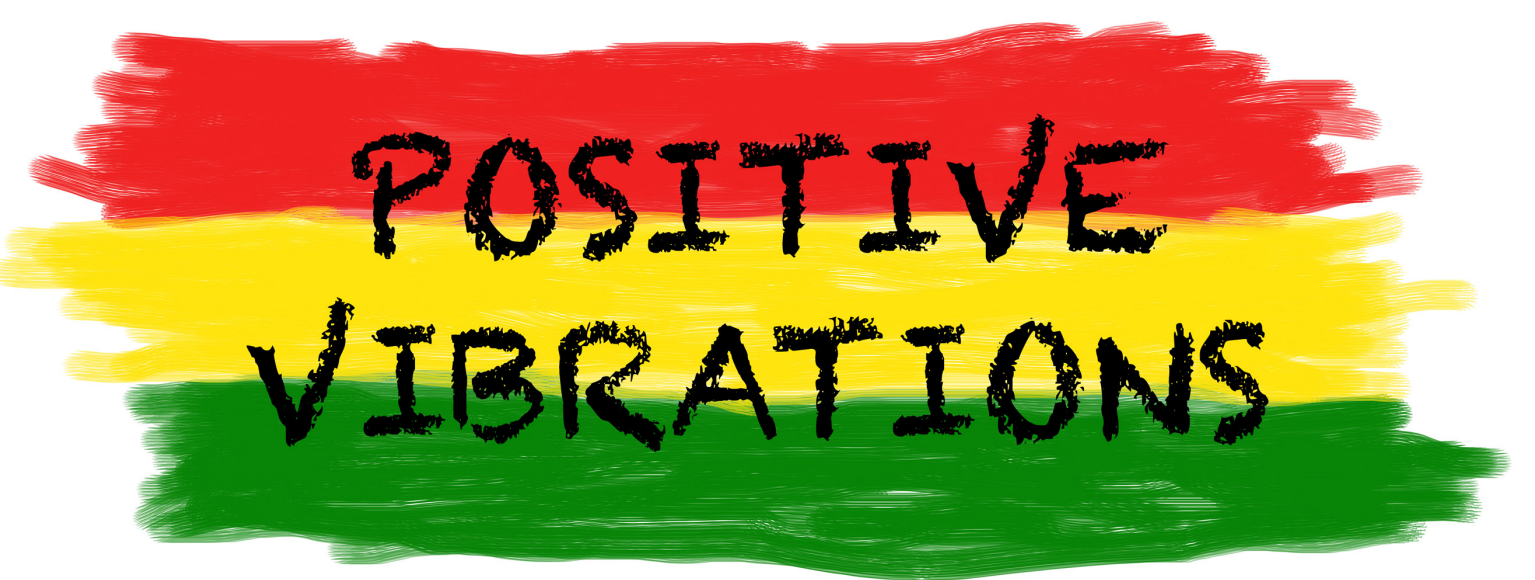

July 21, 2014

Alexandre Reily Rocha

Instituto de Física Teórica, UNESP, Brazil

Pedro Brandimarte Mendonça

Instituto de Física, USP, Brazil

brandimarte@gmail.com 


\section{Contents}

Contents . . . . . . . . . . . . . . . . . . . . 218

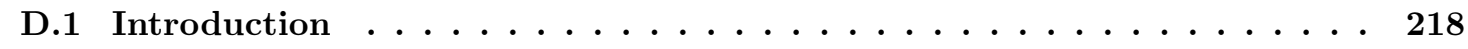

D.2 Compilation . . . . . . . . . . . . . . . . . . . 218

D.3 Execution Steps and Options $\ldots \ldots \ldots \ldots \ldots \ldots \ldots$

D.3.1 Vibrational Modes and Frequencies . . . . . . . . . . . . . . . . . . . . . 219

D.3.2 Electron-Phonon Coupling Matrices … . . . . . . . . . . . . . . . 220

D.4 Problem Handling . . . . . . . . . . . . . . . . . . . . . . . 221

D.5 Acknowledgements . . . . . . . . . . . . . . . . . 222

References . . . . . . . . . . . . . . . . . . . . . 222

\section{D.1 Introduction}

This is a practical description of the code PhOnonS ITeratIVE VIBRATIONS, a tool for computing materials' vibrational modes and frequencies and electron-phonon coupling matrices, from first principles calculations. It is based on code SIESTA [1,2], a density functional theory (DFT) implementation.

With the purpose of calculating the vibrational modes and frequencies, the POSITIVE VIBRATIONS code uses the force constant (FC) matrix as calculated by SIESTA. In order to minimize the effects arising from the use of a discrete grid on FC calculation (such as the so-called egg-box effect [1]), the POSITIVE VIBRATIONS code applies some corrections by enforcing momentum conservation and the FC matrix symmetrization.

To compute the electron-phonon coupling matrices some modifications were performed on SIESTA code, following the methodology described in [3].

The POSITIVE VIBRATIONS source code is stored as a git repository and can be easily downloaded with the line command:

\$ git clone https://brandimarte@bitbucket.org/brandimarte/vibrations.git

\section{D.2 Compilation}

The compilation of the program is done using a Makefile. Some examples of Makefile for different architectures are provided with the code. Basically, the user should set the $C$ compiler and its options, and give the path for LAPACK libraries (Linear Algebra PACKage [4]). 


\section{D.3 Execution Steps and Options}

\section{D.3.1 Vibrational Modes and Frequencies}

The first step is to execute a FC calculation with SIESTA code (for a detailed description on how to perform a force constant calculation in SIESTA and the required flags in input file, refer to the SIESTA user's guide [2]). The usual FC calculation is enough if one is interested only in vibrational frequencies and modes, which then can be calculated with the following command:

\$ vibrations $\sim /$ PathToFCdir input.fdf onlyPh

The first string (vibrations) is the POSITIVE VIBRATIONS executable. The second ( /PathToFCdir) is the path to the directory where the FC calculation was performed. The third argument (input.fdf) is the SIESTA fdf input file used in the FC calculation (refer to SIESTA user's guide for details) and the last one (onlyPh) tells to the code that one is only looking for the vibrational frequencies and modes (i.e. the electron-phonon coupling computation will be neglected).

During the POSITIVE VIBRATIONS code execution the calculation progress is printed on screen. The computed vibrational frequencies are enumerated in ascending order and the corresponding modes follow the same enumeration (i.e. the last mode corresponds to the highest frequency).

One possibility to accelerate the FC calculation on SIESTA is to split over the atoms which are being moved, by performing a FC computation on each atom independently. An example of script for that (runFC.sh) is provided with the code. For each dynamic atom $i$ (those who will be displaced), the script creates the folder $\mathrm{FC} i$ and submit for execution. In this case the total force constant matrix will be split over the individual directories and, in order to inform the POSITIVE VIBRATIONS code about that, one should include an additional splitFC option:

\$ vibrations $\sim /$ PathToFCdir input.fdf onlyPh splitFC

The POSITIVE VIBRATIONS code can generate files for visualizing the vibrational modes according to Jmol viewer format [5]. For that, the file SystemLabel.xyz with non-displaced coordinates must be present on the directory where the FC calculation was performed. For each mode $j$ a file SystemLabelJMOL $j$.xyz is generated, containing the system's coordinates and the mode eigenvector. When opening this file with Jmol viewer one can visualize the corresponding vectors and an animation of the vibrational mode. 


\section{D.3.2 Electron-Phonon Coupling Matrices}

In order to compute the electron-phonon coupling matrices one should include the following flag in the input.fdf file for FC calculation:

\section{FCwriteHS (Boolean):}

When FCwriteHS is set to T, the modified SIESTA will write: a file SystemLabel_d.gHS for each displacement $d$ containing the Hamiltonian and the overlap matrices, a file SystemLabel.ef containing the FC step and the corresponding computed Fermi energy, and a file SystemLabel.orb containing the last orbital index of each atom of the entire system (starting from 0 ).

Default value: $\mathrm{F}$

The generated files will be used by the POSITIVE VIBRATIONS code for computing the derivative of the electronic Hamiltonian with respect to the atoms displacements, which is needed when calculating the electron-phonon coupling [3].

In addition to the FC calculation, one needs to execute the modified SIESTA one more time for computing matrix elements like $\left\langle i^{\prime} \mid k\right\rangle$ and $\left\langle l \mid j^{\prime}\right\rangle$, where $\left|i^{\prime}\right\rangle$ represents the variation of the basis orbital with respect to the displacement of an atom in a given direction. These elements will be used by the POSITIVE VIBRATIONS code for correcting the electronic Hamiltonian derivatives. They can be obtained from the overlap matrix resulting from a SIESTA execution for a duplicated system, where the duplicated atoms are displaced in one of the directions (totaling six different runs: $-x,+x,-y,+y,-z$ and $+z)$.

To automate this procedure, a script runOS. sh was develop. It receives the SIESTA fdf input file (input.fdf), sets the flag FCwriteHS to F and include the following flag as T:

\section{FConlyS (Boolean):}

When FConlys is set to $\mathrm{T}$, the modified SIESTA will write the overlap matrix (which happens at the beginning of the program) at the file SystemLabel.oS and finishes the execution.

Default value: $\mathrm{F}$

The script runOS.sh then duplicates the system and displaces the duplicated atoms in each direction. At each displacement $d$ the system label is modified to SystemLabel_d (where $d$ goes from 1 to 6 meaning $-x,+x,-y,+y,-z$ and $+z$, respectively) and the modified SIESTA is executed. This procedure is very fast, since the overlap matrix is built at the beginning of SIESTA and the execution is finished just after that. 
Once this is done the electron-phonon coupling matrices can be computed (together with the vibrational frequencies and modes) with POSITIVE VIBRATIONS code with the following command:

\$ vibrations $\sim /$ PathToFCdir input.fdf full

Here vibrations is the POSITIVE VIBRATIONS executable, $/$ PathToFCdir is the path to the directory where the FC calculation was performed, input.fdf is the SIESTA fdf input file used in the FC calculation (refer to SIESTA user's guide for details) and full tells to the code that the electron-phonon coupling matrices will be computed besides the vibrational frequencies and modes. Note that the overlap matrix files described above (SystemLabel_d.oS) should be in the FC folder.

If the FC calculation was split over the displaced atoms (see Section D.3.1) one should include an additional splitFC option:

$\$$ vibrations $\sim /$ PathToFCdir input.fdf full splitFC

In addition to the output files described in Section D.3.1, the POSITIVE VIBRATIONS code generates the following file:

\section{SystemLabel.Meph :}

The first line contains integer numbers separated by white spaces corresponding to the following information: the number of spin components, the number of dynamic atoms (those that were displaced on FC calculation), the number of dynamic orbitals, the first dynamic orbital index and the last dynamic orbital index. The next line contains the vibrational frequencies (in $\mathrm{eV}$ ) in descending order separated by white spaces (if it was considered $n$ atoms in the vibrational analysis, then there should have $3 n$ frequencies). The following lines contains the electron-phonon coupling matrices (in $\mathrm{eV}$ ) in the same order as the frequencies (for each frequency there must have one electron-phonon coupling matrix).

\section{D.4 Problem Handling}

The users are encouraged to report problems and bugs to the POSITIVE VIBRATIONS's developers at brandimarte@gmail.com. Patches and fixes will be uploaded to the web-site https://bitbucket.org/brandimarte/vibrations/wiki/Home. 


\section{D.5 Acknowledgements}

PBM thanks CNPq (Conselho Nacional de Desenvolvimento Científico e Tecnológico) for financial support (grant 142792/2010-1).

\section{References}

[1] J. M. Soler, E. Artacho, J. D. Gale, A. García, J. Junquera, P. Ordejón and D. Sánchez-Portal, J. Phys. Cond. Mat. 14, 2745-2779 (2002).

[2] http://departments.icmab.es/leem/siesta/

[3] T. Frederiksen, M. Paulsson, M. Brandbyge and A.-P. Jauho, Phys. Rev. B 75, 205413 (2007).

[4] LAPACK - Linear Algebra PACKage. http://www.netlib.org/lapack/

[5] Jmol: an open-source Java viewer for chemical structures in 3D. http://jmol.sourceforge. net 
Apêndice E

I-SMEAGOL User's Guide

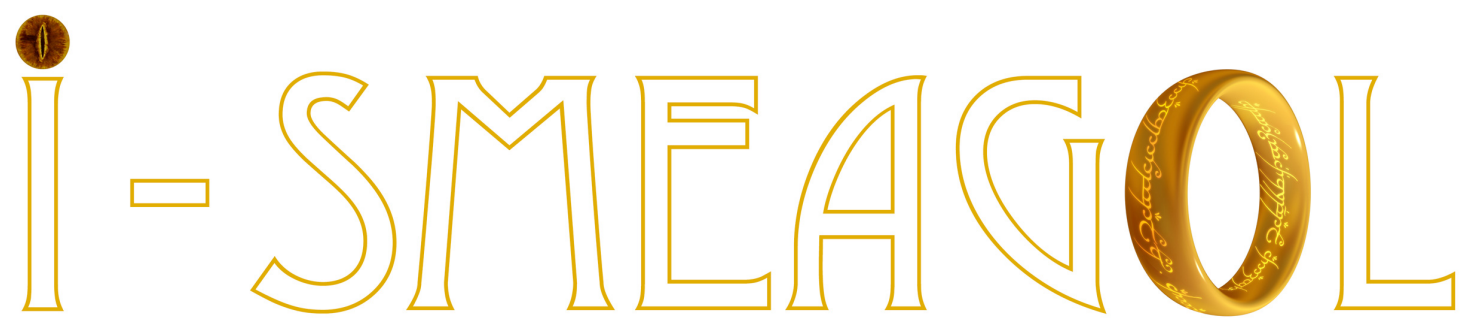

INELASTIC NON-EQUILIBRIUM TRANSPORT 


\title{
Inelastic SMEAGOL (version 2.0)
}

July 21, 2014

\author{
Alexandre Reily Rocha \\ Instituto de Física Teórica, UNESP, Brazil \\ Pedro Brandimarte Mendonça \\ Instituto de Física, USP, Brazil \\ reilya@ift.unesp.br \\ https://bitbucket.org/brandimarte/smeagol-2.0/wiki/Home
}

\section{SMEAGOL (version 1.0)}

June 29, 2005

\author{
Alexandre Reily Rocha and Stefano Sanvito \\ Department of Physics, Trinity College Dublin, IRELAND
}

Víctor Manuel García Suárez and Jaime Ferrer Rodríguez

Departamento de Física, Universidad de Oviedo, SPAIN

Steve Bailey and Colin Lambert

Department of Physics, Lancaster University, Lancaster, LA14YB, UK

smeagol@tcd.ie

http://www.smeagol.tcd.ie 


\section{Contents}

Contents . . . . . . . . . . . . . . . . . . . 225

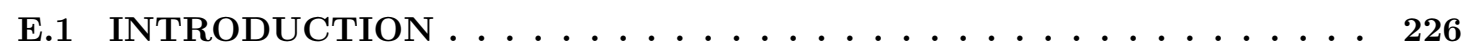

E.2 THE SYSTEM SET UP $\ldots \ldots \ldots \ldots \ldots \ldots \ldots \ldots \ldots$

E.3 THE LEADS CALCULATION $\ldots \ldots \ldots \ldots \ldots \ldots \ldots \ldots$

E.3.1 Input files . . . . . . . . . . . . . . . . . . . . . . . . . . . . . 228

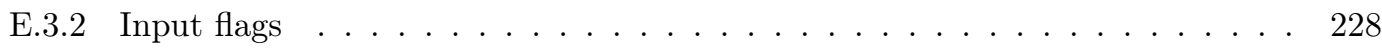

E.3.3 Output files . . . . . . . . . . . . . . . . . . . . . . . . . . 228

E.3.4 Periodic Leads . . . . . . . . . . . . . . . . . . . . . . . . . . . . . 229

E.3.4.1 $\quad$ Input flags $\ldots \ldots \ldots \ldots \ldots$

E.3.4.2 Output files. . . . . . . . . . . . . . . . . . . . 230

E.3.4.3 Example . . . . . . . . . . . . . . . . . . . . 230

E.4 $I-V$ CALCULATION $\ldots \ldots \ldots \ldots \ldots \ldots \ldots \ldots \ldots \ldots$

E.4.1 Input files . . . . . . . . . . . . . . . . . . . . . . . 233

E.4.2 Input flags . . . . . . . . . . . . . . . . . . . . . . 234

E.4.2.1 Matching of the Hartree Potential . . . . . . . . . . . . . 238

E.4.2.2 $\quad$ Flags specific of $k$-point calculations . . . . . . . . . . . . . . 240

E.4.2.3 Flags specific of $\Gamma$ point calculations . . . . . . . . . . . . . 241

E.4.2.4 Flags specific of calculations involving periodic leads . . . . . . . . . 241

E.4.2.5 Example for periodic lead calculation . . . . . . . . . . . . . 242

E.4.3 Output files . . . . . . . . . . . . . . . . . . . . . . . . . . . . . . . . . 242

E.5 INELASTIC $I$ - $V$ CALCULATION $\ldots \ldots \ldots \ldots \ldots \ldots \ldots \ldots \ldots$

E.5.1 Input files . . . . . . . . . . . . . . . . . . . . . . . . . . . 244

E.5.2 Input flags . . . . . . . . . . . . . . . . . . . . . . . . 245

E.5.3 Output files . . . . . . . . . . . . . . . . . . . . . . . . . . . . 247

E.6 MOLECULAR DYNAMICS WITH TRANSPORT $\ldots \ldots \ldots \ldots \ldots \ldots$

E.6.1 Input files . . . . . . . . . . . . . . . . . . . . . . . . . . . . . . 249

E.6.2 Input flags . . . . . . . . . . . . . . . . . . . . . . . . . . . 249

E.6.3 Output files . . . . . . . . . . . . . . . . . . . . . 250

E.7 HOW TO RUN SMEAGOL $\ldots \ldots \ldots \ldots \ldots \ldots \ldots \ldots \ldots$

E.8 PROBLEM HANDLING $\ldots \ldots \ldots \ldots \ldots \ldots \ldots \ldots \ldots \ldots$

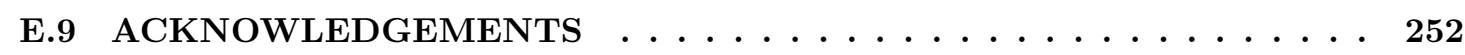

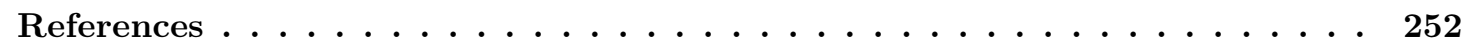




\section{E.1 INTRODUCTION}

This is a comprehensive user's guide to the quantum electronic transport code SMEAGOL (Spin and Molecular Electronics Algorithm on a Generalized atomic Orbital Landscape) [1,2]. SMEAGOL is based on the non-equilibrium Green's function (NEGF) formalism for one-particle Hamiltonian. In its present form it uses density functional theory (DFT) with the numerical implementation contained in the code SIESTA [3,4]. However, SMEAGOL's computational scheme is very general and can be implemented together with any electronic structure methods based on localized basis sets. Alternative implementations are currently under investigation.

The new version of the code, called Inelastic SMEAGOL, has a number of improvements, bug fixes and new implementations. The first major change was the transitioning from the deprecated version 1.3 of SIESTA code to the new version 3.1. The new implementations include the possibility of performing molecular dynamics together with transport calculations, a more efficient calculation of the self-energies from periodic leads and the electronic transport with electron-phonon interaction in the Born approximation [6].

In the next sections we will explain how to set up a typical calculation and we will describe the various options. The reader of this user guide is supposed to have familiarity with the nonequilibrium Green's function formalism $[5,12]$ and with density functional theory [13, 14]. In addition a good knowledge of SIESTA and the SIESTA's input files is necessary since only the I-SMEAGOL's commands are described here, although a complete input file needs the setting of flags proper of SIESTA. For these we refer to the SIESTA user's guide [4].

\section{E.2 THE SYSTEM SET UP}

SMEAGOL is designed for calculating two probe $I-V$ characteristics. The typical system investigated is described in Figure E.1. It comprises two semi-infinite leads (left and right) and an extended molecule, which includes the region of interest and a few atomic layers of the leads. The electronic structure of the leads is not affected by the potential drop and it is computed only once at the beginning of the calculation.

There are two fundamental steps in setting up a SMEAGOL's calculation: 1) calculating the electronic structure for the leads, and 2) calculating the $I-V$ curve for a given system. The evaluation of the electronic structure of the leads is essential and must be performed before attempting the calculation of the $I-V$. The files: SystemLabel.HSL, SystemLabel.DM, bulklft.DAT and bulkrgt.DAT are generated during the evaluation of the electronic structure of the leads. These 


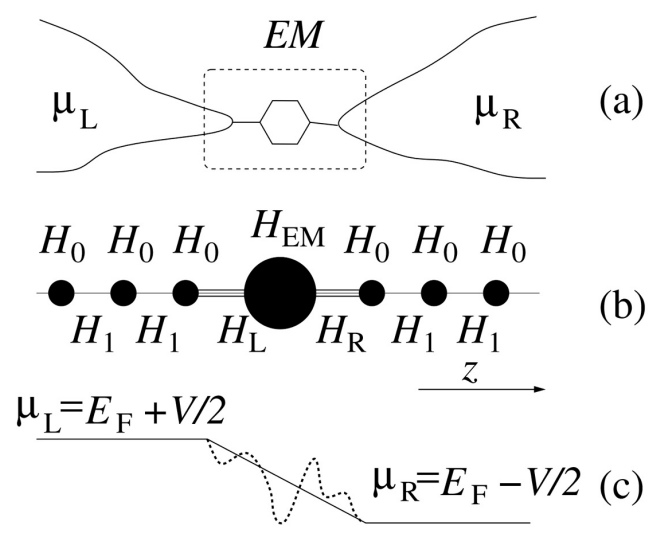

Figure E.1: (a) Schematic two terminal device. Two leads are kept at the chemical potentials $\mu_{L}$ and $\mu_{R}$ and the transport is through the extended molecule EM. (b) The Hamiltonian is an infinite matrix comprising two block diagonal parts describing the leads and a part (finite) describing the extended molecule $H_{E M}$. (c) Typical potential profile.

are necessary for calculating the $I-V$ characteristic.

\section{E.3 THE LEADS CALCULATION}

Here SMEAGOL evaluates the Hamiltonian $\mathcal{H}$, the overlap $\mathcal{S}$ and the density $\rho$ matrices of the current/voltage probes (the leads). The self-energies for the semi-infinite electrodes can be obtained from the knowledge of the Hamiltonian and the overlap matrices of an infinite system, and therefore only a DFT calculation for a bulk system is needed. $\mathcal{H}$ and $\mathcal{S}$ should be written in the tridiagonal form described by Sanvito et al. 1, 15. Figure E.2 shows the typical setup for the electrodes.

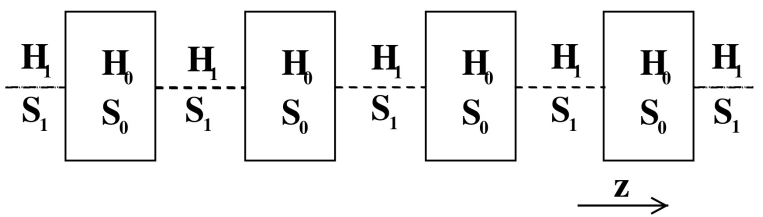

Figure E.2: Periodic structure of the leads. $H_{0}$ and $S_{0}$ are the Hamiltonian and overlap matrix describing the interaction within one unit cell and $H_{1}$ and $S_{1}$ are the coupling and overlap matrix describing the interaction between adjacent unit cells. The arrow shows the direction of transport ( $z$ direction).

The same procedure is used for calculating the matrix elements of the Hamiltonian and overlap matrix in the case of $k$-points calculation (along the direction orthogonal to transport). 


\section{E.3.1 Input files}

There are no additional input files than those used by SIESTA for an ordinary DFT calculation (input file, pseudopotentials ...). For these we refer to the SIESTA user's guide [4].

\section{E.3.2 Input flags}

The following input flags must be supplied to the input.fdf file in order to perform the bulk calculation.

\section{BulkTransport (Boolean):}

When this flag is set to true the leads Hamiltonian and overlap matrix will be written to the file SystemLabel.HSL. Moreover the files bulklft.DAT or bulkrgt.DAT (or both) will be created. These contain information about the system (Fermi energy, System label, unit cell, non-zero elements of the Hamiltonian and information about the super cell).

Default value: $\mathrm{F}$

\section{BulkLeads (String):}

Define whether the calculated electronic structure should be used for the left-hand side lead, for the right-hand side lead of for both. In practise it defines the names and the extensions of the output files. There are three possible options:

- LR or RL (the same output files will be used for both left- and right-hand side lead),

- $\mathrm{R}$ (the output files will be used for the right-hand side lead only),

- L (the output files will be used for the left-hand side lead only).

Default value: LR

\section{E.3.3 Output files}

\section{bulklft.DAT :}

File containing information about the left-hand side lead (number of states, Fermi energy, number of $k$-points, etc).

\section{bulkrgt.DAT :}

File containing information about the right-hand side lead (number of states, Fermi energy, number of $k$-points, etc). 


\section{SystemLabel.HSL :}

File containing the Hamiltonian and overlap matrices of the leads. The string SystemLabel is read from either bulklft.DAT or bulkrgt.DAT. If the two leads are the same one can use the same SystemLabel.HSL file to read the information for both the left and right leads (set the flag BulkLeads to LR). If the two systems are different, then two files with different names must be provided from two different leads calculations (set the flag BulkLeads to either L or R).

\section{SystemLabel.DM :}

File containing the density matrix of the leads. It is needed for calculating the $I-V$. The string SystemLabel is read from either bulklft.DAT or bulkrgt.DAT. If the two leads are made from the same materials and have the same geometrical arrangement one can use the same SystemLabel.DM file to read the information about both the left- and righthand side lead (set the flag BulkLeads to LR). If the two leads are different, then two files with different names must be provided from two independent leads calculations (set the flag BulkLeads to either L or R). The SystemLabel.DM file is used to set the boundary condition at the interface between the scattering region and the electrodes.

\section{E.3.4 Periodic Leads}

In case of leads, which are periodic in all directions, the resources needed for computing the electrodes' self-energies in the non-equilibrium transport calculation (Section E.4) can be reduced if one considers their periodicity in the direction perpendicular to transport. For that purpose one needs to perform another lead calculation but considering only the lead's unit cell and with a different SystemLabel, say SystemLabelSmall.

\section{E.3.4.1 Input flags}

In addition the input flags for bulk calculation described in Section E.3.2, the following input flags must be supplied to the input.fdf file in order to perform the periodic lead calculation.

\section{PeriodicLead (Boolean):}

When this flag is set to true the information about the positions of atomic orbitals for mounting the corresponding lead supercell is written to the file SystemLabelSmall.xij. Default value: $\mathrm{F}$ 
InitTransport (Boolean):

When this flag is set to true the supercell size is expanded to 3 in transverse directions.

Default value: $\mathrm{F}$

\section{E.3.4.2 Output files}

Similarly to the bulk calculation (Section E.3.3 the files bulklft.DAT and bulkrgt.DAT are generated. But since they have the same name, one should rename these files as bulklftsmall.DAT and bulkrgtsmall.DAT, respectively. In addition, the following file is generated:

\section{SystemLabelSmall.HSL :}

File containing the Hamiltonian and overlap matrices of the leads. The string SystemLabelSmall is read from either bulklftsmall. DAT or bulkrgtsmall. DAT. If the two leads are the same one can use the same SystemLabelSmall. HSL file to read the information for both the left and right leads (set the flag BulkLeads to LR). If the two systems are different, then two files with different names must be provided from two different leads calculations (set the flag BulkLeads to either L or R).

\section{SystemLabelSmall.xij :}

File containing the positions of atomic orbitals which are used for mounting the corresponding lead supercell in transport calculations.

\section{E.3.4.3 Example}

In order to understand the periodic lead procedure, let's consider the example of a gold electrode, which has a face-centered cubic crystal structure, with a supercell consisting of 27 atoms (three layers of 9 atoms) as represented in Figure E.3. We note that this supercell can be reproduced by repeating the highlighted atoms 3 times in either $x$ and $y$ directions.

At the usual leads calculation, one should have the following parameters at the input.fdf file (refer to the SIESTA manual for a detailed description of the input flags):

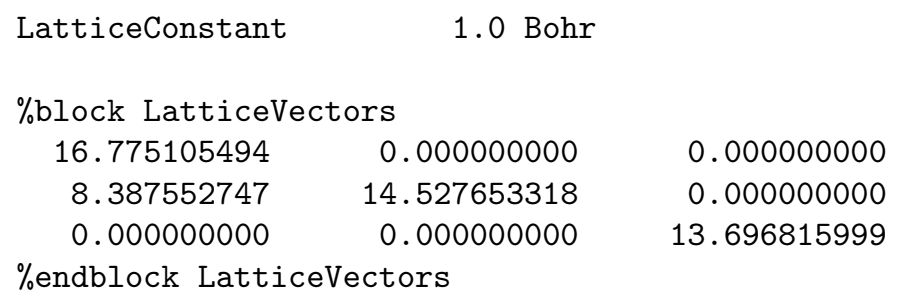




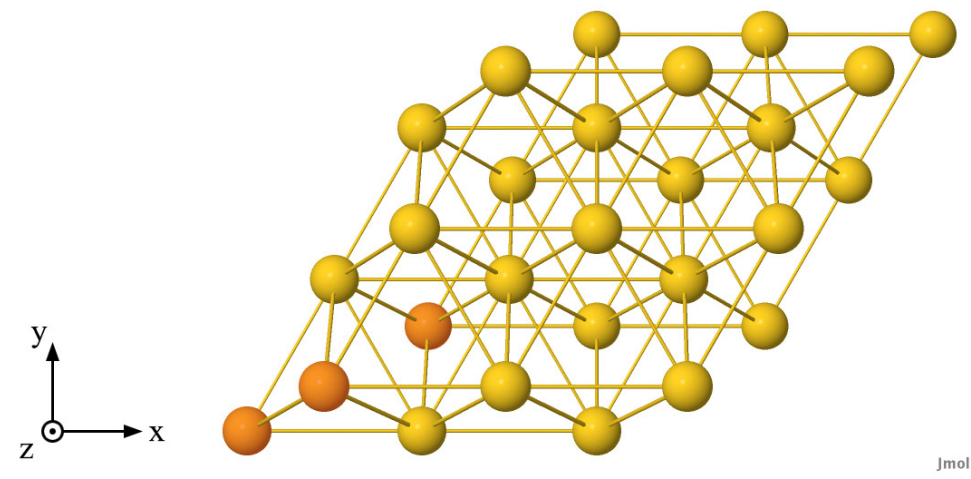

Figure E.3: Example of solid lead supercell constituted by 27 gold atoms which are repeated periodically in all directions. The lead's unit cell containing 3 gold atoms is highlighted.

$\begin{array}{cccc}\text { \%block } & \text { kgrid_Monkhorst_Pack } \\ 1 & 0 & 0 & 0.0 \\ 0 & 1 & 0 & 0.0 \\ 0 & 0 & 100 & 0.0\end{array}$

\%endblock kgrid_Monkhorst_Pack

AtomicCoordinatesFormat NotScaledCartesianBohr

AtomCoorFormatOut NotScaledCartesianBohr

\%block AtomicCoordinatesAndAtomicSpecies

3.779453756

4.565599034

6.575304671

5.393639557

9.131216965

14.962857418

7.007825359

0.000000000

9.371155587

3.779453756

4.565599034

12.167006503

5.393639557

9.131216965

20.554559250

7.007825359

0.000000000

14.962857418

3.779453756

4.565599034

17.758708334

5.393639557

9.131216965

12.167006503

11.850382764

0.000000000

6.575304671

8.622011161

4.565599034

9.371155587

10.236196962

9.131216965

17.758708334

11.850382764

0.000000000

12.167006503

8.622011161

4.565599034

14.962857418

10. 236196962

9.131216965

23.350410165

11.850382764

0.000000000

17.758708334

8.622011161

4.565599034

20.554559250

10.236196962

9.131216965

14.962857418

16.692940169

0.000000000

9. 371155587

13.464568566

4.565599034

12.167006503

15.078754368

9.131216965

20.554559250

16.692940169

0.000000000

4.565599034

14.962857418

13.464568566

9.131216965

17.758708334

15.078754368

0.000000000

26.146261081

16.692940169

4.565599034

13.464568566

9.131216965

23. 350410165

15.078754368

\%endblock AtomicCoordinatesAndAtomicSpecies 
In this example, the lead's unit cell calculation is performed considering only three gold atoms (those highlighted in Figure E.3) and the $k$-points in $x$ and $y$ directions should be multiplied by 3 as follows:

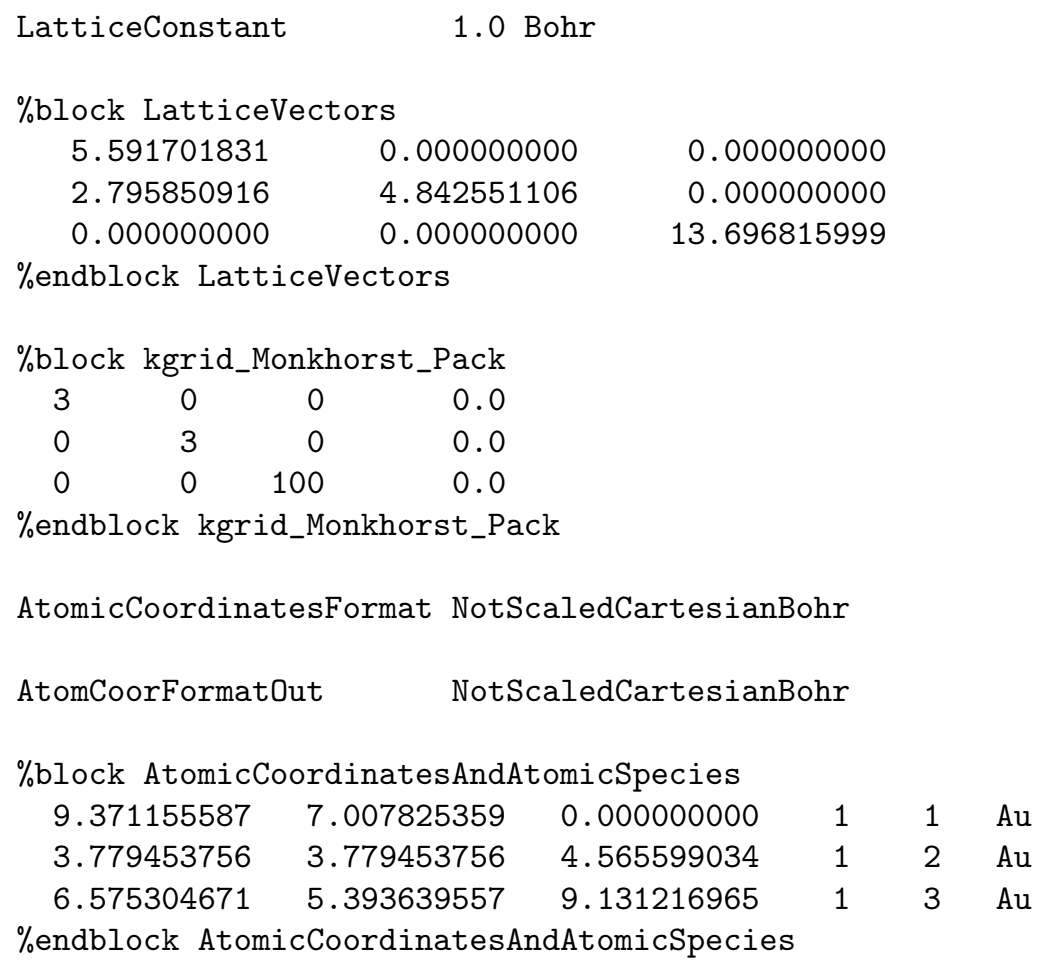

Here, it is important to note that the order within the AtomicCoordinatesAndAtomicSpecies block in the calculations for the large leads should be taken into consideration. It should be built by repeating the small electrodes $n_{1}$ times in the direction of the first lattice vector, and $n_{2}$ times in the direction of the second lattice vector.

It is also important to note that the kgrid_Monkhorst_Pack block for the small leads calculation should be given by

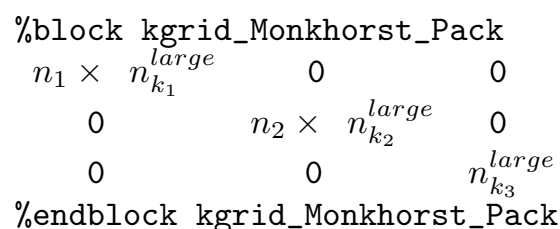

if we define the kgrid_Monkhorst_Pack for the large electrode as

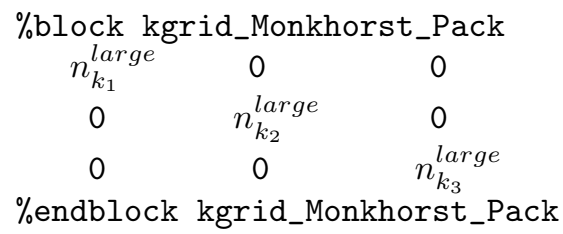




\section{E.4 $I-V$ CALCULATION}

Once the initial calculation for the leads has been performed we can proceed with computing the non-equilibrium transport properties of our system.

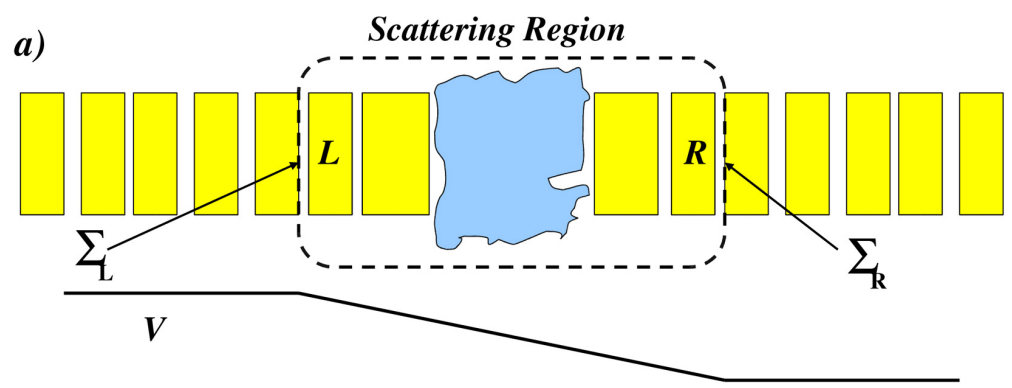

b)
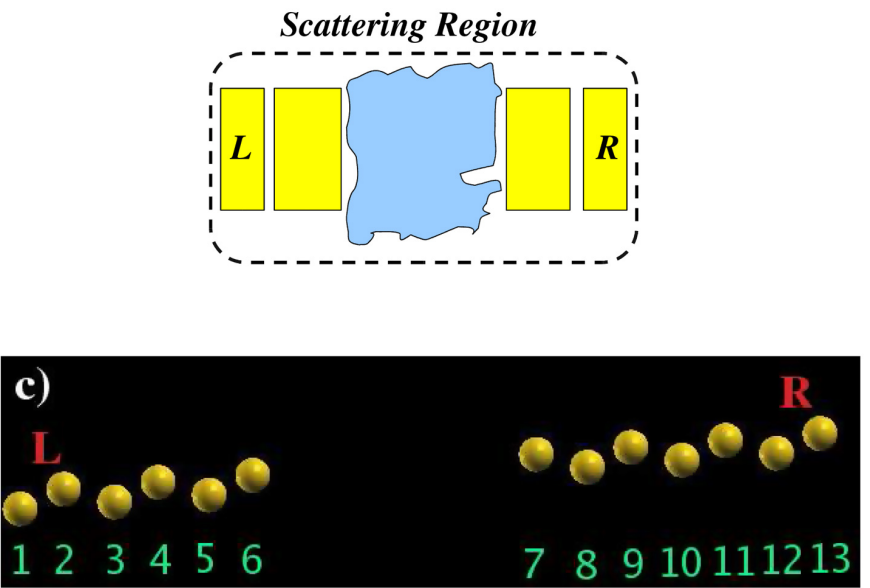

Figure E.4: a) Sketch of a typical system simulated using SMEAGOL: two semi-infinite electrodes connected to a central scattering region. b) The typical unit cell for a SMEAGOL calculation. The reader must note that we include at least one layer of the leads both to the left- and to the right-hand side of the scattering region. The order of the atoms in the input file is only important in these two layers. The first atoms of the list (see AtomicCoordinatesAndAtomicSpecies in the SIESTA user's guide) must be those contained in the left-hand side lead unit cell, and they should be input with the same order than in the bulk leads calculation. In an analogous way, the last atoms of the list must conform with the input file for the right-hand side lead. c) An example of a system setup for two semi-infinite zig-zag wires separated by a vacuum region. The numbers for each atom have been indicated as well as the two atoms on either side corresponding to the the unit cells for the left- and right-hand side lead.

\section{E.4.1 Input files}

Several files generated during the construction of the leads must be used. These are: bulklft.DAT, bulkrgt.DAT, SystemLabel.HSL and SystemLabel.DM. In case of periodic leads (Section E.3.4) one should include also the files bulklftsmall. DAT, bulkrgtsmall.DAT, SystemLabelSmall. HSL and SystemLabelSmall.xij. 


\section{E.4.2 Input flags}

The following input flags must be supplied to the file input.fdf in order to perform the transport $(I-V)$ calculation. If any of these values is not supplied, the default value will be assumed. Special care should be taken for the variables that control the real and contour integrals (Figure E.5), when calculating the non-equilibrium density matrix.

\section{EMTransport (Boolean):}

When EMTransport is set to T, SMEAGOL will perform an NEGF calculation (transport). Otherwise, the code will perform a standard SIESTA equilibrium ground state calculation for either a periodic system or a molecule.

Default value: $\mathrm{F}$

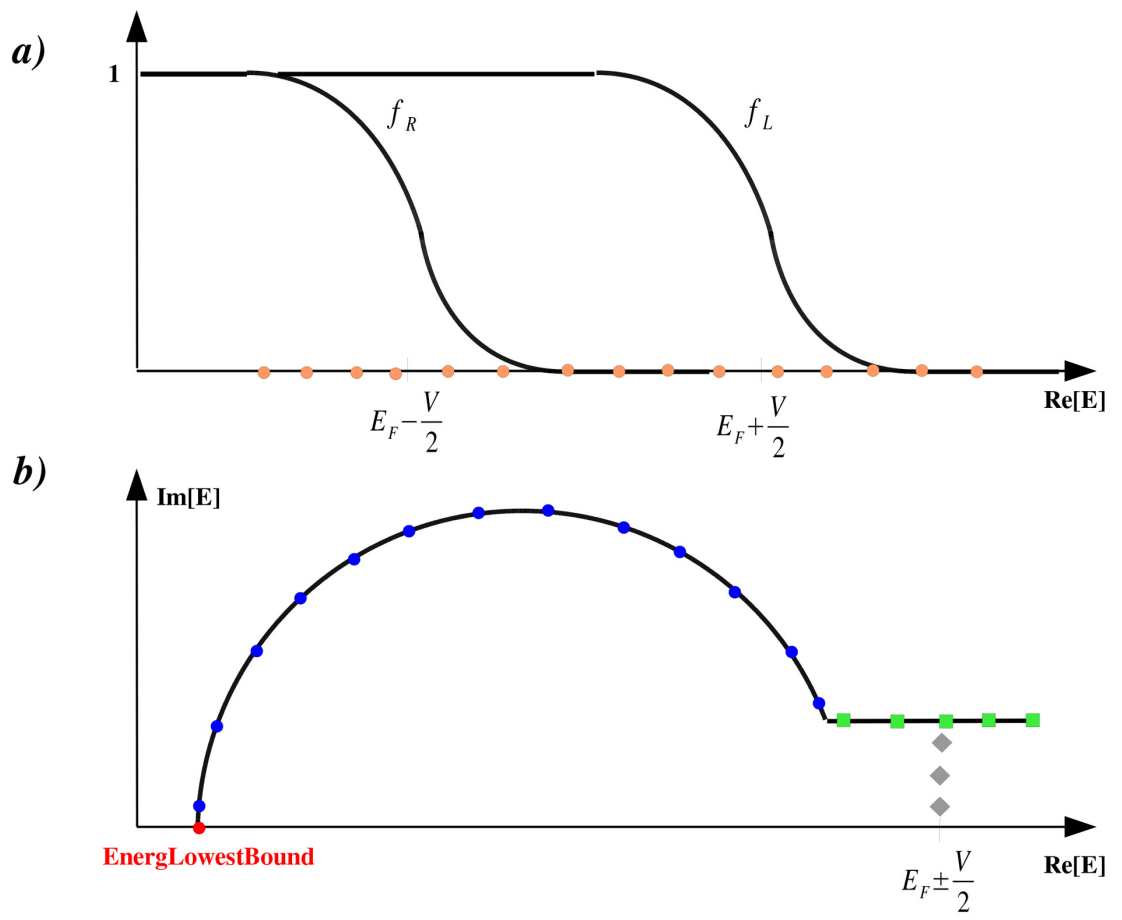

Figure E.5: Sketches for the Green Function integration leading to the non-equilibrium charge density 1.2. a) The non-equilibrium part must be performed along the real energy axis, but is bound by the left- and right-hand side Fermi distributions functions, $f_{L}$ and $f_{R}$. b) The equilibrium component is performed over a semicircular path in the complex energy plane. Here we show the starting position EnergLowestBound, the energy mesh along the two segments of the curve and the poles of the Fermi distribution.

\section{NEnergReal (Integer):}

Number or energy points along the real axis for the integration of the non-equilibrium part of the Green's function. The number of points is extremely sensitive to the type 
of calculation performed. The points are distributed between $-\left(V / 2+30.0 k_{B} T\right)$ and $\left(V / 2+30.0 k_{B} T\right)$. The number of points determines how fine the mesh is. Since the Green's function on the real axis can be ill-behaving (large number of singularities), a very fine mesh might be necessary. For equilibrium calculations, NEnergReal can be set to zero. In Figure E.5 (a) the real axis energy points are sketched as orange circles and NEnergReal $=15$.

Default value: 0

\section{NEnergImCircle (Integer):}

Number of energy points along the semi-circle in the complex plane (equilibrium part of the Green's function integral). The circle starts at the energy EnergLowestBound and finishes at the complex point energy point $\left(E_{F}-30.0 k_{B} T, 2 N_{P o l e s} \pi k_{B} T\right)$. The values for the energy points are calculated using a Gauss-Legendre algorithm along a semicircle. Figure E.5 (b) shows a sketch of the points along the contour in the complex energy plane. In this example, the points along the circle are represented by blue circles and NEnergImCircle $=13$.

Default value: 15

\section{NEnergImLine (Integer):}

Number of energy points along the line in the complex plane where the integral of the equilibrium contribution to the density matrix is performed. This straight line starts at the point $\left(E-30.0 k_{B} T, 2 N_{\text {Poles }} k_{B} T\right)$ and finishes at point $\left(E_{F}+30.0 k_{B} T, 2 N_{\text {Poles }} k_{B} T\right)$ (green squares in Figure E.5 (b), NEnergImLine $=5$ ). The position of the energy points is determined by a Gauss-Legendre algorithm.

Default value: 20

NPoles (Integer):

Number of poles in the Fermi distribution used to compute the contribution to the equilibrium charge density. The Fermi distribution is given by:

$$
f(\epsilon)=\frac{1}{e^{\frac{\epsilon-\mu}{k_{B} T}}+1}
$$

The poles of $f(\epsilon)$ all lay on the complex plane with $i_{n}=\mu+(2 n+1) i \pi$ being the $n$-th pole. The number of poles specifies how far from the real axis the contour integral will 
be performed. The furthest away from the real axis the more well-behaving the Green's function is.

In Figure E.5 (b) the position of the poles are represented by grey diamonds (NPoles $=3)$.

Default value: 5

VInitial (Physical):

Value of the initial bias for the $I-V$ calculation.

Default value: $0.0 \mathrm{eV}$

VFinal (Physical):

Value of the final bias for the $I-V$ calculation.

Default value: $0.0 \mathrm{eV}$

NIVPoints (Integer):

Number of bias steps considered between the two limits $V_{\text {initial }}$ and $V_{\text {final }}$. The current will be calculated only for these biases.

Default value: 0

Delta (Double precision):

Small imaginary part $\delta$ that accounts for the broadening of the localized energy levels of Green function, $G=\left[\epsilon+i \delta-H_{S}-\Sigma_{L}-\Sigma_{R}\right]^{-1}$.

Default value: $10^{-4}$

\section{EnergLowestBound (Physical):}

Energy that specifies the beginning of the contour integral in the complex plane for the equilibrium contribution to the charge density. EnergLowestBound must be below both the lowest band of the leads and the lowest energy level of the scattering region. Red dot in Figure E.5 (b).

Default value: $-5.0 \mathrm{Ry}$

NSlices (Integer):

Number of slices of the bulk Hamiltonian substituted into the left- and right-hand side part of scattering region. The Hamiltonian of these slices is not recalculated self-consistently and these "buffer" layers help the convergence, in particular when the two leads are different. Default value: 1 
AtomLeftVCte (Integer):

Number of the atom used to determine the onset of the bias ramp on the left-hand side. The number of the atom is taken from the order in which the atoms are specified in the SIESTA block AtomicCoordinatesAndAtomicSpecies (refer to the SIESTA manual for a description of this block).

Default value: 1

\section{AtomRightVCte (Integer):}

Number of the atom that is used to determine the onset of the bias ramp on the right-hand side. The number of the atom is taken from the order in which the atoms are specified in the SIESTA block AtomicCoordinatesAndAtomicSpecies (refer to the SIESTA manual for a description of this block).

For $z<z_{\text {AtomLeftVCte }}$ and $z>z_{\text {AtomRightVCte }}$, the potential added to the Hartree potential is a constant equal to $+V / 2$ and $-V / 2$ respectively. For $z_{\text {AtomLeftVCte }}<z<$ $z_{\text {AtomRightVCte }}$ a bias ramp $V\left(z-z_{0}\right)$ is introduced, where $z_{0}$ is the midpoint between $z_{\text {AtomLeftVCte }}$ and $z_{\text {AtomRightVCte. }}$

Default value: Last Atom

\section{TrCoefficients (Boolean):}

If set to true the transmission coefficients as a function of energy $T(\epsilon, V)$ will be calculated and printed to the file SystemLabel.TRC (see Section E.4.3 below) for any bias point. Although the current is defined as:

$$
\int_{-\infty}^{+\infty} d \epsilon T(\epsilon, V)\left(f_{L}-f_{R}\right)
$$

we can calculate the transmission coefficients for a wider range of energy values and a different number of energy points by using the variables InitTransmRange, FinalTransmRange and NTransmPoints which are defined below.

Default value: $\mathrm{F}$

\section{InitTransmRange (Physical):}

Initial energy for the calculation of the transmission coefficients.

Default value: -5.0 Ry 
FinalTransmRange (Physical):

Final energy for the calculation of the transmission coefficients.

Default value: $5.0 \mathrm{Ry}$

\section{NTransmPoints (Integer):}

Number of energy points uniformly distributed between InitTransmRange and FinalTransmRange for which the transmission coefficients are calculated. If TrCoefficients is set to false, this flag is ignored.

Default value: 100

\section{PeriodicTransp (Boolean):}

If the two electrodes are equal one may use periodic boundary conditions over the supercell. This procedure improves convergence and decreases the size of the unit cell. If the two leads are different then PeriodicTransp must be set to F.

Default value: $\mathrm{T}$

\section{SaveBiasSteps (data block):}

Block containing information for printing the Hartree potential (SystemLabel.VH), the charge density (SystemLabel.RHO), the converged density matrix (SystemLabel.DMT), the difference between the self-consistent and the atomic charge densities (SystemLabel.DRHO) and the total DFT potential (SystemLabel.VT) at a specified bias step. The arguments in the block are integers indicating the bias steps at which to print these files. They range from from 0 up to NIVpoints. The appropriate SIESTA flag for printing any of these files must also be set to true.

Example:

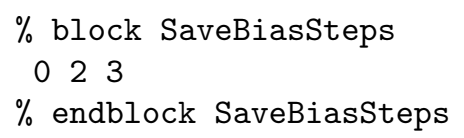

SMEAGOL will print the requested file (for instance SystemLabel.vH if the SIESTA flag SaveElectrostaticPotential is set to true) at the bias steps 0,2 and 3 .

Default value: No Default

\section{E.4.2.1 Matching of the Hartree Potential}

One important information needed from the leads calculation is the average value of the Hartree potential. SMEAGOL uses a Fast Fourier Transform (FFT) algorithm to solve the Poisson 


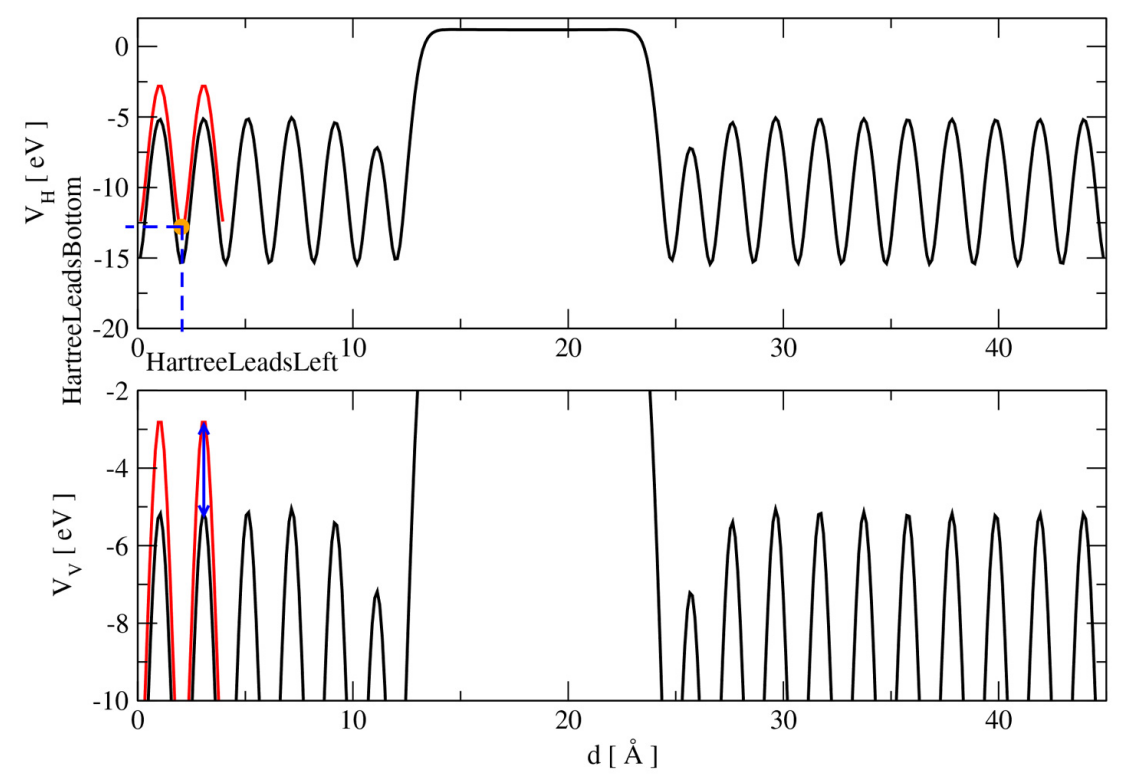

Figure E.6: Shift of the Hartree potential between two SIESTA calculations: bulk gold (the leads) with two atoms in the unit cell (red line) and the parallel plate capacitor (scattering region) depicted in Figure F.1 (3c). Note that the Hartree potential of the scattering region has converged to the bulk value after the first atomic layer except for a constant.

equation in reciprocal space (refer to the SIESTA manual for more details). The $\vec{k}=0$ term is ignored resulting in a Hartree potential that is defined up to a constant. This might lead to a mismatch between the Hartree potential of the leads and that at the edges of the scattering region. This mismatch is better illustrated in Figure E.6 where we have plotted the average Hartree potential on the plane orthogonal to transport for the electrodes and two semi-infinite wires separated by $12 \AA$ (our scattering region; see Figure F.1 (3c)) using the post-processing tool Pot.exe (included in the Utils directory).

In order to compensate for the arbitrary zero of the potential we shift the Hartree potential of the scattering region by a constant (see variables HartreeLeadsLeft, HartreeLeadsRight and HartreeLeadsBottom described below). In this way we force the match of the potential at the scattering region/leads interface.

\section{HartreeLeadsLeft (Physical):}

Position in space in the left-hand side lead where the Hartree potential of the scattering region should match that of the bulk.

Default value: 0.0 Ang

\section{HartreeLeadsRight (Physical):}


Position in space of the right-hand side lead where the Hartree potential in the scattering region should match that of the bulk.

Default value: 0.0 Ang

\section{HartreeLeadsBottom (Physical):}

Average value of the Hartree potential in the leads over a plane perpendicular to the transport direction. This parameter should be evaluated from the bulk leads calculation.

The Fast Fourier Transform algorithm used in SMEAGOL to solve the Poisson's equation disposes the $\vec{k}=0$ term. This defines the Hartree potential up to an arbitrary constant. By defining HartreeLeadsBottom we shift the Hartree potential in the scattering region in order to match that of the leads.

Default value: $0.0 \mathrm{eV}$ (no shift)

\section{E.4.2.2 Flags specific of $k$-point calculations}

These flags are used only when periodic boundary conditions in the direction orthogonal to the transport are considered.

\section{SaveMemTranspK (Boolean):}

By default, SMEAGOL calculates the self-energies during the first iteration and stores them in memory. There are two self-energies (one for the left- and one for right-hand side contact) for each energy point and each $k$-point. If the number of $k$-points and the size of the unit cell of the leads are large, large memory might be needed. In that case, SaveMemTranspK should be set to T, and the self-energies will be re-calculated after each iteration without the need for storing them in memory. Use with caution; this specification might increase considerably the time of the calculation.

Default value: $\mathrm{F}$

\section{TransmissionOverk (Boolean):}

Allow to print the transmission coefficient as a function of both the k-vector in the transverse Brillouin zone and the energy. Note that the flag TrCoefficients provide only the integral of the transmission coefficients over the $k$-points. TrCoefficients must be set to true. The file SystemLabel.TRC.k.up (down) contains the results (see Section E.4.3 below).

Default value: $\mathrm{F}$ 


\section{E.4.2.3 Flags specific of $\Gamma$ point calculations}

\section{UseLeadsGF (Boolean):}

If true the file SystemLabel.LGF is read from disk (see description of output files below). It is particularly useful when one wants to perform numerous calculations with the same set of leads and the same number of real energy points.

Default value: $\mathrm{T}$

\section{UseLeadsGF (Boolean):}

If true the file SystemLabel.LGF is read from disk (see description of output files below). It is particularly useful when one wants to perform numerous calculations with the same set of leads and the same number of real energy points.

Default value: $\mathrm{T}$

\section{E.4.2.4 Flags specific of calculations involving periodic leads}

\section{PeriodicLead (Boolean):}

If true I-SMEAGOL will perform a periodic leads calculation. This requires files SystemLabelSmall.HSL, SystemLabelSmall.xij, bulklftsmall.DAT and bulkrgtsmall.DAT should be present.

Default value: $\mathrm{F}$

\section{LatticeConstantLeads (Physical):}

Lattice constant parameter used at the lead unit cell calculation.

Default value: 1.0 Bohr

LeadsLatticeVectors (data block):

Contains the 3 lattice vectors of the unit cell of the small electrodes.

Default value: No Default

\section{LeadsSuperCell (data block):}

The number of repetitions for the first and cell leads lattice vectors. The values in the block should be integer numbers.

Default value: No Default

It is important to note that each cell vector multiplied by the corresponding LeadsSuperCell factor should be equal to the LatticeVector defined in the input.fdf file. 


\section{E.4.2.5 Example for periodic lead calculation}

Considering the example used to setup the electrodes in Section E.3.4.3, in the input.fdf file, one should use the following flags:

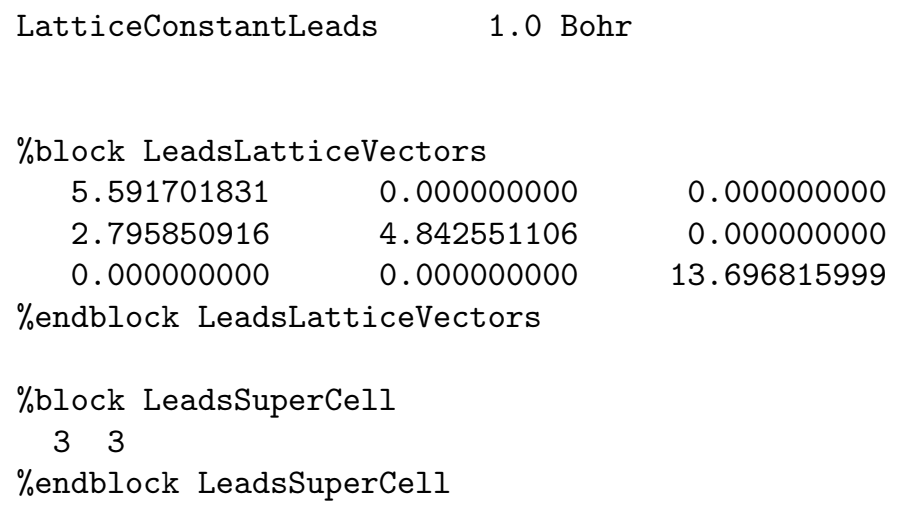

We note that the LeadsLatticeVectors correspond to the lattice vectors used for the small electrodes calculations, and the LeadsSuperCell is given by:

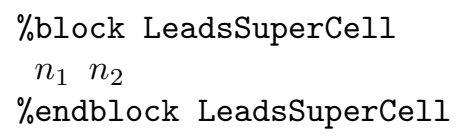

\section{E.4.3 Output files}

\section{SystemLabel.CUR :}

Four column vector containing the $I-V$ characteristics. The first column corresponds to the bias in $\mathrm{eV}$, the second column corresponds to the total current and the third and fourth columns (in the case of a spin-dependent calculation) correspond to the spin-decomposed current.

\section{SystemLabel.TRC :}

Transmission coefficients as a function of energy for different biases. The first column corresponds to the energy, the second to the total transmission for both the spin direction and the following nspin columns are the spin-decomposed transmission coefficients.

\section{SystemLabel.TRC.k.up (down) :}

If TransmissionOverk is set to T, SystemLabel.TRC.k.up (down) contains the transmission coefficients for each $k$-point and energy for up (down) spin. The first two columns contain the perpendicular components of each $k$-point, the third column contains the spin dependent transmission coefficient and the fourth column contains the energy. 


\section{SystemLabel.LGF :}

For a $\Gamma$ point serial calculation, this file contains the NEnergReal self-energies for both the right and left hand-side leads for all the energies lying on the real axis. This file might be useful when restarting a calculation. The user should note that, in this case, both VInitial, VFinal and NEnergReal must be the same as in a previous calculation.

\section{SystemLabel.CHR :}

File containing the total charge inside the scattering region after each iteration. The first column contains the iteration number, the second column contains the charge in units of $e$ (the electron charge), the third column contains the bias in Rydberg and the fourth column contains the temperature also in Rydberg.

\section{IV.SystemLabel.VH :}

If SaveElectrostaticPotential is set to true (see SIESTA user's guide) and the block SaveBiasSteps is present, the file IV.SystemLabel.VH containing the Hartree potential for the biases IV specified in SaveBiasSteps will be generated.

\section{IV.SystemLabel.VT :}

If WriteVT is set to true (see SIESTA user's guide) and the block SaveBiasSteps is present, the file IV.SystemLabel.VT containing the total DFT potential for the biases IV specified in SaveBiasSteps will be generated.

\section{IV.SystemLabel.RHO :}

If WriteRHO is set to true (see SIESTA user's guide) and the block SaveBiasSteps is present, the file IV. SystemLabel. RHO containing the self-consistent charge density for the biases IV specified in SaveBiasSteps will be generated.

\section{IV.SystemLabel.DRHO :}

If WriteDRHO is set to true (see SIESTA user's guide) and the block SaveBiasSteps is present, the file IV. SystemLabel. DRHO containing the difference between the self-consistent and the atomic charge densities for the biases IV specified in SaveBiasSteps will be generated.

\section{IV.SystemLabel.DMT :}

If SaveDMT is set to true and the block SaveBiasSteps is present, the file IV. SystemLabel.DMT containing the converged density matrix at each bias IV specified in SaveBiasSteps will be written. 


\section{E.5 INELASTIC $I-V$ CALCULATION}

In the I-SMEAGOL code, weak electron-phonon interactions can be considered by the so-called first Born approximation (1BA) and self-consistent Born approximation (SCBA) [6]. Their interaction self-energies are represented in diagrammatic form in Figure E.7.
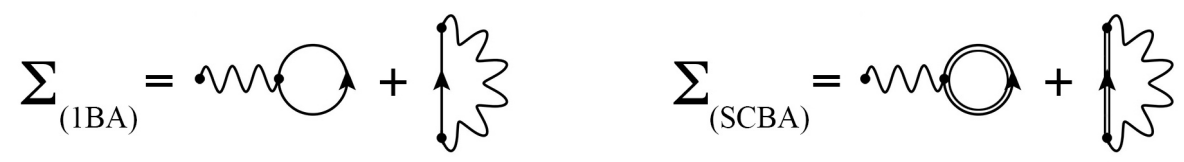

Figure E.7: Diagrammatic representation of the first Born approximation (left) and the self-consistent Born approximation (right).

In the first Born approximation one considers only the first order terms of the electronic Green's function expansion. The two first order diagrams can be identified, from left to right, as the Hartree and the Fock diagrams. In the self-consistent Born approximation, the interaction self-energy is calculated with the perturbed electronic Green's function in a self-consistent way.

\section{E.5.1 Input files}

In addition to the files required for usual $I-V$ calculation (Section E.4.1), another file containing the vibrational analysis (electron-phonon couplings and vibrational frequencies) must be supplied:

\section{FileEPh :}

The first line contains integer numbers separated by white spaces corresponding to the following information: the number of spin components, the number of dynamic atoms (those that were displaced on force constant calculation for the vibrational analysis), the number of dynamic orbitals, the first dynamic orbital index and the last dynamic orbital index. The next line contains the vibrational frequencies (in $\mathrm{eV}$ ) separated by white spaces (if it was considered $n$ atoms in the vibrational analysis, then there should have $3 n$ frequencies). The following lines contains the electron-phonon coupling matrices (in $\mathrm{eV}$ ) in the same order as the frequencies (for each frequency there must have one electron-phonon coupling matrix). 


\section{E.5.2 Input flags}

In addition to the input flags required for usual $I-V$ calculation (Section E.4.1), the following input flags must be supplied to the file input.fdf in order to perform inelastic transport $(I-V)$ calculations.

\section{Inelastic (Boolean):}

If true I-SMEAGOL will perform NEGF transport calculation with inelastic scattering given by the first Born approximation or/and the self-consistent Born approximation. Otherwise, the code will perform the usual elastic transport calculation.

Default value: $\mathrm{F}$

ElectronPhononMatrix (String):

Name of the file containing the electron-phonon coupling matrices and the vibrational mode frequencies as described in Section E.5.1.

Default value: No Default

SCBA (Boolean):

If true the code will compute the self-consistent Born approximation. If false I-SMEAGOL will calculate only the first Born approximation.

Default value: $\mathrm{F}$

SCBAconv (Double precision):

Self-consistent convergence criteria. The self-consistency is achieved when the difference $\left|\Sigma_{S C B A}^{n}-\Sigma_{S C B A}^{n-1}\right|$, between the new self-energy and the one calculated on the last cycle, is lower than SCBAconv.

Default value: $10^{-4}$

SCBAmix (Double precision):

It is a real number in $[0,1]$ which defines the self-energies mixing factor for the selfconsistent procedure. At each cycle, the previous self-energy is redefined as $\Sigma_{S C B A}^{n-1}=$ $(1-S C B A m i x) * \Sigma_{S C B A}^{n-1}+S C B A m i x * \Sigma_{S C B A}^{n}$. A lower value for SCBAmix is better to ensure the convergence, but also reduces the rate of convergence.

Default value: $10^{-1}$

MaxSCBAIterations (Integer): 
Maximum number of self-consistent iterations. If the self-consistency was not achieved after MaxSCBAIterations cycles, the code will stop the self-consistent procedure and will consider the self-energy from the last cycle as a converged one.

Default value: 500

\section{Hartree2 (Boolean):}

If true the code will use calculated density matrix to compute the Hartree diagram contribution. Otherwise it computes the Hartree term independently.

Default value: $\mathrm{F}$

\section{NEnergInel (Integer):}

Number or energy points along the real axis used for computing some integrals at inelastic transport (the current and the non-equilibrium part of the Hartree diagram). If SCBA is false (i.e. only the first Born approximation is considered) the points are distributed between $-\left(V / 2+30.0 k_{B} T+2 \Omega_{\max }\right)$ and $\left(V / 2+30.0 k_{B} T+2 \Omega_{\max }\right)$, where $\Omega_{\max }$ is the highest vibrational frequency. If SCBA is true the points are distributed between $-\left(E B+2 \Omega_{\max }\right)$ and $\left(V / 2+30.0 k_{B} T+2 \Omega_{\max }\right)$, where $E B$ is the EnergLowestBound (see Section E.4.2). In the last case a much large number of points is needed.

Default value: 1000

\section{iNEnergImCircle (Integer):}

As in elastic transport, where the equilibrium part of the Green's function integral is calculated in a contour in the complex plane, in inelastic transport one need to perform the same kind of integrals for computing the equilibrium part of the Hartree diagram and for computing some terms of the Fock diagram. Therefore, the flag iNEnergImCircle indicates the number of energy points along the semi-circle in the complex plane (see Figure E.5 (b) for a sketch of the points along the contour in the complex energy plane, where the points on the semi-circle are represented by blue points).

Default value: 34

iNEnergImLine (Integer):

Number of energy points along the line in the complex plane contour used for computing integrals (see Figure E.5 (b) for a sketch of the points along the contour in the complex energy plane, where the points on the line part are represented by green points).

Default value: 20 
iNPoles (Integer):

Number of poles in the Fermi distribution used for computing integrals on the complex plane. It specifies how far from the real axis the contour integral will be performed (see Figure E.5 (b) for a sketch of the points along the contour in the complex energy plane, where the poles inside the contour are represented by grey diamonds).

Default value: 5

fullDOS (Boolean):

If true the code will compute the total density of states (DOS) with the interacting Green's function. Regardless of this option the code calculates the local density of states of the dynamic atoms.

Default value: $\mathrm{F}$

\section{E.5.3 Output files}

The following files contain the $I-V$ characteristics:

SystemLabel.I0CUR : File containing the elastic $I$ - $V$ characteristics.

SystemLabel.I1BA0CUR : File containing the "elastic" $I$ - $V$ characteristics of the first Born approximation (i.e. the elastic current computed with the interacting 1BA Green's function)

SystemLabel.I1BACUR : File containing the total (elastic and inelastic) $I$ - $V$ characteristics of the first Born approximation.

SystemLabel.ISCBA0CUR : File containing the "elastic" $I-V$ characteristics of the selfconsistent Born approximation (i.e. the elastic current computed with the interacting SCBA Green's function).

SystemLabel.ISCBACUR : File containing the total (elastic and inelastic) $I$ - $V$ characteristics of the self-consistent Born approximation.

In case of spin polarized calculation the $*$ CUR files above contain four columns, where the first corresponds to the bias in $\mathrm{eV}$, the second column corresponds to the total current and the third and fourth columns (in the case of a spin-dependent calculation) correspond to the spindecomposed current. In case of non-polarized spin calculation these files contain only the first two columns. 
The following files contain the local density of states (LDOS) of dynamic atoms computed at bias IV:

SystemLabel_IV.IA0 : Local density of states of dynamic atoms computed at bias IV with non-interacting Green's functions.

SystemLabel_IV.IA1BA : Local density of states of dynamic atoms computed at bias IV with 1BA Green's functions.

SystemLabel_IV.IASCBA : Local density of states of dynamic atoms computed at bias IV with SCBA Green's functions.

In case of spin polarized calculation the $*$ IV. IA $*$ files contain three columns, where the first corresponds to the energy (subtracted by the Fermi energy) and the second and third columns corresponds to the LDOS for up and down spins, respectively. In case of non-polarized spin calculation these files contain only a second column containing the LDOS.

If fullDOS is set to $\mathrm{T}$, the following files contain the total density of states (DOS) computed at bias IV:

SystemLabel_IVf.IA0 : If fuIIDOS is set to T, this file contains the total density of states computed at bias IV with non-interacting Green's functions.

SystemLabel_IVf.IA1BA : If fullD0S is set to T, this file contains the total density of states computed at bias IV with 1BA Green's functions.

SystemLabel_IVf.IASCBA : If fullDOS is set to T, this file contains the total density of states computed at bias IV with SCBA Green's functions.

In case of spin polarized calculation the $* \operatorname{IVf}$. IA $*$ files contain three columns, where the first corresponds to the energy (subtracted by the Fermi energy) and the second and third columns corresponds to the DOS for up and down spins, respectively. In case of non-polarized spin calculation these files contain only a second column containing the DOS.

If fullDOS is set to $\mathrm{T}$, the following files contain the projected density of states computed at bias IV:

SystemLabel_IVp.IA0 : If fullDOS is set to T, this file contains the projected density of states computed at bias IV with non-interacting Green's functions.

SystemLabel_IVp.IA1BA : If fullDOS is set to T, this file contains the projected density of states computed at bias IV with 1BA Green's functions. 
SystemLabel_IVp.IASCBA : If fullDOS is set to T, this file contains the projected density of states computed at bias IV with SCBA Green's functions.

The *IVp. IA* files are structured using spacing and xml tags, and can be processed by the program in Util/pdosxml.

\section{E.6 MOLECULAR DYNAMICS WITH TRANSPORT}

In the I-SMEAGOL code one can perform molecular dynamics (MD) and structural optimizations, as implemented in SIESTA, in non-equilibrium situation together with transport calculations. I-SMEAGOL has an outer bias loop, where the applied bias is changed according to the options described in Section E.4, and an inner geometry loop, where the electronic structure, forces and stress are computed for a given geometry. Subsequently the atomic positions are updated according to the MD or optimization option (refer to the SIESTA manual to check the implemented options for MD and structural optimizations).

For all different MD options allowed by SIESTA, the key point are the forces and stress computations, which in most cases determines the next geometry step. Therefore, in the same way that we need to correct the density matrix in usual transport calculations, in order to include the effects of the applied bias and the leads coupling on MD, one should also correct the energy density, which is used for calculating the forces. The implementation of the non-equilibrium forces follows the methodology described in [16].

\section{E.6.1 Input files}

There are no additional input files besides those used for the $I-V$ calculation (Section E.4.1).

\section{E.6.2 Input flags}

The following input flags must be supplied to the file input.fdf in order to perform MD together with transport $(I-V)$ calculations.

\section{CalculateForces (Boolean):}

If true I-SMEAGOL will calculate the non-equilibrium energy density. Since this specification increases the time of the calculation, it should be used only when the force calculation is relevant (e.g. in MD and structural optimizations) and should be unset in ordinary transport calculations.

Default value: $\mathrm{F}$ 
SaveMDSteps (data block):

Block containing information for printing the Hartree potential (IV_MD.SystemLabel.VH), the charge density (IV_MD.SystemLabel.RHO), the converged density matrix (IV_MD.SystemLabel.DMT), the difference between the self-consistent and the atomic charge densities (IV_MD.SystemLabel.DRHO) and the total DFT potential (IV_MD.SystemLabel.VT) at a specified bias step and MD step. The bias steps are those specified at SaveBiasSteps block (Section E.4.2). The arguments in SaveMDSteps block are integers indicating the MD steps in which these informations should be printed. Their values must be consistent with the chosen MD. Type0fRun (refer to the SIESTA manual for the possible options). The appropriate SIESTA flag for printing any of these files must also be set to true.

Example:

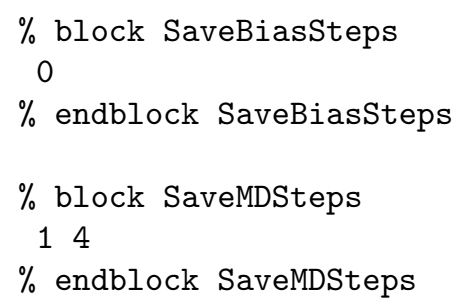

Then, for instance if the SIESTA flag SaveElectrostaticPotential is set to true, ISMEAGOL will print the files 0_1. SystemLabel.VH and 0_4. SystemLabel.VH corresponding to the Hartree potential calculated at bias 0 and MD steps 1 and 4, respectively.

Default value: No Default

\section{E.6.3 Output files}

The output files are the same as those obtained in $I-V$ calculations Section E.4.3. The only difference corresponds to the files prefixed by the IV bias step specified in SaveBiasSteps. In MD they are prefixed by the IV bias step specified in SaveBiasSteps and by the MD step specified in SaveMDSteps as: IV_MD.SystemLabel.

\section{E.7 HOW TO RUN SMEAGOL}

Assuming that the user has already compiled SMEAGOL successfully and saved it as an executable (say i-smeagol.2.0), then we are ready to start a calculation. Initially the user must setup the input file for the left- and right-hand side leads. The input file must include the appropriate SIESTA and SMEAGOL flags as discussed in Section E.3.2 and in the SIESTA Manual. 
Let us assume, as a simple example, that the two leads are equivalent and the leads file is called leads.fdf. In the same directory where this file is, there must also be the pseudopotential files - either in .psf or . vps format. Then the user should type:

$\$$ i-smeagol.2.0 < leads.fdf > leads.out

The calculation will yield the files bulklft.DAT, bulkrgt.DAT, SystemLabel.HSL and SystemLabel.DM. These should be moved to the directory containing the input file for the scattering region - say scattering.fdf. This directory must also contain pseudopotential files for all the atomic species involved in the calculation. It is important to note that the user must be consistent with regards to pseudopotentials, they should be identical to the ones used for the leads calculation.

Before starting the $I-V$ calculation we must obtain HartreeLeadsBottom. For that we use the post-processing tool Pot.exe contained in the Utils directory of the SMEAGOL distribution. The file Potential.dat should be edited in order to process the file SystemLabel.vH (for the leads). The resulting output SystemLabel-VH_AV.dat can be viewed using a plotting tool such as gnuplot or xmgrace.

We are interested in the first two columns of this data file. They contain the planar average of the Hartree potential as a function of the $z$ direction. A value for the Hartree potential (HartreeLeadsBottom) should be chosen (usually the edges of the unit cell) and the position in the unit cell should be recorded and matched with the equivalent position in the scattering region (HartreeLeadsLeft and HartreeLeadsRight).

In case of periodic lead calculation, another bulk calculation should be performed considering only the lead's unit cell:

$\$$ i-smeagol.2.0 < leadSmall.fdf > leadSmall.out

The calculation will yield the files bulklft.DAT, bulkrgt.DAT, SystemLabelSmall. HSL and SystemLabelSmall.xij. Since the files bulklft.DAT and bulkrgt.DAT have the same name as those from the previous bulk calculation, one should rename them as bulklftsmall.DAT and bulkrgtsmall. DAT, respectively. After that all four files should be moved to the directory where the transport calculations will be performed.

Once this is done, we are ready to calculate the $I-V$ characteristics:

\$i-smeagol.2.0 < scattering.fdf > scattering.out

The resulting files can be processed using a variety of plotting programs such as xmgrace for 2D-plots and OpenDX for 3D-plots and isosurfaces or gnuplot for either cases. 


\section{E.8 PROBLEM HANDLING}

The users are encouraged to report problems and bugs to the I-SMEAGOL's developers at reilya@ift.unesp.br. Patches and fixes will be uploaded to the web-site https://bitbucket. org/brandimarte/smeagol-2.0/wiki/Home.

\section{E.9 ACKNOWLEDGEMENTS}

This work is sponsored by Science Foundation of Ireland under the grant SFI02/IN1/I175. JFR and VMGS thank the spanish Ministerio de Educacíon y Ciencia for financial support (grants BFM2003-03156 and AP2000-4454). SB and CJL thank the UK EPSRC and the EU network MRTN-CT-2003-504574 RTNNANO. ARR thanks Enterprise Ireland (grant EI-SC/2002/10) for financial support. Traveling has been sponsored by the Royal Irish Academy under the International Exchanges Grant scheme. PBM thanks CNPq (Conselho Nacional de Desenvolvimento Científico e Tecnológico) for financial support (grant 142792/2010-1).

\section{References}

[1] A. R. Rocha, V. M. García-Suárez, S. W. Bailey, C. J. Lambert, J. Ferrer and S. Sanvito, Nature Materials 4, 335-339 (2005).

[2] A. R. Rocha, V. M. García-Suárez, S. W. Bailey, C. J. Lambert, J. Ferrer and S. Sanvito, Phys. Rev. B 73, 085414 (2006).

[3] J. M. Soler, E. Artacho, J. D. Gale, A. García, J. Junquera, P. Ordejón and D. SánchezPortal, J. Phys. Cond. Mat. 14, 2745-2779 (2002).

[4] http://departments.icmab.es/leem/siesta/

[5] S. Datta, Electronic Transport in Mesoscopic Systems, Cambridge University Press, UK (1995).

[6] H. Haug and A.-P. Jauho, Quantum Kinetics in Transport and Optics of Semiconductors, Springer, Berlin (1994).

[7] C. Caroli, R. Combescot, P. Nozieres and D. Saint-James, J. Phys. C: Solid State Phys. 5, $21(1972)$.

[8] J. Ferrer, A. Martín-Rodero and F. Flores, Phys. Rev. B 38, 10113 (1988).

[9] J. Taylor, H. Guo and J. Wang, Phys. Rev. B 63, 245407 (2001). 
[10] M. Brandbyge, J.-L. Mozos, P. Ordejón, J. Taylor and K. Stokbro, Phys. Rev. B 65, 165401 (2002).

[11] J. J. Palacios, A. J. Pérez-Jiménez, E. Louis, E. SanFabián, and J. A. Vergés, Phys. Rev. B 66, $035322(2002)$.

[12] A. Pecchia and A. Di Carlo, Rep. Prog. Phys. 67, 1497 (2004).

[13] P. Hohenberg and W. Kohn, Phys. Rev. 136(3B), B864-B871 (1964).

[14] W. Kohn and L. J. Sham, Phys. Rev. 140(4A), A1133-A1138 (1965).

[15] S. Sanvito, C. J. Lambert, J. H. Jefferson, and A. M. Bratkovsky, Phys. Rev. B 59, 11936 (1999).

[16] R. Zhang, I. Rungger, S. Sanvito and S. Hou, Phys. Rev. B 84, 085445 (2011). 


\section{Apêndice F}

\section{I-DISORDER User's Guide}

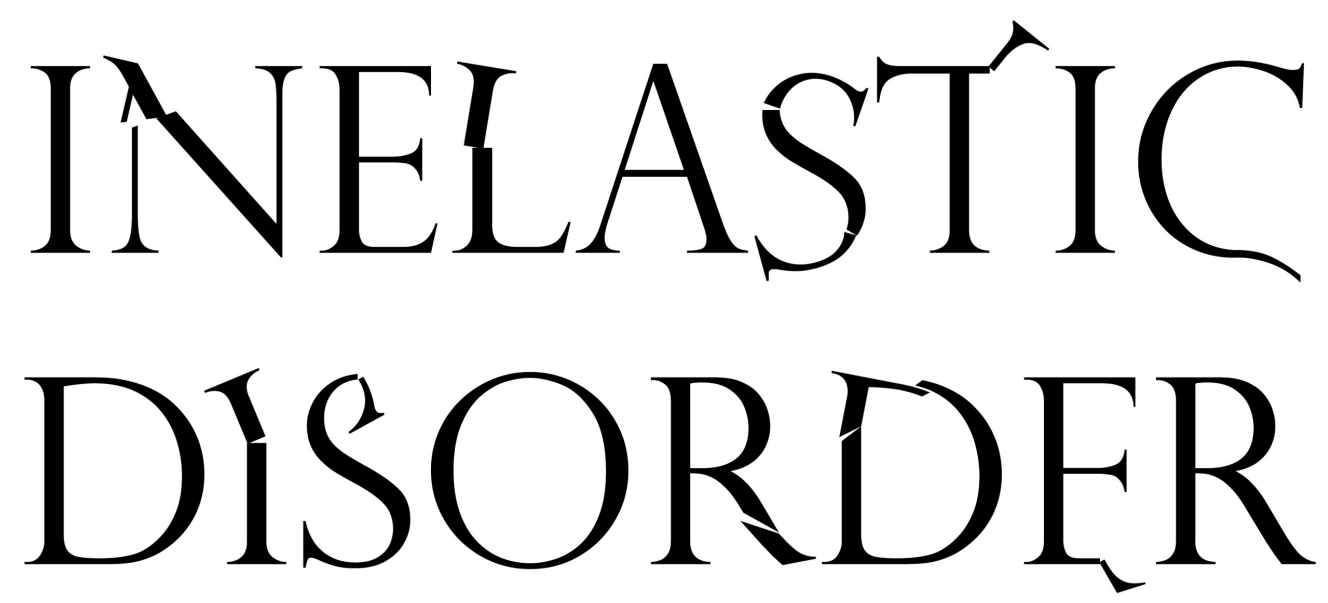

\section{INELASTIC NON-EQUILIBRIUM ELECTRONIC TRANSPORT IN DISORDERED SYSTEMS}

July 21, 2014

Alexandre Reily Rocha

Instituto de Física Teórica, UNESP, Brazil

Pedro Brandimarte Mendonça

Instituto de Física, USP, Brazil

Alberto Torres Riera Junior

Departamento de Física, UFSC, Brazil

brandimarte@gmail.com 


\section{Contents}

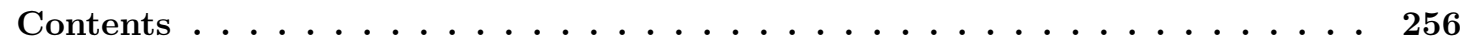

F.1 Introduction . . . . . . . . . . . . . . . . 256

F.2 Compilation . . . . . . . . . . . . . . . . . 256

F.3 The System Set-Up $\ldots \ldots \ldots \ldots \ldots \ldots \ldots \ldots \ldots$

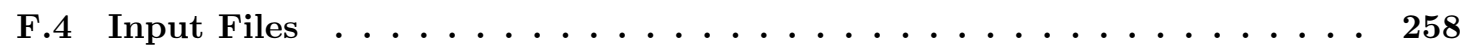

F.5 Input Flags $\ldots \ldots \ldots \ldots \ldots \ldots \ldots \ldots \ldots \ldots \ldots \ldots \ldots$

F.6 Output Files . . . . . . . . . . . . . . . . . . 261

F.7 Running I-DISORDER $\ldots \ldots \ldots \ldots \ldots \ldots \ldots \ldots \ldots \ldots$

F.8 Problem Handling . . . . . . . . . . . . . . . . . . . 263

F.9 Acknowledgements . . . . . . . . . . . . . . . . . . 263

References .............................. 264

\section{F.1 Introduction}

The Inelastic DISORDER code was developed for computing, from first principles methods, the electronic transport properties of 1-dimensional devices with a large number of randomly distributed defects considering the inelastic electron-phonon scattering. It combines electronic structure calculations via density functional theory (DFT) with the non-equilibrium Green's function formalism (NEGF) for the transport.

The electron-phonon interaction is considered to be weak and localized in the defects. Under these circumstances, the current and power expressions can be expanded to second order in the electron-phonon couplings to obtain the so-called Lowest Order Expansion [1,2]. Approximating the contact broadening and the non-interacting retarded Green's functions as energy independent matrices (Wide-Band Limit), the current and power expressions take a simplified form where the integration over energy are calculated analytically.

I-DISORDER uses the Hamiltonian and overlap matrices from DFT calculations performed by the SIESTA code [3, 4], the leads calculations from SMEAGOL code [5] and the electronphonon coupling from POSITIVE VIBRATIONS code [7]. For computing the non-equilibrium Green's function I-DISORDER uses a recursive method [8, 9].

\section{F.2 Compilation}

The compilation of the program is done using a Makefile which, in turn, uses a arch.make file provided by the user, containing the required informations for compiling the code. Several 
examples of arch.make files for different machine architectures are provided at $0 \mathrm{bj} / \mathrm{archmake}$ folder.

Basically, in the arch.make file the user should set the $C$ and Fortran compilers and its options, and should provide the path for BLAS [10] and LAPACK [11] libraries. For parallel computing with Message Passing Interface MPI [12], the user should choose a MPI Fortran compiler and provide the path for MPI libraries. If one wants to use CUDA-enable graphics processing units (GPU) for a GPU-accelerated computing, one should also provide the path for MAGMA [11], CUDA [14] and CUBLAS [15] libraries.

The code compilation takes place at Obj directory, which will hold the resulting object files, modules files and libraries. In order to prepare the building of the code, one should go to $\mathrm{Obj}$ directory and run a script as follows:

$\$$ sh $\ldots /$ Src/obj_setup.sh

Once the set-up is done, to proceed with the compilation just type:

\$ make

This will create an executable file, as defined at arch.make file with the EXEC variable.

\section{F.3 The System Set-Up}

A typical disordered system simulated by I-DISORDER is presented in Figure F.1. It comprises two semi-infinite leads (left and right) and an scattering region with randomly distributed defects.

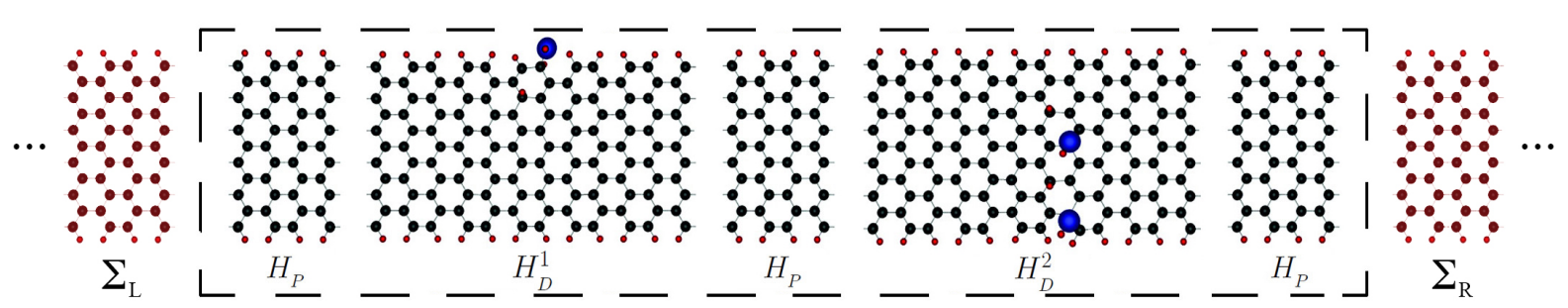

Figure F.1: Representation of a typical disordered system using a graphene nanoribbon as an example. One identifies a scattering region with three pristine segments $\left(H_{P}\right)$ and two with defects $\left(H_{D}^{1}\right.$ and $\left.H_{D}^{2}\right)$. The left and right leads, made by semi-infinite pristine nanoribbons, are coupled to the first and last segments, respectively. 
Before running I-DISORDER there are two preliminary steps: 1. the electronic structure calculating of each type of block that composes the system; 2. the vibrational calculation of each block with electron-phonon interaction.

The first step is carried out with SMEAGOL code with a lead-type computation (refer to SMEAGOL User's Guide to see how to perform this calculation [6]). For each type of block, an electronic structure calculation is performed with the segment periodically repeated in the transport direction. The resulting Hamiltonian and overlap matrices from each unit cell are stored, as well as their respective couplings.

One must take a special care when building the blocks containing defects. They must have at least one pristine segment at each end to ensure that the coupling between two blocks is always equal to the coupling between pristine blocks.

The second step is to compute the vibrational modes and frequencies and the electron-phonon coupling matrices of each block with inelastic scattering. This is carried out with POSITIVE VIBRATIONS code (refer to POSITIVE VIBRATIONS User's Guide to see how to perform this calculation $[7])$.

\section{F.4 Input Files}

From a lead-type computation with SMEAGOL code, the following files are generated: BlockLabel.HSL, bulklft.DAT and bulkrgt.DAT. For the blocks that will constitute the leads one should copy all these files to the directory where the calculation with I-DISORDER will be performed and duplicate the file bulklft.DAT as LeadLabel.DAT. For the other blocks one should copy the file BlockLabel.HSL and copy the file bulklft.DAT as BlockLabel.DAT.

From the vibrational calculation with POSITIVE VIBRATIONS code, one should copy only the file BlockLabel.Meph, which contains all calculated frequencies and electron-phonon coupling matrices.

\section{F.5 Input Flags}

The following input flags must be supplied to the input file (say input.in) in order to perform the transport calculation. If any of these values is not supplied, the default value will be assumed.

SystemLabel (String):

A single word (maximum of 60 characters without blanks) containing a nickname of the system, used to name output files.

Default value: i-disorder 
NumberUnits (Integer):

Total number of blocks in the scattering region (in the example of Figure F.1 the NumberUnits is 5).

Default value: 1

NumberUnitTypes (Integer):

Number of different types of blocks in the scattering region (in Figure F.1 the NumberUnitTypes is 3 , since there are two different types of defects and the pristine block).

Default value: 2

\section{NumberDefects (Integer):}

Number of defects in the entire system (in Figure F.1 the NumberDefects is 2). Note that this number should be $\leq$ NumberUnits.

Default value: 1

\section{UnitsFiles (data block):}

Block containing information about all different types of blocks. It contains NumberUnitTypes +2 rows and 3 columns. The last 2 rows correspond to the left and right leads (in this order) and the antepenultimate (third last) correspond to the pristine block. The first NumberUnitTypes rows correspond to the different types of blocks in the scattering region. The first column consists of real numbers which indicates the probability that the system contains each kind of block (the last three, from the leads and pristine block, are ignored). Note that the sum of the elements of the first column must be equal to 1 . The second column contains an integer 0 or 1 indicating if there will be (1) or not (0) electron-phonon interaction in the block. The last column are strings the with block labels, which are used to find the files BlockLabel.HSL and BlockLabel.DAT.

Example:

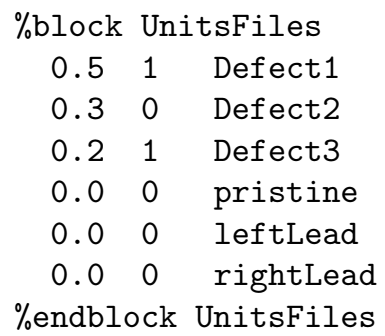

In this example, the NumberUnitTypes is 4 and there are 3 types of defects, with the first one with a higher probability of being present in the system. The electron-phonon 
interaction will be considered only on first and third defects blocks and the program will search for the files Defect1. Meph and Defect3.Meph.

Default value: No Default

SpinPolarized (Boolean):

If SpinPolarized is set to $\mathrm{T}$ it will be considered 2 spin components when reading the input files.

Default value: $\mathrm{F}$

ElectronicTemperature (Physical):

Temperature for Fermi-Dirac distribution.

Default value: $300.0 \mathrm{~K}$

VInitial (Physical):

Value of the initial bias.

Default value: $0.0 \mathrm{eV}$

VFinal (Physical):

Value of the final bias.

Default value: $0.1 \mathrm{eV}$

NIVPoints (Integer):

Number of bias steps considered between the two limits VInitial and VFinal. The current and the dissipated power will be calculated only for these biases.

Default value: 10

TransmInitial (Physical):

Value of the initial energy.

Default value: $-1.0 \mathrm{eV}$

TransmFinal (Physical):

Value of the final energy.

Default value: $1.0 \mathrm{eV}$ 
NumberTransmPoints (Integer):

Number of energy points uniformly distributed between TransmInitial and TransmFinal for which the current and the dissipated power are calculated.

Default value: 100

\section{AsymmGridPts (Integer):}

Number of energy grid points for computing the integral from asymmetric term of the inelastic current in LOE approximation.

Default value: 1000

\section{PhononEquilibrium (Boolean):}

If PhononEquilibrium is set to $\mathrm{T}$ the phonons will be considered in thermodynamic equilibrium and will be described by the Bose-Einstein distribution. Otherwise an non-equilibrium heating of the phonon system will be considered.

Default value: $\mathrm{T}$

\section{PhononDamping (Physical):}

External damping rate. It is a phenomenological parameter related to the inverse of phonon's lifetime and is used when considering non-equilibrium heating of the phonon system. If PhononEquilibrium is set to $\mathrm{T}$, this flag is ignored.

Default value: $0.05 \mathrm{eV}$

\section{GPU.ProcsPerGPU (Integer):}

Number of processes running in each GPU (in case I-DISORDER has been compiled with CUDA-enable graphics processing units for GPU-accelerated computing).

Default value: 1

\section{F.6 Output Files}

\section{SystemLabel ExVxI.CUR :}

File with six columns containing the computed currents. The first column corresponds to the energy in $\mathrm{eV}$ (subtracted by the Fermi energy) and the second column corresponds to the bias in $\mathrm{V}$. The next columns are the computed current in ampere A with the following order: elastic current, symmetric term of inelastic current, asymmetric term of inelastic current and the total current. 


\section{SystemLabel_ExVxdI.dIdV :}

File with six columns containing the computed differential conductances $d I / d V$. The first column corresponds to the energy in $\mathrm{eV}$ (subtracted by the Fermi energy) and the second column corresponds to the bias in V. The next columns are the computed $d I / d V$ in quantum conductance units $\left(G_{0}\right)$ with the following order: elastic $d I / d V$, symmetric term of inelastic $d I / d V$, asymmetric term of inelastic $d I / d V$ and the total $d I / d V$.

\section{SystemLabel_ExVxd2I.d2IdV2 :}

File with six columns containing the computed derivatives $d^{2} I / d V^{2}$. The first column corresponds to the energy in eV (subtracted by the Fermi energy) and the second column corresponds to the bias in $\mathrm{V}$. The next columns are the computed $d^{2} I / d V^{2}$ in quantum conductance per volt $\left(G_{0} \mathrm{~V}^{-1}\right)$ with the following order: elastic $d^{2} I / d V^{2}$, symmetric term of inelastic $d^{2} I / d V^{2}$, asymmetric term of inelastic $d^{2} I / d V^{2}$ and the total $d^{2} I / d V^{2}$.

\section{SystemLabel_ExVxP_nU.PWR :}

File containing the computed dissipated power by the block nU with electron-phonon interaction. The first column corresponds to the energy in eV (subtracted by the Fermi energy) and the second column corresponds to the bias in V. The following columns corresponds to the dissipated power in $\mathrm{eV}$ by each vibrational mode. The last column corresponds to the total dissipated power in $\mathrm{eV}$ (all modes).

\section{SystemLabel_nU.SPCTR :}

File containing the computed spectral function of the block nU with electron-phonon interaction. The file indexed with $\mathrm{nU}+1$ (the number of the blocks with electron-phonon interaction plus 1) corresponds to the spectral function of all blocks with electron-phonon interaction. The first column corresponds to the energy in eV (subtracted by the Fermi energy) and the second column corresponds to the spectral function in $(\mathrm{eV})^{-1}$.

\section{SystemLabel nU.DOS :}

File containing the computed local density of states (LDOS) of the block nU with electronphonon interaction. The file indexed with $\mathrm{nU}+1$ (the number of the blocks with electronphonon interaction plus 1) corresponds to the sum of the LDOS all blocks with electronphonon interaction. The first column corresponds to the energy in $\mathrm{eV}$ (subtracted by the Fermi energy) and the second column corresponds to the LDOS in $(\mathrm{eV})^{-1}$. 


\section{F.7 Running I-DISORDER}

Assuming that the user has already compiled I-DISORDER successfully and saved it as an executable (say i-disorder) and assuming that the user has already performed the two preliminary steps described in Section F.3, then we are ready to start a calculation.

The input files must be copied to the directory where the calculation will be performed, as described in Section F.4 and an file (say input.in) containing the input flags described in Section F.5 must also be present.

Then the user can run I-DISORDER by typing:

\$ i-disorder < input.in > output.out

In case of parallel computing with \$PROCS processes, the user can type:

\$ mpirun -np \$PROCS i-disorder < input.in > output.out

The I-DISORDER code will generate a system with a randomly distributed defects accordingly to the user's options in input.in. The resulting output files can be processed using a variety of plotting programs such as xmgrace for 2D-plots and OpenDX for 3D-plots and isosurfaces or gnuplot for either cases. Some scripts for plotting the results with gnuplot can be find at Scripts/plot directory.

Usually, one is interested in running I-DISORDER code a large number of times and then compute the average values. For that, the user will find some useful scripts at Scripts/analysis and Scripts/submit directories.

\section{F.8 Problem Handling}

The users are encouraged to report problems and bugs to the I-DISORDER's developers at brandimarte@gmail.com. Patches and fixes will be uploaded to the web-sitehttps://bitbucket. org/brandimarte/i-disorder/wiki/Home

\section{F.9 Acknowledgements}

PBM thanks CNPq (Conselho Nacional de Desenvolvimento Científico e Tecnológico) for financial support (grant 142792/2010-1) 


\section{References}

[1] J. K. Viljas, J. C. Cuevas, F. Pauly and M. Häfner, Phys. Rev. B 72, 245415 (2005).

[2] M. Paulsson, T. Frederiksen and M. Brandbyge, Phys. Rev. B 72, 201101(R) (2005).

[3] J. M. Soler, E. Artacho, J. D. Gale, A. García, J. Junquera, P. Ordejón and D. SánchezPortal, J. Phys. Cond. Mat. 14, 2745-2779 (2002).

[4] http://departments.icmab.es/leem/siesta/

[5] A. R. Rocha, V. M. García-Suárez, S. W. Bailey, C. J. Lambert, J. Ferrer and S. Sanvito, Phys. Rev. B 73, 085414 (2006).

[6] SMEAGOL User's Guide comes along with the code at: http://www.smeagol.tcd.ie

[7] PhOnonS ITeratIVE VIBRATIONS. https://bitbucket.org/brandimarte/vibrations/ wiki/Home

[8] C. H. Lewenkopf and E. R. Mucciolo, J. Comput. Electron. 12, 203-231 (2013).

[9] A. R. Rocha, M. Rossi, A. J. R. da Silva and A. Fazzio, J. Phys. D: Appl. Phys. 43, 374002 (2010).

[10] BLAS - Basic Linear Algebra Subprograms. http://www.netlib.org/blas/

[11] LAPACK - Linear Algebra PACKage. http://www.netlib.org/lapack/

[12] M. Snir, S. Otto, S. Huss-Lederman, D. Walker and J. Dongarra, MPI: The Complete Reference, The MIT Press (1996).

[13] MAGMA - Matrix Algebra on GPU and Multicore Architectures. http://icl.cs.utk.edu/ magma/

[14] NVIDIA CUDA Parallel Programming and Computing Platform. http://www.nvidia.com/ object/cuda_home_new.html

[15] CUBLAS - NVIDIA CUDA Basic Linear Algebra Subroutines. https://developer. nvidia.com/cublas 


\section{Referências Bibliográficas}

[1] M. Büttiker. "Role Of Quantum Coherence In Series Resistors". Phys. Rev. B 33(5), 3020-3026 (1986).

[2] E. Artacho, D. Sánchez-Portal, P. Ordejón, A. García e J. M. Soler. "Linear-Scaling ab initio Calculations for Large and Complex Systems". Phys. Stat. Sol. (b) 215, 809-817 (1999).

[3] J. M. Soler, E. Artacho, J. D. Gale, A. García, J. Junquera, P. Ordejón e D. SánchezPortal. "The SIESTA Method for ab initio Order- $N$ Materials Simulation". J. Phys. Cond. Mat. 14, 2745-2779 (2002).

[4] A. R. Rocha, V. M. García-Suárez, S. W. Bailey, C. J. Lambert, J. Ferrer e S. Sanvito. "Spin and Molecular Electronics in Atomically Generated Orbital Landscapes". Phys. Rev. $B$ 73, 085414 (2006).

[5] P. W. Anderson. "Absence of Diffusion in Certain Random Lattices". Phys. Rev. 109, 1492 (1958).

[6] R. H. Smit, Y. Noat, C. Untiedt, N. D. Lang, M. C. van Hemert e J. M. van Ruitenbeek. "Measurement of the conductance of a hydrogen molecule". Nature 419, 906-909 (2002).

[7] N. Agraït, C. Untiedt, G. Rubio-Bollinger e S. Vieira. "Onset of Energy Dissipation in Ballistic Atomic Wires". Phys. Rev. Lett. 88(21), 216803 (2002).

[8] H. Haug e A.-P. Jauho. Quantum Kinetics in Transport and Optics of Semiconductors. Springer, (1994).

[9] A. R. Rocha. Theoretical and Computational Aspects of Electronic Transport at the Nanoscale. Tese de doutoramento, Trinity College Dublin, (2007).

[10] P. A. Lee e T. V. Ramakrishnan. "Disordered Electronic Systems". Rev. Mod. Phys. 57(2), 287-337 (1985). 
[11] A. H. Castro Neto, F. Guinea, N. M. R. Peres, K. S. Novoselov e A. K. Geim. "The Electronic Properties of Graphene". Rev. Mod. Phys. 81, 109-162 (2009).

[12] F. W. King. Hilbert Transforms. Cambridge University Press, volumes 1 e 2 (2009).

[13] G. M. Moore. "Cramming More Components onto Integrated Circuits". Electronics 38(8), 114-117 (1965).

[14] A. Aviram e M. A. Ratner. "Molecular Rectifiers". Chem. Phys. Lett. 29(2), 277-283 (1974).

[15] M. A. Reed, C. Zhou, C. J. Muller, T. P. Burgin e J. M. Tour. "Conductance of a Molecular Junction". Science 278, 252-254 (1997).

[16] C. Joachim, J. K. Gimzewski e A. Aviram. "Electronics Using Hybrid-Molecular and MonoMolecular Devices". Nature 408, 541-548 (2000).

[17] A. Nitzan e M. A. Ratner. "Electron Transport in Molecular Wire Junctions". Science 300, 1384-1389 (2003).

[18] N. J. Tao. "Electron Transport in Molecular Junctions". Nature Nanotech. 1, 173-181 (2006).

[19] N. Agraït, A. L. Yeyati e J. M. van Ruitenbeek. "Quantum Properties of Atomic-sized Conductors". Phys. Rep. 377, 81-279 (2003).

[20] T. Komeda. "Chemical Identification and Manipulation of Molecules by Vibrational Excitation via Inelastic Tunneling Process with Scanning Tunneling Microscopy". Prog. Surf. Sci. 78, 41-85 (2005).

[21] B. C. Stipe, M. A. Rezaei e W. Ho. "Single-Molecule Vibrational Spectroscopy and Microscopy". Science 280, 1732-1735 (1998).

[22] V. Singh, D. Joung, L. Zhai, S. Das, S. Khondaker e S. Seal. "Graphene Based Materials: Past, Present and Future". Prog. Mat. Sci. 56, 1178-1271 (2011).

[23] A. K. Geim e K. S. Novoselov. "The Rise of Graphene". Nature Materials 6, 183-191 (2007).

[24] K. S. Novoselov, A. K. Geim, S. V. Morozov, D. Jiang, Y. Zhang, S. V. Dubonos, I. V. Grigorieva e A. A. Firsov. "Electric Field Effect in Atomically Thin Carbon Films". Science 306, 666-669 (2004). 
[25] Y.-W. Son, M. L. Cohen e S. G. Louie. "Energy Gaps In Graphene Nanoribbons". Phys. Rev. Lett. 97, 216803 (2006).

[26] M. Y. Han, B. Özyilmaz, Y. Zhang e P. Kim. "Energy Band-Gap Engineering of Graphene Nanoribbons". Phys. Rev. Lett. 98, 206805 (2007).

[27] B. Partoens e F. M. Peeters. "From Graphene to Graphite: Electronic Structure Around the K Point". Phys. Rev. B 74, 075404 (2006).

[28] A. Narita, X. Feng, Y. Hernandez, S. A. Jensen, M. Bonn, H. Yang, I. A. Verzhbitskiy, C. Casiraghi, M. R. Hansen, A. H. R. Koch, G. Fytas, O. Ivasenko, B. Li, K. S. Mali, T. Balandina, S. Mahesh, S. De Feyter e K. Müllen. "Synthesis of Structurally WellDefined and Liquid-Phase-Processable Graphene Nanoribbons". Nature Chem. 6, 126-132 (2013).

[29] Y.-Z. Tan, B. Yang, K. Parvez, A. Narita, S. Osella, D. Beljonne, X. Feng e K. Müllen. "Atomically Precise Edge Chlorination of Nanographenes and its Application in Graphene Nanoribbons". Nature Comm. 4(2646) (2013).

[30] A. Hashimoto, K. Suenaga, A. Gloter, K. Urita e S. Iijima. "Direct Evidence for Atomic Defects in Graphene Layers". Nature 430, 870-873 (2004).

[31] A. Eckmann, A. Felten, A. Mishchenko, L. Britnell, R. Krupke, K. S. Novoselov e C. Casiraghi. "Probing the Nature of Defects in Graphene by Raman Spectroscopy". Nano Lett. 12, 3925-3930 (2012).

[32] Y. Zhu, S. Murali, W. Cai, X. Li, J. Suk, J. Potts e R. Ruoff. "Graphene and Graphene Oxide: Synthesis, Properties, and Applications". Adv. Mat. 22, 3906-3924 (2010).

[33] O. C. Compton e S. T. Nguyen. "Graphene Oxide, Highly Reduced Graphene Oxide, and Graphene: Versatile Building Blocks for Carbon-Based Materials". Small 6(6), 711-723 (2010).

[34] T. J. Echtermeyer, M. C. Lemme, M. Baus, B. N. Szafranek, A. K. Geim e H. Kurz. "Nonvolatile Switching in Graphene Field-Effect Devices". IEEE Electron Device Lett. 29(8), 952-954 (2008).

[35] B. Kramer e A. MacKinnon. "Localization: Theory and Experiment". Rep. Prog. Phys. 56, 1469-1564 (1993). 
[36] A. R. Rocha, M. Rossi, A. J. R. da Silva e A. Fazzio. "Realistic Calculations of CarbonBased Disordered Systems". J. Phys. D: Appl. Phys. 43, 374002 (2010).

[37] A. López-Bezanilla, F. Triozon e S. Roche. "Chemical Functionalization Effects on Armchair Graphene Nanoribbon Transport". Nano Lett. 9(7), 2537-2541 (2009).

[38] C. J. Cattena, R. A. Bustos-Marún e H. M. Pastawski. "Crucial Role of Decoherence for Electronic Transport in Molecular Wires: Polyaniline as a Case Study". Phys. Rev. B 82, 144201 (2010).

[39] Natalya A. Zimbovskaya. "Inelastic Electron Transport in Polymer Nanofibers". J. Chem. Phys. 129, 114705 (2008).

[40] S. Stankovich, R. D. Piner, S. T. Nguyen e R. S. Ruoff. "Synthesis and Exfoliation of Isocyanate-Treated Graphene Oxide Nanoplatelets". Carbon 44, 3342-3347 (2006).

[41] M. Acik, G. Lee, C. Mattevi, M. Chhowalla, K. Cho e Y. J. Chabal. "Unusual InfraredAbsorption Mechanism in Thermally Reduced Graphene Oxide". Nature Materials 9, 840845 (2010).

[42] W. Kohn e L. J. Sham. "Self-Consistent Equations Including Exchange and Correlations Effects". Phys. Rev. 140(4A), A1133-A1138 (1965).

[43] K. Stokbro, J. Taylor, M. Brandbyge e P. Ordejón. "TranSIESTA - A Spice for Molecular Electronics". Ann. N. Y. Acad. Sci. 1006, 212-216 (2003).

[44] T. Markussen, A.-P. Jauho e M. Brandbyge. "Electron and Phonon Transport in Silicon Nanowires: Atomistic Approach to Thermoelectric Properties". Phys. Rev. B 79, 035415 (2009).

[45] M. Engelund, M. Brandbyge e A.-P. Jauho. "Atomistic Theory for the Damping of Vibrational Modes in Monoatomic Gold Chains". Phys. Rev. B 80, 045427 (2009).

[46] M. Paulsson, T. Frederiksen e M. Brandbyge. "Inelastic Transport through Molecules: Comparing First-Principles Calculations to Experiments". Nano Lett. 6(2), 258-262 (2006).

[47] F. Haupt, T. Novotný e W. Belzig. "Phonon-Assisted Current Noise in Molecular Junctions". Phys. Rev. Lett. 103, 136601 (2009).

[48] J. Hihath, C. R. Arroyo, G. Rubio-Bollinger, N. Tao e N. Agraït. "Study of ElectronPhonon Interactions in a Single Molecule Covalently Connected to Two Electrodes". Nano Lett. 8(6), 1673-1678 (2008). 
[49] T. Yamamoto e K. Watanabe. "Nonequilibrium Green's Function Approach to Phonon Transport in Defective Carbon Nanotubes". Phys. Rev. Lett. 96, 255503 (2006).

[50] N. Mingo. "Anharmonic Phonon Flow through Molecular-Sized Junctions". Phys. Rev. B 74, $125402(2006)$.

[51] N. Mingo, D. A. Stewart, D. A. Broido e D. Srivastava. "Phonon Transmission through Defects in Carbon Nanotubes from First Principles". Phys. Rev. B 77, 033418 (2008).

[52] C. H. Lewenkopf e E. R. Mucciolo. "The Recursive Green's Function Method for Graphene". J. Comput. Electron. 12, 203-231 (2013).

[53] J. K. Viljas, J. C. Cuevas, F. Pauly e M. Häfner. "Electron-Vibration Interaction in Transport Through Atomic Gold Wires". Phys. Rev. B 72, 245415 (2005).

[54] M. Paulsson, T. Frederiksen e M. Brandbyge. "Modeling Inelastic Phonon Scattering in Atomic- and Molecular-Wire Junctions". Phys. Rev. B 72, 201101(R) (2005).

[55] M. Paulsson, T. Frederiksen e M. Brandbyge. "Phonon Scattering in Nanoscale Systems: Lowest Order Expansion of the Current and Power Expressions". J. Phys. Conference Series 35, 247-254 (2006).

[56] C. Cohen-Tannoudji, B. Diu e F. Laloë. Quantum Mechanics. Hermann e John Wiley \& Sons, $2^{\mathrm{a}}$ edição, (1997).

[57] L. V. Keldysh. "Diagram Technique for Nonequilibrium Processes". Sov. Phys. JETP-USSR 20(4), 1018 (1965).

[58] L. P. Kadanoff e G. Baym. Quantum Statistical Mechanics. W.A. Benjamin, (1962).

[59] A. L. Fetter e J. D. Walecka. Quantum Theory of Many-Particle Systems. Dover Publications, (2003).

[60] G. D. Mahan. Many Particle Physics. Physics of Solids and Liquids. Kluwer Academic / Plenum Publishers, $3^{\mathrm{a}}$ edição, (2000).

[61] R. D. Mattuck. A Guide to Feynman Diagrams in the Many-body Problem. Dover Publications, $2^{\mathrm{a}}$ edição, (1976).

[62] H. Bruus e K. Flensberg. Many-Body Quantum Theory in Condensed Matter Physics - An Introduction. Oxford University Press, (2010). 
[63] S. Datta. Quantum Phenomena. Modular Series on Solid State Devices - Volume VIII. Addison-Wesley, (1989).

[64] D. Langreth. Linear and Nonlinear Electron Transport in Solids. NATO Advanced Study Institutes Series: Series B, Physics. Plenum Press, (1976).

[65] M. Di Ventra. Electrical Transport in Nanoscale Systems. Cambridge University Press, (2008).

[66] J. H. Davies, S. Hershfield, P. Hyldgaard e J. W. Wilkins. "Current and Rate Equation for Resonant Tunneling". Phys. Rev. B 47(8), 4603-4618 (1993).

[67] S. Datta. Electronic Transport in Mesoscopic Systems. Cambridge University Press, (1995).

[68] P. Hohenberg e W. Kohn. "Inhomogeneous Electron Gas". Phys. Rev. 136(3B), B864-B871 (1964).

[69] W. Kohn. Nobel Lecture: Electronic Structure of Matter - Wave Functions and Density Functionals, 213-237, (1999). http://nobelprize.org/nobel_prizes/chemistry/ laureates/1998/kohn-lecture.html.

[70] M. Born e J. R. Oppenheimer. "On the Quantum Theory of Molecules". Ann. Physik 84, 457 (1927). Traduzido por S. M. Blinder com correções de B. Sutcliffe e W. Geppert, em 23 de janeiro de 2002 .

[71] R. M. Martin. Electronic Structure - Basic Theory and Pratical Methods. Cambridge University Press, Nova York, $1^{\mathrm{a}}$ edição, com correções (2008).

[72] J. Kohanoff. Electronic Structure Calculations for Solids and Molecules. Cambridge University Press, (2006).

[73] K. Capelle. "A Bird's-Eye View of Density-Functional Theory". Braz. J. Phys. 36(4A), 1318-1343 (2006).

[74] A. D. Becke. "Perspective: Fifty Years of Density-Functional Theory in Phemical Physics". J. Chem. Phys. 140, 18A301 (2014).

[75] J. P. Perdew, K. Burke e M. Ernzerhof. "Generalized Gradient Approximation Made Simple". Phys. Rev. Lett. 77(18), 3865-3868 (1996).

[76] D. R. Hamann, M. Schlüter e C. Chiang. "Norm-Conserving Pseudopotentials". Phys. Rev. Lett. 43(20), 1494-1497 (1979). 
[77] M. Head-Gordon e J. C. Tully. "Vibrational Relaxation on Metal Surfaces: Molecularorbital Theory and Application to $\mathrm{CO} / \mathrm{Cu}(100)$ ". J. Chem. Phys. 96(5), 3939-3949 (1992).

[78] T. Frederiksen, M. Paulsson, M. Brandbyge e A.-P. Jauho. "Inelastic Transport Theory From First Principles: Methodology and Application to Nanoscale Devices". Phys. Rev. B 75, 205413 (2007).

[79] Jmol: an open-source Java viewer for chemical structures in 3D. http://jmol. sourceforge.net.

[80] G. S. Ohm. The Galvanic Circuit Investigated Mathematically. D. Van Nostrand Company, (1827). Traduzido por W. Francis. Reimpressão de 1969. Disponível online: http://www. archive.org/details/galvaniccircuit00lockgoog.

[81] R. Landauer. "Conductance from Transmission: Common Sense Points". Phys. Scr. T42, 110-114 (1992).

[82] N. W. Ashcroft e N. D. Mermin. Solid State Physics. Brooks Cole, (1976).

[83] S. Datta. Quantum Transport: Atom to Transistor. Cambridge University Press, (2005).

[84] S. Datta. Ece 659 - quantum transport: Atom to transistor. Disciplina ECE 659 - Quantum Transport: Atom to Transistor, Jan (2009). http://nanohub.org/resources/6172.

[85] S. Datta. "Electrical Resistance - An Atomistic View". Nanotech. 15, S433-S451 (2004).

[86] Y. Meir e N. S. Wingreen. "Landauer Formula for the Current through an Interacting Electron Region". Phys. Rev. Lett. 68(16), 2512-2516 (1992).

[87] M. Snir, S. Otto, S. Huss-Lederman, D. Walker e J. Dongarra. MPI: The Complete Reference. The MIT Press, (1996). http://www.netlib.org/utk/papers/mpi-book/ mpi-book.html.

[88] M. Büttiker. "Four-Terminal Phase-Coherent Conductance". Phys. Rev. Lett. 57(14), 1761-1764 (1986).

[89] M. Büttiker, Y. Imry, R. Landauer e S. Pinhas. "Generalized Many-Channel Conductance Formula With Application to Small Rings". Phys. Rev. B 31(10), 6207-6215 (1985).

[90] N. S. Wingreen, K. W. Jacobsen e J. W. Wilkins. "Resonant Tunneling with ElectronPhonon Interaction: An Exactly Solvable Model". Phys. Rev. Lett. 61(12), 1396-1399 (1988). 
[91] V. J. Goldman e D. C. Tsui. "Evidence for LO-Phonon-Emission-Assisted Tunneling in Double-Barrier Heterostructures". Phys. Rev. B 36(14), 7635-7637 (1987).

[92] J. Maassen, F. Zahid e H. Guo. "Effects of Dephasing in Molecular Transport Junctions Using Atomistic First Principles". Phys. Rev. B 80, 125423 (2009).

[93] R. Golizadeh-Mojarad e S. Datta. "Nonequilibrium Green's Function Based Models for Dephasing in Quantum Transport". Phys. Rev. B 75, 081301(R) (2007).

[94] R. L. Burden e J. D. Faires. Numerical Analysis. Brooks/Cole, 10ª edição, (2010).

[95] W. H. Press, S. A. Teukolsky, W. T. Vetterling e B. P. Flannery. Numerical Recipes - The Art of Scientific Computing. Cambridge University Press, $3^{\mathrm{a}}$ edição, (2007).

[96] W. Lee, N. Jean e S. Sanvito. "Exploring the Limits of the Self-consistent Born Approximation for Inelastic Electronic Transport". Phys. Rev. B 79, 085120 (2009).

[97] P. Hyldgaard, S. Hershfield, J. H. Davies e J. W. Wilkins. "Resonant-tunneling With an Electron-Phonon Interaction". Ann. Phys. 236, 1-42 (1994).

[98] J. R. Schrieffer. Theory of Superconductivity. Advanced Books Classics. Westview Press, edição revisada (1999).

[99] L. R. F. Lima. Electronic Transport in Disordered Graphene Nanoribbons: a Recursive Green's Functions Approach. Tese de doutoramento, Universidade Federal do Rio de Janeiro, (2013).

[100] T. Ozaki. "Continued Fraction Representation of the Fermi-Dirac Function for Large-Scale Electronic Structure Calculations". Phys. Rev. B 75, 035123 (2007).

[101] M. Brandbyge, J.-L. Mozos, P. Ordejón, J. Taylor e K. Stokbro. "Density-Functional Method for Nonequilibrium Electron Transport". Phys. Rev. B 65, 165401 (2002).

[102] BLAS - Basic Linear Algebra Subprograms. http://www.netlib.org/blas/.

[103] LAPACK - Linear Algebra PACKage. http://www.netlib.org/lapack/

[104] T. Frederiksen. Inelastic Electron Transport in Nanosystems. Dissertação de Mestrado, Technical University of Denmark, (2004).

[105] T. Frederiksen, M. Brandbyge, N. Lorente e A.-P. Jauho. "Inelastic Scattering and Local Heating in Atomic Gold Wires". Phys. Rev. Lett. 93, 256601 (2004). 
[106] A. Jacobs. "The Pathologies of Big Data". ACM Data 7(6) (2009).

[107] K. S. Novoselov, D. Jiang, F. Schedin, T. J. Booth, V. V. Khotkevich, S. V. Morozov e A. K. Geim. "Two-dimensional Atomic Crystals". Proc. Nat. Acad. Sci. USA 102(30), 10451-10453 (2005).

[108] R. Zhang, I. Rungger, S. Sanvito e S. Hou. "Current-Induced Energy Barrier Suppression for Electromigration from First Principles". Phys. Rev. B 84, 085445 (2011). 